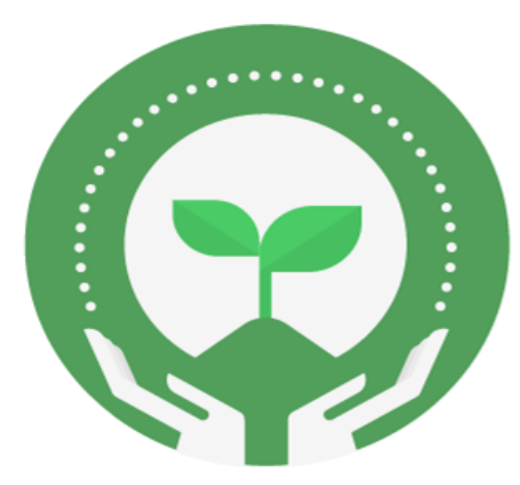

CONGRESO DE DESARROLLO SOSTENIBLE

\title{
DESAFÍOS EN LA CONSTRUCCIÓN DEL DESARROLLO SOSTENIBLE
}

Septiembre 28-29

2020

ENTIDADES ORGAMIZARORAS:
Q) UNVERELLDSANDTONAS (G) icontec ASHRAE

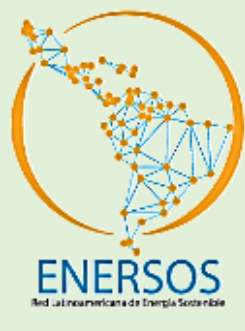




\section{CONGRESO DE DESARROLLD

\section{DESAFÍOS EN LA CONSTRUCCIÓN DEL DESARROLLO SOSTENIBLE}

\section{ORGANIZADORES}

RED LATINOAMERICANA DE ENERGÍA SOSTENIBLE-ENERSOS UNIVERSIDAD SANTO TOMAS UNIVERSIDAD EL BOSQUE ACAIRE ICONTEC

UNIVERSIDAD DEL VALLE CAPÍTULO ASHRAE COLOMBIA 


\section{PRESENTACIÓN}

Desde el año 1987 con la presentación de la Comisión Brundtland el concepto de Desarrollo Sostenible ha venido siendo impulsado a nivel global. Diferentes iniciativas se han venido gestando para lograr que los capitales humano, natural y económico puedan crecer a la par, como un trípode que sostiene a la naturaleza y la humanidad misma. Con un sentido de urgencia, desde el año 2015 la ONU ha promovido los Objetivos de Desarrollo Sostenible (ODS) que deberán mostrar sus resultados desde dicho año hasta el 2030. Todo esto, sumado a las muchas conferencias en diversos temas particulares dan un panorama sobre la importancia del tema para todos.

Por otro lado, el aprovechamiento de nuevas fuentes de energía, optimización de procesos y gestión de recursos son mecanismos que permiten asegurar un desarrollo sostenible disminuyendo el impacto que se genera sobre el ambiente. Los sectores académicos e industriales hacen parte de la fuerza impulsora para promover la investigación en sistemas de adaptación y diseño de alternativas que permitan suplir las necesidades actuales sin comprometer los recursos y procesos futuros. Por el contrario, ayudan a la identificación y potencialización de capitales que contribuyan con la explotación sostenible del capital natural, de tal manera que crezcan el capital social y económico.

Nada de esto sería posible sin el apoyo de la Ingeniería Industrial, la cual desde su perspectiva holística contribuye notablemente en el desarrollo y consolidación de procesos. Este PRIMER CONGRESO INTERNACIONAL DE DESARROLLO SOSTENIBLE, organizado por la Universidad Santo Tomás, Universidad El Bosque, 
Universidad del Valle, Acaire y el capítulo Ashrae-Colombia trabajando dentro de la Red de Energía Sostenible-ENERSOS ha sido el espacio propicio para identificar avances a nivel local e internacional. Dadas las circunstancias del COVID ha sido la primera versión virtual, lo que permitió reunir de forma sincrónica expertos internacionales, científicos, investigadores, estudiantes, docentes y numerosos profesionales del sector publico y privado interesados en el desarrollo sostenible.

Las jornadas del evento se realizaron de manera virtual, durante los días 28 y 29 de septiembre y contaron con la participación de destacados expertos investigadores, representantes de sectores gubernamentales e industriales provenientes de Argentina, Colombia, Costa rica, México, Estados Unidos, Francia y Brasil. La presencia de invitados nacionales e internacionales permite establecer un punto de referencia para la discusión en temas de investigación conjunta y fortalecimiento de redes de investigación a nivel interdisciplinar e interinstitucional.

\title{
COMITÉ ORGANIZADOR
}

\author{
M.Sc. Diana Catalina Londoño, Universidad El Bosque \\ M.Sc. Diana Fajardo, Universidad El Bosque \\ Ph.D. Carlos Herrera, Universidad del Valle-Ashrae \\ Ing. Fabio Clavijo, Acaire-Ashrae \\ M.Sc. Bolívar Monroy, Acaire-Ashrae \\ Ph.D. Dionisio Humberto Malagón, Universidad Santo Tomás- Ashrae
}

EDICIÓN Y DIAGRAMACIÓN: MSc. Ing. Carolina Hernández.

Publicado en Bogotá, Colombia

2020 
Analysis of thermal comfort in classrooms in equatorial region

Energy efficiency analysis in nonlinear triphasic systems using ieee 1459-2010

Tendencias en la generación de nuevos materiales a partir de fibras naturales y residuos poliméricos

Tendencias del aprovechamiento de la cascarilla de arroz con matrices poliméricas recicladas para la formulación de nuevos materiales y sus posibles aplicaciones

Propuesta diseño sistema de gestión ambiental enfocado en la disminución de co2 en el laboratorio de motores diesel, como contribución a la reducción de la huella de carbono en la universidad ecci

Manufactura de monturas oftálmicas personalizadas mediante fabricación aditiva con pet recuperado

Métodos de degradación de las mezclas polipropileno y poliestireno expandidos recuperados y polipropileno, polietileno de alta densidad recuperados, cascarilla de arroz y agente de acople silano

Anaerobic digestion of orange, tangerine and lemon peel

The hydrogen production as a base of green chemistry

Optimization of a route for collecting waste cooking oil in bogotá

ENERGÍAS ALTERNATIVAS

Energy use of biomass in colombia from a latin american compared perspective

Evaluar el comportamiento de una batería recargable con energía solar, diseñada a base de elementos alcalinos, alcalinotérreos, metales de transición y grafeno 
Evaluation of biodiesel production in a hydrodynamic cavitation reactor from waste cooking oil $\quad 146$

Construction of an electric low power generator using piezoelectric cells 157

EMPRENDIMIENTO SOSTENIBLE

Transformation of the biogas produced in filling the el carrasco sanitary in bucaramanga, colombia

AMBIENTES Y EDIFICACIONES SOSTENIBLES

Pedestrian mobility plan for social distancing in chapinero centro

Caracterización de las propiedades térmicas del bahareque embutido en tierra con esterilla de guadua

Caracterización térmica de envolventes arquitectónicas en esterilla laminada de guadua

Climate zones and sub-zones in the equatorial region

Análisis con algoritmo k-means de la fuerza laboral en el sector comercio colombiano 218

Modelo de gobernanza inteligente para la gestión de procesos en las instituciones de educación superior: caso universidad de santander

Modelo de metabolismo industrial aplicado a una startup de alimentación en canadá

The strategic direction of the organization and the management systems. Towards a research agenda

Tool for internal audits in local governments

Diseño de un prototipo para el manejo de datos en el museo de la universidad de la salle 
CONGRESO DE DESARROLLO

SOSTENIBLE

\section{EFICIENCIA Y AUDITORÍA}




\title{
ANALYSIS OF THERMAL COMFORT IN CLASSROOMS IN EQUATORIAL REGION
}

\section{Análisis de confort térmico en aulas de región ecuatorial}

\author{
Montoya, Olga1; Herrera, Carlos², San Juan, Gustavo ${ }^{3}$ \\ ${ }^{1}$ San Buenaventura University-Cali-Architecture, Art and Design Faculty-Architecture, Aesthetic Urbanism Research Group. \\ Colombia. \\ ${ }^{2}$ Valle University, Cali, Habitat and Sustainable Development Research Group. Colombia \\ ${ }^{3}$ La Plata National University. Architecture and Urbanism Faculty. Institute of Research and \\ Policies of Constructed Environment (IIPAC in Spanish). Laboratory of Models and Environmental Design (LAMbDA-K). \\ Argentina.
}

\begin{abstract}
The environmental quality of inhabitable spaces implies to enjoy comfort, and obtaining it by natural means is the objective of sustainability. The prediction of comfort in equatorial places is complex and rarely precise. A case of special attention is the naturally ventilated school classrooms, densely populated, very common in developing countries. Appropriate design models that give some certainty on the real behavior of the construction are expected. In order to do so, it is necessary to determine the validity of its predictions and correlate them with the appreciation by their users. This study compared the predictions of comfort under the analytical (PMV and PPD) and adaptive model, with Thermal Sensation Votes (TSV) of users analyzing 8 classrooms during two months in 2017, according to the methodology of User Perception Environmental Audit. This methodology has two parts: objective component (measurements of environmental conditions using specialized equipment), and subjective component (survey to 535 students). It was found that both models proposed by the international ASHRAE standard and Colombian Technical Standard NTC 5316, slightly fit the situation observed in the study and therefore, they do not allow taking proper design decisions to create comfortable spaces
\end{abstract}

Keywords: Thermal comfort, analytical model, adaptive model, naturally ventilated classrooms.

${ }^{1}$ Corresponding E-mail:olmontoy@usbcali.edu.co 


\section{INTRODUCTION}

The Colombian Technical Standard (NTC in Spanish) 5316: Thermal Environmental Conditions of Buildings for People [1], identical translation (IDT) of Standard ASHRAE 55 [2], regulates the comfort conditions in constructions. The NTC defines, for Colombia, a summer comfort rank regarding to operative temperature (To) of $22.5^{\circ} \mathrm{C}<$ Tor $<26^{\circ} \mathrm{C}$, relative humidity (RH) $60 \%$ and metabolic activity met $\leq 1.2$, where maximum $10 \%$ of population is dissatisfied.

Standard ASHRAE 55 uses the thermal sensation scale: -3 cold, -2 cool, -1 slightly cool, 0 neutral, +1 slightly warm, +2 warm, +3 hot, and establishes two methods to determine comfort: analytical and adaptive. This last one is based on the average exterior temperature and it only applies to naturally ventilated conditions. On the other hand, the analytical model is based on the predicted mean vote, Predicted Mean Vote (PMV) and on the expected dissatisfaction percentage, Predicted Percentage of Dissatisfied (PPD), developed by Fanger [3], which allows to predict the average value of thermal sensation of occupants and the percentage of them that finds it satisfactorily comfortable. For this standard, the comfort rank is defined between $-0.5<\mathrm{PMV}<+0.5$; with a PPD $<10 \%$.

Tablada et al [4] states that studies carried out on the last three decades in naturally ventilated schools in warm climates, demonstrated that the thermal sensation in comfort is warmer than the information indicated in the standards [5], and in those environments the students can report $90 \%$ acceptability at a temperature of $30^{\circ} \mathrm{C}$ [6] and $78 \%$ at $30.7^{\circ} \mathrm{C}$ [7]. Although in the international panorama there are several publications on the comfort in ventilated classrooms [8], at a local level, particularly for tropical or equatorial conditions these publications are recent [9], [10] and rare. In addition, it is necessary to research if predictions with the standard methods correspond with the reality, and to what extent, in order to eventually propose adjustments or improvements.

\section{MATERIALS AND METHODS.}

The methodology used in the study is the Environmental Audit of User Perception, carried out in 2017 in 8 school classrooms simultaneously in the city of Cali. Corresponding to climate zone 1A: very hot humid $\left(5000<\right.$ Cooling Degree Days $\left.10^{\circ} \leq 6000\right)$ [11], for two months. This methodology has previously been validated in studies in classrooms in different countries of Latin America [12]. The audit has two components: the objective component based on the records of environmental parameters, through continuous measurements during 55 days every 10 minutes using specific equipment according to the analysis variable, and precise measurements inside the classrooms when filling out the opinion survey. Table 1 , shows the specifications of the equipment used 
Table 1 Specifications of equipment used in the objective audit. Source: Authors

\begin{tabular}{|c|c|c|c|c|c|}
\hline $\begin{array}{c}\text { Type of } \\
\text { measurement }\end{array}$ & Equipment & $\begin{array}{c}\text { Environmental } \\
\text { Parameter }\end{array}$ & $\begin{array}{c}\text { Rank of } \\
\text { measurement }\end{array}$ & Accuracy & Resolution \\
\hline $\begin{array}{l}\text { Exterior } \\
\text { Continuous } \\
\text { measurements }\end{array}$ & $\begin{array}{l}\text { Data logger, model HOBO } \\
\text { U23 Pro V2 }\end{array}$ & \multirow{2}{*}{$\begin{array}{l}\text { Temperature } \\
\left({ }^{\circ} \mathrm{C}\right) \text { and relative } \\
\text { humidity }(\%)\end{array}$} & $\begin{array}{l}0^{\circ} \text { to } 50^{\circ} \mathrm{C} \\
10 \% \text { to } 90 \%\end{array}$ & $\begin{array}{l} \pm 0.21^{\circ} \mathrm{C} \\
\pm 2.5 \%\end{array}$ & $\begin{array}{l}0.02^{\circ} \mathrm{C} \text { to } \\
25^{\circ} \mathrm{C}, \quad \text { and } \\
0.05 \%\end{array}$ \\
\hline $\begin{array}{l}\text { Interior } \\
\text { Continuous } \\
\text { measurements }\end{array}$ & $\begin{array}{l}\text { Data logger, model HOBO } \\
\text { U12-012 }\end{array}$ & & $\begin{array}{l}0^{\circ} \text { to } 50^{\circ} \mathrm{C} \\
10 \% \text { to } 90 \%\end{array}$ & $\begin{array}{l} \pm 0.35^{\circ} \mathrm{C} \\
\pm 2.5 \%\end{array}$ & $\begin{array}{l}0.03^{\circ} \mathrm{C} \text { to } \\
25^{\circ} \mathrm{C}, \quad \text { and } \\
0.05 \%\end{array}$ \\
\hline \multirow{2}{*}{$\begin{array}{l}\text { Interior precise } \\
\text { measurement }\end{array}$} & $\begin{array}{l}\text { Anemometer model LM-8000 } \\
\text { Lutron }\end{array}$ & Airspeed (m/s) & 0.4 to $30.0 \mathrm{~m} / \mathrm{s}$ & & $0.1 \mathrm{~m} / \mathrm{s}$ \\
\hline & $\begin{array}{l}\text { Digital laser thermometer } \\
\text { model NUBBE }\end{array}$ & $\begin{array}{l}\text { Surface } \\
\text { temperature } \\
\left({ }^{\circ} \mathrm{C}\right)\end{array}$ & -50 to $380{ }^{\circ} \mathrm{C}$ & $\pm 2 \%$ & \\
\hline
\end{tabular}

The subjective component is based on the study of the occupant perception, based on the implementation of participative workshops, where 535 students between 8 and 16 years old were involved. It was carried out through an opinion survey based on the evaluation and diagnosis tool [11], governed by the Ethics committee of the San Buenventura University, Cali. The survey inquired the students about their thermal perception: i) Thermal sensation [2] which is represented by Thermal Sensation Votes - TSV and ii) the strategies to adapt to thermal conditions such as opening and closing windows, switching places in the classroom, iii) wrapping up more or less, perception of inner ventilation and the most ventilated place of the classroom.

For the subsequent comfort analysis, the following values were calculated:

Mean TSV: Mean thermal sensation votes

Mean radiant temperature (MRT): Through factors of form, emissivity and temperatures of each surface [14].

Operative Temperature $(\mathrm{To})=\mathrm{A}^{*} \mathrm{To}+(1-\mathrm{A}) \mathrm{MRT}$ Where: To $=$ Operative Temperature; Ta = Air Temperature $/$ MRT =Mean Radiant Temperature and A (value based on air speed) $=0.5(<0.2 \mathrm{~m} / \mathrm{s})$; $=0.6(0.2$ to $0.6 \mathrm{~m} / \mathrm{s}) ;=0.7(0.6$ to $1.0 \mathrm{~m} / \mathrm{s})$, [2]

PMV, PPD and Acceptability under the adaptive model: data recorded by CBE Thermal Comfort Tool [15], validated by ASHRAE for the analysis of thermal comfort [16].

The audited classrooms correspond to four public schools in Cali based on the NTC 4595 [17], which governs the design conditions for comfortable classrooms from year 2000. Two classrooms 
in each school were selected, located in the ground floor and in the top floor (exposed roof) in order to identify possible differences. The general characteristics of surrounding are block of ceramic and concrete brick, with high percentage of permeability to the exterior through windows, blinds and openworks ( Figure 1).
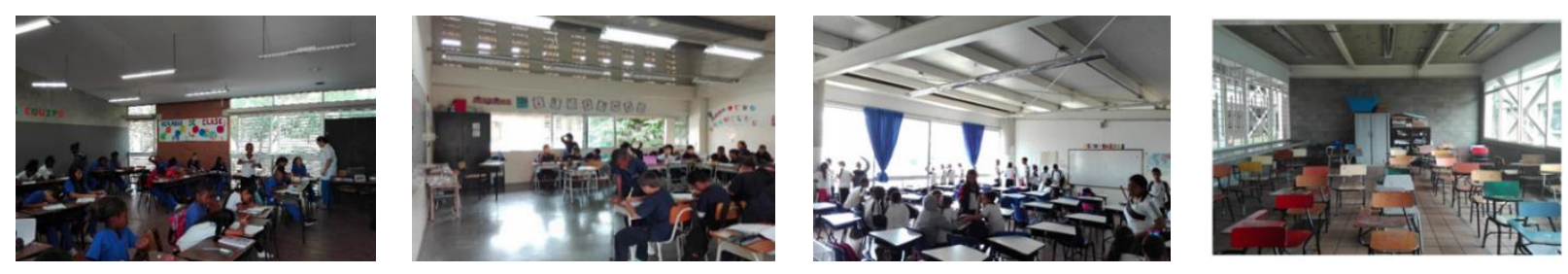

Figure 1 General characteristics of the studied classrooms. Source: Authors

\section{RESULTS AND ANALYSIS RESULTS}

During the measurement period, the average exterior room temperature (Ta) recorded was $25.3^{\circ} \mathrm{C}$, with Ta maximum $32.3^{\circ} \mathrm{C}$ and minimum $23.1^{\circ} \mathrm{C}$, while the average Relative Humidity (RH) was $58 \%$, with $\mathrm{RH}$ maximum $61 \%$ and minimum $39.4 \%$, in agreement with the semi-arid warm climate defined by the Institute of Hydrology, Meteorology and Environmental Studies IDEAM, for the city of Cali.

The 256 TSV of those classrooms located in the ground floor, represent $47.8 \%$ of the total, and 279 votes from the classrooms located in the top floor amount $52 \%$. Table 2 and 3 show the information regarding to the distribution of votes, which showed similar results on the three central options (comfort) 122 and 141, in the ground floor and the top floor respectively, also the votes in discomfort by cold 84 and 88 , and discomfort by heat 50 in the ground floors as well as the classrooms located in the top floors

Table 2 Data of fieldwork (TSV, Mean TSV, MRT and To) and PMV ground floor classrooms. Source: Authors

\begin{tabular}{|c|c|c|c|c|c|c|c|c|c|c|c|c|c|}
\hline \multicolumn{14}{|c|}{ GROUND FLOOR CLASSROOMS } \\
\hline \multirow{2}{*}{\multicolumn{2}{|c|}{ School/classroom }} & \multirow{2}{*}{$\begin{array}{c}\text { MRT } \\
\left({ }^{\circ} \mathrm{C}\right)\end{array}$} & \multirow{2}{*}{$\begin{array}{c}\text { To } \\
\left({ }^{\circ} \mathrm{C}\right)\end{array}$} & \multirow{2}{*}{$\begin{array}{c}\text { PMV } \\
\text { (analytical } \\
\text { and } \\
\text { theoretical) }\end{array}$} & \multirow[b]{2}{*}{ Mean TSV (perception) } & \multicolumn{7}{|c|}{ TSV (student perception) } & \multirow[b]{2}{*}{ Total } \\
\hline & & & & & & 3 & 2 & 1 & 0 & 1 & $?$ & 3 & \\
\hline 1 & \multirow{4}{*}{ MONTH 1} & 26.1 & 26.4 & 0.92 & 0.17 & 0 & 9 & 0 & 8 & 3 & 10 & 0 & 30 \\
\hline 2 & & 24.3 & 25.8 & 0.54 & -0.03 & 1 & 8 & 7 & 8 & 6 & 5 & 3 & 38 \\
\hline 3 & & 29.5 & 29.6 & 1.59 & 1.14 & 0 & 4 & 3 & 0 & 13 & 7 & 8 & 35 \\
\hline 4 & & 22.4 & 22.9 & -0.12 & -2.33 & 16 & 11 & 1 & 1 & 1 & 0 & 0 & 30 \\
\hline
\end{tabular}




\begin{tabular}{|c|c|c|c|c|c|c|c|c|c|c|c|c|c|}
\hline 1 & \multirow{4}{*}{ MONTH 2} & 25.8 & 26.6 & 0.77 & -0.42 & 1 & 5 & 4 & 7 & 7 & 0 & 0 & 24 \\
\hline 2 & & 24.5 & 26.7 & 0.77 & -0.20 & 1 & 10 & 3 & 5 & 5 & 3 & 3 & 30 \\
\hline 3 & & 26.2 & 26.8 & 0.65 & -0.55 & 1 & 11 & 4 & 5 & 4 & 3 & 1 & 29 \\
\hline 4 & & 23.4 & 24.3 & 0 & 0.35 & 0 & 6 & 4 & 9 & 14 & 5 & 2 & 40 \\
\hline & \multirow{2}{*}{\multicolumn{5}{|c|}{ Total Classrooms Floor 1}} & 20 & 64 & 26 & 43 & 53 & 33 & 17 & \multirow{2}{*}{256} \\
\hline & & & & & & \multicolumn{2}{|c|}{84} & \multicolumn{3}{|c|}{122} & \multicolumn{2}{|c|}{50} & \\
\hline
\end{tabular}

Where: green color represents the votes in comfort, pink represents slightly warm and orange represents warm votes. Clear blue represents slightly fresh votes and dark blue represents the fresh votes.

Table 3 Data of fieldwork (TSV, Mean TSV, MRT and To) and PMV top floor classrooms. Source: Authors

\begin{tabular}{|c|c|c|c|c|c|c|c|c|c|c|c|c|c|}
\hline \multicolumn{14}{|c|}{ TOP FLOOR CLASSROOMS } \\
\hline & & \multirow{2}{*}{$\begin{array}{c}\text { MRT } \\
\left({ }^{\circ} \mathrm{C}\right)\end{array}$} & \multirow{2}{*}{$\begin{array}{c}\text { To } \\
\left({ }^{\circ} \mathrm{C}\right)\end{array}$} & \multirow{2}{*}{$\begin{array}{c}\text { PMV } \\
\text { (analytical } \\
\text { and } \\
\text { theoretical) }\end{array}$} & \multirow[b]{2}{*}{ Mean TSV (perception) } & \multicolumn{7}{|c|}{ TSV (student perception) } & \multirow[b]{2}{*}{ Total } \\
\hline Sche & 1/classroom & & & & & & & & & & & & \\
\hline 1 & \multirow{4}{*}{ MONTH 1} & 28.3 & 28.4 & 1.24 & 1.29 & 0 & 3 & 4 & 3 & 5 & 8 & 11 & 34 \\
\hline 2 & & 26.9 & 27.0 & 0.75 & -0.25 & 1 & 11 & 3 & 8 & 9 & 2 & 2 & 36 \\
\hline 3 & & 30.2 & 30.4 & 1.81 & 0.76 & 0 & 5 & 2 & 4 & 16 & 8 & 3 & 38 \\
\hline 4 & & 23.2 & 23.2 & 0.15 & -1.81 & 12 & 14 & 2 & 7 & 1 & 0 & 0 & 36 \\
\hline 1 & \multirow{4}{*}{ MONTH 2} & 25.5 & 26.1 & 0.6 & -0.71 & 1 & 12 & 5 & 9 & 6 & 1 & 0 & 34 \\
\hline 2 & & 24.3 & 25.6 & 0.31 & 0.07 & 0 & 9 & 5 & 12 & 8 & 6 & 2 & 42 \\
\hline 3 & & 27.8 & 28.4 & 1.18 & -0.53 & 1 & 12 & 1 & 10 & 6 & 1 & 1 & 32 \\
\hline 4 & & 26.1 & 26.5 & 0.65 & 0.00 & 1 & 6 & 2 & 6 & 7 & 5 & 0 & 27 \\
\hline \multirow{2}{*}{\multicolumn{6}{|c|}{ Total Classrooms Floor 1}} & 16 & 72 & 24 & 59 & 58 & 31 & 19 & \multirow{2}{*}{279} \\
\hline & & & & & & \multicolumn{2}{|c|}{88} & \multicolumn{3}{|c|}{141} & \multicolumn{2}{|c|}{50} & \\
\hline
\end{tabular}

Where: green color represents the votes in comfort, pink represents slightly warm and orange represents warm votes.

Clear blue represents slightly fresh votes and dark blue represents the fresh votes.

Results of PMV (theoretical model), predict a warmer thermal sensation than the one reported in the study. It can be observed in Tables 2 and 3 and in Figure 2 under the PMV, that most of the classrooms would have sensations between Slightly fresh to Warm (from -0.5 to 2.0), while the TSV of the students spread in the thermal scale with votes between Cool to Slightly warm (-2.5 to 1.5) (Figure 3). Regarding the Neutral Temperature (Tn), considered in thermal comfort, resulting from the intersection of the linear regression line between both variables under the theoretical model (PMV and To) it is $23.5^{\circ} \mathrm{C}$, while the Tn result of the surveys (TSV and To) is $27^{\circ} \mathrm{C}$, implying a difference of $3.5^{\circ} \mathrm{C}$. This value agrees with the Tn reported by 11 year old children in classrooms in Costa Rica [18], this situation reinforces the findings of other studies in schools where a warmer thermal sensation was found respecting to the reports on the standards (Figure 2 and 3 ). 


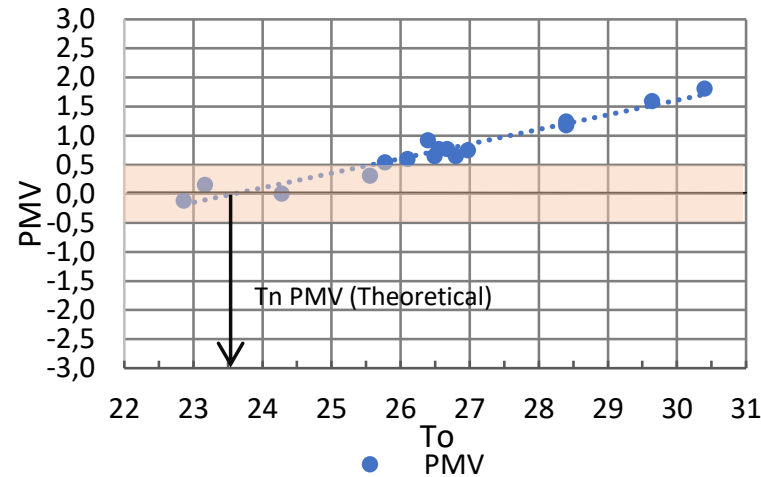

Figure 2 PMV (theoretical model) based on To.

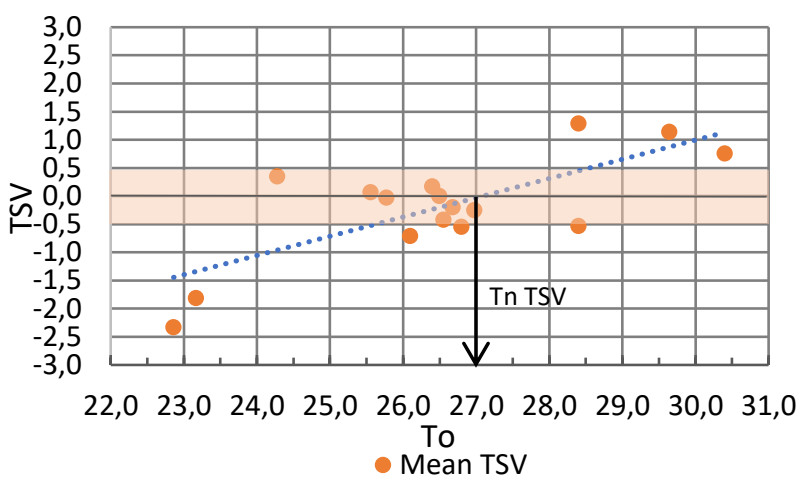

Figure 3 TSV (student perception) based on To

Source: Authors

The analysis under the PMV is more sensible to the environmental conditions of the classrooms in the different levels where the classrooms are located, than to personal conditions the students may have such as gender. The Tn among the classrooms located on the ground floor $\left(\mathrm{Tn}=26.6^{\circ} \mathrm{C}\right)$ is slightly inferior to the Tn reported in the top floor $\left(\mathrm{Tn}=27.4^{\circ} \mathrm{C}\right)$ as it is shown in Figure 4, while the Tn differentiated according to the gender is $27^{\circ} \mathrm{C}$ (Figure 5) not displaying differences, opposite situation to the findings of studies where the Tn of girls and boys between 11 to 17 years displayed a difference of $0.9^{\circ} \mathrm{C}[19]$.

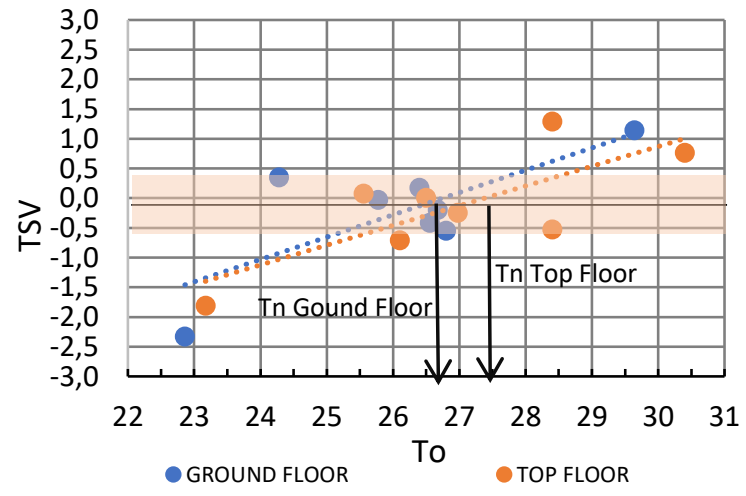

Figure 4 TSV based on the location of the classroom

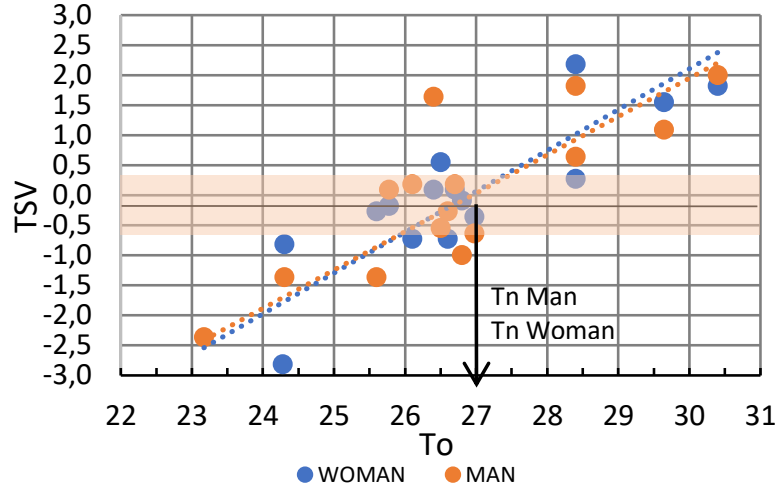

Figure 5 TSV based on the gender

Source: Authors

On the other hand, for the adaptive model it will be assumed that acceptability is present in the percentage of those votes in three central categories $(-1,0,1)$, that is to say, $47.7 \%$ and $50.5 \%$ in ground floor and top floor classrooms respectively (4 Table), which is different from the $80 \%$ acceptability predicted by the theoretical model in an Operative Temperature rank between $22.5^{\circ} \mathrm{C}$ to $30.5^{\circ} \mathrm{C}$ as it appears in Figure 6 . The results of this study, contradict others made in mild 
climates where high percentages of acceptability with high temperatures were reported, as the $90 \%$ acceptability to $30^{\circ} \mathrm{C}$ in Taiwan [7] and $78 \%$ to $30.7^{\circ} \mathrm{C}$ in Tezpur, India [6].

Table $4 \%$ PPD in the classrooms. Source: Authors

\begin{tabular}{|c|c|c|c|c|c|}
\hline \multicolumn{2}{|c|}{$\begin{array}{l}\text { Ground } \\
\text { floor } \\
\text { classrooms }\end{array}$} & $\begin{array}{c}\text { Total } \\
\text { votes in } \\
\text { comfort } \\
(-1,0,+1)\end{array}$ & $(\%)$ & $\begin{array}{c}\text { Total } \\
\text { votes in } \\
\text { discomfort }\end{array}$ & $\begin{array}{l}\text { PPD } \\
(\%)\end{array}$ \\
\hline \multirow{4}{*}{3} & \multirow{4}{*}{$\begin{array}{c}\text { MONTH } \\
1\end{array}$} & 11 & 36.7 & 19 & 63.3 \\
\hline & & 21 & 55.3 & 17 & 44.7 \\
\hline & & 16 & 45.7 & 19 & 54.3 \\
\hline & & 3 & 10.0 & 27 & 90.0 \\
\hline \multirow[b]{4}{*}{4} & \multirow{4}{*}{$\begin{array}{c}\text { MONTH } \\
2\end{array}$} & 18 & 75.0 & 6 & 25.0 \\
\hline & & 13 & 43.3 & 17 & 56.7 \\
\hline & & 13 & 44.8 & 16 & 55.2 \\
\hline & & 27 & 67.5 & 13 & 32.5 \\
\hline \multicolumn{2}{|c|}{ Total (256) } & 122 & 47.7 & 134 & 52.7 \\
\hline
\end{tabular}

\begin{tabular}{|c|c|c|c|c|c|}
\hline \multirow{2}{*}{\multicolumn{2}{|c|}{$\begin{array}{l}\text { Top floor } \\
\text { classrooms }\end{array}$}} & $\begin{array}{c}\text { Total } \\
\text { votes in } \\
\text { comfort }\end{array}$ & \multirow[t]{2}{*}{$\begin{array}{c}\text { Comfort } \\
(\%)\end{array}$} & \multirow[t]{2}{*}{$\begin{array}{c}\text { Total } \\
\text { votes in } \\
\text { discomfort }\end{array}$} & \multirow[t]{2}{*}{$\begin{array}{l}\text { PPD } \\
\text { (\%) }\end{array}$} \\
\hline & & $(-1,0,+1)$ & & & \\
\hline \multirow{4}{*}{\multicolumn{2}{|c|}{$\begin{array}{c}\text { MONTH } \\
1\end{array}$}} & 12 & 35.3 & 22 & 64.7 \\
\hline & & 20 & 55.6 & 16 & 44.4 \\
\hline & & 22 & 57.9 & 16 & 42.1 \\
\hline 4 & & 10 & 27.8 & 26 & 72.2 \\
\hline 1 & \multirow{4}{*}{$\begin{array}{c}\text { MONTH } \\
2\end{array}$} & 20 & 58.8 & 14 & 41.2 \\
\hline 2 & & 25 & 59.5 & 17 & 40.5 \\
\hline 3 & & 17 & 53.1 & 15 & 46.9 \\
\hline 4 & & 15 & 55.6 & 12 & 44.4 \\
\hline \multicolumn{2}{|c|}{ Total (279) } & 141 & 50.5 & 138 & 49.5 \\
\hline
\end{tabular}

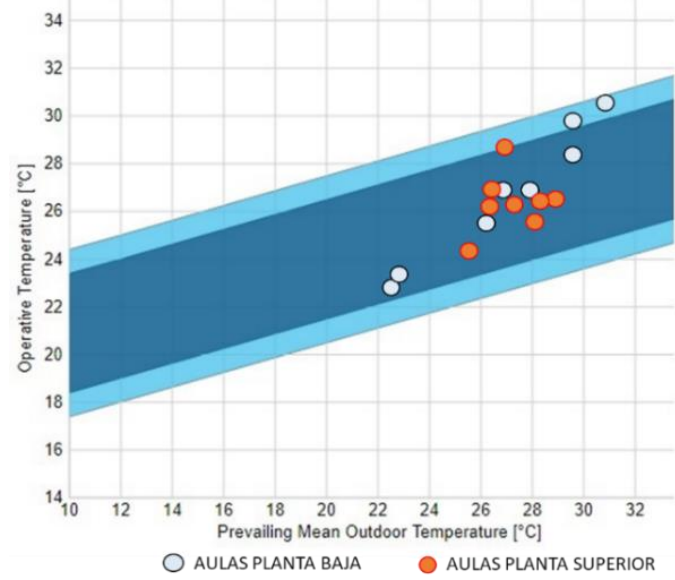

Figure 6 Comfort rank according to the adaptive model. Source: CBE Comfort Tools

\section{CONCLUSIONS}

Both theoretical models for the thermal comfort analysis were inaccurate when evaluating the comfort. In naturally ventilated classrooms such as the ones studied here, students showed a Tn up to $3.5^{\circ} \mathrm{C}$ higher than the Tn predicted by the analytical model, which in addition, foretold a predicted average vote of discomfort by heat, which does not agree with the records from the 
study. On the other hand, the students showed an acceptability close to $50 \%$ in the rank of $22.5^{\circ} \mathrm{C}$ (2) To॰ $30.5^{\circ} \mathrm{C}$, which is far below the $80 \%$ acceptability predicted by the adaptive model in the same conditions.

The analysis methodology used from the application of an environmental audit -considering the objective and subjective components- confronting the results with the standards and theoretical methods in order to determine the thermal comfort, provides excellent information, to adjust the standard, of implemented models, as well as for evaluating building responses, in this case for warm, equatorial climates

\section{REFERENCES}

[1] ICONTEC, “Norma Técnica Colombiana NTC 5316," Thermal environmental conditions for human occupancy. p. 34, 2004.

[2] ANSI/ASHRAE Standard 55-, "Thermal Environmental Conditions for Human Occupancy." 2017.

[3] P. O. Fanger, Thermal comfort. Analysis and applications in environmental engineering. 1972.

[4] A. Tablada, A. M. De La Peña, and F. De Troyer, "Thermal comfort of naturally ventilated buildings in warm-humid climates: field survey," in 22 Conference on Passive and Low Energy Architecture, 2005, pp. 1-6.

[5] H. H. Liang, T. P. Lin, and R. L. Hwang, "Linking occupants' thermal perception and building thermal performance in naturally ventilated school buildings," Appl. Energy, vol. 94, pp. 355-363, 2012.

[6] P. Baruah, M. Kumar Singh, and S. Mahapatra, "Thermal Comfort in Naturally Ventilated Classrooms," PLEA 2014 - 30th Int. Plea Conf., no. December, pp. 1-8, 2014.

[7] R.-L. Hwang, T.-P. Lin, C.-P. Chen, and N.-J. Kuo, "Investigating the adaptive model of thermal comfort for naturally ventilated school buildings in Taiwan," Int. J. Biometeorol., vol. 53, no. 2, pp. 189-200, 2009.

[8] A. K. Mishra and M. Ramgopal, "Thermal comfort in undergraduate laboratories - A field study in Kharagpur, India," Build. Environ., vol. 71, pp. 223-232, Jan. 2014.

[9] V. Iglesias, C. A. Herrera, and M. E. Rosillo, Confort ambiental en escuelas públicas de Cali. Universidad del Valle, 2016.

[10] C. M. Zapata et al., Comodidad ambiental en aulas escolares. Incidencia en la salud docente y en el rendimiento cognitivo de los estudiantes en colegios públicos de Bogotá, Medellín y Cali, 1st ed. Editorial Bonaventuriana; Ediciones Unisalle; Universidad Nacional de la Plata, 2018.

[11] ASHRAE, Proposed Addendum b to Standard 169, ClimaticData for Building Design Standards. 2012, p. 77.

[12] G. San Juan, S. Hoses, and I. Martini, “Aprendizaje en las escuelas del siglo XXI: Nota 5: Auditoría ambiental y condiciones de confort en establecimientos escolares," 2014.

[13] G. A. San Juan, S. Hoses, D. Rojas, and J. M. Moreno, "Integración de la opinión de los usuarios en la evaluación ambiental de aulas escolares," Av. en energías Renov. y medio Ambient., vol. 3, no. 1, pp. 169172, 1999.

[14] F. J. Forbes, "Diseño de una aplicación computacional para el calculo de factor de vision y TMR en espacios arquitecónicos," Universidad Del Valle, 2017.

[15] T. Hoyt, S. Schiavon, A. Piccioli, T. Cheung, D. Moon, and K. Steinfeld, "CBE Thermal Comfort Tool," Center for the Built Environment, University of California Berkeley, 2017. [Online]. Available: http://comfort.cbe.berkeley.edu/.

[16] ANSIASHRAE, Standard 55: Thermal environmetal conditions for human occupancy. ASHRAE, 2013, p. 52.

[17] Instituto Colombiano de Normas Técnicas y Certificación (ICONTEC), “Norma Técnica Colombiana 4595: Ingeniería Civil y Arquitectura Planeamiento y Diseño de Instalaciones y Ambientes Escolares," Bogotá, 2000.

[18] J. A. Porras-Salazar, D. P. Wyon, B. Piderit-Moreno, S. Contreras-Espinoza, and P. Wargocki, "Reducing 
classroom temperature in a tropical climate improved the thermal comfort and the performance of elementary school pupils," Indoor Air, 2018.

[19] K. E. Al-Rashidi, D. L. Loveday, and N. K. Al-Mutawa,
"Investigating the applicability of different thermal comfort models in Kuwait classrooms operated in hybrid air-conditioning mode," in Sustainability in energy and buildings, Springer, 2009, pp. 347-355. 


\title{
ENERGY EFFICIENCY ANALYSIS IN NONLINEAR TRIPHASIC SYSTEMS USING IEEE 1459-2010
}

\section{Análisis de eficiencia energética en sistemas trifásicos no lineales usando IEEE 1459-2010}

\author{
Forero, Edwin 1 \\ Universidad Santo Tomás
}

\section{Abstract}

The energy efficiency measurement according to the IEEE 1459-2010 standard applied to static power converters in three-phase systems, constitutes the axis of this research. The methodology to measure the efficiency developed in the Matlab Simulink program is presented according to the parameters of the IEEE 1459-2010 applied to a three-phase bridge-type inverter. Then, the method is experimentally demonstrated, applying it to a variable speed drive. Finally, the efficiency measurement is compared in a traditional way with the one proposed thanks to the IEEE 1459-2010 standard. The result of the proposed methodology with respect to the traditional one showed that with the application of the IEEE1459-2010 standard, the efficiency value can be more accurately specified, since the standard includes inefficient energy associated with the presence of harmonics in the signals of current and voltage that are generated by the static converters in the process.

Keywords: Measurement, three-phase converter, simulation, energy efficiency.

\section{Resumen}

La medición de eficiencia energética según la norma IEEE 1459-2010 aplicada a convertidores estáticos de potencia en sistemas trifásicos, constituye el eje de esta investigación. Se presenta la metodología para medir la eficiencia desarrollada en el programa Simulink de Matlab según los parámetros de la IEEE 1459-2010 aplicada a un inversor trifásico tipo puente. Acto seguido, se demuestra experimentalmente el método, aplicándolo a un variador de velocidad. Para finalizar se compara la medición de eficiencia de forma tradicional con la propuesta gracias a la norma IEEE 1459-2010. El resultado de la metodología propuesta respecto a la tradicional demostró que

${ }^{1}$ Correo electrónico de contacto: edwinforero@usantotomas.edu.co 
con la aplicación de la norma IEEE1459-2010 se puede precisar con más exactitud el valor de la eficiencia, por cuanto el estándar incluye energía ineficiente asociada a la presencia de armónicos en las señales de corriente y voltaje que son generadas por los convertidores estáticos en el proceso.

Palabras clave: Medición, convertidor trifásico, simulación, eficiencia energética.

\section{INTRODUCCIÓN}

La mayoría de los equipos y dispositivos modernos desarrollados por la creatividad del ser humano requieren energía para funcionar, sin embargo, a pesar de que ellos generan bienestar, no todos los sistemas creados funcionan de una manera energéticamente eficiente. Existe infinidad de artefactos que necesitan ser alimentados por dispositivos de potencia, que son controlados electrónicamente como los convertidores estáticos, éstos fueron fabricados con la intención de trabajar con el mínimo consumo de energía eléctrica, no obstante con la evolución de los cálculos matemáticos y la implementación de metodologías para su comprobación se ha demostrado que la estimación de energía no es la señalada por cuanto los componentes intrínsecos en el sistema eléctrico no contienen esta nueva formulación matemática [1].

La implementación de la metodología se patentiza en varias entidades de orden internacional que validan sus principios, para nuestro análisis tomamos como referencia la establecida en el Instituto de Ingeniería Eléctrica y Electrónica denominada IEEE 1459-2010 [2] la cual plantea y explica de manera coherente la forma de calcular todas las potencias discriminándolas de manera adecuada, tanto para sistemas monofásicos como trifásicos: balanceados, lineales, no lineales o desequilibrados. Se escogió esta norma como referencia por cuanto en su estándar se proporcionan definiciones para medir y diseñar instrumentación para la cuantificación de energía y potencia, utiliza el diseño del control en tiempo real de compensadores dinámicos y el diagnóstico de instrumentación para identificar un tipo específico de evento molesto o armónico, además de presentar la estimación de la eficiencia de convertidores estáticos de potencia usando programas de simulación y pruebas de campo que permiten corroborar la metodología planteada. Como resultado de la aplicación de la metodología se logró establecer con la medición de las potencias en la entrada y salida del convertidor en una red trifásica que existe una mayor pérdida de energía. 


\section{Estándar IEEE 1459-2010 en trifásicos}

Esta norma extiende las definiciones de potencia aparente propuesta por Buchholz [3], y la rebautiza como potencia aparente efectiva; asimismo, divide los sistemas trifásicos en dos, tres hilos y cuatro hilos con neutro. La figura 1, muestra un sistema trifásico con una fuente convencional y con diferentes cargas conectadas en un punto común PPC [4]. Este puede ser balanceado, no balanceado, lineal y no lineal, sin caídas de tensión o pérdidas.

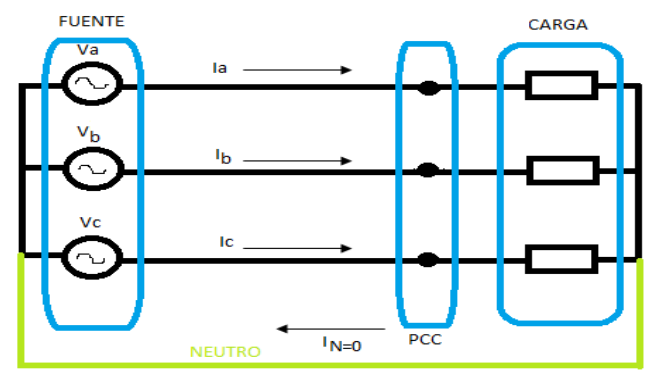

Figura 1 Resultados de la simulación. Fuente: Elaboración propia del autor

El voltaje equivalente del circuito de 4 hilos se obtiene según la IEEE 1459-2010 [2], suponiendo que los componentes activos de la carga consisten en tres resistencias $R_{y}$ conectadas en estrella en los 4 hilos y disipando una potencia activa $P_{y}$ [5]. La carga activa restante consiste en tres resistencias $R_{\Delta}$ conectadas en delta que disipan la potencia $P_{\Delta}$. Entonces la potencia equivalente entre el sistema actual y el equivalente será:

$$
\begin{gathered}
P_{y}=\frac{V_{a}^{2}+V_{b}^{2}+V_{c}^{2}}{R_{y}}=\frac{3 V_{e}^{2}}{R_{y}} \\
P_{\Delta}=\frac{V_{a b}^{2}+V_{b c}^{2}+V_{c a}^{2}}{R_{\Delta}}=\frac{\left(3 \sqrt{3} V_{e}\right)^{2}}{R_{y}}
\end{gathered}
$$

Donde $V_{e}$, es al valor efectivo de voltaje entre línea y neutro. Y la relación $\xi$ entre $P_{y}$ y $P_{\Delta}$ es:

$$
\xi=\frac{P_{\Delta}}{P_{y}}=\frac{3 R_{y}}{R_{\Delta}}
$$

El estándar recomienda $\xi=1.0$ si no se conoce su valor. Luego despejando obtenemos que $V_{e}$ es igual a:

$$
V_{e}=\sqrt{\frac{3\left(V_{a}^{2}+V_{b}^{2}+V_{c}^{2}\right)+\left(V_{a}^{2}+V_{b}^{2}+V_{c}^{2}\right)}{18}}
$$


Para calcular la corriente efectiva $I_{e}$, se basa en las pérdidas de de potencia de la red en sistema de distribución. Para sistemas de cuatro líneas se expresan así:

$$
r\left(I_{a}^{2}+I_{b}^{2}+I_{c}^{2}+\rho I_{n}^{2}\right)=3 r I_{e}^{2}
$$

Donde r es la resistencia de línea, $I_{n}$ es el valor rms de la corriente de neutro y la relación $\rho=\frac{r_{n}}{r}$ , con $r_{n}$ siendo la resistencia del cable de neutro. Si $\rho$ es desconocido el estándar recomienda 1.0. Con esto se obtiene que:

$$
I_{e}=\sqrt{\frac{1}{3}\left(I_{a}^{2}+I_{b}^{2}+I_{C}^{2}+I_{n}^{2}\right)}
$$

La potencia efectiva aparente se relaciona con esta ecuación.

$$
S_{e}=V_{e} I_{e}
$$

$S_{e}$, muestra los fenómenos físicos, refleja las pérdidas en el conductor de neutro, el fenómeno del desequilibrio, además de ser la máxima potencia transmitida por un sistema de distribución trifásico a una carga perfectamente equilibrada, alimentada con una tensión efectiva igual a Ve, manteniendo las mismas pérdidas en las líneas. [6]

La IEEE1459 -2010 [2] define y específica los sistemas trifásicos lineales y desequilibrados y, trifásicos lineales equilibrados, como se observa en las figuras 2 y 3 : 


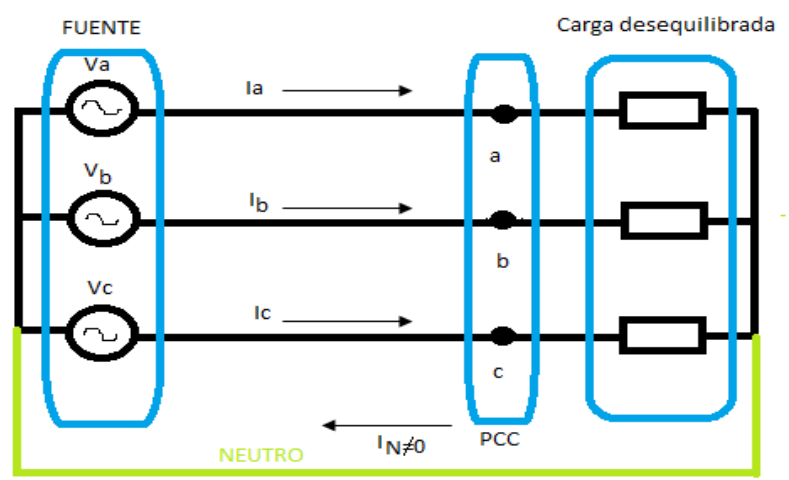

$$
\begin{gathered}
\text { Voltajes Asimétricos } \\
v_{a}=\sqrt{2} V \sin \left(w t+\alpha_{a}\right) \\
v_{b}=\sqrt{2} V \sin \left(w t+\alpha_{b}-120\right) \\
v_{c}=\sqrt{2} V \sin \left(w t+\alpha_{b}+120\right) \\
\text { Corrientes Desequilibradas } \\
i_{a}=\sqrt{2} V \sin \left(w t+\beta_{a}\right) \\
i_{b}=\sqrt{2} V \sin \left(w t+\beta_{b}-120\right) \\
i_{c}=\sqrt{2} V \sin \left(w t+\beta_{c}+120\right)
\end{gathered}
$$

Figura 2 Sistema trifásico lineal y desequilibrado. Fuente: Elaboración propia

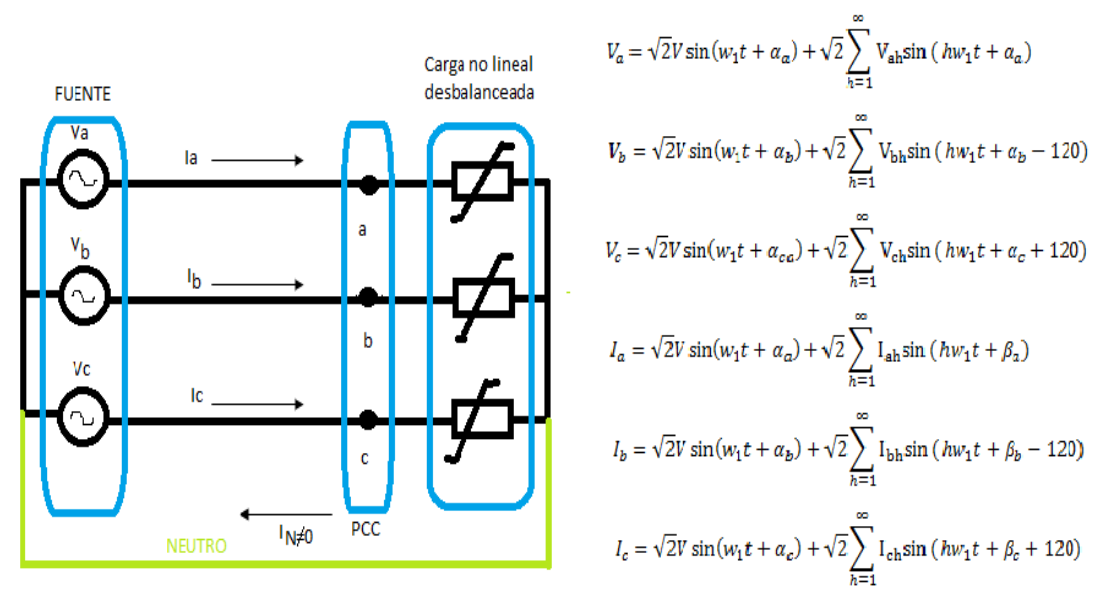

Figura 3 Sistema trifásico con cargas no lineales o con armónicos, fuentes no simétricas y corrientes desequilibradas. Fuente: Elaboración propia del autor

\section{MATERIALES Y MÉTODOS.}

La metodología propuesta consistió en medir la potencia trifásica de un sistema convertidor estático (inversor), tanto en la entrada como en la salida, usando los parámetros de la norma IEEE 1459 -2010 [2], para determinar la eficiencia energética de este tipo de sistemas a través de la plataforma Simulink.

Para estimar la eficiencia del convertidor trifásico se excluyó la parte reactiva [7], considerando solo la potencia activa fundamental de salida y la utilización de la potencia efectiva aparente de entrada según la norma IEEE 1459-2010 [2], la cual incluye de manera intrínseca las potencias debido a los armónicos. 
Su estimación se basa en la ecuación 8:

$$
\text { Eficiencia }(\%)=\frac{P_{1}}{S_{e}} \times 100
$$

Para desarrollar la metodología se utilizó un inversor trifásico de 6 pulsos controlado por PWM como se evidencia en la figura 4:

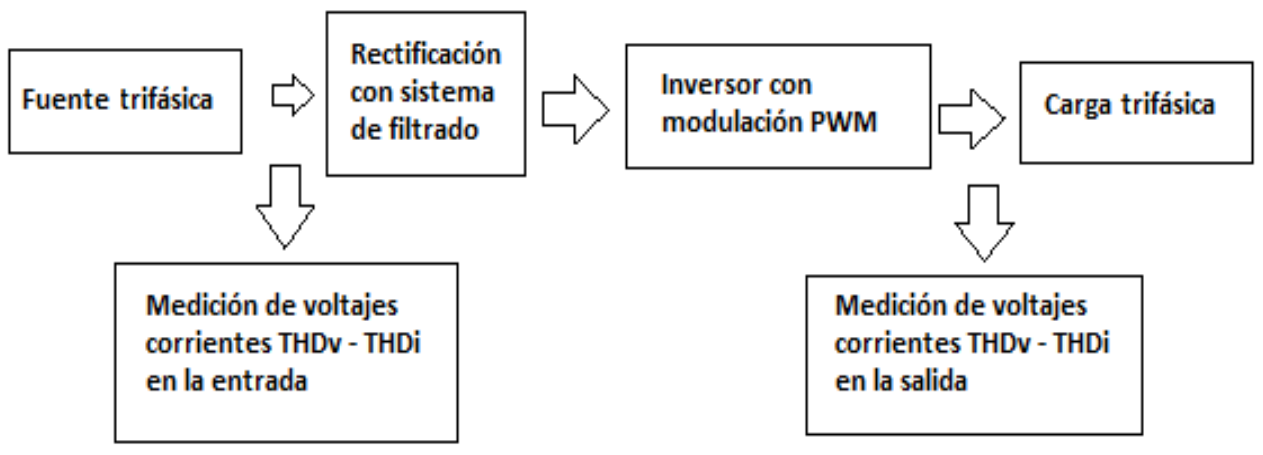

Figura 4 Esquema del circuito de simulación. Fuente: Elaboración propia

Para su diseño se tuvo en cuenta la siguiente relación denominada índice de modulación de frecuencia [8]:

$$
M_{f}=\frac{f_{p}}{f_{m}}
$$

Donde fm es la frecuencia de la señal modulante que en nuestro caso es una onda seno a $60 \mathrm{HZ}$ y fp de $4 \mathrm{khz}$ es una onda triangular a $4 \mathrm{khz}$, esto debido a que el variador usado en las pruebas experimentales funciona a esta frecuencia.

El PWM se trabajó en modo asíncrono a alta frecuencia para minimizar los subarmónicos producidos y evitar sonidos sensibles que detecta el oído humano ( 2 a $3 \mathrm{Khz}$ ). Al aumentar la frecuencia de switcheo se producen efectos de sobretensiones y sobrecorrientes que afectan al motor; sin embargo, en variadores modernos éstos son controlables hasta frecuencias de $16 \mathrm{Khz}$. [9]

\section{Simulación}

En el programa Simulink se implementó el inversor trifásico y se midieron los parámetros de corrientes , voltajes, distorsión armónica en la entrada y la salida como lo muestra la figura 5: 


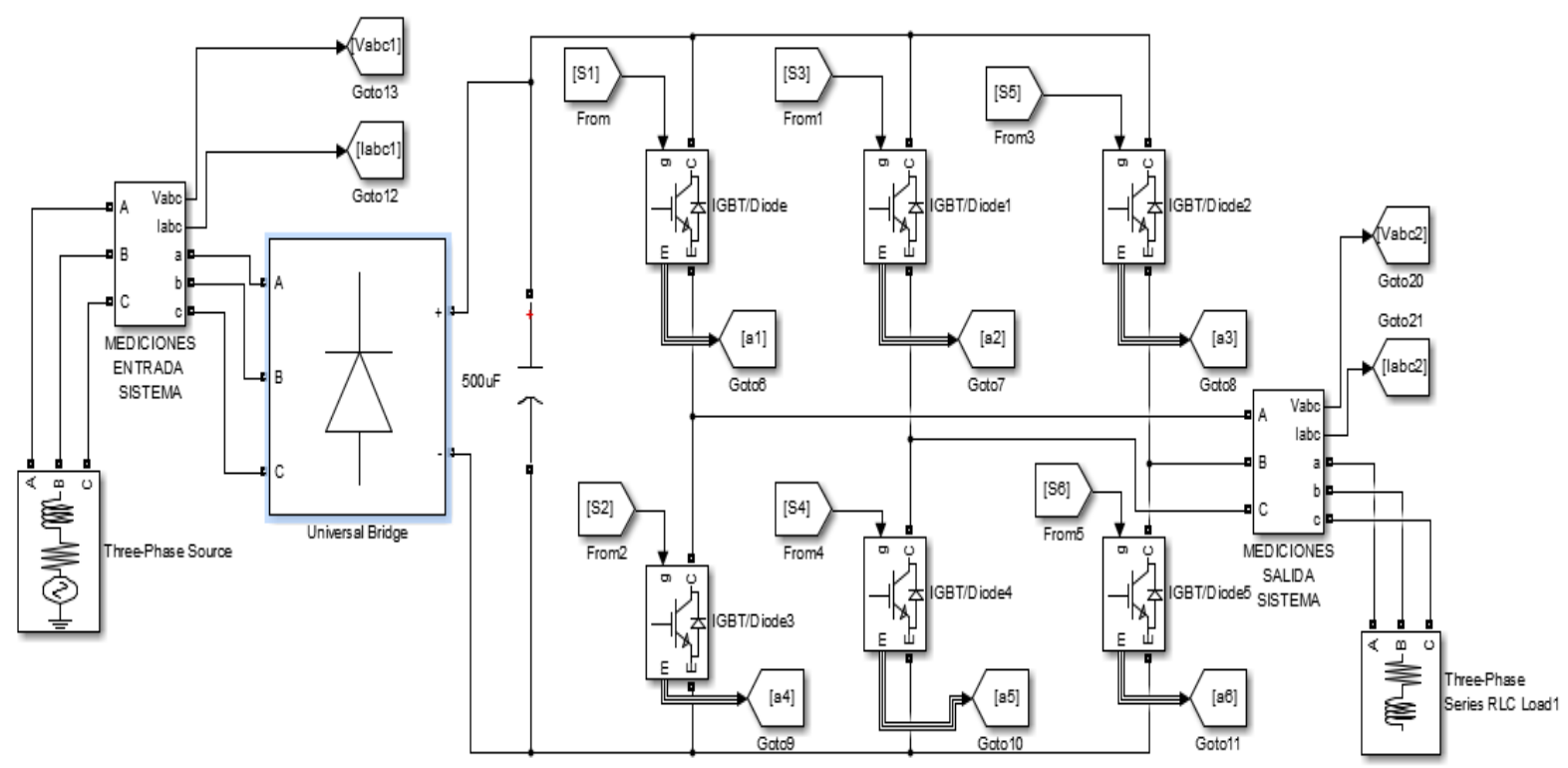

Figura 5 Resultados de la simulación. Fuente: Elaboración propia a partir de Simulink

De otra parte, en la presentación de los trabajos originales del IEEE Working Group, se descompone la potencia aparente efectiva en dos términos denominados voltaje efectivo y corriente efectiva [10], que, para sistemas trifásicos de tres hilos con cargas no lineales, que es el caso de estudio, se calculan tanto para la entrada como para la salida con sus es el caso de estudio, se calculan tanto para la entrada como para la salida con sus respectivos valores fundamentales. La norma también tiene implícito el cálculo de una corriente efectiva equivalente, por supuesto con su fundamental [11], tanto para la entrada como para la salida. En la figura 6 se muestra cómo se calcularon los valores de corriente y voltaje efectivos: 

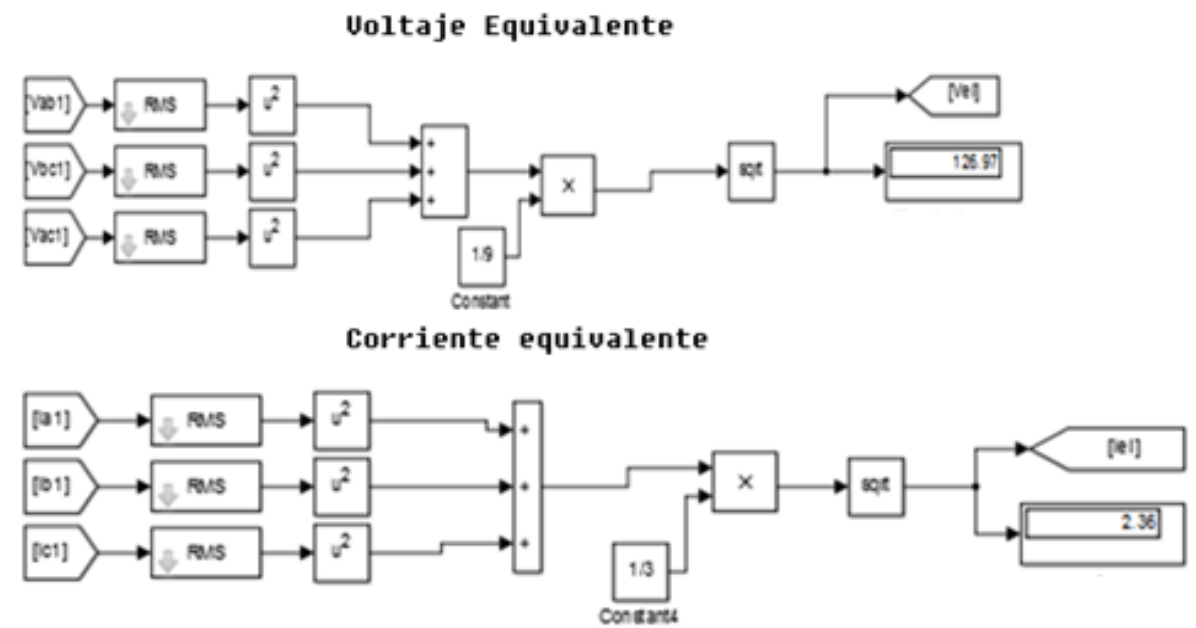

Figura 6 Cálculo voltaje y corriente equivalente. Fuente: Elaboración propia a partir de Simulink

Así mismo, para ver la influencia de los armónicos tanto de voltaje y corriente la IEEE 1459-2010 [2] tiene en cuenta el voltaje Veh y la corriente Ieh, ambos de tipo armónico y la tasa de distorsión armónica, tanto de la corriente THDie como del voltaje THDve del circuito equivalente [12]. En la figura 7 podemos observar la forma en que se hallan estos elementos:

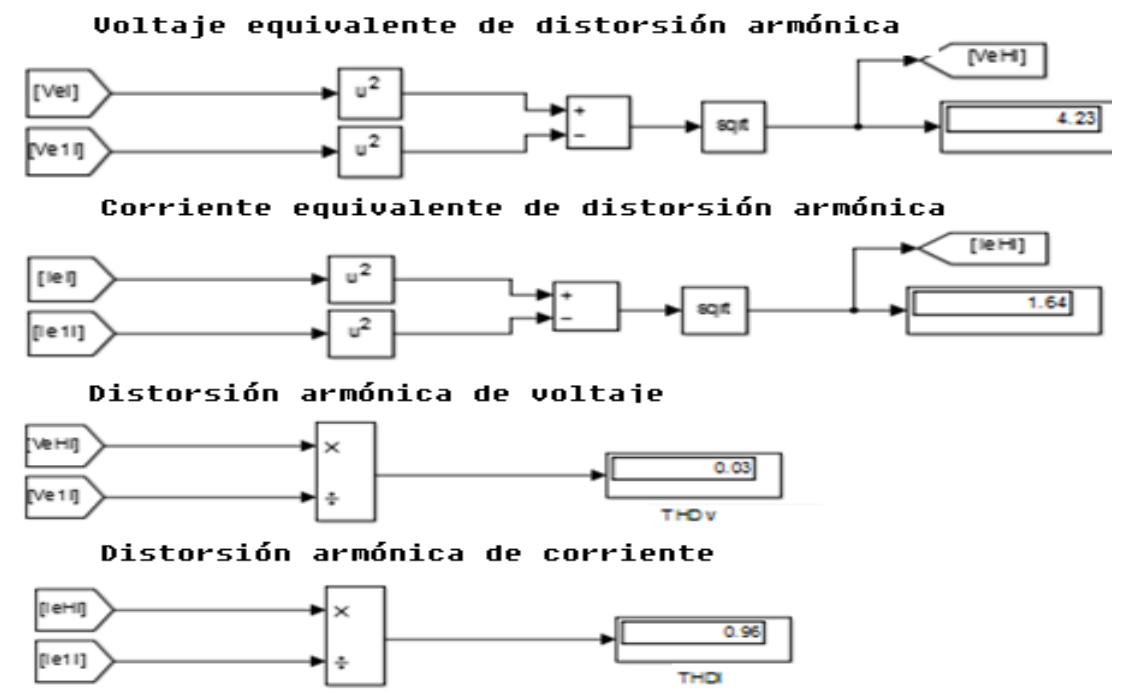

Figura 7 Distorsiones armónicas de voltaje y corriente. Fuente: Elaboración propia a partir de Simulink

Con todos los datos de voltaje y corriente del sistema equivalente propuesto por la norma IEEE 1459-2010 [2], se halló la potencia aparente total Se y fundamental Se1 equivalente tanto para la entrada como para la salida del inversor. De igual forma, se calculó la potencia activa total. Ver 
figuras 8 y 9 :
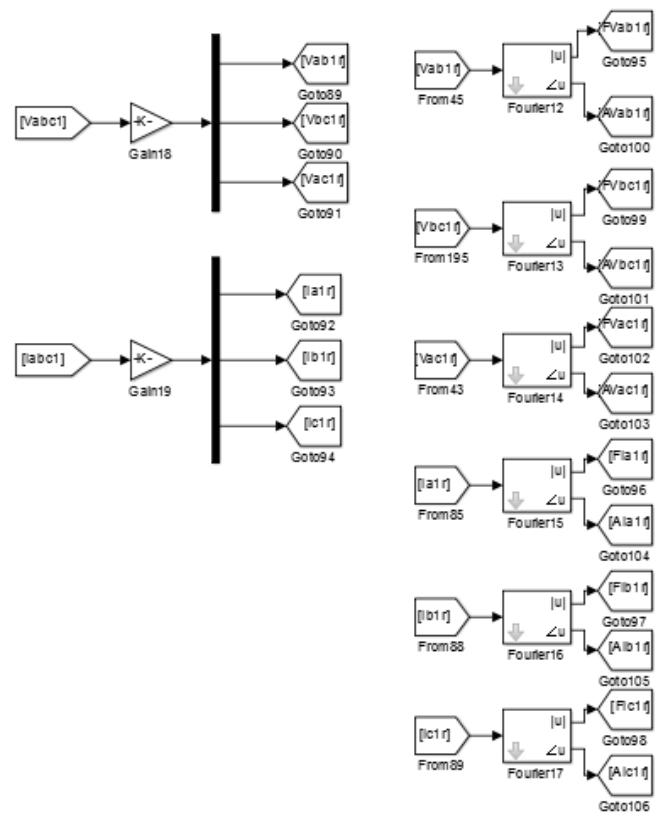

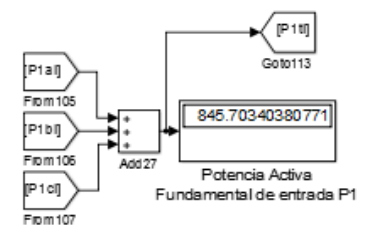

Figura 8 Potencia activa y reactiva fundamental. Fuente: Elaboración propia a partir de Simulink

\section{Potencia aparente}

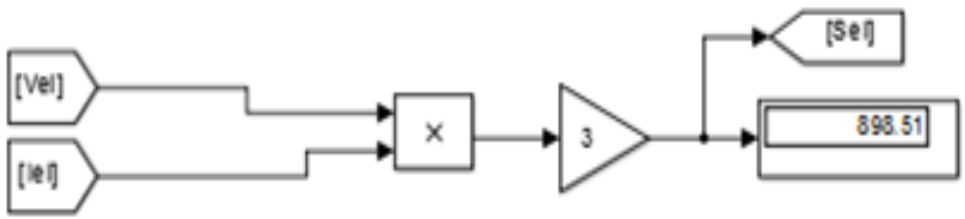

Potencia aparente fundamental

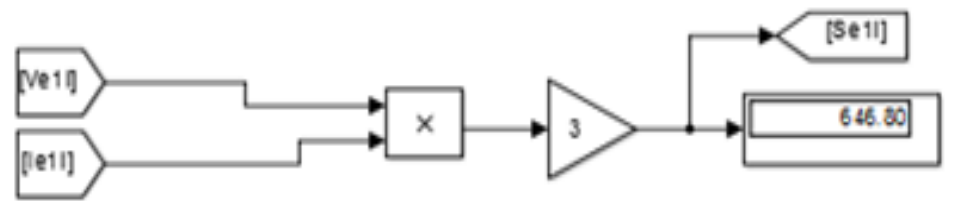

Figura 9 Potencias aparentes Se y fundamental Se1. Fuente: Elaboración propia a partir de Simulink

Otros factores influyentes en el análisis de potencia aparente no fundamental o en su propio cálculo son la potencia de distorsión de corriente DeI, la potencia de distorsión de voltaje $\mathrm{DeV}$ y la potencia aparente armónica SeH [13], como se observa en la figura 10: 
Potencia de distorsión de corriente

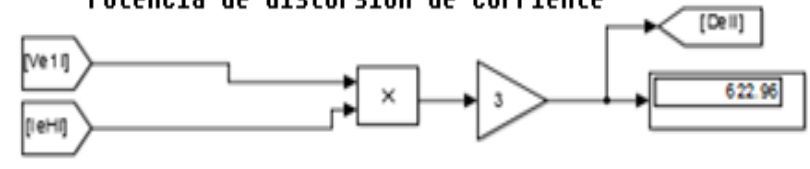

Potencia de distorsión de voltaje

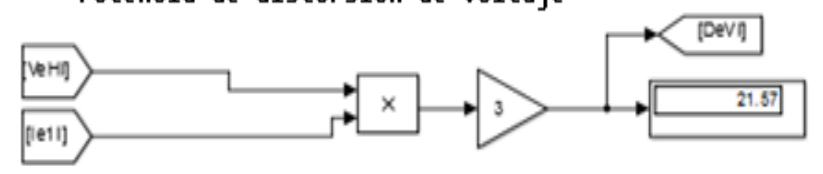

Potencia aparente armónica

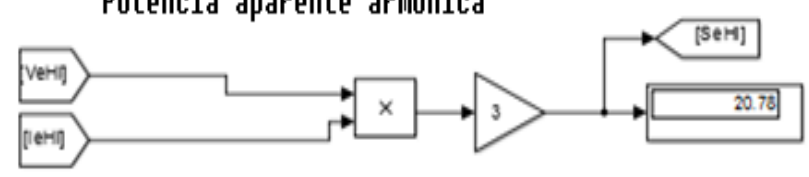

Figura 10 Potencia DeI, DeV y SeH Fuente: Elaboración propia a partir de Simulink

\section{Comprobación metodología con sistema experimental}

La metodología implementada en la plataforma Simulink se corroboró con la realización de un experimento utilizando un motor trifásico en configuración en delta y conectado a un variador de velocidad de referencia Altivar ATV312HU15M3 de Schenider Electric, siguiendo el esquema de la figura 11.

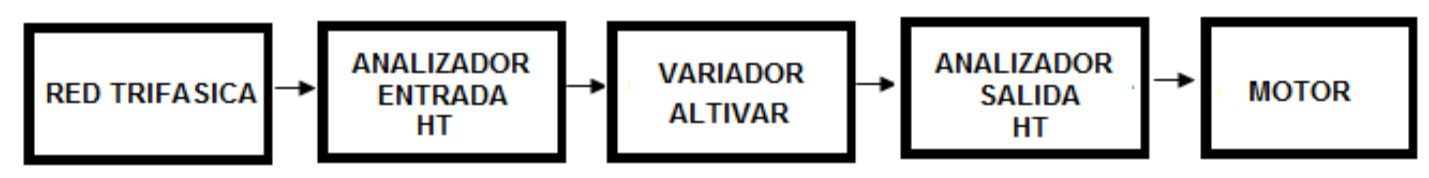

Figura 11 Esquema del experimento. Fuente: Elaboración propia a partir de Simulink

El montaje realizado se pude ver en la figura 12: 


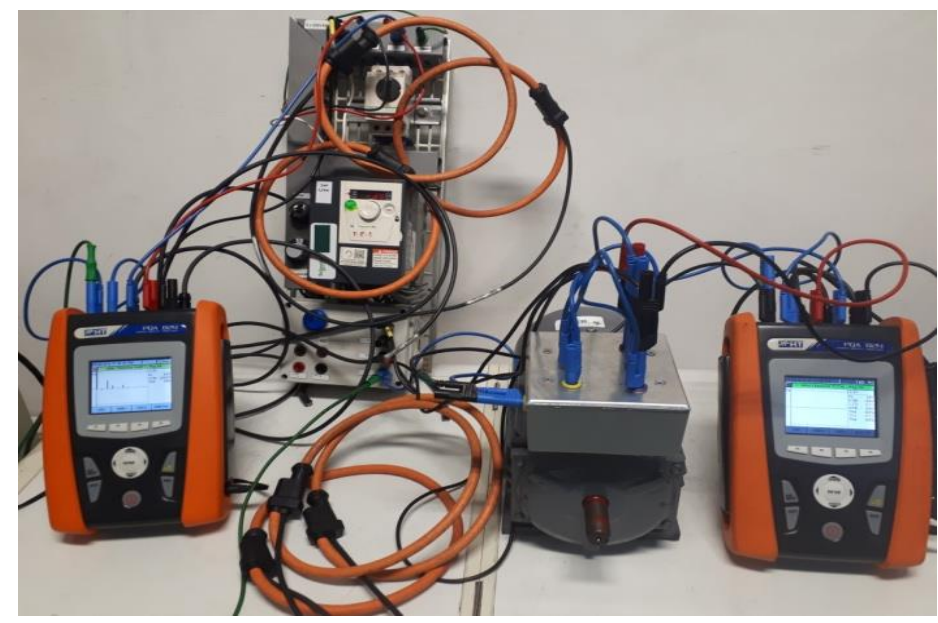

Figura 12 Montaje experimental. Fuente: Elaboración propia del autor

Para medir la eficiencia según la metodología implementada en este trabajo, se adquirieron los siguientes datos desde los analizadores de redes marca HT con número de serie PQA 824, a saber: voltaje fase a fase, corrientes de línea, componentes fundamentales de voltaje, componentes fundamentales de corriente y potencia activa, y, el programa TOPVIEW para la adquisición de los datos al computador.

Se configuraron los analizadores marca HT con número de serie PQA 824, como lo indica la figura 13:

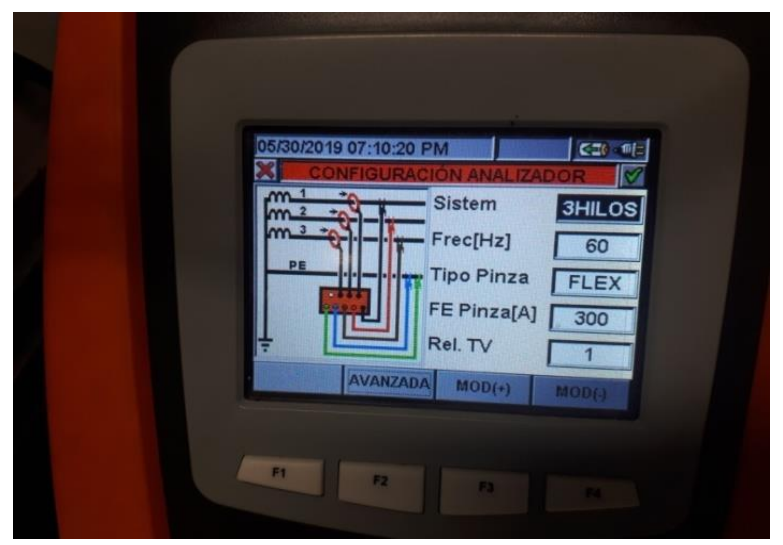

Figura 13 Configuración del variador. Fuente: Elaboración propia del autor

La figura anterior contiene la configuración apropiada del sistema teniendo en cuenta las diferentes conexiones y los valores de voltaje y corriente aproximados a medir, además de la frecuencia de trabajo. En este caso se utiliza un transductor de corriente tipo FLEX tal como nos indica el fabricante en la figura 14: 
TRMS AC Current with Flex transducer clamp - Range 300A

\begin{tabular}{|c|c|c|c|c|}
\hline Range & Accuracy & Resolution & Input impedance & Overload protection \\
\hline $0.0 \div 49.9 \mathrm{~A}$ & $\pm(0.5 \% \mathrm{rdg}+0.24 \% \mathrm{FS})$ & \multirow{2}{*}{$0.1 \mathrm{~A}$} & $510 \mathrm{k} \Omega$ & $5 \mathrm{~V}$ \\
\cline { 1 - 2 } $50.0 \div 300.0 \mathrm{~A}$ & $\pm(0.5 \% \mathrm{rdg}+0.06 \% \mathrm{FS})$ & & & \\
\hline
\end{tabular}

Measurements performed with HTFLEX33 flexible clamp

Max crest factor $=3$

Current values $<1 \mathrm{~A}$ are considered zero by meter

Figura 14 Configuración del Transductor FLEX. Fuente: Manual analizador HT [14]

\section{Comparación metodología con sistema tradicional de medición}

Una metodología tradicional para medir la eficiencia incluye tanto la potencia reactiva como la activa para un óptimo funcionamiento del sistema de manera intrínseco. Lo que significa que debemos medir la potencia total tanto de entrada como de salida de la siguiente manera:

$$
\text { Eficiencia }(\%)=\frac{S_{\text {out }}}{S_{\text {in }}} \times 100
$$

Teniendo en cuenta lo anterior, y, usando la simulación planteada en la figura 15, se aplicó la metodología tradicional, para lo cual se utilizó el método de tres vatímetros para medir potencias trifásicas en sistemas trifilares sin acceso a la carga como se observa en la figura 16 que precede.

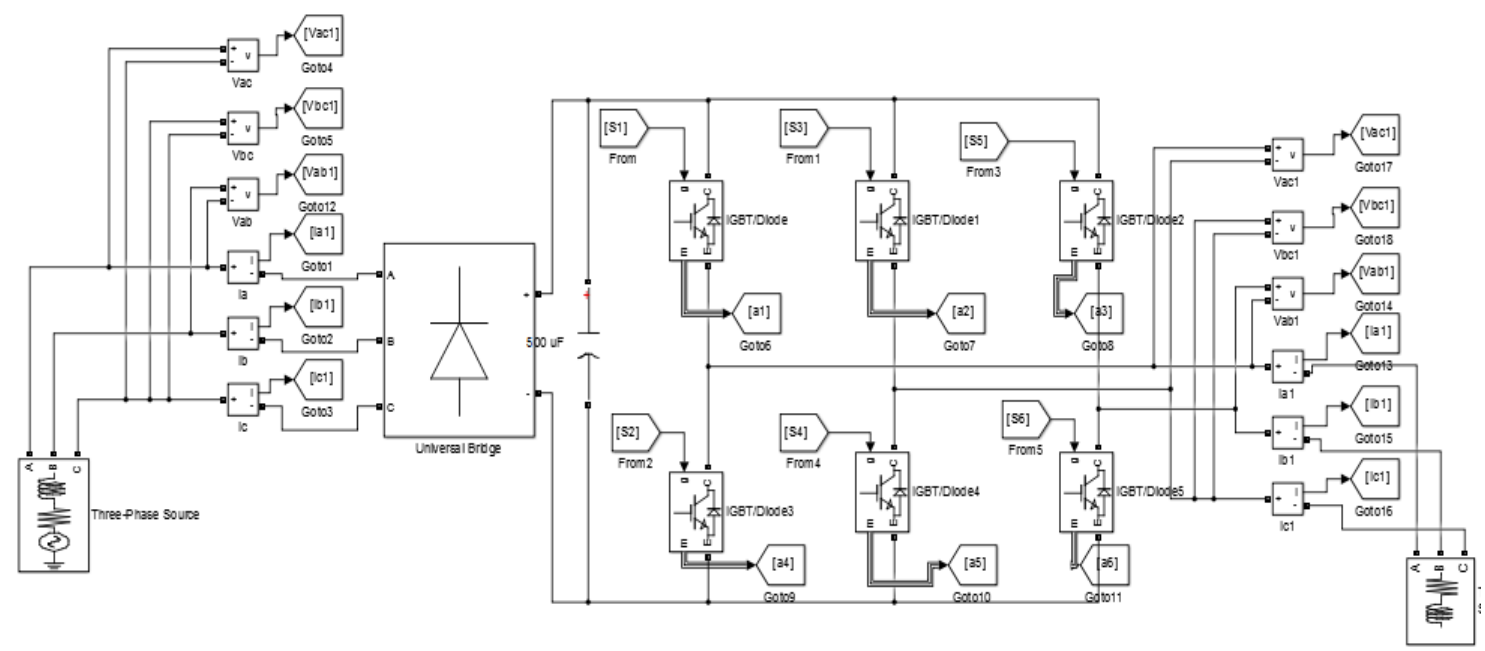

Figura 15 Circuito de medición de potencia. Fuente: Elaboración propia a partir de Simulink 


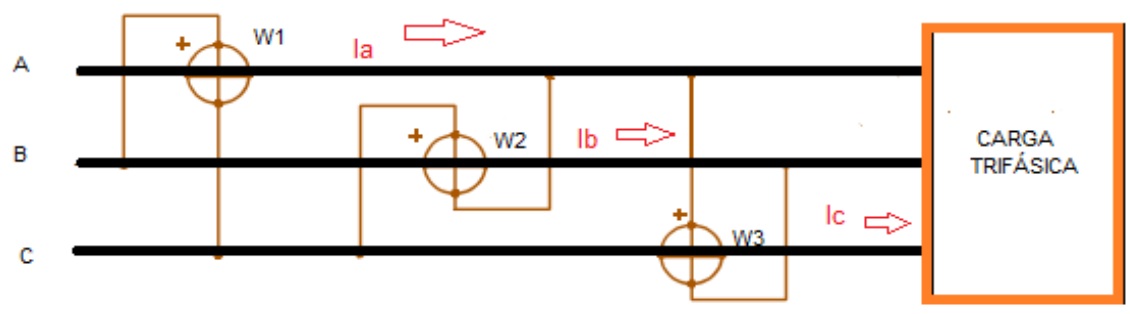

Figura 16 Método de medición de potencia. Fuente: Elaboración propia del autor

Al aplicar el sistema de los tres vatímetros y sabiendo que la potencia aparente de cada fase [15] es igual a:

$$
S_{A=} I_{r m s} \cdot V_{r m s}
$$

Se obtuvo una potencia de entrada y de salida del sistema como se pude ver en las figuras $17 \mathrm{y}$ 18:

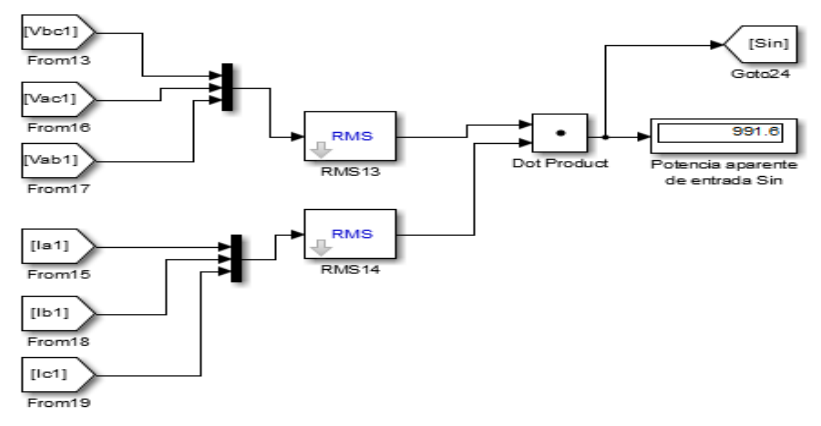

Figura 17 Potencia aparente de entrada

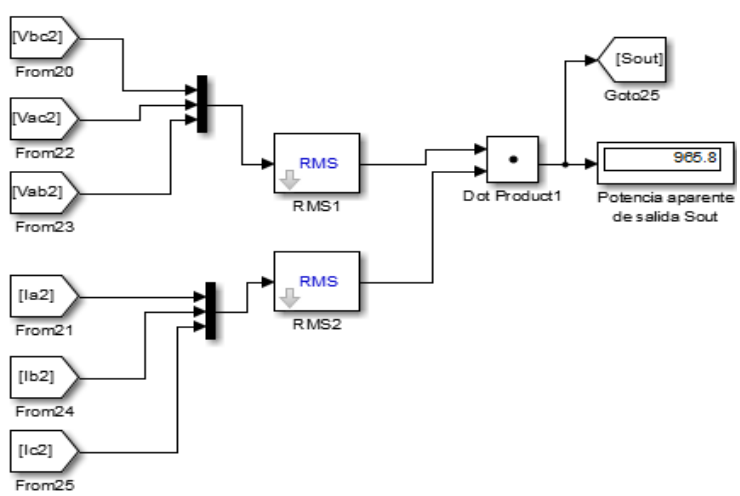

Figura 18 Potencia aparente de salida

Fuente: Elaboración propia a partir de Simulink

\section{RESULTADOS}

\section{Simulación}

Los resultados obtenidos de la simulación del circuito de la figura 5 se muestran en la tabla 1: 
Tabla 1 Datos de entrada y salida del inversor trifásico frecuencia $60 \mathrm{HZ}$.

\begin{tabular}{|c|c|c|}
\hline Parámetros & Entrada & Salida \\
\hline$V_{a b}$ & $219.8 \mathrm{~V}$ & $231.2 \mathrm{~V}$ \\
\hline$V_{b c}$ & $219.8 \mathrm{~V}$ & $232 \mathrm{~V}$ \\
\hline$V_{a c}$ & $219.8 \mathrm{~V}$ & $231.4 \mathrm{~V}$ \\
\hline$I_{a}$ & $2.374 \mathrm{~A}$ & $2.18 \mathrm{~A}$ \\
\hline$I_{b}$ & $2.343 \mathrm{~A}$ & $2.273 \mathrm{~A}$ \\
\hline$I_{c}$ & $2.36 \mathrm{~A}$ & $2.177 \mathrm{~A}$ \\
\hline$T H D_{V a b}$ & $3.36 \%$ & $61.38 \%$ \\
\hline$T H D_{V b c}$ & $3.34 \%$ & $62.55 \%$ \\
\hline$T H D_{V a c}$ & $3.38 \%$ & $61.31 \%$ \\
\hline$T H D_{I a}$ & $96.52 \%$ & $5.313 \%$ \\
\hline$T H D_{I b}$ & $96.45 \%$ & $5.439 \%$ \\
\hline$T H D_{I c}$ & $95.98 \%$ & $5.422 \%$ \\
\hline$V_{a b 1}$ & $219.8<29.66$ & $197.1<29.69$ \\
\hline$V_{b c 1}$ & $219.8<-90.34$ & $196.7<-90.17$ \\
\hline$V_{a c 1}$ & $219.8<149.7$ & $197.3<149.9$ \\
\hline$I_{a 1}$ & $1.708<-11.65$ & $2.177<-32.07$ \\
\hline$I_{b 1}$ & $1.686<-131.4$ & $2.17<-152.1$ \\
\hline$I_{c 1}$ & $1.703<109.1$ & $2.173<88.1$ \\
\hline
\end{tabular}

Fuente: Creación propia del autor

La tabla 2 muestra el consolidado de los resultados de cada uno de los valores obtenidos al aplicar la norma IEEE 1459-2010 [2]:

Tabla 2 Resultados de la simulación

\begin{tabular}{|c|c|c|}
\hline Parámetros & Entrada & Salida \\
\hline$V_{e}$ & $126.97 \mathrm{~V}$ & $133.69 \mathrm{~V}$ \\
\hline$V_{e 1}$ & $126.90 \mathrm{~V}$ & $113.76 \mathrm{~V}$ \\
\hline$I_{e}$ & $2.36 \mathrm{~A}$ & $2.18 \mathrm{~A}$ \\
\hline$I_{e 1}$ & $1.70 \mathrm{~A}$ & $2.17 \mathrm{~A}$ \\
\hline$I_{e H}$ & $1.64 \mathrm{~A}$ & $0.12 \mathrm{~A}$ \\
\hline$V_{e H}$ & $4.23 \mathrm{~A}$ & 70.24 \\
\hline$T H D_{V e}$ & $3 \%$ & $62 \%$ \\
\hline $1 T H D_{I e}$ & $96 \%$ & $5 \%$ \\
\hline$S_{e}$ & $898.51 \mathrm{VA}$ & $872.91 \mathrm{VA}$ \\
\hline$S_{e 1}$ & $646.80 \mathrm{VA}$ & $741.67 \mathrm{VA}$ \\
\hline$S_{e N}$ & $623.68 \mathrm{VA}$ & $460.33 \mathrm{VA}$ \\
\hline$D_{e I}$ & $622.96 \mathrm{VAR}$ & $36.87 \mathrm{VAR}$ \\
\hline
\end{tabular}




\begin{tabular}{|c|c|c|}
\hline Parámetros & Entrada & Salida \\
\hline$D_{e V}$ & $21.57 \mathrm{VAR}$ & $457.94 \mathrm{VAR}$ \\
\hline$S_{e H}$ & $20.78 \mathrm{VA}$ & $24.62 \mathrm{VA}$ \\
\hline$P_{1}$ & $845.70 \mathrm{~W}$ & $607.08 \mathrm{~W}$ \\
\hline$Q_{1}$ & $734.68 \mathrm{VAR}$ & $1132.10 \mathrm{VAR}$ \\
\hline
\end{tabular}

Fuente: Creación propia del autor

Según la ecuación (8) el valor de la eficiencia es 67.57 \% se puede evidenciar en la figura 19:

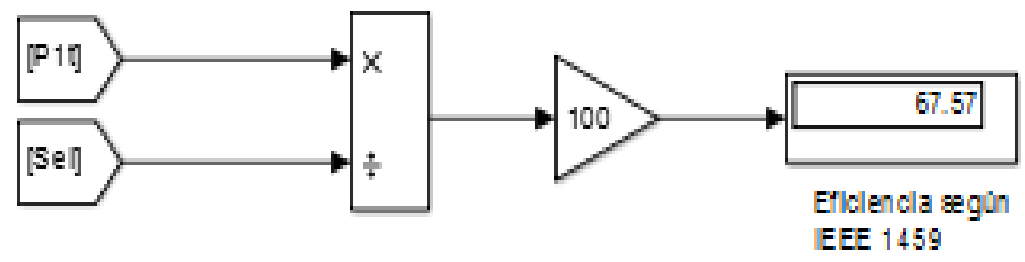

Figura 19 Resultado de la eficiencia según IEEE 1459. Fuente: Elaboración propia a partir de Simulink

\section{Sistema experimental}

La tabla 3 muestra los datos tomados en los analizadores marca HT con número de serie PQA 824:

Tabla 3. Datos de los analizadores marca HT con número de serie PQA 824

\begin{tabular}{|c|c|c|}
\hline Parámetros & Entrada & Salida \\
\hline$V_{a b}$ & $221.9 \mathrm{~V}$ & $221.2 \mathrm{~V}$ \\
\hline$V_{b c}$ & $219.2 \mathrm{~V}$ & $221.5 \mathrm{~V}$ \\
\hline$V_{a c}$ & $220.1 \mathrm{~V}$ & $220.0 \mathrm{~V}$ \\
\hline$I_{a}$ & $3.0 \mathrm{~A}$ & $2.8 \mathrm{~A}$ \\
\hline$I_{b}$ & $2.5 \mathrm{~A}$ & $2.3 \mathrm{~A}$ \\
\hline$I_{c}$ & $3.3 \mathrm{~A}$ & $2.8 \mathrm{~A}$ \\
\hline$V_{a b 1}$ & $220.9 \mathrm{~V}$ & $217 \mathrm{~V}$ \\
\hline$V_{b c 1}$ & $220 \mathrm{~V}$ & $216.8 \mathrm{~V}$ \\
\hline$V_{a c 1}$ & $218.5 \mathrm{~V}$ & $215.3 \mathrm{~V}$ \\
\hline$I_{a 1}$ & $3.1 \mathrm{~A}$ & $2.5 \mathrm{~A}$ \\
\hline$I_{b 1}$ & $2.3 \mathrm{~A}$ & $2.6 \mathrm{~A}$ \\
\hline$I_{c 1}$ & $3.0 \mathrm{~A}$ & $2.6 \mathrm{~A}$ \\
\hline $\mathrm{Pt}$ & $629.5 \mathrm{~W}$ & $497.3 \mathrm{~W}$ \\
\hline
\end{tabular}

Fuente: Creación propia del autor

Los resultados obtenidos al aplicar las ecuaciones de la norma IEEE 1459-2010 para el caso experimental los podemos observar en la tabla 4: 


\section{CONGRESO DE DESARROLLD \\ SOSTENIBLE}

Tabla 4. Cálculos resultados experimentales

\begin{tabular}{|c|c|c|c|}
\hline Parámetros & Ecuación & Entrada & Salida \\
\hline$V_{e}$ & $\sqrt{\frac{1}{9}}\left[\left(V_{a b}^{2}+V_{b c}^{2}+V_{c a}^{2}\right)\right]$ & $127.2 \mathrm{~V}$ & $127.5 \mathrm{~V}$ \\
\hline$V_{e 1}$ & $\sqrt{\frac{1}{9}}\left[\left(V_{a b 1}^{2}+V_{b c 1}^{2}+V_{c a 1}^{2}\right)\right]$ & $126.9 \mathrm{~V}$ & $124.9 \mathrm{~V}$ \\
\hline$I_{e}$ & $\sqrt{\frac{1}{3}\left[\left(I_{a}^{2}+I_{b}^{2}+V_{c}^{2}\right)\right]}$ & $2.95 \mathrm{~A}$ & $2.64 \mathrm{~A}$ \\
\hline$I_{e 1}$ & $\sqrt{\frac{1}{3}}\left[\left(I_{a 1}^{2}+I_{b 1}^{2}+I_{c 1}^{2}\right)\right]$ & $2.82 \mathrm{~A}$ & $2.56 \mathrm{~A}$ \\
\hline$I_{e H}$ & $\sqrt{\left[\left(I_{e}^{2}-I_{e 1}^{2}\right)\right]}$ & $0.86 \mathrm{~A}$ & $0.64 \mathrm{~A}$ \\
\hline$V_{e H}$ & $\sqrt{\left[\left(V_{e}^{2}-V_{e 1}^{2}\right)\right]}$ & $8.73 \mathrm{~V}$ & $25.61 \mathrm{~V}$ \\
\hline$T H D_{V e}$ & $\frac{V_{e H}}{V_{e 1}}$ & $6.8 \%$ & $20 \%$ \\
\hline$T H D_{I e}$ & $\frac{I_{e H}}{I_{e 1}}$ & $30 \%$ & $25 \%$ \\
\hline$S_{e}$ & $3 V_{e} I_{e}$ & 1125.7 VA & $1009.8 \mathrm{VA}$ \\
\hline$S_{e 1}$ & $3 V_{e 1} I_{e 1}$ & 1072.5 VA & $959.23 \mathrm{VA}$ \\
\hline$S_{e N}$ & $\sqrt{\left[\left(S_{e}^{2}-S_{e 1}^{2}\right)\right]}$ & $341.97 \mathrm{VA}$ & 315.55 VA \\
\hline$D_{e I}$ & $D_{e I}=3 V_{e 1} I_{e H}$ & 327.4 VAR & 239.8 VAR \\
\hline$D_{e V}$ & $D_{e V}=3 V_{e H} I_{e 1}$ & 73.85 VAR & 196.68 VAR \\
\hline$S_{e H}$ & $3 V_{e H} I_{e H}$ & 22.52 VA & 49.17 VA \\
\hline
\end{tabular}

Fuente: Creación propia del autor

Donde el valor de la eficiencia se calcula según la ecuación 12:

$$
\text { Eficiencia }(\%)=\frac{P_{t}}{S_{e}} x 100=44 \%
$$

\section{Comparación metodología con sistema tradicional de medición}

El resultado del sistema tradicional se evidencia en la figura 20, el cual equivale a $97.40 \%$ : 


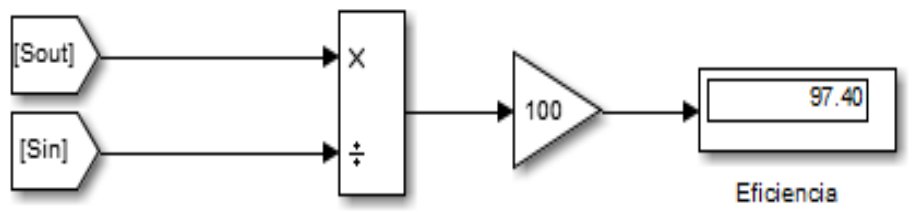

Figura 20 Eficiencia metodología tradicional Fuente: Elaboración propia a partir de Simulink

La comparación de los resultados de las metodologías lo podemos observar en la tabla 5:

Tabla 5. Comparación de resultados

\begin{tabular}{|l|l|l|}
\hline Metodologías & Eficiencia & Figura \\
\hline IEEE-1459 Simulada & 67.57 & No. 19 \\
\hline IEEE-1459 Experimental & 44.00 & N.A. \\
\hline Tradicional & 97.40 & No. 20 \\
\hline
\end{tabular}

Fuente: Elaboración propia a partir de Simulink

\section{ANÁLISIS DE RESULTADOS}

En la tabla 2 se ven los datos de las pérdidas y distorsiones provocadas por componentes armónicos y también se comprueba que el circuito equivalente propuesto por la norma es considerablemente acertado. Si se revisa el valor de la tasa de distorsión armónica de voltaje (THDv) y corriente (THDi) medida directamente con el bloque que trae Simulink (Ver tabla 2) es muy parecido al hallado con la norma.

Así mismo, se observa que con la metodología propuesta la eficiencia equivale a 67.57 \% según el programa Simulink, mientras que con el método experimental su valor es igual a $44 \%$, esta diferencia se debe a las pérdidas producidas por los elementos reales y calibración de los instrumentos de medición.

De otra parte, el valor de la eficiencia por el método tradicional fue del $97.40 \%$, debido a que en su cálculo no se incluyeron distorsiones de tipo armónico, lo cual difiere en un $29.83 \%$ con la eficiencia calculada por la metodología propuesta en este artículo. 


\section{CONCLUSIONES}

La metodología para la estimación y análisis de eficiencia energética propuesta proporcionó resultados satisfactorios respecto a la metodología tradicional de tres vatímetros, por cuanto se demostró que con la aplicación de la norma IEEE1459-2010 el valor de la eficiencia es equivalente al $67.57 \%$ y el resultado con la metodología tradicional es de $97.40 \%$.

Para dar cabida a la simulación se requirió de un diseño matemático previo del inversor PWM.

La medición de la eficiencia del convertidor trifásico se realizó con éxito y según los resultados de Simulink, el nivel de eficiencia considerando la norma IEEE 1459-2010, disminuye 29.83\% con respecto al método tradicional.

Los resultados muestran que la eficiencia estimada usando el estándar IEEE 1459-2010 proporciona una eficiencia de menor valor; esto se debe a que el estándar incluye energía ineficiente asociada con la presencia de armónicos en las señales de corriente y voltaje, que son generadas por los convertidores estáticos en el proceso.

Calculando la eficiencia según el método implementado en las pruebas reales se obtuvo un valor del $44 \%$, este valor es menor al resultado simulado, y es el esperado debido a las pérdidas en los diferentes dispositivos y a la calibración del instrumento.

En los convertidores estáticos PWM sobremodulados los voltajes de salidas se incrementan de una forma no lineal con respecto a la frecuencia, debido a que la señal de control es mayor que la señal moduladora. Si no se sobremodula, el voltaje de salida nunca llegaría a un valor aproximado de $220 \mathrm{~V}$.

La aplicación de los resultados en nuestra cotidianidad se puede medir en los campos económico, social y ambiental, en términos de sostenibilidad en favor de la humanidad y el planeta tierra.

La globalización ha convertido al hombre en un ser energívoro, condición que avanza de forma desmedida, requiriéndose una señal de PARE para acondicionar las ventajas de la eficiencia energética en su propio beneficio y como mecanismo de mitigación de la devastación de la naturaleza y los recursos naturales. 


\section{REFERENCIAS}

[1] H. Akagi, Trends in active power line conditioners. IEEE Transactions on Power Electronics, 9 (3), 1994, pp. $263-$ 268. https://doi.org/10.1109/63.311258

[2] IEEE Standard Defintitions for the Measurement of Elcetric Power Quantities Under Sinusoidal, Nonsinusoidal, Balanced, or Undalanced Conditions, IEEE Standard 1459, 2010.

F. Buchholz, "Die Drehstrom-Scheinleistung bei Ungleichmassiger Belastung Der Drei Zweige", Alemania; Licht und Kraft, núm. 2, 1922, pp. 9-11. Budeanu, C.I.: 'Reactive and fictitious powers' (in Romanian) (Inst. Romain de l'Energie, Bucharest, Romania, 1927)

J.C. Alfonso Gil, S. Orts Grau, N. Munoz Galeano, F.J. Gimeno Sales, S. Segui Chilet, Measurement System for a Power Quality Improvement Structure Based on IEEE Std.1459. IEEE Transactions on Instrumentation and Measurement, 2013, 62 (12), pp. 3177-3188. https://doi.org/10.1109/TIM.2013.2270901

[6] J.C. Alfonso Gil (2010). Contribución a la mejora de los sistemas de medida basados en el IEEE Std 1459-2000 [Tesis doctoral no publicada]. Universitad Politécnica de

Valencia.

https://doi.org/10.4995/Thesis/10251/8345

[7] C. Torres, E. Forero, D. Tibaduiza. Energy efficiency analysis in static converters using IEEE 1459. En VI Simposio Internacional sobre Energía y Foro de Innovación tecnológica. Gurabo-Puerto Rico, enero, 2014.

[8] D. Hart. Electrónica de potencia. España: Pearson Educación S.A. 2001, pp.343-345.
J. G. Barrero, A. J. Bautista, C.A. Duarte, G. Ordoñez. "Medición de las magnitudes de potencia y energía eléctrica bajo las nuevas condiciones de los sistemas eléctricos." Dialnet [En línea]. Disponible en: https://dialnet.unirioja.es/descarga/articulo/6299759. pdf. [Accedido: 14-mar-2020] 
CONGRESO DE DESARROLLD

SOSTENIBLE

\section{PROCESOS SOSTENIBLES}




\title{
TENDENCIAS EN LA GENERACIÓN DE NUEVOS MATERIALES A PARTIR DE FIBRAS NATURALES Y RESIDUOS POLIMÉRICOS
}

\section{Trends in the generation of new materials from natural fibers and polymeric residues}

\author{
Ortega, Mayerly; Rios, Deyner ${ }^{1}$; Orjuela, David; Solano, Johanna; Gómez, Zully \\ Universidad Santo Tomas.
}

\section{Resumen}

En los últimos años las problemáticas asociadas a la gestión de residuos agrícolas como lo son diversas fibras (bagazo de caña, kenaf, yute, sisal, kapok, salvado de sorgo, aserrín de madera, cascarilla de arroz, bambú, coco, palmera datilera, lino, mengkuank, diss, cáñamo, yerba mate, etc) y residuos poliméricos (polipropileno, polietileno tereftalato y polietileno de alta y baja densidad) han tenido un crecimiento excesivo y a la vez un bajo índice de aprovechamiento; razón por la cual el uso de dichos residuos en la fabricación de materiales compuestos ha entrado en auge durante los últimos años. Por lo que el presente documento es una revisión que pretende analizar las tendencias en la generación de nuevos materiales a partir de fibras naturales y residuos poliméricos mediante la comparación de resultados experimentales, los cuales han sido caracterizados a través de pruebas físico-mecánicas, y como resultado, los compuestos presentan un aumento en propiedades como la resistencia a la tracción, resistencia al impacto, estabilidad térmica y dureza, además, en algunos casos se han incluidos agentes de acople y tratamientos químicos realizados a las fibras que permiten mejorar la adhesión del compuesto.

Palabras claves: fibras naturales, polímeros reciclados, materiales compuestos

\section{INTRODUCCIÓN}

Las fibras naturales de origen vegetal son resultado de procesos agroindustriales, las cuales en su mayoría son tratadas como residuos y cuyas cantidades anuales tienden a aumentar con el paso del tiempo, generando gran preocupación a nivel medioambiental [1], [2]. En general, las fibras naturales están compuestas de celulosa, hemicelulosa, lignina y pectina, siendo la celulosa la más abundante con un $65 \%$ del total de sus componentes [3], [4]; los compuestos con fibras como

${ }^{1}$ Correo electrónico de contacto: deynerrios@usantotomas.edu.co 
refuerzo han sido de gran interés en los últimos años debido a su bajo peso específico, la resistencia a la abrasión, la fatiga, la rigidez y su bajo costo relacionado con su abundancia son características que llaman la atención de estas e inducen a reutilizarlas [1], [5], [6]. Por otra parte, la problemática asociada a la contaminación por desechos plásticos ocasiona gran afectación al medio ambiente debido a su generación en masa y baja biodegradabilidad. En Colombia se generan alrededor de 30.081 Ton/día de residuos sólidos, de los cuales gran porcentaje corresponden a plásticos (polietileno, polipropileno, poliéster, etc.) generados por el consumo desmedido de la sociedad, además del bajo aprovechamiento de los residuos [7]; razón por la cual los compuestos utilizan materiales poliméricos reciclados como matriz [8]-[10].

Dentro de los polímeros más utilizados para la fabricación de compuestos se encuentran el polipropileno (PP), polietileno tereftalato (PET) y polietileno de alta densidad (HDPE) [8], [9]; encontrando también varias mezclas de polímeros en las que predomina la fracción porcentual de polietileno de alta densidad [11]-[13]. En cuanto a las fibras más utilizadas se encuentran el bagazo de caña, el aserrín y palmera datilera, entre otras. Todas utilizadas en diferentes proporciones, de 5 - 80\% con el fin de mejorar las propiedades mecánicas y térmicas de las respectivas matrices [10], [11].

\section{MATERIALES Y METODOS}

En primera instancia se realizó la revisión bibliográfica, etapa para la cual se llevaron a cabo dos procedimientos; el primero fue la delimitación y organización del tipo de artículos a escoger, donde la elección del operador lógico más adecuado fue de vital importancia a la hora de generar ecuaciones de búsqueda tales como: "composite materials" AND "natural fibers", "composite materials" AND "recycled polymers", "composite materials with natural fiber" AND "recycled polymers", entre otras. El segundo, fue la selección de aquellas bases de datos que suministraran la mayor cantidad de información acerca del tema de investigación, siendo estas Science Direct, SpringerLink, Sage Journals y Scopus, en las cuales se depuró la búsqueda según los criterios establecidos previamente, seleccionando aquellos artículos que proporcionaban mayor información acerca de los procesos efectuados para la fabricación de materiales compuestos, tales como tratamientos previos y el tamaño tanto de la fibra como del polímero, proporciones de mezcla, el uso de agentes de acople, el método de fabricación, los métodos de caracterización y resultados enfocados al análisis de las propiedades mecánicas, físicas y térmicas como lo son la resistencia a la tracción, resistencia a la flexión, resistencia al impacto, adhesión y estabilidad térmica. Además, la herramienta de gestión bibliográfica ProQuest RefWorks fue la plataforma 
elegida para realizar el control y seguimiento de los artículos seleccionados.

Al finalizar la revisión, la información obtenida de cada documento fue agrupada de acuerdo a la matriz base clasificándose en cuatro grandes grupos: polipropileno (PP), polietileno (PE), polietileno tereftalato (PET) y mezclas. Con la finalidad de comparar las condiciones de fabricación y encontrar posibles similitudes que estandaricen el proceso según algunas variables

\section{RESULTADOS Y ANALISIS DE RESULTADOS}

Como resultado a la revisión bibliográfica realizada, se encontró que el tema de reforzamiento de polímeros reciclados con fibras naturales ha sido estudiado desde el año 2003, el cual ha experimentado un crecimiento en el ámbito de la investigación a partir del año 2013, además, se identificó que países como Malasia, India y Brasil son los que más investigaciones y publicaciones realizan. Donde se puede identificar la tendencia a trabajar con ciertas fibras como el aserrín de madera, hojas de palmera datilera, sisal, bagazo de caña y kenaf. Asimismo, se identificó que dentro de las matrices más usadas están el polipropileno, el polietileno y el PET, estudiadas tanto de manera individual como en mezclas con diversas proporciones.

También se identificó la tendencia a usar tratamientos químicos y agentes de acople para la fabricación de dichos compuestos, ya que permiten eliminar lignina y hemicelulosas, además de otras impurezas parciales o superficiales, aumentando la interacción interfacial entre la fibra y matriz al mejorar el comportamiento hidrofílico de las fibras hidrofóbico de la matriz polimérica [5], [8], [14]; reduciendo así la porosidad, comportamiento que mejora directamente la resistencia a la tracción. Dentro de los tratamientos, es habitual encontrar el uso de $\mathrm{NaOH}$ y Silano durante la adecuación de la fibra y anhídrido maléico, polietileno y polipropileno injertados con anhídrido maléico como agentes de acople.

Otro factor de vital de importancia es la carga volumétrica de las fibras, ya que al incorporar entre el 10 y el $40 \%$ de peso en fibra se evidencia el aumento más significativo de las propiedades mecánicas (la tracción, flexión e impacto), y al pasar esta fracción volumétrica las fibras tienden a aglomerarse y no logran adherirse correctamente, ocasionando pérdidas en cuanto a propiedades mecánicas y estructurales [15]. Además de las propiedades mecánicas, también son estudiadas propiedades térmicas tales como la estabilidad térmica, resistencia al calor y propiedades morfológicas; las cuales tienden a variar su comportamiento según la fibra y matriz utilizadas.

Por último, cabe resaltar la importancia del uso de polímeros reciclados y no vírgenes dentro de la fabricación de materiales compuestos; esto, en gran parte dada la problemática ya mencionada, 
la facilidad de encontrar dicha materia prima y factores de influencia durante el proceso de fabricación de polímeros. Además, una matriz reciclada puede ofrecer casi los mismos resultados que una virgen tal como lo demuestran algunos autores durante sus investigaciones, las cuales arrojan que a pesar de no superar el porcentaje de mejoría en cuanto a propiedades físico mecánicas, la diferencia no es muy significativa a nivel cuantitativo pero sí a nivel medioambiental.

Por último, cabe resaltar la importancia del uso de polímeros reciclados dentro de la fabricación de materiales compuestos; esto, dada la problemática ya mencionada, la facilidad de encontrar dicha materia prima y factores de influencia durante el proceso de fabricación de nuevos polímeros. Además, una matriz reciclada puede ofrecer casi los mismos resultados e incluso mejores en algunos casos que una virgen, tal como lo demuestran algunos autores durante sus investigaciones [5], [16]. Las cuales arrojan, que en los casos que no superar el porcentaje de mejoría en cuanto a propiedades físico - mecánicas, la diferencia no es muy significativa a nivel cuantitativo pero sí a nivel medioambiental [17].

A continuación, en la tabla 1 se reúnen las descripciones de los artículos más detallados encontrados durante la revisión. En la cual se especifican el tipo de matriz y fibras usadas, proporciones de fibra, tratamientos químicos y agentes de acople utilizados; asimismo, las propiedades físico, mecánicas y térmicas que mejoraron con sus respectivos valores.

Tabla 3 Principales resultados

\begin{tabular}{|c|c|c|c|c|}
\hline Matriz & Fibras & $\begin{array}{l}\text { Proporciones y } \\
\text { tratamientos a fibra }\end{array}$ & Agentes de acople & $\begin{array}{c}\text { Resultados } \\
\text { (propiedades que } \\
\text { mejoraron) }\end{array}$ \\
\hline \multirow{5}{*}{$(\mathrm{PP})$} & Sisal & $\begin{array}{l}-30-40 \% \\
-\mathrm{NaOH} \text { por } 18 \mathrm{~h} .\end{array}$ & MAPP (5\%) & $\begin{array}{l}\text { RT. } 12 \% \text {, MT. } 280 \% \\
\text { RF. } 55 \% \text { y MF. } 274 \% \text { [18]- } \\
\text { [20]. }\end{array}$ \\
\hline & Yute & $\begin{array}{l}-50 \% \\
-S i l a n o\end{array}$ & Silano $(6 \%)$ & RF. $7,4 \%$, y MF. $22,7 \%$ [21]. \\
\hline & Bambú & $\begin{array}{l}-35-40 \% \\
-\mathrm{NaOH} \text {, etanol y anhídrido } \\
\text { acético. }\end{array}$ & MAPP (10\%) & RI. $32 \%$ y CR. $21,4 \%$ [22]. \\
\hline & $\begin{array}{l}\text { Palma } \\
\text { aceitera }\end{array}$ & $\begin{array}{l}-30 \% \\
-S L N \text { de tolueno, etanol y } \\
\text { acetona por } 3 \mathrm{~h} .\end{array}$ & $\begin{array}{l}\text { Anhídrido maléico }(10 \%) \\
\text { e hidroquinona }(5 \%)\end{array}$ & $\begin{array}{l}\text { RT. } 84 \% \text {, TE, y WA. } \\
258 \text { [23]. }\end{array}$ \\
\hline & Mengkuang & $\begin{array}{l}-40 \% \\
-\mathrm{NaOH} \text { por } 24 \mathrm{~h} .\end{array}$ & MAPP (5\%) & $\begin{array}{l}\text { RT. 27,6\%, MT. 224\%, RF, y } \\
\text { MF. [18] }\end{array}$ \\
\hline \multirow[t]{2}{*}{ (HDPE) } & $\begin{array}{l}\text { Bagazo de } \\
\text { caña }\end{array}$ & $\begin{array}{l}-30 \% \\
- \text { Sin tratamiento }\end{array}$ & $\operatorname{MPP}(0,6-4,5 \%)$ & $\begin{array}{l}\text { RT. } 40 \% \text {, RI. } 40 \% \text { y AM. } \\
50 \% .[1]\end{array}$ \\
\hline & Cáñamo & $-40 \%$ & $\mathrm{NaOH}$ & RT. 34\%, RF y RI. [24] \\
\hline
\end{tabular}




\begin{tabular}{|c|c|c|c|c|}
\hline & & $-\mathrm{NaOH}$ & & \\
\hline (LDPE) & Sisal & $\begin{array}{l}-5-20 \% \\
- \text { Sin tratamiento }\end{array}$ & Sin agente & RT. 15\%, RI. [25] \\
\hline \multirow{2}{*}{ (PET) } & $\begin{array}{l}\text { Palmera } \\
\text { datilera }\end{array}$ & $\begin{array}{l}-5 \% \\
-\mathrm{NaOH} \\
\end{array}$ & MAPE & $\begin{array}{l}\text { RT. } 42 \%, \text { RF. } 28 \% \text {, RI, D. } \\
{[10]}\end{array}$ \\
\hline & $\begin{array}{l}\text { Racimos de } \\
\text { fruta }\end{array}$ & $\begin{array}{l}-30 \% \\
- \text { Silano }\end{array}$ & Anhídrido maléico (10\%) & RT. 21\%, MT, RI y TD. [26] \\
\hline $\begin{array}{l}\text { HDPE, } \\
\text { LDPE y } \\
\text { PP } \\
\end{array}$ & $\begin{array}{l}\text { Palmera } \\
\text { datilera }\end{array}$ & $\begin{array}{l}-10-20 \% \\
-\mathrm{NaOH} \text { por } 1 \mathrm{~h} .\end{array}$ & Anhídrido maléico (1\%) & $\begin{array}{l}\text { RT } 8,3 \% \text {, MT 33,15\%, D y } \\
\text { TE. [8] }\end{array}$ \\
\hline $\begin{array}{l}\text { HDPE y } \\
\text { PET }\end{array}$ & $\begin{array}{l}\text { Cascarilla de } \\
\text { arroz }\end{array}$ & $\begin{array}{l}-70 \% \\
-3 \% \text { organoarcilla } \\
\end{array}$ & $\begin{array}{l}\text { MAPE }(3 \%) \\
\text { GMA }(5 \%)\end{array}$ & $\begin{array}{l}\text { RT. } 22 \% \text {, MF. } 258 \% \text {, RC. } 9 \% \text {, } \\
\text { TE y MT. [27] }\end{array}$ \\
\hline $\begin{array}{l}\text { HDPE y } \\
\text { PP }\end{array}$ & Lino & $\begin{array}{l}-43-45 \% \\
- \text { Sin tratamiento }\end{array}$ & MAPP (3\%) & $\begin{array}{l}\text { MF. 15\%, MT. } 83 \% \text { y RI. } \\
63 \% \text { [18]. }\end{array}$ \\
\hline
\end{tabular}

Fuente: Autores.

RT: Resistencia a la tracción, MT: módulo de tracción, RF: resistencia a la flexión, MF: módulo de flexión, RI: resistencia al impacto, CR: cristalización, TE: estabilidad térmica, WA: absorción de agua, AM: módulo de almacenamiento, TD: temperatura a la descomposición, D: dureza, RC: resistencia al calor.

*Los porcentajes presentados en las columnas 3 y 4 están expresados en función de peso.

\section{CONCLUSIONES}

En general, la adición de fibras naturales en entre 10\% y $40 \%$ de peso en fibra como refuerzo en la fabricación de materiales compuestos, aumenta propiedades mecánicas tales como la resistencia a la tracción, resistencia al impacto y por ende algunas propiedades como la rigidez, dureza y rugosidad; así como también conlleva a la mejora de la estabilidad térmica y resistencia al calor de los compuestos fabricados. Por otra parte se encuentra que la presencia de un agente de acople así como el tratamiento previo de las fibras ayuda a incrementar las propiedades físico - mecánicas al aumentar la adhesión entre las fibras y la matriz. Además, se observa que durante la fabricación de los compuestos los métodos más usados son moldeo por compresión e inyección; por último, debido a que todas la investigaciones se llevaron a cabo utilizando como matriz polímeros reciclados los cuales demuestran muy buenos resultados, evidenciando un incremento de estudios durante los últimos años, debido al interés técnico de usar matrices recicladas en la fabricación de materiales compuestos 


\section{REFERENCIAS}

[1] Y. Lei et al, "Preparation and properties of recycled HDPE/natural fiber composites," Composites Part A: Applied Science and Manufacturing, vol. 38, (7), pp. 16641674, $2007 . \quad$ Available: http://www.sciencedirect.com.craiustadigital.usantotomas.edu.co/science/article/pii/S135 9835X07000310. DOI: https://doi-org.craiustadigital.usantotomas.edu.co/10.1016/j.compositesa.2 007.02.001.

[2] K. B. Adhikary, S. Pang and M. P. Staiger, "Dimensional stability and mechanical behaviour of wood-plastic composites based on recycled and virgin high-density polyethylene (HDPE)," Composites Part B: Engineering, vol. 39, (5), pp. 807-815, 2008. Available: http://www.sciencedirect.com.crai-

ustadigital.usantotomas.edu.co/science/article/pii/S135 983680700145X. DOI: https://doi-org.craiustadigital.usantotomas.edu.co/10.1016/j.compositesb.2 007.10.005

[3] C. Wu and C. Wu, "Enhanced Interfacial Adhesion and Characterisation of Recycled Natural Fibre-Filled Biodegradable Green Composites," J Polym Environ, vol. 26, (7), pp. 2676-2685, 2018. Available: https://search.proquest.com/docview/1973290625. DOI: 10.1007/s10924-017-1160-9.

[4] R. M. de Sá, C. S. de Miranda and N. M. José, "Preparation and Characterization of Nanowhiskers Cellulose from Fiber Arrowroot (Maranta arundinacea)," Materials Research, vol. 18, (2), pp. 225229, $2015 . \quad$ Available: https://www.openaire.eu/search/publication?articleId= od__ 3056::b326e7eb240014ea7b17eee1ca7699f7. DOI: $10.1590 / 1516-1439.366214$.

[5] D. B. Rocha and D. d. S. Rosa, "Coupling effect of starch coated fibers for recycled polymer/wood composites," Composites Part B: Engineering, vol. 172, pp. 1-8, 2019. Available: http://www.sciencedirect.com.craiustadigital.usantotomas.edu.co/science/article/pii/S135 9836818343051. DOI: https://doi-org.craiustadigital.usantotomas.edu.co/10.1016/j.compositesb.2 019.05.052

[6] M. Rokbi et al, "Effect of processing parameters on tensile properties of recycled polypropylene based composites reinforced with jute fabrics," International Journal of Lightweight Materials and Manufacture, vol. 3, (2), pp. 144-149, 2020. Available: http://www.sciencedirect.com.crai-

ustadigital.usantotomas.edu.co/science/article/pii/S258 8840419301076. DOI: https://doi-org.craiustadigital.usantotomas.edu.co/10.1016/j.ijlmm.2019.09. 005.

[7] Superintendencia de Servicios Públicos Domiciliarios, "Informe de Disposición Final de Residuos Sólidos,"
2019.

[8] P. Noorunnisa Khanam and M. A. AlMaadeed, "Improvement of ternary recycled polymer blend reinforced with date palm fibre," Materials \& Design, vol. 60, pp. 532-539, 2014. Available: http://www.sciencedirect.com.crai-

ustadigital.usantotomas.edu.co/science/article/pii/S026 130691400301X. DOI: https://doi-org.craiustadigital.usantotomas.edu.co/10.1016/j.matdes.2014.0 4.033 .

[9] A. Bourmaud, A. Le Duigou and C. Baley, "What is the technical and environmental interest in reusing a recycled polypropylene-hemp fibre composite?" Polymer Degradation and Stability, vol. 96, pp. 1732-1739, 2011.

[10] A. Dehghani et al, "Mechanical and thermal properties of date palm leaf fiber reinforced recycled poly (ethylene terephthalate) composites," Materials \& Design (19802015), vol. 52, pp. 841-848, 2013.

[11] R. S. Chen et al, "Effect of polymer blend matrix compatibility and fibre reinforcement content on thermal stability and flammability of ecocomposites made from waste materials," Thermochimica Acta, vol. 640, pp. 52-61, 2016. Available: http://www.sciencedirect.com.craiustadigital.usantotomas.edu.co/science/article/pii/S004 0603116302027. DOI: https://doi-org.craiustadigital.usantotomas.edu.co/10.1016/j.tca.2016.08.00 5.

[12] K. M. Zadeh, D. Ponnamma and M. Al Ali Al-Maadeed, "Date palm fibre filled recycled ternary polymer blend composites with enhanced flame retardancy," Polymer Testing, vol. 61, pp. 341-348, 2017. Available: http://www.sciencedirect.com.craiustadigital.usantotomas.edu.co/science/article/pii/S014 2941817302210. DOI: https://doi-org.craiustadigital.usantotomas.edu.co/10.1016/j.polymertestin g.2017.05.006.

[13] K. Zadeh et al, "Effects of date palm leaf fiber on the thermal and tensile properties of recycled ternary polyolefin blend composites," Fibers Polym, vol. 18, (7), pp. 1330-1335, 2017. Available: https://search.proquest.com/docview/1925068279. DOI: 10.1007/s12221-017-1106-9.

[14] M. J. Taufiq, M. R. Mansor and Z. Mustafa, "Characterisation of wood plastic composite manufactured from kenaf fibre reinforced recycledunused plastic blend," Composite Structures, vol. 189, pp. 510-515, 2018.

Available: http://www.sciencedirect.com.craiustadigital.usantotomas.edu.co/science/article/pii/S026 3822317327976. DOI: https://doi-org.craiustadigital.usantotomas.edu.co/10.1016/j.compstruct.20 
18.01.090.

[15] J. O. Agunsoye and V. S. Aigbodion, "Bagasse filled recycled polyethylene bio-composites: Morphological and mechanical properties study," Results in Physics, vol. 3, pp. 187-194, 2013. Available: http://www.sciencedirect.com.crai-

ustadigital.usantotomas.edu.co/science/article/pii/S221 1379713000296. DOI: https://doi-org.craiustadigital.usantotomas.edu.co/10.1016/j.rinp.2013.09.0 03.

[16] N. Lu and S. Oza, "A comparative study of the mechanical properties of hemp fiber with virgin and recycled high density polyethylene matrix," Composites Part B: Engineering, vol. 45, (1), pp. 1651-1656, 2013. Available: http://www.sciencedirect.com.craiustadigital.usantotomas.edu.co/science/article/pii/S135 9836812006403. DOI: https://doi-org.craiustadigital.usantotomas.edu.co/10.1016/j.compositesb.2 012.09.076.

[17] J. A. Foulk et al, "Enzyme-Retted Flax Fiber and Recycled Polyethylene Composites," Journal of Polymers and the Environment, vol. 12, (3), pp. 165-171, 2004. Available: https://link-springer-com.craiustadigital.usantotomas.edu.co/article/10.1023/B:JOOE. 0000038548.73494.59.

DOI:

10.1023/B:JOOE.0000038548.73494.59

[18] A. Gupta et al, "Mechanical and thermal degradation behavior of sisal fiber (SF) reinforced recycled polypropylene (RPP) composites," Fibers Polym, vol. 15, (5), pp. 994-1003, 2014. Available: https://search.proquest.com/docview/1530762892. DOI: 10.1007/s12221-014-0994-1.

[19] S. Nayak, G. Dixit and K. Appu Kuttan, "Mechanical properties of eco-friendly recycled polymer composites: a comparative study of theoretical and experimental results," Int J Plast Technol, vol. 17, (1), pp. 75-93, 2013. Available:

https://search.proquest.com/docview/1400587951. DOI: 10.1007/s12588-013-9050-z.

[20] M. Naushad et al, "Mechanical and damage tolerance behavior of short sisal fiber reinforced recycled polypropylene biocomposites," Journal of Composite Materials, vol. 51, (8), pp. 1087-1097, 2017. Available: https://journals.sagepub.com/doi/full/10.1177/00219983 16658945. DOI: 10.1177/0021998316658945.

[21] K. Das et al, "Development of Recycled Polypropylene Matrix Composites Reinforced with Waste Jute Caddies," Journal of Reinforced Plastics and Composites, vol. 29, (2), pp. 201-208, 2010. Available: https://journals.sagepub.com/doi/full/10.1177/07316844 08096929. DOI: $10.1177 / 0731684408096929$.

[22] T. P. Nguyen, C. Sollogoub and A. Guinault, "Relationship between fiber chemical treatment and properties of recycled pp/bamboo fiber composites," Journal of Reinforced Plastics \& Composites, vol. 29, (21), pp. 3244-3256, 2010. Available: https://search.ebscohost.com/login.aspx?direct=true\&d $\mathrm{b}=\mathrm{a} 9 \mathrm{~h} \& \mathrm{AN}=55060828 \&$ lang $=$ es\&site $=$ ehost-live. $\quad$ DOI: 10.1177/0731684410370905.

[23] O. A. Saputra et al, "Improvement of ThermoMechanical Properties of Short Natural Fiber Reinforced Recycled Polypropylene Composites through Double Step Grafting Process," IOP Conference Series: Earth and Environmental Science, vol. 75, pp. 12023, 2017. . DOI: 10.1088/1755-1315/75/1/012023.

[24] T. Zi-Ni et al, "Characteristics of Metroxylon sagu Resistant Starch Type III as Prebiotic Substance," Journal of Food Science, vol. 80, (4), pp. H875-H882, 2015. Available: https://onlinelibrary.wiley.com/doi/abs/10.1111/17503841.12817. DOI: 10.1111/1750-3841.12817.

[25] A. Raju and M. Shanmugaraja, "Recent researches in fiber reinforced composite materials: A review," Materials Today: Proceedings, 2020. Available: http://www.sciencedirect.com.crai-

ustadigital.usantotomas.edu.co/science/article/pii/S221 4785320308920. DOI: https://doi-org.craiustadigital.usantotomas.edu.co/10.1016/j.matpr.2020.02 141.

[26] C. Tan, I. Ahmad and M. Heng, "Characterization of polyester composites from recycled polyethylene terephthalate reinforced with empty fruit bunch fibers," Materials \& Design, vol. 32, (8), pp. 4493-4501, 2011. Available: $\quad$ http://www.sciencedirect.com.craiustadigital.usantotomas.edu.co/science/article/pii/S026 1306911002007. DOI: https://doi-org.craiustadigital.usantotomas.edu.co/10.1016/j.matdes.2011.0 3.037 .

[27] R. S. Chen and S. Ahmad, "Mechanical performance and flame retardancy of rice husk/organoclayreinforced blend of recycled plastics," Materials Chemistry and Physics, vol. 198, pp. 57-65, 2017. Available: http://www.sciencedirect.com.craiustadigital.usantotomas.edu.co/science/article/pii/S025 4058417304236. DOI: https://doi-org.craiustadigital.usantotomas.edu.co/10.1016/j.matchemphys .2017.05.054. 


\title{
TENDENCIAS DEL APROVECHAMIENTO DE LA CASCARILLA DE ARROZ CON MATRICES POLIMÉRICAS RECICLADAS PARA LA FORMULACIÓN DE NUEVOS MATERIALES Y SUS POSIBLES APLICACIONES
}

\section{Trends in the use of rice husk with recycled polymeric matrices for the formulation of new materials and their possible applications}

\author{
Diaz, Natalia; Mora, Laura1 ; Ospina, Sebastián; Orjuela, David; Solano, Johanna \\ Universidad Santo Tomás, Facultad de Ingeniería Ambiental
}

\section{Resumen}

La generación de residuos como el plástico y la cascarilla de arroz (CA) ha aumentado con el paso del tiempo, ocasionando problemas de contaminación por su inadecuado manejo y falta de aprovechamiento. El enfoque de esta investigación es consultar mezclas entre polímeros y CA que permitan obtener compuestos que cumplan con las propiedades térmicas, mecánicas y químicas, óptimas para construir prototipos que puedan ingresar al mercado y generar beneficios tanto ambientales como económicos. En la búsqueda realizada en bases de datos se encontraron estudios sobre mezclas compuestas principalmente por polietileno de alta densidad (HDPE), polipropileno (PP), poliestireno (PS), entre otros, y CA, y que a su vez contaban con la presencia o ausencia de un agente de acoplamiento (AGC), cuyo fin era modificar la superficie de la CA y mejorar la adhesión entre la matriz polimérica y la fibra. Por medio de este trabajo se determinó que la influencia tanto de los AGC como de la CA sobre las matrices poliméricas mejora propiedades como la dureza, la temperatura de deflexión térmica, resistencia a la tracción, el módulo de Young, resistencia térmica, flexión, lo que permite usar los compuestos en industrias como la automotriz y la de construcción.

Palabras claves: Matriz polimérica, cascarilla de arroz, aprovechamiento, compuestos.

\footnotetext{
${ }^{1}$ Correo electrónico de contacto: laurajmora@usantotomas.edu.co
} 


\section{INTRODUCCIÓN}

En Colombia, la producción de CA es de aproximadamente 400.000 toneladas al año [1], a pesar de que dicho volumen es considerable, el aprovechamiento que se le da es inadecuado generando tanto problemas ambientales como económicos, ya que las empresas deben asumir los costos de gestión ambiental de este [2]. Cabe mencionar que la CA se caracteriza por ser un desecho de difícil biodegradación por sus propiedades fisicoquímicas y aunado a esto, representa aproximadamente el $20 \%$ en peso de la producción de arroz, generando grandes volúmenes de residuo que además al tener baja densidad $100 \mathrm{~kg} / \mathrm{m}^{3}$, ocasiona problemas de espacio, por lo tanto, se hace necesaria su pronta evacuación, implicando costos elevados por el transporte de este [3]. Adicionalmente, desde el año 2012 se ha incrementado la producción de arroz en Colombia a tal punto que en el año 2017 se obtuvo una producción de 2’059.035 toneladas de arroz [4], indicando que entre el 2012 y 2017 hubo un aumento de aproximadamente el 37\% [5], estas cifras son preocupantes, porque a mayor producción, más residuo se genera; además, la disposición final que se le suele dar es en los rellenos sanitarios, lo que conlleva a la rápida disminución de la vida útil de estos.

Sin embargo, en los últimos años se ha despertado un interés particular por la valorización de la CA, ya que puede ser utilizada como materia prima o insumo de otro proceso productivo por las propiedades que posee, esto implica que se pueden generar productos de valor agregado [6], [7] por ello, deja de ser un problema y se convierte en un ingreso adicional para la industria que lo produce. La CA ha sido aprovechada para lograr materiales que se utilizan de manera inmediata o en diferentes formas, entre ellas: producción de aglomerados, material aislante en construcción, para camas en avicultura, porcicultura y transporte de ganado, compostaje y como lecho filtrante en aguas residuales, obtención de etanol por vía fermentativa, obtención de cerámicas, concreto y cemento, entre otros [8].

Por otro lado, un residuo que genera problemas importantes es el plástico. Se estima que para el año 2015 se produjo 6.300 millones de ton métricas de plásticos vírgenes, de los cuales alrededor del $9 \%$ se reciclaron, el 12\% se incineró y el 79\% se acumularon en vertederos o en el ambiente natural [9]. Esto se debe al uso masivo e indiscriminado y que además no es aprovechado a pesar de que su ingreso a la cadena de reciclaje es sencillo; esto demuestra que los programas de reciclaje y reutilización de residuos plásticos son ineficientes [10].

En estudios realizados anteriormente se evidenció que la CA se ha mezclado con una variedad de polímeros en los cuales se destacan el PP [11]-[15], HDPE [16]-[20], PS [21],[22], policloruro de 
vinilo PVC [23], entre otros. A partir de esta información se determinó que los compuestos que se elaboraron presentan una mejora en sus propiedades mecánicas, químicas, físicas pero especialmente en sus propiedades térmicas debido a la adición de esta fibra. Sin embargo, el porcentaje de CA que se emplea en los compuestos tiene un efecto variable dependiendo de la matriz polimérica usada como se evidencia en la tabla 1.

\section{MATERIALES Y MÉTODOS}

Para la elaboración de este artículo de revisión se hizo una búsqueda de artículos científicos, de investigación y libros, en diferentes tipos de bases de datos como lo son: Sciencedirect $14 \%$, Scopus $27 \%$, Google académico 10\%, Web Of Science 10\%, SciELO 7\% y repositorios universitarios 32\%, con el fin de realizar la compilación de información relacionada con las mezclas hechas a base de CA y polímeros reciclados, además se verificó que la fecha de publicación se encontrara en un rango entre los años 2005 y 2020. Como criterios de búsqueda en las bases de datos, se utilizaron los siguientes descriptores: cascarilla de arroz, polímeros reciclados, mezcla de polímeros y cascarilla de arroz, matriz polimérica, compuestos poliméricos. En total para la construcción del artículo se tuvieron en cuenta 42 referencias bibliográficas las cuales fueron elaboradas por medio de la herramienta de gestión bibliográfica RefWorks, ya que en dichos artículos se encontró información que coincidía con los parámetros de búsqueda establecidos anteriormente, a partir de esto se procedió a hacer la debida elaboración del artículo de revisión plasmando de manera clara y concisa la información para al final realizar un análisis adecuado y así poder formular las conclusiones respectivas.

Además de esto, para obtener mejores resultados en la investigación se planteó la pregunta orientadora ¿Cuál es la influencia de la CA sobre los compuestos elaborados en comparación con los materiales originales? Con el fin de hacer más compleja la resolución de dicha pregunta se plantearon interrogantes como ¿Cuáles son las propiedades que obtienen una mejora con la adición de la CA?, ¿Cuáles son los AGC y su influencia?, ¿Cuáles son los posibles usos con las mezclas identificadas?.

\section{RESULTADOS Y ANÁLISIS DE RESULTADOS}

Se han desarrollado diferentes matrices poliméricas usando la CA como complemento y sus proporciones en la mezcla tal como se evidencia en la tabla 1, varían de acuerdo con las características a las que se quiera llegar para ser aplicado en un uso específico [24]. Así, cuando el uso está enfocado a exteriores, en la mezcla se realizan pruebas de absorción de agua (WA) y foto envejecimiento, con el fin de determinar qué tanto se afectan las propiedades mecánicas y 
químicas del compuesto. Esta clase de pruebas se hacen principalmente en lugares donde hay estaciones, por el cambio brusco en la humedad del ambiente o cuando el compuesto se aplicará en interiores (como baldosas) donde se someterá a presión y humedad constante. La literatura reporta que la exposición a los rayos UV genera decoloración del compuesto, sin embargo, las propiedades mecánicas no se ven afectadas significativamente, por otro lado, se evidencia que cuando hay un AGC la WA disminuye, debido a que hay mejor interacción o adhesión entre la matriz polimérica y la fibra [18],[25]-[28].

En diversos estudios de matrices poliméricas con CA como fibra, se han utilizado AGC, con el fin de ver su efecto sobre las propiedades físico-mecánicas, químicas y en algunos casos térmicas de los compuestos, a partir de esto, se pudo identificar que uno de los agentes más usado es el anhídrido maléico (MA), ya que al mezclarlo con los polímeros, permite que la dispersión de las fibras mejore, además, se evidenció que en las mezclas mejora las propiedades mecánicas y disminuye el porcentaje de WA; en general, los AGC mejoran la interfaz matriz-fibra, lo que se traduce en un mayor rendimiento de las propiedades de los compuestos[11], [16],[18], [23],[29][31].

Las proporciones de carga en peso de cada compuesto deben estar o bien direccionadas hacia un producto específico o elegir la mezcla que con cierta combinación de polímero-fibra proporcione las mejores características mecánicas, térmicas y demás posible, para que su aplicación sea más amplia. Sin embargo, de acuerdo a las proporciones que se presentan en la Tabla 1, el rango de adición de CA más adecuado cuando el polímero usado es PP varía entre 25 y 50\%, cuando se utiliza HDPE la proporción de CA es mayor, entre 30 y 70\% y la proporción cuando se usa PS es de 20 y 30\% . La literatura reporta que cuanto más alta es la carga en peso de la fibra en el compuesto, sin emplearse un AGC, propiedades como la resistencia al impacto y la elongación a la rotura disminuyen, esto se debe a que en el compuesto se desmejora la capacidad de adhesión entre la fibra y la matriz, produciendo fragilidad por el aumento en la rigidez [32]-[35]

Tabla 1. Característica de compuestos poliméricos y cascarilla de arroz

\begin{tabular}{|c|c|c|c|c|}
\hline Matriz polimérica & $\begin{array}{c}\text { Tratamiento } \\
\text { previo a la CA }\end{array}$ & Pruebas realizadas & $\begin{array}{c}\text { Efecto de la CA y agentes } \\
\text { compatibilizantes sobre las } \\
\text { propiedades }\end{array}$ & Ref. \\
\hline $\begin{array}{c}\text { Compatibilizador } \\
\text { Polipropileno MA } \\
\text { (MAPP) y AGC } \\
\text { Titanato (Ti) } \\
50 \text { PP / } 50 \text { CA, AGC } 0,3 \\
\text { a } 0,6 \text {. }\end{array}$ & $\begin{array}{l}\text { Tamaño de } \\
\text { partícula malla } \\
\qquad 24\end{array}$ & $\begin{array}{c}\text { Temperatura de deflexión } \\
\text { térmica (HDT), temperatura de } \\
\text { degradación, entalpía de fusión } \\
\text { y degradación }\end{array}$ & $\begin{array}{l}\text { Mejora de adhesión entre la CA y el } \\
\text { PP con la adición de MAPP y Ti } \\
\text { Mejora HDT y temperatura de } \\
\text { degradación, disminuye entalpía de } \\
\text { fusión y degradación. El punto de } \\
\text { fusión de compuestos CA-PP no }\end{array}$ & [11] \\
\hline
\end{tabular}




\begin{tabular}{|c|c|c|c|c|}
\hline Compatibilizante: $3 \%$ & & & $\begin{array}{l}\text { muestra cambios significativos } \\
\text { Uso: Industria automotriz, } \\
\text { doméstica, electrónica y deportiva }\end{array}$ & \\
\hline $\begin{array}{l}\text { Cargas } 50 \mathrm{CA} / 48 \mathrm{PP} / 2 \\
\text { Struktol }(\% \mathrm{p} / \mathrm{p})\end{array}$ & \multirow{2}{*}{$\begin{array}{l}\text { Tamaño de } \\
\text { partícula 100- } \\
500 \mu \mathrm{m}\end{array}$} & $\begin{array}{l}\text { Resistencia a la tracción, a la } \\
\text { flexión y módulo de flexión y } \\
\text { microscopía electrónica de } \\
\text { barrido (SEM) }\end{array}$ & $\begin{array}{l}\text { Propiedades mecánicas óptimas y a } \\
\text { mayor carga de CA se producen más } \\
\text { vacíos }\end{array}$ & \multirow[t]{2}{*}{ [12] } \\
\hline $\begin{array}{l}\text { Cargas } 35 \mathrm{CA} / 63 \mathrm{PP} / 2 \\
\quad \text { Struktol }(\% \mathrm{p} / \mathrm{p})\end{array}$ & & $\begin{array}{l}\text { Resistencia al impacto y a la } \\
\text { flexión, módulo de flexión y de } \\
\text { Young y SEM }\end{array}$ & $\begin{array}{l}\text { Mejor rendimiento de propiedades } \\
\text { mecánicas y morfológicas }\end{array}$ & \\
\hline $\begin{array}{c}59.5 \% \text { PP; } \\
\text { 37\% CA Y } 3.5 \% \text { PP con } \\
\text { MA }\end{array}$ & \multirow{3}{*}{$\begin{array}{l}\text { Disminución del } \\
\text { tamaño de } \\
\text { partícula } \\
\text { aproximadamen } \\
\text { te } 400 \mu \mathrm{m}\end{array}$} & \multirow{3}{*}{$\begin{array}{l}\text { Análisis Dinámico Mecánico } \\
\text { (DMA), SEM, ensayos de } \\
\text { impacto y rigidez, módulo de } \\
\text { almacenamiento y tangente de } \\
\text { pérdidas }\end{array}$} & $\begin{array}{l}\text { Aumento del módulo de } \\
\text { almacenamiento, mejora en la } \\
\text { rigidez y disminución de la tangente } \\
\text { de pérdidas e impacto }\end{array}$ & \multirow{3}{*}{ [13] } \\
\hline $\begin{array}{c}59.5 \% \text { PP; } 37 \% \text { CA y } \\
3.5 \% \text { estireno-etileno- } \\
\text { butadieno con MA }\end{array}$ & & & $\begin{array}{l}\text { Mejora de la tensión de impacto y } \\
\text { reducción de la rigidez }\end{array}$ & \\
\hline $\begin{array}{c}61.5 \% \text { PP } \\
37 \% \text { CA } \\
1.5 \% \text { Silano }(\mathrm{Si})\end{array}$ & & & $\begin{array}{l}\text { Aumento del módulo de } \\
\text { almacenamiento y rigidez, } \\
\text { disminución de la tangente de } \\
\text { pérdidas e impacto }\end{array}$ & \\
\hline $\begin{array}{c}92 \% \text { PP; } 5 \% \text { CA y } 3 \% \\
\text { MAPP con } \\
\text { polipropileno } \\
\text { maleizado (MAPP) }\end{array}$ & $\begin{array}{l}\text { Tamaño entre } \\
250 \text { a } 500 \mu \mathrm{m}\end{array}$ & $\begin{array}{l}\text { Ensayos de tracción, flexión, } \\
\text { impacto (IZOD), dureza, } \\
\text { análisis termogravimétrico } \\
\text { (TGA), degradación }\end{array}$ & $\begin{array}{l}\text { Aumento de deformación, rigidez, } \\
\text { resistencia a la temperatura, a la } \\
\text { tracción, flexión y al impacto y } \\
\text { degradación completa }\end{array}$ & [14] \\
\hline $5 \%, 10 \%$ y $15 \%$ PP y CA & $\begin{array}{l}\text { Tamaño entre } \\
250 \text { a } 150 \mu \mathrm{m}\end{array}$ & $\begin{array}{l}\text { Ensayo de tracción, flexión, } \\
\text { impacto, } \\
\text { diferencia de barrido y TGA }\end{array}$ & $\begin{array}{l}\mathrm{Al} 10 \% \text { en peso se tiene mayor } \\
\text { porcentaje de deformación y mejor } \\
\text { resistencia a la tracción }\end{array}$ & [15] \\
\hline $\begin{array}{c}46 \% \mathrm{HDPE}, 4 \% \text { MA y } \\
50 \% \text { CA }\end{array}$ & \multirow{2}{*}{$\begin{array}{l}\text { Secar CA a } 105 \\
{ }^{\circ} \mathrm{C} \text { por } 24 \mathrm{~h} \text { y } \\
\text { tamizado malla } \\
\quad 600 \mu \mathrm{m}\end{array}$} & \multirow{2}{*}{$\begin{array}{l}\text { Resistencia al impacto, WA e } \\
\text { índice de fluidez }\end{array}$} & \multirow{2}{*}{$\begin{array}{l}\text { Reducción del índice de fluidez y } \\
\text { aumento de la WA a mayor } \\
\text { contenido de CA }\end{array}$} & \multirow{2}{*}{ [16] } \\
\hline $\begin{array}{c}66 \% \text { HDPE, } 4 \% \text { MA y } \\
30 \% \text { CA }\end{array}$ & & & & \\
\hline $\begin{array}{c}0 \%, 10 \% \text { y } 15 \% \text { de } \\
\text { CCA, } 0 \%, 10 \%, 20 \% \text { y } \\
30 \% \text { de Polybond y } \\
\text { HDPE }\end{array}$ & $\begin{array}{l}\text { Incineración a } \\
500^{\circ} \mathrm{C} \text { por } 30 \\
\text { min. }\end{array}$ & $\begin{array}{l}\text { Resistencias a la tracción y el } \\
\text { módulo de Young y SEM }\end{array}$ & $\begin{array}{l}\text { Al emplear } 10 \% \text { de Polybond y una } \\
\text { mayor dosis de CCA se aumenta la } \\
\text { resistencia de la mezcla }\end{array}$ & [17] \\
\hline $\begin{array}{c}\text { Matriz HDPEr 50/ PETr } \\
50 \text { (p/p) Carga CA 40, } \\
60 \text { y } 80 \% \\
\text { Metacrilato de glicidilo } \\
8 \% \\
\text { MAPE 3\% en peso }\end{array}$ & $\begin{array}{l}\text { Tamaño de } \\
\text { partícula malla } \\
\text { de } 100\end{array}$ & $\begin{array}{l}\text { Resistencia a la tracción y } \\
\text { flexión, elongación a la rotura y } \\
\text { resistencia al impacto, WA de } \\
\text { mar y destilada y SEM }\end{array}$ & $\begin{array}{l}\text { Aumenta el módulo de tracción, } \\
\text { flexión y la WA y disminuye la } \\
\text { elongación a la rotura y la resistencia } \\
\text { al impacto a mayor } \\
\text { CA, menor WA con agua destilada. } \\
\text { Mejor unión entre matriz-matriz y } \\
\text { matriz-fibra con compatibilizantes } \\
\text { Uso: aplicaciones en exteriores }\end{array}$ & [18] \\
\hline $\begin{array}{l}65 \% \text { PS, } 5 \% \text { injerto de } \\
\text { CA y } 30 \% \text { CA y HDPE }\end{array}$ & & $\begin{array}{l}\text { Resistencias a la tracción y } \\
\text { flexión y WA }\end{array}$ & $\begin{array}{l}\text { Mejoras significativas frente a la } \\
\text { resistencia a la tracción y WA } \\
\text { Usos: aislantes acústicos }\end{array}$ & [21] \\
\hline $\begin{array}{c}20,30,40 \text { y } 50 \% \text { CA } \\
\text { PS }\end{array}$ & & $\begin{array}{l}\text { Resistencia a la tracción, } \\
\text { flexión e impacto }\end{array}$ & $\begin{array}{l}\text { Mejora módulo de tracción y } \\
\text { disminuye deformación con }\end{array}$ & [22] \\
\hline
\end{tabular}




\begin{tabular}{|c|c|c|c|c|}
\hline & & & $\begin{array}{l}\text { aumento de CA } \\
\text { Usos: elaboración de cielo rasos }\end{array}$ & \\
\hline $\begin{array}{c}\text { rHDPE / rPET / E-GMA } \\
\text { a 75/25/5 relación en } \\
\text { peso } \\
\text { Carga de CA } \\
\text { 40,50,60,70 Y } 80 \% \\
\text { MAPE 3\% }\end{array}$ & $\begin{array}{l}\text { Tamaño de } \\
\text { partícula malla } \\
\text { de } 100\end{array}$ & $\begin{array}{l}\text { Resistencia a la tracción y el } \\
\text { módulo de Young, WA, } \\
\text { estabilidad dimensional y } \\
\text { entalpía de combustión }\end{array}$ & $\begin{array}{l}\text { Mejor rendimiento de resistencia a } \\
\text { tracción y módulo de Young a } 70 \% \\
\text { de CA. La WA aumentó en función } \\
\text { de la CA, estabilidad dimensional } \\
\text { aceptable. Entalpía de combustión } \\
\text { disminuye con mayor carga de CA. }\end{array}$ & [19] \\
\hline $\begin{array}{l}\text { Cargas de CA de 10,20 } \\
\text { y 30\% con PP y HDPE }\end{array}$ & ---------- & $\begin{array}{l}\text { Resistencia a la compresión, a } \\
\text { la tracción, densidad del } \\
\text { material y a agentes químicos }\end{array}$ & $\begin{array}{l}\text { Mejor resistencia a compresión y } \\
\text { tracción con mezclas del } 30 \% \text { y } 10 \% \\
\text { de CA respectivamente } \\
\text { disminución de densidad con } \\
\text { aumento CA y baja afectación por } \\
\text { agentes químicos } \\
\text { Uso: postes para uso agropecuario }\end{array}$ & [20] \\
\hline
\end{tabular}

Sin embargo, hay otras propiedades que mejoran con el aumento de la cascarilla como la dureza, la temperatura de deflexión térmica, resistencia a la tracción y el módulo de Young, resistencia térmica, flexión y la estabilidad dimensional del compuesto [14], [16], [18],[36].

Otro factor que influye en el rendimiento de las propiedades de los compuestos es el tamaño de partícula de la CA, ya que al disminuir su tamaño se obtienen mejores propiedades físicomecánicas en el compuesto [14],[18],[19],[30],[37]. Por otro lado, Rezaur Rahman et al, sostienen que tratar químicamente la CA en medios alcalinos mejora las propiedades mecánicas de los compuestos respecto a la cascarilla cruda [38]. Un estudio previo evidenció que al calcinar la CA se obtienen mejores propiedades de refuerzo en los compuestos a causa de que la ceniza es amorfa, ya que esta posee baja energía de activación, es reactiva y absorbente para formar compuestos [17].

Las aplicaciones de las matrices poliméricas con cargas de cascarilla de arroz son muy variadas y dependen de los polímeros usados y de las proporciones de las cargas en peso en la mezcla, sin embargo, muchas están direccionadas hacia productos en el área de construcción, el mercado automotriz, en electrónica, usos deportivos, usos en ambientes húmedos, usos domésticos, en materiales de amortiguación [11], [16],[25], [37], [39], [40]-[42].

\section{CONCLUSIONES}

De acuerdo con la revisión realizada, los compuestos elaborados a partir de las matrices poliméricas, la CA y AGC, se evidenció que estos presentan una mejora principalmente sobre las propiedades térmicas de los compuestos debido a que este residuo agroindustrial posee buenas 
condiciones de resistencia térmica, por la presencia de sílice, es por esto que para la construcción de un prototipo para la venta en el mercado se recomienda que se realicen mezclas con termoplásticos como el polipropileno para así potencializar los compuestos y que se puedan emplear en sectores industriales donde se requiera un material altamente resistente como aislantes térmicos y acústicos, en usos domésticos como piezas de hornos de microondas y en la industria automotriz para la construcción de partes que se ubiquen debajo del capó. No obstante, las mezclas realizadas con polímeros diferentes a los termoplásticos presentan propiedades viables para ser empleadas en el sector de la construcción y en el diseño de interiores a un bajo costo de ejecución. Sin embargo, los posibles usos que pueden proporcionar las mezclas se pueden explorar con más detalle, con el fin de aumentar la cartera de opciones de aplicación de los compuestos.

\section{REFERENCIAS}

[1] G. A. Velasco, A. M. Campos, J. Proaños, Y. Piñeros Castro, W. Cortés, y Á. M. Otálvaro, Aplicación de tecnologías para el aprovechamiento de la cascarilla de arroz. 2011.

[2] S. M. Yepes, L. J. Montoya Naranjo and F. Orozco Sánchez, "Valoración de residuos agroindustriales frutas - en Medellín y el sur del Valle de Aburrá, Colombia," Revista Facultad Nacional De Agronomía Medellín, vol. 61, (1), pp. 4422-4431, 2008. Available: http://www.scielo.org.co/scielo.php?script=sci_arttex t\&pid=S0304-28472008000100018\&lng=en\&tlng=en.

[3] Jaider Sierra Aguilar, "Alternativas de aprovechamiento de la cascarilla de arroz en Colombia." $2009 . \quad$ Available: https://pdfs.semanticscholar.org/640e/26eec51fc43a8c c5dba67ced0d423ecc941a.pdf.

[4] Fedearroz, "Encuesta Nacional de Arroz Mecanizado (ENAM)", feb. 08, 2019.

[5] A. E. Anaya Vargas, "Análisis Del Sector Arrocero Colombiano Frente Al TLC Con Estados Unidos.", Universidad Santo Tomás, Bucaramanga, 2019.

[6] N. Mejias Brizuela, E. Orozco Guillen and N. Galán Hernández, "Aprovechamiento de los residuos agroindustriales y su contribución al desarrollo sostenible de México," Revista De Ciencias Ambientales Y Recursos Naturales, vol. 2, (6), pp. 27-41, 2016. Available:

https://www.researchgate.net/publication/323959087 Aprovechamiento de los residuos agroindustriale s_y_su_contribucion_al_desarrollo_sostenible_de_M exico.

[7] S. S. Hossain and P. K. Roy, "Fabrication of sustainable insulation refractory: Utilization of different wastes," Boletín De La Sociedad Española De Cerámica Y Vidrio, vol. 58, (3), pp. 115-125, 2019. Available: http://dx.doi.org/10.1016/j.bsecv.2018.09.002. DOI: 10.1016/j.bsecv.2018.09.002.

[8] A. Prada and C. E. Cortés, "La descomposición térmica de la cascarilla de arroz: una alternativa de aprovechamiento integral," Orinoquia, vol. 14, (suppl 1), pp. 155-170, 2010. Available: http://www.scielo.org.co/scielo.php?script=sci arttex t\&pid=S0121-37092010000300013\&lng=en\&tlng=en.

[9] R. Geyer, J. R. Jambeck and K. L. Law, "Production, use, and fate of all plastics ever made," Sci Adv, vol. 3,
(7), pp.
e1700782,
2017.
Available:

http://advances.sciencemag.org/content/3/7/e1700782. abstract. DOI: 10.1126/sciadv.1700782.

[10] Clínica Jurídica de Medio Ambiente y Salud Pública (MASP) de la Facultad de Derecho de la Universidad de los Andes and Greenpeace Colombia, "Situación actual de los plásticos en Colombia y su impacto en el medio ambiente," Noviembre de. 2019.

[11] V. Kumar et al, "Study of Thermal Properties of Rice Husk Polypropylene (RHPP) Composites," Advanced Composites Letters, vol. 22, (6), pp. 96369351302200 , 2013.. DOI: $10.1177 / 096369351302200601$.

[12] N. A. M. Aridi et al, "Mechanical and morphological properties of injection-molded rice husk polypropylene composites," International Journal of Polymer Analysis and Characterization, vol. 21, (4), pp. 305-313,

2016. Available:http://www.tandfonline.com/doi/abs/10.10 80/1023666X.2016.1148316.DOI:10.1080/1023666X.201 6.1148316

[13] F. Vilaplana, A. Ribes Greus, F. Martí Ferrer, A. Benedito Borrás, y C. Sanz Box, "Efecto de diferentes agentes compatibilizantes sobre el comportamiento viscoelástico de materiales compuestos en base polipropileno reforzados con cáscara de arroz", p. 8. 
[14] T. Sánchez, "Caracterización de un material compuesto de polipropileno reforzado con cascarilla de arroz obtenido por extrusión", Escuela Politécnica Nacional, Quito - Ecuador, 2019.'

[15] T. Sánchez, "Estudio del comportamiento mecánico de un material compuesto con matriz de polipropileno al añadir partículas de cascarilla de oryza sativa (arroz)", Escuela Politécnica Nacional, Quito - Ecuador, 2019.

[16] J. Cevallos, "Estudio Comparativo De Las Propiedades Mecánicas y Reológicas De Compuestos De Polietileno De Alta Densidad Con Cascarilla De Arroz y Bagazo De Caña", Escuela Superior Politécnica del Litoral, Guayaquil - Ecuador, 2008.

[17] V. A. B. Paredes, "Estudio del comportamiento termo mecánico de compuestos de polietileno de alta densidad (HDPE) reciclado y ceniza de cascarilla de arroz (CCA)", Universidad Nacional de San Agustín, Arequipa-Perú, 2018.

[18] R. S. Chen et al, "Mechanical, water absorption, and morphology of recycled polymer blend rice husk flour biocomposites," Journal of Applied Polymer Science, vol. 132, (8), pp. n/a, 2015. Available:

https://onlinelibrary.wiley.com/doi/abs/10.1002/app.41494. DOI: 10.1002/app.41494

[19] R. S. Chen et al, "High loading rice husk green composites: Dimensional stability, tensile behavior and prediction, and combustion properties," Journal of Thermoplastic Composite Materials, pp. 89270571881553, 2019.. DOI: $10.1177 / 0892705718815536$.

[20] J. M. Baller and A. Ríos, "Evaluación del uso de cascarilla de arroz, polietileno y polipropileno en la producción de postes para uso agropecuario," Informador Técnico, vol. 80, (2), pp. 40, 2016. Available:

https://search.proquest.com/docview/1898618327.

[21] M. E. Tawfik, S. B. Eskander, y G. A. Nawwar, "Hard wood-composites made of rice straw and recycled polystyrene foam wastes", Journal of Applied Polymer Science, may 10, 2017. https://onlinelibrary.wiley.com/doi/abs/10.1002/app.4 $\underline{4770}$ (accedido jun. 19, 2020).

[22] S. I Wayan, I Gusti Agung Kade, Suriadi and K. Arnis, "Mechanical Properties of Rice Husks Fiber Reinforced Polyester Composites," International Journal of Materials, Mechanics and Manufacturing, vol. 2, (2), pp. 165-168, 2014. Available: https://pdfs.semanticscholar.org/1491/c618f515c17495 1e2032c2b08bfc9f249fed.pdf..DOI:

10.7763/IJMMM.2014.V2.121.

[23] Navin Chand and Bhajan Das Jhod, "Mechanical, electrical, and thermal properties of maleic anhydride modified rice husk filled PVC composites," BioResources, vol. 3, (4), pp. 1228-1243, 2008. Available:

https://doaj.org/article/6ee315bd79244769bf234c7ff5fa 20cf.

[24] Reza Arjmandi et al, "Rice Husk Filled Polymer Composites," International Journal of Polymer Science, vol. 2015, pp. 1-32, 2015. Available: http://dx.doi.org/10.1155/2015/501471. DOI: 10.1155/2015/501471.

[25] Mohd Hafizuddin Ab Ghani and Sahrim Ahmad, "The Comparison of Water Absorption Analysis between Counter Rotating and Co Rotating TwinScrew Extruders with Different Antioxidants Content in Wood Plastic Composites," Advances in Materials Science and Engineering, vol. 2011, pp. 1-4, 2011. Available: $\quad$ http://dx.doi.org/10.1155/2011/406284. DOI: $10.1155 / 2011 / 406284$..

[26] S. Panthapulakkal, S. Law and M. Sain, "Effect of water absorption, freezing and thawing, and photoaging on flexural properties of extruded HDPE/rice husk composites," J Appl Polym Sci, vol. 100, (5), pp. 3619-3625, 2006. Available: https://doi.org10.1002/app.23152. DOI: 10.1002/app.3152.

[27] A. Mora ortilla, "Propuesta de diseño y desarrollo preliminares de un prototipo de baldosa para piso a partir del PET reciclado y la cascarilla de arroz para una vivienda de interés social," Revista Científica YACHANA, vol. 3, (1), pp. 84-90, 2014.

[28] M. A. ovoa Galeano, L. D. Becerra León and M. P. Vásquez Piñeros, "La ceniza de cascarilla de arroz y su efecto en adhesivos tipo mortero," Avances Investigación En Ingeniería, vol. 11, (2), 2016.. DOI: 10.18041/1794-4953/avances.2.233.

[29] S. Panthapulakkal, M. Sain and S. Law, "Effect of coupling agents on rice-husk-filled HDPE extruded profiles," Polymer International, vol. 54, (1), pp. 137142, $2005 . \quad$ Available: https://onlinelibrary.wiley.com/doi/abs/10.1002/pi.16 57. DOI: 10.1002/pi.1657.

[30] R. Arjmandi et al, "Effects of ammonium polyphosphate content on mechanical, thermal and flammability properties of kenaf/polypropylene and rice husk/polypropylene composites," Construction and Building Materials, vol. 152, pp. 484-493, 2017. Available: http://dx.doi.org/10.1016/j.conbuildmat.2017.07.052. DOI: 10.1016/j.conbuildmat.2017.07.052.

[31] V. Amigó et al, Materiales Compuestos 05. (05th ed.) España: Editorial de la UPV, 2005.

[32] S. G. Nunes et al, "Study of Composites Produced with Recovered Polypropylene and Piassava Fiber," Materials Research, vol. 20, (1), pp. 144-150, 2016. Available:

http://www.scielo.br/scielo.php?script=sci arttext\&pi $\mathrm{d}=S 1516-14392017000100144 \& \operatorname{lng}=e n \& t \operatorname{lng}=$ en. DOI: 10.1590/1980-5373-mr-2016-0659.

[33] Rahman, W. A. W. A. et al, "Rice husk/high density polyethylene bio-composite: Effect of rice husk filler 
size and composition on injection molding processability with respect to impact property," Adv. Mater. Res., vol. 83-86, pp.367374,2010.Available:https://www.scopus.com/inward/ record.uri?eid $=2$-s2.0

75849147082\&doi $=10.4028 \% 2$ fwww.scientific.net $\% 2 \mathrm{f}$ AMR.8386.367\&partnerID $=40 \& m d 5=c c f f 22 b b 83 a 1737$ e3599cfc6eecf81d4.

DOI:

10.4028/www.scientific.net/AMR.83-86.367.

[34] M. Ismail et al, "Mechanical properties of hybrid glass fiber/rice husk reinforced polymer composite," Materials Today: Proceedings, vol. 27, pp. 1749-1755, 2020. Available: http://dx.doi.org/10.1016/j.matpr.2020.03.660. DOI: 10.1016/j.matpr.2020.03.660.

[35] C. Ganchozo and G. Zambrano, "Aprovechamiento De La Cascarilla De Arroz Y Plástico PET En La Fabricación De Eco-Bloques En La ESPAM MFL.", Escuela Superior Politécnica Agropecuaria de Manabí Manuel Félix López, 2018.

[36] Y. Xu et al, "Natural Fiber Reinforced Poly (vinyl chloride) Composites: Effect of Fiber Type and Impact Modifier," J Polym Environ, vol. 16, (4), pp. 250-257, 2008.

Available:

https://search.proquest.com/docview/226078857.

DOI: 10.1007/s10924-008-0113-8.

[37] S. M. L. Rosa et al, "Studies on the properties of ricehusk-filled-PP composites: effect of maleated PP," Materials Research (São Carlos, São Paulo, Brazil), vol.12, (3), pp. 333-338, 2009. Available: https://search.datacite.org/works/10.1590/s151614392009000300014. $\quad$ DOI: $10.1590 / \mathrm{s} 1516-$
14392009000300014.

[38] Md. Rezaur Rahman et al, "Effect of chemical treatment on Rice Husk (RH) reinforced Polyethylene (PE) composites," Bioresources, vol. 5, (2), pp. 854-869, 2010.

Available: https://doaj.org/article/41121cc4a0774bd5b909d5b130 $\underline{3764 \mathrm{c} 5}$

[39] E. F. Llaleo Galarza, "Caracterización mecánica del material compuesto de matriz poliéster reforzada con cascarilla de arroz para determinar las propiedades mecánicas en aplicaciones industriales," Universidad Técnica de Ambato Ecuador, 2016.

[40] G. P. Otto et al, "Mechanical properties of a polyurethane hybrid composite with natural lignocellulosic fibers," Compos Part B: Eng, vol. 110, pp. 459-465, 2017. Available: https://www.scopus.com/inward/record.uri?eid=2$\underline{\mathrm{s} 2.0-}$ $\underline{84998865645 \& \text { doi }=10.1016 \% 2 \text { fi. } . \text { compositesb. } 2016.11 .0}$ 35\&partnerID $=40 \& m d 5=c 9 b 34 a 5094 d d a 5 c e c 50 a 16 c d 7$ 65d2d96. DOI: 10.1016/j.compositesb.2016.11.035.

[41] Y. A. Vargas Corredor and L. I. Peréz Pérez, "Aprovechamiento de residuos agroindustriales en el mejoramiento de la calidad del ambiente," Revista Facultad De Ciencias Básicas, vol. 14, (1), pp. 59-72, 2018.. DOI: $10.18359 /$ rfcb.3108.

[42] P. A. Fowler, J. M. Hughes and R. M. Elias, "Biocomposites: technology, environmental credentials and market forces," J. Sci. Food Agric., vol. 86, (12), pp. 1781-1789, 2006. Available: https://doi.org/10.1002/jsfa.2558. DOI: 10.1002/jsfa.2 


\title{
PROPUESTA DISEÑO SISTEMA DE GESTIÓN AMBIENTAL ENFOCADO EN LA DISMINUCIÓN DE CO2 EN EL LABORATORIO DE MOTORES DIESEL, COMO CONTRIBUCIÓN A LA REDUCCIÓN DE LA HUELLA DE CARBONO EN LA UNIVERSIDAD ECCI
}

\section{Design of an environmental management system for the Diesel engine laboratory contributing to the reduction of the carbon footprint in ECCI University}

\author{
Sarmiento, Alejandro; Aparicio, Andrea ${ }^{1}$ \\ Universidad ECCI, Semillero de Investigación UERSIS
}

\section{Resumen}

La propuesta del semillero de investigación UERSIS - Utilización de Energías Renovables Sostenibles para la Industria y la Sociedad- en conjunto con el grupo del Sistema de Gestión Ambiental y Energético de la Universidad ECCI, surge de la necesidad de controlar la huella de carbono generada por la institución, desde la utilización de sus laboratorios, especialmente los pertenecientes al área automotriz del programa de ingeniería mecánica. Al observar el trabajo práctico que se realiza con los estudiantes y el docente al interior del laboratorio de motores Diesel, se evidencia que el espacio se llena de humo tóxico y aunque se tienen extractores, se debe abrir la puerta, generando contaminación ambiental hacia el exterior donde se encuentran cafeterías y viviendas familiares.

Se hace el diagnóstico simulando una sesión práctica, utilizando un analizador de gases midiendo emisiones de $\mathrm{CO}_{2}, \mathrm{CO}$, Oxígeno e hidrocarburos, en ambiente limpio, motor encendido-aceleradoapagado. Evidenciando que el encendido y apagado del motor generó aumento en el $\mathrm{CO}_{2}$ del ambiente creando molestias en las personas que se encuentran en él, adicional a esto, aunque se está certificado en la norma ISO 9001 ésta no se está cumpliendo.

Se hace la propuesta de elaborar un sistema de gestión ambiental para el laboratorio de motores Diesel con el fin de generar un estándar de normativas que permita las buenas prácticas al interior

${ }^{1}$ Correo electrónico de contacto: aapariciog@ecci.edu.co, Líder de semillero de investigación UERSIS 
del laboratorio y su cumplimiento, adicional será puesto a consideración ante el Sistema de Gestión Ambiental y Energético de la Universidad ECCI, para que sean ellos quienes lo implementen.

Palabras claves: Dióxido de Carbono, Medio Ambiente, Monóxido de carbono, Normas Ambientales, Analizador de gases.

\section{INTRODUCCIÓN}

El presente trabajo de investigación hace parte de un grupo de proyectos que se están llevando a cabo al interior del semillero de investigación UERSIS - Utilización de Energías Renovables Sostenibles para la Industria y la Sociedad, perteneciente al programa de ingeniería industrial y al grupo de Innovación Pedagógica-IP, apoyando los procesos que se vienen desarrollando de forma interdisciplinar en el grupo de trabajo del Sistema de Gestión Ambiental y Energético de la Universidad ECCI. El objetivo principal es trabajar en la disminución de la emisión de dióxido de carbono al ambiente, principalmente en el laboratorio de motores Diesel de la Institución, donde se realizan las prácticas del programa de tecnología en mecánica automotriz. Para iniciar el proceso de recolección de información, se realizaron una serie de entrevistas a estudiantes, docentes, auxiliar y director del laboratorio, en las cuales se evidencia que durante las prácticas en este laboratorio, especialmente cuando se utiliza un motor tipo Diesel, el ambiente se llena de gas tóxico que a pesar de tener los extractores y algunas medidas de evacuación de estos gases no son suficientes, teniendo que abrir las puertas del laboratorio, lo que ocasiona que los lugares aledaños (algunos restaurantes, cafeterías y edificio de apartamentos) se vean afectados por estos gases. Para continuar con el proceso y con ayuda de ingenieros mecánicos de la Universidad, se llevaron a cabo algunas mediciones de dióxido de carbono, hidrocarburos y nivel de oxígeno al interior del laboratorio, inicialmente con un solo motor Diesel. Se debe aclarar en este punto que no se lograron realizar más pruebas debido a las condiciones de salubridad nacional que se han presentado desde el mes de marzo de 2020, las cuales no permitieron ingresar nuevamente a la universidad para terminar de hacer las mediciones, pero con los datos recolectados se realiza un análisis de los niveles de los gases mencionados: dióxido de carbono, hidrocarburos y nivel de oxígeno, observando que con el encendido de un solo motor Diesel hay una afectación considerable del aire respirable al interior del laboratorio, y que el tiempo necesario para dispersar estos gases es de mínimo 40 minutos. Adicional a lo anterior se observa que, aunque se está certificado en la norma ISO 9001 en su apartado de gestión de los recursos en la cual se hace referencia a la infraestructura y normas de seguridad en este caso en el laboratorio, no se cumplen 
algunas como nomenclaturas o demarcaciones.

Después de analizar las falencias que se tienen especialmente en cuanto a la emisión de gases al ambiente, especialmente el dióxido de carbono se llega a la conclusión de que debe haber una concientización por parte de la comunidad universitaria: docentes, estudiantes, auxiliares y directores de laboratorio, para seguir las normas y estar monitoreando permanentemente estas emisiones. Por lo tanto, este trabajo concluye con la propuesta de un Sistema de Gestión Ambiental para Emisiones de Gases al ambiente con el objetivo de dar cumplimiento a la norma NTC ISO 14001 de 2015 [1] y presentar esta propuesta al Sistema de Gestión Ambiental y Energético de la Institución para su correspondiente implementación.

\section{MATERIALES Y MÉTODOS}

El tipo de investigación desarrollado en esta propuesta es de tipo cuantitativo no experimental, debido a que se pueden manipular aleatoriamente todas las variables, lo cual quiere decir que es una investigación en la cual no se hace variación de variables independientes de manera intencional [2]. Teniendo un alcance de estudio cuantitativo descriptivo, "Con los estudios descriptivos se busca especificar las propiedades, las características y los perfiles de personas, grupos, comunidades, procesos, objetos o cualquier otro fenómeno que se someta a un análisis. Es decir, únicamente pretenden medir o recoger información de manera independiente o conjunta sobre los conceptos o las variables a las que se refieren, esto es, su objetivo no es indicar cómo se relacionan éstas." [2]. Para recolectar los datos correspondientes a la emisión de gases, se utiliza el analizador de gases marca Hanatech que entrega el número de partículas por millón emitidas de: $\mathrm{CO} 2, \mathrm{CO}$, Oxígeno e hidrocarburos, adicional se utiliza la entrevista a un grupo focal de estudiantes, al auxiliar del laboratorio y al director de los laboratorios del programa de Ingeniería Mecánica como instrumento que permite recopilar los puntos de vista de las personas que utilizan el laboratorio. También se hace la búsqueda de información al interior de la universidad referente a si se encuentran sistemas de gestión ambiental vigentes o certificación en alguna normativa que tengan que ver con salud y seguridad industrial. Por último, se lleva a cabo el análisis de los resultados y hallazgos.

El procedimiento para la medición de gases se realiza por un período de 2 horas simulando una sesión de clase. Se toman datos con un analizador de gases en el laboratorio de motores Diésel, donde se hace la medición de la cantidad de carbono, monóxido, hidrocarburos y oxígeno, 
durante 10 minutos con tomas cada dos segundos antes de encender un carro con motor turbo Diésel (tabla Antes). Luego se enciende el vehículo y se realiza el mismo proceso de recolección de datos por 10 minutos cada 2 segundos (tabla Durante), pero en la mitad de la actividad se acelera el motor con el fin de observar si hay cambios en la cantidad de los gases medidos.

En seguida se debe observar el comportamiento y extracción de los gases dentro del laboratorio una vez se apaga el motor para saber si hubo o no contaminación; para esto, se espera 35 minutos se realiza una medición por 10 minutos con tomas de 2 segundos (tabla D35) y finalmente se esperan otros 15 minutos y nuevamente se hace la medición por 10 minutos con tomas de 2 segundos (Tabla 15+).

\section{RESULTADOS Y ANÁLISIS DE RESULTADOS}

Se generaron los siguientes hallazgos:

- De acuerdo con la tabla de datos recolectada correspondiente al monóxido de carbono se puede decir que antes de iniciar con las pruebas se tiene una línea continua y constante de 0,05\%, creando una variabilidad cuando se enciende el motor y se acelera podemos y aún después de 50 minutos no se puede volver a tener la línea continua inicial mostrando picos distanciados de $0,06 \%$.

- En la tabla de datos recolectada de los hidrocarburos se evidencia como antes de empezar las pruebas el nivel de este tipo de gases está demasiado bajo y luego asciende notoriamente al encender el motor y al acelerarlo no se nota la diferencia, esta se ve después de apagar el motor y realizar la medición después de 35 minutos como llega a los picos más altos y vuelve a descender después de 50 minutos, pero aun así no vuelven a su punto de partida después de la hora.

- Teniendo en cuenta que el más importante es el análisis para el dióxido de carbono $\left(\mathrm{CO}_{2}\right)$ se muestra a continuación su resumen: 


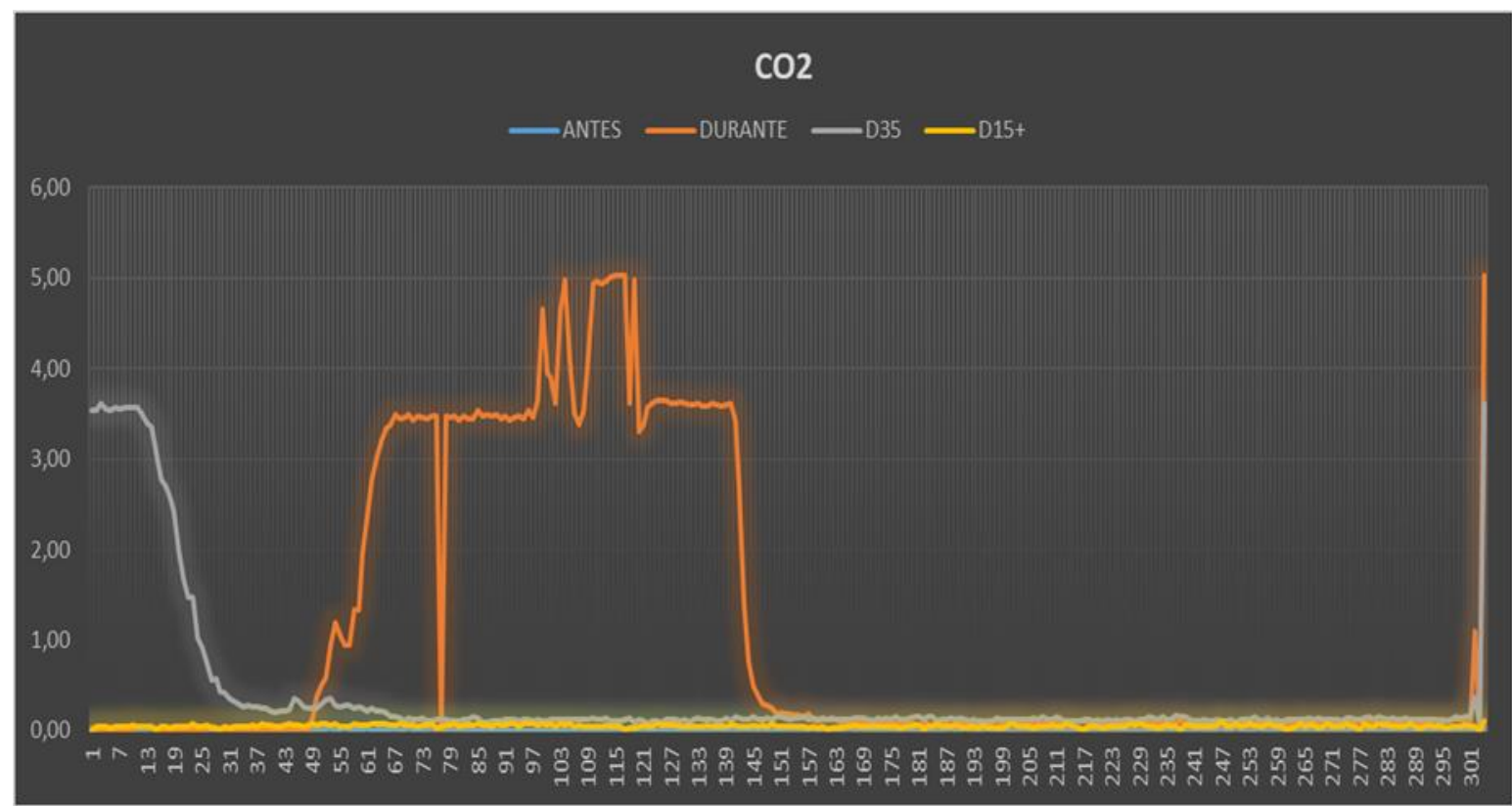

Figura 21 Respuesta del CO2 en todos los momentos de medición. Fuente: El autor

En el caso del $\mathrm{CO}_{2}$ se observa como la línea del durante realiza un gran aumento y más cuando se tiene acelerado el motor, lo cual quiere decir que, al aumentar el dióxido de carbono se reduce el monóxido de carbono y disminuye el nivel de oxígeno respirable; lo cual puede generar asfixia teniendo efectos sistémicos agudos independientemente de la concentración del oxígeno. Si la concentración de $\mathrm{CO}_{2}$ en el aire ambiente se incrementa, el intercambio pulmonar de gas en los pulmones se pone en peligro, provocando un incremento de la concentración de $\mathrm{CO}_{2}$ en sangre y tejidos. Este tipo de intoxicación es algo a lo que el cuerpo humano es extremadamente sensible. Un ejemplo: el aire ambiente en un depósito (espacio confinado) consiste en más del 18\% de oxígeno y más del 10\% de dióxido de carbono. Esto significa que incluso si hay suficiente oxígeno en la atmósfera del depósito para que haya vida, los humanos no podemos sobrevivir en una atmósfera con más del $10 \%$ de $\mathrm{CO}_{2}[3]$.

Según Messer Gases for Life en Colombia[4] los efectos de inhalar dióxido de carbono 1-1,5 \% de $\mathrm{CO}_{2}$ en el aire ambiente: Leve efecto en el metabolismo químico tras una exposición de varias horas. 3 \% de $\mathrm{CO}_{2}$ en el aire ambiente: El gas es ligeramente narcótico a estos niveles, dando lugar a una respiración más profunda y rápida, reducción de la capacidad auditiva, dolor de cabeza e incremento de la presión sanguínea y el pulso. 4-5\% de $\mathrm{CO}_{2}$ en el aire ambiente: Respiración más profunda y rápida como resultado de la estimulación del aparato respiratorio. Aparecen signos evidentes de intoxicación tras 30 minutos de exposición. 5-10 \% de $\mathrm{CO}_{2}$ en el aire ambiente: La 
respiración se vuelve pesada, con dolor de cabeza y pérdida de la razón. 10-100 \% de $\mathrm{CO}_{2}$ en el aire ambiente: Inconsciencia en menos de un minuto $\mathrm{y}$, a menos que se tomen medidas de inmediato, el resultado puede ser mortal[5]

Tabla 4 Datos promedio medidos CO2

\begin{tabular}{|c|c|c|c|c|c|}
\hline \multirow{2}{*}{\multicolumn{2}{|c|}{ Messer }} & \multicolumn{4}{|c|}{ Datos Promedio del Dióxido de Carbono } \\
\hline & & Antes & Durante & Después +35 & Después +15 \\
\hline $1-1,5 \%$ & $\begin{array}{l}\text { Leve efecto en el metabolismo químico tras una } \\
\text { exposición de varias horas. }\end{array}$ & 0,03 & ----- & ----- & 0,1 \\
\hline $3 \%$ & $\begin{array}{l}\text { El gas es ligeramente narcótico a estos niveles, } \\
\text { dando lugar a una respiración más profunda y } \\
\text { rápida, reducción de la capacidad auditiva, } \\
\text { dolor de cabeza e incremento de la presión } \\
\text { sanguínea y el pulso. }\end{array}$ & ----- & ----- & 3,61 & ----- \\
\hline $4-5 \%$ & $\begin{array}{l}\text { Respiración más profunda y más rápida como } \\
\text { resultado de la estimulación del aparato } \\
\text { respiratorio. Aparecen signos evidentes de } \\
\text { intoxicación tras } 30 \text { minutos de exposición. }\end{array}$ & ----- & ----- & ----- & ----- \\
\hline $5-10 \%$ & $\begin{array}{l}\text { La respiración se vuelve pesada, con dolor de } \\
\text { cabeza y pérdida de la razón. }\end{array}$ & ----- & 5,03 & ----- & ----- \\
\hline $\begin{array}{c}10- \\
100 \%\end{array}$ & $\begin{array}{l}\text { Inconsciencia en menos de un minuto y, a } \\
\text { menos que se tomen medidas de inmediato, el } \\
\text { resultado puede ser mortal. }\end{array}$ & -- & ----- & & ----- \\
\hline
\end{tabular}

Podemos evidenciar que durante los 20 minutos desde que se enciende el motor llegamos al 4 nivel de 5, donde es perjudicial para la salud de docente, instructor y estudiantes que estén en las prácticas, esos datos los pueden evidenciar en la tabla de datos DURANTE $\mathrm{CO}_{2}$. Donde en la TABLA DESPUÉS + 35 sigue con altos índices, el cual nos da 55 minutos de practica donde la inhalación de este gas es perjudicial para la salud. Es decir que por una práctica donde se enciende 20 minutos el motor (en este caso solo para pruebas) 15 minutos de encendido reflejan 30 minutos de reposo para que bajen los niveles de Dióxido de Carbono perjudicial para la salud. También dependiendo la cantidad de estudiantes que tenga cada curso, en el cual la instalación es amplia dependiendo del número de estudiantes, ya que al aumento de dióxido disminuye el oxígeno afectando más el factor del espacio y la aglomeración que tendría el espacio alrededor del motor.

Por los motivos anteriores se propone desarrollar un sistema de gestión ambiental que generará 
una mejora considerable en las buenas prácticas para la utilización de los equipos y cumplimiento de la normatividad en salud, seguridad industrial y medio ambiente.

\section{CONCLUSIONES}

Aunque el espacio en el cual se desarrollan las prácticas de motores Diesel para el programa de tecnología automotriz e ingeniería mecánica es el adecuado; al momento de llevar a cabo las prácticas no se pueden encender todos los motores con los que cuenta el laboratorio porque el lugar se satura de dióxido de carbono e hidrocarburos que disminuyen considerablemente el oxígeno respirable. Por esto se genera la propuesta de diseño de un sistema de gestión ambiental (un modelo) que permitiría la realización de mejoras en cuanto a infraestructura física y ambiental, cumplimiento de las normas ambientales, de salud y seguridad. Adicional a esto la Universidad sigue mostrando ante los entes competentes que busca seguir siendo amigable y sostenible con el medio ambiente en todos sus aspectos.

\section{REFERENCIAS}

[1] ISO, "ISO 14001:2015(es), Sistemas de gestión ambiental - Requisitos con orientación para su uso," 2015.

https://www.iso.org/obp/ui/es/\#iso:std:iso:14001:ed3:v1:es (accessed Sep. 11, 2020).

[2] R. Hernández S., C. Fernandez C., and M. Baptista L., Metodología de la Investigación, 6ta. 2014.

[3] Observatorio Ambiental de Bogotá, "PLAN AMBIENTAL LOCAL TEUSAQUILLO 2017-2020,"
2017.

https://oab.ambientebogota.gov.co/?post_type=dlm_d ownload\&p=3186 (accessed Sep. 09, 2020).

[4] Messer gases for life, "index - Messer," 2020. https://www.messer-co.com/ (accessed Sep. 11, 2020).

[5] Ministerio de Salud Pública de Tucumán, "El monóxido de carbono es un asesino silencioso," 2015. https://msptucuman.gov.ar/el-monoxido-de-carbonoes-un-asesino-silencioso/ (accessed Sep. 09, 2020). 


\title{
MANUFACTURA DE MONTURAS OFTÁLMICAS PERSONALIZADAS MEDIANTE FABRICACIÓN ADITIVA CON PET RECUPERADO
}

\section{Manufacture of customized ophthalmic frames through additive manufacturing with recovered pet}

\author{
Buitrago, Rubén ; Díaz, Luis \\ Universidad ECCI, Grupo de investigación GIII-ECCI
}

\section{RESUMEN}

El consumo de materiales plásticos se ha extendido además de la manufactura de envases, a otros sectores como la fabricación de componentes de la industria automotriz, vivienda, y todo tipo de bienes de consumo. Lo anterior a ocasionado que gran parte de este material no sea aprovechado de forma óptima, generando un incremento en los rellenos sanitarios, por tanto, es necesario pensar que algunos de estos materiales pueden volver a incorporarse en un proceso productivo. El objetivo de esta investigación es elaborar monturas oftálmicas mediante fabricación aditiva Aprovechando el material plástico recuperado. El paradigma de investigación que permite alcanzar el propósito es de tipo aplicada con un enfoque cuantitativo, dado que permite generar conocimientos a partir de otras investigaciones y de la experimentación mediante el desarrollo del prototipo elaborado. Los resultados permitieron obtener una montura personalizada basada en las medidas antropométricas. Con base en lo anterior, se concluye que la implementación de la impresión 3D como proceso de manufactura sostenible, facilita la personalización de productos, asimismo, se valida el proceso de fabricación de monturas oftálmicas personalizadas a base de PET recuperado, teniendo en cuenta el contexto normativo en cuanto a factores de resistencia y criterios de calidad. Finalmente, esta investigación contribuye a ampliar el estado del arte existente en el campo.

Palabras claves: Monturas oftálmicas, PET, Fabricación aditiva, Impresión 3D.

\footnotetext{
${ }^{1}$ Correo electrónico de contacto: rbuitragop@ecci.edu.co
} 


\section{INTRODUCCIÓN}

La industria 4.0 busca la transformación digital e industrial, generando avances tecnológicos donde es necesario personalizar y crear nuevos productos, ofreciendo servicios inteligentes, donde el cliente paga por el servicio adquirido, buscando eliminar pasos intermedios para la adquisición de los elementos requeridos.

Para lograr que la industria este interconectada, las tecnologías de la información y la comunicación permiten enlazar el mundo físico y el digital mediante dispositivos, productos, equipos e instalaciones, logrando sistemas colaborativos y productos interrelacionados. Para este caso, una de las herramientas utilizadas en estos procesos corresponde a la impresión 3D o fabricación por deposición fundida, donde un conjunto de características de fabricación por adición permite que un objeto tridimensional en un software específico sea creado mediante la superposición de capas sucesivas de material [1].

La fabricación aditiva o fabricación por adición es un concepto nuevo de producción, mediante el cual el material plástico o metal es superpuesto capa a capa de una manera controlada en secciones donde se hace necesario [2]. En comparación con otras técnicas de fabricación tradicional, reduce los procesos intermedios, como la producción de utillajes, por lo que permite obtener piezas hasta un $90 \%$ más rápido, reduciendo desechos y lo más importante, ahorrando energía.

A primera vista, se suele relacionar los términos de impresión 3D y fabricación aditiva como sinónimos, sin embargo, no son exactamente lo mismo. La fabricación aditiva hace referencia a las técnicas de fabricación por adición de diferentes materiales empleadas con el fin de producir productos nuevos complejos y durables. Por otra parte, la impresión 3D, como consecuencia del prototipado rápido, hace referencia a la fabricación de modelos y piezas de con limitaciones que habitualmente se vinculan al utilizar un tipo concreto de tecnología aditiva [3].

De acuerdo con las necesidades de cada sector, el proceso de manufactura aditiva puede producirse o desarrollarse a partir de diferentes tecnologías, en donde la elección entre ellas dependerá de diversos factores como la funcionalidad que tendrá la pieza final producida, o si se desea fabricar a partir de la inclusión de polímeros o aleaciones metálicas.

Las principales tecnologías de la fabricación aditiva son la fusión de lecho de polvo, el modelado por deposición fundida, el material Jetting o Impresión de resina y, la impresión 3D [4]. Esta última, es un proceso por el cual se elaboran objetos físicos colocando un material determinado por capas con base en un modelo digital creado con anterioridad. Todos los procesos que intervienen dentro de la impresión 3D requieren que el software, el hardware y los materiales trabajen conjuntamente. Esta tecnología, es aplicada con posibilidades infinitas y en diversos 
sectores de la industria, en donde desde sitios más cómodos, los diseños y prototipos llevados a la realidad, brindan una solución económica para todas aquellas personas que cuenten con alguna necesidad [5].

Los estudios que relacionan el proceso de manufactura aditiva enfocado a la producción de monturas oftálmicas son pocos, sin embargo, vienen ganando interés por el factor de la personalización de productos. En [6] se llevó a cabo un diseño de gafas de sol, consiguiendo que sea apto para cualquier usuario. Este trabajo integro la impresión 3D y la manufactura con control numérico computarizado (CNC) con un programa libre de tipo CAD, logrando reducir los costos de producción. Por otra parte, en [7] se lleva cabo una investigación que valida el uso de materiales poliméricos como el ácido poliláctico (PLA) y el acrilonitrilo butadieno estireno (ABS), y su compatibilidad al rostro humano, implementándolo en usuarios que necesitan correcciones ópticas. Otros estudios, desarrollaron análisis de factibilidad [8] y personalización de gafas para niños con anomalías craneofaciales [9].

Desde este punto de vista, se identifica la oportunidad de aprovechar las herramientas de producción de la industria 4.0; donde con ayuda de la fabricación aditiva se pueden incorporar materiales de desecho y volverlos útiles, por tanto, esta investigación tiene como propósito producir monturas oftálmicas mediante fabricación aditiva aprovechando el material plástico recuperado.

\section{MATERIALES Y MÉTODOS}

Para la impresión de prototipos se implementa una Impresora Híbrida similar a la referencia Prusa I3, con una cama de impresión de $20 \mathrm{~cm} X 20 \mathrm{~cm} X 20 \mathrm{~cm}$ con alcance de temperatura maxima de $70^{\circ} \mathrm{C}$, extrusor con temperatura maxima de $250^{\circ} \mathrm{C}$ y una boquilla de extrusión de $0.4 \mathrm{~mm}$. En cuanto a la materia prima se implementa filamento de Pet Recuperado con diámetro de $1.75 \mathrm{~mm}$. En la máquina mencionada fueron manufacturadas seis probetas con las dimensiones establecidas por la norma NTC 5607. Bajo esta perspectiva, la norma sugiere aplicar las pruebas de estabilidad dimensional a elevadas temperaturas, ensayo para la resistencia al sudor y el ensayo de resistencia a la ignición. Lo anterior fue llevado a cabo en el laboratorio de la Universidad ECCI.

Asimismo, para el desarrollo de las pruebas de flexión el referente fue la norma ASTM D790, en el caso del ensayo para tensión, la norma ASTM D638 y finalmente, el ensayo de resistencia al impacto a través de la norma ASTM D256-10. El equipo utilizado para este ensayo es de propiedad del laboratorio de materiales de la Universidad Nacional, de marca SHIMADZU, MODELO AGIS-5 KN Y SERIAL NO. 130104403667.

Para determinar cuál es material y el método de fabricación adecuado, se implementó el método 
de análisis jerárquico (AHP) el cual es una herramienta que permite tomar decisiones que facilitan lograr un adecuado resultado; su implementación se refiere a solucionar problemas que presentan una alta complejidad y en los cuales se deben tener en cuenta múltiples criterios. Este método exige que la persona que toma la decisión establezca evaluaciones subjetivas frente a la relevancia de los criterios definidos y luego se debe aclarar la preferencia basada en cada una de las opciones de decisión; el resultado de este proceso es una jerarquización priorizada que muestra preferencia para las alternativas de decisión definidas con anterioridad [10].

\section{ANALISIS DE RESULTADOS}

\section{A. Selección de material plástico y del método de fabricación aditiva para la elaboración de las monturas oftálmicas}

Una vez identificados los posibles materiales con los cuales se puede proceder a la elaboración de las monturas por medio de la impresión 3D, el desarrollo del método AHP permitió establecer que el PET RECUPERADO es el filamento ideal para la impresión de las piezas destinadas al contacto con el rostro humano. A su vez se determina que en cuanto al área de la impresión 3D su rango de temperatura de impresión es de 215 a $250^{\circ} \mathrm{C}$. Estos parámetros varían de acuerdo con las especificaciones con las que cuente la impresora a utilizar. Para el caso de la investigación, la temperatura óptima de impresión es de $230^{\circ} \mathrm{C}$ y requiere que la cama caliente se encuentre a $65^{\circ} \mathrm{C}$ aproximadamente para asegurar una adhesión del material a la placa y garantizar que las capas de impresión sean las mejores.

En cuanto a las puntuaciones obtenidas por el método AHP para evaluar los diferentes métodos de fabricación aditiva, se establece que el modelado por deposición fundida favorece las condiciones de funcionalidad, costo y aporte de material.

\section{B. Ensayos para la estabilidad dimensional a elevadas temperaturas, resistencia al sudor y resistencia a la ignición}

La norma NTC 5607 en el numeral 4.6 establece una tolerancia. de + / - 5mm para la prueba de estabilidad dimensional a elevadas temperaturas, obteniéndose que la variación de las medidas frontal $(0.097 \mathrm{~mm})$, alto $(0.047 \mathrm{~mm})$ y de los brazos $(-0.409 \mathrm{~mm})$ están dentro de la tolerancia especificada. En cuanto al ensayo para la resistencia al sudor, la norma indica en el numeral 4.7 que no debe presentarse un cambio de color en la montura excluyendo las bisagras y tornillos después de $8 \mathrm{~h}$. al respecto, se puede determinar que la muestra expuesta a la prueba no presenta variación significativa de coloración en sus superficies (frontal y brazos) y en especial en aquellas que tendrán contacto con la piel del usuario final. Sin embargo, si se evidencia que en algunos sectores de la muestra se presenta separación de algunas de sus capas de impresión. Finalmente, los resultados para el ensayo de resistencia a la ignición, la norma en el numeral 4.9 indica que el 
material no debe continuar combustión una vez la barra de ensayo sea retirada. Al ejecutar la prueba permite identificar que la probeta no presenta ignición luego de retirar la barra metálica, adicional a esto, se puede observar una deformación en el material a causa de la temperatura elevada de la barra.

\section{Ensayo de resistencia a la tensión (resistencia y elongación a tracción)}

Este ensayo se desarrolló teniendo en cuenta la norma ASTM D638 con probeta tipo I $(\sigma<5000 \mathrm{~N})$. Los resultados presentan en las seis probetas una DT=110,654 con valor máximo de 1522,97 N y un mínimo de $1221,56 \mathrm{~N}$. La tabla 1 evidencia los resultados obtenidos, así como los valores de referencia de los materiales comerciales con los que se manufactura las monturas oftálmicas.

Tabla 1. Valores de referencia de resistencia a la tensión en materiales para monturas oftálmicas

\begin{tabular}{|c|c|c|c|c|c|c|}
\hline & $\begin{array}{c}\text { Acetato de } \\
\text { celulosa }\end{array}$ & $\begin{array}{c}\text { Propinato } \\
\text { de } \\
\text { celulosa }\end{array}$ & $\begin{array}{c}\text { Resina } \\
\text { epoxi }\end{array}$ & Poliamida & Fibra de carbono & $\begin{array}{c}\text { Pet } \\
\text { recuperado }\end{array}$ \\
\hline Material & CA & $\mathrm{CP}$ & $\mathrm{EP}$ & PA & $\mathrm{CF}$ & PETR \\
\hline $\begin{array}{c}\text { Resistencia } \\
\text { tracción } \\
(\mathrm{N} / \mathrm{mm} 2)\end{array}$ & $30-50$ & $30-50$ & 75 & 75 & 1800 & 15,1 \\
\hline
\end{tabular}

Al ejecutar la prueba de resistencia a la tracción se evidencia la obtención de valores que son inferiores a los comerciales, lo anterior responde a que los polímeros son moléculas formadas por las uniones repetidas de una o varias moléculas enlazadas por enlaces. Dentro de estos enlaces, la polaridad y el volumen de estos átomos afectaran especialmente a las fuerzas de cohesión entre cadenas, que, a su vez establecen la flexibilidad del material, su temperatura de fusión y su capacidad de cristalización, es decir, cuanto mayor sean las fuerzas de cohesión entre cadenas, el polímero obtendrá más rigidez y temperatura de fusión, aplicándose para polímeros cristalinos como el PET.

A su vez, es necesario resaltar que en los procesos de polimerización los monómeros se unen de la misma forma, esto para el caso del PET. Dentro de esta estructura se forman ramificaciones que proporcionan un volumen libre y separación entre cadenas, relacionado con la densidad del polímero y su cristalización, formando moléculas químicamente y geométricamente regulares en su estructura [11].

Por otra parte, en estado fundido o líquido, las moléculas de este polímero se encuentran encogidas y enredadas si no hay una fuerza externa que obligue a orientarlas en una dirección. A partir de lo anterior, se establece que es necesario realizar un cambio estructural en el enmallado de la pieza al momento de imprimirla, puesto que la configuración de malla triangular como en 
los prototipos impresos y probados, la distribución del esfuerzo aplicado no es proporcional, lo que influye principalmente en la capacidad de resistencia y en los valores obtenidos en la prueba efectuada.

\section{Ensayo de resistencia a la Flexión}

Este ensayo se desarrolló teniendo en cuenta la norma ASTM D790 con geometría rectangular de $100 \mathrm{~mm}$ de largo, $15 \mathrm{~mm}$ de ancho y $3.75 \mathrm{~mm}(\sigma<5000 \mathrm{~N})$. Los resultados en las seis probetas presentan una $\mathrm{DT}=5,940$ con valor máximo de $132,594 \mathrm{~N}$ y un mínimo de $114,406 \mathrm{~N}$. La tabla 2 evidencia los resultados obtenidos, así como los valores de referencia de los materiales comerciales con los que se manufactura las monturas oftálmicas.

Tabla 2. Valores de referencia de resistencia flexión en materiales para monturas oftálmicas

\begin{tabular}{ccccccc}
\hline & $\begin{array}{c}\text { Acetato de } \\
\text { celulosa }\end{array}$ & $\begin{array}{c}\text { Propinato de } \\
\text { celulosa }\end{array}$ & $\begin{array}{c}\text { Resina } \\
\text { epoxi }\end{array}$ & Poliamida & $\begin{array}{c}\text { Fibra de } \\
\text { carbono }\end{array}$ & $\begin{array}{c}\text { Pet } \\
\text { recuperado }\end{array}$ \\
Material & CA & CP & EP & PA & CF & PETR \\
\hline $\begin{array}{c}\text { Resistencia } \\
\text { flexión } \\
(\mathrm{N} / \mathrm{mm} 2)\end{array}$ & $24-71$ & $40-60$ & 110 & 70 & 70 & 44,1 \\
\hline
\end{tabular}

Los resultados permiten identificar que el PET presenta un mejor comportamiento en comparación al material comercial más utilizado y resistente, conocido como acetato. Este fenómeno se puede explicar gracias a que el nivel de cristalinidad y la orientación de las fibras no es igual en los materiales extruidos que en los moldeados por inyección, lo que resulta en diferencias importantes en los valores.

\section{E. Ensayo de resistencia al Impacto}

Este ensayo se desarrolló teniendo en cuenta la norma ASTM D256-10 con probeta de capacidad máxima de 22 Jules. Los resultados presentan en las seis probetas una $\mathrm{DT}=0,001$ con valor máximo de 0,018 y un mínimo de 0,007 tenacidad a la fractura. La tabla 3 evidencia los resultados obtenidos, así como los valores de referencia de los materiales comerciales con los que se manufactura las monturas oftálmicas.

Tabla 3. Valores de referencia de resistencia al impacto en materiales para monturas oftálmicas

$\begin{array}{cccccc}\begin{array}{c}\text { Acetato de } \\ \text { celulosa }\end{array} & \begin{array}{c}\text { Propinato } \\ \text { de } \\ \text { celulosa }\end{array} & \begin{array}{c}\text { Resina } \\ \text { epoxi }\end{array} & \text { Poliamida } & \text { Fibra de carbono } & \begin{array}{c}\text { Pet } \\ \text { recuperado }\end{array}\end{array}$




\begin{tabular}{ccccccc} 
Material & CA & CP & EP & PA & CF & PETR \\
\hline $\begin{array}{c}\text { Resistencia } \\
\text { impacto } \\
(\mathrm{N} / \mathrm{mm} 2)\end{array}$ & $50-80$ & $50-80$ & 130 & 130 & 70 & $15-35$ \\
\hline
\end{tabular}

Los resultados obtenidos en la prueba de resistencia al impacto indican que el material seleccionado presenta valores inferiores a los materiales de referencia comercial. Cabe indicar que estos resultados sufren variaciones por influencia de variables como la temperatura a la que se encuentre el polímero al momento de la ejecución de la prueba, incidiendo directamente en la tenacidad del polímero sometido y la velocidad de la carga aplicada, puesto que a mayor la velocidad el polímero tendrá mayor probabilidad de fallar al convertirse en un material quebradizo, esto como consecuencia de la falta de tiempo para que la fuerza intermolecular sea efectiva, es decir, si se realiza la prueba a bajas temperaturas y a altas velocidades, el material obtendrá la menor resistencia al impacto posible [12].

Adicional a lo anterior, es necesario tener como referencia la ductilidad y fragilidad del material. La primera permite una deformación plástica antes de romperse, y por otra parte, la fragilidad corresponde a una ruptura microscópicamente lisa o estriada al ser expuesto a escasa fuerza. Esta última, guarda estrecha relación con la velocidad con la que se propaga el crecimiento de grietas en toda su estructura [13].

Por último y no menos importante, dentro del proceso de moldeo de piezas con ayuda de materia prima a base de material recuperado, se encuentran factores que inciden directamente en su resistencia, ya que debido al reproceso del material las cadenas moleculares son modificadas, se generan tensiones residuales, se generan líneas defectuosas de soldadura (adhesión entre capas) y cristalización de este en algunas secciones. A su vez, las probetas realizadas y como se mencionó anteriormente, dentro de su estructura interna cuenta con un enmallado triangular que deja como consecuencia huecos internos lo que ocasiona la deficiencia de volumen que ayude a la absorción y disipación del impacto al que se somete [14].

\section{CONCLUSIONES}

La caracterización del material mediante el método AHP, permitió definir que el PET cuenta con las propiedades mecánicas, de termoestabilidad y de bajo costo, necesarias para implementarse en procesos de impresión 3D. Asimismo, estos procesos pueden llevarse a cabo mediante dispositivos operados con software libre, cuyas prestaciones favorecen la manufactura flexible, y mantienen las condiciones de acabado superficial. Finalmente, las pruebas de laboratorio basadas en el marco normativo nacional e internacional, demuestran que la resistencia a la flexión es mayor en comparación a los material comerciales, sin embargo, los resultados del ensayo de 
resistencia al impacto se encuentran por debajo de los mismos materiales comparados, encontrándose que durante el proceso de moldeo de piezas con ayuda de materia prima a base de material recuperado, las cadenas moleculares son modificadas, generando tensiones residuales y minimizando los valores para esta prueba.

\section{REFERENCIAS}

[1] M. K. J. E. Exconde, J. A. A. Co, J. Z. Manapat, and E. R. Magdaluyo, "Materials Selection of 3D Printing Filament and Utilization of Recycled Polyethylene Terephthalate (PET) in a Redesigned Breadboard," Procedia CIRP, vol. 84, pp. 28-32, 2019, doi: https://doi.org/10.1016/j.procir.2019.04.337.

[2] L. S. Dalenogare, G. B. Benitez, N. F. Ayala, and A. G. Frank, "The expected contribution of Industry 4.0 technologies for industrial performance," Int. J. Prod. Econ., vol. 204, pp. 383-394, Oct. 2018, doi: 10.1016/j.ijpe.2018.08.019.

[3] M. A. Gibson et al., "3D printing metals like thermoplastics: Fused filament fabrication of metallic glasses," Mater. Today, vol. 21, no. 7, pp. 697-702, 2018, doi: https://doi.org/10.1016/j.mattod.2018.07.001.

[4] H. Bikas, P. Stavropoulos, and G. Chryssolouris, "Additive manufacturing methods and modeling approaches: A critical review," Int. J. Adv. Manuf. Technol., vol. 83, no. 1-4, pp. 389-405, 2016, doi: 10.1007/s00170-015-7576-2.

[5] S. Ford and M. Despeisse, "Additive manufacturing and sustainability: an exploratory study of the advantages and challenges," J. Clean. Prod., vol. 137, pp. 1573-1587, 2016, doi: 10.1016/j.jclepro.2016.04.150.

[6] R. Corbatón Celia, "Planteamiento de proceso de diseño para la personalizacion de producto. Caso: montura de gafas de sol.," Universidad Politécnica de Valencia. Escuela Técnica Superior de Ingeniería del Diseño, 2015.

[7] S. C. Hereida Pichucho and M. Gomez, "Estudio de materiales polímeros y su compatibilidad con el rostro humano en pacientes usuarios de correcciones ópticas en la ciudad de quito, 2017-2018. Creación de monturas en $3 \mathrm{~d}$ con dos tipos de polímeros y su compatibilidad con el rostro humano," Tecnológico Superior Cordillera, 2018.

[8] R. M. Zaki et al., "Direct 3D-printing of phosphate glass by fused deposition modeling," Mater. Des., vol. 194, p. 108957, 2020, doi: 10.1016/j.matdes.2020.108957.

[9] F. L. Brodie et al., "Computed tomography-based 3D modeling to provide custom 3D-printed glasses for children with craniofacial abnormalities," J. AAPOS, vol. 23, no. 3, pp. 165-167.e1, Feb. 2019, doi: 10.1016/j.jaapos.2019.01.010.

[10] M. J. José María, “El proceso analítico jerárquico (AHP). Fundamentos, metodología y aplicaciones," Universidad de Zaragoza, Zaragoza, 2016.

[11] Beltrán Rico Maribel and Marcilla Gomis Antonio, Tecnología de polímeros - Publicaciones y ediciones digitales de la Universidad de Alicante. 2012.

[12] G. B. Castro, L. O. Carmona, and J. O. Florez, "Production and characterization of the mechanical and thermal properties of expanded polystyrene with recycled material," Ing. y Univ., vol. 21, no. 2, pp. 177194, 2017, doi: 10.11144/javeriana.iyu21-2.mtpe.

[13] R. Singh, R. Kumar, I. Farina, F. Colangelo, L. Feo, and F. Fraternali, "Multi-material additive manufacturing of sustainable innovative materials and structures," Polymers (Basel)., vol. 11, no. 1, pp. 1-14, 2019, doi: 10.3390/polym 11010062

[14] P. Frontini, "Resistencia al impacto de Materiales Plásticos:¿Qué ensayo elegir?," Mar del Plata (Argentina), 2017. [Online]. Available: http://www3.fi.mdp.edu.ar/ingpolimeros/grupo.html. 


\section{MÉTODOS DE DEGRADACIÓN DE LAS MEZCLAS POLIPROPILENO Y POLIESTIRENO EXPANDIDOS RECUPERADOS Y POLIPROPILENO, POLIETILENO DE ALTA DENSIDAD RECUPERADOS, CASCARILLA DE ARROZ Y AGENTE DE ACOPLE SILANO}

\section{Degradation methods of recovered expanded polypropylene and polystyrene mixtures and recovered polypropylene, high density polyethylene, rice husk and silane coupling agent}

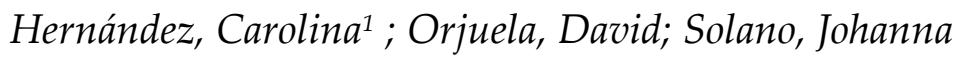

Universidad Santo Tomas, Grupo de investigación, semillero de gestión y valorización de residuos sólidos.

\section{RESUMEN}

Los residuos poliméricos son materiales que hacen parte de una problemática actual dentro de los rellenos sanitarios y centros de acopio, por tanto, son materiales de interés e importancia por su valor y composición, de allí parte el interés de identificar sus propiedades de degradabilidad. La degradación puede ser realizada de forma química, física o biológica, y lo que se busca con ella es que se deteriore el material lo máximo posible, para así evitar problemas e impactos ambientales generados por este tipo de residuos. El objetivo de esta revisión es identificar los métodos de degradación más adecuados para la mezcla de polipropileno y poliestireno expandido recuperados y la mezcla de polipropileno y polietileno de alta densidad recuperados, cascarilla de arroz y agente de acople silano, dentro de la revisión se logro identificar que para la mayoría de los materiales es necesario hacer un pretratamiento, para posteriormente iniciar con las pruebas de degradación, en el caso del poliestireno es posible realizar degradación biológica, térmica y fotodegradación, para el polipropileno es necesario realizar pretratamiento, y posteriormente fotodegradación o degradación química, el polietileno de alta densidad y la

${ }^{1}$ Correo electrónico de contacto: leidyhernandezh@usantotomas.edu.co 
cascarilla de arroz también requieren pretratamiento antes de iniciar las pruebas de biodegradación. Se pudo concluir que de acuerdo con el material polimérico es necesario identificar el tipo de ensayo a realizar, para así, medir la degradabilidad de cada mezcla, se debe hacer la combinación de los diferente métodos y pruebas de acuerdo con los materiales que componen cada una de estas

Palabras clave: degradación, biodegradación, polímeros, cascarilla de arroz.

\section{INTRODUCCIÓN}

Los polímeros son bien conocidos por su larga duración, generando así un impacto ambiental significativo, ya que a través de los años se ha convertido en un desecho industrial de gran magnitud [1], y así mismo, son materiales que se han vuelto indispensables en la vida diaria. Es tal el problema, que se han realizado evaluaciones sobre métodos de biodegradabilidad en rellenos sanitarios por exceso de polímeros, y la implementación de sistemas tratamiento de residuos sólidos [2]. Es de allí donde parten los estudios realizados sobre la degradabilidad o biodegradabilidad de los plásticos, con el fin de obtener métodos y mecanismos que logren la reducción de este tipo de residuos después de su vida útil.

Por lo general, los plásticos son muy resistentes al ataque microbiano, ya que son producidos a través de petróleo, estos son conocidos como polímeros sintéticos [3], también existen polímeros naturales como: la celulosa, colágeno, quitosano, alginato [4], entre otros. En cualquiera de las dos formas, ya sea natural o sintético, lo ideal es conseguir su degradación en el menor tiempo posible y de la forma más adecuada de acuerdo con las características fisicoquímicas de cada uno. Para el presente trabajo, se tuvo en cuenta diferentes estudios realizados sobre la degradabilidad de polímeros, se trabajará especialmente con pruebas o métodos que sean útiles para la degradación de las mezclas de polipropileno y poliestireno expandido recuperados y polipropileno, polietileno de alta densidad recuperados, cascarilla de arroz y agente de acople silano. Se utiliza polipropileno en las dos mezclas puesto que, este es uno de los polímeros más usados y estudiados debido a su gran interés comercial e industrial [5].

En una de las mezclas se trabaja cascarilla de arroz debido a que, hoy en día es uno de los residuos agrícolas más importantes y con diferentes usos, gracias a su composición y sus características químicas [6], además, es considerada como un residuo de baja degradabilidad ambiental [7], y uno de los usos que se le ha dado a este residuo, es que sea mezclado con polietileno de alta 
densidad y polipropileno con el fin de elaborar otro material, que sea capaz de sustituir los polímeros sintéticos que se conocen hoy en día [8], se trabaja con estas mezclas puesto aún no se encuentran estudios de degradabilidad con la composición de las mismas, teniendo en cuenta el residuo agrícola de una de ellas. Por tal motivo es importante determinar qué tan degradable puede llegar a ser el material polimérico, para así determinar si la mezcla es apta en la elaboración de distintos materiales.

\section{MATERIALES Y MÉTODOS.}

Este artículo se desarrolla a través de revisión bibliográfica en diferentes artículos de revistas académicas, artículos científicos y de investigación, los cuales fueron buscados en bases de datos especializadas como: Scopus y Sciencedirect, encontrando así, 38 estudios de los cuales se utilizaron como referencia los que fueron publicados desde el 1998, que en total fueron 26, con información relacionada a la degradabilidad y biodegradabilidad de polímeros como son: polietileno, poliestireno, el polipropileno, y así mismo el residuo agrícola cascarilla de arroz. La selección de los estudios fue realizada por las palabras clave "degradación de polímeros" y "degradación de cascarilla de arroz" excluyendo la degradación de los polímeros que no serán trabajados.

Dentro de la búsqueda se tuvieron en cuenta los estudios realizados, bien sea, con problemáticas en la industria polimérica, experimentos o métodos de degradación o biodegradación, artículos de revisión donde se realizan la compilación de varios métodos, artículos de las características y composición de cada uno de los materiales a trabajar. Se procede a realizar el artículo de revisión teniendo en cuenta los métodos que se podrían llegar a trabajar dentro de un laboratorio, al momento de poner en práctica la degradación de las mezclas.

\section{RESULTADOS}

Existen diferentes métodos para la degradación de los polímeros, entre los cuales se encuentra: la degradación fotooxidativa [5], termooxidación [9], degradación oxidativa, hidrolítica, mecánica causada por turbulencia de agua [10], degradación microbiana [11], entre otras. En la degradación van involucrados factores ambientales como el calor, humedad, luz, actividad biológica y química, los cuales son capaces de generar cambios estructurales en el polímero que favorecen la degradación [11]. De acuerdo con un estudio realizado por Arutchelvi et al. [12] la biodegradación se lleva a cabo a través de los siguientes pasos: fijación de microorganismos al polímero, 
crecimiento de los microorganismos, degradación primaria y degradación final [12]. Cuando el polímero se degrada de forma aeróbica se produce agua y dióxido de carbono, mientras que cuando se realiza de forma anaeróbica se produce agua, dióxido de carbono y metano [11].

Los microorganismos solo se adhieren al polímero si la superficie es hidrofílica [1], pero existen polímeros con alto nivel hidrofóbico y alto peso molecular como el polietileno, y por lo tanto no es posible que se degrade de forma natural [11]. Así como el polietileno, el polipropileno también es hidrofóbico y para ambos deberá realizarse un pretratamiento debido a que contienen metileno - CH2 [12]. Esto indica que los polímeros, se deben despolimerizar a monómeros más pequeños para que así sean biodegradados o absorbidos por células microbianas [11]. Para el pretratamiento es conveniente utilizar, las fuerzas físicas como el calentar/enfriar, humedecer/secar, entre otras, ya que estas causan agrietamientos en los polímeros, y las fuerzas biológicas, que pueden generar hinchazón y estallido en los sólidos poliméricos [11], lo cual podría ser una posibilidad para posteriormente hacer la biodegradación. Otro de los pretratamientos posibles podría ser mecánico, ya que da lugar a la tensión, compresión o fuerzas de corte, estas fuerzas se ven relacionadas con el envejecimiento por carga, turbulencias de agua o aire [13], entre otras.

Anteriormente se nombraron los pasos para lograr la biodegradabilidad, ahora se mencionan las etapas de desarrollo, estas son: biodeterioro, biofragmentación y asimilación [13], usualmente esas son las etapas que debería tomar el polímero para lograr la biodegradabilidad. En el caso de la biodegradación del polietileno es necesario modificar, el nivel cristalino, propiedades mecánicas y su peso molecular, ya que estas son las que se encargan de la resistencia del material en el proceso de degradación [11]. De acuerdo con lo expuesto por Bikiaris [14] citado por Shah et al. [11], reducir el nivel cristalino se puede lograr mejorando el nivel hidrofílico o utilizando la oxidación para reducir la cadena del polímero. Lucas et al. Biodegradation of polyethylene and polypropylene anuncia que los daños al polímero no se ven de inmediato, pero no quiere decir que no hubiese empezado la degradación [12].

En cuanto a la degradación térmica de los polímeros, esta se da gracias al deterioro de las moléculas causado por el sobrecalentamiento, esto se presenta ya que a altas temperaturas el polímero principal empieza a separarse y a reaccionar entre si [11]. Esta degradación se da cuando el polímero se lleva a la temperatura de fusión, por ello no es muy usual que sean degradados a temperatura ambiente, puesto que esta suele ser menor que la de fusión [13].

En el caso de la fotodegradación, esta usa la luz UV para degradar el producto final [11], además 
gracias a que los fotones transportan la energía se crea inestabilidad en algunas moléculas [13]. En otro estudio se determinó que, la degradación fotooxidativa generalmente implica degradación fotolitica pura y oxidación fotoinducida [9]. Es necesario realizar fotodegradación o degradación química para que polímeros como el polietileno puedan ser biodegradados bien sea por oxo-biodegradación e hidro-biodegradación [11].

Por otra parte, la degradación química es uno de los métodos más importantes de la degradación abiótica; el oxígeno es el químico más poderoso para lograr este tipo de degradación, puesto que este interfiere entre los enlaces covalentes para producir radicales libres [13]. Además de esto, el oxígeno es importante debido a que los microorganimos los usan para oxidar el carbono y formar dióxido de carbono, esta formación es un buen indicador de degradación [11]. Para el caso del poliestireno inicia su degradación formando un radical, y como resultado del procesamiento catalítico se forma benceno y metilindano [15].

La cascarilla de arroz es un residuo agrícola conocido como lignocelulósico con variedad de usos [1], este material tiene una estructura cerrada, lo cual dificulta la combustión y gracias a su alto contenido de sílice, es de muy baja biodegradabilidad en condiciones de ambiente natural [7], es importante identificar que la biomasa lignocelulósica está compuesta de tres polímeros, estos vienen siendo: celulosa, hemicelulosa y lignina [15], a través de esto, se han venido desarrollando una serie de estudios relacionados con la degradación de materiales lignocelulósicos. Algunos estudios declaran que la biodegradación de estos materiales está directamente limitada por una serie de factores como: la cristalinidad de la celulosa, el contenido de lignina y el área de superficie biodisponible [16], [17], y por ello es necesario un pretratamiento para reducir estos factores [16]. Existen diferentes tipos de pretratamiento como lo son: el mecánico, térmico, ácido, alcalino [17], oxidativo [17],[18], o la combinación de estos [17].

Existen diferentes técnicas para obtener la información de degradación, en el caso de la térmica, podría realizarse a través de calorimetría diferencial de barrido y análisis termogravimétrico [13], otros métodos para evaluar la degradación existentes son: microscopía electrónica de barrido, microscopía de fuerza atómica [11], espectroscopía infrarroja por transformada de Fourier (FTIR) [19], [11], [2] y espectroscopia de masas [11], [12], las anteriormente nombradas son aquellas más comunes dentro de los estudios revisados para detectar las modificaciones químicas de los polímeros.

En este trabajo, se realizó la revisión de diferentes estudios sobre la degradación de polímeros, 
especialmente los de las mezclas a trabajar, en la tabla 1 se encuentran algunas de las pruebas que realizaron diferentes autores para determinar la degradación.

Tabla 5 Descripción general y resultados de algunas pruebas de degradación con su respectivo material

\begin{tabular}{|c|c|c|}
\hline Tipo de degradación & Material & $\begin{array}{l}\text { Descripción general y resultados } \\
\end{array}$ \\
\hline Biodegradación & Polietileno & $\begin{array}{l}\text { Se uso de Phanerochaete chrysosporium con limitaciones en carbono o } \\
\text { nitrógeno y manganeso de peroxidasa, después de la prueba se obtuvo } \\
\text { que el polietileno fue degradado por hongos que son capaces de } \\
\text { degradar lignina, en condiciones limitantes [1]. }\end{array}$ \\
\hline Biodegradación & Polietileno & $\begin{array}{l}\text { Identificación de microorganismos capaces de degradar el polietileno, } \\
\text { el estudio arrojo que los microorganismos idóneos son Brevibacillus } \\
\text { borstelensis, Penicillium simplicissimum, y Caucho de Rhodococcus [11]. }\end{array}$ \\
\hline Biodegradación & Polietileno & $\begin{array}{l}\text { Prueba realizada con } T \text {. versicolor y } P \text {. chrysosporium, con adición de } \\
\text { óxido de manganeso (II), los cultivos fueron preparados con limitantes } \\
\text { de nitrógeno y carbono, para lo cual la degradación fue positiva, se } \\
\text { comprobó que la adición de óxido de manganeso (II) mejora la } \\
\text { degradabilidad del polietileno y que la degradación se relaciona } \\
\text { directamente con los hongos capaces de degradar lignina [20]. }\end{array}$ \\
\hline Fotocatalítica & Poliestireno & $\begin{array}{l}\text { Prueba simulada con rayos UV por medio de una lampara halógena de } \\
500 \mathrm{~W} \text {, haciendo uso de Oxicloruro de bismuto }(\mathrm{BiOCl}) \text {, al pasar } 90 \\
\text { horas de la irradiación, evidenciaron pequeños orificios en la película } \\
\text { de poliestireno y los picos de intensidad en la cristalinidad del material } \\
\text { se redujeron [21]. }\end{array}$ \\
\hline Térmica y bacteriana & Poliestireno & $\begin{array}{l}\text { Se usó poliestireno licuado a punto de fusión } 240^{\circ} \mathrm{C} \text { con ayuda de las } \\
\text { bacterias } P \text {. putida y dalmonella, como resultado se tuvo que el polímero } \\
\text { se degrado a moléculas más pequeñas como el naftaleno y el benceno } \\
\text { [14]. }\end{array}$ \\
\hline Fotodegradación & Polipropileno & $\begin{array}{l}\text { Se realizo a través de una cámara de envejecimiento con lampara de } \\
500 \mathrm{~W} \text { e intensidad hasta de } 365 \mathrm{~nm} \text {, con intervalos de muestreo entre } \\
24 \text { y } 192 \text { horas, se observaron cambios en la cristalización del polímero, } \\
\text { pero no se consideran óptimas para el estudio, se recomendó aumento } \\
\text { de temperatura para pruebas futuras [22]. }\end{array}$ \\
\hline Fotooxidación & Polipropileno & $\begin{array}{l}\text { Pruebas de degradación para la mezcla de polipropileno y } \\
\text { politereftalato de butileno, se utilizaron composiciones diferentes para } \\
\text { la mezcla con } 30 \%, 50 \% \text { y } 70 \% \text { de polipropileno, con el fin de lograr } \\
\text { hacer una comparación del comportamiento de los polímeros, los } \\
\text { resultados indicaron que las reacciones fotolíticas puras, no son } \\
\text { compatibles con el politereftalato de butileno, mientras que para el } \\
\text { polipropileno si, lo cual favorece la degradación [9]. }\end{array}$ \\
\hline Fotooxidación & Polipropileno & $\begin{array}{l}\text { Prueba realizada en la superficie del mar, con diferentes } \\
\text { profundidades entre } 50 \text { y } 70 \mathrm{~cm} \text {, en temporada de calor, se obtuvo que } \\
\text { la degradación es óptima entre mayor disponibilidad de luz solar y de } \\
\text { oxígeno se tenga, combinados con la naturaleza hidrolítica del agua. El } \\
\text { polímero presenta fragmentación y a } 50 \mathrm{~cm} \text { de profundidad y presenta } \\
\text { reducción en el contenido de carbono en un } 6,67 \% \text { [10]. }\end{array}$ \\
\hline & & Pretratamiento térmico hidrotermal a temperaturas de $160,180,200 \mathrm{y}$ \\
\hline
\end{tabular}




\begin{tabular}{|c|c|l|}
\hline Pretratamiento & $\begin{array}{c}\text { Cascarilla de } \\
\text { arroz }\end{array}$ & $\begin{array}{l}220^{\circ} \mathrm{C} \text { y pretratamiento no térmico (molienda), observaron que el } \\
\text { pretratamiento térmico obtuvo mejor rendimiento de degradación a } \\
\text { temperatura } 180^{\circ} \mathrm{C}(43-63 \%) \text {, con el no térmico la degradación no fue } \\
\text { representativa [19]. }\end{array}$ \\
\hline Pretratamiento & $\begin{array}{l}\text { Mejorar el rendimiento de la degradabilidad por medio de la } \\
\text { Cascarilla de } \\
\text { arroz } \\
\text { pruebas se obtuvo que la combinación CaO-LFD (Óxido de calcio - } \\
\text { fracción líquida de digestato) fue la que mostró mejores resultados de } \\
\text { acuerdo con las otras pruebas. La reducción de lignocelulosa fue de } \\
20,73 \% \text { en comparación con la muestra de control lignina [23]. }\end{array}$ \\
\hline Térmica & Cascarilla de \\
arroz & $\begin{array}{l}\text { Comparación de la degradación sin pretratamiento o con él, a través de } \\
\text { ácido y alcalino, concluyen que la degradación es más efectiva con } \\
\text { pretratamiento, puesto que se evidencia alteración en la hemicelulosa } \\
\text { y en la lignina [24]. }\end{array}$ \\
\hline
\end{tabular}

Fuente: Elaboración propia basado en [1], [5], [9],[10], [11], [14], [16], [17], [21], [23], [24], [27].

A través de la revisión realizada y expuesta en la tabla 1, se pueden identificar los posibles métodos de degradación para cada uno de los compuestos, se puede decir que en el caso de la mezcla de polipropileno y poliestireno expandido recuperados las mejores opciones de degradación vendrían siendo degradación térmica y fotodegradación, y para el caso de la mezcla de polipropileno, polietileno de alta densidad recuperados, cascarilla de arroz y agente de acople silano, es importante realizar un pretratamiento para posteriormente hacer degradación biológica y fotodegradación, de acuerdo con la revisión, la degradación se debe realizarla en diferentes fases, puesto que se encontraron pocos estudios donde se realice la degradación de la misma forma para los diferentes compuestos de las mezclas.

En un estudio declaran que ningún pretratamiento es del todo efectivo, y se propone que lo mejor es combinar los métodos, ya que, ni el físico, ni químico, ni biológico, por sí solos muestran resultados favorables para la degradación [25]. A partir de dichos pretratamientos la mayor información encontrada acerca de la degradabilidad de la cascarilla de arroz es por degradación térmica [24], [26].

En cuanto a las mezclas, es importante tener en cuenta que la degradación debe ir directamente relacionada con el componente que se degrade más fácilmente [12], puesto que el proceso de degradación inicia principalmente interfiriendo en la cadena estructural del polímero, por lo cual aumenta el área de ataque de las enzimas [11], seguir esta base hace que la degradación se realice de forma adecuada y en menos tiempo. En el estudio realizado por Valverde et.al [6] se identificó que la biodegradación es más optima cuando hay contenido de lignina, esto es favorable para el presente estudio, puesto que, una de las mezclas es polipropileno y polietileno de alta densidad recuperados, cascarilla de arroz y agente de acople silano, lo que quiere decir que favorece la degradación de la cascarilla de arroz por su contenido de lignina [6]. 
Este trabajo fue basado en una revisión bibliográfica, para dar un aporte acerca de la degradación de diferentes polímeros y así mismo de la cascarilla de arroz, dando respuesta a la degradación de las mezclas a trabajar, puesto que no se conocen estudios para degradarlas, se hizo compilando datos de diferentes estudios realizados, da tal manera que sea de utilidad a futuros trabajos, con el fin de que no se realicen experimentaciones innecesarias, y por lo contrario se puedan articular y complementar nuevos experimentos y métodos a realizar.

\section{CONCLUSIONES}

La degradación de los polímeros es indispensable para lograr reducir el impacto ambiental generado por estos, pero, a pesar de que existen una gran variedad de métodos y experimentos, no todos son útiles para cualquier tipo de material y menos tratándose de polímeros. Ahora bien, para el caso de los materiales analizados, es menos probable que un solo método sirva para todos los materiales, y aun más, debido a que una de las mezclas contiene un residuo agrícola como lo es la cascarilla de arroz. La mejor opción para lograr la degradación de estas mezclas es realizar la combinación de los diferentes métodos expuestos en este artículo, en el caso de la primera mezcla se podrían utilizar métodos de degradación térmica y fotodegradación, y para la segunda es necesario realizar pretratamiento para posteriormente hacer degradación biológica y fotodegradación. Esto se expone debido a que, la mayoría de los estudios sobre este tema son propios por cada tipo de polímero, y es necesario realizar pruebas específicas dependiendo del tipo de componentes que contiene la mezcla

\section{REFERENCIAS}

[1] M. Shimao, «Biodegradation of plastics», Current Opinion in Biotechnology, vol. 12, n. ${ }^{\circ}$ 3, pp. 242-247, jun. 2001, doi: 10.1016/S0958-1669(00)00206-8.

[2] Y. Orhan y H. Büyükgüngör, «Enhancement of biodegradability of disposable polyethylene in controlled biological soil», International Biodeterioration $\mathcal{E}$ Biodegradation, vol. 45, n. 1 , pp. 49-55, ene. 2000, doi: 10.1016/S0964-8305(00)00048-2.

[3] R.-J. Mueller, «Biological degradation of synthetic polyesters-Enzymes as potential catalysts for polyester recycling», Process Biochemistry, vol. 41, n. ${ }^{\circ} 10$, pp. 21242128, oct. 2006, doi: 10.1016/j.procbio.2006.05.018.

[4] S. Tabasum et al., "A review on blending of corn starch with natural and synthetic polymers, and inorganic nanoparticles with mathematical modeling», International Journal of Biological Macromolecules, vol. 122, pp. 969-996, feb. 2019, doi: 10.1016/j.ijbiomac.2018.10.092.

[5] Q. Liu et al., «Effect of annealing-induced microstructure on the photo-oxidative degradation behavior of isotactic polypropylene», Polymer Degradation and Stability, vol. 162, pp. 180-195, abr. 2019, doi: 10.1016/j.polymdegradstab.2019.02.023.

[6] A. Valverde, L. Sarria, y J. Monteagudo, «Análisis comparativo de las características fisicoquímicas de la cascarilla de arroz», Scientia et Technica, vol. 1, n. ${ }^{\circ} 37$, pp. 255-260, dic. 2007, doi: 10.22517/23447214.4055.

[7] A. Prada y C. E. Cortés, «La descomposición térmica de la cascarilla de arroz: una alternantiva de aprovechamiento integral», Revista Orinoquia, vol. 14, n. ${ }^{\circ}$ 1, p. 17, 2010.

[8] D. Munar, J. Solano, y D. Orjuela, «Caracterización mecánica, térmica y morfológica de la matriz de 
polietileno de alta densidad/polipropileno con cascarilla de arroz y determinación de posibles usos», Santo Tomás, Bogotá, Colombia.

[9] A. Rivaton, F. Serre, y J. L. Gardette, «Oxidative and photooxidative degradations of PP/PBT blends», Polymer Degradation and Stability, vol. 62, n. ${ }^{\circ} 1$, pp. 127-143, ene. 1998, doi: 10.1016/S0141-3910(97)00271-1.

[10] A. Khoironi, H. Hadiyanto, S. Anggoro, y S. Sudarno, «Evaluation of polypropylene plastic degradation and microplastic identification in sediments at Tambak Lorok coastal area, Semarang, Indonesia», Marine Pollution Bulletin, vol. 151, p. 110868, feb. 2020, doi: 10.1016/j.marpolbul.2019.110868.

[11] A. A. Shah, F. Hasan, A. Hameed, y S. Ahmed, «Biological degradation of plastics: A comprehensive review», Biotechnology Advances, vol. 26, n. ${ }^{\circ}$ 3, pp. 246-265, may 2008, doi: 10.1016/j.biotechadv.2007.12.005.

[12] J. Arutchelvi, M. Sudhakar, A. Arkatkar, M. Doble, S. Bhaduri, y P. Veera, "Biodegradation of polyethylene and polypropylene», Indian Journal of Biotechnology, vol. 7, p. 14, ene. 2008.

[13] N. Lucas, C. Bienaime, C. Belloy, M. Queneudec, F. Silvestre, y J.-E. Nava-Saucedo, «Polymer biodegradation: Mechanisms and estimation techniques - A review», Chemosphere, vol. 73, n. ${ }^{\circ} 4$, pp. 429-442, sep. 2008, doi: 10.1016/j.chemosphere.2008.06.064.

[14] D. Bikiaris, J. Aburto, I. Alric, M. Botev, C. Betchev, y C. Panayiotou, «Mechanical Properties and Biodegradability of LDPE Blends with Fatty-Acid Esters of Amylose and Starch», Journal of Applied Polymer Science, vol. 71, n. ${ }^{\circ}$ 7, pp. 1089-1100, feb. 1999, doi: 10.1002/(sici)1097-4628(19990214)71:7<1089::aidapp7>3.0.co;2-i.

[15] J. Savoldelli, D. Tomback, y H. Savoldelli, «Breaking down polystyrene through the application of a two-step thermal degradation and bacterial method to produce usable byproducts», Waste Management, vol. 60, pp. 123126, feb. 2017, doi: 10.1016/j.wasman.2016.04.017.

[16] G. Botelho, A. Queirós, A. Machado, P. Frangiosa, y J. Ferreira, «Enhancement of the thermooxidative degradability of polystyrene by chemical modification», Polymer Degradation and Stability, vol. 86, n. ${ }^{\circ} 3$, pp. 493497, dic. 2004, doi: 10.1016/j.polymdegradstab.2004.05.022.

[17] L. Vargas y P. Alvarado, «Caracterización del subproducto cascarilla de arroz en búsqueda de posibles aplicaciones como materia prima en procesos», Revista Científica de la Facultad de Ciencias Químicas y Farmacia, vol. 23, n. ${ }^{\circ}$ 1, pp. 86-101, 2013.

[18] J. Lee, J. R. Kim, S. Jeong, J. Cho, y J. Y. Kim, «Long-term performance of anaerobic digestion for crop residues containing heavy metals and response of microbial communities», Waste Management, vol. 59, pp. 498-507, ene. 2017, doi: 10.1016/j.wasman.2016.10.005.

[19] J. Lee y K. Y. Park, «Impact of hydrothermal pretreatment on anaerobic digestion efficiency for lignocellulosic biomass: Influence of pretreatment temperature on the formation of biomass-degrading byproducts», Chemosphere, vol. 256, p. 127116, oct. 2020, doi: 10.1016/j.chemosphere.2020.127116.

[20] Y. Liyoshi, Y. Tsutsumi, y T. Nishida, «Polyethylene degradation by lignin-degrading fungi and manganese peroxidase», Journal of Wood Science, vol. 44, n. ${ }^{\circ} 3$, pp. 222229, 1998, doi: 10.1007 / BF00521967.

[21] A. T. W. M. Hendriks y G. Zeeman, «Pretreatments to enhance the digestibility of lignocellulosic biomass», Bioresource Technology, vol. 100, n. ${ }^{\circ}$ 1, pp. 10-18, ene. 2009, doi: 10.1016/j.biortech.2008.05.027.

[22] B. Sarwan, A. D. Acharya, S. Kaur, y B. Pare, «Visible light photocatalytic deterioration of polystyrene plastic using supported $\mathrm{BiOCl}$ nanoflower and nanodisk", European Polymer Journal, vol. 134, p. 109793, jul. 2020, doi: 10.1016/j.eurpolymj.2020.109793.

[23] A. Kumar y R. Chandra, «Ligninolytic enzymes and its mechanisms for degradation of lignocellulosic waste in environment», Heliyon, vol. 6, n. ${ }^{\circ}$ 2, p. e03170, feb. 2020, doi: 10.1016/j.heliyon.2020.e03170.

[24] M. Kumar, P. K. Mishra, y S. N. Upadhyay, «Thermal degradation of rice husk: Effect of pre-treatment on kinetic and thermodynamic parameters», Fuel, vol. 268, p. 117164, may 2020, doi: 10.1016/j.fuel.2020.117164.

[25] R. Guan et al., «Enhancing anaerobic digestion performance and degradation of lignocellulosic components of rice straw by combined biological and chemical pretreatment», Science of The Total Environment, vol. 637-638, pp. 9-17, oct. 2018, doi: 10.1016/j.scitotenv.2018.04.366.

[26] K. G. Mansaray y A. E. Ghaly, «Thermal degradation of rice husks in nitrogen atmosphere», Bioresource Technology, vol. 65, n. ${ }^{\circ} 1$, pp. 13-20, jul. 1998, doi: 10.1016/S0960-8524(98)00031-5 


\title{
ANAEROBIC DIGESTION OF ORANGE, TANGERINE AND LEMON PEEL
}

\section{Digestión anaeróbica de cascara de naranja, mandarina y limón}

\author{
Milquez-Sanabria, Harvey ${ }^{1}$; Escarraga-Parra, Karen; Espinosa-González, Nicolas \\ Universidad de América, Grupo de Investigación en Procesos de Separación no Convencionales
}

\begin{abstract}
The agro-industrial sector in Colombia is the first non-oil related product to be exported in the country. It contributes $31 \%$ of the national GDP. However, agro-industrial waste is a problem that affects the entire community. In 2019, 283,815 tons of citrus fruits were produced, of which $20 \%$ are organic waste. Anaerobic digestion is a technology that has been used in different industries, however the complexity of the substrate is an element to consider in the design of industrial processes. In the present project, anaerobic digestion of citric residues (orange, tangerine and lemon) was carried out, with a high concentration of volatile fatty acids. The results indicated that although anaerobic digestion can be used to reduce the volume of the residue, energy production is affected by the high concentration of VFA, which was greater than $1000 \mathrm{mg} / \mathrm{L}$, alternatives must be proposed to improve the process. However, the biogas production in this project was lower than that reported by other researchers for citrus residues, reaching accumulated values of $88 \mathrm{ml}$. This indicates an obvious inhibition of the process, which can be improved through a two-stage digestion process.
\end{abstract}

Keywords: Anaerobic digestion, citrus waste, VFA inhibition.

\section{Resumen}

El sector agroindustrial en Colombia es el primer sector no petrolero que exporta en el país, aporta el 31\% del PIB nacional. Sin embargo, los residuos agroindustriales son un problema que afecta a toda la comunidad. En 2019 se produjeron 283.815 toneladas de cítricos. La digestión anaeróbica es una tecnología que se ha utilizado en diferentes industrias, sin embargo, la complejidad del sustrato es un elemento que debe considerarse en el diseño de procesos industriales. En el presente proyecto se realizó la digestión anaeróbica de residuos cítricos (naranja, mandarina y limón), con alta concentración de ácidos grasos volátiles. Los resultados indicaron que, si bien se puede utilizar la digestión anaeróbica para reducir el volumen del residuo, la producción de

\footnotetext{
${ }^{1}$ Corresponding E-mail: harvey.milquez@profesores.uamerica.edu.co
} 
energía se ve afectada por la alta concentración de AGV, que fue superior a $1000 \mathrm{mg} / \mathrm{L}$, por lo cual se deben proponer alternativas para mejorar el proceso. Sin embargo, la producción de biogás en este proyecto fue menor a la reportada por otros investigadores para residuos de cítricos, alcanzando valores acumulados de $88 \mathrm{ml}$. Esto indica una obvia inhibición del proceso, que puede mejorarse mediante un proceso de digestión en dos etapas.

Palabras clave: digestión anaeróbica, residuos cítricos, inhibición de AGV.

\section{INTRODUCTION}

According with the Colombian Residential Public Utility Superintendency (SSPD), the disposition of waste in Colombia was 30.081 ton/day; only in Bogota daily was disposed around 6.700 tons in the local landfill, of which $53.22 \%$ was organic waste [1]. The agro-industrial sector in Colombia generates more that $20 \%$ of the jobs, represent $5 \%$ of the GDP, only in 2016 the agro-industrial production was 75.518 million of pesos, equivalent to $31.7 \%$ of the national production; and in 2017 exportation of food and commodities were USD 7.309 million, in 164 countries and with a participation of $49 \%$ of the exportation non relatives to oil and coal [2]. However, agro-industrial wastes are a problem for the sector, because the high cost of its disposition, taxes and transport [3].

In 2019 the production of fruits in Colombia reached 441.267 ha, of which production of citrus was 283.815 tons [4]. The main destinations of citrus in the country are domestic consumption, in industrial production of juices, jams and food additives. However, about $20 \%$ of the weight of the fruit becomes waste [5]. For this reason, a valorization of this wastes are becoming an strategic alternative, using different technologies as extraction of organic compounds [6], animal food [7], raw material [8] and transformation in biofuels [9].

Anaerobic digestion is a process by which a highly complex carbon source is broken down by different metabolic pathways to produce methane, carbon dioxide, water, and hydrogen [10]. The two main objectives of the process are the reduction of the volume and complexity of the substrate [11], and the production of biofuels [12]. Different studies have been published related to the anaerobic digestion of citrus residues, Eryildiz et al., 2020 studied production of VFA and its inhibition of the process [13]; Lotito et al., 2018 show that the age of the residue and the type of citric storage affect the anaerobic digestion process [14]. Anaerobic digestion has been researched in recent years, mainly due to the advantages related to the diversity of substrates to be used [15], modification of metabolic pathways [16], redesign of reactors [17], inhibitor identification [18] and 
mathematical modeling [19]. The aim of this study is applying anaerobic digestion as a technology for the reduction of citric residues in the Colombian industry.

\section{MATERIALS AND METHODS.}

\section{Characterization of citrus residues}

Citric residues (lemon, tangerine, and orange) were provided by a company that produces pulps and juice in Bogota; and is mainly composed of shells, seeds, and bagasse. For the development of the project, the three residues were mixed, to establish a strategy that minimizes the separation operations, in proportions of $40 \%$ lemon residues, 35\% tangerine and $25 \%$ orange. For experimental development were used ratios 1:2, 1: 3 and 1: 4 between citric residues and water $(\mathrm{S} / \mathrm{W})$. These mixtures were analyzed for volatile fatty acids (VFA), alkalinity, total solids. VFA and alkalinity were measured according Bote-Tello (2013) [20] and total solids agreeing Zagal et al., (2007) [21].

\section{Reactor system characterization}

Reactors used were made of glass, a total volume of 41 and effective volume of 31 ; upper cover was adjusted to allow coupling of the biogas outlet, and leak tests were made. The reactor was filled with a mix of carbon source $(44 \%)$ and manure $(66 \%)$, and only carbon source without manure at proportions described above. Reactors were maintained between 37.4 and $38.1{ }^{\circ} \mathrm{C}$, in an isolated medium with constant temperature control.

\section{Anaerobic digestion process}

Experimentation was divided into two phases, in the first 12 experiments were realized, with the conditions described above, for 30 days. In the second, only reactors with manure were reinoculated with a granular inoculum from an active methanogenic reactor, in a relation $2 / 3$, both phases duplicated. Figure 1 presents experimental setup for phase 1. Variables measured for monitoring anaerobic digestion were $\mathrm{pH}$ (RongZhan $\mathrm{pH} 009$ IA), temperature, AGV , alkalinity [22]. The volume of biogas was measured daily, through a fluid displacement system, with a solution of $\mathrm{NaOH}$, like that reported by Salazar-Gomez, 2019 [23] 


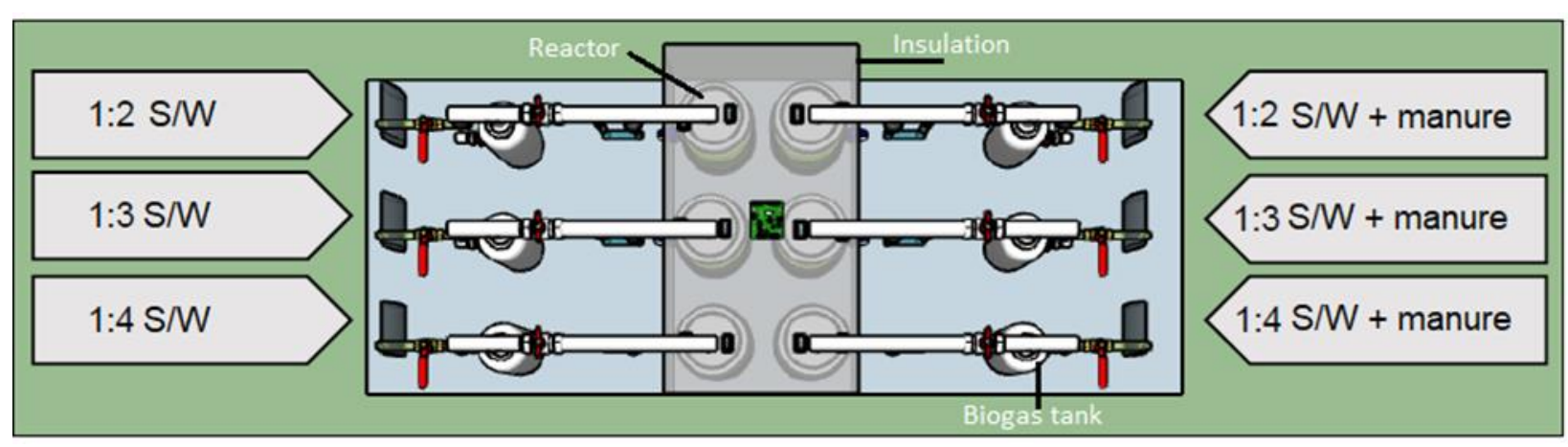

Figure 7 Reactors configuration

\section{RESULTS AND DISCUSSION}

\section{Characterization of citrus waste}

Table 1 presents the results of the characterization of carbon source. Citric residues and water are reported for different ratios. According to Varnero [22] in an anaerobic system, optimal concentration of VFA in carbon source should be between 50 and $200 \mathrm{mg} / \mathrm{l}$, since higher concentrations can affect the microbial activity of methanogenic bacteria, increasing the hydrolysis of the material. This information agrees with report by Palomino et al., where they mentioned that AGV concentrations greater than $1400 \mathrm{mg} / \mathrm{l}$ can inhibit the growth of microorganisms [24]. For the present study, the minimum concentration of AGV identified was $420 \mathrm{mg} / \mathrm{l}$ and the maximum $3972 \mathrm{mg} / \mathrm{l}$, so the process of methanization could be inhibited.

Table 5 Report carbon source.

\begin{tabular}{|c|c|c|c|c|c|c|c|c|c|}
\hline \multirow{2}{*}{} & \multicolumn{3}{|c|}{ Orange } & \multicolumn{3}{c|}{ Tangerine } & \multicolumn{3}{c|}{ Lemon } \\
\cline { 2 - 10 } & $\begin{array}{c}\text { VFA } \\
(\mathrm{mg} \\
\mathrm{Ac} / \mathrm{l})\end{array}$ & $\begin{array}{c}\text { Alkalinity } \\
(\mathrm{mg} \\
\left.\mathrm{CaCO}_{3} / \mathrm{l}\right)\end{array}$ & $\begin{array}{c}\mathrm{TS} \\
(\%)\end{array}$ & $\begin{array}{c}\text { VFA } \\
(\mathrm{mg} \\
\mathrm{Ac} / \mathrm{l})\end{array}$ & $\begin{array}{c}\text { Alkalinity } \\
(\mathrm{mg} \\
\left.\mathrm{CaCO}_{3} / \mathrm{l}\right)\end{array}$ & $\begin{array}{c}\mathrm{TS} \\
(\%)\end{array}$ & $\begin{array}{c}\text { VFA } \\
(\mathrm{mg} \\
\mathrm{Ac} / \mathrm{l})\end{array}$ & $\begin{array}{c}\text { Alkalinity } \\
(\mathrm{mg} \\
\left.\mathrm{CaCO}_{3} / \mathrm{l}\right)\end{array}$ & $\begin{array}{c}\text { TS } \\
(\%)\end{array}$ \\
\hline $1: 2$ & 1488 & 2330 & 12 & 420 & 1500 & 6 & 3972 & 1660 & 25 \\
\hline $1: 3$ & 1500 & 2650 & 6 & 1380 & 2100 & 4 & 3204 & 1570 & 16 \\
\hline $1: 4$ & 1284 & 2330 & 5 & 852 & 1090 & 3 & 2471 & 1500 & 5 \\
\hline
\end{tabular}

\section{Anaerobic digestion phase 1}

Figure 2 shows the results of the $\mathrm{pH}$ monitoring for phase 1. For all three cases (1:2, 1: 3 and 1:4) 
solutions $\mathrm{pH}$ were reduced approximately 10 days after the start of the process. In the same way, it can be observed that the mixtures with the addition of pig manure have a higher $\mathrm{pH}$ compared to the system without the addition of this compound. However, after 30 days of operation, the $\mathrm{pH}$ of both systems reaches relatively similar values. The reduction of $\mathrm{pH}$ in anaerobic systems is also reported by other authors, mainly when citric residues are used as a carbon source. Zema et al 2018., observed a rapid reduction in the $\mathrm{pH}$ of the solution to a minimum of 3.55, which can affect the production of biogas and methane in the reactor [25].
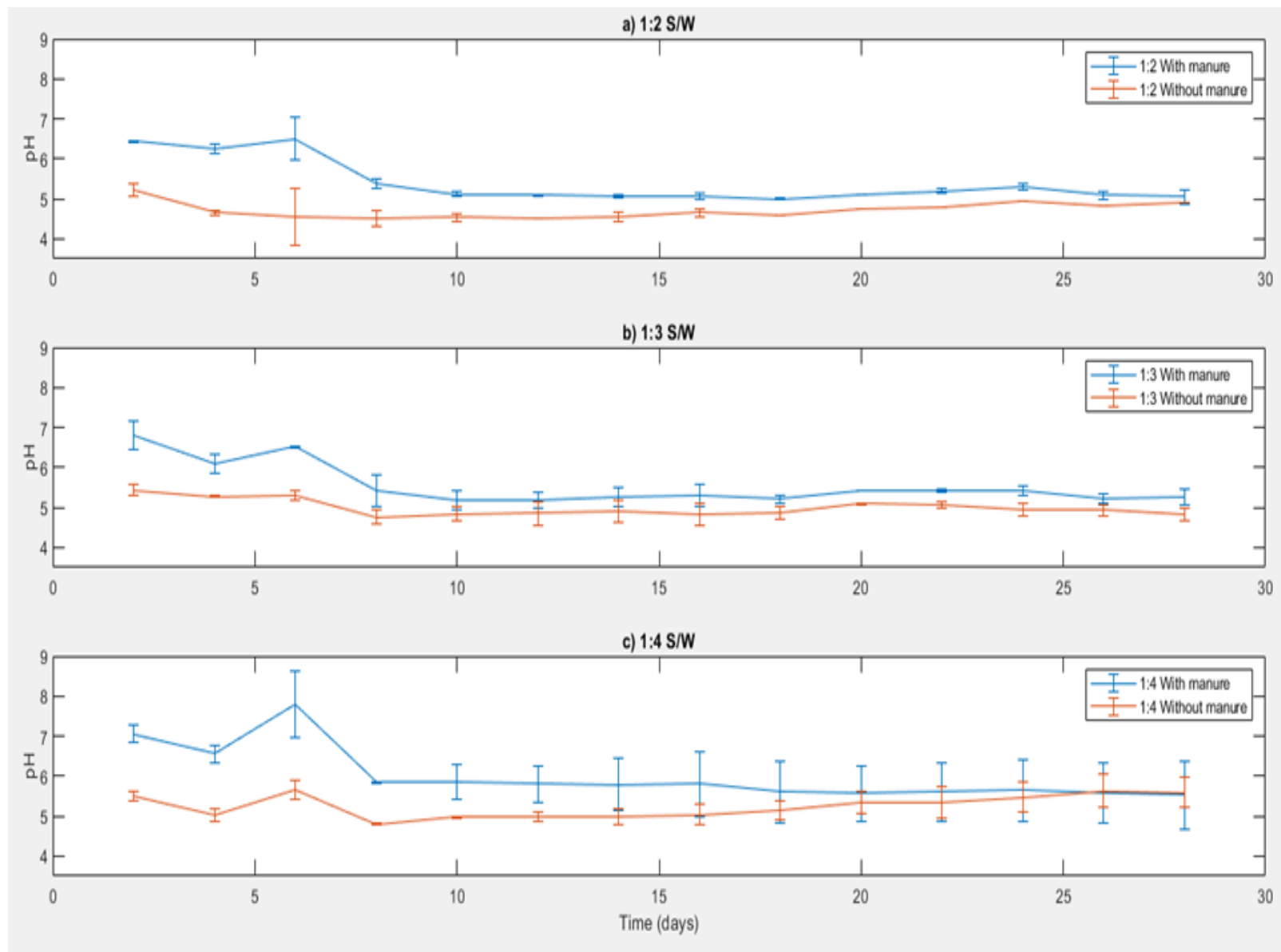

Figure 8 pH phase 1

Monitoring of the VFA and alkalinity relationship is presented in figure 3. An increase in the VFA concentration can be seen in the first 8 days, which is related to an adaptation phase; and agrees with the $\mathrm{pH}$ peak reported in figure 1 . There is a direct relationship between the VFA and the $\mathrm{pH}$, which can be seen in Figure 1 and Figure 2, both for the system with and without manure. Ruiz and Float, 2014 reported inhibition of anaerobic digestion process when citric residues are used 
as a carbon source, mainly related to high concentration of VFA together with the presence of essential oils, characteristic of citrus peels [26]. No biogas was presented in this phase, this characteristic was agreeing with the report of Yin et al., 2014 were indicated that acetic acid was the main inhibitor in methanogenesis, due few species grew well under excessive organic loads [27].
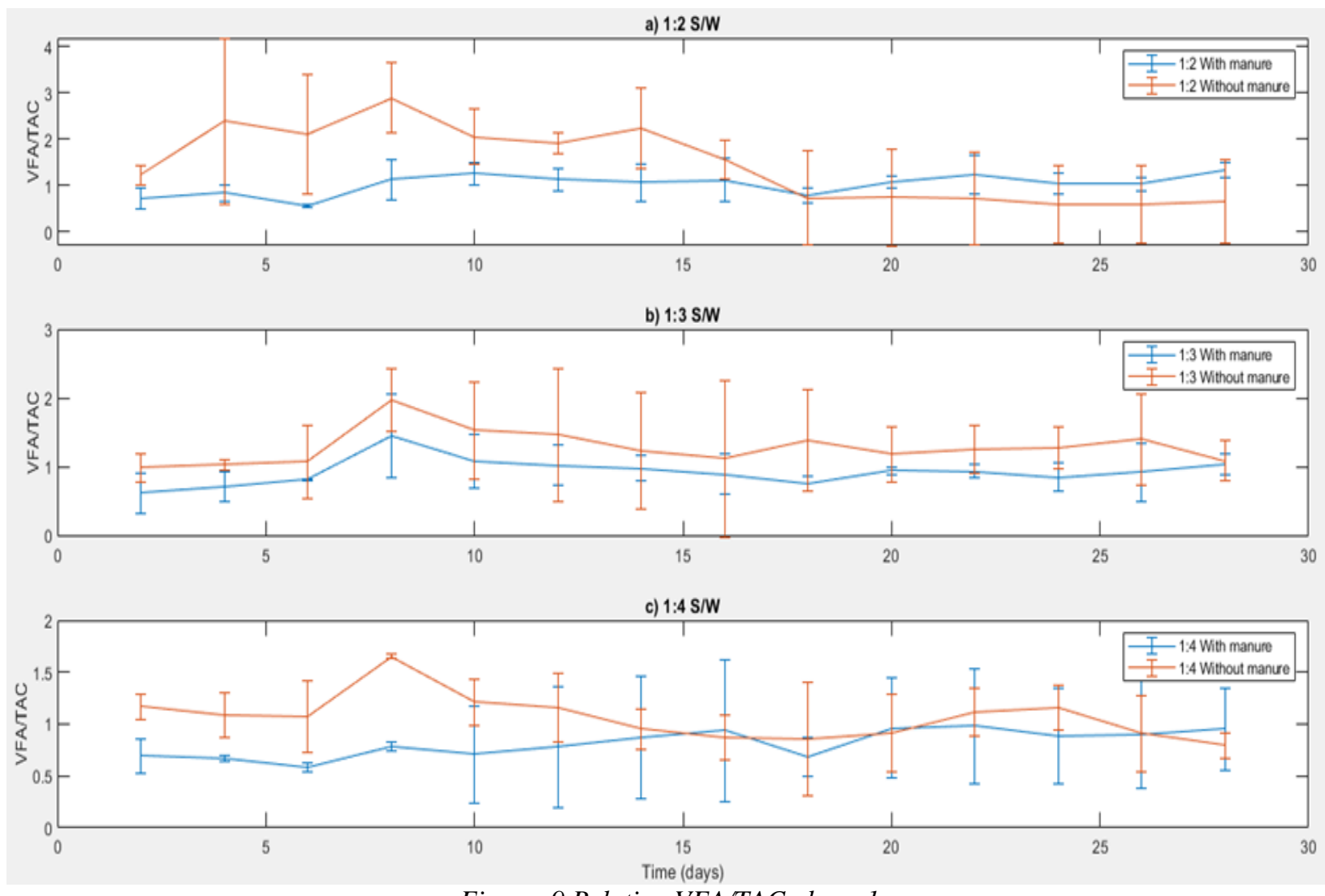

Figure 9 Relation VFA/TAC phase 1

\section{Anaerobic digestion phase 2}

Figure 4 shows the monitoring of $\mathrm{pH}$ in the anaerobic system for phase 2 . It is observed that addition of sludge from a wastewater treatment plant increases the $\mathrm{pH}$ of the system, placing it in the case of ratio 1:2. close to the recommended value of literature. After first 10 days, an increase was observed, which may be due to the consumption of VFA by the methanogenic bacteria. 

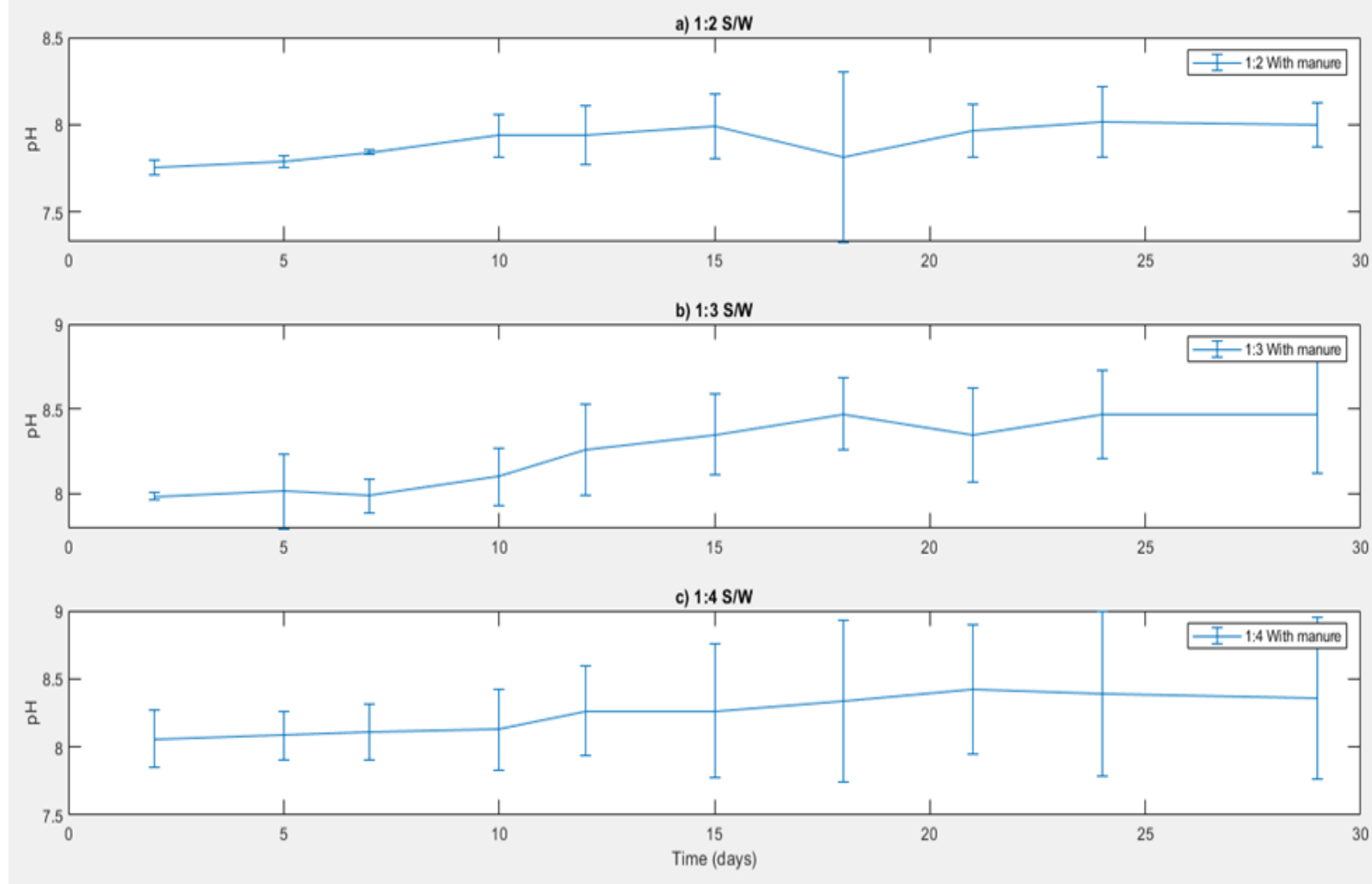

Figure $10 \mathrm{pH}$ phase 2

Report of AGV profile is presented in figure 5, reduction of organic acids in the first days of the process is confirmed, which implies that addition of sludge improves the performance of the reactor. According to Julio Guerrero et al 2016., increase in the organic load and the co-digestion of sludges of a wastewater treatment plant increases the production of methane, rising the availability of volatile solids. However, the same authors indicate that if the sludge has not been acclimatized, operating time of the process may increase [28]. 

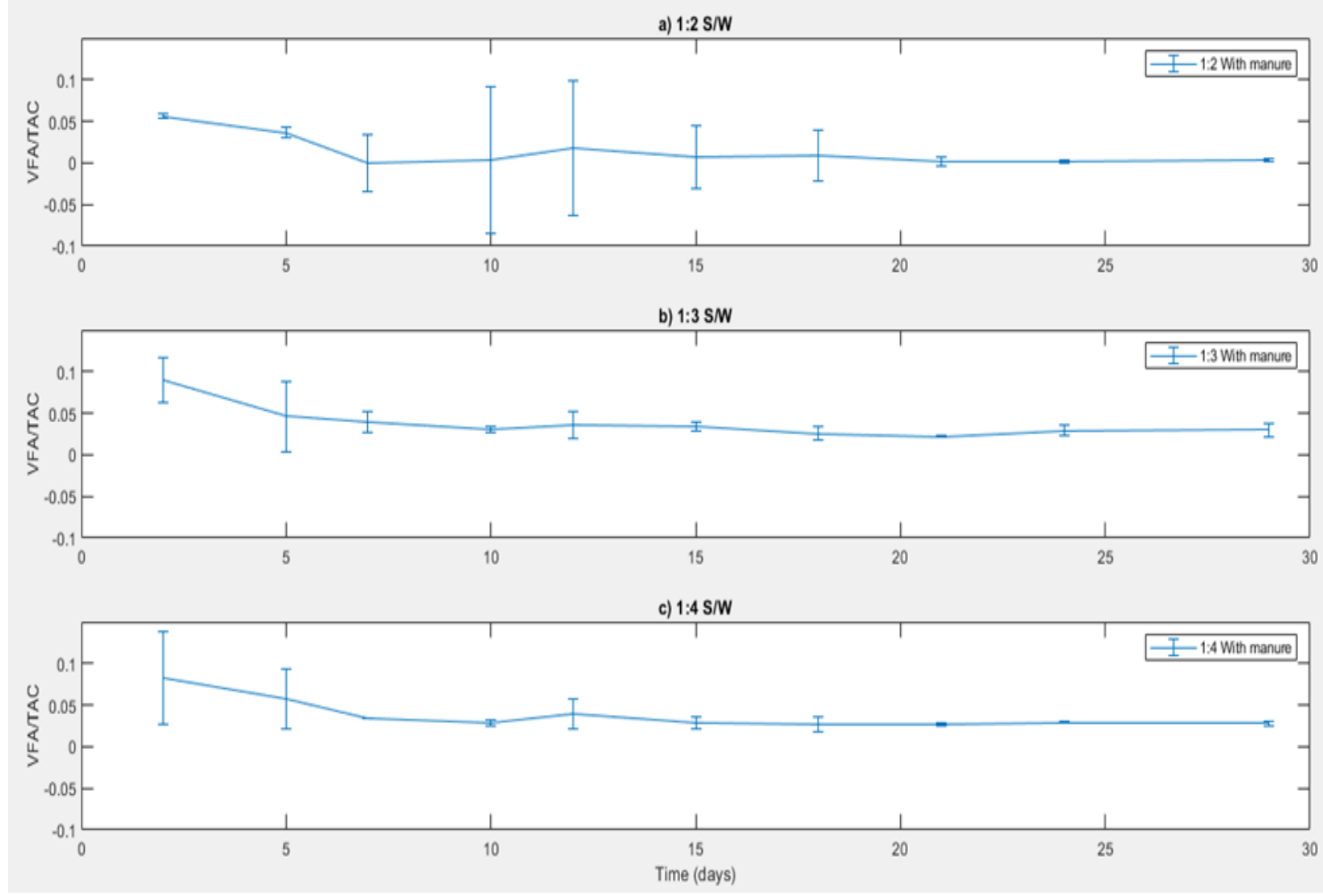

Figure 11 Relation VFA/TAC phase 2

Finally, after 30 days, maximum accumulated volume of biogas produced was $88 \mathrm{~mL}$, as show figure 6. Starting the experiment there is a typical tendency of acclimatization of the bacterial consortium to environmental and substrate conditions. However, the production of biogas is very low in the analyzed period, which is related to the inhibition presented in the reactor due to the increase in the VFA concentration. This behavior is reported by other researchers, such as RuizFuertes (2015), who indicate that d-limonene, present in citrus peel, affects the growth of methanogenic bacteria, mainly due to the reduction in the $\mathrm{pH}$ of the solution due to the concentration of VFA [29]. Although, from a theoretical point of view, and taking into account the concentration of VFA, operating conditions must be improved to reach methanogenic theorical potential that were $0.56,0.42$ and $0.34 \mathrm{~m}^{3} \mathrm{CH}_{4} / \mathrm{kg}$ TS for ratios 1:2, 1:3 and 1:4, respectively 

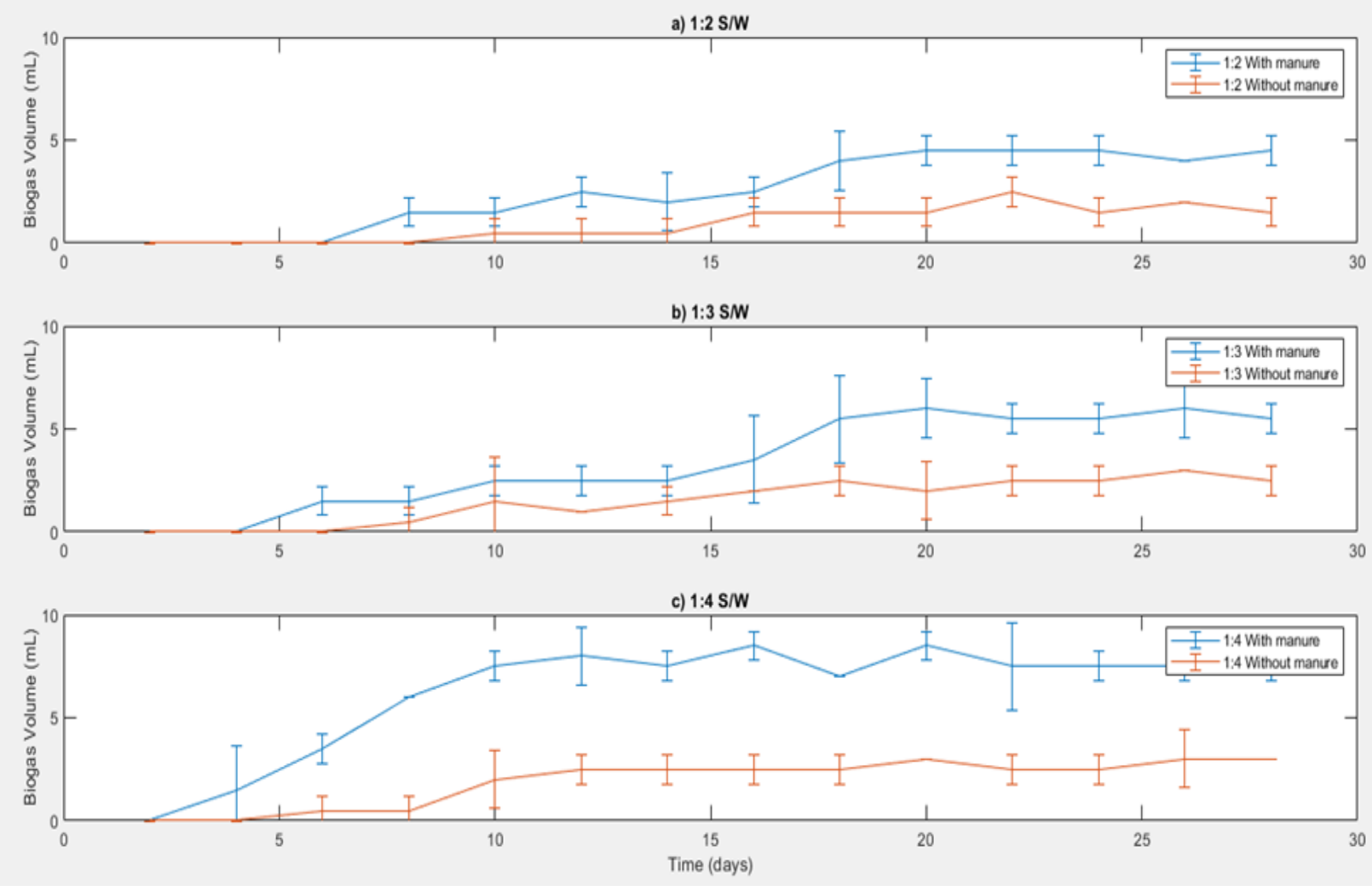

Figure 12 Biogas volume phase 1.

\section{CONCLUSIONS}

Agro-industrial waste, including citric, is a potential source of energy through biological transformations; that not only allow the use but also the reduction of the volume disposed in sanitary landfills or open dumps. Although the citrus residues have a high concentration of volatile fatty acids, traditional anaerobic digestion cannot reach methanogenic phase. For those reason, the degradation of citrus waste must be studied from the concept of digestion in two phases, in such hydrolysis processes are accelerated in a first stage, and in a second one methanogenic bacterium increase their productivity. From the theoretical point of view, the methane production potential of citrus waste, considering the VFA concentration for the systems $1: 2,1: 3$ and $1: 4$, was $0.56,0.42$ and $0.34 \mathrm{~m}^{3} \mathrm{CH}_{4} / \mathrm{kg}$ TS. Variables used for indirect monitoring of anaerobic digestion process can be applied to other projects, in which availability for direct analysis are limited, which allows the anaerobic digestion process to be applied in locations far from research centers 


\section{ACKNOWLEDGMENTS}

The authors appreciate the company sponsoring the project. In addition to the GPS research group at the University of America

\section{REFERENCES}

[1] Superservicios, "Informe de Disposición Final de Residuos Sólidos - 2017." Superintendencia de Servicios Publicos. Departamento Nacional de Planeacion, Bogota, p. 177, 2018, [Online]. Available: https://www.superservicios.gov.co/sites/default/archi vos/Publicaciones/Publicaciones/2018/Dic/2._disposici on_final_de_residuos_solidos_-_informe_2017.pdf.

[2] Procolombia, "Inversión en el sector Agroindustria en Colombia," Bogota, 2017. [Online]. Available: https:/www.inviertaencolombia.com.co/SECTORAG ROINDUSTRIA2016.pdf.

[3] Y. A. Vargas Corredor and L. I. Peréz Pérez, "Aprovechamiento de residuos agroindustriales en el mejoramiento de la calidad del ambiente," Rev. Fac. Ciencias Básicas, vol. V, no. 1, pp. 59-72, 2018, doi: 10.18359/rfcb.3108.

[4] DANE, “Encuesta Nacional Agropecuaria," Bogota, 2019. [Online]. Available: https://www.dane.gov.co/files/investigaciones/agrope cuario/enda/ena/2019/boletin_ena_2019-I.pdf.

[5] F. C. Rincón Alicia M., Vásquez, A. Marina, Padilla, “Composicion quimica y compuestos bioactivos de las harinas de cascaras de naranja (citrus sinensis), mandarina (citrus reticulata) y toronja (citrus paradisi) cultivadas en Venezuela," Arch. Latinoam. Nutr., vol. 55, no. 3, 2005.

[6] I. Leite, P., Silva, C., Salgado, J. M., \& Belo, "Simultaneous production of lignocellulolytic enzymes and extraction of antioxidant compounds by solid-state fermentation of agro-industrial wastes.," Ind. Crops Prod., no. 137, pp. 315, 322, 2019.

[7] J. S. Sadh, P. K., Duhan, S., \& Duhan, “Agro-industrial wastes and their utilization using solid state fermentation: a review," Bioresour. Bioprocess., vol. 5, p. 1, 2018.

[8] S. Panesar, P. S., \& Kaur, "Bioutilisation of agroindustrial waste for lactic acid production," Int. J. food Sci. Technol., vol. 50, no. 10, pp. 2143, 2151, 2015.

[9] \& A.-G. L. Milquez-Sanabria, Harvey., Blanco-Cocom,
Luis., "A fast linear predictive adaptive model of packed bed coupled with UASB reactor treating onion waste to produce biofuel," Microb. Cell Fact., vol. 15, no. 1, p. 167, 2016.

[10] G. Mao, C., Feng, Y., Wang, X., \& Ren, "Review on research achievements of biogas from anaerobic digestion," Renew. Sustain. energy Rev., vol. 45, pp. 540, 555, 2015.

[11] Y. Yin, Y., Liu, Y. J., Meng, S. J., Kiran, E. U., \& Liu, "Enzymatic pretreatment of activated sludge, food waste and their mixture for enhanced bioenergy recovery and waste volume reduction via anaerobic digestion," Appl. Energy, vol. 179, pp. 1131, 1137, 2016.

[12] J. Li, Y., Chen, Y., \& Wu, "Enhancement of methane production in anaerobic digestion process: A review," Appl. Energy, vol. 240, p. 120,137, 2019.

[13] B. Eryildiz, Lukitawesa, and M. J. Taherzadeh, “Effect of $\mathrm{pH}$, substrate loading, oxygen, and methanogens inhibitors on volatile fatty acid (VFA) production from citrus waste by anaerobic digestion," Bioresour. Technol., vol. 302, no. December 2019, p. 122800, 2020, doi: 10.1016/j.biortech.2020.122800.

[14] A. M. Lotito, M. De Sanctis, C. Pastore, and C. Di Iaconi, "Biomethanization of citrus waste: Effect of waste characteristics and of storage on treatability and evaluation of limonene degradation," J. Environ. Manage., vol. 215, pp. 366-376, 2018, doi: 10.1016/j.jenvman.2018.03.057.

[15] F. Carlsson, M., Lagerkvist, A., \& Morgan-Sagastume, "The effects of substrate pre-treatment on anaerobic digestion systems: a review," Waste Manag., vol. 32, no. 9, pp. 1634, 1650, 2012.

[16] B. K. Khan, M. U., \& Ahring, "Lignin degradation under anaerobic digestion: Influence of lignin modifications-A review.," Biomass and Bioenergy, vol. 128, 2019.

[17] L. Hassanein, A., Witarsa, F., Guo, X., Yong, L., Lansing, S., \& Qiu, "Next generation digestion: complementing anaerobic digestion (AD) with a novel 
microbial electrolysis cell (MEC) design," Int. J. Hydrogen Energy, vol. 42, no. 48, 2017.

[18] A. L. Amha, Y. M., Anwar, M. Z., Brower, A., Jacobsen, C. S., Stadler, L. B., Webster, T. M., \& Smith, “Inhibition of anaerobic digestion processes: applications of molecular tools," Bioresour. Technol., vol. 247, pp. 999, 1014, 2018.

[19] J. Batstone, D. J., Puyol, D., Flores-Alsina, X., \& Rodríguez, "Mathematical modelling of anaerobic digestion processes: applications and future needs," Environ. Sci. Bio/Technology, vol. 14, no. 4, p. 595,613, 2015.

[20] P. A. Bote-Tello, D. Prast-Rico, and A. Seller-Suarez, “Estudio de los efectos del $\mathrm{pH}$ extremo por adición de $\mathrm{NaOH}$ sobre la producción y calidad del biogás y otros parámetros de la digestión anaerobia urbana," Instituto Universitario del Agua y de las Ciencias Ambientales, 2013.

[21] A. ZAGAL, Erick; SADZAWKA, "PROTOCOLO DE MÉTODOS DE ANÁLISIS PARA SUELOS Y LODOS.” Facultad de Agronomía Chillán. Universidad de Concepción, Concepcion, p. 12, 2007, [Online]. Available:

http://www.sag.cl/sites/default/files/METODOS_LOD OS_SUELOS.pdf.

[22] M. Varneno, "Manual del Biogas." Organización de las Naciones Unidas para la Alimentación y la Agricultura (FAO), Santiago, p. 112, 2011, [Online]. Available: http://www.fao.org/3/as400s/as400s.pdf.

[23] L. M. Salazar-Galvis and I. D. Porras-Gomez, “MEDICIÓN DE LA PRODUCCIÓN DE BIOGÁS Y LA REMOCIÓN DE DQO Y DBO5 A PARTIR DE LA DIGESTIÓN DEL MUCÍlAGO DE CAFÉ INOCULADO CON ESTIÉRCOL BOVINO EN EL MUNICIPIO DE SUATA, SANTANDER,"
Universidad Industrial de Santander, 2019.

[24] H. L. Palomino, M. A., Ortegón, M. F., Betancourt, T. R., Martínez, J., Valderrama, J., Barragán, R., ... \& Wandurraga, "Evaluación del potencial acidogénico para producción de AGV de melaza de la industria azucarera como valorización de este subproducto," Rev. Ion, vol. 29, no. 1, pp. 71-80, 2016.

[25] D. A. Zema, A. Fòlino, G. Zappia, P. S. Calabrò, V. Tamburino, and S. M. Zimbone, "Anaerobic digestion of orange peel in a semi-continuous pilot plant: An environmentally sound way of citrus waste management in agro-ecosystems," Sci. Total Environ., vol. 630, pp. 401-408, 2018, doi: 10.1016/j.scitotenv.2018.02.168.

[26] B. Ruiz and X. Flotats, "Citrus essential oils and their influence on the anaerobic digestion process: An overview," Waste Manag., vol. 34, no. 11, pp. 2063-2079, 2014, doi: 10.1016/j.wasman.2014.06.026.

[27] Z. Xu, M. Zhao, H. Miao, Z. Huang, S. Gao, and W. Ruan, "In situ volatile fatty acids influence biogas generation from kitchen wastes by anaerobic digestion," Bioresour. Technol., vol. 163, pp. 186-192, 2014, doi: 10.1016/j.biortech.2014.04.037.

[28] I. C. Julio Guerrero, C. A. Peláez Jaramillo, and F. J. Molina Perez, "Evaluación de la co-digestión anaerobia de lodos de aguas residuales municipales con residuos de alimentos TT - Anaerobic co-digestion of municipal sewage sludge with food waste TT - Avaliação do codigestão anaeróbia de lodo de esgotos locais com resíduo," Rev. ION, vol. 29, no. 1, pp. 63-70, 2016, doi: 10.18273/revion.v29n1-2016005.

[29] M. B. Ruiz-Fuertes, "Effect of limonene on anaerobic digestion of citrus waste and pretreatments for its improvement," Universitat Politecnica de Valencia, 2015. 


\title{
THE HYDROGEN PRODUCTION AS A BASE OF GREEN CHEMISTRY
}

\section{Producción de hidrógeno como base de la química verde}

\author{
Lopez, Alejandro ${ }^{1}$; Morales-Fonseca, Diana ${ }^{2}$ \\ Fundación Universidad de América, Department Chemical Process
}

\begin{abstract}
Hydrogen has been called as a potential energy carrier for satisfying power demand of the world in the future. This document is a compilation of the information from many others papers about the hydrogen production. In addition, an account of the methods developed for the purification and improvement of production is presented. In this document was used a bibliometric analysis (VOSviewer) with a statistical method to reveal the characteristics of the quantity. According with the research publications related to hydrogen production was increased in a factor of 7.5 in the last decade with China, USA and India as leader of publication, where USA has the leadership in cited documents. Taking into account that alternative energy sources has become a pressing agenda due to recent global energy concern as a result a new tendency of environmental responsibility the research has shown that water splitting and bio-hydrogen production are the future of hydrogen production.
\end{abstract}

Key words: state of art; hydrogen; future tendency

\section{Resumen}

El hidrógeno ha sido considerado como el portador de energía potencial para satisfacer la demanda energética en el futuro. Este documento comprende una recopilación de información de otros artículos de investigación sobre la producción de hidrógeno. Así mismo, presenta una reseña de los métodos desarrollados para la depuración y mejora de la producción de hidrogeno. Para este trabajo empleó un análisis bibliométrico (VOSviewer) con un método estadístico para revelar las características de cantidad de producción bibliografica. De acuerdo con las publicaciones de investigación relacionadas con la producción de hidrógeno, el factor de publicaciones incrementó en 7.5 en la última década, siendo China, Estados Unidos e India los líderes de publicación científica en estos temas, donde Estados Unidos lidera en cuanto a documentos citados. Teniendo en cuenta que las fuentes de energía alternativas se han convertido en una agenda apremiante

\footnotetext{
${ }^{1}$ Corresponding E-mail: alejandro.lopez2@estudiantes.uamerica.edu.co

${ }^{2}$ Corresponding E-mail: diana.morales@profesores.uamerica.edu.co
} 
debido a la reciente preocupación mundial en temas energéticos y como resultado de una nueva tendencia de responsabilidad ambiental, la investigación ha demostrado que la división del agua y la producción de biohidrógeno son el futuro de la producción de hidrogeno.

Palabras clave: estado del arte; hidrógeno; tendencia futura

\section{INTRODUCTION}

Hydrogen has been called as a potential energy carrier for satisfying power demand of the world in the future, because its advantages as fuel are numerous: it is ecofriendly, efficient, renewable, and during its production and utilization no $\mathrm{CO} 2$ [1]. Organizations as HIS Chemical expects an increase of the demand of hydrogen by $5 \%$ where China, Middle East and United Stated are going to have more than middle of the domestic market [2]. The major applications of hydrogen is its ability to work as building block for many products as ammonia, methanol and for hydrogenation of liquid oils. [3]

Hydrogen is not in a free state in normal Earth condition, so it is necessary to convert natural resources as water, fossil hydrocarbons, biomass, hydrogen sulfide and anthropogenic wastes [4]. At present, the most common sources of hydrogen are nonrenewable fossil fuels, such as crude oil $(30 \%)$, natural gas $(48 \%)$, coal $(18 \%)$, and electrolysis of water $(4 \%)$; therefore, actually hydrogen cannot be consider a green technology [5].

Taking into account that alternative energy sources has become a pressing agenda due to recent global energy concern as a result a new tendency of environmental responsibility. The goal of this paper is to present a network analysis and a review about various types of proposed green hydrogen processes. For the methodology of search was used the work of Xu Tian [6] and Anbesh [7] with an adaptation of hydrogen production as parameter.

\section{METHODS}

A state of art represents a review of papers and research documents of a specific topic during a period of time with the goal to help to direct new documents. Normally, this documents follow a series of steps. First, topically relevant keywords are needed for electronic database searches. Secondly some literature analysis tools (such as VOSviewer) was applied for identifying basic features of the selected papers. Thirdly, a network analysis was made with a complex amongst the publications and their various characteristics. Fourthly, a discussion of the topics taking in 
count the main ideas.

VOSviewer is a software tool for construction of bibliometric networks with journals, researchers. For this document, it was helpful in evaluating the research status and trends of various disciplines and topics, which allows for identifying future research direction. A excel file with .CVS extension was downloaded with basic information from each publication, such as title, keywords, publication year, journal, authors' information (names, countries and institutions), and citations. In this document, databases from Scopus; Springer Link; ScienceDirect and Ebsco Host were used to make a bibliometric analysis because all these pages have good credibility and a connection algorithm of search. On the other hand, the search equation used is: "hydrogen production" AND "future"; with a change in the last part as "green hydrogen", "catalysis" and "fermentation".

Network analysis was used in these literature reviews by establishing nodes that may be represented by research papers, keywords, countries, institutions, or authors. Arcs, which are relationships, such as collaborations and influences of these nodes, can also be evaluated.

To clarify the type of data and obtain more credible results, our search criteria only included peerreviewed journal articles (selected "articles" and "review" from the "document types" option) and excluded books, book chapter and conference paper. English was selected as the principal language for further analysis in this paper. For this paper, network analysis was used to evaluate various relationships among interacting units, furthermore, the paper of Tsay was employed as reference for the characteristic of hydrogen energy related literatures between 1965 and 2015 [8]

\section{RESULTS}

\section{General performance of selected publication}

The topic of hydrogen production was divided in three main groups according with their relation: General (optimization process); Water splitting (catalysis process and electrolysis); and Fermentation (Dark-Photo fermentation). The per year total number of publications and published countries from 2011 to 2020 are shown in Fig.1

On the other hand, a cording with Wenjing Liu the number of annual publications related to hydrogen production was increased in a factor of 7.5 in the last decade [9]. Furthermore, in the 
period of 2004-2018 a total of 86 countries has published articles related by hydrogen production where China, USA and Japan have the leader hood of publications. Furthermore, these countries have carried out specific and detailed development plan to guide the hydrogen economy development.

As can be seen few publications about water splitting and hydrogen optimization process were published before 2014, while the number increased rapidly from 2014 to 2019. Overall, during this period, topic about hydrogen fermentation process kept up the number of official papers with an increase of $20 \%$

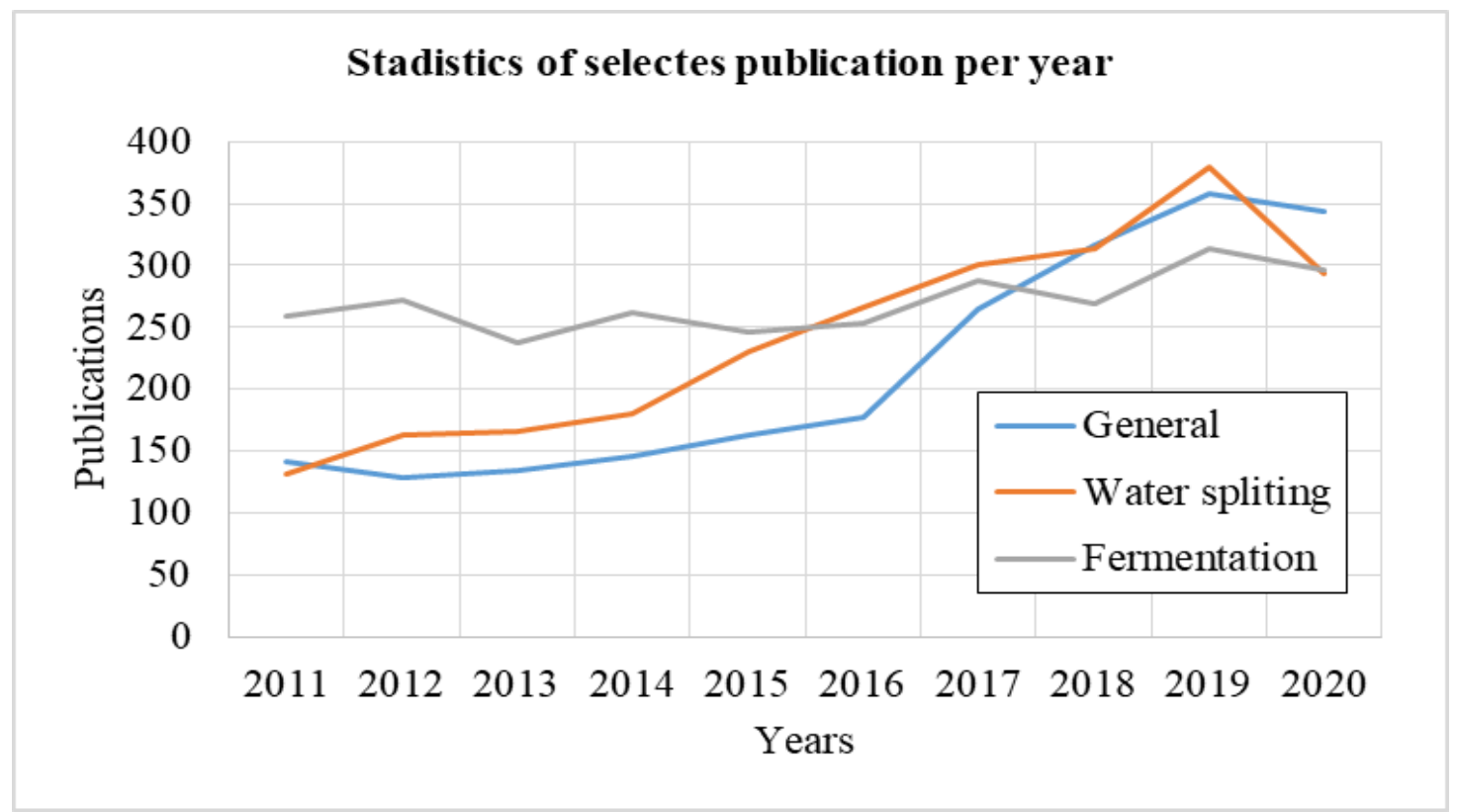

Figure 13 General statistics of selected publications from 2011 to 2020

\section{Country influences}

Each Country has his own social/economical/political needs that can help provide insight level interest of a specific investigation topic. For example, if a nation is under pressure to address environmental concerns, the embodied flows perspective may provide valuable insights on green circular economy in that nation. The number of publication shows a tendency and a political investigation support to reach a political goal. Fig. 2 summarizes this information 


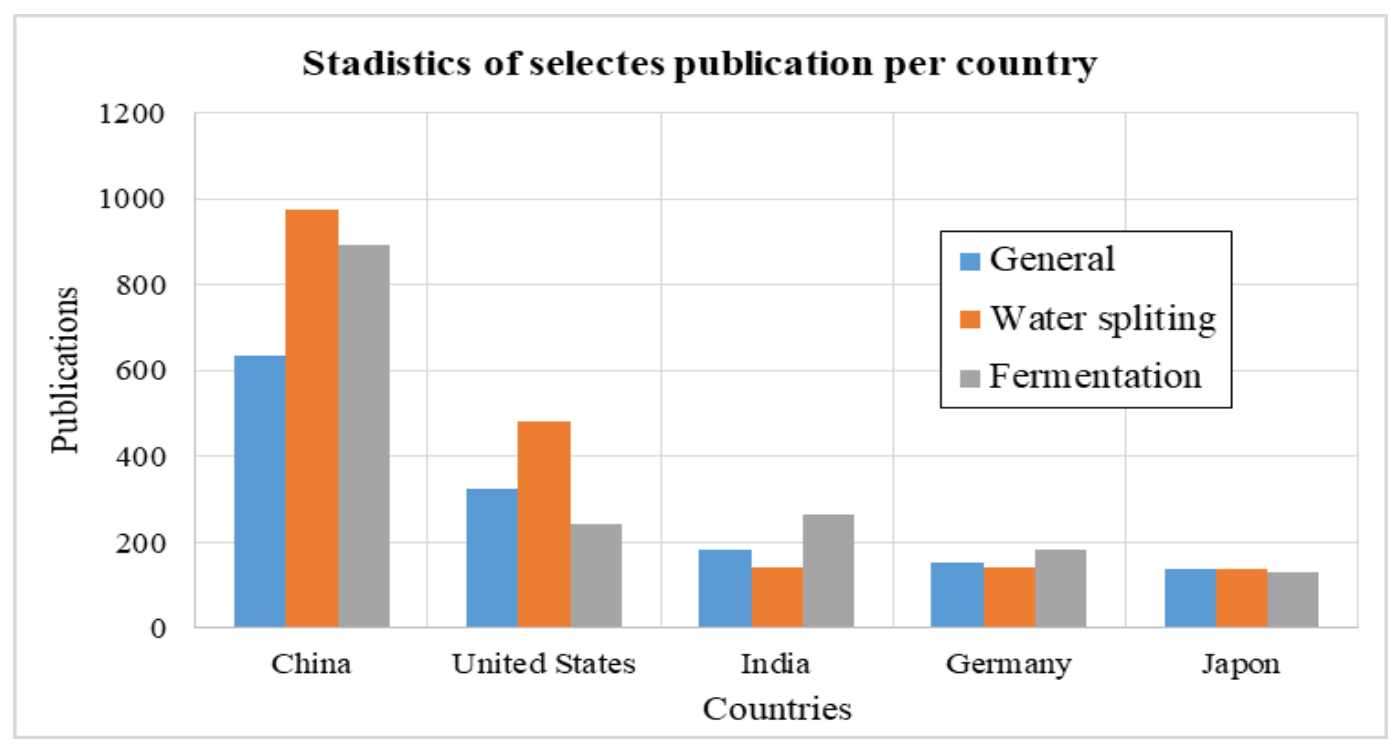

Figure 14 General statistics of selected publications per countries from 2011 to 2020

Nevertheless, the quantity of publications may not be equivalent to the quality and importance of the publications[10]. Fig. 3 shows a relation of citation by document, where it is evident that there is a inflection point that present how despite the fact that the US has fewer publications than China, US's papers double China's number of citations

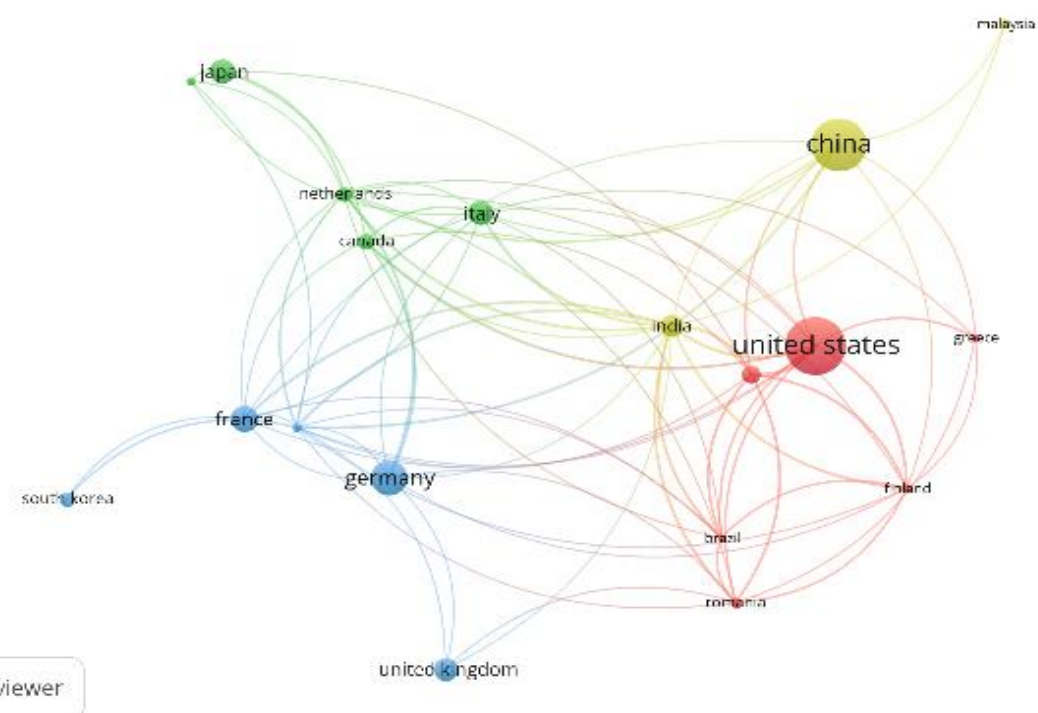

Figure 15 Performance of influence and co-occurrence publication per country from 2011 to 2020

A total of 91 countries have been represented in publications on embodied flows in international trade research from 2011 to 2020. Among these countries, USA, China, Germany, France, Italy, 
Japan, the United Kingdom, India, Spain and Canada are the top ten with the relation quality/quantity productive countries in that order; respectively they account for $22.8 \%, 20.3 \%$, $12.2 \%, 8.77 \%, 8.07 \%, 7.7 \%, 7.3 \%, 7.01 \%, 5.6 \%$ and $4.91 \%$ of the total selected publications. In addition, from an hindex perspective, USA (65), China (58), Germany (35), France (25) and Italy (23) are the top five countries in terms of influential publications.

Moreover, some papers are cooperative across countries. Fig.3 shows a co-authors analysis of the top countries in four main groups. The USA has the most collaborative publications with international partners for a total 165 papers with the other 10 countries/regions. This result shows a strong USA-centric focus of the research

\section{Keywords analysis}

A total of 1235 keywords with a minimum number of occurrences of 10 per keywords occurrences appeared from the 63 selected papers. From these keywords, $64.6 \%$ appeared only five times. Only $5.6 \%$ of the keywords appeared more than 10 times each. Some keywords may have similar ultimate meanings, such as "bio-hydrogen" and "fermentative hydrogen"; so a filter was used to regard as one keyword. Keywords information in one publication may include objectives, methods, study areas, and purposes[8]. Keywords are classified into these four areas. Fig. 4 illustrates the main topics which are extracted from keywords of the 63 selected publications, this nodal analysis shows there are three main topics that of hydrogen production.

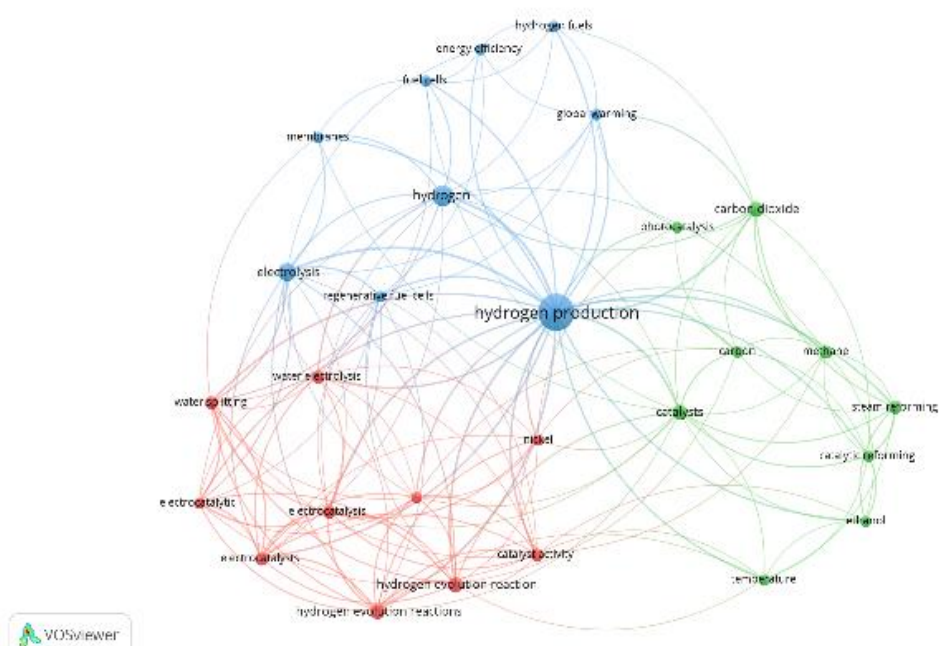

Figure 16 Nodal analysis of hydrogen production 
In order to segment the main groups. It has been made a time nodal analysis for hydrogen production Fig. 5, Fig. 6 and Fig. 7, summarize this information, where in Fig. 5 it is found that since the last 3 years, electro catalysis and metal catalysis have increased a $20 \%$ in publications, while in Fig. 6 it is found that for fermentative hydrogen an increase of $34 \%$ papers about new design of bioreactor and mix photo-dark fermentation.

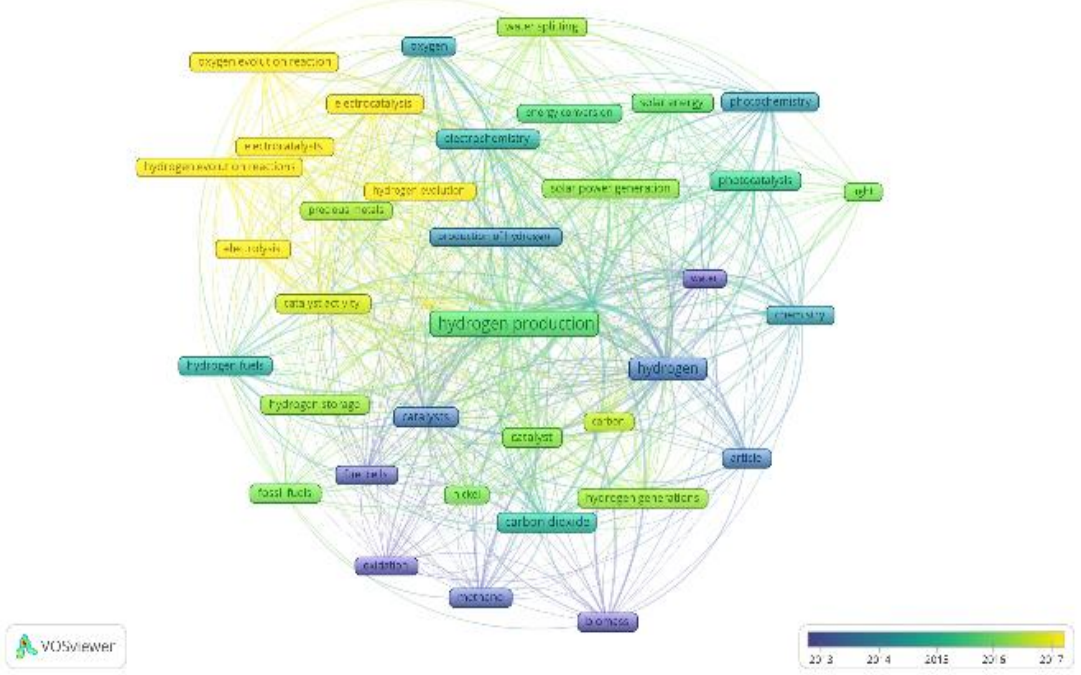

Figure 17 Time Nodal analysis of water splitting

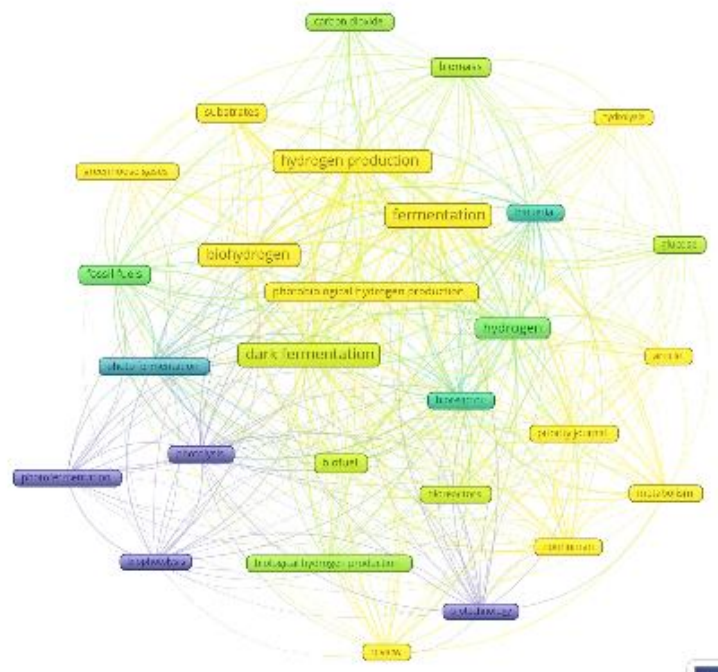

Figure 18 Time Nodal analysis of photo and dark fermentation 


\section{DISCUSSIONS}

The research on green hydrogen production has focused on two big groups: optimization process; Water splitting and Fermentation

\section{Water Splitting}

Water splitting is the process where oxygen and hydrogen are separated by electricity or in catalytic reaction. This process is needed when high purity hydrogen is demanded[11]. Through, high efficiency cells which work in low temperature, a hydrogen production rate with high purity could be obtained [12].

In contrast, efficiency of water splitting process is around the $50 \%$ and with high electricity consumption, which is not an efficient way to produce hydrogen [9]. To solve the key efficiency problem, some researches has proposed the use of renewable energy as sun light [13]. For example: Photocatalytic decomposition of water has turned very attractive for many countries due to the simplicity of the process. One of the qualities of interest is that here are many studies about the topic and are classified according with the catalyst metals: $\mathrm{TiO} 2$, sulphides represented by CdS and organic compounds represented by g-C3N4.

Other of the most recent process that has captured the attention is the thermochemical watersplitting cycle [14]. This process consists in two continues reactions where a metal oxide is used as catalyst. First, it is reduced to lower valence metal oxide at high temperature. Subsequently, a hydrogen stream is produced by water and the lower valence metal oxide, this reaction regenerate the catalyst[15][16][17][18][19]. This technology is highly research because solar light could be used to reach the temperature required in the reduction of the metal oxide. Actual tendency is operate the process near-isothermal operation.

However, one of the biggest limits consists of the selection of the metal oxide and the development of a suitable reactor.

\section{Bio-Hydrogen production}

Bioprocess can be used to produces hydrogen through many microorganisms and with different kind of substrates. Some researchers prefer biological methods over chemical methods because it 
use sunlight, $\mathrm{CO} 2$ and organic wastes for benign conversion[20],[21]. All processes of biological hydrogen production are fundamentally dependent upon the presence of a hydrogen-production enzyme. The three enzymes carrying out this reaction are known: nitrogenase, Fe-hydrogenase and NiFe hydrogenase[1].

One of the principal arguments for chosen fermentative hydrogen as the support of $\mathrm{H}_{2}$ economy is due to there are many abundant renewable biomass with a wide range of carbohydrates which offers a highest potential[22][23]. For waste feedstock substrates, a careful pretreatment is necessary to be selected to liberate glucose and make possible the process. In the case of cellulosic substrates, some authors have probe various chemical, physical and enzymatic pretreatment[24], [25].

For an improvement of the process hypothetically it is possible that the quantity or inherent activity of this enzyme could limit the overall process. [5] However, identifying novel hydrogenases and metabolic pathways through genetic engineering may assist to make biological hydrogen production more economical, practical and commercially feasible.

Microorganisms always represent a hard and fussy work. A change in the $\mathrm{pH}$, temperature, pressure or a low sterilization represent a reduction of yield [26], [27]

Bio-hydrogen is classified in three main groups: Dark fermentation, photo fermentation and a mix of the last both

\section{Dark fermentation}

Normally a bacteria uses oxygen reduction to degrade their substrates and maintain electrical neutrality. But in an anoxic environment, a bacteria has to reduce protons to molecular hydrogen and cogenerate organic [28].

Many literature sources show the microorganism species of the Clostridium as their principal study subject because these bacteria group has a high growth rate [29] and high hydrogen production rates[11].

One the other hand, self-inhibition once hydrogen partial pressure surpasses a critical values, which force the bacteria to produce acetyl-CoA and CO2 [30] 
One method that have been recently implemented is mixed cultures which allow use of feedstocks without pre-treatment or sterilization [31]. Nevertheless, mixed cultures have lower yields of hydrogen because some of the microorganisms consume a proportion of the substrate and use the formed $\mathrm{H} 2$ as electron donor[32].

\section{Photo fermentation}

Hydrogen Photo fermentation can be classified in two groups: direct and indirect where direct photolysis is inhibited by oxygen generated from water so it is necessary to implement a fastgrowing host organism to overcome the inhibition through string expression[33]; while indirect photolysis produces individually oxygen and hydrogen[34], [35].

A new method to increase rates of $\mathrm{H} 2$ production by direct photolysis consists in a nutrient deprivation [36] where cyanobacterias and microalgae express hydrogenase and nitrogenase for remains a high respiration rate [37], [38], [39]

\section{Mix of fermentation}

Individually Dark fermentation have a low hydrogen yield and high production cost [40]. For that reason some researchers proposed a combinations between dark fermentation and photo fermentative hydrogen production process [41], [42], [43], which has shown an improvement of yields from industrial wastes because it reduces co-products of the fermentation process[44], [45], [46].

One advantages of this method is that dual system can be operated in continuous mode over extended periods[47]. However, combined fermentation also faces limitations with the reactor design and high energy consumption.

\section{CONCLUSIONS}

This study made a bibliometric analysis on hydrogen production and storage publications since 2011, where China, USA and India are the principal countries that have contributed and planed a hydrogen economy, where US has the leadership with a referenced research. 
According with the network analysis for hydrogen production research, the future of green hydrogen production is focus in three main groups: general optimization process, water splitting and fermentation, which the first one is transversal optimization process and the last two are the principal tendencies.

For water splitting process, photocatalytic decomposition of water to hydrogen has attracted more and more research in the last two year, due to the control of the reaction conditions has important effect on the efficiency of photocatalytic decomposition of water. On the other hand, bio-hydrogen production has a low efficiency but there is a good change of improvement with CRISPR modification. It is crucial to continue additional research and improve the efficiency of hydrogen generation

\section{REFERENCES}

[1] P. Tamagnini, R. Axelsson, P. Lindberg, F. Oxelfelt, R. Wünschiers, and P. Lindblad, "Hydrogenases and Hydrogen Metabolism of Cyanobacteria," Microbiol. Mol. Biol. Rev., vol. 66, no. 1, pp. 1-20, 2002, doi: 10.1128/mmbr.66.1.1-20.2002.

[2] I. Markit, “Chemical Economics Handbook," 2018. [Online]. Available: https://ihsmarkit.com/products/hydrogen-chemicaleconomics-handbook.html.

[3] C. A. Grimes, O. K. Varghese, and S. Ranjan, From Hydrocarbons to Hydrogen: Towards a Sustainable Future. 2007.

[4] I. Dincer and C. Zamfirescu, Hydrogen and Its Production. 2016.

[5] P. Sinha and A. Pandey, "An evaluative report and challenges for fermentative biohydrogen production," Int. J. Hydrogen Energy, vol. 36, no. 13, pp. 7460-7478, 2011, doi: 10.1016/j.ijhydene.2011.03.077.

[6] Z. Kasapi and A. Mihiotis, "Management as applied to New Products Penetration in the Competitive Environment of Pharmaceutical Industry.," Interdiscip. J. Res. Bus., vol. 1, no. 10, pp. 73-85, 2011.

[7] A. Jamwal, R. Agrawal, M. Sharma, G. . Dangayach, and S. Gupta, "Application of optimization techniques in metal cutting operations: A bibliometric analysis," Mater. Today Proc., no. xxxx, pp. 7-12, 2020, doi: 10.1016/j.matpr.2020.07.425.

[8] X. Tian, Y. Geng, J. Sarkis, and S. Zhong, "Trends and features of embodied flows associated with international trade based on bibliometric analysis," Resour. Conserv. Recycl., vol. 131, no. September 2017, pp. 148-157, 2018, doi: 10.1016/j.resconrec.2018.01.002.

[9] W. Liu and L. Sun, "Trends and future challenges in hydrogen production and storage research," vol. 2050, 2020.

[10] L. Bornmann, R. Mutz, S. E. Hug, and H. D. Daniel, “A multilevel meta-analysis of studies reporting correlations between the $\mathrm{h}$ index and 37 different $\mathrm{h}$ index variants," J. Informetr., vol. 5, no. 3, pp. 346-359, 2011, doi: 10.1016/j.joi.2011.01.006.

[11] J. Baeyens et al., "Reviewing the potential of biohydrogen production by fermentation," Renew. Sustain. Energy Rev., vol. 131, no. June, p. 110023, 2020, doi: 10.1016/j.rser.2020.110023.

[12] F. Barbir, "PEM electrolysis for production of hydrogen from renewable energy sources," Sol. Energy, vol. 78, no. 5, pp. 661-669, 2005, doi: 10.1016/j.solener.2004.09.003.

[13] S. Shiva Kumar and V. Himabindu, "Hydrogen production by PEM water electrolysis - A review," Mater. Sci. Energy Technol., vol. 2, no. 3, pp. 442-454, 2019, doi: 10.1016/j.mset.2019.03.002.

[14] B. Cui, J. Zhang, S. Liu, X. Liu, Z. Zhang, and J. Sun, “A low-temperature electro-thermochemical watersplitting cycle for hydrogen production based on LiFeO2/Fe redox pair," Int. J. Hydrogen Energy, vol. 45, no. 41, pp. 20800-20807, 2020, doi: 10.1016/j.ijhydene.2020.02.087. 
[15] C. L. Muhich, B. D. Ehrhart, I. Al-Shankiti, B. J. Ward, C. B. Musgrave, and A. W. Weimer, "A review and perspective of efficient hydrogen generation via solar thermal water splitting," Wiley Interdiscip. Rev. Energy Environ., vol. 5, no. 3, pp. 261-287, 2016, doi: 10.1002/wene.174.

[16] D. Yadav and R. Banerjee, "A review of solar thermochemical processes," Renew. Sustain. Energy Rev., vol. 54, pp. 497-532, 2016, doi: 10.1016/j.rser.2015.10.026.

[17] Z. Chen, Q. Jiang, J. Tong, M. Yang, Z. Jiang, and C. Li, "Enhancement effects of dopants and $\mathrm{SiO} 2$ support on mixed metal ferrites based two-step thermochemical water splitting," Sol. Energy, vol. 144, pp. 643-659, 2017, doi: 10.1016/j.solener.2017.01.049.

[18] H. Xing, Y. Yuan, Z. Huiyuan, S. Yong, L. Bingxi, and T. Heping, "Solar thermochemical hydrogen production using metallic oxides," Energy Sources, Part A Recover. Util. Environ. Eff., vol. 39, no. 3, pp. 257-263, 2017, doi: 10.1080/15567036.2013.878769.

[19] H. I. Villafán-Vidales, C. A. Arancibia-Bulnes, D. Riveros-Rosas, H. Romero-Paredes, and C. A. Estrada, "An overview of the solar thermochemical processes for hydrogen and syngas production: Reactors, and facilities," Renew. Sustain. Energy Rev., vol. 75, no. October 2015, pp. 894-908, 2017, doi: 10.1016/j.rser.2016.11.070.

[20] D. Dutta, D. De, S. Chaudhuri, and S. K. Bhattacharya, "Hydrogen production by Cyanobacteria," Microb. Cell Fact., vol. 4, pp. 1-11, 2005, doi: 10.1186/1475-2859-4-36.

[21] M. D. Redwood, M. Paterson-Beedle, and L. E. MacAskie, "Integrating dark and light bio-hydrogen production strategies: Towards the hydrogen economy," Rev. Environ. Sci. Biotechnol., vol. 8, no. 2, pp. 149-185, 2009, doi: 10.1007/s11157-008-9144-9.

[22] S. Blanchette, "A hydrogen economy and its impact on the world as we know it," Energy Policy, vol. 36, no. 2, pp. 522-530, 2008, doi: 10.1016/j.enpol.2007.09.029.

[23] P. Tseng, J. Lee, and P. Friley, "A hydrogen economy: Opportunities and challenges," Energy, vol. 30, no. 14, pp. 2703-2720, 2005, doi: 10.1016/j.energy.2004.07.015.

[24] M. Cui, Z. Yuan, X. Zhi, L. Wei, and J. Shen, "Biohydrogen production from poplar leaves pretreated by different methods using anaerobic mixed bacteria," Int. J. Hydrogen Energy, vol. 35, no. 9, pp. 4041-4047, 2010, doi: 10.1016/j.ijhydene.2010.02.035.

[25] L. Laureano-Perez, F. Teymouri, H. Alizadeh, and B. E. Dale, "Understanding Factors that Limit Enzymatic Hydrolysis of Biomass," Twenty-Sixth Symp. Biotechnol. Fuels Chem., vol. 121, pp. 1081-1099, 2009, doi:
10.1007/978-1-59259-991-2_91.

[26] R. L. Orozco, M. D. Redwood, G. A. Leeke, A. Bahari, R. C. D. Santos, and L. E. MacAskie, "Hydrothermal hydrolysis of starch with $\mathrm{CO} 2$ and detoxification of the hydrolysates with activated carbon for bio-hydrogen fermentation," Int. J. Hydrogen Energy, vol. 37, no. 8, pp. 6545-6553, 2012, doi: 10.1016/j.ijhydene.2012.01.047.

[27] N. T. Eriksen, T. M. Nielsen, and N. Iversen, "Hydrogen production in anaerobic and microaerobic Thermotoga neapolitana," Biotechnol. Lett., vol. 30, no. 1, pp. 103-109, 2008, doi: 10.1007/s10529-007-9520-5.

[28] R. Nandi and S. Sengupta, "Microbial Production of Hydrogen: An Overview," vol. 24, no. 1, pp. 61-84, 1998.

[29] M. Krupp and R. Widmann, "Biohydrogen production by dark fermentation: Experiences of continuous operation in large lab scale," Int. J. Hydrogen Energy, vol. 34, no. 10, pp. 4509-4516, 2009, doi: 10.1016/j.ijhydene.2008.10.043.

[30] K. Nath and D. Das, "Improvement of fermentative hydrogen production: Various approaches," Appl. Microbiol. Biotechnol., vol. 65, no. 5, pp. 520-529, 2004, doi: 10.1007/s00253-004-1644-0.

[31] D. L. H. G. Kyazze, R. Dinsdale, A.J. Guwy, F.R. Hawkes, G.C. Premier, “Performance Characteristics of a Two-Stage Dark Fermentative System Producing Hydrogen and Methane Continuously," J. Anat., vol. 12, no. Ii, pp. 503-505, 2006, doi: 10.1002/bit.

[32] F. R. Hawkes, I. Hussy, G. Kyazze, R. Dinsdale, and D. L. Hawkes, “Continuous dark fermentative hydrogen production by mesophilic microflora: Principles and progress," Int. J. Hydrogen Energy, vol. 32, no. 2, pp. 172-184, 2007, doi: 10.1016/j.ijhydene.2006.08.014.

[33] J. Miyake, M. Miyake, and Y. Asada, "Biotechnological hydrogen production" research for efficient light energy conversion," Prog. Ind. Microbiol., vol. 35, no. C, pp. 89-101, 1999, doi: 10.1016/S0079-6352(99)80103-9.

[34] D. B. Levin, L. Pitt, and M. Love, "Biohydrogen production: Prospects and limitations to practical application," Int. J. Hydrogen Energy, vol. 29, no. 2, pp. 173-185, 2004, doi: 10.1016/S0360-3199(03)00094-6.

[35] D. Madamwar, N. Garg, and V. Shah, "Cyanobacterial hydrogen production," World J. Microbiol. Biotechnol., vol. 16, no. 8-9, pp. 757-767, 2000, doi: 10.1023/A:1008919200103.

[36] D. D. Wykoff, J. P. Davies, A. Melis, and A. R. Grossman, "The regulation of photosynthetic electron transport during nutrient deprivation in Chlamydomonas reinhardtii," Plant Physiol., vol. 117, no. 1, pp. 129-139, 1998, doi: 10.1104/pp.117.1.129. 
[37] J. Benemann, "Hydrogen Production:Progress and prospects," Nat. Biotechnol., vol. 14, pp. 1101-1103, 1996.

[38] J. H. Jo, D. S. Lee, and J. M. Park, "Modeling and optimization of photosynthetic hydrogen gas production by green alga Chlamydomonas reinhardtii in sulfur-deprived circumstance," Biotechnol. Prog., vol. 22, no. 2, pp. 431-437, 2006, doi: 10.1021/bp050258z.

[39] T. V. Laurinavichene, A. S. Fedorov, M. L. Ghirardi, M. Seibert, and A. A. Tsygankov, "Demonstration of sustained hydrogen photoproduction by immobilized, sulfur-deprived Chlamydomonas reinhardtii cells," Int. J. Hydrogen Energy, vol. 31, no. 5, pp. 659-667, 2006, doi: 10.1016/j.ijhydene.2005.05.002.

[40] S. Ghosh, R. Chowdhury, and P. Bhattacharya, "A review on single stage integrated dark-photo fermentative biohydrogen production: Insight into salient strategies and scopes," Int. J. Hydrogen Energy, pp. 2091-2107, 2018, doi: 10.1016/j.ijhydene.2017.12.018.

[41] K. Y. Show, D. J. Lee, and J. S. Chang, "Bioreactor and process design for biohydrogen production," Bioresour. Technol., vol. 102, no. 18, pp. 8524-8533, 2011, doi: 10.1016/j.biortech.2011.04.055.

[42] H. Argun and F. Kargi, "Bio-hydrogen production by different operational modes of dark and photofermentation: An overview," Int. J. Hydrogen Energy, vol. 36, no. 13, pp. 7443-7459, 2011, doi: 10.1016/j.ijhydene.2011.03.116.

[43] E. Özkan, B. Uyar, E. Özgür, M. Yücel, I. Eroglu, and U. Gündüz, "Photofermentative hydrogen production using dark fermentation effluent of sugar beet thick juice in outdoor conditions," Int. J. Hydrogen Energy, vol. 37, no. 2, pp. 2044-2049, 2012, doi: 10.1016/j.ijhydene.2011.06.035.

[44] P. K. Rai, S. P. Singh, and R. K. Asthana, “Biohydrogen production from cheese whey wastewater in a two-step anaerobic process," Appl. Biochem. Biotechnol., vol. 167, no. 6, pp. 1540-1549, 2012, doi: 10.1007/s12010-0119488-4.

[45] Y. Tao, Y. Chen, Y. Wu, Y. He, and Z. Zhou, "High hydrogen yield from a two-step process of dark- and photo-fermentation of sucrose," Int. J. Hydrogen Energy, vol. 32, no. 2, pp. 200-206, 2007, doi: 10.1016/j.ijhydene.2006.06.034.

[46] H. Yang, L. Guo, and F. Liu, "Enhanced bio-hydrogen production from corncob by a two-step process: Darkand photo-fermentation," Bioresour. Technol., vol. 101, no. 6, pp. 2049-2052, 2010, doi: 10.1016/j.biortech.2009.10.078.

[47] E. Franchi, C. Tosi, G. Scolla, G. Della Penna, F. Rodriguez, and P. M. Pedroni, "Metabolically engineered Rhodobacter sphaeroides RV strains for improved biohydrogen photoproduction combined with disposal of food wastes," Mar. Biotechnol., vol. 6, no. 6, pp. 552-565, 2004, doi: 10.1007/s10126-004-1007y. 


\title{
OPTIMIZATION OF A ROUTE FOR COLLECTING WASTE COOKING OIL IN BOGOTÁ
}

\section{Optimización de una ruta de recolección de aceite de cocina en Bogotá}

\author{
Bernal, Angie1; León. P; Fernández, C2; Malagón-Romero, Dionisio ${ }^{3}$ \\ Universidad ECCI \\ Universidad Santo Tomás
}

\section{Abstract}

Waste cooking oil (WCA) has been an environmental problem in different cities around the world. It has been reported that 1 liter of OMA could contaminate 1000 liters of water. A possible solution is collection for recycling and integration into different chemical processes, although, due to related logistical aspects, collection in large cities is difficult. In this work, a combinatorial optimization model based on the Vehicule Routing Problem is proposed that seeks to obtain the optimal cost of the WCA in the city of Bogotá produced in restaurants, shopping centers and food services. It was determined that in this city there are 17,008 commercial establishments located in 19 localities that produce 720,000 liters per month. This oil could be collected for the production of biodiesel, for which a representative sample of each locality was taken and a total of 289 collection points were used. For the programming of the model, an adaptation of the VRP Solver application was carried out, which allowed obtaining the optimal collection routes from which a value of US \$ 0.22-0.25 was determined as the optimal cost of collection in the city of Bogotá. In addition, a cost of obtaining US \$ 0.078-0.095/ km and a distance traveled ranging between $308.81 \mathrm{~km}-478.39 \mathrm{~km}$ was deducted. The results have shown that by applying route optimization, WCA could be collected in Bogotá at a competitive cost compared to virgin palm oil and under the results it is considered a very convenient option for the partial substitution of oil as a raw material in the biodiesel production process.

Keywords: WCO,collecting,optimization.

\section{Resumen}

El aceite de cocina usado (OMA) ha sido un problema ambiental en diferentes ciudades del

\footnotetext{
${ }^{1}$ Corresponding E-mail: angieberc@gmail.com

${ }^{2}$ Corresponding E-mail: clapafer@yahoo.com

${ }^{3}$ Corresponding E-mail: dionisiomalagon@usantotomas.edu.co
} 
mundo. Se ha informado que 1 litro de OMA podría contaminar 1000 litros de agua. Una posible solución es la recolección para su reciclaje e integración en diferentes procesos químicos, aunque, debido a los aspectos logísticos relacionados, la recolección en grandes ciudades resulta dificil. En este trabajo, se propone un modelo de optimización combinatoria basado en el Vehicule Routing Problem que busca obtener el costo óptimo del OMA en la ciudad de Bogotá producido en restaurantes, centros comerciales y servicios de alimentación. Se determinó que en esta ciudad hay 17.008 establecimientos comerciales ubicados en 19 localidades que producen 720.000 litros mensuales. Este aceite podría recolectarse para la producción de biodiesel, por lo que se tomó una muestra representativa de cada localidad y en total se utilizaron 289 puntos de recolección. Para la programación del modelo se realizó una adaptación de la aplicación VRP Solver, la cual permitió obtener las rutas óptimas de recolección a partir de lo cual se determinó un valor de US \$ 0,22-0,25 como el costo óptimo de recolección en la ciudad de Bogotá. Además, se dedujo un costo de obtención de US \$ 0,078-0,095 / km y una distancia recorrida que oscila entre 308,81 km-478,39 km. Los resultados han demostrado que aplicando la optimización de rutas, el OMA podría recolectarse en Bogotá con un costo competitivo en comparación con el aceite de palma virgen y bajo los resultados se plantea como una opción muy conveniente para la sustitución parcial de aceite como materia prima en el proceso de producción de biodiesel.

Palabras clave: OMA, Recolección,optimización.

\section{INTRODUCTION}

Waste cooking oil (WCA) is a liquid that originates from the transformation of vegetable oil when it is used at high temperatures [1], this process changes its organoleptic and physicochemical properties, generating new compounds [2]. Worldwide, AOM is the second most polluting liquid in wastewater [3], because concentrations of $1 \mathrm{mg}$ of oil / 1 in water make it unsuitable for human consumption [4]. Recycling the OMA and then using it in the production of biofuels could solve the problems of final disposal of this compound [5]. The reported yield is 1 liter of biodiesel per 1.2 liters of OMA used as raw material [6], [7], [8]. Furthermore, due to its high availability and low acquisition costs [9], this compound is an excellent alternative for industrial applications. However, collecting this material from restaurants, shopping malls, food services, and other sources is a major limitation.

So it is essential to determine the logistics costs for an accurate economic evaluation[9]. Once 
established the availability of the waste, the next step in the development of the process, aiming to the cost reduction is the optimization of the route[10]. Collection and optimization are associated with policies implemented in each country around the world. Different laws of WCO resources are the main issues, such as the penalty and, rewarding mechanisms, and the kitchen waste disposal fee systems[10]. However, these mechanisms have not increased the colleting due to the absence of restaurant recycling facilities and lack of knowledge about of the recycling process.

Optimization consists in the search for values of certain variables so that, fulfilling a set of requirements, represented by equations and / or algebraic inequalities, they provide the best possible value for a function that is used to measure the studied performance[11]. Optimization is achieved through mathematical strategies known as methods, which serve as a tool for decision making that maximizes or minimizes an objective function [12]. Different optimization models can be found in the literature. One of the optimization models, which has done with routing, is the model of the vehicle routing problem (VRP, vehicle route problem). VRP emerged as an extension of the Travelling Salesman Problem (TSP), in which the restriction of the limited capacity of vehicles is considered, making it necessary to use several vehicles covering different routes to satisfy all customers.

VRP has been applied extensively to transportation and logistics fields to solve the generic problem of satisfying spatially dispersed customer requests using a fleet of vehicles[13]. VRP models suppose that all vehicles depart from an initial location called depot, visit the customers according to the selected order, and once their capacities are attained they go back to the depot[13]. VRP has been used since 1959 [14], when was applied to the delivery of gasoline to service stations, setting the mathematical foundation of this problem. VRP is a complex combinatorial optimization problem, which starts from a warehouse and has a fleet of vehicles that must serve a set of customers dispersed in a geographical area, having some specific constraints of the problem[15]. Constraints could be imposed by market dynamics. VRP is a highly complex mathematical method, because the number of solutions grows exponentially according to the number of nodes, which are represented by clients or warehouses [16].

For waste cooking oil collection, the route starts and ends at the same point [9], so the cost of the service is minimized respecting different constrains. The aim of this paper is the developing of a mathematical optimization model for collecting waste cooking oil in Bogotá, Colombia. 


\section{MATERIALS AND METHODS.}

\section{Determination of volume of WCO in Bogotá.}

The collection points of the city of Bogotá used in the programming of the model were classified into three groups: restaurants, fast food places and bakeries. The location of each eating place (address, latitude and longitude) was determined using Google Maps and the points were selected from the 19 urban locations in Bogotá. The sample size was determined for $95 \%$ of the level of confidence in each locality, taking into account the number of establishments in Bogotá, for this the following equation was used.

$$
n=\frac{\left(k^{2} * p * q * N\right)}{\left.\left(e^{2} *(N-1)\right)+k^{2} * p * q\right)}
$$

Equation 1. Equation to determine the sample size.

Where:

- $\mathrm{N}$ : It is the size of the population or universe (total number of possible respondents).

- $\mathrm{k}$ : It is a constant that depends on the level of confidence assigned. The one that indicates the probability that the research results are true, for the case the confidence level used is $95 \%$ of $Z=1.96$

- e: It is the margin of error allowed. The one that for the case has been $5 \%$.

- p: Proportion of individuals who possess the study characteristic in the population. Since this data is unknown, it has been assumed that $\mathrm{p}=\mathrm{q}=0.5$ to guarantee it as the safest option.

- q: Proportion of individuals that do not have this characteristic, that is, it is 1-p. n: Sample size (number of surveys to be carried out).

The questionnaire was made thorough an interview with producer of WCO for asking: oil cost, amount of oil produced and collection frequency. From the number of places interviewed was taken a sample with a confidence level of $95 \%$, having the same percentage of cases per location. Table 1 shows the information about number of food places interviewed in each locality. 
Table 6 Results of the questionnaire sent to food places in different Bogotá's locality.

\begin{tabular}{|c|c|c|c|c|}
\hline Locality & $\begin{array}{l}\text { Percentage of number } \\
\text { of restaurants, fast } \\
\text { foods and bakeries }\end{array}$ & $\begin{array}{l}\text { Number of } \\
\text { food places }\end{array}$ & $\begin{array}{l}\text { Number of } \\
\text { interviews }\end{array}$ & $\begin{array}{c}\text { Number of } \\
\text { dates of } \\
\text { optimization } \\
\text { algorithm }\end{array}$ \\
\hline 1. Usaquén & $6 \%$ & 1133 & 67 & 17 \\
\hline 2. Chapinero & $12 \%$ & 1863 & 136 & 35 \\
\hline 3. Santafé & $4 \%$ & 788 & 52 & 12 \\
\hline 4. San Cristóbal & $2 \%$ & 455 & 29 & 6 \\
\hline 5. Usme & $1 \%$ & 114 & 12 & 3 \\
\hline 6. Tunjuelito & $3 \%$ & 456 & 33 & 9 \\
\hline 7. Bosa & $3 \%$ & 467 & 33 & 9 \\
\hline 8. Kennedy & $9 \%$ & 1730 & 104 & 26 \\
\hline 9. Fontibón & $6 \%$ & 979 & 68 & 17 \\
\hline 10. Engativá & $11 \%$ & 1665 & 132 & 32 \\
\hline 11. Suba & $13 \%$ & 1932 & 148 & 38 \\
\hline 12. Barrios Unidos & $4 \%$ & 1012 & 51 & 12 \\
\hline 13. Teusaquillo & $4 \%$ & 580 & 41 & 12 \\
\hline 14. Mártires & $3 \%$ & 648 & 40 & 9 \\
\hline 15. Antonio Nariño & $4 \%$ & 654 & 43 & 12 \\
\hline 16. Puente Aranda & $9 \%$ & 1417 & 106 & 26 \\
\hline 17. Candelaria & $1 \%$ & 258 & 13 & 3 \\
\hline 18. Rafael Uribe & $4 \%$ & 644 & 47 & 12 \\
\hline 19. Ciudad Bolívar & $1 \%$ & 213 & 11 & 3 \\
\hline Total & $100 \%$ & 17008 & 1166 & 289 \\
\hline
\end{tabular}

Source: Authors

\section{Model and Mathematical backgound.}

The objective of the model is to minimize the operational costs implicit in the collection, defining the most relevant costs in the process and the distances between the different nodes, in order to design the optimal route that allows to collect the largest amount of OMA at a low cost. In the mathematical formulation, a series of restrictions were taken into account that influence the OMA collection process, which are subject to the proposed model.

For the mathematical formulation, the variables and parameters described below were taken into account: 


\section{Variables}

$\mathrm{i}=$ Point of Origin $\mathrm{i}=1,2,3$

$\mathrm{j}=$ Collection points

$\mathrm{j}=1,2,3, \ldots \mathrm{n}$.

$\mathrm{K}=$ Vehicles $\mathrm{K}=1.2$

$\mathrm{Xji}=$ Liters of OMA produced at each collection point $\mathrm{j}$ to transport to point of origin $\mathrm{i}$.

Yij $=$ Distance from the point of origin $\mathrm{i}$ to the collection points $\mathrm{j}$ (taking into account the distance between the collection points).

\section{Parameters}

CXij $=$ Cost of the liter collected at each collection point $\mathrm{j}$.

$\mathrm{CKmij}=$ Cost per $\mathrm{Km}$ traveled from the point of origin $\mathrm{i}$ to the collection points $\mathrm{j}$. CTKij $=$ Number of vehicles leaving the point of origin $i$ towards the collection points $j$.

$\mathrm{QKij}=$ Capacity of each vehicle $\mathrm{K}$ that leaves from the point of origin $\mathrm{i}$ towards the collection points j.

$\mathrm{Ei}=$ Capacity in liters that the point of origin $\mathrm{i}$ can process.

$\mathrm{F} j=$ Capacity of the containers delivered to each of the collection points $j$.

$\mathrm{TMij}=$ Maximum time in working hours for the journey from the point of origin $\mathrm{i}$ to the collection points $\mathrm{j}$.

$\mathrm{TRJ}=$ Collection time according to the capacity of the container delivered to the collection point.

$\mathrm{TPj}=$ Average collection time according to the number of liters to be collected at each collection point.

$\mathrm{TFij}=$ Frequency time to carry out the collection at each collection point $\mathrm{j}$.

The mathematical model was developed in accordance with others presented in the consulted literature [9], which were designed from the VRP model. In this model, $\mathrm{Z}$ is the OMA collection operating cost. The operational cost was defined as an objective optimization function, according to equation 2 . 


$$
F . O . Z=\left\{\sum_{j=1}^{n} X_{j i} \cdot C X_{j}+\sum_{i=1}^{1} \sum_{j=1}^{n} Y_{i j} \cdot C K_{i j}\right\}
$$

\section{Constraint}

$$
\sum_{i j=1}^{n} Q K_{i j} X>\sum_{j}^{n} F_{j i} . X \forall j=1,2,3, \ldots n \text { (1) }
$$

Restriction (1) is subject to the sum of the capacity in liters of OMA that the vehicles have that leave from the points of origin $i$ towards the collection points $j$, which must be greater than the sum of the maximum capacity of the containers in liters of OMA that is collected at the collection points $\mathrm{j}$ assigned to transport to the points of origin i. For the programming of the mathematical model, a fleet of vehicles with a capacity of 800 liters was used to optimize the function.

$$
\sum_{i j=1}^{n} Q K_{i j} X \leq E_{i} X \forall i=1
$$

Restriction (2) is subject to the sum of the capacity in liters of OMA of the vehicles that leave the points of origin $\mathrm{i}$ towards the collection points $\mathrm{j}$ must be less than or equal to the capacity in liters that the points of origin $i$.

$$
\sum_{j=1}^{n} T P_{j} X_{j i}<T M_{i j} \forall j=1,2,3, \ldots n \text { (3) }
$$

Restriction (3) is subject to the sum of the average collection time for each liter of OMA produced at each collection point $j$ to transport to the plant of origin $i$ must be less than the maximum time available for the journey from the point of origin $i$ up to collection points $j$ for all collection points.

In this work, 3 deposits were considered: Biogras S.A.S, Bioils Colombia S.A.S and Biominerals Colombia S.A.S. For the collection process, the average time was assigned according to the amount of OMA collected per point, this time was assigned for the collection operator to receive the OMA, fill out the certificate and load the vehicle. Therefore, a collection time of 15 minutes was assigned for the collection of 30,50 and 70 liters of OMA and a collection time of 25 minutes for the collection of 90, 120 and 150 liters of OMA. 


$$
\sum_{j}^{n} T F_{i j} \geq T R . X . F_{j} \forall j=1,2,3, \ldots n
$$

The restriction (4) the time of collection frequency from the point of origin $i$ to the collection points $\mathrm{j}$ must be greater than or equal to the collection time of liters of OMA according to the capacity of the container delivered to each collection point. According to the collection frequency of the 289 points used, the frequency for programming the model was established in the following three groups and under the following parameters: weekly (between 5 to 7 days), biweekly (10 to 15 days) and monthly (between 20 to 30 days).

\section{Model programming}

The model is based on the well-known Travelling Salesman Problem (TSP) [17] , considering constraints that turn the TSP into a Capacitated Vehicle Routing Problem (C-VRP). Routines that are based on two classical algorithms: a classical heuristic method known as the Clarke and Wright Savings Algorithm [18] and the Branch and Bound (BnB) algorithm [19] were used. The "Savings Algorithm" is characterized for performing a relatively limited exploration; the solution found may not be optimal but typically has a good quality taking into account the relatively short computing time, hence this method is still generally employed in most commercial packages [20]. The BnB algorithm is employed in order to verify the optimal order of the sub-routes that form part of the solution provided by the savings algorithm.

In the present work, the algorithm was coded in $\mathrm{R}$ language. The algorithm calculates the cost and duration between the nodes of the network and the collection points. The application takes as arguments: the spatial ubication of the origin points, the volume of oil to be collected and, the vehicles load capacity. The analysis of the road network was taken into account characteristics as direction of traffic, hierarchy and turn restrictions. An interactive map showing the route is generated by application. The data flow within the application is summarized on figure 1 . 


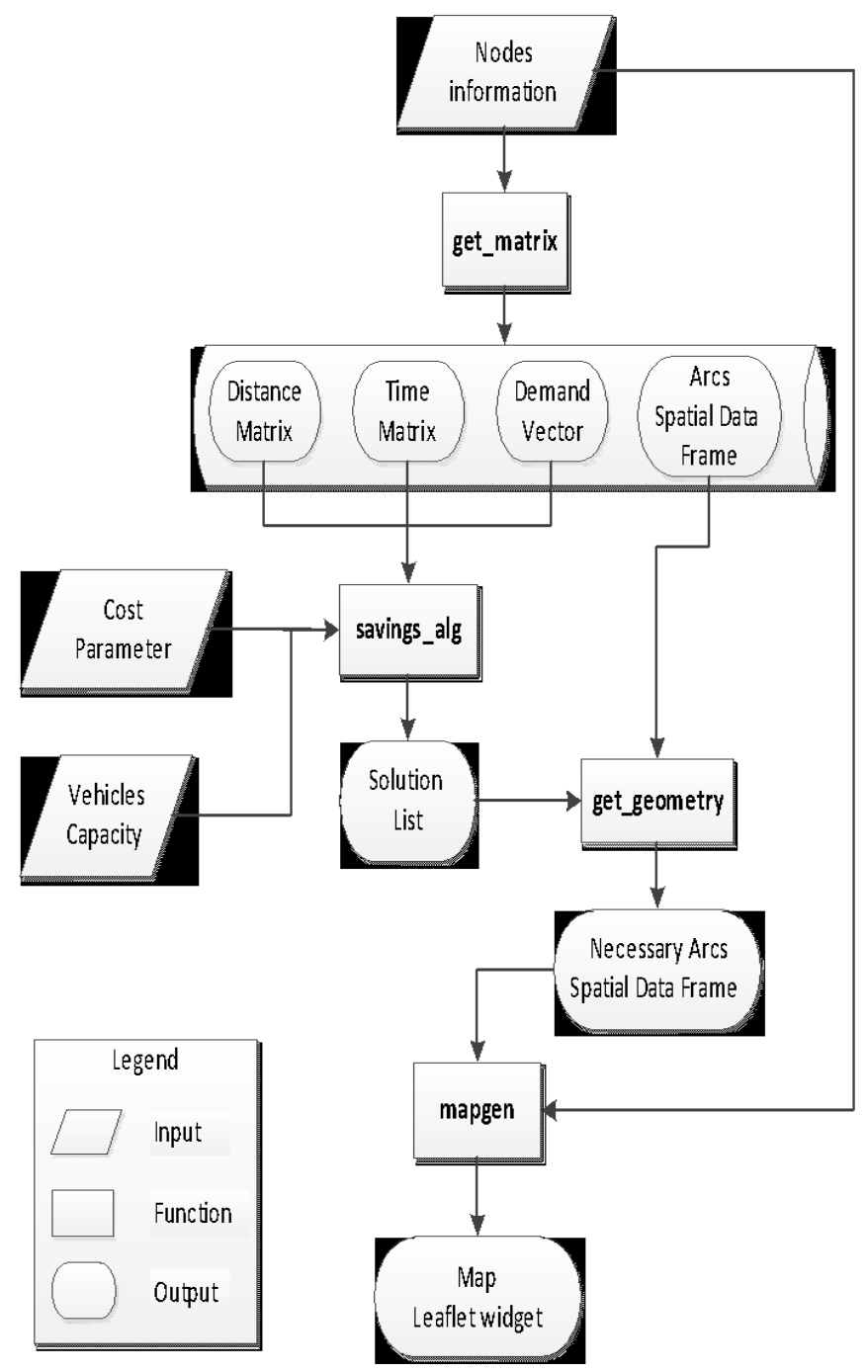

Figure 19 Flow chart of the VRP SOLVER app. Source: Authors

Once the data is loaded as a matrix in the software, this one generates a function with the attributes and geometry of the shortest/fastest route between the nodes (origin/collection points). This function was structured using the OSRM (Open Source Routing Machine) project, which uses a contraction hierarchies algorithm for computing the shortest or fastest path and as designed to work with data from OpenStreetMap. Under this configuration the OSMR project provides a Spatial object with 4 fields of information containing: its origin and destination Ids, travel time (minutes) and travel distance $(\mathrm{km})$. This information is used as an argument in another function that gets the optimal solution to the CVRP. The users can be to choose the type of calculation: Distance or time. The mathematical model were created base for 3 vehicles, 3 collection points and 289 origin points. 


\section{RESULTS}

It was determined that in Bogotá there are 17,008 commercial establishments of the type restaurants, fast food places and cafeterias. From the surveys carried out, the amount of oil collected by each surveyed point was determined, which was extrapolated to the quantity offered by locality and for the whole city. In this way it was determined that the amount of oil produced in Bogotá is 720,000 liters / month. Each of the surveyed points was associated in a matrix with a geographical position, quantity of oil (liters), cost of oil (US \$0.13-0.2). Figure 1 show the quantity of waste cooking oil produced in each locality in Bogotá.

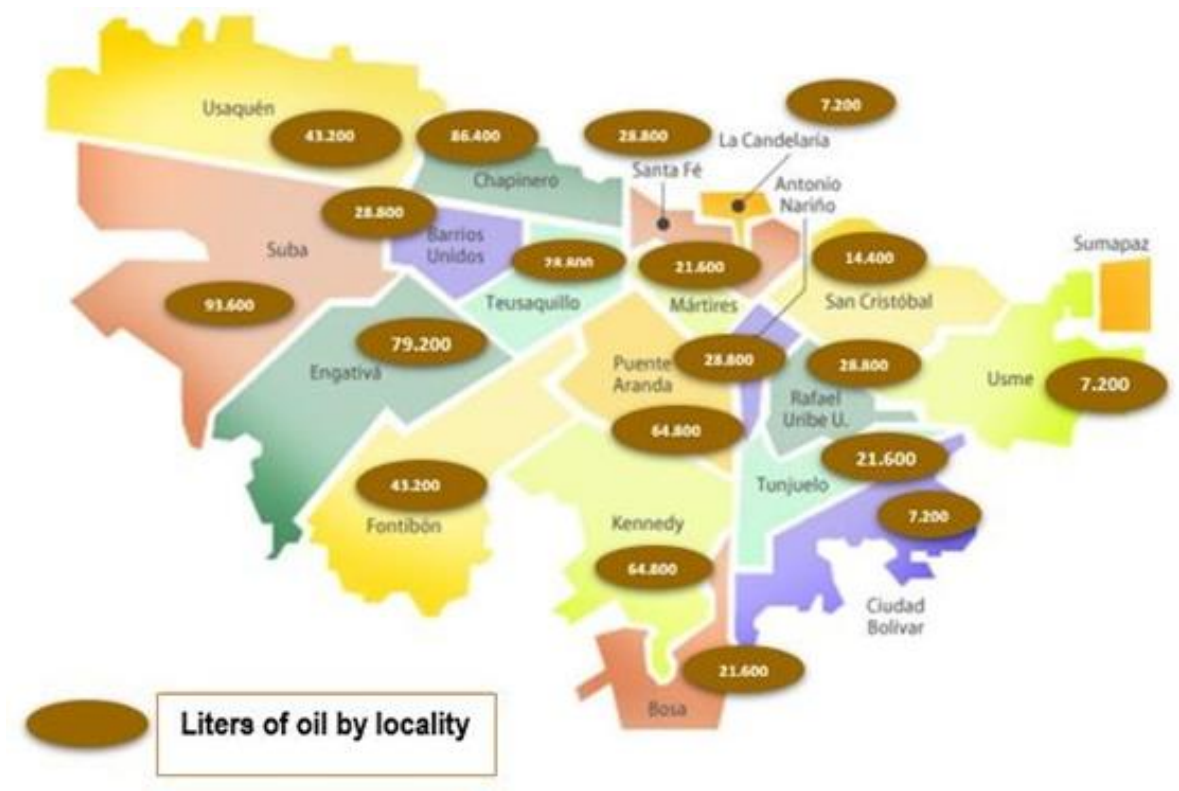

Figure 20 Distribution of oil collected in Bogotá. Sougrce: Authors.

The total quantity of oil collected would be sufficient for installing a small biodiesel plant [25], because the demand of industrial plant is near to 8,000 ton/month[26]. So, oil collected could be offered to industrial plant as raw material for mixing with palm oil in the biodiesel production, according to publish previously[7], [8]. Collecting and recycling WCO contributes to solve three environ- mental problems: waste reduction by product reuse/recovery, reduction of the fossil fuels energy dependence and reduction of pollutants emissions[29]. Apart of biodiesel production, the collected oil could be used in the chemical industry to produce soap, detergents, lubricants, paint, grease, among others[29]; other possibility is for producing polymers as polyurethane[27]. In this way, it is necessary to establish a posterior treatment of filtration, reduction of free fatty acid and reduction of water[30].

The optimization route showed that is a good approximation for solution of collecting wastes. 
This alternative could identify the source of the waste and trace the best route for transporting it to the collection point. There are several applications that address the same problem with different mathematical approaches, as the ones reported previously [22], [23]. This alternative of calculus had been used in a lot of commercial packages provide tools to solve the CVRP(Capacitated Vehicle Routing Problem) [24]. Different advantages of VRP Solver over similar software are: the use of open source tools and free sources of information that are updated periodically, the inclusion of a graphical solution through an interactive map, and free access.

The results obtained from the collection routes are shown in tables 2, 3 and 4 . Table 2 shows the results of the collection of the weekly frequency by the manager Biogras S.A.S. According to the table, it is evidenced that 6 routes were generated and 4125 liters of OMA were collected with a total cost of US \$ 820.82 in 81 collection points. Figure 3 shows information about the 6 routes.

Table 7 Weekly frequency

\begin{tabular}{|c|c|c|c|c|c|c|c|c|c|c|c|c|}
\hline \multicolumn{13}{|c|}{ Weekly frequency } \\
\hline Route no. & $\begin{array}{l}\text { No. } \\
\text { collected } \\
\text { points }\end{array}$ & Demand(Lts) & Distance $(\mathrm{km})$ & $\begin{array}{l}\text { Route Duration } \\
\text { (min) }\end{array}$ & $\begin{array}{c}\text { Collection } \\
\text { duration (min) }\end{array}$ & $\begin{array}{l}\text { Total time } \\
\text { (min) }\end{array}$ & & $\begin{array}{l}\text { on cost } \\
\text { D) }\end{array}$ & & $\begin{array}{l}\text { neters } \\
\text { (USD) }\end{array}$ & & cost \\
\hline No. 1 & 16 & 793 & 91.57 & 257.89 & 280 & 537.89 & $\$$ & 107.86 & $\$$ & 84.34 & $\$$ & 192.20 \\
\hline No. 2 & 17 & 798.5 & 43.57 & 145.03 & 275 & 420.03 & s & 107.68 & $\$$ & 40.13 & $\$$ & 147.82 \\
\hline No. 3 & 12 & 651 & 23.67 & 82.39 & 230 & 312.39 & $\$$ & 82.87 & $\$$ & 21.80 & $\$$ & 109.72 \\
\hline No. 4 & 15 & 797.5 & 79.19 & 225.69 & 285 & 510.69 & $\$$ & 105.21 & $\$$ & 72.94 & $\$$ & 178.15 \\
\hline No. 5 & 9 & 414.5 & 40.96 & 110.7 & 145 & 255.7 & $\$$ & 58.13 & $\$$ & 37.73 & $\$$ & 95.86 \\
\hline \multirow[t]{2}{*}{ No. 6} & 12 & 671 & 29.85 & 93.88 & 210 & 303.88 & $\$$ & 69.57 & $\$$ & 27.49 & $\$$ & 97.07 \\
\hline & 81 & 4125.5 & 308.81 & 915.58 & 1425 & 2340.58 & $\$$ & 531.32 & $\$$ & 284.43 & $\$$ & 820.82 \\
\hline
\end{tabular}

Source: Authors

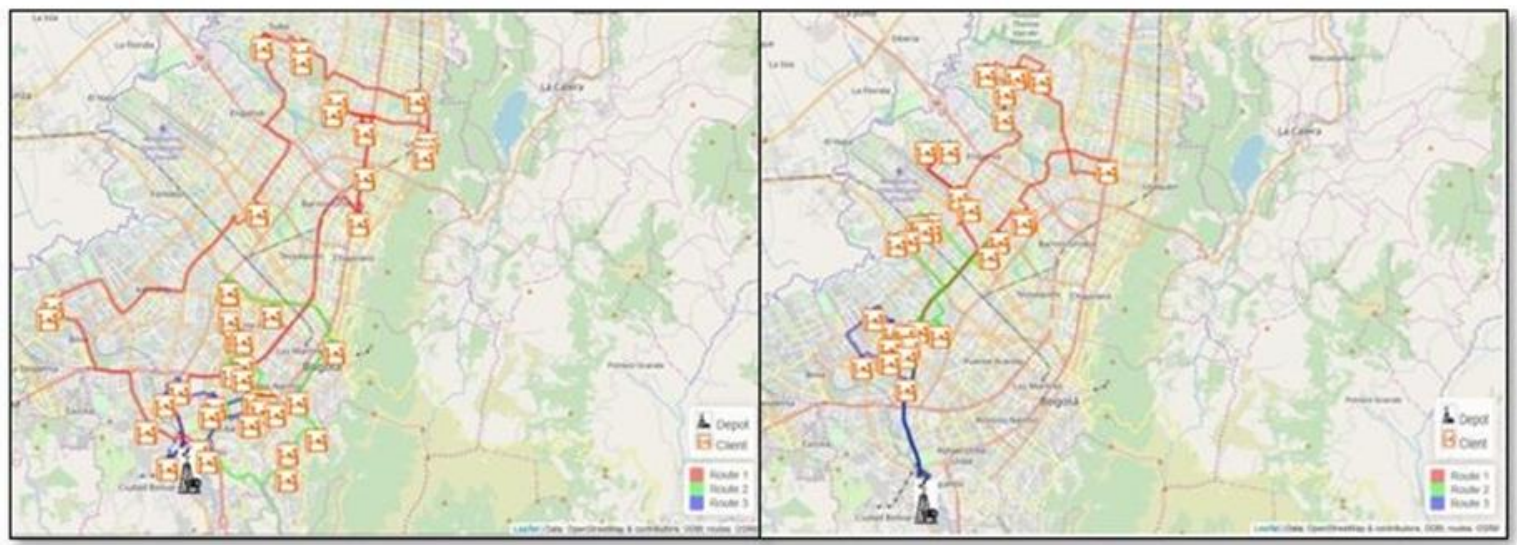

Figure 21 Weekly route map. Source: VRP Solver application results. 


\section{CONGRESO DE DESARROLLO SOSTENIBLE}

On the other hand, the manager Biolis Colombia S.A.S was programmed for the biweekly collection. According to the table 3, it is evident that 6 routes were generated and 4,456 liters of OMA were collected with a total cost of US \$ 889.02 in 92 collection points. Figure 4 shows information about the 6 routes.

Table 8 Biweekly frequency of WCO collected.

\begin{tabular}{|c|c|c|c|c|c|c|c|c|c|c|c|c|}
\hline \multicolumn{13}{|c|}{ Biweekly frequency } \\
\hline Route no. & $\begin{array}{c}\text { No. } \\
\text { collected } \\
\text { points }\end{array}$ & Demand(Lts) & Distance $(\mathrm{km})$ & $\begin{array}{l}\text { Route Duration } \\
\text { (min) }\end{array}$ & $\begin{array}{c}\text { Collection } \\
\text { duration (min) }\end{array}$ & $\begin{array}{c}\text { Total time } \\
\text { (min) }\end{array}$ & & $\begin{array}{l}\text { on cost } \\
\text { D) }\end{array}$ & & $\begin{array}{l}\text { neters } \\
\text { USD) }\end{array}$ & & cost \\
\hline No. 1 & 19 & 779.5 & 62.84 & 176.34 & 315 & 491.34 & $\$$ & 104.05 & $\$$ & 57.88 & $\$$ & 161.93 \\
\hline No. 2 & 15 & 747.5 & 38.27 & 125.44 & 255 & 380.44 & $\$$ & 96.73 & $\$$ & 35.25 & $\$$ & 131.98 \\
\hline No. 3 & 12 & 571 & 61.66 & 176.87 & 190 & 366.87 & $\$$ & 75.31 & \$ & 56.79 & s & 132.10 \\
\hline No. 4 & 20 & 790 & 71.72 & 218.75 & 330 & 548.75 & $\$$ & 104.79 & $\$$ & 66.06 & $\$$ & 170.85 \\
\hline No. 5 & 14 & 797 & 46.91 & 135.29 & 270 & 405.29 & $\$$ & 104.23 & $\$$ & 43.21 & $\$$ & 147.44 \\
\hline \multirow[t]{2}{*}{ No. 6} & 12 & 771 & 48.7 & 143.86 & 220 & 363.86 & $\$$ & 99.86 & $\$$ & 44.86 & $\$$ & 144.72 \\
\hline & 92 & 4456 & 330.1 & 976.55 & 1580 & 2556.55 & $\$$ & 584.97 & $\$$ & 304.05 & $\$$ & 889.02 \\
\hline
\end{tabular}

\section{Source: Authors}

Finally, Table 4 shows the results of the Biominerales Colombia SAS manager. According to the table 4 , it is evidenced that 8 routes were generated and 5203 liters of OMA were collected with a total cost of US \$1,136.27 in 116 collection points. Figure 4 shows information about the 8 routes.

Table 9 Monthly frecuency of WCO collected.

\begin{tabular}{|c|c|c|c|c|c|c|c|c|c|c|c|c|}
\hline \multicolumn{13}{|c|}{ Monthly frequency } \\
\hline Route no. & $\begin{array}{l}\text { No. } \\
\text { collected } \\
\text { points }\end{array}$ & Demand(Lts) & Distance(km) & $\begin{array}{l}\text { Route Duration } \\
\text { (min) }\end{array}$ & $\begin{array}{c}\text { Collection } \\
\text { duration (min) }\end{array}$ & $\begin{array}{c}\text { Total time } \\
\text { (min) }\end{array}$ & & $\begin{array}{l}\text { on cost } \\
\text { D) }\end{array}$ & & $\begin{array}{l}\text { neters } \\
\text { USD) }\end{array}$ & & al cost \\
\hline No. 1 & 12 & 576 & 74.83 & 212.22 & 210 & 422.22 & s & 80.32 & $\$$ & 68.92 & $\$$ & 149.25 \\
\hline No. 2 & 19 & 744.5 & 71.5 & 220.66 & 315 & 535.66 & $\$$ & 98.28 & $\$$ & 65.86 & $\$$ & 164.13 \\
\hline No. 3 & 17 & 693.5 & 49.48 & 172.18 & 285 & 457.18 & $\$$ & 92.49 & $\$$ & 45.57 & $\$$ & 138.06 \\
\hline No. 4 & 10 & 665 & 59.46 & 156.6 & 190 & 346.6 & $\$$ & 84.11 & $\$$ & 54.77 & $\$$ & 138.87 \\
\hline No. 5 & 23 & 781.5 & 83.07 & 249.25 & 385 & 634.25 & $\$$ & 105.45 & $\$$ & 76.51 & $\$$ & 181.97 \\
\hline No. 6 & 10 & 785 & 55.3 & 165.7 & 210 & 375.7 & $\$$ & 103.97 & $\$$ & 50.94 & $\$$ & 154.90 \\
\hline No. 7 & 20 & 710 & 73.76 & 220.94 & 300 & 520.94 & $\$$ & 97.23 & $\$$ & 67.94 & s & 165.17 \\
\hline \multirow[t]{2}{*}{ No. 8} & 5 & 247.5 & 10.99 & 40.35 & 95 & 135.35 & $\$$ & 33.80 & $\$$ & 10.12 & $\$$ & 43.92 \\
\hline & 116 & 5203 & 478.39 & 1437.9 & 1990 & 3427.9 & $\$$ & 695.65 & $\$$ & 440.63 & $\$$ & $1,136.27$ \\
\hline
\end{tabular}

\section{Source: Authors}




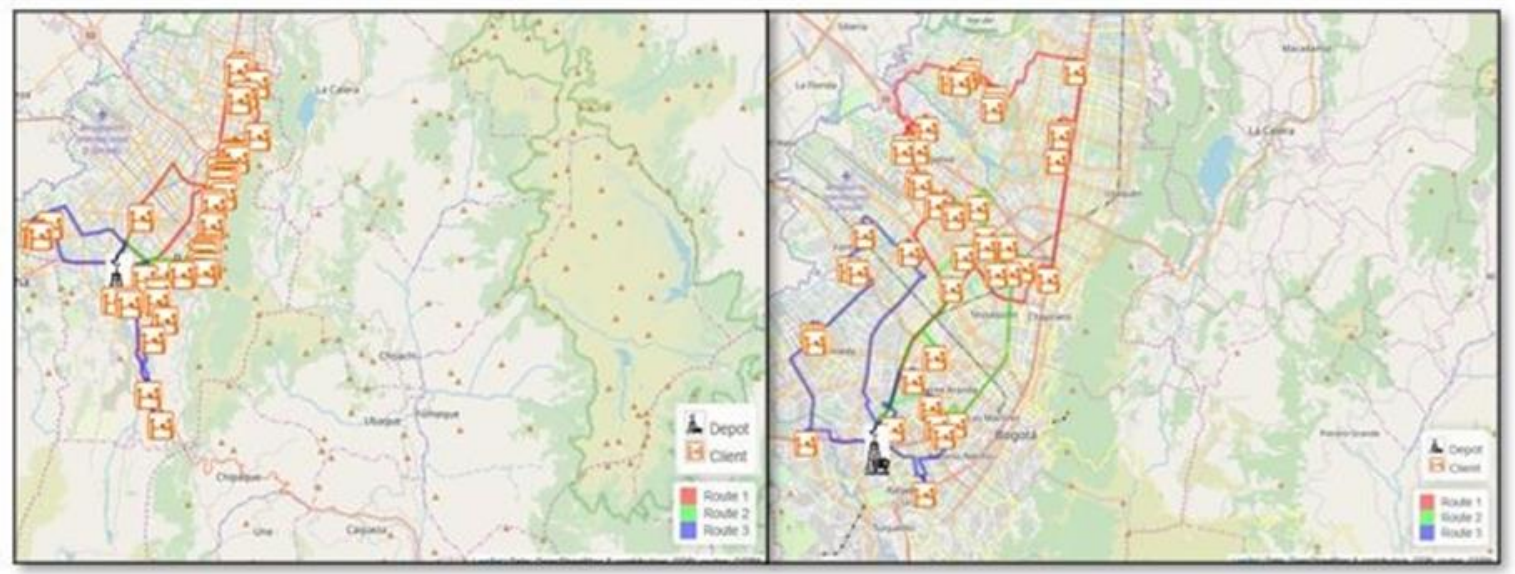

Figure 22 Biweekly route map.Source: VRP Solver application results

According to the information in Table 5, the average cost to collect 1 liter of WCO in Bogotá was US\$ 0.23; this value is lower than palm oil (US\$ 0.5 per liter). This value is similar to reported in Rio de Janeiro (US \$0.22 per liter) [9]. The obtained cost offers an opportunity for collecting the WCO for partial substitution of virgin palm oil. In this way, costs associated with raw materials could be diminished due to the feedstock represents up to $75 \%$ of the total manufacturing cost of biodiesel [28]. On the other hand, the collection had importance in the total cost of WCO, near to $32-40 \%$ of the total cost is associated with logistics aspects. This aspect had been reported as an important factor in the biodiesel production from WCO[9]. Likewise, having the information of the routes, the data of the demand, the time and the total cost for each of the routes will be calculated, as well as the total cost for collecting 13,783 liters of oil from the 289 generating points.

Table 10 Results of the optimization algorithm.

\begin{tabular}{|l|c|c|c|}
\hline \multicolumn{1}{|c|}{ Parameters } & Biogras S.A.S & $\begin{array}{c}\text { Bioils Colombia } \\
\text { SAS }\end{array}$ & $\begin{array}{c}\text { Biominerales } \\
\text { Colombia SAS }\end{array}$ \\
\hline Liters of WCO collected & 4125 & 4456 & 5203 \\
\hline Vehicle capability & $800 \mathrm{~kg}$ & $800 \mathrm{~kg}$ & $800 \mathrm{~kg}$ \\
\hline $\begin{array}{l}\text { Cost of obtaining the oil } \mathrm{x} \\
\text { liter }\end{array}$ & $\mathrm{US} \$ 0,15$ & $\mathrm{US} \$ 0,15$ & $\mathrm{US \$ 0,15}$ \\
\hline Collection cost $x$ liter & US\$ $0,078 / \mathrm{km}$ & $\mathrm{US} \$ 0,077 / \mathrm{km}$ & $\mathrm{US} \$ 0,095 / \mathrm{km}$ \\
\hline Number of routes & 6 & 6 & 8 \\
\hline Number of origin points & 81 & 92 & 116 \\
\hline Distance traveled & $308,81 \mathrm{~km}$ & $330,1 \mathrm{~km}$ & $478,39 \mathrm{Km}$ \\
\hline Number of vehicles & 2 & 2 & 3 \\
\hline
\end{tabular}




\begin{tabular}{|l|c|c|c|}
\hline $\begin{array}{l}\text { Total Cost per liter of } \\
\text { waste oil }\end{array}$ & US\$ 0.22 & US\$ 0.22 & US\$ 0.25 \\
\hline
\end{tabular}

\section{Source: Authors}

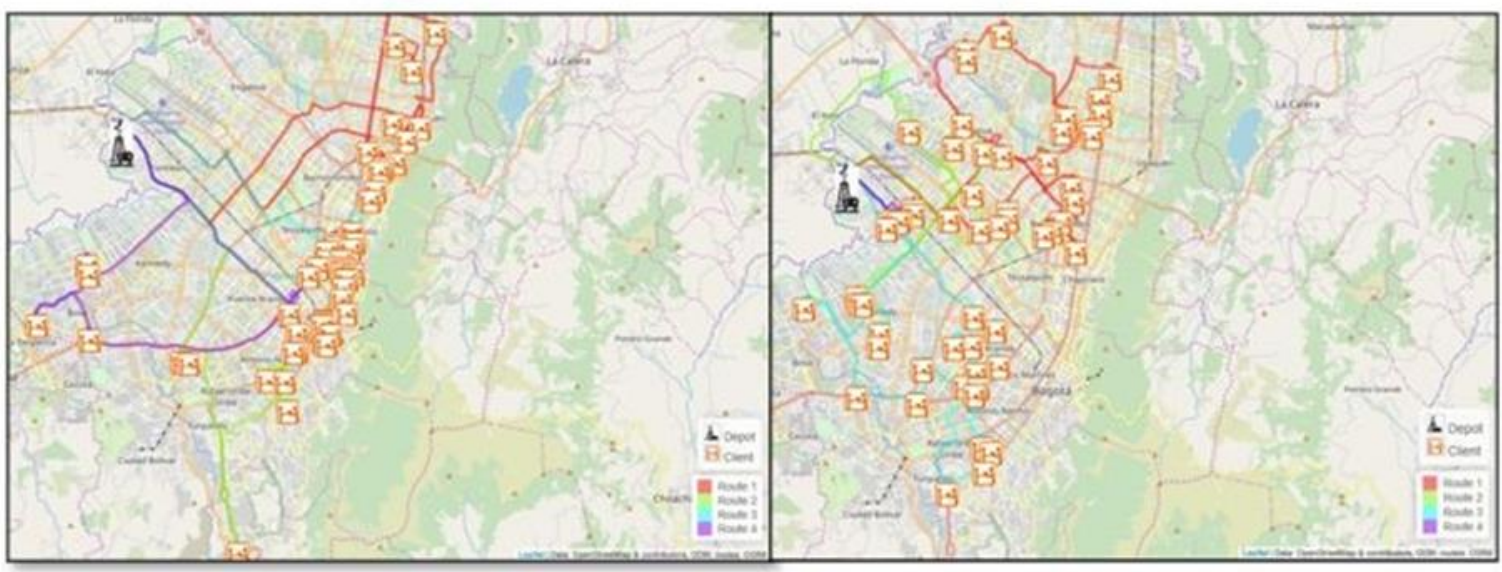

Figure 23 Monthly roadmap. Source: VRP Solver application results

\section{CONCLUSIONS}

Optimization of the costs associated with producer, collecting and transport of WCO in Bogotá have shown that it is a profitable process. The cost obtained could be competitive with palm oil in biodiesel production. The integration of virgin and collected oil had been evaluated, so technical aspects have been overcome. Additionally, WCO collected could be integrated to other process as soap or polyurethane production. The integration of heuristics models for optimization allowed an advantage for establishing the optimal point in VRP and TSP.

\section{REFERENCES}

[1] E. Alptekin, M. Canakci, and H. Sanli, "Biodiesel production from vegetable oil and waste animal fats in a pilot plant," Waste Manag., vol. 34, no. 11, pp. 2146-2154, 2014, doi: 10.1016/j.wasman.2014.07.019.

[2] C. Varella, K. Oliveira, and D. Oliveira, “Crude glycerol by transesterification process from used cooking oils : Characterization and potentialities on hydrogen bioproduction," Int. J. energy, vol. 41, pp. 14641-51, 2016, doi: 10.1016/j.ijhydene.2016.06.209.

[3] L. M. Verdade, C. I. Piña, and L. M. Rosalino, "Biofuels and biodiversity: Challenges and opportunities," Environ. Dev., vol. 15, pp. 64-78, 2015, doi: 10.1016/j.envdev.2015.05.003.

[4] L. E. Rincón, J. J. Jaramillo, and C. A. Cardona, "Comparison of feedstocks and technologies for biodiesel production: An environmental and techno-economic evaluation," Renew. Energy, vol. 69, pp. 479-487, 2014, doi: 10.1016/j.renene.2014.03.058.

[5] F. Ezzati, R. Babazadeh, and A. Donyavi, "Optimization of multimodal, multi-period and complex biodiesel supply chain systems: Case 
study," Renew. Energy Focus, vol. 26, no. 00, pp. 8192, 2018, doi: 10.1016/j.ref.2018.07.005.

[6] L. López, J. Bocanegra, and D. Malagón-romero, "Production of biodiesel from waste cooking oil by transesterification," Ing. y Univ., vol. 19, no. 1, pp. 155-172, 2015, doi: 10.11144/Javeriana.iyu19-1.sprq.

[7] R. Alarcón, D. Malagón-Romero, and A. Ladino, "Biodiesel production from waste frying oil and palm oil mixtures," Chem. Eng. Trans., vol. 57, pp. 571-576, 2017, doi: 10.3303/CET1757096.

[8] D. Rodríguez, J. Riesco, and D. Malagon-Romero, "Production of Biodiesel from Waste Cooking Oil and Castor Oil Blends," Chem. Eng. Trans., vol. 57, pp. 679-684, 2017, doi: 10.3303/CET1757114.

[9] V. K. W. S. Araujo, S. Hamacher, and L. F. Scavarda, "Economic assessment of biodiesel production from waste frying oils," Bioresour. Technol., vol. 101, no. 12, pp. 4415-4422, 2010, doi: 10.1016/j.biortech.2010.01.101.

[10] Y. Jiang and Y. Zhang, "Supply Chain Optimization of Biodiesel Produced from Waste Cooking Oil," Transp. Res. Procedia, vol. 12, no. June 2015, pp. 938-949, 2016, doi: 10.1016/j.trpro.2016.02.045.

[11] R. Kampf, "Optimization of Delivery Routes using the Little's Algorithm," Naše more, vol. 65, no. 4, pp. 237-239, 2018, doi: 10.17818/NM/2018/4SI.13.

[12] P. Belavenutti, C. Romero, and L. Diaz-Balteiro, "A critical survey of optimization methods in industrial forest plantations management," Sci. Agric., vol. 75, no. 3, pp. 239-245, 2018, doi: 10.1590/1678-992x2016-0479.

[13] Z. Bouyahia, H. Haddad, N. Jabeur, and A. N. Sidi Moh, "Optimization of Chartered Buses Routes under Uncertainties Using Probabilistic Vehicle Routing Problem Modeling," Procedia Comput. Sci., vol. 130, pp. 644-651, 2018, doi: 10.1016/j.procs.2018.04.115.

[14] G. Laporte, P. Toth, and D. Vigo, "Vehicle routing: historical perspective and recent contributions," EURO J. Transp. Logist., vol. 2, no. 1-2, pp. 1-4, 2013, doi: 10.1007/s13676-013-0020-6.

[15] J. Sepúlveda, J. W. Escobar, and W. Adarme-Jaimes, "Un algoritmo para el problema de ruteo de vehículos con entregas divididas y ventanas de tiempo (SDVRPTW) aplicado a las actividades de distribución de PYMEs del comercio al por menor," Dyna, vol. 81, no. 187, pp. 223-231, 2014, doi: 10.15446/dyna.v81n187.46104.
[16] B. Domínguez-Martín, I. Rodríguez-Martín, and J. J. Salazar-González, "The driver and vehicle routing problem," Comput. Oper. Res., vol. 92, pp. 56-64, 2018, doi: 10.1016/j.cor.2017.12.010.

[17] X.-S. Yang, Introduction to Mathematical Optimization: From Linear Programming to Metaheuristics. Cambridge, UK: Cambridge International Science Publishi, 2008.

[18] G. Clarke and J. W. Wright, "Scheduling of Vehicles from a Central Depot to a Number of Delivery Points," Oper. Res., vol. 12, no. 4, pp. 568-581, 1964, doi: 10.1287/opre.12.4.568.

[19] M. Bellmore and J. C. Malone, "Pathology of Traveling-Salesman Subtour-Elimination Algorithms," Oper. Res., vol. 19, no. 2, pp. 278-307, Apr. 1971, doi: 10.1287/opre.19.2.278.

[20] P. Toth and D. Vigo, The Vehicle Routing Problem. Philadelphia: Society for Industrial and Applied Mathematics, 2002.

[21] J. D. C. Little, K. G. Murty, D. W. Sweeney, and C. Karel, "An algorithm for the traveling salesman problem," Oper. Reseach, vol. 11, no. 6, pp. 972-989, 1963.

[22] S. R. Thangiah, K. E. Nygard, and P. L. Juell, "GIDEON: a genetic algorithm system for vehicle routing with time windows," in The Seventh IEEE Conference on Artificial Intelligence Application [1991] Proceedings, 1991, vol. i, pp. 322-328, doi: 10.1109/CAIA.1991.120888.

[23] L. M. Gambardella, R. Taillard, and G. Agazzi, "MACS-VRPTW: A multiple ant colony system for vehicle routing problems with time windows," Aug. 2001.

[24] J. Lysgaard, A. N. Letchford, and R. W. Eglese, "A new branch-and-cut algorithm for the capacitated vehicle routing problem," Math. Program., vol. 100, no. 2, pp. 423-445, Jun. 2004, doi: 10.1007/s10107003-0481-8.

[25] B. Galeano, J. Alexander, and D. Malagón-romero, "Parametric Algorithm for the Study of Technical and Economic Feasibility of Biodiesel Production Plants at Small and Medium Scale in Colombia," Chem. Eng. Trans., vol. 65, 2018.

[26] Y. Zhang, M. a. Dubé, D. D. McLean, and M. Kates, "Biodiesel production from waste cooking oil: 1 . Process design and technological assessment," Bioresour. Technol., vol. 89, pp. 1-16, 2003, doi: 10.1016/S0960-8524(03)00040-3. 


\section{CONGRESO DE DESARROLLD SOSTENIBLE}

[27] D. Moreno, M. Velasco, and D. Malagón-Romero, "Production of polyurethanes from used vegetable oil-based polyols," Chem. Eng. Trans., vol. 79, pp. 337-342, 2020, doi: 10.3303/CET2079057.

[28] Y. Zhang, M. A. Dubé, D. D. McLean, and M. Kates, "Biodiesel production from waste cooking oil: 2. Economic assessment and sensitivity analysis," Bioresour. Technol., vol. 90, no. 3, pp. 229-240, 2003, doi: 10.1016/S0960-8524(03)00150-0.

[29] T. R. P. Ramos, M. I. Gomes, and A. P. Barbosa-Póvoa, "Planning waste cooking oil collection systems," Waste Manag., vol. 33, no. 8, pp. 1691-1703, 2013, doi: 10.1016/j.wasman.2013.04.005.

[30] I. D. Casallas et al., "Pre-Treatment of Waste Frying Oils for Biodiesel Production," Chem. Eng. Tra, vol. 65, pp. 1-6, 2018, doi: 10.5539/mas.v9n7p99. 
CONGRESO DE DESARROLLD

SOSTENIBLE

\section{ENERGIAS \\ ALTERNATIVAS}




\title{
ENERGY USE OF BIOMASS IN COLOMBIA FROM A LATIN AMERICAN COMPARED PERSPECTIVE
}

\section{Uso energético de biomasa en Colombia desde una perspectiva latinoamericana}

\author{
Sánchez-Gómez, Sebastián"; Medellín, Lizeth \\ Universidad de los Andes, Observatorio Regional ODS.
}

\section{Abstract}

This research presents a systematic review of the main technological trends regarding biomass and its use for the production of renewable energy, in order to discuss the influence of international environmental law on the implementation of these in Colombia. The main findings were related to the lack of knowledge about international conventions and mechanisms for the mitigation and adaptation of climate change in the Latin American countries studied. In the Colombian case, given the need to have more interconnected areas, it is necessary to empower rural communities to strengthen their projects on technologies for the energy use of biomass. This would be possible if these communities know the described international green funds and understand the application processes for that their community projects are supported and empowered at the economic, technological and Know-How levels.

Keywords: Biomass, international environmental law, international cooperation..

\section{INTRODUCTION}

Since the United Nations Conference on the Human Environment in Stockholm in 1972, environmental issues have been placed on the international agenda. According to researcher Paula Caballero [1], the foundation of Modern International Environmental Law is the Stockholm Declaration. In addition to this declaration, around 1992 the United Nations Conference on Environment and Development was held in Rio de Janeiro. These summits were fundamental foundations for issues on the environment, sustainable development and international cooperation for the protection of the environment worldwide [1]. It is from these international legal spaces that the issue of the use of biomass has gradually come into prominence, tending to face the difficulties that the empire of fossil fuel use represents. Biomass was one of the main fuels for humans before the arrival of fossil fuels and the industrial revolution [2]. In particular, biomass

\footnotetext{
${ }^{1}$ Corresponding E-mail: js.sanchez14@ uniandes.edu.co
} 
was used for cooking, heating homes, and making ceramics. Despite the fact that fossil fuels became more relevant worldwide, the international community has developed international legal mechanisms that promote a greater development and use of biomass than fossils.

One of the most relevant international frameworks is international environmental law, from which the problems of fossil fuel use have been discussed and spaces have been created in which biomass is presented as the necessary sustainable alternative in the world to be able to adapt to climate change. The United Nations Conference on Environment and Development held in Rio de Janeiro is the event where bioenergy tooks relevance [3]. It was in this scenario that Programa 21 was created, which establishes the need to promote bioenergy to reduce CO2. These approaches to biomass as an opportunity for sustainable development are strengthened in the negotiations of the Kyoto Protocol and those of the Paris Agreement, since it is argued that "energy production, energy markets, and the use of energy are generating in our times serious and visible environmental problems, since energy-related activities represent $84.3 \%$ of the planet's anthropogenic greenhouse gases" [4]. These advances in the international legal field gave rise to the "promotion of bioenergy from biomass [...] through subsidies and in some cases from mandatory targets in Europe and North America". Therefore, support for the use of biomass focused on bioenergy advances comes largely from financing and policies of developed countries, especially the European Union and the United States [5], since they are the most consuming energy and are subject to the energy crisis due to the decrease in fossil fuels.

The purpose of this research is to present a systematic review of the main technological trends regarding biomass and its use for the production of renewable energy, in order to discuss the influence of international environmental law on the implementation of these in Colombia. In this sense, it will be shown that the strengthening and support of the use of biomass, through the mechanisms of international environmental law, in Colombia can enhance sustainable development initiatives led by rural communities in the country. The international theoretical and legal development of biomass in international environmental law will be presented first from the perspective of soft law. Next, the main advances in biomass in Latin America will be presented, taking as reference countries such as Argentina, Brazil, Peru, Chile and Uruguay, which use biomass produced from agricultural residues. Third, the biomass energy use technologies that are used in rural projects in Colombia will be exposed. 


\section{MATERIALS AND METHODS.}

This research is a comparative qualitative study, with a descriptive approach based on a documentary design of specialized literature. This is based on the collection of data from the consultation of books, articles and records, which were interpreted from an analytical perspective, to demonstrate the limitations and possibilities in the use and implementation of international environmental law and its mechanisms regarding the use energy of biomass in Colombia

\section{RESULTS AND ANALYSIS OF RESULTS}

\section{Soft Law and Biomass}

Soft law is part of the called non-traditional sources of international environmental law. This "soft" component is shown from the normative dimension under two meanings: processes of gestation of legal norms and in instruments without legal force such as resolutions, declarations, programs, final acts of international conferences, reports of groups of experts and strategic plans [6]. Likewise, this soft law in its applicative dimension is expressed when resolutions are issued in the application of the Conventions, which are generally not mandatory. In accordance with the above, the Stockholm Declaration on the Human Environment (1972), the Rio Declaration on Environment and Development (1992) and a large part of the Conventions specific to the development of international environmental law are part of soft law.

According to this view of soft international law, the progress made on the use of biomass as a form of sustainable development is inserted in the negotiations and agreements made between States without legal force. This indicates that each State assumes this issue taking into account the negotiated guidelines and its national priorities and needs. International environmental law is a roadmap that guides the global energy sector with the support of the research and reports of the Intergovernmental Panel on Climate Change. This group of experts affirms that "with the appropriate policies, $77 \%$ of the demand in 2050 could be covered with energy from renewable sources. One of the technologies examined by the Panel has been Bioenergy, and in particular, energy crops; forest, agricultural and livestock waste, and the called "second generation biofuels [7]. According to Blasco [8], renewable sources covered $12.9 \%$ of global energy demand in 2008, with biomass being the most important $(10.2 \%)$, followed by hydro $(2.3 \%)$, wind $(0.2 \%)$, geothermal and solar (0.1\%).

In international environmental law, developed countries promote and finance projects related to 
the use of biomass in developing countries, based on national and regional projects that work with bioenergy and bioenergy crops. This international dynamic has gradually allowed developing countries to carry out their biomass projects from different dimensions. The support from developed countries to developing countries is framed in the Principle of Common but Differentiated Responsibilities proper to international environmental law [9], [10]. An example of this are the "EU's renewable energy targets and the subsidies support the large agricultural and forestry industry and the generation of bioenergy, as they give confidence and stability to the market" [5].

\section{Emerging mechanisms of soft law}

In international environmental law, support mechanisms have emerged for the use of biomass through economic, technological and Know-How support from developed countries to developing countries. Some of the institutions and mechanisms include: The World Renewable Energy Assembly, the World Renewable Energy Forum and its World Renewable Energy Agenda, the International Renewable Energy Agency and the process for the development of global regulations and standards for the renewable energy industry. These international mechanisms seek to move towards the use of biomass to achieve the goals of the 2030 agenda in line with the Sustainable Development Goals and the necessary reduction of $\mathrm{CO} 2$ emissions proposed by the IPCC for not to reach $2^{\circ} \mathrm{C}$. International tools that seek for both developed and developing countries to support and carry out projects that do not depend on fossil fuels, the main source of $\mathrm{CO} 2$ and global pollution.

\section{Biomass in Latin America}

Latin America presents great energy efficiency, this is due, according to Coviello [11], to renewable energies, since these represent great potential, which mitigates the effects of energy consumption as a direct consequence of social growth. Some of the strategies that are being carried out to take advantage of this energy potential are the development and creation of policies that contemplate the use of renewable energies, among which is the use of biomass. In this case, the creation of the regional initiative "Energy and Sustainable Development in Latin America and the Caribbean" which was promoted by the Latin American Energy Organization, ECLAC and the German Society for Technical Cooperation [12]. Next, the actions of five Latin American countries that were chosen as a sample are evaluated, given that they have been pioneers in the generation of alternative energy sources from biomass. 
Argentina is a country with great electricity potential, according to Leandro, Evelyn and Balbina [13] in 2009, the Planning Ministry launched the GENREN I program with a bidding system which was intended to cover $1000 \mathrm{MW}$ of electricity generation, where $200 \mathrm{MW}$ will be produced through biomass. In addition, in 2012 the Ministry of Agriculture, Livestock and Fisheries and the Ministry of Federal Planning, Public Investment and Services, with the technical assistance of FAO launched the project PROBIOMASA for the promotion of energy derived from biomass taking advantage of waste, mostly forest. It even has a unique opportunity defined by its territorial breadth, geographic diversity, and great potential for available biomass resources and waste for its energy use [14]. It is for the above that it can be seen how Argentina uses the biomass potential to generate energy.

In the case of Brazil, it is considered to be a great producer of electrical energy worldwide, according to Verdesio [15], which produces a wide range of biofuels for transportation, such as ethyl alcohol. It should be noted that the use of biomass in electricity generation is outstanding worldwide despite only corresponding to a small part of its potential. It is a highly developed country in the agricultural sector as Pereira [16] explains Brazil's approach to creating rural extension projects, tropical and temperate fruit growing, cereals, olive growing, viticulture, livestock, fishing, erosion control, among others . In addition, a great domain in the elaboration of energy from biomass, especially biodiesel and ethanol fuels, so it can be said that this country presents great success in this energy area.

In Chile, biomass generation is focused above all on electricity generation, and this is because 16 million cubic meters of firewood are consumed annually, of which more than $60 \%$ comes from native forest [17]. Pontt and Guiñez [18] specify how biomass is very important in the national energy matrix, it is carried out by the pulp and paper industry, which uses waste from its raw materials and processes to use it as fuel, including the company Energía Verde generates electricity based on forest waste.

Peru, like other developing countries, uses firewood as one of the energy promoters, since more than $20 \%$ of the primary energy corresponds to firewood and dung. By burning this raw material, biomass is generated to produce heat, gas and biofuels. The potential of biomass is not limited to processes with direct combustion of biomass, but energy can also be obtained through the gasification of the residues of certain crops, whose properties such as calorific value and chemical structure promotes a favorable process and efficient chemical reactions that take place in this process [19]. There are 11 sugar mills in Peru, of which at least 9 of them use bagasse in their 
cogeneration plants [20].

In Uruguay, there are only a few experimental results of energy plantations whose information has not been for public use and has not been transferred to commercial ventures [21]. Uruguay has development potential, since according to Bittencourt and Reig, is very important the direct production in its different stages and territorial integration with recovery of areas excluded from the relative national development. It is even stated that the cultivation of summer oilseeds such as sunflower and soy is predominant in the country, while the cultivation of rapeseed is still incipient [22].

Latin America and the Caribbean can establish themselves as important producers of advanced biofuels in the global context. However, it will be necessary to stimulate the incorporation of innovative processes and adequate technological development adapted to the characteristics of the region. In this sense, the existence of directed public and private institutions for the aggregation of knowledge to the biofuel production chain, especially advanced ones, is essential. Naturally, there are already 2nd and 3rd generation Biofuels in progress within the region [23].

\section{Biomass in Colombia}

Colombia has half of its territory without connection to the National Interconnected System. According to the Institute for the Planning and Promotion of Energy Solutions for NonInterconnected Zones, in 2018 non-interconnected zones (ZNI) represented 51\% of Colombian territory, with $96.6 \%$ of non-renewable energy sources and $3.4 \%$ of renewable energy [24]. In these ZNI zones propane gas is mainly used as combustion for cooking, which is expensive and dangerous. For this reason, Castro et al. propose anaerobic digestion as a renewable source with energy potential [25]. It is possible to identify the energy supply of the biomass contained in the species that are most representative of the agricultural, livestock and urban organic waste sectors. In these sectors, the following are reported as sources of residual biomass: i) agricultural crop residues and agro-industrial residues in the agricultural sector, ii) bovine, pig and poultry manure in the livestock sector, iii) residues from market places, centers of supplies and pruning in the urban organic waste sector [26]. In this context, it could be seen that the reported studies make use of biomass derived from anaerobic biodigestion for the production of biogas as a renewable energy source. On the one hand, anaerobic digestion of livestock residues would be a better alternative to traditional biomass, by improving rural productivity, reducing the use of physical and agrochemical fuels, and therefore reducing the environmental impact [27]. Next, productive 
projects for the generation of renewable energy from anaerobic biomass digestion in rural settings will be presented.

Palm oil is the most widely used input to produce biodiesel in Colombia, so the effluents generated, even though they are pollutants, could be used for the production of biogas through anaerobic digestion [28]. Additionally, on average $93 \%$ of solid waste is in sanitary landfills, which could be used to generate renewable energy through sustainable clean technology businesses [29]. Likewise, Escalante, Guzmán and Castro studied the production of biogas by means of the fique bagasse, using the ruminal fluid and porcine manure as inoculum, demonstrating the reuse of juice and bagasse that contaminate by being discarded in the fique processing [30] . Cendales and Jiménez developed a computational model of anaerobic digestion of bovine manure and citrus residues, to demonstrate its potential as renewable energy [31]. Likewise, Castro, Escalante, Gómez and Jiménez reported the biomethanisation of wastewater from a bovine slaughter plant with pre-incubated bovine manure as a source of inoculum [32]. In the municipalities of Fómeque, Guacheta and Anapoima, agro-industrial waste was used for the production of biogas, through anaerobic digestion [33]. Marín, Salazar, Castro and Escalante investigated the co-digestion of residual vinasse and cage manure from the agro-industry [34], while Fernández, Martínez, Morán and Gómez recorded the use of whey derived from cheese to produce biofuels [35].

Finally, for the production of biogas, food residues and pruning are used as substrates by anaerobic digestion in bioreactors at laboratory scale [36]. Likewise, Rodríguez, Colmenares, Barragán and Mayorga studied the energy use of the aquatic weed Eichhornia crassipes, which is processed to obtain bi-ethanol and biogas, as it is a renewable energy source that improves agricultural productivity [37]. Finally, Alzate, Restrepo and Jaramillo studied the use of urban solid waste for the production of electrical energy in the Cundiboyacense region, through thermal conversion by incineration and biological conversion by anaerobic digestion [38].

\section{CONCLUSIONS}

The $51 \%$ of the Colombian territory is not connected to the National Interconnected System, given this state absence, the empowerment of the rural population is required to create alternative energy sources that are friendly to the environment. These projects represent a high cost for these populations, so they need financing, technology and knowledge through international cooperation. This cooperation seeks to make Latin America the recipient of the financing, technology transfer, and knowledge granted by developed countries. However, the literature 
review showed that this international cooperation has only been seen in Argentina and Brazil, leaving Peru, Chile, Uruguay and Colombia alone. In particular, the Colombian initiatives studied tend to be community projects without national and international financial support. This represents an opportunity to learn about the benefits of international agreements and their cooperation mechanisms, in order to strengthen existing renewable energy projects in Colombia and make them sustainable over time.

In this sense, the most important international funds are the Green Fund of the European Union and the Green Climate Fund of the UN Framework Convention on Climate Change. Likewise, there are subsidies for adaptation and mitigation against climate change, which work as compensation mechanisms, through which developed countries that exceed the $\mathrm{CO} 2$ emission limit, finance initiatives related to mass planting and forest conservation in developing countries. These funds also involve technology exchange initiatives and Know-How, to share expert experiences, transmit knowledge and generate projects in communities in developing countries.

Finally, in most of the Latin American countries studied, international conventions and mechanisms are not being used to finance projects related to the mitigation and adaptation of climate change. In the Colombian case, there is a trend that makes it evident that rural communities are unaware of these international conventions and mechanisms, which could enhance and strengthen their projects for the generation of alternative energy. Therefore, the need to empower these rural Colombian communities is observed, through an educational proposal that provides them with the technological tools and knowledge required to strengthen their initiatives and projects for generating alternative energy

\section{REFERENCES}

[1] P. Caballero, “Colombia y la agenda ambiental internacional", $\quad$ 1997. Available: https://revistas.uniandes.edu.co/doi/pdf/10.7440/colom biaint38.1997.02

[2] Energías Renovables, “Energía Biomasa”, 2008. Available:

http://www.energia.gov.ar/contenidos/archivos/public aciones/libro_energia_biomasa.pdf

[3] FAO, "La biomasa en los países en desarrollo: Potencialidades y restricciones". Available: http://www.fao.org/3/t2363s/t2363s08.htm

[4] Z. Drnas, "El derecho ambiental y el derecho de la energía, ¿Paradigmas compatibles o contrapuestos?",
2016. Available: http://www.acaderc.org.ar/doctrina/elderecho-ambiental-y-el-derecho-de-la-energiaparadigmas-compatibles-o-contrapuestos

[5] WRM, "Biomasa y Bioenergía", 2013. Available: https://wrm.org.uy/es/articulos-del-boletinwrm/seccion1/biomasa-y-bioenergia/

[6] V. Pierre, "Soft law y derecho internacional ambiental algunas implicaciones nacionales", 2008. Available: https://www.redalyc.org/pdf/4275/427539905005.pdf

[7] IRENA, “Transformación energética mundial”, 2018. Available:

https://www.irena.org//media/Files/IRENA/Agency/Pu blication/2018/Apr/IRENA 
Global_Energy_Transformation_2018_summary_ES.pd $\mathrm{f} ? \mathrm{la}=\mathrm{en} \&$ hash $=$ A5492C2AAC7D8E7A7CBF71A460649A 8DEDB48A82

[8] E. Blasco, "La biomasa, una energía renovable que escala peldaños. A propósito del plan regional de ámbito sectorial de la bioenergía de Castilla y León", 2011.

Available:

https://www.actualidadjuridicaambiental.com/wpcontent/uploads/2011/06/2011_06_Blasco-

Eva_Biomasa.pdf

[9] P. Aristegui, "Evolución del principio responsabilidades comunes pero diferenciadas en el régimen internacional del cambio climático, 2016. Available: http://derecho.udp.cl/wpcontent/uploads/2016/08/28_Aristegui.pdf

[10] C. Cerdas, "El Principio de Responsabilidades Comunes pero Diferenciadas", Revista Tribuna Internacional, vol. 5, no. 10, 2016-

[11] M. Coviello, "Entorno internacional y oportunidades de desarrollo de las fuentes renovables de energía en los países de América Latina y el Caribe", 2003. Available: https://repositorio.cepal.org/bitstream/handle/11362/64 28/S039641_es.pdf?sequence $=1 \&$ isAllowed $=y$

[12] ONU, "Energía y desarrollo sustentable en América Latina y el Caribe. Guía para la formulación de políticas energéticas", 2003.2 Available: https://repositorio.cepal.org/bitstream/handle/11362/27 838/S2003004_es.pdf?sequence $=1 \&$ isAllowed $=y$

[13] M. Leandro, G. Evelyn, G. Balbina, “Generación de energía eléctrica a partir de biomasa, experiencias y actualidad en Argentina", 2016. Available: http://unsam.edu.ar/escuelas/economia/Ciepe/pdf/bio masa.pdf

[14] L. Pérez, "Residuos Forestales. Argentina con energías renovables", $2014 . \quad$ Available: http://www.scielo.org.ar/pdf/ria/v40n2/v40n2a03.pdf

[15] J. Verdesio, "Políticas públicas para la difusión de las Nuevas Energías Renovables (NER) en Brasil", 2003. Available: http://132.247.149.1/p-cientifica/coloquioerdal/27HJuanjoseverdesioLtt.pdf

[16] L. Pereira, “La visión de Brasil sobre la cooperación internacional. La cooperación internacional como instrumento de la política exterior brasileña", Revista Española de Desarrollo y Cooperación, no. 22, pp. 63-77, 2008. Available: https://www.academia.edu/download/39623603/La_vis ion_de_Brasil_sobre_la_coop_II.pdf\#page $=63$

[17] A. Altamirano, B. Schlegel, O. Thiers, A. Miranda, B. Pilquinao, R. Orrego, C. Rocha, “Disponibilidad y potencial energético de la biomasa del bosque nativo para el desarrollo de la dendroenergía en el centro-sur de Chile", vol. 36, no. 2, pp. 223-237, 2015. Available: https://dx.doi.org/10.4067/S0717-92002015000200008

C. Pontt, C. Guiñez, "Potencial de Biomasa en Chile. Estudio de contribución de las ERNC al SIC al 2025", 2008. Available: http://www.produccionanimal.com.ar/Biodigestores/32-Biomasa_Chile.pdf

[19] M. Aldana, W. Bizzo, M. Viera, "Evaluación del potencial energético de los residuos de la caña de azucar en el Perú", 2016. Available: http://xxiiispes.perusolar.org/wpcontent/uploads/2016/10/Ponencia-N\%C2\%AA-26Viernes-304.pdf

[20] H. García, "Matriz energética en el Perú y energías renovables". Lima: Fundación Friedrich Ebert (FES) y Derecho, Ambiente y Recursos Naturales (DAR), 2013.

[21] F. Resquin, C. Rachid, L. Carrasco, D. Vázquez, "Biomasa: Fuente de energía renovable para la matriz energética del Uruguay", 2011. Available: http://www.inia.uy/Publicaciones/Documentos\%20com partidos/18429130112134100.pdf

[22] G. Bittencourt, N. Lorenzi, "La industria de biocombustibles en Uruguay: situación actual y perspectivas", $2009 . \quad$ Available: https://www.colibri.udelar.edu.uy/jspui/bitstream/20.5 00.12008/2128/1/DT\%20E\%202009-11.pdf

[23] C. Machado, "Situación de los Biocombustibles de 2da y 3era Generación en América Latina y Caribe", 2010 Available: http://www.olade.org/sites/default/files/CIDA/Biocom ustibles/SITUACION\%20DE\%20BIOCOMBUSTIBLES \%20EN\%20ALC.pdf

[24] Instituto de Planificación y Promoción de Soluciones Energéticas para las Zonas No Interconectadas - IPSE, “Informe de Gestión 2017-2018", 2018. Available: http://www.ipse.gov.co/atencion-al-

ciudadano/rendicion-de-cuentas/category/291rendicion-de-cuentas-2018?download=1331:informede-gestion-2017-2018

[25] L. Castro-Molano, H. Escalante, J. Jaimes-Estévez, L. Díaz, K. Vecino, G. Rojas, L. Mantilla, “Low cost digester monitoring under realistic conditions: Rural use of biogas and digestate quality", Bioresource Technology, no. 239, pp. 311-317, 2017. Available: http://dx.doi.org/10.1016/j.biortech.2017.05.035

[26] Sistema de Información de Eficiencia Energética y Energías Alternativas - SI3EA, "Atlas del potencial energético de la biomasa residual en Colombia", 2010. Available:

http://www.si3ea.gov.co/si3ea/documentos/documenta cion/Biodiesel/1Indice_Generalidades.pdf

[27] D. Parra-Ortiz, M. Botero-Londoño, J. Botero-Londoño, 
"Biomasa residual pecuaria: revisión sobre la digestión anaerobia como método de producción de energía y otros subproductos", Revista UIS Ingenierías, vol. 18, no. 1, pp. 149-160, 2018. Available: https://doi.org/10.18273/revuin.v18n1-2019013

[28] D. Nabarlatz, L. Arenas-Beltrán, D. Herrera-Soracá, D. Niño-Bonilla, "Biogas production by anaerobic digestion of wastewater from palm oil mill industry", Ciencia, Tecnología y Futuro, vol. 5, no. 2, pp. 73-84, 2013. Available: http://dx.doi.org/10.29047/01225383.58

[29] M. Acosta, J. Pasqualino, "Potencial de uso de biogás en Colombia", Teknos Revista Científica, vol. 14, no. 2, pp. 2733, 2014. Available: https://doi.org/10.25044/25392190.468

[30] H. Escalante, C. Guzmán, L. Castro, "Anaerobic digestion of fique bagasse: An energy alternative", DYNA, vol. 81 , no. 183, pp. 74-85, 2014. Available: https://doi.org/10.15446/dyna.v81n183.34382

[31] E. Cendales, S. Jiménez, “Modelamiento computacional de la producción de energía renovable a partir del biogás mediante la codigestión anaeróbica de la mezcla de residuos cítricos y estiércol bovino", Revista Escuela De Administración De Negocios, no. 77, pp. 42-62, 2014. Available:

https://doi.org/10.21158/01208160.n77.2014.814

[32] L. Castro-Molano, H. Escalante-Hernández, O. GómezSerrato, D. Jiménez-Piñeros, "Análisis del potencial metanogénico y energético de las aguas residuales de una planta de sacrificio bovino mediante digestión anaeróbica", DYNA, vol. 83, no. 199, p. 41, 2016. Available: http://dx.doi.org/10.15446/dyna.v83n199.56796

[33] K. Montenegro, A. Rojas, I. Cabeza, M. Hernández, "Potencial de biogás de los residuos agroindustriales generados en el departamento de Cundinamarca", Revista Ion, vol. 29, no. 2, pp. 23-37, 2016. Available: http://dx.doi.org/10.18273/revion.v29n2-2016002

[34] J. Marín-Batista, L Salazar, L. Castro, H. Escalante, “Codigestión anaerobia de vinaza y gallinaza de jaula: alternativa para el manejo de residuos agrícolas colombianos", Revista Colombiana de Biotecnología, vol. 18, no. 2, p. 6, 2016. Available: https://doi.org/10.15446/rev.colomb.biote.v18n2.53

[35] C. Fernández, E. Martínez, A. Morán, X. Gómez, "Procesos biológicos para el tratamiento de lactosuero con producción de biogás e hidrógeno", Revista ION, vol. 29, no. 1, pp. 47-62, 2016. Available: http://dx.doi.org/10.18273/revion.v29n1-2016004

[36] J. Solarte, J. Mariscal, B. Aristizábal, “Evaluación de la digestión y co-digestión anaerobia de residuos de comida y de poda en bioreactores a escala laboratorio", Revista ION, vol. 30, no. 1, pp. 105-116, 2017. Available: http://dx.doi.org/10.18273/revion.v30n1-20170

[37] A. Rodríguez, F. Colmenares, J. Barragán, M. Mayorga, "Aprovechamiento Energético Integral De La Eichhornia Crassipes (Buchón De Agua)", Ingenium, vol. 18, no. 35, pp. 134-152, 2017.

[38] S. Alzate, B. Restrepo-Cuestas, A. Jaramillo-Duque, "Electricity generation potential from solid waste in three Colombian municipalities", TecnoLógicas, vol. 21, no. 42, pp. 111-128, 2018. Available: https://doi.org/10.22430/22565337.782 


\title{
EVALUAR EL COMPORTAMIENTO DE UNA BATERÍA RECARGABLE CON ENERGÍA SOLAR, DISEÑADA A BASE DE ELEMENTOS ALCALINOS, ALCALINOTÉRREOS, METALES DE TRANSICIÓN Y GRAFENO
}

\section{Assessing rechargeable battery's behavior with solar energy, battery's design is based on alkaline, alkaline-earth metals, transition metals and graphen}

\author{
Aparicio, Andrea ${ }^{1}$ \\ Universidad Internacional Iberoamericana - UNINI (México), Linea de Investigación: Desarrollo sostenible y Energías \\ Renovables
}

\section{Resumen}

Teniendo en cuenta que las baterías recargables de litio son las más utilizadas ya que permiten un buen almacenamiento de energía en un área reducida, también presentan desventajas como una vida útil relativamente corta, sobrecalentamiento, su producción es de alto costo y en este momento el material se encuentra en etapa de agotamiento en el planeta. Por ello, a nivel mundial se experimenta con diferentes materiales y aleaciones creando nuevas baterías de elementos como el níquel, cadmio, hidrógeno, sodio, potasio, manganeso y su combinación con otros materiales y aglutinantes. Esta investigación en curso pretende evaluar el comportamiento de una batería que pueda ser recargable con energía solar, diseñada a través de biomimética, con base en el principio de intercambio de iones que generan potenciales eléctricos a nivel celular (bomba sodio-potasio-calcio). En donde el ánodo será construido a partir de las aleaciones de sodio-potasio, calcio-sodio, magnesio-calcio en diferentes concentraciones; el cátodo será de Nitinol y el dieléctrico que completará el sistema será grafeno. De las pruebas realizadas con cada una de las aleaciones se escoge la que presente mayor conductividad y electronegatividad implementando el prototipo final y llevando a cabo las pruebas correspondientes. Con el desarrollo de este proyecto se espera encontrar una forma de almacenar mayor cantidad de energía por área, acrecentando la vida útil de la batería al ser recargada con energía solar, lo cual genera

\footnotetext{
${ }^{1}$ Correo electrónico de contacto: andreaapaga74@gmail.com.
} 
disminución de: costos de producción y niveles de contaminación, además los metales utilizados aún se encuentran presentes de forma abundante en la naturaleza.

Palabras claves: Biomimética, acumulación de energía, corriente, diferencia de potencial.

\section{INTRODUCCIÓN}

Dado que las diferentes revoluciones industriales que se han desarrollado a lo largo de los últimos siglos han dejado como resultado grandes inventos y grandes desarrollos a nivel industrial, científico, técnico, tecnológico, social y de formas de pensar; también es cierto que han generado un sin número de daños al medio ambiente y a un agotamiento acelerado de los recursos naturales que existen en nuestro planeta; es así como la aparición de las máquinas de vapor (su base el carbón), luego las de combustión (su base el petróleo) y por último el descubrimiento de la electricidad, generaron grandes desarrollos en sectores económicos como el agrícola, automotriz, manufacturero entre otros, hasta tal punto que en este momento la energía eléctrica es parte fundamental en la vida diaria.

Es así como la necesidad de innovar y tecnificar aún más la industria automotriz permitió que la pila de Alexandro Volta pasara a ser una batería recargable a manos del físico Francés Gastón Planté quien descubrió la celda de plomo-ácido, que tenía el potencial para ser cargada nuevamente después de ser utilizada, esta es la precursora de las baterías húmedas. Después de esto vienen otros experimentos haciendo que aparezca la batería de níquelcadmio y posteriormente la más efectiva y más utilizada batería de litio.

La batería de ión litio fue creada en 1985 por Akira Yoshino, la cual hasta ahora ha seguido siendo perfeccionada y modificada para una mayor efectividad con una mejor densidad de energía[1]. Desde entonces se sabe que este tipo de baterías en su producción tienen un costo elevado debido a que los polímeros con los que son recubiertas deben tener condiciones muy específicas lo que hace costosa su fabricación final, además siguen siendo poco seguras, presentando sobrecalentamientos en ciertas circunstancias, su reutilización es muy costosa porque se necesita mano de obra especializada, además tanto en su proceso de fabricación como de reutilización es poco amigable con el medio ambiente.

Teniendo en cuenta estas características los científicos a nivel global buscan la forma de reemplazar el litio para la generación de pilas y/o baterías, lo que ha permitido una evolución e innovación en este aspecto, es así como el desarrollo de las baterías de 
combustión se ha generalizado sobre todo en la industria automotriz debido a que este tipo de batería tiene la capacidad de transformar por medio de una reacción química la energía que se encuentra almacenada en un combustible en energía eléctrica; para esta finalidad se tiene la batería de combustión de hidrógeno que es un gran aliado del medio ambiente puesto que no genera emisiones de $\mathrm{CO} 2$ al medio ambiente porque su resultado es vapor de agua y es abundante en la naturaleza, pero una de sus grandes debilidades es que debe ser comprimido a 700 bares para poderlo introducir en el espacio a utilizar[2]. De este tipo de batería se presentan varios tipos a saber: membrana de polímero sólido, utilizada para portátiles y transporte; de solución alcalina con una mayor eficiencia y utilizadas en la industrial espacial; las de ácido fosfórico (combinadas con el hidrógeno), carbonatos fundidos y óxido de sólido que se utilizan para generar energía eléctrica distribuida y calor; por último se tienen las de membrana de polímero sólido que son utilizadas en los portátiles[3].

Otro tipo de batería prometedora es la de Magnesio - magnesio/sodio, inicialmente porque este material es muy abundante en la naturaleza, presenta una alta capacidad de volumen y no forma denditras durante el ciclo electroquímico, adicional a esto se han realizado nanocintas de vanadato de sodio $(\beta-\mathrm{NaV6O15})$ bajo un proceso hidrotermal; este material permite acomodar de forma reversible sodio y magnesio en una misma estructura, pero esta experimentación no fue tan exitosa como se esperaba porque se generan reacciones que no se pueden revertir propias de la descomposición del electrolito que generan unas láminas que no permiten el paso a la superficie lo que hace que el electrodo sea electroquímicamente inerte. (nanocintas)[4].

De lo anterior se tienen varias investigaciones como es el caso de la batería híbrida de Magnesio-Sodio, donde el ánodo está conformado por el magnesio metálico y su cátodo es Sodio, de tal forma que se aprovechan la capacidad que tiene el Magnesio de depositarse en el ánodo sin que se formen dendríticas (ramificaciones que forman corto circuito) y su rápida interacción en el cátodo de Sodio permitiéndole acumular y recoger energía de forma alternada. En este experimento la batería híbrida entregó $183 \mathrm{Wh} / \mathrm{kg}$ con un voltaje de 2,3 V promediado en 50 ciclos[5]

En esta carrera por la innovación y creación de nuevas baterías también se han realizado estudios utilizando otros tipos de electrodos como lo es el caso de la utilización del floruro de manganeso para las baterías de iones de sodio realizado por investigadores del Instituto de Ciencia de Materiales de Barcelona, el Departamento de Química Inorgánica, de la 
Universidad Complutense de Madrid y el Departamento de Química de la Universidad Autónoma de Barcelona; quienes analizaron los compuestos Na2MnF5 y NaMnF3 utilizando un medio húmedo (acuoso), se centró la atención en el NaMnF3 observándose su comportamiento en una celda electroquímica interactuando con el $\mathrm{Na}+$ : comparando los resultados con los obtenidos utilizando electrodos sodio y metal, esperando que el NaMnF3 se preveía una entrega de densidad de energía de $2,7 \mathrm{eV}$ de acuerdo con sus propiedades pero esto no fue alcanzado debido a que sus características de aislante logran inducir una polarización celular bastante alta, dando como resultado una actividad electroquímica que no es posible alcanzar con los electrolitos que se encuentran comúnmente[6].

Otro caso de estudio han sido los nanotubos de carbono como acumuladores de energía, estos al estar formados por moléculas de carbono que forman una red de hexágonos son el diseño perfecto para que la energía se mueva a través de él otorgándole una conductividad bastante alta, además de que esta composición hace que tenga una muy buena resistencia, y adicional a esto presenta una gran capacidad para autoregenerase. Las investigaciones están centradas en el almacenamiento de energía solar ya que esta puede ser atrapada en los enlaces que forma su estructura molecular, una vez la almacena a través de un estímulo externo, la estructura vuelve de forma rápida a su forma habitual, lo cual permite liberar la energía acumulada en forma de calor. Se puede decir que las ventajas de este tipo de batería es que no tendrá un tiempo limitado de uso, pues su capacidad de regeneramiento la hará prácticamente infinita, también puede someterse a cambios abruptos de temperatura, vibraciones y golpes y lo mejor de todo es que en un espacio muy pequeño se podrá almacenar gran cantidad de energía[7].

Se han realizado varios experimentos utilizando iones multivalentes, pero estos presentan una velocidad de difusión bastante lenta, por lo que se debe trabajar en materiales catódicos adecuados para poder construir baterías recargables. Así mismo en las baterías de calcio el inconveniente es que no hay una buena compatibilidad entre electrodos y electrolitos y cuando reacciona genera una lámina de residuos que lo que hace es bloquear la acción de los iones de calcio. También se ha trabajado baterías de iones de aluminio donde sus propiedades de carga y descarga son inestables debido a que durante estos procesos el voltaje es inestable[8].

Hay un punto de vista que, aunque es muy conocido no se ha trabajado mucho como punto de partida para la generación de una batería y es la forma en la que las células al interior del cuerpo humano generan potenciales eléctricos, esto hace que nos traslademos a la 
biomimética (el arte de imitar las funciones biológicas) en el área de intercambio de iones al interior de las células justo en la membrana celular. A lo anterior se le denomina gradiente electroquímico, generado cuando se presenta el transporte activo de iones tanto al interior como al exterior de las células, donde el número de iones cargados positivamente en el exterior de la célula doblan en número a los que se encuentran en el interior de la célula lo cual vendría a ser una especie de batería ya que las cargas en la parte externa serían un polo positivo, mientras que el interior se comportaría como un polo negativo a esto es a lo que se le denomina potencial de membrana el cual permite la conducción eléctrica a través de las células nerviosas[9].

Con la información anterior se evidencia el trabajo que realizan los investigadores por lograr una batería que pueda reemplazar a la batería de litio, siendo más o igual de eficiente, más segura (sin fluctuaciones por temperatura), con mayor capacidad y densidad de energía. También se observa que se ha trabajado con materiales como lo son el sodio y el potasio pertenecientes al grupo I: Metales alcalinos en la tabla periódica, donde se encuentra el litio, obteniendo buenos resultados en cuanto a seguridad y almacenamiento, pero sacrificando densidad de energía. En conclusión, se desea buscar un conjugado de materiales que sean abundantes en la naturaleza, que permitan una mayor capacidad para acumulación de energía, que sea seguro, que su costo de fabricación no sea tan elevado y por último que la densidad de energía no decaiga con la electrólisis.

\section{MATERIALES Y MÉTODOS}

Se llevará a cabo una investigación de tipo experimental en la que se pretende utilizar los elementos alcalinos como el sodio y el potasio en combinación con el magnesio y el calcio que son elementos alcalinotérreos pero que al igual que los metales alcalinos presentan buenas propiedades de conductividad, electronegatividad y resistividad, con el fin de generar el electrodo positivo de la batería. Para crear el electrodo negativo se utilizará la aleación de NiTi en hilo conductor de $2 \mathrm{~mm}$ de espesor y por último como dieléctrico utilizar el grafeno que por sus propiedades conductoras gracias a su efecto túnel y a su estructura atómica permitirá tanto el movimiento de electrones como el almacenamiento de energía proveniente de la radiación solar para su carga.

Lo anterior para mejor entendimiento se expresa en la siguiente Figura 1: 


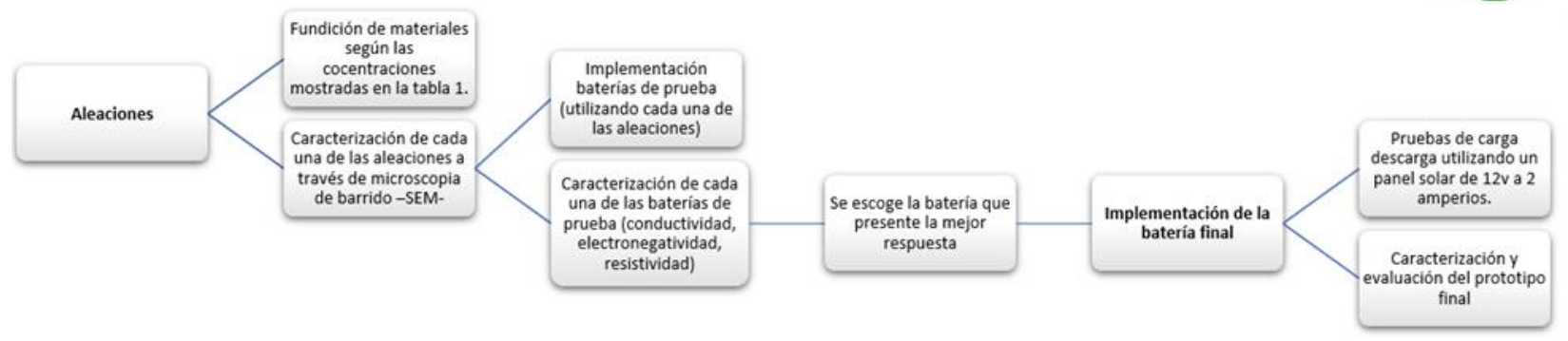

Figura 22 Etapas consideradas para el desarrollo y evaluación de la batería

Para el desarrollo del proyecto es necesario contar con una mufla de atmósfera controlada ya que es necesario rodear a los metales que se van a trabajar con un gas inerte (nitrógeno o helio) para que no deteriore la superficie del metal creando una atmósfera que protege el material para que no se contamine de aire, agua o vapor entre otros[10]. El rango de temperatura de trabajo para la mufla debe estar entre 1000 a $1200^{\circ} \mathrm{C}$ para llevar a cabo las aleaciones de los materiales alcalinos y alacalinotérreos a saber: $\mathrm{Na}, \mathrm{K}, \mathrm{Mg}$ y $\mathrm{Ca}$ en las cantidades y combinaciones que se muestran en la tabla:

\section{Tabla 6 Combinaciones}

\begin{tabular}{|c|c|c|}
\hline $\mathrm{CaMg}$ & $\mathrm{MgCa}$ & $\mathrm{CaMg}$ \\
$20 / 80$ & $50 / 50$ & $80 / 20$ \\
\hline $\mathrm{KNa}$ & $\mathrm{NaK}$ & $\mathrm{KNa}$ \\
$20 / 80$ & $50 / 50$ & $80 / 20$ \\
\hline $\mathrm{CaNa}$ & $\mathrm{NaCa}$ & $\mathrm{CaNa}$ \\
$20 / 80$ & $50 / 50$ & $80 / 20$ \\
\hline
\end{tabular}

Nota: Las concentraciones se encuentran en tanto por ciento.

Una vez fundidas las aleaciones y caracterizadas a través de microscopia electrónica de barrido SEM se da inicio al diseño de la batería experimentando como electrodo positivo de la misma, cada una de las aleaciones, como electrodo negativo el NiTi y como dieléctrico el grafeno; para cada experimento se utilizará $1 \mathrm{~g}$ de grafeno. Este modelado de la batería se llevará a cabo utilizando el software de simulación de procesos Matlab y su complemento Simulink de la empresa MathWorks.

Después de realizar y analizar los diferentes montajes, se determina si se cumplió o no el modelado planteado a través de la simulación de acuerdo con el cual algunos o todos los electrodos (aleaciones) utilizados genera un potencial eléctrico en mayor o menor proporción. Enseguida se procederá a conectar el panel solar de 12 voltios 2 amperios para observar si hay una inversión en el proceso efectuado anteriormente (si lo hay quiere decir 
que el proceso se da en doble vía y podría ser el principio de una batería recargable). Los parámetros por medir y comparar son: Potencial eléctrico, resistividad, conductividad, densidad de energía, ciclos de carga y descarga. Los instrumentos de medición a utilizar serán un multímetro marca fluke, pinza amperimétrica marca fluke, osciloscopio digital marca tecktronics.

Cuando las pruebas se hayan completado se utilizará el software SPSS con el fin de saber cuál de las pruebas realizadas ha brindado mejores resultados en cuanto a densidad de energía, conductividad, ciclos de carga y descarga, consiguiendo resultados que llevarán a un análisis final generando la conclusión del experimento.

\section{RESULTADOS Y ANÁLISIS DE RESULTADOS}

Aún no se cuenta con resultados concretos debido a que no se ha realizado el fundido de los materiales para realizar las aleaciones, por lo tanto, el análisis de los resultados se hará con base en la comparación de la respuesta de la batería propuesta con las características de las diferentes clases de batería de litio funcionales desarrolladas hasta el momento, las cuales se muestran en la siguiente tabla:

Tabla 7 Características de las baterías de litio

\begin{tabular}{|l|c|c|c|c|c|c|}
\hline \multicolumn{1}{|c|}{ Química } & $\begin{array}{c}\text { Densidad } \\
\text { Energética }\end{array}$ & $\begin{array}{c}\text { Temperatura } \\
\text { de trabajo }\end{array}$ & ciclos & seguridad & $\begin{array}{c}\text { Respetuoso } \\
\text { con el medio } \\
\text { ambiente }\end{array}$ & $\begin{array}{c}\text { Costo en } \\
\text { base a } \\
\text { ciclos } \mathbf{x} \text { Wh }\end{array}$ \\
\hline LiFePO4 & $>120 \mathrm{wh} / \mathrm{kg}$ & $-20-60^{\circ} \mathrm{C}$ & $>2000(0.2 \mathrm{C})$ & segura & si & $0,15-25$ \\
\hline Plomo ácido & $>35 \mathrm{wh} / \mathrm{kg}$ & $-20-40^{\circ} \mathrm{C}$ & $>200$ & segura & no & 1 \\
\hline NICd & $>40 \mathrm{wh} / \mathrm{kg}$ & $-20-50^{\circ} \mathrm{C}$ & $>1000$ & segura & no & 0,7 \\
\hline $\mathrm{NIMH}$ & $>80 \mathrm{wh} / \mathrm{kg}$ & $-20-50^{\circ} \mathrm{C}$ & $>500$ & segura & si & $1,2-1,4$ \\
\hline $\mathrm{LiMnxNiyCozO} 2$ & $>160 \mathrm{wh} / \mathrm{kg}$ & $-20-40^{\circ} \mathrm{C}$ & $>500$ & No segura & no & $1,5-2,0$ \\
\hline $\mathrm{Li} \mathrm{CoO2}$ & $>35 \mathrm{wh} / \mathrm{kg}$ & $-20-40^{\circ} \mathrm{C}$ & $>200$ & segura & no & 1 \\
\hline
\end{tabular}

Uno de los alcances de esta experimentación debe ser el aumento en la densidad energética, la cual experimentalmente deberá alcanzar entre 80 a $100 \mathrm{wh} / \mathrm{kg}$ y en aumento, en cuanto a la temperatura de trabajo no deberá superar los $-20^{\circ} \mathrm{C}$ y debe generar aproximadamente de 500 a 1000 ciclos. Adicional a esto también debe analizarse, qué sucede con estos parámetros al momento de incorporar el panel solar. 


\section{CONCLUSIONES}

Se espera que una vez realizada la experimentación y caracterizados todos los materiales, el prototipo final de batería recargable con energía solar propuesta sea capaz de generar una densidad energética que se encuentre en un rango entre 80 a $100 \mathrm{wh} / \mathrm{kg}$, además de tener una temperatura de trabajo lo suficientemente baja para que no exista sobrecalentamiento y de pronto una posible chispa, por lo tanto, sería muy segura, también se espera que pueda realizar de 500 a 1000 ciclos y por último al realizar el análisis costo beneficio de generación de 1ciclo por wh, se espera que el tiempo de recuperación no sea mayor a 5 años.

\section{AGRADECIMIENTOS}

Agradezco el apoyo de mi tutor el doctor Misael Díaz Asencio, por su apoyo y colaboración para iniciar este proyecto, a los docentes de la universidad Internacional Iberoaméricana UNINI de México. Además, al MsC. Ingeniero Químico Camilo Posada, quien ha impulsado la experimentación de este proyecto. También agradezco la colaboración de la Ingeniera Mónica Ballén coordinadora del laboratorio de química de la Universidad ECCI.

\section{Referencias}

[1] A. Pintos et al., "Herramienta educativa para la formación en baterías de Litio-Ión," 2012.

[2] "Pilas de Combustible," 2015. https://ingemecanica.com/tutorialsemanal/tutorial n115.html (accessed Jul. 13, 2020).

[3] "Pilas de Combustible - Centro Nacional de Hidrógeno," 2018. https://www.cnh2.es/pilas-decombustible/ (accessed Jul. 13, 2020).

[4] M. Cabello, F. Nacimiento, R. Alcântara, P. Lavela, G. Ortiz, and J. Luis Tirado, “NANOCINTAS DE $\beta$ $\mathrm{NaV} \quad 6 \quad \mathrm{O} \quad 15$ COMO ELECTRODO PARA BATERÍAS DE MAGNESIO Y DUALES MAGNESIO-SODIO," 2016.

[5] R. Zhang, O. Tutusaus, R. Mohtadi, and C. Ling, "Magnesium-sodium hybrid battery with high voltage, capacity and cyclability," Front. Chem., 2018, doi: 10.3389/fchem.2018.00611.

[6] J. Nava-Avendaño, M. E. Arroyo-de Dompablo, C. Frontera, J. A. Ayllón, and M. R. Palacín, “Study of sodium manganese fluorides as positive electrodes

106-113, Oct. 2015, doi: 10.1016/j.ssi.2015.05.023.
"Nanotubos de carbono para el almacenamiento de energía | Twenergy," 2015. https://twenergy.com/eficienciaenergetica/almacenamiento-deenergia/nanotubos-de-carbono-para-elalmacenamiento-de-energia-2405/ (accessed Jul. 13, 2020).

[8] P. Rubio G., Raúl; Ortíz, Gregorio F.; Tirado C.,José L.; Lavela C., "Magnesio, calcio, sodio... ¿qué elemento sustituirá al litio en las baterías?," 2020. https://theconversation.com/magnesio-calciosodio-que-elemento-sustituira-al-litio-en-lasbaterias-130810 (accessed May 18, 2020).

[9] "Bomba de sodio-potasio । CK-12 Foundation." https://www.ck12.org/book/ck-12-conceptosbiología/section/2.16/ (accessed Jul. 13, 2020).

[10] Y. Diaz, Diseño de un horno de mufla para la fusión de aleaciones Ni-Co con fines estomatológicos. 2016. 


\title{
EVALUATION OF THE PRODUCTION OF BIO- OIL OBTAINED THROUGH PYROLYSIS OF BANANA
}

\section{Evaluación de la Producción de Bio-Oil obtenido a partir de la Pirólisis del Banano}

\author{
Ayala, Nathaly1; López, Hernán²; Malagón-Romero, Dionisio ${ }^{3}$. \\ Universidad Santo Tomás
}

\begin{abstract}
Banana harvesting only uses 20 to $30 \%$ of its mass, leaving 70 to $80 \%$ as waste, studies have indicated that the wastes of the banana are a potential feedstock for pyrolysis due to the volatile matter and fixed carbon that has been found. Peels were milled for reducing the size and submitted to granulometric analysis using the Tyler series sieve. The experimental design was factorial by duplicate. Pyrolysis was performed at four different temperatures $\left(300,400,500\right.$, and $\left.600{ }^{\circ} \mathrm{C}\right)$, three heating rates $\left(5,10\right.$ and $\left.15^{\circ} \mathrm{C} / \mathrm{min}\right)$ and residence time of three hours. Samples were physically and chemically characterized. The granulometric analysis showed an average diameter of $2 \mathrm{~mm}$. The banana powder had a moisture content of $8.16 \% \pm 0.8$. The content of lignin (3.24 \%), cellulose $(6.15 \%)$ and hemicellulose $(10.46 \%)$ was identified, and the ash content was $14.92 \%$. The obtained bio-oils were characterized using GC. At a heating rate of $10{ }^{\circ} \mathrm{C} / \mathrm{min}$, bio-oil present a better yield of $6.43 \%$ at $300{ }^{\circ} \mathrm{C}$. The better gas yield was at $600{ }^{\circ} \mathrm{C}$ with $70.30 \%$ at $5{ }^{\circ} \mathrm{C} / \mathrm{min}$ and $67.24 \%$ at $10{ }^{\circ} \mathrm{C} / \mathrm{min}$. The percent of Pentadecane ( $\mathrm{C} 15 \mathrm{H} 32)$ was the highest with a concentration of $30.018 \mathrm{ppm}$ at 26.4 min of retention time. The bio-oil obtained during pyrolysis experiments had a viscosity of $0.03 \pm 0.011 \mathrm{~Pa} \bullet$ s determined at $40{ }^{\circ} \mathrm{C}$ and density $1118 \pm 98 \mathrm{~kg} / \mathrm{m} 3$ measured at $16{ }^{\circ} \mathrm{C}$. These results showed that banana peels could be used as raw material for solvents, fuels, or bioenergy production
\end{abstract}

Keywords: Pyrolysis, granulometric analysis, biomass, bio-oil.

${ }^{1}$ Corresponding E-mail:nathalyayala@usantotomas.edu.co

${ }^{2}$ Corresponding E-mail:hernanlopezr@usantotomas.edu.co

${ }^{3}$ Corresponding E-mail:dionisiomalagon@usantotomas.edu.co 
Resumen

La cosecha de banano sólo utiliza del 20 al 30\% de su biomasa, quedando de un 70 al 80\% como desperdicio, estudios han indicado que estos desperdicios de banano son materia prima potencial para la pirolisis debido al material volátil y el carbón activado que ha sido encontrado. Las cáscaras fueron molidas para reducir su tamaño y expuestas a un análisis granulométrico utilizando las series de tamizado de Tyler. El diseño experimental fue factorial y se realizó por duplicado. La pirolisis se realizó a 4 temperaturas diferentes (300, 400 , 500, y $\left.600{ }^{\circ} \mathrm{C}\right)$, tres velocidades de calentamiento $\left(5,10\right.$ y $15^{\circ} \mathrm{C} / \mathrm{min}$ ) a un tiempo de residencia de tres horas. Las muestras fueron caracterizadas física y químicamente. El análisis granulométrico mostró un diámetro promedio de $2 \mathrm{~mm}$. El polvo del banano tuvo una humedad $8.16 \% \pm 0.8$. Los contenidos de lignina $(3.24 \%)$, celulosa $(6.15 \%)$ and hemicelulosa (10.46\%) fueron identificados, y el contenido de cenizas fue de $14.92 \%$. El biooil obtenido fue caracterizado utilizando la cromatografía de gases. A la velocidad de $10{ }^{\circ} \mathrm{C}$ $/ \mathrm{min}$, el mejor rendimiento del bio-oil fue de $6.43 \%$ a $300{ }^{\circ} \mathrm{C}$. El mejor rendimiento del gas fue a los $600{ }^{\circ} \mathrm{C} \operatorname{con} 70.30 \%$ a $5{ }^{\circ} \mathrm{C} / \mathrm{min}$ y $67.24 \%$ at $10{ }^{\circ} \mathrm{C} / \mathrm{min}$. El porcentaje del Pentadecano (C15H32) fue la mayor concentración de $30.018 \mathrm{ppm}$ at $26.4 \mathrm{~min}$ de retención. El bio-oil obtenido durante los experimentos tuvo una viscosidad de $0.03 \pm 0.011 \mathrm{~Pa} \bullet$ s determinada a $40{ }^{\circ} \mathrm{C}$ y una densidad de $1118 \pm 98 \mathrm{~kg} / \mathrm{m} 3$ medida a $16^{\circ} \mathrm{C}$. Estos resultados mostraron que las cáscaras de banano pueden ser usadas como materia prima solventes, combustibles o para la producción de bioenergía.

Palabras clave: Pirólisis, análisis granulométrico, biomasa, bio-oil.

\section{INTRODUCTION}

Since some years ago, climate change has been one of the main concerns due to the emission of greenhouse gases caused by the combustion of fossil fuels, causing an increase in the earth's temperature. Burning fossil fuels produce compounds such as particulate material $(\mathrm{PM})$, nitrogen oxides (NOX), carbon dioxide (CO2), and sulfur dioxide (SO2), which have impacted people's health [1], [2]. This problem has generated the search for new sources of energy to replace traditional sources. Biomass is one source for obtaining energy due to the possibility of obtaining renewable energy [3], and for reducing atmospheric emissions [4], making biomass one of the best alternatives to reduce greenhouse emissions and also remove wastes.

Biomass is considered as a natural source of energy that biologically transforms inorganic compounds in organic [5] and can be extracted from forestry, agricultural, or industrial residues [6]. However, agricultural wastes are not being properly treated as most of these 
wastes are being left on the ground, becoming a risk for transmission of diseases and production of greenhouse gases as they decompose [7]. One of the industries that generate a huge amount of wastes is the banana crop industry, which has been increasing over the years. In 2017, banana production dedicated about 5.6 million hectares of land globally, generating 114 million tons around the world [8]. Nevertheless, banana harvesting only uses 20 to $30 \%$ of its mass, leaving 70 to $80 \%$ of waste [9] and most of these wastes are being burned at an open field, polluting soils and degrading ecosystems that increase carbon footprint [10] . One possibility for reusing banana wastes is their transformation in solvents and fuels though pyrolysis [11].

Pyrolysis is a thermochemical technology that transforms biomass in different products with superior properties that could be used as fuels or solvents, so obtained products could be harnessed as an energy source or for chemical supplies [12]. Heating rate and residence time are parameters that classified pyrolysis in slow, fast and flash [13]. Pyrolysis has been extensively studied because of its advantages as having a higher conversion rate to produce different bio-product and reduce the environmental risk of the contaminants [14]. In fact, several of the processes for obtaining chemical compounds such as benzaldehydes, cyclopentane, and pyridine [15] use agricultural wastes as feedstock with pyrolysis due to the simplicity of performance, high warming speed, easy control [16].

South American countries as Brazil, Colombia, Ecuador, and Costa Rica, are the principal producers of banana Cavendish [17];[18]. Therefore, wastes produced by harvesting are bigger in these countries; for that reasons, there are different studies about how to process those wastes and what added value can obtain from them. Some studies have indicated that processing rachis of banana by pyrolysis can generate biochar [19], parallel that, other investigations have establish that pseudo stem and leafs of banana, are a potential feedstock to used it in process of combustion[20]. Hence, banana wastes are a potential feedstock for pyrolysis since it was found volatile matter of $88.02 \%$ and fixed carbon of $2.7 \%$ in them [21]. Additionally, other studies have found different yields of products obtained from banana peels pyrolysis, at a heating rate of $10{ }^{\circ} \mathrm{C} / \mathrm{min}$, temperatures from 350 to $550{ }^{\circ} \mathrm{C}$ and residence time between 45 and 90 minutes $35.64 \%$ of char was obtained [22]. Other authors obtained with a heating rate of and $7^{\circ} \mathrm{C} / \mathrm{min}$, temperature between $400{ }^{\circ} \mathrm{C}$ and $700{ }^{\circ} \mathrm{C}$ and residence time of 20 minutes, yields of $28.03 \%$ of bio-oil and more than $30 \%$ of gas at 700 ${ }^{\circ} \mathrm{C}$ [23]. Besides, in the literature, it is reported the potential of production of char with a yield of $47.7 \%$ [24]. 
The aim of this paper is to expand the studies that already exist and give an additional value to wastes, specifically peels, from banana crops and determinate if this process could be an energy opportunity for banana producers as Colombia.

\section{MATERIALS AND METHODS}

\section{Biomass preparation and characterization}

Banana peel samples were obtained from an urban zone of Garagoa- Boyacá, Colombia. There, $25 \mathrm{~kg}$ of mature peels were collected. The initial moisture content of banana peels was determined by drying according to ASTM E1757-19. Then, samples were dried for reducing the moisture content in an oven (Memmert, Germany) for 24 hours at $110{ }^{\circ} \mathrm{C}$. Once dried, peels were milled in domestic equipment and sieved using Tyler series sieves according to ASTM C136. $2400 \mathrm{~g}$ of banana peel powder were isolated in sealed plastic bags, at room temperature $\left(19^{\circ} \mathrm{C}\right)$, to avoid biological contamination. The banana powder was characterized by proximate and ultimate analyses. Moisture and ash content were determined following methods ASTM (ASTM E1358-97) and ASTM E1755-01, respectively. Elemental composition of banana powder carbon $(\mathrm{C})$, hydrogen $(\mathrm{H})$, nitrogen $(\mathrm{N})$, oxygen (O), and sulfur (S), were characterized according to ASTM D3178. The lignocellulose analysis was developed according to Van Soest Method for determining cellulose, hemicellulose and lignin fraction. Thermogravimetric Analysis (TGA) of banana powder was carried out in Mettler Toledo equipment. Samples were heated up from room temperature to $900{ }^{\circ} \mathrm{C}$ at three heating rates of 5,10 , and $15^{\circ} \mathrm{C} / \mathrm{min}$.

\section{Pyrolysis equipment}

The experiments were developed in the pyrolysis equipment designed by Universidad Santo Tomás (Bogotá, Colombia). Figure 1 shows a diagram of the equipment. The equipment consists of three elements: reactor, condenser and control system. The reactor was built in stainless steel AISI 316, equipped with an electrical resistance for heating (1.6 $\mathrm{kW})$. The reactor volume has a capacity of $1 \mathrm{~L}$. The reactor has an insulating material in the ceramic fiber blanket. The reactor is connected with a pipe schedule 40 to a condenser. The condenser has a capacity of $4.5 \mathrm{~L}$ and has two valves to regulate the amount of water. The equipment has an activated carbon filter responsible for the adsorption of volatiles substances [25]. The equipment is controlled by Maxthermo MC 5438 trade controller, which allows programming the time and heating speed of the reactor. 


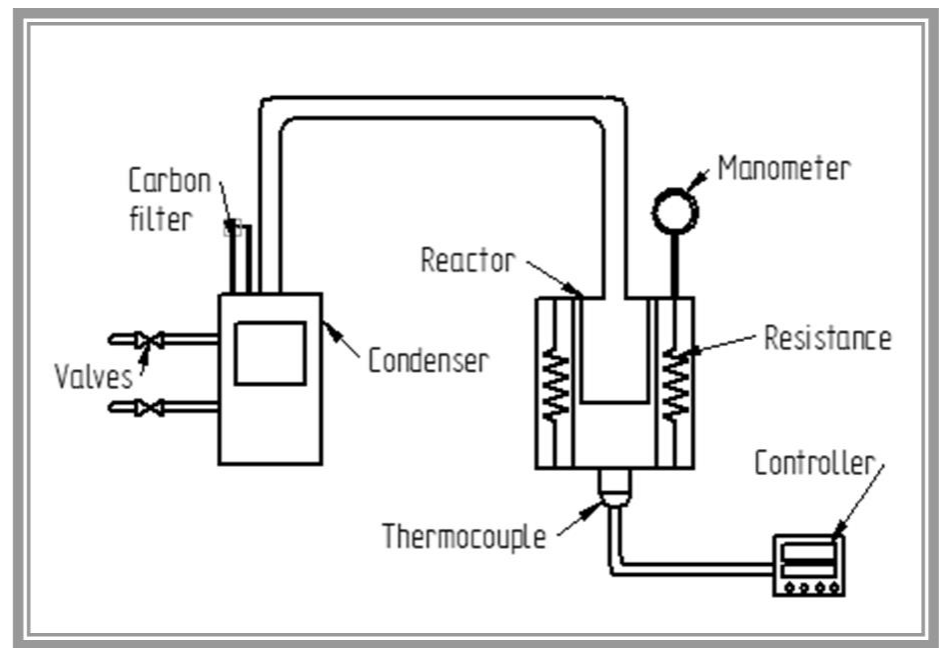

Figure 24 Schematic diagram of the pyrolysis apparatus. Source: Authors own creation.

\section{Experimental design}

The pyrolysis assays were carried out under a factorial design by duplicated. Two factors were evaluated: temperature $\left(300,400,500\right.$, and $\left.600{ }^{\circ} \mathrm{C}\right)$ and heating rates $\left(5,10\right.$ and $15^{\circ} \mathrm{C}$ $/ \mathrm{min})$; these conditions were selected according to the literature reviewed [26]. For each assay, samples of $200 \mathrm{~g}$ of banana powder were put inside the reactor and hermetically sealed, after the reactor reached the run temperature, samples were kept at a residence time of three hours. The heating rate was programmed with the control system. During residence time, condensable gaseous products were collected in the condenser for its quantification and analysis. Mass yield of the products was determined according to the quantity of product generated: char, bio-oil and gas.

\section{Products analysis}

Density analysis was evaluated to the liquid product of pyrolysis in a pycnometer, according to ASTM D 6822-12. The kinematic viscosity was developed at a temperature of $40{ }^{\circ} \mathrm{C}$ in a viscometer according to ASTM D-445-06. The chemical composition of the bio-oil collected from the condenser was characterized according to ASTM D2887-16a using a standard mixture of n-alkanes (ASTM D2887 - 12). The liquid was injected directly into Shimadzu GC 2010 chromatograph, equipped with a quadrupole mass spectrometer Shimadzu QP 2020 and a SH-Rxi-5 sil column MS, 30m L x 0.25 mm I.D. x $0.25 \mu \mathrm{m}$. Helium was used as a gas carrier at a flow rate of $1 \mathrm{~mL} / \mathrm{min}$ and injector temperature of $250^{\circ} \mathrm{C}$. The column conditions were at an initial temperature of $40^{\circ} \mathrm{C}$ for 10 minutes at $8{ }^{\circ} \mathrm{C} / \mathrm{min}$ until 
$250{ }^{\circ} \mathrm{C}$, then at $10{ }^{\circ} \mathrm{C} / \mathrm{min}$ until $300{ }^{\circ} \mathrm{C}$ for 10 minutes. The percentages compositions were determinate by an alkane standard Resket brand catalog number 560295, which contained the series listed for 27 hydrocarbons.

\section{RESULTS}

\section{Characterization of biomass}

The content of Moisture in banana peels samples was $44 \% \pm 31$ due to dry process, this content was reduced to $8.16 \% \pm 0.8$, which is similar to other studies that present moisture ranged between $6.7 \%$ to $11.6 \%$ [21]. This water content indicated that the feedstock is acceptable for pyrolysis experiments since biomass with higher moisture content requires more thermal energy to vaporize the contained water [21], and water could change the product obtained due to heating value decreases with moisture content [27]. The granulometric analysis of powder showed an average diameter of $2 \mathrm{~mm} \pm 0.5$. This size was selected because a large size represents decomposition delay in pyrolysis, known as the thermal resistance effect [28], that causes temperature gradients inside the particle. Meanwhile, a size particles from $0.4 \mathrm{~mm}$ to $2 \mathrm{~mm}$ do not influence in products obtained, presenting only small differences due to experimental errors [26]. The banana powder had hemicellulose, cellulose and lignin contents of $10.46 \%, 6.15 \%$ and $3.24 \%$, respectively. The content of hemicellulose and cellulose are lower than the presented in other studies which have contents of hemicellulose and cellulose of $41.38 \%$ and $9.9 \%$ [21];[29] respectively and can be attributed to the microbial action in the degradation of peels due to the exposed time to the environment [29]. Moreover, the lignin content (3.24\%) is also lower than other papers with contents of $17 \%$ in banana leaves and $8.9 \%$ in banana peels [21]. The ash content of $14.92 \%$ is high compared to content of $9.28 \%$ presented by [22], but it is not a problem as long as it remains below $20 \%$ [29]. Even this content can be an advantage for pyrolysis and for gasification [27]. The low content of sulfur $(0.16 \%)$ and nitrogen $(1.12 \%)$ are like the presented in literature, with content of $0.49 \%$ of sulfur and $0.98 \%$ of nitrogen [30] and are the expected since these compounds during combustion generate toxic gases for the environment [27] and are undesirable for a thermochemical conversion design [29]. The high content of carbon (38.54\%) and oxygen (40.13\%), were as expected and desire since they are the main compounds of fuels and influence the higher heating value [29] and are similar to the presented in literature with contents of $47.5 \%$ of carbon and $45.5 \%$ of oxygen [24]. 


\section{Thermogravimetric analysis}

Thermogravimetric analysis, presented in figure 2, showed the degradation of banana powder with three different heating rates, $5 \mathrm{~K} / \mathrm{min}, 10 \mathrm{~K} / \mathrm{min}$ and $15 \mathrm{~K} / \mathrm{min}$. From room temperature to $100{ }^{\circ} \mathrm{C}$ the sample presents a loss of weight of $12 \%$ due to evaporation of moisture. Also, the sample could lose formic acid and acetic acid from inside the particle [29]. The most important weight loss occurs between $200{ }^{\circ} \mathrm{C}$ and $400{ }^{\circ} \mathrm{C}$ with a mass loss from $85 \%$ to $45 \%$ that can be attributed to thermal degradation of volatile matter, including hemicelluloses, small lignin and cellulose fraction [29]. Therefore, from 400 to $500{ }^{\circ} \mathrm{C}$, occurs the complete loss of cellulose being similar at the three heating rates. Above $550^{\circ} \mathrm{C}$, the degradation of lignin is complete [11]. The samples at the three heating rates present up to $500{ }^{\circ} \mathrm{C}$ a similar performance. However, above $500{ }^{\circ} \mathrm{C}$ at $10 \mathrm{~K} / \mathrm{min}$, the degradation rate of biomass becomes relatively constant with the complete degradation of lignin. At the end of the degradation of the mass at heating rate of $5 \mathrm{~K} / \mathrm{min}$, there was $25 \%$ solid waste, at the end of $10 \mathrm{~K} / \mathrm{min}$ by $30 \%$ and the largest mass loss was at the $15 \mathrm{~K} / \mathrm{min}$ rate leaving $15 \%$ solid waste [31], which is a similar result compared to other studies that present that after $550{ }^{\circ} \mathrm{C}$ the weight loss occurs essentially in a range of $50 \%$ and $40 \%$ [23]. Experiment at 15 $\mathrm{K} / \mathrm{min}$ is the best condition for generating lower content of solid waste, and the quantity of solids is similar to ash content.

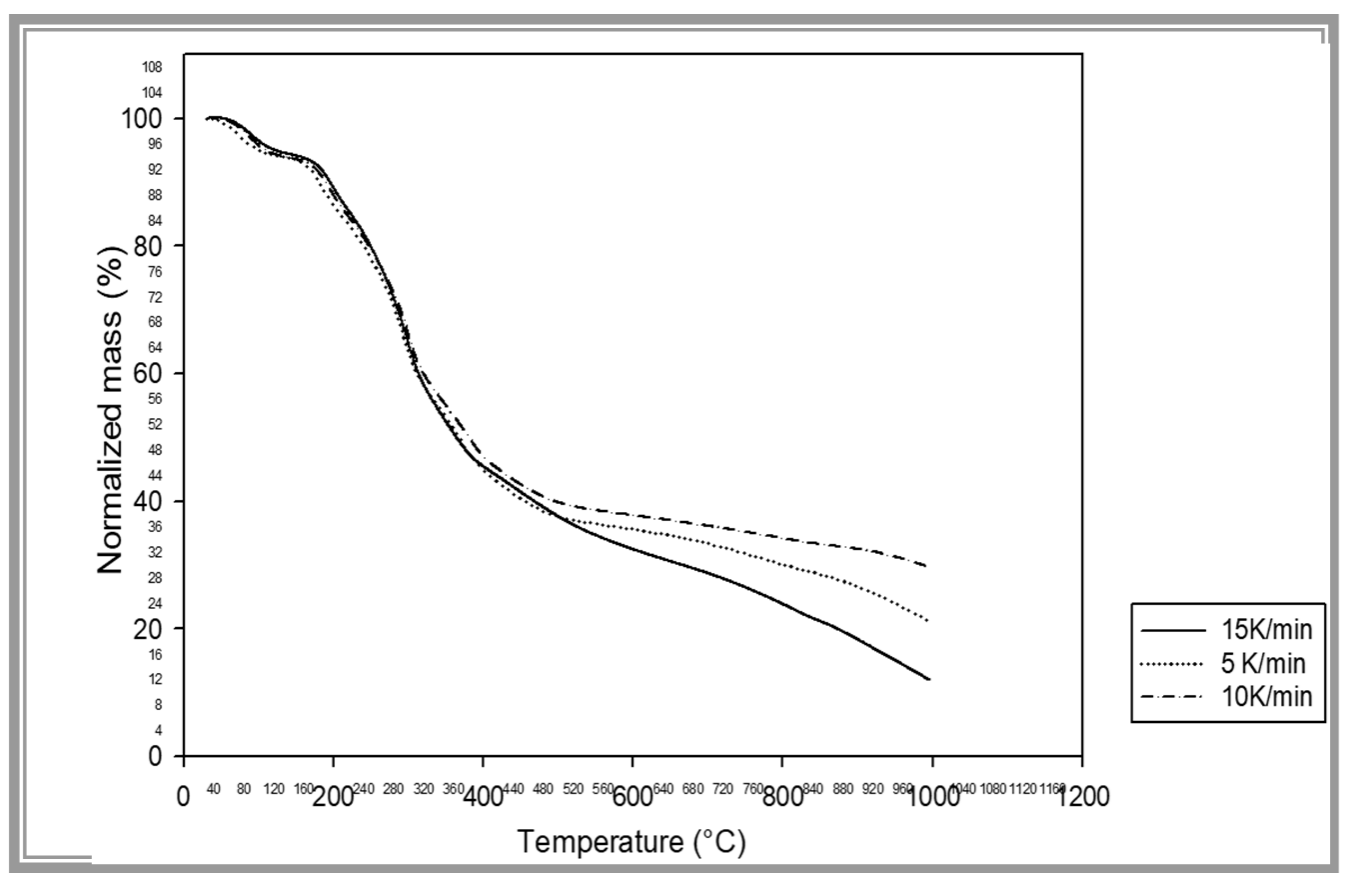

Figure 25 Thermal behavior of banana peels by TGA 


\section{Mass yield of pyrolysis products}

Table 1 show the yield of each product, char, bio-oil and gas, obtained at four different temperatures $\left(300,400,500\right.$ and $\left.600{ }^{\circ} \mathrm{C}\right)$ and three heating rates at $5{ }^{\circ} \mathrm{C} / \mathrm{min}, 10{ }^{\circ} \mathrm{C} / \mathrm{min}$ and $15{ }^{\circ} \mathrm{C} / \mathrm{min}$ in pyrolysis equipment. Most products corresponding to gas, and the bio-oil content is the lowest for all experiments. When the temperature increased, at the same heating rate, the gas percentage increased too. The best gas yield was obtained at $600{ }^{\circ} \mathrm{C}$ $(70.30 \%)$ at $5{ }^{\circ} \mathrm{C} / \mathrm{min}$, which is different to other studies with a gas yield of $35 \%$ at $700{ }^{\circ} \mathrm{C}$ and heating rate of $7^{\circ} \mathrm{C} / \mathrm{min}$ [23]. The best yield of bio-oil $(6.43 \%)$ was at $300{ }^{\circ} \mathrm{C}$ and 10 ${ }^{\circ} \mathrm{C} / \mathrm{min}$ of heating rate. However, the amount of bio-oil is low compared to other studies where bio-oil yield was $28.03 \%$ at $550{ }^{\circ} \mathrm{C}$ and heating rate of $7^{\circ} \mathrm{C} / \mathrm{min}$ [23], the low yield of bio-oil can be attributed for the lignocellulosic fraction; cellulose and hemicellulose are polysaccharides essential in ethanol production [21], for that, it can be assumed that those contents were the reason for the low yield of bio-oil. The amount of char, for all experiments, was higher than the ash content reported, indicating that increasing the temperature up to $700{ }^{\circ} \mathrm{C}$ would reduce this content. Differences between this paper and other reports consulted are due to the amount of feedstock and the design of the pyrolysis equipment [22], so the scale-up of the pyrolysis process it is difficult for the variability of the results.

Table 11. Mass yield of pyrolysis

\begin{tabular}{|c|c|c|c|c|}
\hline Heating rate & Temperature $\left({ }^{\circ} \mathrm{C}\right)$ & Yield of char & Yield of bio-oil & Yield of gas \\
\hline \multirow{4}{*}{$5{ }^{\circ} \mathrm{C} / \mathrm{min}$} & 300 & $33.11 \pm 5.34$ & $6.13 \pm 9.83$ & $60.76 \pm 15.17$ \\
\cline { 2 - 5 } & 400 & $33.37 \pm 0.63$ & $4.89 \pm 3.67$ & $61.72 \pm 3.03$ \\
\cline { 2 - 5 } & 500 & $33.08 \pm 5.03$ & $4.75 \pm 1.69$ & $62.17 \pm 6.73$ \\
\hline & 600 & $26.38 \pm 13.90$ & $3.31 \pm 0.03$ & $70.30 \pm 13.87$ \\
\hline \multirow{4}{*}{$10^{\circ} \mathrm{C} / \mathrm{min}$} & 300 & $31.48 \pm 11.77$ & $6.43 \pm 5.69$ & $60.07 \pm 17.47$ \\
\cline { 2 - 5 } & 400 & $36.00 \pm 0.56$ & $4.53 \pm 4.89$ & $59.47 \pm 4.32$ \\
\cline { 2 - 5 } & 500 & $28.17 \pm 19.29$ & $4.15 \pm 8.52$ & $64.55 \pm 15.54$ \\
\hline \multirow{3}{*}{$15^{\circ} \mathrm{C} / \mathrm{min}$} & 600 & $32.77 \pm 1.48$ & $4.57 \pm 3.32$ & $67.24 \pm 22.62$ \\
\cline { 2 - 5 } & 300 & $35.25 \pm 7.37$ & $6.02 \pm 1.53$ & $61.00 \pm 0.10$ \\
\cline { 2 - 5 } & 400 & $40.55 \pm 22.77$ & $3.02 \pm 1.49$ & $61.70 \pm 8.90$ \\
\hline & 500 & $34.15 \pm 0.54$ & $3.76 \pm 1.02$ & $55.70 \pm 23.80$ \\
\hline
\end{tabular}

Source: Authors own creation.

\section{Products characteristics and properties}

Bio-oil obtained during pyrolysis experiments had a viscosity of $0.03 \pm 0.011 \mathrm{~Pa} \bullet \mathrm{s}$ determined at $40{ }^{\circ} \mathrm{C}$, according to ASTM D-445-06. Samples present a density of $1118 \pm 98$ 
$\mathrm{kg} / \mathrm{m} 3$ measured at $16^{\circ} \mathrm{C}$. Other studies about products obtained from biomass pyrolysis, as sawdust and wheat straw, present similar results even though they are different feedstock; density is within the same range with variation from 918 to $1239 \mathrm{~kg} / \mathrm{m} 3$ [32].

The chemical composition of bio-oil was determined by GC-MS analysis, and this analysis showed a mixture of linear alkanes (C7 - C33), as shown in Figure 3. The compounds were grouped in low molecular weight (LMW; C7 - C11), medium molecular weight (MMW; C12 - C22), and high molecular weight (HMW; C23 - C33). The best conditions corresponding to $5^{\circ} \mathrm{C} / \mathrm{min}$ and $300^{\circ} \mathrm{C}$, in which the percent of pentadecane $(\mathrm{C} 15 \mathrm{H} 32)$ and decane $(\mathrm{C} 10 \mathrm{H} 22)$ were important with a concentration of $30.018 \mathrm{ppm}$ and $24.699 \mathrm{ppm}$, respectively. The experiments that present the less concentration of alkanes were at a heating rate of $10^{\circ} \mathrm{C} / \mathrm{min}$ at $500{ }^{\circ} \mathrm{C}$, where the only significant concentration was of pentadecane $(\mathrm{C} 15 \mathrm{H} 32)$ with 3.322 ppm. The heating rate is an important factor for concentration since concentrations at 5 ${ }^{\circ} \mathrm{C} / \mathrm{min}$ are better than the presented at a heating rate of 10 and $15^{\circ} \mathrm{C} / \mathrm{min}$, the highest peaks occur equally in the LMW and MMW sectors. Lower temperature ranges have a higher concentration. These results have shown that banana peels could be as raw material for solvents, fuels or bioenergy production [33].

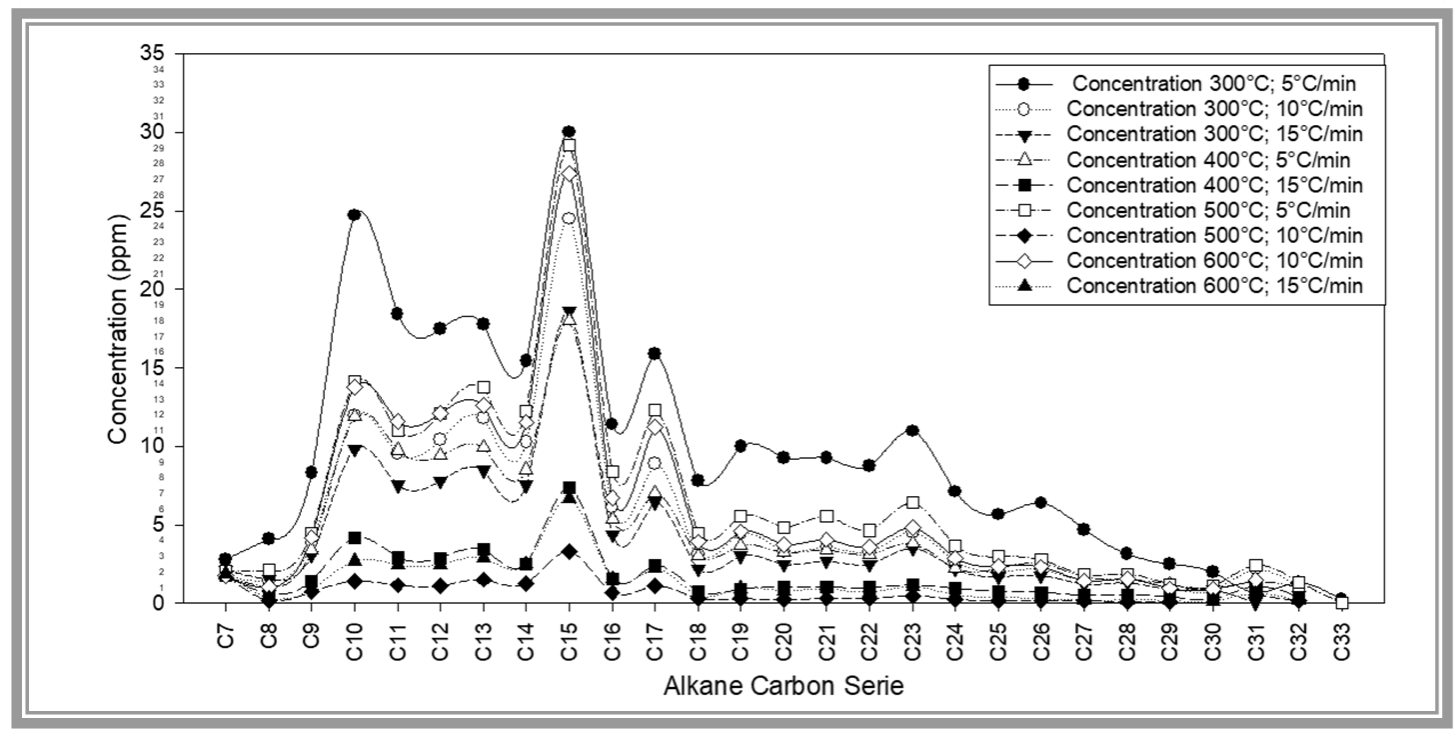

Figure 26 Chromatogram of bio-oil. Source: Authors 


\section{CONCLUSIONS}

Banana peels presented a high content of oxygen, carbon and low content of sulfur and nitrogen, indicating the potential as a feedstock for conversion to fuel by pyrolysis. Results showed that temperature and heating rate influenced gas, liquid, and char products. The best gas yield was at $600{ }^{\circ} \mathrm{C}$ and $5{ }^{\circ} \mathrm{C} / \mathrm{min}$, while the bio-char yield was at $400{ }^{\circ} \mathrm{C}$ and 10 ${ }^{\circ} \mathrm{C} / \mathrm{min}$. However, the bio-oil yield in each test presented lower data than the presented in other studies, due to the low content of cellulose and hemicellulose obtained in the feedstock. The chemical compounds found in bio-oil with the highest concentration were Pentadecane $(\mathrm{C} 15 \mathrm{H} 32)$ and Decane $(\mathrm{C} 10 \mathrm{H} 22)$, which are components for industrial purposes. This result confirms that banana peels could be used as solvents and fuel production. Therefore, for future investigations, it is expected to identify more compounds of the bio-oil obtained.

\section{REFERENCES}

[1] N. Ashikin, B. Mabahwi, O. Ling, H. Leh, and D. Omar, "Human Health and Wellbeing: Human health effect of air pollution," Procedia - Soc. Behav. Sci., vol. 153, pp. 221-229, 2014.

[2] J. S. Gaffney and N. A. Marley, "The impacts of combustion emissions on air quality and climate From coal to biofuels and beyons," Atmos. Environ., vol. 43, no. 1, pp. 23-36, 2009.

[3] P. McKendry, "Energy production from biomass (part 1): overview of biomass," Bioresour. Technol., vol. 83, no. 1, pp. 37-46, 2002.

[4] X. Shi, X. Pang, Y. Mu, H. He, and S. Shuai, "Emission reduction potential of using ethanol biodiesel - diesel fuel blend on a heavy-duty diesel engine," Atmos. Environ., vol. 40, pp. 2567-2574, 2006.

[5] C. Bonechi et al., "Biomass: An overview," in Bioenergy Systems for the Future, 2017, p. 595.

[6] C. T. Wright, R. D. Boardman, N. A. Yancey, and S. Sokhansanj, "A Review on Biomass Classification and Composition , Co-Firing Issues and Pretreatment Methods," 2011.

[7] E. Cohen and G. Yom Din, "Agricultural Waste Management: Case Study of a Waste Treatment Plant for Animal Manure," Electron. J., pp. 1-17, 2010.

[8] Food and Agriculture Organization of the United Nations, "Banana facts and figures," 2019.

[9] M. Mazzeo M., L. León Agatón, L. F. Mejía Gutierrez, L. E. Guerrero Mendieta, and J. D. Botero López, “Aprovechamiento industrial de residuos de cosecha y poscosecha del plátano en el departamento de Caldas," Rev. Educ. en Ing., vol. 9, pp. 128-139, 2010.

[10] P. Kumar and L. Joshi, "Pollution Caused by Agricultural Waste Burning and Possible Alternate Uses of Crop Stubble: A Case Study of Punjab," in Knowledge Systems of Societies for Adaptation and Mitigation of Impacts of Climate Change, 2013, pp. 367-385.

[11] N. Sellin, D. Ricardo, C. Marangoni, and O. Souza, "Oxidative fast pyrolysis of banana leaves in $\mathrm{fl}$ uidized bed reactor," Renew. Energy, vol. 96, pp. 56-64, 2016.

[12] D. Mohan, C. U. Pittman Jr, and P. H. Steele, "Pyrolysis of Wood/Biomass for Bio-oil: A Critical Review," Energy fuels, vol. 20, no. 3, pp. 848-889, 2006.

[13] T. Kan, V. Strezov, and T. J. Evans, "Lignocellulosic biomass pyrolysis: A review of product properties and effects of pyrolysis parameters," Renew. Sustain. Energy Rev., vol. 57, pp. 1126-1140, 2016.

[14] Z. Gong, P. Fang, Z. Wang, X. Li, Z. Wang, and F. Meng, "Pyrolysis characteristics and products distribution of haematococcus pluvialis microalgae and its extraction residue," Renew. Energy, vol. 146, pp. 2134-2141, 2019.

[15] A. V. Bridgwater and G. V. C. Peacocke, "Fast pyrolysis processes for biomass," Renew. Sustain. energy Rev., vol. 4, no. 1, pp. 1-73, 2000.

[16] A. Heidari, R. Stahl, H. Younesi, A. Rashidi, N. Troeger, and A. Asghar, "Effect of process 
conditions on product yield and composition of fast pyrolysis of Eucalyptus grandis in fluidized bed reactor," J. Ind. Eng. Chem., vol. 20, no. 4, pp. 2594-2602, 2014.

[17] P. Arias, C. Dankers, P. Liu, and P. Pilkauskas, LA ECONOMÍA MUNDIAL DEL BANANO 1985-2002. 2005.

[18] M. Soto, "Situación y avances tecnologicos en la producción bananera mundial," Rev. Bras. Fruct., vol. 33, no. spe1, pp. 13-28, 2011.

[19] J. Marín, R. García, and S. Barrezueta-Unda, "Elaboracion de biocarbón obtenido a partir de la cáscara del cacao y raquis del banano," Rev. Científica Agroecosistemas, vol. 6, no. 3, pp. 75-81, 2018.

[20] N. I. Giraldo Cano and N. Montoya Martínez, "Caracterización de residuos de banano (pseudotallo y hojas) mediante análisis termogravimétrico para uso potencial como biocombustible sólido," Universidad de Medellín, 2015.

[21] I. Kabenge, G. Omulo, N. Banadda, J. Seay, A. Zziwa, and N. Kiggundu, "Characterization of Banana Peels Wastes as Potential Slow Pyrolysis Feedstock," Sustain. Dev., vol. 11, no. 2, pp. 14-24, 2018.

[22] G. Omulo, N. Banadda, I. Kabenge, and J. Seay, "Optimizing slow pyrolysis of banana peels wastes using response surface methodology," Environ, vol. 24, no. 2, pp. 354-361, 2019.

[23] N. Ozbay, A. S. Yargic, R. Zerrin, Y. Sahin, and E. Yaman, "Valorization of banana peel waste via insitu catalytic pyrolysis using Al-Modified SBA-15," Renew. Energy, vol. 140, pp. 633-646, 2019.

[24] S. Shiung et al., "Pyrolysis production of fruit peel biochar for potential use in treatment of palm oil mill ef fl uent," J. Environ. Manage., vol. 213, pp. 400-408, 2018.

[25] A. Demirbas, "Pyrolysis of municipal plastic wastes for recovery of gasoline-range hydrocarbons," J. Anal. Appl. Pyrolysis, vol. 72, no. 1, pp. 97-102, Aug. 2004.

[26] P. Taylor, Y. Niu, H. Tan, Y. Liu, X. Wang, and T. $\mathrm{Xu}$, "The Effect of Particle Size and Heating Rate on Pyrolysis of Waste Capsicum Stalks Biomass The Effect of Particle Size and Heating Rate on Pyrolysis of Waste Capsicum Stalks Biomass," Energy Sources, pp. 1663-1669, 2014.

[27] R. García, C. Pizarro, A. G. Lavín, and J. L. Bueno, "Characterization of Spanish biomass wastes for energy use," Bioresour. Technol., vol. 103, pp. 249258, 2012

[28] V. Seebauer, J. Petek, and G. Staudinger, "Effects of particle size, heating rate and pressure on measurement of pyrolysis kinetics by thermogravimetric analysis," Fuel, vol. 76, no. 13, pp. 1277-1282, 1997.

[29] E. R. K. Fernandes, C. Marangoni, O. Souza, and N. Sellin, "Thermochemical characterization of banana leaves as a potential energy source," Energy Convers. Manag., vol. 15, pp. 603-608, 2013.

[30] N. Sellin, B. Goulart, D. O. Maia, C. Marangoni, A. Pedro, and N. Oliveira, "Use of Banana Culture Waste to Produce Briquettes," Chem. Eng. Trans., vol. 32, pp. 349-354, 2013.

[31] H. Yang, R. Yan, H. Chen, D. Ho Lee, and C. Zheng "Characteristics of hemicellulose, cellulose and lignin pyrolysis," Fuel, vol. 86, pp. 1781-1788, 2007.

[32] R. Azargohar, K. L. Jacobson, E. E. Powell, and A K. Dalai, "Evaluation of properties of fast pyrolysis products obtained, from Canadian waste biomass," J. Anal. Appl. Pyrolysis, vol. 104, pp. 330340, 2013

[33] M. Hussain, Z. Zhao, J. Ren, T. Rasool, and S. Raza, "Thermo-kinetics and gaseous product analysis of banana peel pyrolysis for its bioenergy potential," Biomass and Bioenergy, vol. 122, pp. 193-201, 2019. 


\title{
EVALUATION OF BIODIESEL PRODUCTION IN A HYDRODYNAMIC CAVITATION REACTOR FROM WASTE COOKING OIL
}

\section{Evaluación de la producción de biodiesel en un reactor de cavitación hidrodinámica a partir de aceite residual de cocina}

\author{
Contreras, Daniel1'; Gómez, Luis²; Malagón-Romero, Dionisio ${ }^{3}$. \\ Universidad Santo Tomás
}

\begin{abstract}
Production of biodiesel from waste cooking oil (WCO) is obtained by transesterification reaction. Traditional process is carried out in an agitated reactor at $65^{\circ} \mathrm{C}$ during 2 hours. One alternative for improving is using a hydrodynamic cavitation reactor. In this paper, response surface methodology (RSM) was used for searching the best experimental conditions for biodiesel production. Two response surfaces were adjusted: yield (biodiesel mass to oil mass) and energy consumed per kilogram of biodiesel produced (ECPKB). Experimental variables were: the methanol-oil molar ratio $(6: 1-5: 1-4: 1)$ and the potassium hydroxide catalyst percentage $(1 \%$ and $0.5 \%$ ). The biodiesel was analyzed through a gas chromatography, a cetane index and heat value laboratory tests according to ASTM D6751 standard. Results exhibit methyl esters above 98\%, a heat of combustion of $39.52 \mathrm{MJ} / \mathrm{kg}$ and a cetane index of 55.3. The optimal conditions for the hydrodynamic cavitation process were selected as 5:1 $\mathrm{MR}$ and $0.5 \% \mathrm{KOH}$. These conditions lead to the highest yield of 1.07 and $74.07 \mathrm{~kJ} / \mathrm{kg}$ of ECPKB, respectively. Additionally, the reaction time was less than 10 minutes for all conditions. These results showed it's viable to implement this process in an industrial level as an alternative to transesterification with less production times and energy consumption.
\end{abstract}

Key words: biodiesel, cavitation hydrodynamic, waste cooking oil, biofuels production, response surface methodology.

Resumen

${ }^{I}$ Corresponding E-mail: danielcontreraso@ usantotomas.edu.co

${ }^{2}$ Corresponding E-mail: luisgomezm@usantotomas.edu.co

${ }^{3}$ Corresponding E-mail: dionisiomalagon@usantotomas.edu.co 
La producción de biodiesel a partir de aceite de cocina residual (WCO) es obtenida mediante la reacción de transesterificación. El proceso tradicional se lleva a cabo en un reactor agitado a $65^{\circ}$ $\mathrm{C}$ durante 2 horas. Una alternativa para mejorar es usar un reactor de cavitación hidrodinámica. En este documento, se utilizó la metodología de superficie de respuesta (RSM) para buscar las mejores condiciones experimentales para la producción de biodiesel. Se ajustaron dos superficies de respuesta: rendimiento (masa de biodiesel a masa de aceite) y energía consumida por kilogramo de biodiesel producido (ECPKB). Las variables experimentales fueron: la relación molar metanol-aceite (6: 1 - 5: 1 - 4: 1) y el porcentaje de catalizador de hidróxido de potasio (1\% y $0,5 \%)$. El biodiesel se analizó mediante una cromatografía de gases, índice de cetano y pruebas de laboratorio de poder calorífico acorde al estándar ASTM D6751. Los resultados muestran ésteres metílicos superiores al 98\%, un calor de combustión de 39,52 MJ / kg y un índice de cetano de 55,3. Las condiciones óptimas para el proceso de cavitación hidrodinámica se seleccionaron como 5: $1 \mathrm{MR}$ y $0.5 \% \mathrm{KOH}$.

Estas condiciones conducen al mayor rendimiento de 1.07 y $74.07 \mathrm{~kJ} / \mathrm{kg}$ de ECPKB, respectivamente. Además, el tiempo de reacción fue inferior a 10 minutos para todas las condiciones. Estos resultados mostraron que es viable implementar este proceso a nivel industrial como alternativa a la transesterificación con menos tiempos de producción y consumo de energía

Palabras clave: biodiesel, cavitación hidrodinámica, aceite residual de cocina, producción de biocombustibles, metodología de superficie de respuesta.

\section{INTRODUCTION}

The energetic needs of the world mostly rely on production and extraction of fossil fuels, natural gas and coal [1]. Currently the world is facing two challenges: reducing the petroleum dependency and controlling contamination rates [2]. Due to these, the duty of environmental protection and expansion of alternative fuel resources arises [3]. Increasing demand for unconventional energetic resources has caused that the implementation of biofuels, such as biodiesel, gains popularity mainly because of its cleanliness and the fact that it can be produced out of domestic and renewable resources [4].

Conventional biodiesel is a fuel obtained by transesterification, which is the conversion of triglycerides coming from animal fats and vegetable oils to fatty acid methyl esters [5], this method requires a heating and then a mixing step of the blend between the fat or oil, the methanol and the catalyst, this whole process spends between two to three hours [6].

Biodiesel has proven to be environmentally friendly because it produces $83 \%$ less greenhouse 
gasses presenting itself as a great and viable alternative with similar characteristics to fossil fuels [4]. This is one of the main reasons why there's a need to explore non-conventional biodiesel production methods such as cavitation.

Cavitation is a phenomenon characterized by the formation of tiny steam "bubbles" in the flow or in the solid surfaces in contact with said flow [7]. The most common physical requirement for cavitation is that the absolute pressure of the fluid drops below the value of vapor pressure at the current temperature [8]. This could be used to increase turbulence during a chemical reaction in a hydrodynamic equipment [9]. This instrument uses a reactor which induces the ideal conditions for cavitation with the objective of generating a chemical reaction between the reactants [10]. There are two configurations of hydrodynamic cavitation reactors, sonochemical reactors, and hydrodynamic cavitation reactors; Of these two categories, the first has four different configurations of its reactors, and the last one has only developed orifice plate reactors to generate the phenomenon [11]. From the first reactors, yields around $98 \%$ have been obtained, in a time of $25 \mathrm{~min}$ in the conversion of WCO, but with high energy consumption [12]. On the other hand, hydrodynamic reactors obtain almost equal yields, $96 \%$, with less energy consumption under the same conditions and times less than $15 \mathrm{~min}$. [13]. The most common configuration consists of a pump that pushes the flow through a nozzle-orifice plate configuration where cavitation takes place [14], so changes in the normal flow conditions generate high speeds and shear flows resulting in cavitation's aggressive and fast instigation [15]. Application of hydrodynamic technology decreases reaction time and can be easily scaled up to meet the industrial demands when compared with other cavitation methods [16].

The purpose of alternative methods like the previously mentioned hydrodynamic cavitation is to optimize production by eliminating the heating and mixing steps [17]. Chitsaz et al (2018) produced biodiesel through hydrodynamic cavitation, reaching an optimal point of production with a molar ratio of $6: 1,1.1 \%$ of $\mathrm{KOH}$, a pressure of 3.27 bar and a reaction time of 8 minutes. Chuah et al (2017) obtained in 15 minutes a conversion rate of $98.1 \%$ in an orifice plate hydrodynamic cavitation reactor with $6: 1$ molar ratio, $1 \% \mathrm{KOH}, 60^{\circ} \mathrm{C}$ of reaction temperature and an inlet pressure of 2 bar.

The main objective of this article is to evaluate the production of biodiesel in a hydrodynamic cavitation reactor from collected WCO. This evaluation should lead to the optimal conditions for biodiesel production by the means of a RSM. 


\section{MATERIALS AND METHODS}

\section{Materials}

140 liters of WCO were collected in various fast food restaurants in Bogotá (Colombia) for the purposes of this study. Methanol (99\% purity, Panreac, Spain) was utilized as the alcohol for the reaction. Potassium hydroxide (85\%, Panreac, Spain) was used as the alkali catalyst.

Also for the WCO's characterization were implemented phenolphthalein $(99.5 \%$, Bioquigen, Colombia), potassium iodide (99\%, Panreac, Spain), starch from potato soluble (Panreac, Spain), chloroform (Chemi, Colombia), sodium thiofulsate (99.5\%, Bioquigen, Colombia), Wijs' reagent (Panreac, Spain) and Ethanol (99.5\%, Panreac, Spain).

\section{Filtration and characterization of WCO}

To remove particulate matter and impurities that might affect the experiment, the WCO was filtrated using a grade $3 \mathrm{hw}$ filter paper (Boeco, Germany) and was later stored in plastic containers at room temperature $\left(13^{\circ} \mathrm{C}\right)$. Density (ASTM D-5), acidity index (ASTM D-1980), iodine index (ASTM D-5554), saponification index (ASTM D-5558) and peroxide number (ASTM D-1832) where determined according to the mentioned standards. Also, viscosity was reported at a temperature of $40^{\circ} \mathrm{C}$ using a Cannon viscometer LV model 2020.

\section{Test and reaction system}

Figure 1 shows the setup of the reactor in which the production process was carried out. This reactor was designed by Santo Tomás University (Bogotá, Colombia) and is composed of a 5 liter tank (A), an IHM JET1100G1 pump (B), a LFM SM6004 flow meter - Thermocouple (C), two gate valves $(D)$ and $(H)$, two pressure gauges $(E)$ and $(G)$, a reactor $(F)$ constituted by a $171.5 \mathrm{~mm}$ diameter orifice plate connected to a nozzle and a diffuser and a needle valve (I). The whole reactor has a capacity of 8 liters and was built with 1-inch diameter pipes of SCH 40 INOX 304 steel. The blend of $\mathrm{WCO}$ and methanol with the $\mathrm{KOH}$ already diluted were poured in (A), (A) is then sealed and (B) starts working. (B) Pushes the fluid through (C) which measures flow and temperature; then, the current goes into $(\mathrm{D})$ which along with $(\mathrm{H})$ set the working pressure. Before and after the reactor pressure was measured by the means of $(\mathrm{E})$ and $(\mathrm{G})$. As the flow goes through 
(F) transesterification produced by cavitation begins. Finally, the flowing substance goes back to (A) and recirculates across the setup until predefined time is reached. (I) has the purpose of emptying the machine as well as taking samples when required.

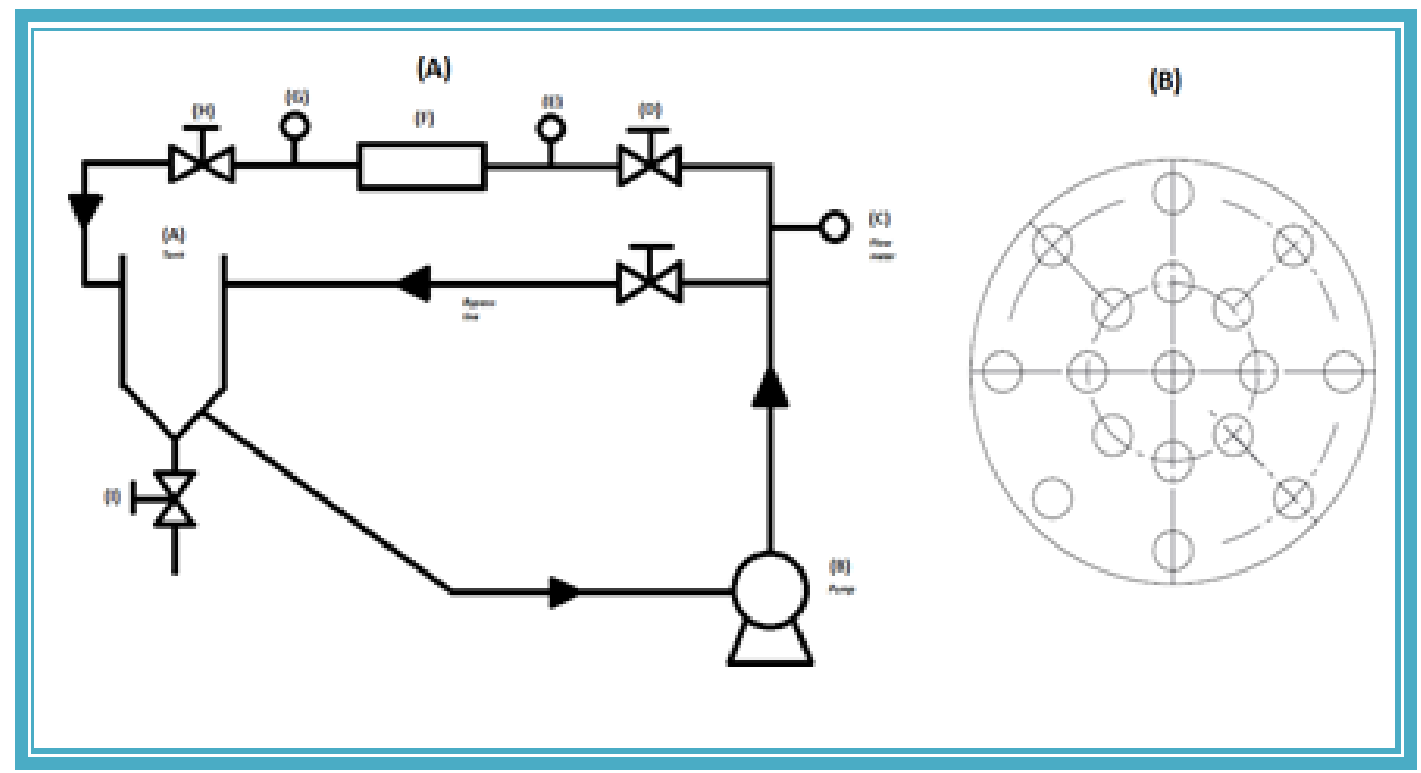

Figure 27 (A) The setup of the hydrodynamic cavitation reactor. (B) The orifice-plate configuration that was used in the reactor. Source: Authors own creation.

\section{Design of experiments}

The experiment was built around the goal of determining the optimal conditions for ECPKB (Energy Consumed per Kilogram of Biodiesel) and yield in biodiesel production by a hydrodynamic cavitation process. Variables were potassium hydroxide concentration (1\% and $0.5 \%)$ and methanol to WCO molar ratio (6:1, 5:1 and 4:1). Difference between input and output pressures of the reactor was maintained at 2 bar according to consulted literature [10]. Each experiment was carried out for 6 liters of WCO. $100 \mathrm{ml}$ samples of the product were collected at time 0 and every 5 minutes for 30 minutes for subsequent separation with a decantation funnel. Three phases were obtained into decantation funnel: Glycerol phase (at the bottom layer), biodiesel phase (intermediate layer) and oil phase (at the top layer). The biodiesel phase was collected and then was washed three times with distilled water until $\mathrm{pH}$ neutral. This process allowed us to eliminate glycerol, methanol and excess catalyst. Temperature, flow, pressure in gauge 1, pressure in gauge 2, voltage and electric current were monitored. The RSM analysis 
was conducted for two output variables: ECPKB and Yield (biodiesel mass to oil mass ratio) [20]. Input variables were molar ratio and $\mathrm{KOH}$ concentration. The data was adjusted to a quadratic model with the free trial of the Minitab software. This, with the goal of finding a surface that best suits the production process and shows the optimal point of production in terms of MR (molar ratio) and $\mathrm{KOH} \%$. The adjusted second order model was:

$$
\eta=\beta_{0}+\beta_{1} x_{1}+\beta_{2} x_{2}+\beta_{3} x_{1}^{2}+\beta_{4} x_{2}^{2}+\beta_{5} x_{1} x_{2}
$$

Where $\eta$ is the output variable, $x 1$ is the molar relation and $x 2$ is the catalyst concentration.

\section{Characterization of the biodiesel}

This test was performed under the ASTM D240 standard and with the following materials: an IKA C 2000 Basic S1 calorimeter and a Mettler-Toledo AB 204 (Switzerland) analytic balance employed in isoperbolic mode, extra dry industrial oxygen grade 2.7 at 30 bars. A Julabo F12 Thermostat at $25^{\circ} \mathrm{C}$ controlled the calorimeter jacket's temperature. As instructed in ASTM D 240 sulfur content was determined with a Spectronic Genesys 5 spectrophometer (Thermo Scientific, Masachusetts, USA) to make the necessary corrections. The gas chromatography was performed with Agilent 6820 (Agilent technologies, China) equipped with a SGE capillary column SGE 12m $X 0.53 \mathrm{~mm} \times 0.15 \mu \mathrm{m}$ and a flame ionization detector (FID). After the $1 \mu \mathrm{l}$ is injected, the oven keeps a $120^{\circ} \mathrm{C}$ temperature for 1 minute and then heats at a rate of $2.25^{\circ} \mathrm{C} / \mathrm{min}$ until a $380^{\circ} \mathrm{C}$ temperature is reached. Injection port and detector temperature was $259^{\circ} \mathrm{C}$. Nitrogen with a flow rate of $6 \mathrm{ml} / \mathrm{s}$ was utilized as the drag gas. The corresponding Cerity QA/QC (Agilent technologies, Germany) software acquired and processed all the data. This test was performed under the ASTM D4737 using the following equipment: Precision PS Scientific Series $10 Z 9$ (Chicago, USA), a CASIO (China) chronometer and a Silver Brand (USA) thermometer.

\section{RESULTS}

\section{Oil characterization}

Results for oil characterization are shown in Table 1. WCO's obtained density, saponification value and viscosity are close to the ones reported in previous WCO characterizations [21]. However high iodine number's value (96.18) points out a great content of carbon-carbon double 
bonds due to the high amount of unsaturated acids in the WCO, producing high reactivity [22]. Acid value was significantly higher than the ones reported by López (2015) and Rodriguez (2017) (approximately 1.0\%), representing a high content of free fatty acids in the WCO used in this investigation and also indicating a high degree of re-usage [20]. Oil's exposure to humidity, high temperatures and oxygen for long periods of time in addition to hydrolysis causes the liberation of free fatty acids, leading then to a restrain in the biodiesel's production process when using an alkaline catalyst [24]. On the other hand, as the acid value is higher than $1 \%$ the reaction should usually be performed in two stages, nevertheless as the mixture's MR is low on WCO and free fatty acids it is possible to perform the process without a neutralization stage [25].

Table 12 Results for WCO's characterization.

\begin{tabular}{|l|l|}
\hline Property & $\begin{array}{l}\text { Waste cooking } \\
\text { oil }\end{array}$ \\
\hline Density $(\mathrm{g} / \mathrm{ml})$ & $0.931 \pm 0.02$ \\
\hline Acid value & $2.11 \pm 0.2$ \\
\hline Iodine $(\mathrm{g} / 100 \mathrm{~g}$ of substance) & $96.18 \pm 1.35$ \\
\hline Saponification $(\mathrm{mg}$ of $\mathrm{KOH} / \mathrm{g})$ & $208.21 \pm 1.07$ \\
\hline Humidity percentage $(\%)$ & 0.2 \\
\hline Viscosity $(\mathrm{cP})$ & $56.4 \pm 0.1$ \\
\hline
\end{tabular}

Source: Authors

\section{Response surface methodology (RSM) for biodiesel production}

According to the obtained experimental results, it was determined that all analysis will be conducted on samples taken ten minutes into the production process because it was noted that by this time the mixture has fully reacted (data not shown). These results are similar to other reports where reaction times for hydrodynamic cavitation production using WCO were between 8 and 15 minutes [18] [10] [19]. Two response surface models (Figure 2) were developed for ECPKB and yield. The yield surface was adjusted to the model: YIELD $=1.053+0.093 \mathrm{MR}-0.291 \mathrm{KOH}-0.0135$ $M R 2+0.0178 \mathrm{MR} * \mathrm{KOH}$ with $\mathrm{R} 2=0.96$ and a $\mathrm{p}$-value $=0.295$. The $\mathrm{ECPKB}$ was adjusted to: $\mathrm{ECPKB}$ $=167.4-32.4 \mathrm{MR}-64.9 \mathrm{KOH}+2.66 \mathrm{MR} 2+13.85 \mathrm{MR} * \mathrm{KOH}$ with $\mathrm{R} 2=0.985$ and a $\mathrm{p}$-value $=0.183$. RSM models show a desirable correlation between the dependent and independent variables and a non-significant lack of fit, meaning that the model fulfills the acceptance criteria [26]. In accordance with the RSM, yield has an inversely proportional relation with $\mathrm{MR}$ and $\mathrm{KOH}$ while 
ECPKB has a directly proportional relationship with both parameters.

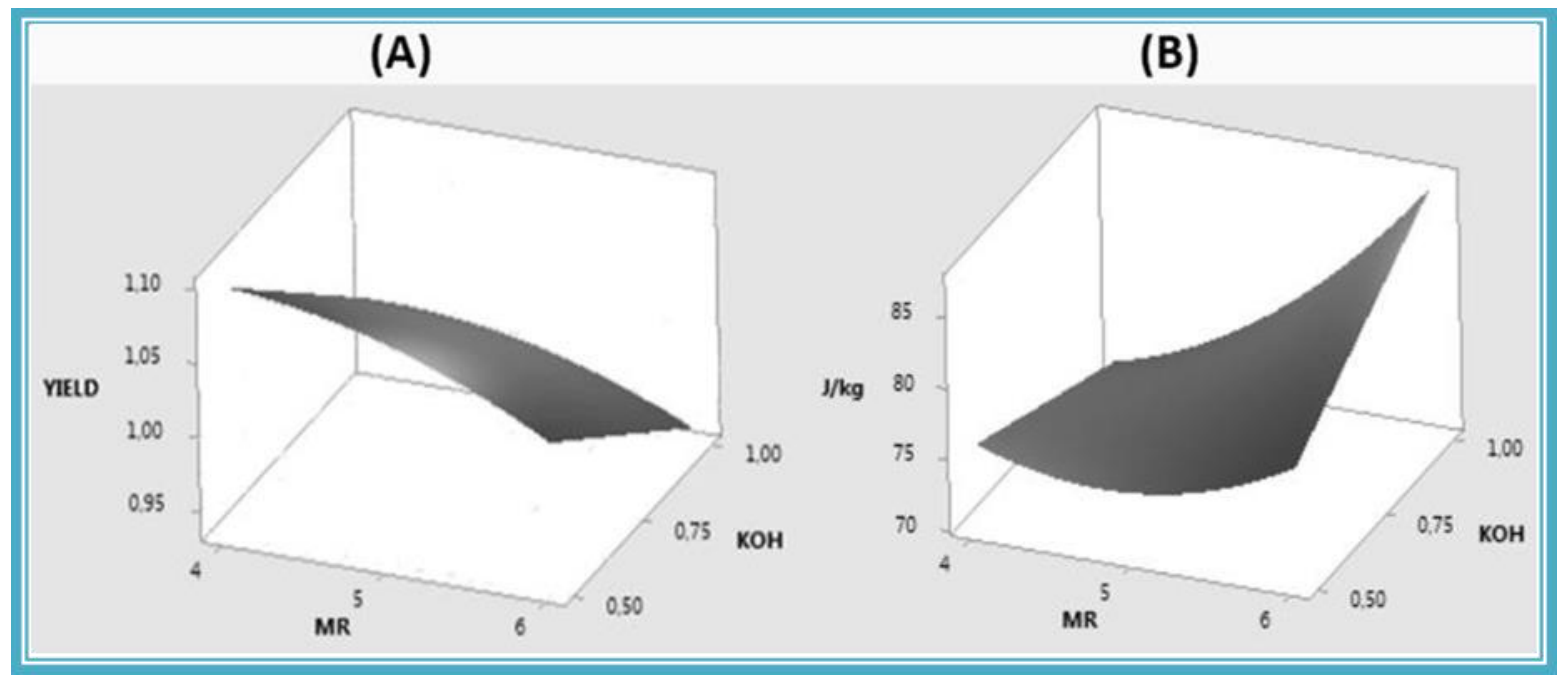

Figure 28 (A) Surface plot obtained by RSM of MR and KOH versus YIELD. (B) Surface plot obtained by RSM of $M R$ and $K O H$ vs ECPKB. Source: Authors own creation.

In compliance with the adjusted models, an optimization for maximizing yield and minimizing ECPKB was developed resulting in (MR 4.5:1 and $0.5 \% \mathrm{KOH})$ as conditions for expecting values of 1.09 for yield and $74.33 \mathrm{~kJ} / \mathrm{kg}$ for ECPKB. Gogate (2008) achieved an experimental value of 296 $\mathrm{kJ} / \mathrm{kg}$ ECPKB and a yield of 0.98 with a reaction time of 15 min under the following conditions: 4:4 ratio (w/v) of oil to alcohol, $1 \%$ of $\mathrm{NaOH}$ and a plate with 16 orifices, each with a diameter of $2 \mathrm{~mm}$. Chuah (2015) reported $800 \mathrm{~kJ} / \mathrm{kg}$ of ECPKB, 0.984 yield and a reaction time of 15 min under the following conditions: $\mathrm{MR}$ of $6: 1,1 \%$ of $\mathrm{KOH}$, an inlet pressure of 2 bar and $60^{\circ} \mathrm{C}$ reaction temperature with a plate of 21 orifices, each with a diameter of $1 \mathrm{~mm}$ in a 50 liters reactor. A considerably less reaction time of 10 min was obtained in the current work, indicating that the proposed operational conditions for production resulted in a fast instigation of cavitation and subsequently in a quicker conversion.

On the other hand, laboratory tests showed maximum results of methyl esters content of $98.6 \%$, cetane number of 55.3 and higher heating value of $39.52 \mathrm{MJ} / \mathrm{kg}$, these correspond to the parameters 5:1 $\mathrm{MR}$ and $0.5 \% \mathrm{KOH}$; furthermore, the lowest methyl esters, cetane number and higher heating value were $83 \%, 39.7$ and $39.25 \mathrm{MJ} / \mathrm{kg}$ respectively. Literature reports values above $96.5 \%$ for methyl esters, between 50 and 60 cetane number and a higher heating value of $39.99 \mathrm{MJ} / \mathrm{kg}$ for 
biodiesel produced from WCO, demonstrating that our results are promising and satisfactory [28][29].

Finally, soap could be produced during reaction due to presence of fatty acids but the intensity of the reaction and the washing process eliminated this soap [12]. This process did not require two steps (acid and basic catalyst) for improving yield reaction as has been reported [30]. Hydrodynamic cavitation is an alternative for biodiesel production from WCO in one step.

\section{CONCLUSIONS}

RSM was a technique appropriated for adjusting of experimental results. Even though the RSM predicted that the optimal parameters of production for both ECPKB minimization and yield maximization are 4.5:1 $\mathrm{MR}$ and $0.5 \% \mathrm{KOH}$, gas chromatography, cetane index and calorific value showed that for this condition the reaction is not fully completed and it doesn't fulfill the minimum quality requirements for biodiesel, indicating that the optimal value for production is 5:1 MR and $0.5 \% \mathrm{KOH}$ considering the high quality of the product $(98.6 \%$ of methyl esters, 55.3 of cetane number and $39.52 \mathrm{MJ} / \mathrm{kg}$ ). For this condition RSM derives in 1.07 yield and $74.07 \mathrm{~kJ} / \mathrm{kg}$ of ECPKB. Finally, low results on ECPKB, high yield, elevated quality of the product, the omission of pre heating and mixing steps, great decrease in reaction time and reducing overall energy consumption justify the idea of taking this process to an industrial level. It is planned to continue carrying out tests with another type of molar ratio and alcohol, which will allow us to have a broader map of these yields, supporting the benefits in the implementation of this technology.

\section{REFERENCES}

[1] S. Khan et al., "Biodiesel Production From Algae to Overcome the Energy Crisis," HAYATI Journal of Biosciences. 2017, doi: 10.1016/j.hjb.2017.10.003.

[2] M. Rehan et al., "Waste to biodiesel: A preliminary assessment for Saudi Arabia," Bioresour. Technol., vol. 250, no. November 2017, pp. 17-25, 2018, doi: 10.1016/j.biortech.2017.11.024.

[3] F. Moazeni, Y. C. Chen, and G. Zhang, "Enzymatic transesterification for biodiesel production from used cooking oil, a review," J. Clean. Prod., vol. 216, pp. 117128, 2019, doi: 10.1016/j.jclepro.2019.01.181.

[4] M. Suresh, C. P. Jawahar, and A. Richard, "A review on biodiesel production, combustion, performance, and emission characteristics of non-edible oils in variable compression ratio diesel engine using biodiesel and its blends," Renew. Sustain. Energy Rev., vol. 92, no. April 2017, pp. 38-49, 2018, doi: 10.1016/j.rser.2018.04.048.

[5] M. H. Hassan and M. A. Kalam, "An overview of biofuel as a renewable energy source: Development and challenges," in Procedia Engineering, 2013, vol. 56, pp. 39-53, doi: 10.1016/j.proeng.2013.03.087.

[6] L. López, J. Bocanegra, and D. Malagón-Romero, "Obtención de biodiesel por transesterificación de aceite de cocina usado," Ing. y Univ., 2015, doi: 10.11144/Javeriana.iyu19-1.sprq.

[7] M. S. Plesset and A. Prosperetti, "Bubble Dynamics and Cavitation," Annu. Rev. Fluid Mech., vol. 9, no. 
1, pp. 145-185, Jan. 1977, doi: 10.1146/annurev.fl.09.010177.001045.

[8] A. Sharma, P. R. Gogate, A. Mahulkar, and A. B. Pandit, "Modeling of hydrodynamic cavitation reactors based on orifice plates considering hydrodynamics and chemical reactions occurring in bubble," Chem. Eng. J., vol. 143, no. 1-3, pp. 201-209, 2008, doi: 10.1016/j.cej.2008.04.005.

[9] J. Alexander Ladino, J. Herrera, D. H. Malagon, M. Prisciandaro, V. Piemonte, and M. Capocelli, “Biodiesel Production Via Hydrodynamic Cavitation: Numerical Study of New Geometrical Arrangements," 2016.

[10] H. Resendiz, "Producción de biodiesel por cavitación hidrodinámica," Rev. Sist. Exp., vol. 3, no. 9, pp. 16-23, 2016.

[11] P. R. Gogate, R. Tayal, and A. Pandit, "Cavitation: A technology on the horizon," Curr. Sci., pp. 35-46, 2006.

[12] A. Talebian-Kiakalaieh, N. A. S. Amin, and H. Mazaheri, "A review on novel processes of biodiesel production from waste cooking oil," Appl. Energy, vol. 104, pp. 683-710, 2013, doi: 10.1016/j.apenergy.2012.11.061.

[13] A. Bokhari, L. F. Chuah, S. Yusup, J. J. Klemeš, and R. N. M. Kamil, “Optimisation on pretreatment of rubber seed (Hevea brasiliensis) oil via esterification reaction in a hydrodynamic cavitation reactor," Bioresour. Technol., vol. 199, pp. 414-422, 2016, doi: 10.1016/j.biortech.2015.08.013.

[14] D. Ghayal, A. Pandit, and V. K. Rathod, “Optimization of biodiesel production in a hydrodynamic cavitation reactor using used frying oil," Ultrason. Sonochem., vol. 20, no. 1, pp. 322-328, 2013.

[15] P. R. Gogate, "Cavitational reactors for process intensification of chemical processing applications: A critical review," Chem. Eng. Process., vol. 47, pp. 515527, 2008

[16] P. Wu, L. Bai, W. Lin, and X. Wang, "Mechanism and dynamics of hydrodynamic-acoustic cavitation (HAC)," Ultrason. Sonochem., vol. 49, pp. 89-96, Dec. 2018, doi: 10.1016/j.ultsonch.2018.07.021.

[17] M. Tabatabaei et al., "Reactor technologies for biodiesel production and processing: A review," Prog. Energy Combust. Sci., vol. 74, pp. 239-303, 2019, doi: 10.1016/j.pecs.2019.06.001.

[18] H. Chitsaz, M. Omidkhah, B. Ghobadian, and M. Ardjmand, "Optimization of hydrodynamic cavitation process of biodiesel production by response surface methodology," J. Environ. Chem. Eng., vol. 6, no. 2, pp.
2262-2268, 2018, doi: 10.1016/j.jece.2018.02.047.

[19] L. F. Chuah, J. J. Klemeš, S. Yusup, A. Bokhari, M. M. Akbar, and Z. K. Chong, "Kinetic studies on waste cooking oil into biodiesel via hydrodynamic cavitation," J. Clean. Prod., vol. 146, pp. 47-56, 2017, doi: 10.1016/j.jclepro.2016.06.187.

[20] R. Alarcón, D. Malagón-Romero, and A. Ladino, "Biodiesel production from waste frying oil and palm oil mixtures," Chem. Eng. Trans., vol. 57, pp. 571-576, 2017, doi: 10.3303/CET1757096.

[21] G. L. Maddikeri, P. R. Gogate, and A. B. Pandit, "Intensified synthesis of biodiesel using hydrodynamic cavitation reactors based on the interesterification of waste cooking oil," Fuel, vol 137, pp. 285-292, 2014, doi: 10.1016/j.fuel.2014.08.013.

[22] D. Y. C. Leung, X. Wu, and M. K. H. Leung, "A review on biodiesel production using catalyzed transesterification," Appl. Energy, vol. 87, no. 4, pp. 1083-1095, 2010, doi: 10.1016/j.apenergy.2009.10.006.

[23] D. Rodríguez, J. Riesco, and D. Malagon-Romero, "Production of Biodiesel from Waste Cooking Oil and Castor Oil Blends," Chem. Eng. Trans., vol. 57, pp. 679684, 2017, doi: 10.3303/CET1757114.

[24] A. Mohammadshirazi, A. Akram, S. Rafiee, and E. Bagheri Kalhor, "Energy and cost analyses of biodiesel production from waste cooking oil," Renew. Sustain. Energy Rev., vol. 33, pp. 44-49, 2014, doi: 10.1016/j.rser.2014.01.067.

[25] Z. Helwani, M. R. Othman, N. Aziz, W. J. N. Fernando, and J. Kim, "Technologies for production of biodiesel focusing on green catalytic techniques: A review," Fuel Process. Technol., vol. 90, no. 12, pp. 15021514, 2009, doi: 10.1016/j.fuproc.2009.07.016.

[26] H. R. F. Masoumi, M. Basri, W. S. Samiun, Z. Izadiyan, and C. J. Lim, "Enhancement of encapsulation efficiency of nanoemulsion-containing aripiprazole for the treatment of schizophrenia using mixture experimental design," Int. J. Nanomedicine, vol. 10, pp. 6469-6471, 2015, doi: 10.2147/IJN.S89364.

[27] L. F. Chuah, S. Yusup, A. R. Abd Aziz, A. Bokhari, J. J. Klemeš, and M. Z. Abdullah, "Intensification of biodiesel synthesis from waste cooking oil (Palm Olein) in a Hydrodynamic Cavitation Reactor: Effect of operating parameters on methyl ester conversion," Chem. Eng. Process. Process Intensif., vol. 95, pp. 235240, 2015, doi: 10.1016/j.cep.2015.06.018.

[28] B. Murcia Ordóñez, L. Chaves Moreno, W. Rodríguez 
Pérez, M. Murcia, and E. Alvarado, "Caracterización de biodiesel obtenido de aceite residual de cocina," Rev. Colomb. Biotecnol., vol. 15, no. 1, pp. 61-70, 2013.

[29] L. F. Chuah, S. Yusup, A. R. Abd Aziz, A. Bokhari, and M. Z. Abdullah, "Cleaner production of methyl ester using waste cooking oil derived from palm olein using a hydrodynamic cavitation reactor," J. Clean. Prod., vol.
112, pp. 4505-4514, 2016, doi: 10.1016/j.jclepro.2015.06.112.

[30] A. Pal, A. Verma, S. S. Kachhwaha, and S. Maji, "Biodiesel production through hydrodynamic cavitation and performance testing," Reneweable Energy, pp. 619-624, 


\title{
CONSTRUCTION OF AN ELECTRIC LOW POWER GENERATOR USING PIEZOELECTRIC CELLS
}

\section{Construcción de un generador eléctrico de baja potencia con células piezoeléctricas}

\author{
Forero-García, Edwin; Rojas-Luna, Milton²; Cuéllar-Guarnizo, Jairo ${ }^{3}$. \\ ${ }^{1}$ Santo Tomás University, Electronic Engineering Master's Program, Research Group MEM \\ 2 Santo Tomás University, Master in Electronic Engineering \\ ${ }^{3}$ Corporación Universitaria Minuto de Dios - UNIMINUTO, Research Group GITSAI
}

\begin{abstract}
This paper shows the design of an electric power generator system, the system bases its operation on the conversion of mechanical energy into electrical energy, taking advantage of the piezoelectric principle; the device designed converts the energy of the footprints of pedestrians in electrical energy using a tile with piezoelectric elements. In first place, the modeling of the piezoelectric transducers was carried out, as well as the identification of their parameters; these parameters will able a future structural and mechanical studies on this device of alternative energy. Likewise, different electrical topologies were considered to construct piezoelectric transducer arrays, although in the study carried out, it was possible to verify that the highest efficiency is obtained with a single piezoelectric disk. Finally, the implementation of an AC/DC converter was carried out, using a commercial solution, the integrated LTC3588, with which it was possible to obtain, with 35 or more steps, a regulated voltage of $3.3 \mathrm{~V}$, useful for a large number of electronic devices.
\end{abstract}

Keywords: Piezoelectric systems, modelling piezoelectric device, piezoelectric energy

\section{Resumen}

Este trabajo presenta el diseño de un sistema generador, el sistema basa su funcionamiento en la conversión de energía mecánica en energía eléctrica, aprovechando el principio piezoeléctrico; el dispositivo diseñado convierte la energía de las pisadas de los peatones en energía eléctrica

${ }^{1}$ Corresponding E-mail: edwinforero@usantotomas.edu.co

${ }^{2}$ Corresponding E-mail: miltonrojas@usantotomas.edu.co

${ }^{3}$ Corresponding E-mail: jairo.cuellar@uniminuto.edu 
mediante una baldosa con elementos piezoeléctricos. En primer lugar, se realizó el modelado de los transductores piezoeléctricos, así como la identificación de sus parámetros; estos parámetros permitirán realizar futuros estudios estructurales y mecánicos sobre este dispositivo de energía alternativa. Asimismo, se consideraron diferentes topologías eléctricas para construir arreglos de transductores piezoeléctricos. En el estudio realizado se pudo comprobar que la mayor eficiencia se obtiene con un solo disco piezoeléctrico. Finalmente, se llevó a cabo la implementación de un convertidor AC / DC, utilizando una solución comercial, el LTC3588 integrado, con el cual fue posible obtener, con 35 o más pasos, una tensión regulada de $3.3 \mathrm{~V}$, útil para una gran cantidad de dispositivos electrónicos.

Palabras clave: Sistemas piezoeléctricos, dispositivo piezoeléctrico de modelado, energía piezoeléctrica

\section{INTRODUCTION}

Piezoelectric systems include intelligent materials for vibration control, aeronautical and aerospace applications of flexible surfaces and structures, robotic sensors, and innovative Energy Harvesting prototypes among others. In the USA a study of a test prototype built with piezoelectrics was carried out. The size of the prototype included inserting it into a shoe, in this way whoever uses them, generates energy through their own mechanical energy [1]. In Israel, the company Innowattech, in collaboration with the Technion - Israel Institute of Technology, developed a device for the generation of energy from the transit of vehicles. The system consisted of a series of generators placed under the asphalt of the roads and generating power when the vehicles circulated. The system can produce up to 2000 watt-hours [2]. In Italy this same technology was used on the motorway connecting Venice with the Trieste Autostrada [3]. On the other hand, the british company PavegenSystems launched in 2009 by Laurence Kemball-Cook, designed and built the Pavegens that were installed at the corporate headquarters of Johnnie Walker and WWF [3]. One of the most representative projects of Pavegen Systems was the one carried out during the London Olympic Games in 2012 where those that were used in the tunnel that connects the West Ham underground station with Olympic Park [4]. During the last ten years, research studies on piezoelectric energy harvesting have been extensively conducted. Some of these studies are: Modeling and experimental research of a piezoelectric energy collector driven by human footsteps [4], Design of a new piezoelectric energy collector based on ferroelectric secondary impact [5], Study of the efficiency of a piezoelectric energy harvesting system using rectifier and configuration of a ferroelectric arrangement [6], Designing and building a piezoelectric tile to collect energy from steps [7], Modeling the energy harvesting potential from piezoelectrics in a educational construction [8]. It was observed that the most widely used 
technique for obtaining electrical energy from the piezoelectric principle is by vibration and by force. In the literature many articles related to the piezoelectric phenomenon can be seen. However, many of these focus on generating portable devices such as shoes or backpacks, these can be seen as sources of micro electricity for auxiliary power.

The mentioned cases are not considered macro sources to their limited area of installation, on the other hand, the tiles can be planted over a larger area; therefore, they can be used as macro power sources. In Colombia there is research on this topic such as: Piezoelectric tile to power lighting systems with low energy consumption [9], Design of a Piezoelectric Energy Collector (Energy Harvesting) by means of topological optimization that maximizes the transformation of mechanical energy into electricity generated by a Being Human When Walking [10]. The first of them was carried out in Antioquia in 2014, which consisted of the design of a piezoelectric tile. The second was a research project at the National University, Medellín headquarters in 2014, where some prototypes for shoes were designed and built, so that when pressing on the heel, electricity was generated. Most of the cases studied have been designs without implementation and in which it has been done, this has had a different approach to that proposed in this research work. According to the research, there are no local companies that are dedicated to the manufacture or distribution of piezoelectric generators, since the main focus it has regarding the use of renewable energies is on the wind and solar part.

\section{MATERIALS AND METHODS}

The work was carried out in experimental steps, in the following sections, each of them is described in detail.

\section{Modeling of the piezoelectric cell}

The model that describes the behavior of the piezoelectric cell to be used is the circuit of Figure 1. In this model, the effects of the load resistance and the parameter $\mathrm{d} 33$ (relationship between the force exerted and the generated electric current), are considered as mentioned in [11] y [12]. 


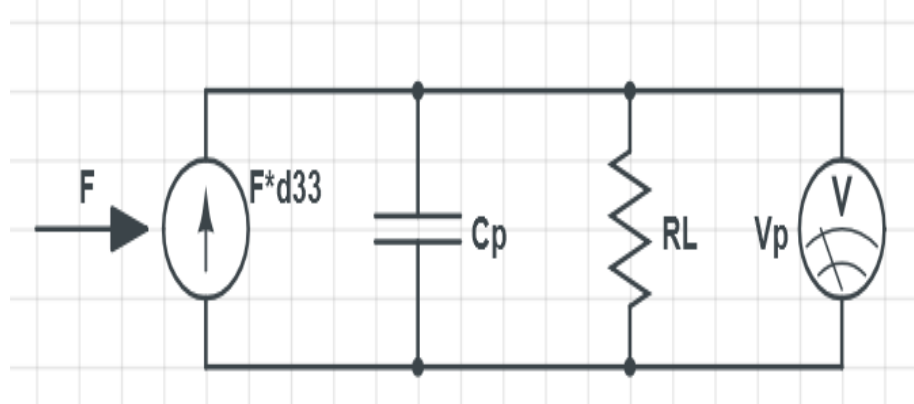

Figure 29 Equivalent circuit.. Source: Authors.

The voltage versus force transfer function of this circuit can be obtained by combining the following equations:

$$
I=d_{33} \frac{d f}{d t}, \quad I_{c}=C_{p} \frac{d v_{c}}{d t}, \quad v_{\rho}=v_{c}=v_{R}=R_{L} \cdot I_{R}
$$

Using a simple circuit analysis of nodes, applying algebra and finally using the Laplace transform, we obtain the relation of equation 2. This is the transfer function and mathematical model of the piezoelectric cell.

$$
\frac{v_{p}}{f}=d_{33} \frac{R S}{R C S+1}
$$

\section{Effect of force application point}

It is possible to appreciate in studies that the constants of a piezoelectric material $(\mathrm{d} 33)$ are not invariant, conversely, they depend on the geometry and location of the force actuators [13][14][15]. At this stage, a solenoid actuator was added to exert pressure at different points on the piezoelectric. The purpose of this exercise is to determine the point of application and magnitude of the force, to achieve better efficiency in the generation of the tile. The comparison should be made taking into account the power delivered by the piezoelectric as it has been oriented in previous studies [16]. Therefore the input variable would be the derivative of the applied force (using the discrete measurements already obtained) and as the output variable it would be the power calculated with (3).

$$
P_{T}=\frac{1}{L R} \sum_{k=0}^{L} v_{[k]}^{2}
$$

The relation of the total power against the derivative of the applied force is observed in figure 2 , 


\section{CONGRESO DE DESARROLLD SOSTENIBLE}

this graph was parameterized with respect to the distance to the center of the piezoelectric.

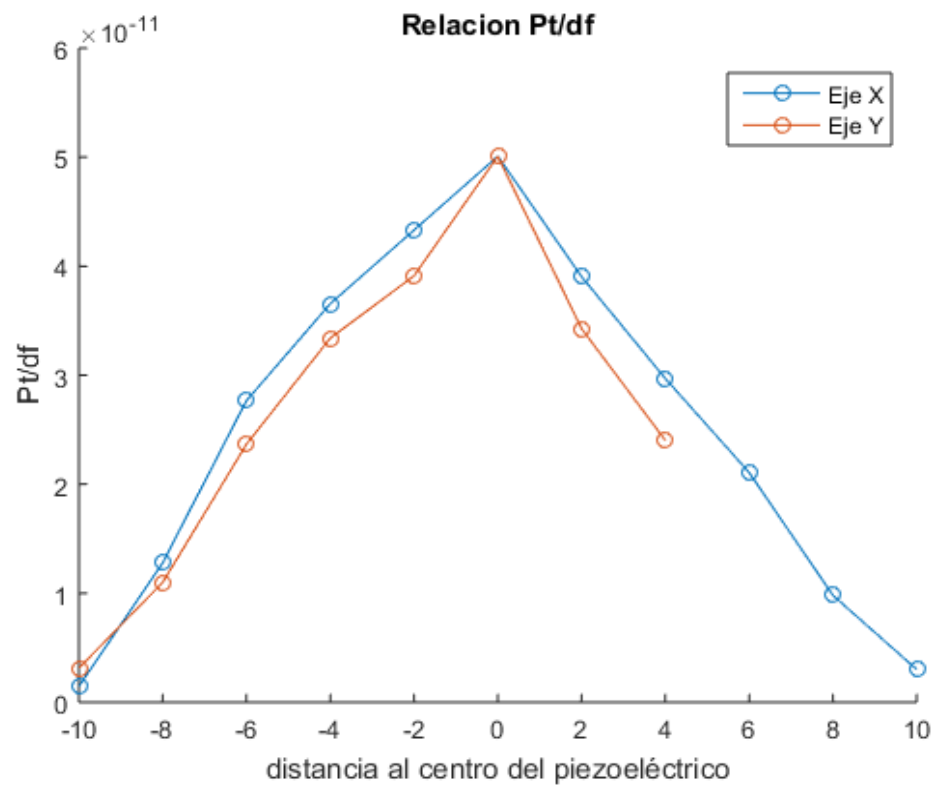

Figure 30 Pt/df vs distance to the center. Source: Authors.

This graph can be approximate to the quadratic function shown in Equation 4.

$$
y=-4.3 \cdot 10^{-13} x^{2}-1.9 \cdot 10^{-13} x+4.2 \cdot 10^{-11}
$$

The variable " $y$ " is the $\mathrm{Pt} / \mathrm{df}$ ratio, and " $\mathrm{x}$ " is the distance to the center of the piezoelectric transducer. In this way, a three-dimensional representation of the power efficiency of the piezoelectric is obtained according to the point where the force is exerted, the result can be seen in figure 3.

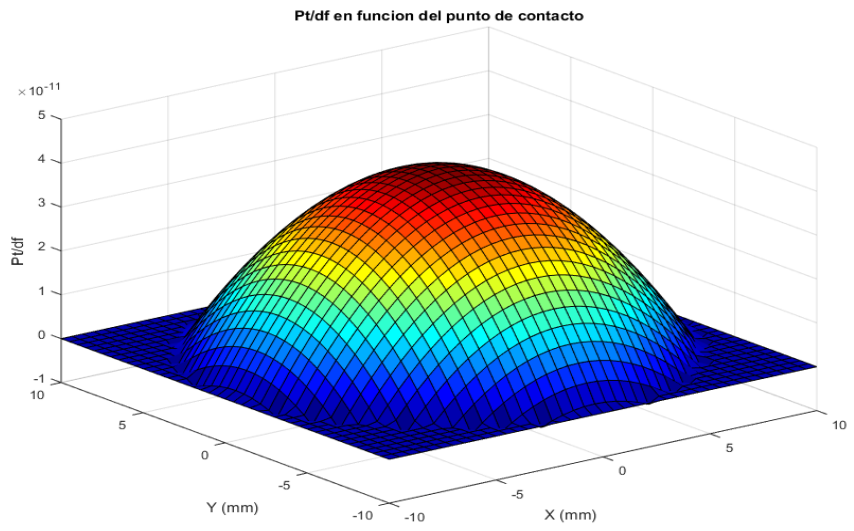

Figure 31 Pt/df vs Force point.. Source: Authors. 
Due to the effect of the force point and analysis of the captures and simulations carried out, it is decided that in the design of the final device, it is necessary that the applied force falls on the center of the capsule and in the smallest possible area.

\section{Characterization of the piezoelectric cell}

Three references were considered during the study and construction of the piezoelectric system: the TB-27-4 from the manufacturer Murata (large), the SKU 392264 model from the manufacturer Hesentec (medium) and the SKU 318189 model from Piezo Hannas (small). To characterize these cells and test their response, it was used an electronic system with a microcontroller to capture the value of the force exerted and the voltage generated. It was tested with $10 \mathrm{M} \Omega, 1 \mathrm{M} \Omega$ and $100 \mathrm{~K} \Omega$ load resistors. In order to compare the piezoelectric capsules, the relationship between the voltage obtained and the derivative of the force exerted was made.

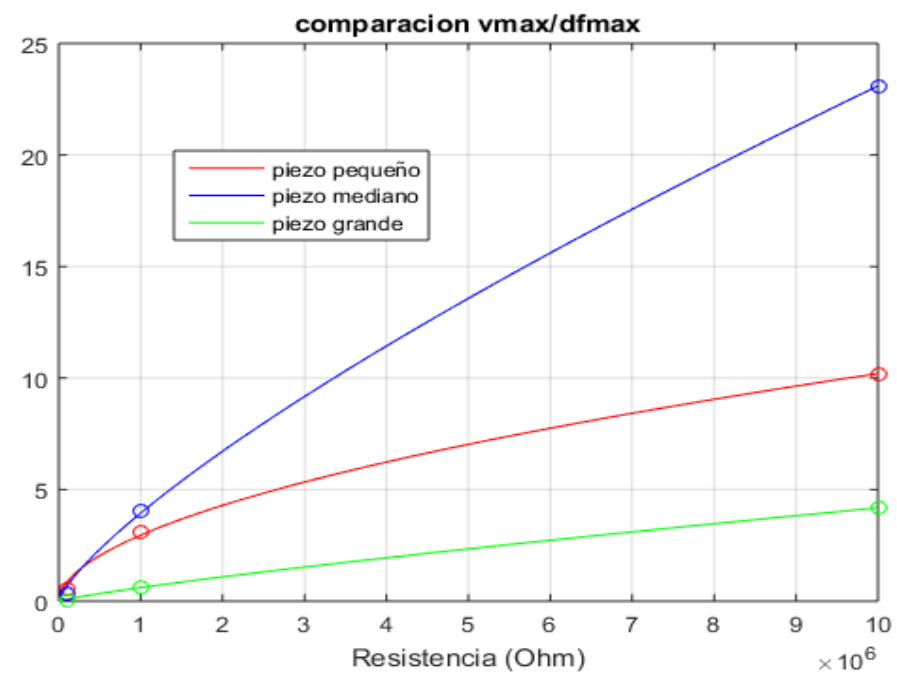

Figure 32 Vmax/dfmax vs Load- Source: Authors.

By tabulating the data obtained with the different loads, figure 4 is obtained. From this comparison it is possible to observe that the median piezoelectric, is one of the three, which most efficiently transforms mechanical energy into voltage, so this will be the one used in the construction of the generator. After using the measurements made in the parametric identification of the piezoelectric capsule, the transfer function was obtenied and can be seen in the equation 5 .

$$
\frac{v}{f}=\frac{0,2301 S}{S+9,869}
$$


Comparing with equation 2, the following constant values was reached: $R=1 \mathrm{M} \Omega, C=3,469 \mathrm{e}^{-8} \mathrm{~F}$ y $\mathrm{d} 33=1,3 \mathrm{e}^{-8}$. Finally, to collect energy from the piezoelectric generator system, the "Energy Harvesting Solution to Go" system from the Würth Elektronik company was used. This system is made up of two fundamental parts: Energy capture card, which contains four linear technology converters, optimized for different alternative energy sources, including piezoelectric. Giant Gecko Kit that has a very low consumption EFM32 microcontroller, accompanied by an LCD screen, programmed for the measurement of generated energy. The system is intended for use in the design and testing of alternative power generators. Using this system, a piezoelectric generator is controlled and configured, with voltage regulation at $3.3 \mathrm{~V}$ in $\mathrm{DC}$, and which is consistent with the simulations carried out.

\section{RESULTS}

The current measured in the piezoelectric cell is compared with the current transformation from the obtained model, it is necessary to convert the model from $\mathrm{V} / \mathrm{f}$ to I / $\mathrm{f}$. The comparison between the measurement and the evaluation by the model was implemented in simulink and the capture can be seen in figure 5. This results allows validate the mathematical model of the tile, wich was development in this article.

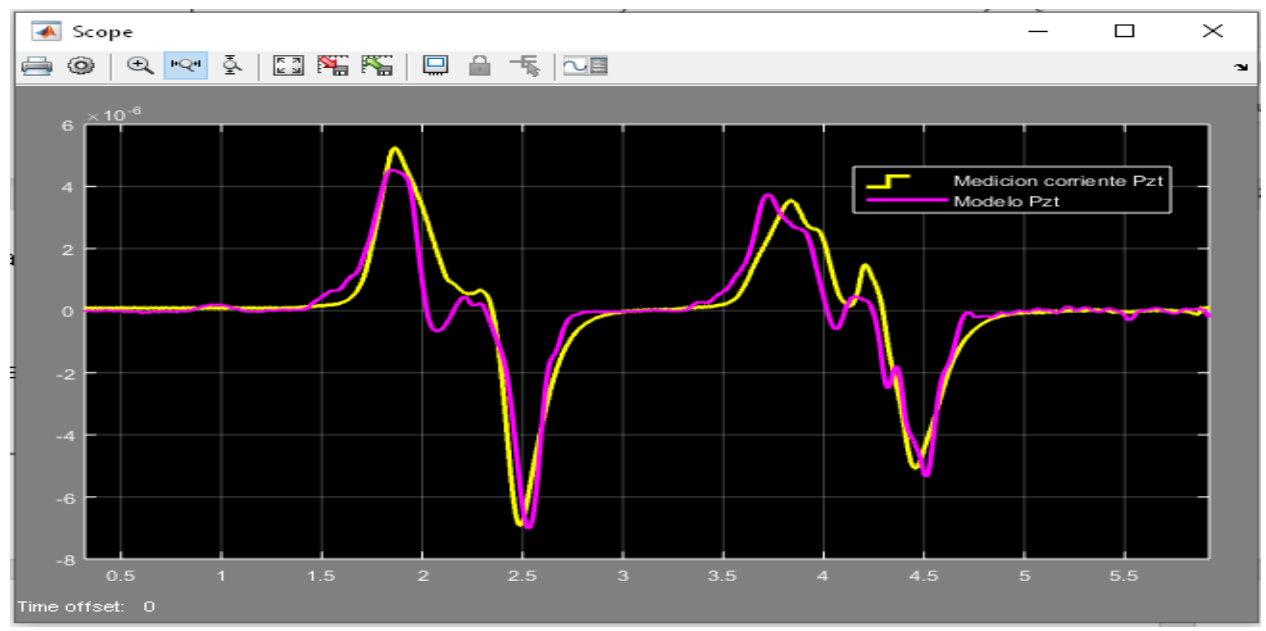

Figure 33Measured current and estimated current. Source: Authors.

From laboratory tests it was determined that if all the piezo disks in the system receive the same force, the option that generates higher voltage peaks even with small load resistances is a single disk; all this is because when having more mechanically coupled piezoelectrics, the force is 
repaired between all the discs, generating less energy in each of them. For laboratory tests performed, a load of $1 \mathrm{M} \Omega$ was used. In figure 6 it can be seen that the generator provides full charge for $5 \mathrm{~V}$ mobiles, starting from thirty steps, similar to the calculations made where 35 steps were determined to reach full load. This difference is due to the non-linearities of the system that were not considered in the modeling: operating voltage of the input rectifier diodes, consumption of the sensing and regulation circuits, and stray capacitances and leaks.

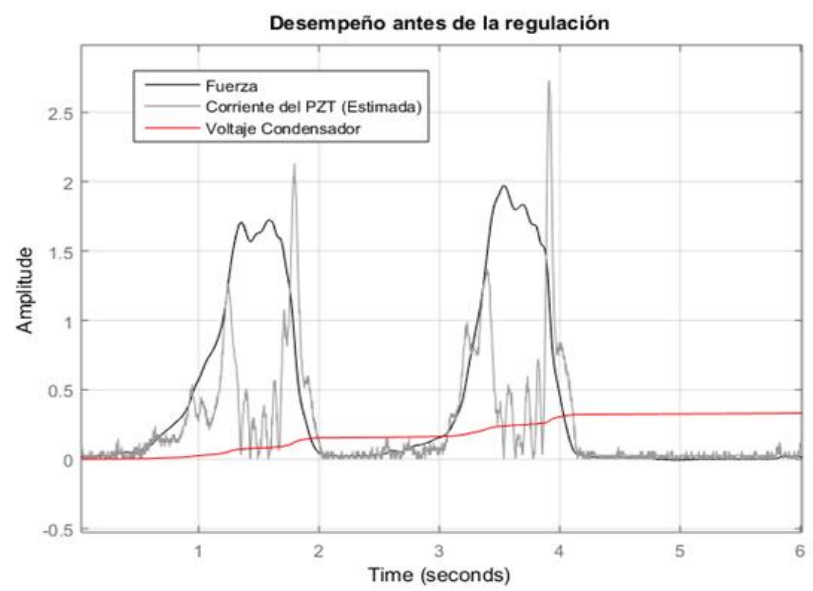

Figure 34 Capacitor charge before regulation. Source: Authors.

Once the system is loaded, for each step taken on the generator, the voltage increases, approximately by 0.3 volts. It was observed that the self-shutdown system of the TLC3588 regulator protects the output circuit, but makes it necessary that to reach the programmed voltage of $(3.3 \mathrm{~V})$ several charges are required in the input capacitor, that is, starting from the fully discharged system, several charge cycles are needed which implies a greater number of steps, this relationship of steps against the voltages in $\mathrm{Ci}$ and $\mathrm{Co}$ is illustrated in figure 7. 


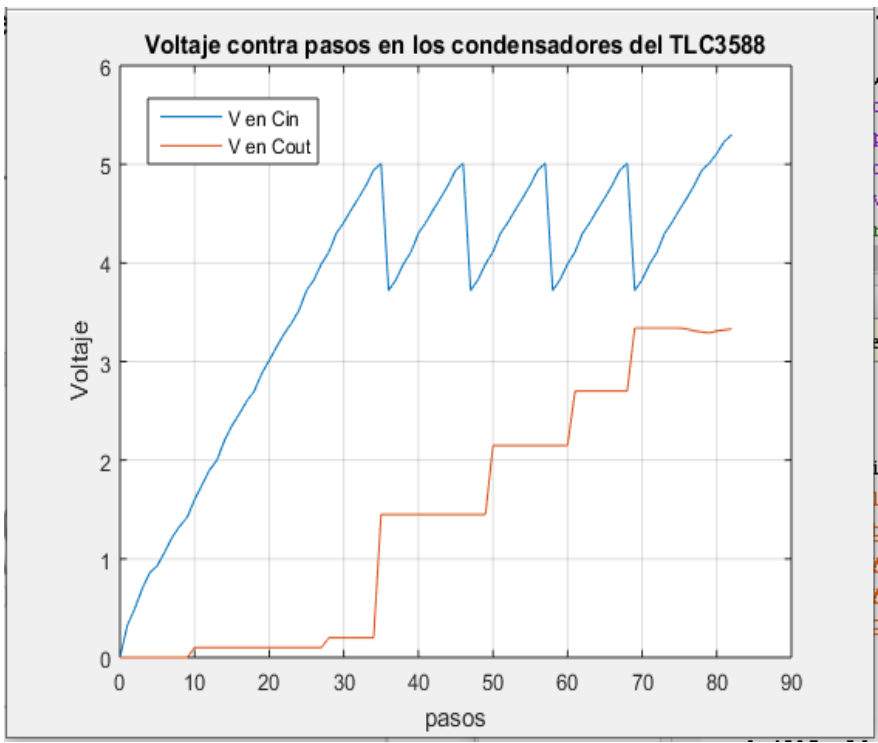

Figure 35 Voltage vs. Steps.. Source: Authors.

It should be noted that after obtaining the operating voltage, about eleven steps are required (according to the measurements made) to reach the regulated voltage of 3.3V again. Said voltage with a load of $1 \mathrm{M} \Omega$ will remain stable for around 4 seconds, maintaining a voltage between 3.4 and $2.95 \mathrm{~V}$, that is, a ripple close to $10 \%$. This results shows that the made tile can be used like a low power generator and this same device could be used to create paralel or series arrays to obtein more output power.

\section{CONCLUSIONS}

The piezoelectric elements are lightweight and solid systems and without mechanical parts that allow their wear by friction, which makes them ideal for low maintenance environments. Piezoelectricity is one of the simplest ways of generating electricity, however, the power delivered by this type of system is also one of the most limited among unconventional energies. So its application is recommended for low consumption loads that support power for short periods of time, such as sensors and data transmitters in the IoT field. Due to their simplicity and robustness, piezo disks are an excellent solution for tight spaces and complex environments. It is necessary to consider the mechanical limits, since being crystals, they are relatively fragile and therefore require mechanical protections to protect them from shocks or over stress. Lastly, the electrical load applied to the piezoelectric generators has a significant impact on their performance, during the study carried out it was possible to obtain peak voltages close to seventy volts, however, this 
was only possible with $10 \mathrm{M} \Omega$ resistors, as the value of this resistance, the maximum amplitudes obtained decreased considerably. Although the arrangements of multiple piezoelectric disks in series and parallel improve the electrical performance (higher voltage or higher current, respectively), they produce an undesired reduction in efficiency since the applied force must be distributed among several elements, therefore in structures of a single block, it is more efficient to use a single generator disk.

\section{REFERENCES}

[1] C. A. Howells, "Piezoelectric energy harvesting," Energy Convers. Manag., vol. 50, no. 7, pp. 1847-1850, Jul. 2009.

[2] Libertad Digital, “Una empresa israelí logra generar electricidad gracias al tráfico - Libertad Digital," 2009.

[3] Off grid energy independence, "Piezoelectric roads for California I Off Grid Energy Independence," 2011. .

[4] S. Wei, H. Hu, and S. He, "Modeling and experimental investigation of an impact-driven piezoelectric energy harvester from human motion," Smart Mater. Struct., vol. 22, no. 10, p. 105020, Oct. 2013.

[5] H. J. Jung, K. H. Baek, S. Hidaka, D. Song, S. Bin Kim, and T. H. Sung, "Design of a New Piezoelectric Energy Harvester Based on Secondary Impact," Ferroelectrics, vol. 449, no. 1, pp. 83-93, Jan. 2013.

[6] K. H. Baek, S. K. Hong, S. Bin Kim, J. H. Kim, and T. H. Sung, "Study of Charging Efficiency of a Piezoelectric Energy Harvesting System Using Rectifier and Array Configuration," Ferroelectrics, vol. 449, no. 1, pp. 42-51, Jan. 2013.

[7] S. J. Hwang et al., "Designing and manufacturing a piezoelectric tile for harvesting energy from footsteps," Curr. Appl. Phys., vol. 15, no. 6, pp. 669-674, Jun. 2015.

[8] X. Li and V. Strezov, "Modelling piezoelectric energy harvesting potential in an educational building," Energy Convers. Manag., vol. 85, pp. 435-442, Sep. 2014.

[9] J. A. Cifuentes Gutierrez, "Baldosa Piezoeléctrica Para Alimentar Sistemas De Iluminación De Bajo Consumo
Energético" Escuela de Ingeniería de Antioquia, 2014

[10] E. Sepúlveda Orozco, "Diseño de un Colector de Energía Piezoeléctrico (Energy Harvesting) Mediante Optimización Topológica que Maximice la Transformación de Energía Mecánica en Eléctrica Generada por un Ser Humano al Caminar," 2014.

[11] E. Lefeuvre, D. Audigier, C. Richard, and D. Guyomar, "Buck-Boost Converter for Sensorless Power Optimization of Piezoelectric Energy Harvester," IEEE Trans. Power Electron., vol. 22, no. 5, pp. 2018-2025, Sep. 2007.

[12] P. M. Mayrhofer, E. Wistrela, M. Schneider, A. Bittner, and U. Schmid, "Precise Determination of d33 and d31 from Piezoelectric Deflection Measurements and 2D FEM Simulations Applied to ScxAl1-xN," Procedia Eng., vol. 168, pp. 876-879, Jan. 2016.

[13] P. Ordoñes, "Estudio del comportamiento de una cerámica piezoelectrica mediante elementos finitos," Universidad politecnica de Valencia, 2011.

[14] A. E. Guennam, "Modelo para compuestos piezoeléctricos utilizados en control activo," Universidad nacional de Tucumán, 2005.

[15] Z. Lašová and R. Zemčík, "Comparison of Finite Element Models for Piezoelectric Materials," Procedia Eng., vol. 48, pp. 375-380, Jan. 2012.

[16] J. Luo, "Energy Harvesting Using Flexible Piezoelectric Materials," 2016 
CONGRESO DE DESARROLLO

SOSTENIBLE

\section{EMPRENDIMIENTO SOSTENIBLE}




\title{
TRANSFORMATION OF THE BIOGAS PRODUCED IN FILLING THE EL CARRASCO SANITARY IN BUCARAMANGA, COLOMBIA
}

\section{Transformación del biogas producido en el relleno sanitario "El Carrasco" en Bucaramanga, Colombia}

\author{
Verjel, Elissa'; Carrión, Sergio \\ Universidad El Bosque, Grupo de investigación Producción Limpia Choc Izone
}

\begin{abstract}
The general objective of this work is to design an environmental business management model for the biogas recovery process, generated in the municipality of Bucaramanga-Santander, in the closure phase of the El Carrasco sanitary landfill. The environmental diagnosis of the current state of the landfill began through the collection of primary and secondary information. The variables that allowed the calculation of the generation and recovery of biogas, using the US EPA model called the "Colombian biogas model" and the technical study of the transformation processes from biogas to energy, was also carried out.
\end{abstract}

The consolidation of the proposed environmental business management model is based on the diagnosis of the macro, micro and internal environment of the business in which the idea of entrepreneurship is framed. The proposal for a Business Management Plan, the guidelines of the Operational Plan and the Environmental Management Plan, that arise from the swot analysis of the DOFA of the information collected to comply with the scope and objectives of the project, are presented, among other analyses.

The model allowed establishing an efficiency of the capture system of $52 \%$, a biogas generation of $555 \mathrm{~m}^{3} / \mathrm{h}$ and a recovery of $551 \mathrm{~m}^{3} / \mathrm{h}$ for 2019 , as well as the maximum capacity of the electricity plant of 0.9 MW. These results allowed to establish the appropriate technology to give an energy use to the biogas and mitigate the socio-environmental impact on the population surrounding the landfill.

Key words: Biogas, Landfill, GHG 


\section{Resumen}

El objetivo general de este trabajo consiste en diseñar un modelo de gestión empresarial ambiental para el proceso de recuperación de biogás generado en el municipio de Bucaramanga-Santander, en la fase de cierre del relleno sanitario El Carrasco. El diagnóstico ambiental del estado actual del relleno sanitario se inició mediante la recolección de información primaria y secundaria. También se establecieron las variables que permitieron el cálculo de la generación y recuperación de biogás, utilizando el modelo US EPA denominado "modelo de biogás colombiano " así mismo se realizó el estudio técnico de los procesos de transformación de biogás a energía.

La consolidación del modelo de gestión empresarial ambiental propuesto se basa en el diagnóstico del entorno macro, micro e interno de la empresa en el que se enmarca la idea de emprendimiento. Se presenta la propuesta de Plan de Gestión Empresarial, los lineamientos del Plan Operativo y el Plan de Gestión Ambiental, que surgen del análisis DOFA de la información recolectada para cumplir con el alcance y objetivos del proyecto.

El modelo permitió establecer una eficiencia del sistema de captación del 52\%, una generación de biogás de $555 \mathrm{~m} 3$ / hy una recuperación de $551 \mathrm{~m} 3$ / h para 2019, así como la capacidad máxima de la planta eléctrica de 0,9 MW. Estos resultados permitieron establecer la tecnología adecuada para darle un uso energético al biogás y mitigar el impacto socioambiental en la población aledaña al relleno sanitario.

Palabras clave: Biogás, Vertedero, GEI.

\section{INTRODUCTION}

The present work includes the study on the estimation of biogas generation in a sanitary landfill, since the urban solid waste deposited in these sites are not properly managed and can cause air, water and soil contamination issues, along with the consequent public health risk. In addition, the contribution to the greenhouse effect of methane, one of the gases present in the biogas that is generated in landfills, is 21 times more potent compared to carbon dioxide $\left(\mathrm{CO}_{2}\right)$ [1].

Consequently, concepts such as generation and recovery of methane gas in sanitary landfills and climate change were deepened, since biogas receives this name given the anaerobic decomposition process of the waste confined at the final disposal site, being a direct contributor to the generation and emission of greenhouse gases that is linked to climate change - thus, additional environmental and social problems. 
One of the benefits currently observed in sanitary landfills is the adequate collection and use of biogas to be used as energy or gas. This is to mitigate the environmental and social problems caused by the activity of the sanitary landfill to influence zones.

The methodology developed is based on a mixed approach. It is characterized by being quantitative and qualitative - by using the Colombian biogas model, and by collecting and analyzing the information needed to guide projects of this nature, respectively. To estimate the final use of biogas in the closing phase of the El Carrasco sanitary landfill, an environmental diagnosis was taken into account to show its current state and the use of variables that allowed the calculation of biogas and thus the pertinent technologies were described for the use of biogas for energy purposes.

In order to meet the objectives, it was necessary to have information provided by the Bucaramanga EMAB waste disposal company. Provided by EMAB, the reports on the disposal of solid waste, chemical, physical composition of waste, waste production and per capita production by municipalities allowed for a general and up-to-date environmental diagnosis of the El Carrasco landfill.

Based on the above-mentioned, it is worth asking this question: How does the design of the environmental business management model for the transformation of biogas contribute to the mitigation of the socio-environmental impacts produced at the El Carrasco sanitary landfill in Bucaramanga-Santander?

In this study, an environmental management strategy for the use of biogas at the internal level of the sanitary landfill is proposed. The ecological, social and economic benefits can be identified through the analysis of the results obtained for each specific objective raised.

\section{MATERIALS AND METHODS}

In order to carry out the first specific objective, "Prepare an environmental diagnosis of the sanitary landfill to find the variables that allow estimating the generation and recovery of biogas using the Colombian biogas model software," the sanitary landfill was diagnosed environmentally, taking into account the impact the landfill had in the surface and underground hydrography, the geospheric component, the weather conditions at the site, and the behavior of 
the wind, were analyzed using air quality monitoring data.

The Colombian Biogas Model provides an automatic tool for estimating the generation and recovery of biogas in municipal sanitary landfills. Its purpose is to provide owners and operators with tools to assess the feasibility and benefits of recovering and using the generated biogas [2]. By using the model, it was essential to have sources that provide real data, since this is the basis for the veracity of the estimation of biogas. This model not only calculates the generation of biogas but also its recovery.

The necessary information to carry out the calculation are as follows: the geographical region in order to determine the average annual precipitation of the department where the landfill is located, the year of its opening, the data found in Megagrams (tons) in the annual disposition of the most recent year, the volume of waste disposed in the landfill, the current year of closure of the landfill or the projected year of closure, the estimated growth in annual disposal, the average depth of the landfill, and the design and practice management of the sanitary landfill (average historical conditions), the biogas capture system from the beginning of the year, and the estimated capture efficiency (maximum of $85 \%$ ).

For the second objective, "Define the technical study of the transformation of biogas into electrical energy", a descriptive methodology was developed for the different advantages and disadvantages of the technologies used to generate electricity from biogas recovered in landfills of solid urban waste (RSU).

In the methodology, as shown in Table I, it is mentioned that the majority of projects in operation use internal combustion engines (reciprocating), turbines and microturbines.

Table 13 Advantages and disadvantages of the technologies studied

\begin{tabular}{|c|c|}
\hline Technology & Characteristics \\
\hline & - High efficiency \\
& - Low cost per $\mathrm{kW}$ \\
& - Engine efficiency varies between $25 \%$ and $35 \%$ \\
- High costs of maintenance
\end{tabular}




\begin{tabular}{|c|c|}
\hline Gas turbine & $\begin{array}{l}\text {-Large-scale projects where there is a flow of biogas to generate } 3 \mathrm{MW} \text { and more } \\
\text { than 5MW (biogas flows greater than } 40 \mathrm{~m}^{3} / \mathrm{min} \text { ) } \\
\text { - The cost of } \mathrm{kW} \text { decreases with the increase in turbine size } \\
\text { - Efficiency between } 20 \text { and } 28 \% \\
\text { - Disadvantage in terms of the removal of siloxanes and other impurities from } \\
\text { biogas. }\end{array}$ \\
\hline Microturbines & $\begin{array}{l}\text { - Biogas flow of less than } 8 \mathrm{~m}^{3} / \mathrm{min} \text { with minimum methane content of up to } 35 \% \\
\text { - High cost per } \mathrm{kW} \\
\text { - Requires primary biogas treatment including siloxane removal, moisture and } \\
\text { particulate material } \\
\text { - Reduced cost of capital and low cost of maintenance and facilities. }\end{array}$ \\
\hline
\end{tabular}

Note: The different advantages and disadvantages of the technologies available for the generation of electricity from biogas from landfills are mentioned. [3].

As for the third objective, "Design a business management model that allows managing electric energy projects as an alternative energy source," the Political, Economic, Social, Technological, Ecological and Legal analyses were carried out by PESTEL, the five forces of Michael Porter, the diagnosis of the necessary components for the design of the environmental business management system in which the project is circumscribed, and the use of the methods presented in "Mastering the Management System by R. S. Kaplan and D. P. Norton" to design the organization's strategic processes, strategic plan and operational plan.

\section{RESULTS AND ANALYSIS OF RESULTS}

The generation and recovery of biogas projected in the El Carrasco sanitary landfill, are among the results obtained, as shown in Figure1. 


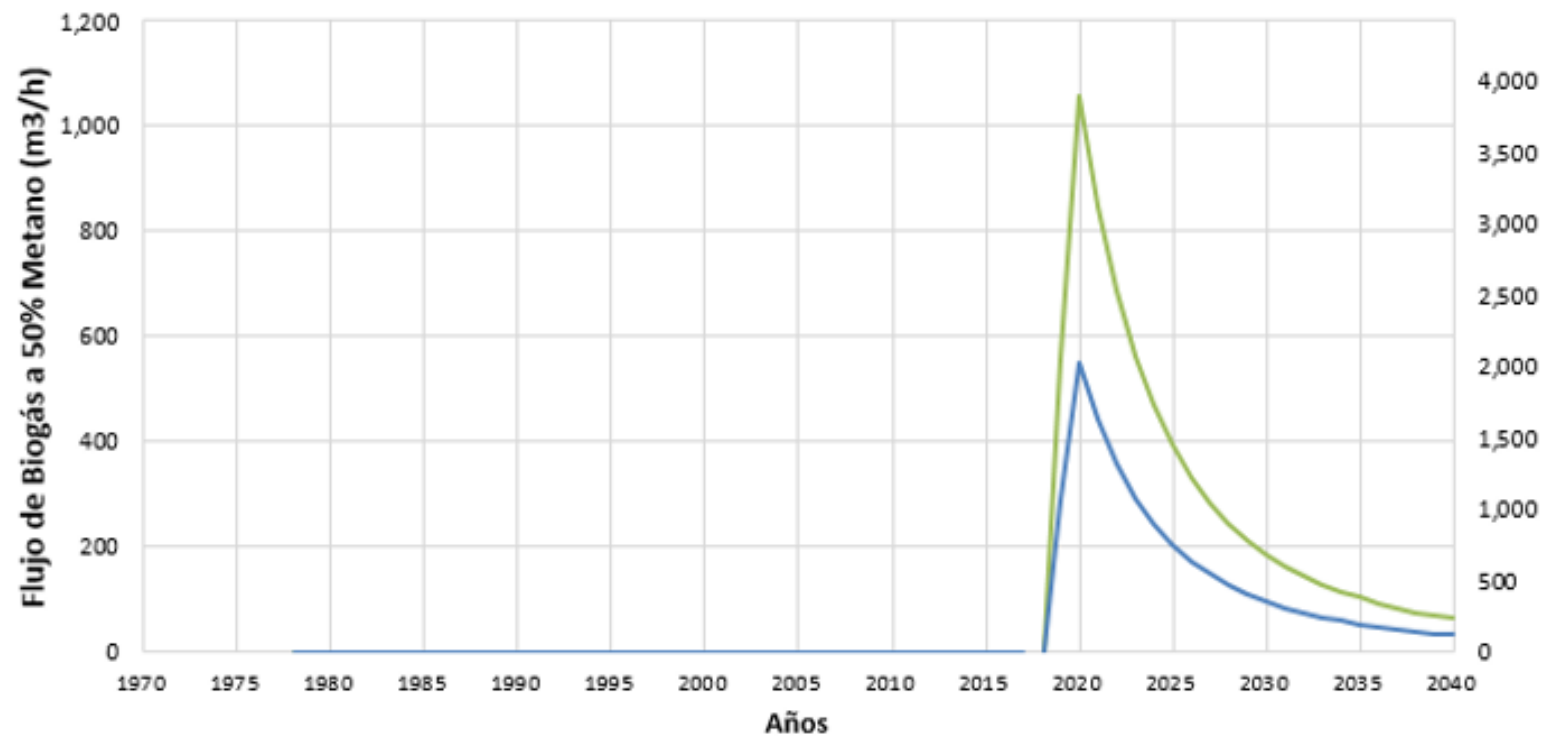

—Generacion de Biogas — Recuperacion de Biogas - Recuperacion de Biogas Actual

Figure 36 Projections of biogas generation and recovery.

Based on the results obtained by the model, which is within the guidelines established by the EPA, the capture efficiency for the El Carrasco landfill is 52\%. It establishes capture efficiencies of 50\%, with a very good system installed.

According to the table of results produced by the Colombian biogas model, it is shown that biogas generation has decreased due to less waste disposal, going from $555 \mathrm{~m}^{3} / \mathrm{h}$ in 2019 to $186 \mathrm{~m}^{3} / \mathrm{h}$ by year 2030 .

Biogas recovery is the fraction of biogas generation that can be trapped by the capture system and that can be recovered for combustion or beneficial use using the capacity of the power plant. In 2020, it reaches the highest amount of biogas of $551 \mathrm{~m}^{3} / \mathrm{h}$, a maximum capacity of the electricity plant of $0.9 \mathrm{MW}$, and by 2030, a decrease of $78 \%$ is shown, taking the maximum capacity ( $0.9 \mathrm{MW})$ of $100 \%$. As per the figures above, by 2020 , it is estimated annually to reduce an amount of 1,727 tons of $\mathrm{CH} 4$ and 36,257 tons of $\mathrm{CO}_{2}$ eq, which would help the goal established within the United Nations Framework Convention on Change Climate (UNFCCC) a reduction of 20\% of GHG by 2030 . 
Figure 1 coincides with the theoretical model of biogas behavior in a landfill cell, as organic matter decomposes, methane stops being produced, reaching the year 2030 at $186 \mathrm{~m}^{3} / \mathrm{h}$. This verifies that the simulation results can be correlated with the theoretical models, since the behavior of the biogas in a sanitary landfill decreases with time and then in the closure phase, it remains constant for a certain time until the landfill meets its useful life and stops emitting gases and leachates.

By extending the years on the graph until 2077, which is the maximum year shown by the model, it is evident that both the generation and recovery of biogas remains constant with a generation of $7 \mathrm{~m}^{3} / \mathrm{h}$, which could not be recovered in energy.

The Carrasco landfill will generate biogas until 2030, i.e. 10 years in which the fill can offer energy to the surrounding neighborhoods such as El Porvenir, Dangond, Tejares and Manuela Beltrán, the above means that with a capacity of $0,9 \mathrm{MW}$ could light up 6,667 homes up to 1,481 homes with a capacity of $0.2 \mathrm{MW}$.

Given the above, it is believed that the population that does not have electricity is around 6,608 inhabitants. Therefore, this type of strategy is aimed at improving the quality of life of these individuals.

\section{CONCLUSIONS}

Implementing the biogas use model to generate electricity is environmentally sustainable. Biogas being emitted through chimneys increases the emission of GHG into the atmosphere, affecting the health of the surrounding population. According to data from the Santander Ministry of Health, the causes of death in children under the age of 1 are respiratory related, with an annual average rate of 55.2 cases per 100,000 inhabitants. In children ages 1 to 4 , the main causes of mortality were external causes such as accidents that obstruct breathing, an annual rate of 7.4 cases per 100,000 inhabitants [4]. This shows the problems caused by the burning of biogas. It is recommended to implement technologies that take advantage of that percentage of methane to generate energy.

The methodology executed in this work, thanks to the use of tools developed by the EPA, estimates of the amounts of biogas that are recovered from energy production. Determining specific activities in an estimated time and obtaining periodic results to establish opportunities 
for improvement, are methodological contributions to the model.

With the environmental business management model, this proposal achieves the mission, vision, corporate values and objectives of the business organization. Its intent is to organize the efforts of all actors and stakeholders in the solid waste value chain, as well as the strategic axises of the business management plan. The differential factor through which the competitive advantage would be achieved by the implementation of new areas of performance in terms of technology and projects in which innovation is implemented and invested in new alternative services for the community surrounding landfills. In addition to the key processes to be developed, guidelines for customer management, human talent management, organizational capital, and the foundations of technological capital should be prioritized.

The evaluation of the technical-economic viability of the technology is still in development, as this study is currently being performed by a graduate student.

\section{REFERENCES}

[1] Q. Aguilar, P. Taboada y B. Ojeda, «Modelo mexicano para la estimación de la generación de biogás,» Ingeniería, vol. 15, no 1, pp. 37-45, 2011.

[2] G. Blanco, E. Santalla, V. Córdoba y A. Levy, «Generación de electricidad a partir de biogás capturado de residuos sólidos urbanos: Un análisis teórico-práctico,» Banco Interam. Desarro, vol. 52, 2017.

[3] Secretaría de Salud de Santander, «Análisis de situación de salud con el modelo de los determinantes sociales de salud,» 2014. [En línea]. Available: http://web.observatorio.co/publicaciones/Bucaramang a_dg.pdf. [Último acceso: 28 Febrero 2019].

[4] Agencia para la Protección del Ambiente EPA, «Manual de usuario Modelo Colombiano de Biogás,» Septiembre 2009. [En línea]. Available: https://globalmethane.org/documents/models/pdfs/M anualdeUsuarioModeloColombiano.pdf.] [Último acceso: 17 Febrero 2019]. 
CONGRESO DE DESARROLLD

SOSTENIBLE

\section{AMBIENTES Y EDIFICACIONES SOSTENIBLES}




\title{
PEDESTRIAN MOBILITY PLAN FOR SOCIAL DISTANCING IN CHAPINERO CENTRO
}

\section{Plan de movilidad peatonal de distancia social en chapinero centro}

\author{
Sánchez-Gómez, Sebastián'; Gutiérrez, Laura². \\ ${ }^{1}$ Universidad de los Andes, Colombia. \\ 2 Universidad Santo Tomás, Colombia.
}

\section{Abstract}

Given the pandemic situation generated by Covid-19, it's necessary to rethink the lifestyle of society and its quality of life around public and urban spaces, researching in 2019 mobility Bogotá survey's; on average, they record trips is 14,069 of foot trips, between 10 and 20 minutes according to the travel patterns in the distinctive transport analysis areas at the zonal planning unit Chapinero Centro in the city of Bogotá. In spite of having great social and economic dynamics, this UPZ has very small platforms that make it difficult to comply with social distancing, necessary to mitigate the Coronavirus pandemic. For this reason, this article carried out a geographical analysis of this area, using the data provided by the vulnerability map of the Coronavirus website in Colombia of the Departamento Nacional de Planeación, the Instituto de Evaluación Tecnológica en Salud and the Departamento Administrativo Nacional de Estadística. This geographical analysis allowed calculating the variables of the population indicator per block, the maximum population per platform and the criterion of compliance with social distance. These variables allowed designing the vulnerability and social distancing maps. It was evident that $37 \%$ of the platforms do not comply the 2-meter distance between people, so it was proposed to expand them by 3.5 meters and reduce this same value in vehicle space, to design the effective social distance map. It is concluded that the extension of the platforms makes it possible to make flexible use of the streets oriented to a healthy distance, which could be complemented by an accessibility study and a vehicle traffic study that measure the impact of this intervention.

Keywords: Pedestrian mobility, Chapinero, social distancing, coronavirus.

\section{Resumen}

Dada la situación de pandemia actual que genera Covid-19, es necesario reevaluar el estilo y

${ }^{1}$ Corresponding E-mail: js.sanchez14@uniandes.edu.co 
calidad de vida de la sociedad en torno a los espacios públicos y urbanos. Investigando en la encuesta de movilidad de Bogotá 2019, en promedio se registran 14.069 viajes a pie de entre 10 y 20 minutos de acuerdo al análisis de los patrones en las áreas de análisis de transporte distintivas de la unidad de planificación zonal Chapinero Centro de la ciudad de Bogotá. A pesar de tener una gran dinámica social y económica, esta UPZ cuenta con plataformas muy pequeñas que dificultan el cumplimiento del distanciamiento social, necesario para mitigar la pandemia de Coronavirus. Por tal motivo, este artículo realizó un análisis geográfico de esta zona, utilizando los datos proporcionados por el mapa de vulnerabilidad del sitio web de Coronavirus en Colombia del Departamento Nacional de Planeación, el Instituto de Evaluación Tecnológica en Salud y el Departamento Administrativo Nacional de Estadística. . Este análisis geográfico permitió calcular las variables del indicador de población por cuadra, la población máxima por plataforma y el criterio de cumplimiento de la distancia social. Estas variables permitieron diseñar los mapas de vulnerabilidad y distanciamiento social. Se evidenció que el 37\% de las plataformas no cumplen con la distancia de 2 metros entre personas, por lo que se propuso ampliarlas en 3,5 metros y reducir este mismo valor en el espacio vehicular, para diseñar el mapa de distancia social efectivo. Se concluye que la ampliación de los andenes permite hacer un uso flexible de las calles orientadas a una distancia saludable, lo que podría complementarse con un estudio de accesibilidad y un estudio de tráfico de vehículos que midan el impacto de esta intervención. Palabras clave: Movilidad peatonal, chapinero, distanciamiento social, coronavirus

\section{INTRODUCTION}

The locality of Chapinero is made up of five zonal planning units (UPZ), which are divided into fifty neighborhoods and the village of El Verjón Bajo as a rural planning unit. The five UPZs in Chapinero are El Refugio (UPZ 88), San Isidro Patios (UPZ 89), Pardo Rubio (UPZ 90), Chicó Lago (UPZ 97) and Chapinero Centro (UPZ 99) [1]. According to the statistical bulletin of Business Dynamics of Bogotá in December 2019, Chapinero is the third locality in Bogotá with the highest number of registered companies, adding $10.1 \%$ of business participation in 2019. The Bogota companies that are in Chapinero represent the following percentages according to the economic sector, occupying the first place in the professional services sector with $15.3 \%$ and the sixth position in the commerce sector with $6 \%$. Likewise, the companies that are in the locality according to their size, that is to say by the staff plant and the level of assets, occupies the fourth place of the micro-enterprises with $9.8 \%$, the first place of the small companies with a $18.1 \%$, first place for medium-sized companies with $21.5 \%$ and first place for large companies with $24.9 \%$ [2]. These figures show that Chapinero Centro is a commercial UPZ, located in the southwest of the locality of Chapinero, with an extension of 159.3 hectares that represents $12.1 \%$ of the total area of the locality. Regarding its limits, Chapinero Centro limits to the north with UPZ Chicó Lago, to 
the south with UPZ Sagrado Corazón, to the west with UPZ Los Alcázares, Galerías and Teusaquillo and to the east with UPZ Pardo Rubio [3].

Now, according to the Statistical Bulletin number 5 of the District Planning Secretariat [4], this UPZ is of a commercial nature when presenting as activities according to the international uniform industrial classification (ISIC), i) 56 companies of agriculture, livestock, hunting, forestry and fishing, ii) 34 mining and quarrying companies, iii) 665 manufacturing industries, iv) 448 construction companies, v) 2350 wholesale and retail trade establishment, vi) 197 transport and storage companies, vii) 868 lodging and food service company, viii) 606 information and communication companies, ix) 371 companies of financial and insurance activities, x) 520 companies of real estate activities, xi) 1352 companies of professional, scientific and techniques, xii) 622 companies of administrative and support services activities, and xiii) 169 companies of education activities.

According to the socioeconomic dynamics of this UPZ, the present study seeks to present an effective social distancing map, in which an intervention per block is proposed that allows to have platforms for pedestrian traffic and connectivity with the public space, as well as where they have enough area for pedestrian mobility based on social distancing that allows mitigating even a high level of vulnerability, given the growing contagion of coronavirus at Chapinero, also given that due to this situation has opted for the adherence of the trade to the public space and / or on the sidewalk, within the framework of the "Bogotá under the open sky" strategy.

The inverted mobility pyramid [5], proposes the prioritization of vulnerable road users headed by pedestrians, which is why complying with the geometric design of streets for pedestrians with commercial corridors stipulated by NACTO in the Global Street Design Guide, it's necessary to specify a clear path of 3 meters or more to allow a continuous flow of pedestrians and enable people to comfortably pass one another, providing flexibility and dedicated space on the sidewalk adjacent to the clear path [6].

\section{MATERIALS AND METHODS.}

The maps to show the urban design proposal were created in ArcGIS, a suite of programs from the Environmental Systems Research Institute. This software suite was selected for containing applications for the capture, edition, analysis and design of the geographic information that was collected from UPZ 99 Chapinero Centro. This geographic information was obtained from the Vulnerability Map of the Coronavirus Colombia website [7], created by the Departamento 
Nacional de Planeación (DNP), the Instituto de Evaluación Tecnológica en Salud (IETS) and the Departamento Administrativo Nacional de Estadística (DANE).

This vulnerability map uses the Vulnerability index suggested by DeCaprio, Gartner, Bugess, Kothari, Sayed and McCall [6] and UNDP [8] as a methodology. In this sense, the DANE methodological note [9] presents its own index and names it the Vulnerability Index per block, which uses demographic variables and comorbidities to identify the population in Colombian municipalities, which could suffer greater complications in case of getting COVID-19, due to its demographic characteristics and health conditions. These variables were obtained through information of the administrative records from the Archivo Nacional de Identificación (ANI), the Registro Civil de Nacimiento (RCN), the Registraduría Nacional del Estado Civil, the Base de Datos Única de Afiliación en Salud (BDUA) and the Registros Individuales de Prestación de Servicios de Salud (RIPS).

Once the information from the vulnerability map has been extracted, an information crossing is carried out in relation to the platforms, identifying the accessibility in pedestrian mobility to access or interact under the current restrictions of social distancing where a minimum of 1 metre is established for interaction with other individuals, because as a result of the health problems brought about by the COVID-19 virus pandemic, it is sought that the probability of contagion is reduced, avoiding all types of agglomeration. From this, we worked with minimum distances for the three types of vulnerabilities (low, medium and high) found in the UPZ. In this sense, this research seeks to analyze the geographic information of the UPZ Chapinero Centro to evaluate its compliance with the proposed measures and restrictions.

From these data, two maps are designed, a first vulnerability map and a second distance map. Finally, according to the vulnerability map and the distance map, it is proposed to design a third intervention map, in which the solution of a flexibility of the streets will be proposed to expand the pedestrian space to half of the current vehicular stream, thus achieving that the platforms that do not comply with the social distance manage to comply

\section{RESULTS AND ANAYLYSIS OF RESULTS}

The daily modal distribution in the transport analysis areas of Chapinero Centro, in Figure 1. show clear data about the dynamics of pedestrians; As around 14,069 daily trips on foot are registered, with records of average travel times between 10 to 20 minutes. This means that the generation of trips in the study area is carried out by the main mode of walking on foot, which 
affects that due to connectivity the inhabitants of the area require passable public spaces, with connectivity and comfort for them. [10]

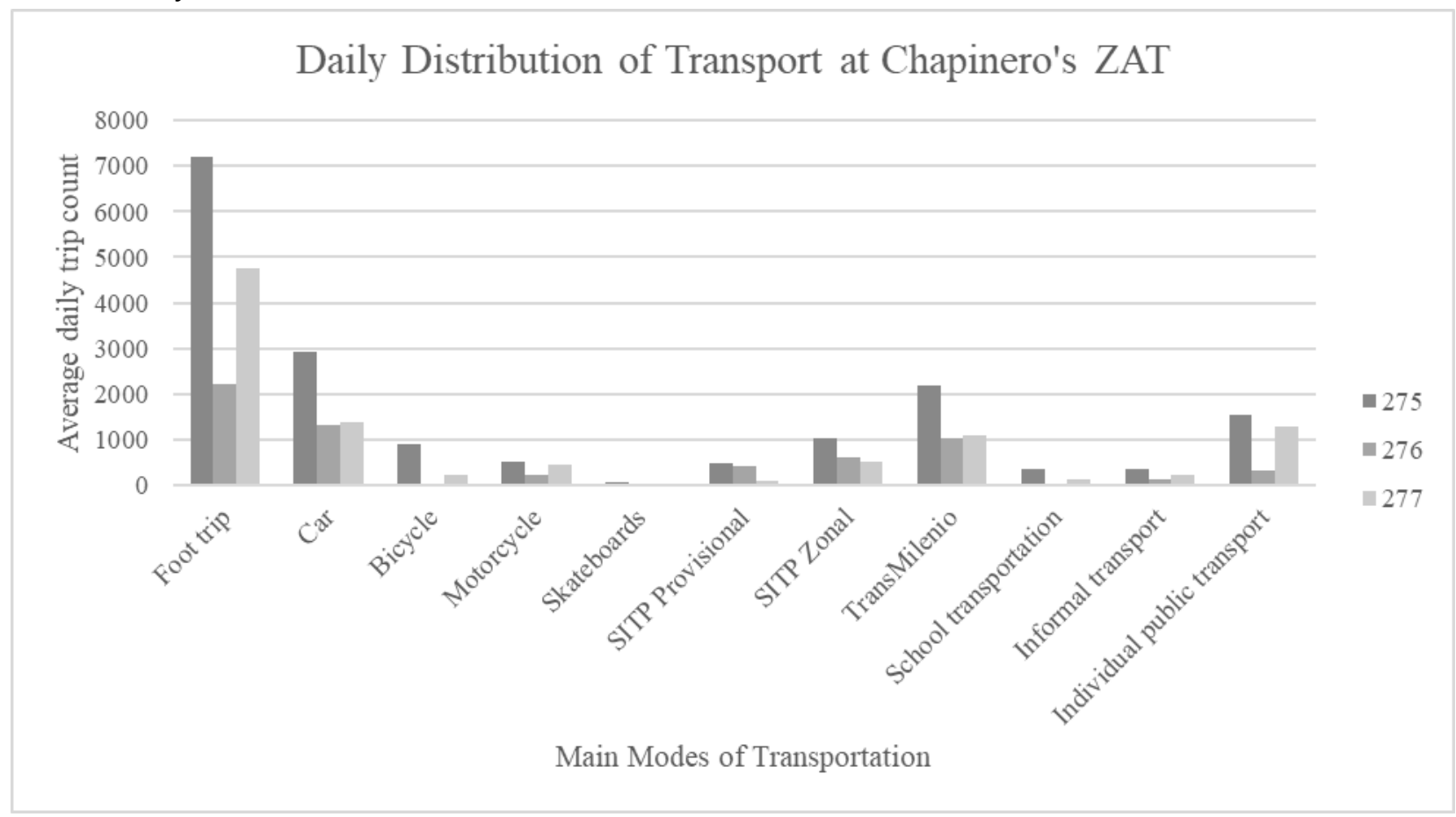

Figure 37 Daily modal distribution at Chapinero's ZAT. Authors' elaboration.

The UPZ Chapinero Centro has 102 blocks, which can be classified by the level of vulnerability, whose classification was taken from the vulnerability map of DANE [11], with new levels of vulnerability of its own making, which were low vulnerability (green ), medium (yellow) and high (red) as can be seen in Figure 1-a. In this regard, it was observed that $49 \%$ of the apples present low vulnerability, $36.2 \%$ medium vulnerability and $14.7 \%$ high vulnerability. 

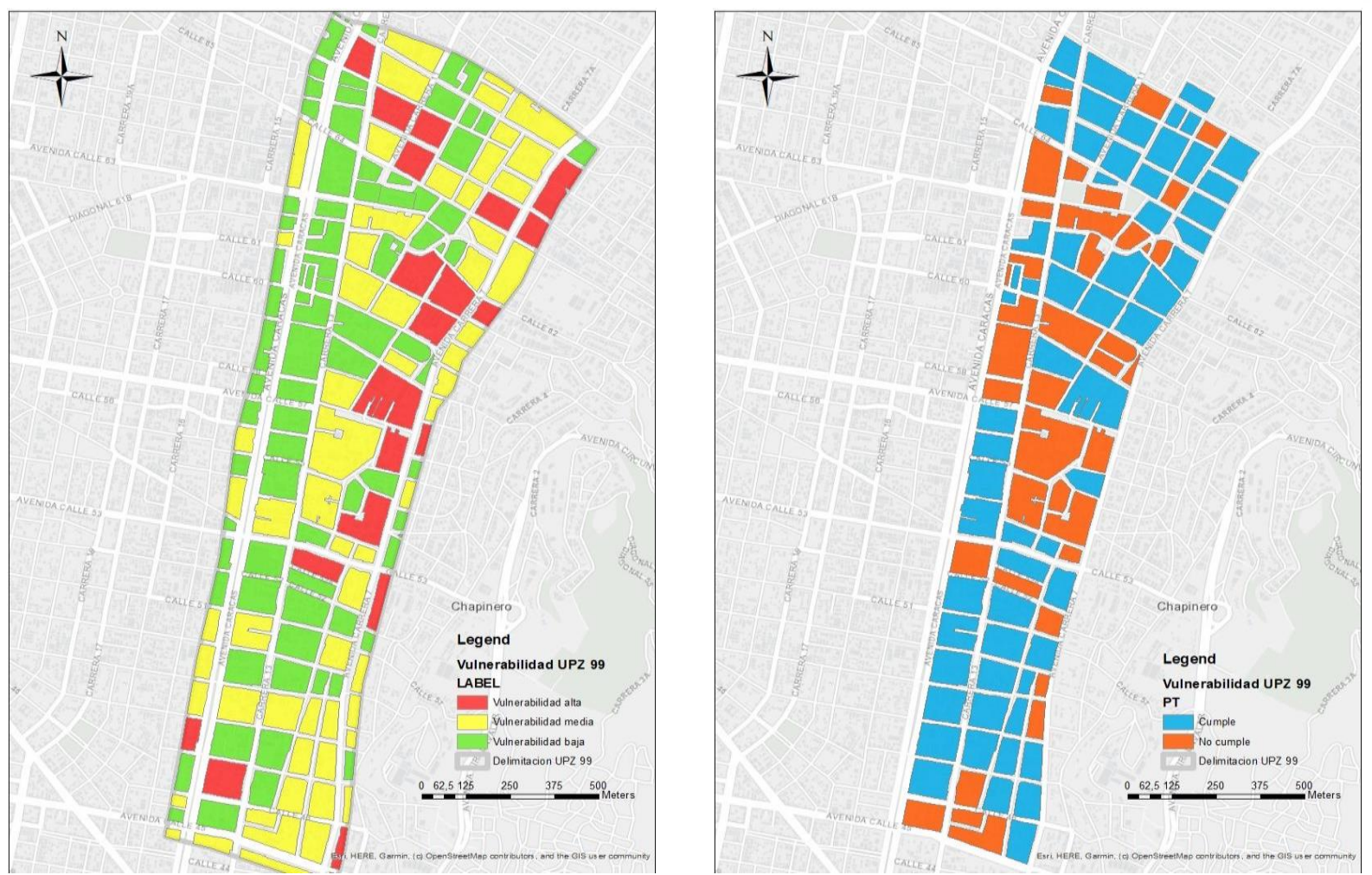

Figure 38 Maps created from UPZ 99 a) vulnerability and b) distancing. Authors' elaboration.

Modeling the platforms to the same number of blocks, there are 102 platforms with areas from 4 $\mathrm{m}^{2}$ to $1955 \mathrm{~m}^{2}$. Therefore, according to the area of each platform and the total population of the UPZ (18,121 habitants), it was possible to estimate the resident population residing in each block. The population indicator was obtained from equation 1, whose dividend is the area of the platform and the divisor is its distance indicator. This indicator of distance is understood as the square meters of distance that each person should have according to the vulnerability of the block, being $1 \mathrm{~m}^{2}$ for low vulnerability, $2 \mathrm{~m}^{2}$ for medium vulnerability and $3 \mathrm{~m}^{2}$ for high vulnerability. The population indicator (in number of habitants) showed that the individual population of each platform ranges between 4 and 978 habitants.

\section{Area $\left(m^{2}\right)$ / Indicator of distance $\left(m^{2}\right)$}

Equation 1. Population indicator of each block.

Likewise, to determine the average capacity of the UPZ 99 platforms, the maximum population per platform was estimated using equation 2, which has as a dividend the total population of the UPZ which is 18121 and as a divisor the total number of platforms which is 102, which results in 177.65 habitants. 
Total population in the UPZ 99 / Total number of platforms in the UPZ 99

Equation 2. Maximum population per platform.

Now, by articulating the area, the population indicator and the distance indicator of each platform, it was possible to model the social distance map. The criterion of compliance with social distancing allowed identifying those blocks that comply the minimum social distancing, which was estimated from equation 3.

\section{Population indicator $\leq$ Maximum population per platform}

Equation 3. Criterion for compliance with social distancing.

The distancing map (see Figure 1-b) showed that in UPZ 99, 63\% of the platforms comply with social distancing (blue color) while $37 \%$ of the platforms do not comply with social distancing (orange color).
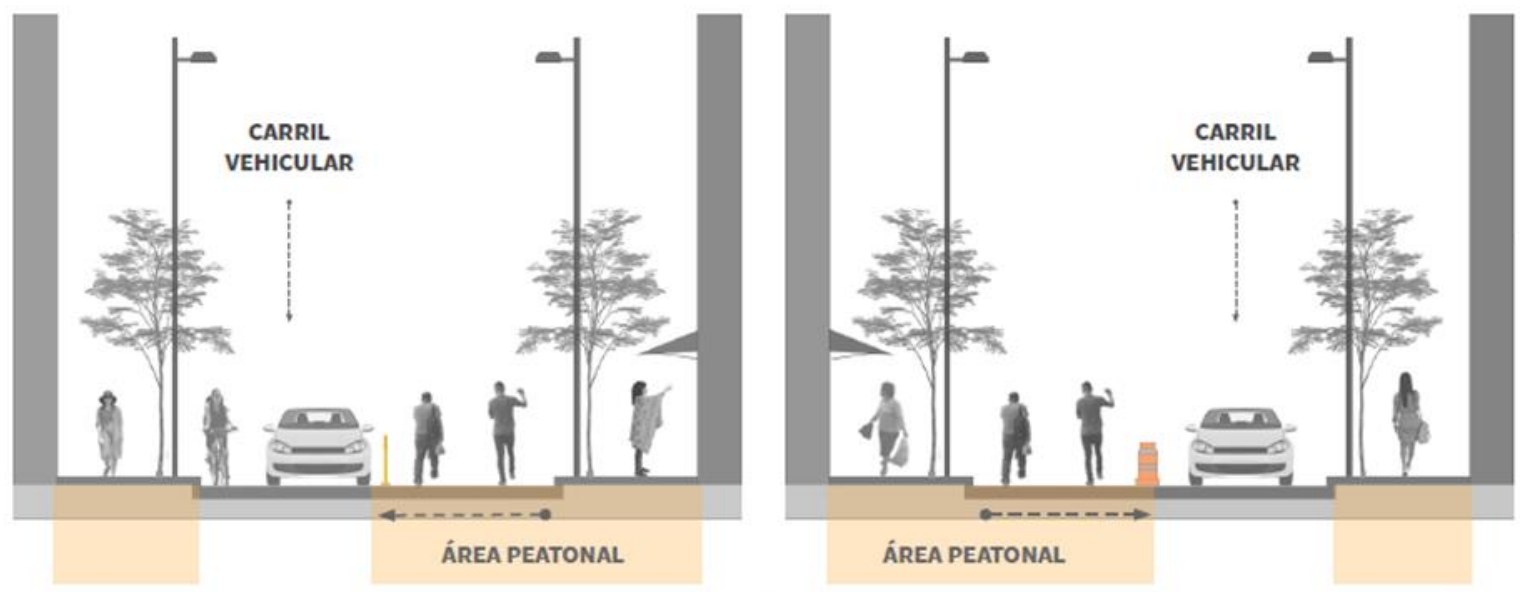

Figure 39 Flexibility of streets in the perimeter of action for pedestrians. Taken from [9].

From a realignment of the platforms that did not comply the distance, as presented in Figure 1-b, the expansion is carried out at approximately about 3.5 meters added to the trafficable public space for pedestrians as exemplified in Figure 2. This figure is part of the proposal for a flexibility of the streets in its perimeter of action of the Plan to reopen the Historic Center of CDMX [12], a proposal that served as a reference to obtain proper compliance with social distancing in pedestrians in UPZ Chapinero Centro (see Figure 3). 


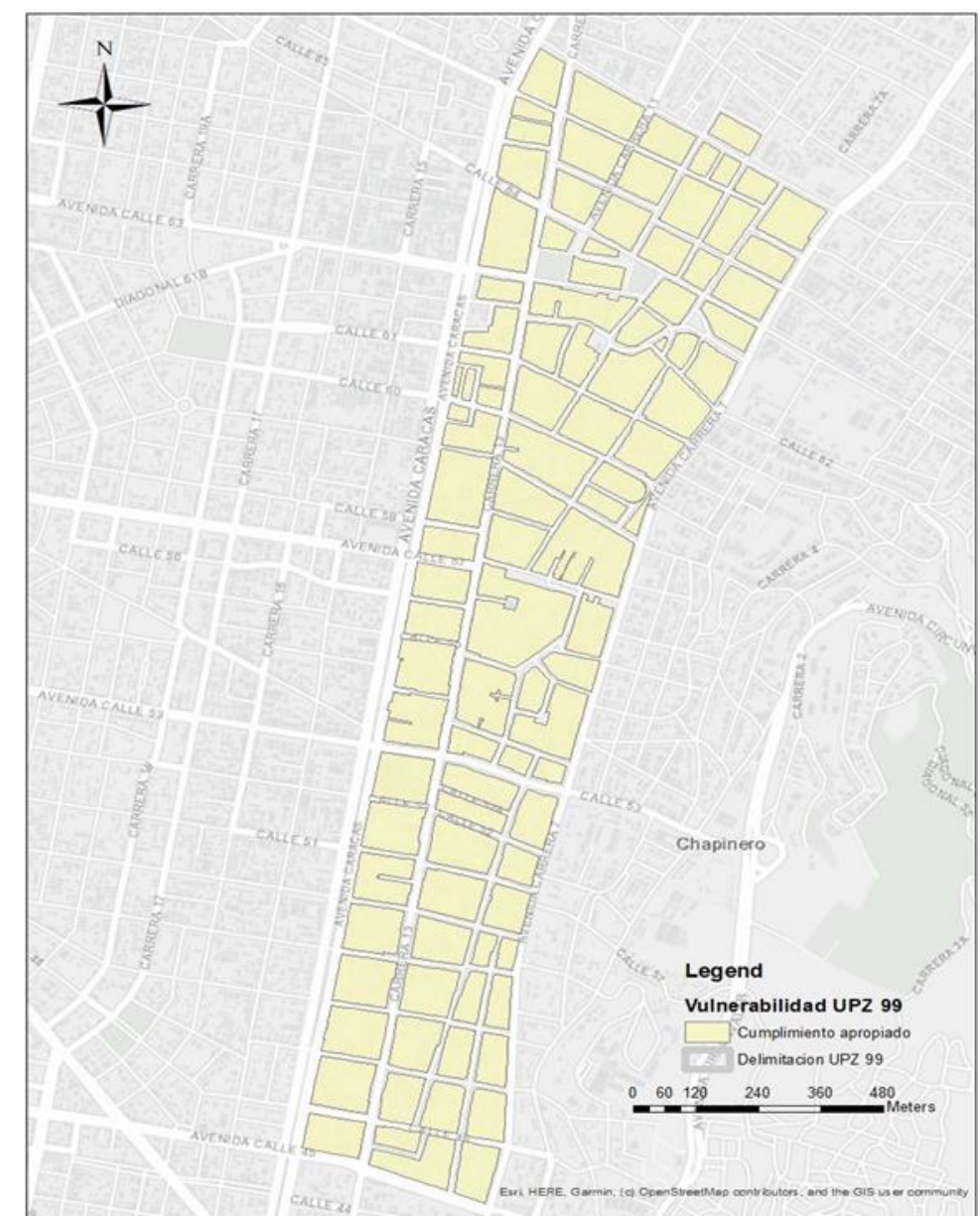

Figure 40 Map of effective social distancing in UPZ 99. Authors' elaboration.

\section{CONCLUSIONS}

A geographical analysis of the UPZ Chapinero Centro was achieved, which allowed the creation of vulnerability and distance maps. Based on these maps, an intervention proposal was made to create scenarios with enough space to face high levels of vulnerability and comply with the social distancing norm. This resulted in an effective social distancing map, with an extension of 3.5 meters on those platforms that did not comply the criteria for compliance with social distancing, which represented $37 \%$ of the total platforms studied. This proposal could be complemented with a study of vehicular traffic that is relevant for those road corridors that were modified as a result of the widening of the platforms, so that not only the social distancing of pedestrians is analyzed, but also the vehicular traffic in these spaces. Finally, as future work, it is proposed to carry out an accessibility study on these extended platforms, in order to guarantee the pedestrian mobility of people with disabilities. 


\section{REFERENCES}

[1] Alcaldía local de Chapinero, "UPZ de la localidad de Chapinero", $2020 . \quad$ Available: http://www.chapinero.gov.co/content/upz-la-localidadchapinero

[2] Secretaría Distrital de Planeación, “Boletín estadístico Dinámica Empresarial de Bogotá IV trimestre de 2019", 2020. Available: http://www.sdp.gov.co/system/tdf/repositoriodice/dice201-dinamicaempresarial31122019.pdf?file $=1 \&$ type $=$ node $\&$ id $=22271 \&$ force $=1$

[3] Secretaría Distrital de Planeación, "Diagnóstico de los principales aspectos territoriales, de infraestructura, demográficos y socioeconómicos: Chapinero 2017", 2018. Available:

http://www.sdp.gov.co/system/tdf/repositoriodice/dice064-monografiachapinero2017_vf.pdf?file=1\&type=node\&id=18966\&force $=1$

[4] Secretaría Distrital de Planeación, "Boletín estadístico número 5", $2018 . \quad$ Available: http://www.sdp.gov.co/gestion-estudiosestrategicos/informacion-cartografia-yestadistica/consulta?page $=1$

[5] Robledo Restrepo, J. M., \& Galarza Molina, D, "Movilidad sostenible en acción", 2017.

[6] NACTO., I, “Urban Street Design Guide”, 2013.
[7] Coronavirus Colombia, "Mapa de Vulnerabilidad", $2020 . \quad$ Available: https://coronaviruscolombia.gov.co/Covid19/estadistic as-covid-19/mapa-vulnerabilidad.html

[8] D. DeCaprio, J. Gartner, T. Burgess, S. Kothari, S. Sayed, C. McCall, "Building a COVID-19 Vulnerability Index", 2020. Available: https://arxiv.org/abs/2003.07347

[9] Programa de las Naciones Unidas para el Desarrollo (PNUD), “UNDP GRANDATA", 2020. Available: https://covid.grandata.com/methodology

[10] Secretaría Distrital de Movilidad, "Encuesta de movilidad", 2019.

[11] Departamento Administrativo Nacional de Estadística (DANE), “Nota metodológica del Índice de Vulnerabilidad por Manzana", 2020. Available: https://www.dane.gov.co/files/comunicados/Nota_met odologica_indice_de_vulnerabilidad.pdf

[12] Secretaria de Movilidad del Gobierno de la Ciudad de México, "Plan de apertura del Centro Historico de la CDMX - hacia la nueva normalidad en el $\mathrm{CH}^{\prime \prime}, 2020$. Available:

https:/semovi.cdmx.gob.mx/storage/app/media/200627 _MovilidadJDG_NuevaMovilidadCH.pdf 


\title{
CARACTERIZACIÓN DE LAS PROPIEDADES TÉRMICAS DEL BAHAREQUE EMBUTIDO EN TIERRA CON ESTERILLA DE GUADUA
}

\section{Characterization of the thermal properties of the bahareque embuided on earth with guadua mat}

\author{
Gómez, María1; Molina, Daniella; Silva, Héctor; Herrera, Carlos ${ }^{2}$ \\ Universidad del Valle
}

\section{Resumen}

El bahareque tradicional embutido en tierra es una técnica constructiva mixta que utiliza materiales renovables como la guadua, madera, tierra y es mezclado ocasionalmente con fibras vegetales y estiércol de vaca. Se busca dar a conocer la efectividad del aislamiento térmico que posee el bahareque tradicional embutido en tierra con esterilla de guadua, sin tener en cuenta el acabado final o revoque, demostrando su capacidad de ahorro energético para brindar confort térmico al interior de edificaciones. Mediante pruebas de laboratorio, se analizan diferentes mezclas de tierra recolectadas en visitas de campo y de esterilla de guadua. Posteriormente se construyen probetas de ensayo, fabricadas con la tierra más óptima, que son sometidas a la prueba de placa caliente resguardada. El resultado determina que el coeficiente de conductividad térmica de un muro de bahareque de $13 \mathrm{~cm}$ de espesor con doble cara de esterilla de Guadua es de 0,303 $\mathrm{W} / \mathrm{m}^{\circ} \mathrm{C}$. Una vez calculado este valor, se procede a validar la ecuación teórica de Fourier sobre la conducción del calor, encontrando así la constante de conductividad térmica del bahareque. Con este dato, ( $\mathrm{K}=$ constante) se hallan los valores de resistencia térmica $1.17^{\circ} \mathrm{C} / \mathrm{W}$ y de transferencia térmica $11.1 \mathrm{~W}$ del sistema constructivo. Se propone incluir la esterilla de guadua como componente del sistema, lo que mejorará la eficacia del confort y potenciará la utilización de materia prima local y reciclable, legitimando el valor social, cultural, económico, ambiental y de confort térmico de la construcción con materiales vernáculos, haciéndolo viable para implementarlo rural y masivamente.

Palabras clave: bahareque, tierra, guadua, constante térmica, resistencia térmica

\footnotetext{
${ }^{1}$ Correo electrónico de contacto: arq.camilag@gmail.com

${ }^{2}$ Correo electrónico de contacto: daniellam1923@gmail.com
} 


\section{INTRODUCCIÓN}

¿Qué tan efectivo es el confort térmico del bahareque tradicional embutido en tierra con esterilla y cómo influye en las condiciones percibidas al interior de edificaciones construidas con esta técnica?

El material de construcción más aprovechado alrededor del mundo ha sido la tierra, utilizado en diversas técnicas existentes como el adobe, la tapia y el bahareque. A pesar de ello, durante los últimos siglos, se ha producido un desuso considerable del material aun conociendo los beneficios y propiedades de carácter social, económico y de confort que posee.

La aparición de nuevas tecnologías y la industrialización ha apartado al hombre de la tradición de construir con tierra ya que se ha estigmatizado su aprovechamiento, convirtiéndolo en un material sinónimo de pobreza. Las comunidades indígenas y campesinas están transformando sus antiguas viviendas y lugares de encuentro en muros "de material" (ladrillo cocido, hierro y concreto). La especulación de estos nuevos materiales y tecnologías ha debilitado a niveles exagerados la tradición constructiva.

Sin embargo, en los últimos tiempos, se ha despertado un interés creciente e importante alrededor del mundo por la utilización de recursos renovables y sostenibles, lo que implicaría el deber de recuperar las técnicas de construcción ancestrales que no generan un impacto negativo en el medio ambiente.

La variabilidad climática es evidente, la sobrepoblación y con ella la sobreexplotación de los recursos naturales, superan la capacidad de sus sistemas, produciendo un exagerado desperdicio de residuos sólidos [1]. Las actividades humanas están causando alteraciones en estos sistemas, como el aumento de la temperatura global, el deshielo de los glaciares que lleva al aumento del nivel del mar y la acidificación de los océanos, todo esto como consecuencia de los gases efecto invernadero que quedan atrapados en la biosfera terrestre.

La industria de la construcción es responsable de consumir casi el 40\% de la energía del planeta y además produce el 30\% de los gases efecto invernadero [1]. Adicional a esto, el 50\% de los recursos no renovables son utilizados para construir y para crear sensaciones de confort internas óptimas, mediante sistemas artificiales de enfriamiento o calefacción. Por esta razón la arquitectura tiene la 
obligación de crear estrategias que contrarresten el impacto negativo a través del diseño o del uso de materiales que contengan un alto aislamiento térmico, sean locales y no necesariamente requieran mano de obra capacitada.

La tierra como material de construcción sólo necesita agua para su transformación, haciendo que las arcillas, que hacen parte de su granulometría, se activen y cumplan la función de aglomerantes. A continuación, el secado es natural, por medio de evaporación del agua y las corrientes de aire que transformarán el barro de estado plástico a estado sólido. El ladrillo cocido es, igualmente, una mezcla de tierra con agua y aditivos que posteriormente estará sometida a altas temperaturas que producirán reacciones físicas y químicas logrando el resultado mecánico deseado. Sin embargo, el grado de contaminación de los hornos y el combustible utilizados en las ladrilleras es muy elevado [1].

La tierra como materia prima tiene más beneficios que perjuicios: está en todos los lugares de la superficie terrestre, necesita solo el 1\% de gasto energético [2], evita el uso de la madera y con esto la deforestación de los bosques, es completamente reciclable para ser reutilizado en otra construcción o para ser reabsorbido por el medio ambiente, está libre de sustancias tóxicas si no ha tenido contacto con agentes contaminantes, no produce emisiones de $\mathrm{CO} 2$, es un excelente aislante térmico y acústico y regula la humedad interior, entre otras. No obstante, la tierra tiene algunos inconvenientes como la limitante de altura de las edificaciones, el mantenimiento constante para su conservación y la perdida de la tradición de este tipo de materiales y construcciones. Ahora bien, arquitectos como Gabriel Barbeta ha investigado la estabilización de la tierra [3] como material constructivo, definiendo guías que potencien y fomenten el interés por estos sistemas constructivos vernáculos.

Si bien es cierto que el mercado desarrolla nuevos métodos de aislamiento de la temperatura y el ruido, la tierra sigue teniendo vigencia y ayuda a ahorrar energía que es utilizada para crear sensaciones favorables, estando directamente relacionado con el desarrollo de construcciones más sostenibles y con altos estándares de calidad. Por estas razones, la propuesta es desarrollar y documentar cuantitativamente y cualitativamente, la efectividad de la tierra y la esterilla de guadua para aislar el calor, de esta manera poder hallar la conductividad, la resistencia y la transferencia térmica del bahareque tradicional embutido en tierra con esterilla de guadua, datos que no se han calculado hasta la fecha. Posteriormente se comparan resultados del bahareque con los sistemas constructivos actuales y de esta manera poder llegar a conclusiones que respalden las 
afirmaciones sensoriales sobre el confort térmico del bahareque descritas por quienes habitan lugares construidos con estas técnicas.

\section{MATERIALES Y METODOS}

Durante la investigación se llevan a cabo tres fases que componen la metodología y hacen referencia a los objetivos específicos de la investigación. El proceso es una sucesión de actividades y técnicas que llevarán a métodos de ensayo en laboratorios para determinar el coeficiente de conductividad térmica, necesario para conocer la transferencia de calor del exterior al interior de un muro en bahareque embutido en tierra con esterilla.

\section{Fase 1:}

- Visitas de campo a 5 municipios de los departamentos del Cauca y Valle del CaucaColombia y determinación del tamaño de las partículas que componen el suelo:

Comienza con visitas de campo a municipios de la región sur occidente de Colombia, comprendida en los departamentos de Cauca y Valle del Cauca. Los lugares escogidos tienen características geográficas diferentes, tales como valle geográfico del río Cauca, ladera y alta montaña. Los municipios seleccionados fueron: Santander de Quilichao en el departamento del Cauca y Calima - El Darién, El Cairo, Robles, y Cali en el departamento del Valle del Cauca.

En cada uno de los municipios visitados se recolecta una muestra de tierra del embutido de aproximadamente $6 \mathrm{Kg}$, la cual se extrae de edificaciones existentes que hayan sido construidas con la técnica de bahareque embutido en tierra.

La prueba de granulometría se realiza para determinar la distribución por tamaños de partículas que tiene la masa del suelo en peso y porcentaje, correspondiente a suelo grueso (grava y arena) y suelo fino (limos y arcillas).

Para esto se emplean los siguientes métodos de laboratorio, basados en sus respectivas normas: Granulometría por tamizado (I.N.V. E - 123 - 13[4], ASTM D 422-63[5], AASHTO T 88 del 2004)[6], Hidrometría (ASTM D-422-63 Revisión 2007)[5], Gravedad específica (I.N.V. E - 128 13, ASTM D 854-10, AASHTO T100- 70)[6], Limites de Atterberg o de consistencia: Limite líquido, límite plástico e Índice de plasticidad (ASTM D 4318-10)[5]. Cada una de estas pruebas se realiza en el Laboratorio de Suelos y Pavimentos de la Facultad de Ingeniería en la Universidad del Valle. 


\section{Fase 2:}

- Prueba de placa caliente resguardada (ASTM C177). Toma de mediciones de transferencia de calor para las dos materias primas: tierra y esterilla de guadua:

Una vez escogida la tierra mejor gradada durante el proceso de determinación de los tamaños de las partículas, Darién 2 (2), se fabrican cuatro probetas de tierra, extraída del mismo lugar, de $30 \mathrm{~cm} \times 30 \mathrm{~cm} \times 10 \mathrm{~cm}$ de espesor y se adquieren seis probetas de esterilla de guadua angustifolia de $30 \mathrm{~cm} \mathrm{X} 30 \mathrm{~cm} \mathrm{X} 2 \mathrm{~cm}$ de espesor. Para llevar a cabo la prueba de conductividad térmica, se ubican las probetas de tierra y de esterilla entre la placa caliente y la placa fría, una al lado izquierdo y otra al lado derecho. Finalmente, es indispensable aislar el perímetro de la muestra para evitar la pérdida del calor por la periferia. La prueba cuenta con un voltaje constante que será aplicado para suministrar el calor y un caudal de agua que debe mantener las placas frías a una temperatura baja.

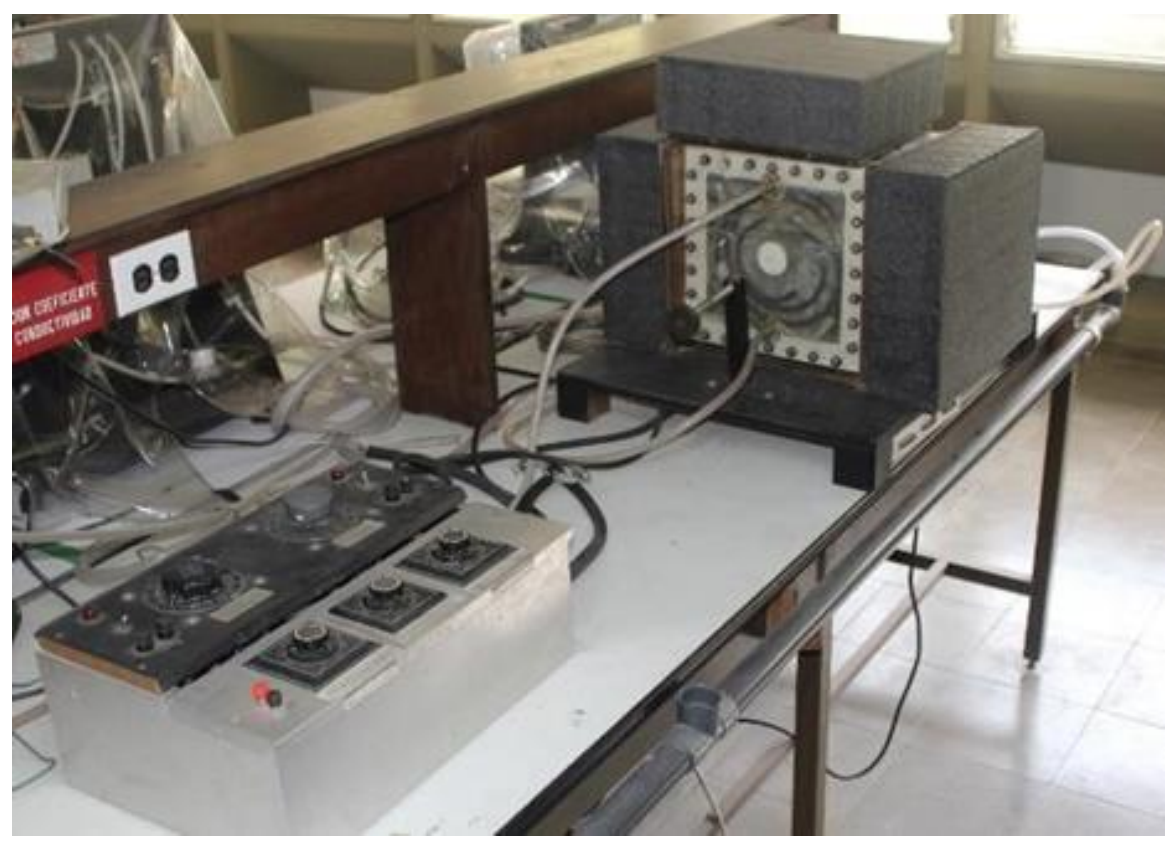

Figura 23 Máquina de conductividad térmica. Molina, D. 2017

El ensayo se llevó a cabo bajo la norma "ASTM C177: Standard Test Method for Steady-State Heat Flux Measurements and Thermal Transmission Properties by Means of the Guarded Hot-Plate Apparatus" el método tuvo en cuenta los rangos de incertidumbre (la inexactitud de los equipos, las condiciones ambientales dentro del laboratorio y la elaboración de las probetas). También se realizó la prueba para hallar el estado estable de las probetas y así determinar la temperatura y la cantidad de horas en las cuales las muestras no ganaban más calor. 
El procedimiento se realizó en el laboratorio de Transferencia de calor y frio de la Facultad de Ingeniería en la Universidad del Valle, el cual se ejecutó en condiciones ambientales iguales para cada probeta a ensayar.

Durante la prueba se tomaron mediciones de caudal, voltaje, temperatura de placa caliente izquierda, placa caliente derecha, placa fría derecha y placa fría izquierda. Al iniciar el ensayo de la probeta de tierra, la toma de datos se realizó cada hora durante las primeras 8 horas, después se tomaron cada media hora, hasta finalizar la prueba. Para las probetas de esterilla se tomaron los datos cada media hora, desde el inicio hasta el final de la prueba.

- Cálculos del coeficiente de conductividad térmica de los dos materiales, basados en la ecuación de la Ley de Fourier $K=\left(Q^{*} L\right) /\left(A^{*} \Delta T\right)$ :

El coeficiente de conductividad térmica se halla mediante la Ley de Fourier, despejando la variable K, así:

$$
K=\frac{Q * L}{A * \Delta T}
$$

Posteriormente se promedian los datos arrojados por la placa caliente y se determina un valor final.

Fase 3:

- Cálculo de la resistencia térmica de los dos materiales (Tierra y esterilla) y de todo el sistema constructivo:

La resistencia es la propiedad física que tienen los materiales de oponerse al flujo del calor y se determina por medio de la siguiente ecuación:

$$
R=\frac{L}{K * A}
$$

- Cálculo de la transferencia del calor y comparación de resultados obtenidos con otros sistemas constructivos:

Finalmente se calcula la cantidad de calor que es transferido de una cara de la probeta a la otra, 
en ambos materiales y a todo el sistema constructivo de bahareque embutido en tierra con esterilla de guadua a través de la siguiente ecuación:

$$
Q=\frac{\Delta T}{R}
$$

\section{RESULTADOS Y ANALISIS DE RESULTADOS}

La síntesis completa de los resultados obtenidos durante el proceso de determinación de los tamaños de las partículas y clasificación plástica del suelo se encuentra en la Tabla 1, en la cual se muestra detalladamente el porcentaje que proyectó cada uno de los componentes que conforman el suelo (Grava, arena, limo, arcilla) y la clasificación según el Sistema Unificado de Clasificación de Suelos (CH, CL, MH, ML, NP)

La tierra mejor gradada, teniendo en cuenta los porcentajes más cercanos a la literatura [7] fue la denominada Darién 2 (2), que se clasifica como un limo de alta plasticidad según su ubicación en la Carta de Casagrande.

Tabla 8 Resumen de fracciones granulométricas

\begin{tabular}{|c|c|c|c|c|c|c|}
\hline \multicolumn{7}{|c|}{ RESULTADOS FRACCIONES GRANULOMÉTRICAS } \\
\hline \multirow{2}{*}{ LUGAR } & \multirow{2}{*}{ MUESTRA } & \multicolumn{4}{|c|}{ FRACCIONES GRANULOMETRICAS \% } & \multirow{2}{*}{ CLASIFICACIÓN } \\
\hline & & GRAVA & ARENA & LIMO & ARCILLA & \\
\hline \multirow{5}{*}{ Darién } & Darien 1 & 1 & 24 & 38 & 36 & $\mathrm{MH}$ \\
\hline & Darien 2 & 4 & 51 & 44 & 2 & ML \\
\hline & Darien 2(2) & 15 & 58 & 15 & 12 & $\mathrm{MH}$ \\
\hline & Darien 3 & 1 & 72 & 25 & 2 & NO PLASTICO \\
\hline & Darien 4 & 5 & 27 & 49 & 19 & $\mathrm{MH}$ \\
\hline \multirow{6}{*}{ Cairo } & Cairo 1 & 23 & 57 & 16 & 4 & $\mathrm{ML}$ \\
\hline & Cairo 2 & 13 & 25 & 57 & 5 & ML \\
\hline & Cairo 3 & 1 & 31 & 66 & 2 & NO PLASTICO \\
\hline & Cairo 4 & 0 & 15 & 78 & 7 & NO PLASTICO \\
\hline & Cairo 5 & 1 & 55 & 43 & 1 & NO PLASTICO \\
\hline & \begin{tabular}{|l|} 
Cairo 6 \\
\end{tabular} & 0 & 31 & 50 & 19 & $\mathrm{ML}$ \\
\hline \multirow{3}{*}{$\begin{array}{c}\text { Santander de } \\
\text { Quilichao }\end{array}$} & Santander 1 & 1 & 34 & 47 & 18 & $\mathrm{MLCL}$ \\
\hline & Santander 2 & 1 & 15 & 68 & 16 & $\mathrm{ML}$ \\
\hline & Santander 3 & 1 & 14 & 52 & 33 & $\mathrm{ML}$ \\
\hline \multirow{5}{*}{ Robles } & Robles 1 & 1 & 41 & 46 & 13 & $\mathrm{MH}$ \\
\hline & Robles 2 & 0 & 25 & 45 & 30 & $\mathrm{ML}$ \\
\hline & Robles 3 & 0 & 6 & 64 & 30 & MH \\
\hline & Robles 4 & 1 & 9 & 56 & 34 & $\mathrm{MH}$ \\
\hline & Robles 5 & 3 & 29 & 39 & 29 & $\mathrm{ML}$ \\
\hline Cañas Gordas & Cali 1 & 0 & 16 & 58 & 26 & $\mathrm{MLCL}$ \\
\hline
\end{tabular}

La placa caliente está compuesta por dos superficies isotérmicas frías y una placa caliente entre 
ellas, todas protegidas por un solo material aislante que proporciona las condiciones térmicas apropiadas dentro del volumen de prueba para reducir el flujo de calor dentro de la máquina (disipación del calor). (Figura 2)

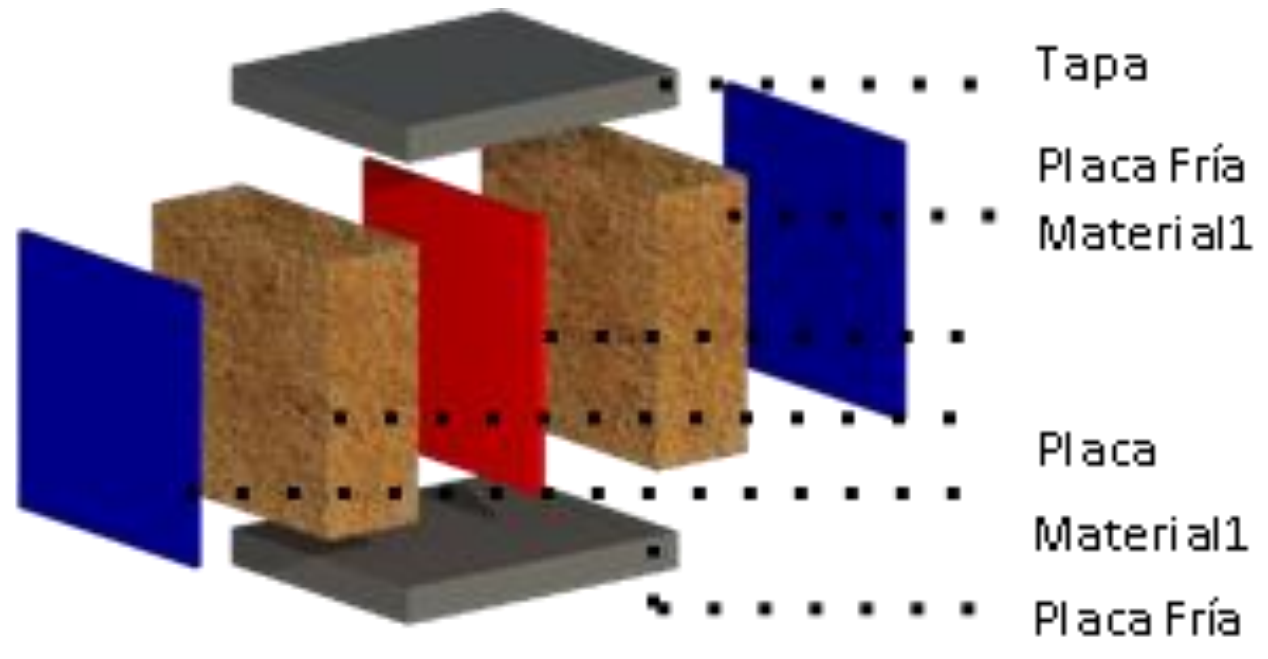

Figura 24 Esquema del equipo de placa caliente

Los resultados arrojados por la placa caliente son datos de temperatura que son promediados y finalmente se aplica la Ley de Fourier despejando K (Constante de conductividad térmica) especificados así:

La resistencia térmica para el material tierra, con un espesor de $9 \mathrm{~cm}$ y área de $1 \mathrm{~m} 2$ es 0,35 $\mathrm{m} 2 .{ }^{\circ} \mathrm{C} / \mathrm{W}$; y para el material esterilla de guadua con espesor de $2 \mathrm{~cm}$ y $1 \mathrm{~m} 2$ es $0,41 \mathrm{~m} 2 .{ }^{\circ} \mathrm{C} / \mathrm{W}$. En este sentido, la esterilla de guadua es superior comparada con la tierra en el momento de evitar el paso del calor

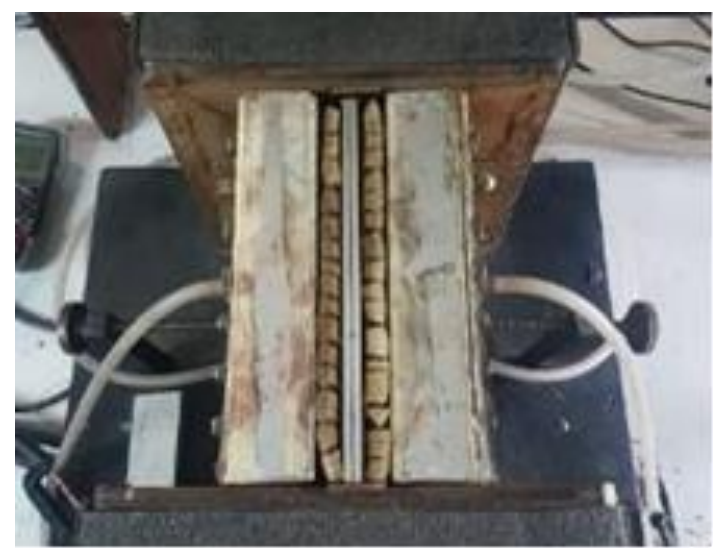

\begin{tabular}{|c|c|}
\hline PROBETAS & CONSTANTE (K) W/m.K \\
\hline P1-A & 0,047 \\
\hline P2-A & 0,045 \\
\hline P1-B & 0,051 \\
\hline P2-B & 0,050 \\
\hline P1-C & 0,051 \\
\hline P2-C & 0,051 \\
\hline PROMEDIO & 0,049 \\
\hline
\end{tabular}

Figura 25 Resultado de la constante de conductividad térmica 


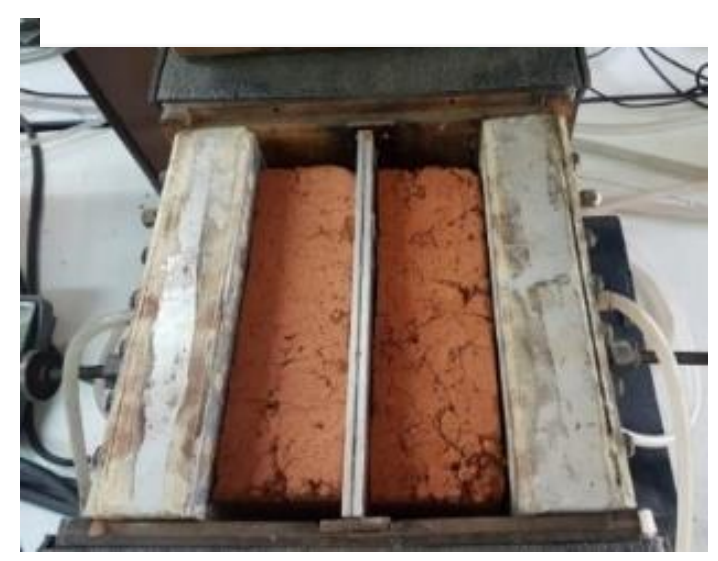

\begin{tabular}{|c|c|c|}
\hline \multirow{8}{*}{$\frac{\overleftarrow{\alpha}}{\frac{\alpha}{\underline{\underline{w}}}}$} & PROBETAS & CONSTANTE (K) W/m.K \\
\hline & $\mathrm{Pl}-\mathrm{A}$ & 0,258 \\
\hline & P2-A & 0,259 \\
\hline & P1-B & 0,264 \\
\hline & P2-B & 0,254 \\
\hline & $\mathrm{Pl}-\mathrm{C}$ & 0,249 \\
\hline & P2-C & 0,247 \\
\hline & PROMEDIO & 0,254 \\
\hline
\end{tabular}

Figura 26 Resultados de la constante de conductividad

Teniendo en cuenta los valores obtenidos para cada material, se simplifica el valor total en una suma de resistencias y de esta manera lograr el valor final del sistema constructivo, que está compuesto por una cara de esterilla a cada lado y en medio el embutido de tierra, así: $\mathrm{Rt}=\mathrm{R} 1 * 2+\mathrm{R} 2$, siendo $\mathrm{R} 1$ el valor de la esterilla de guadua y $\mathrm{R} 2$ el de la tierra

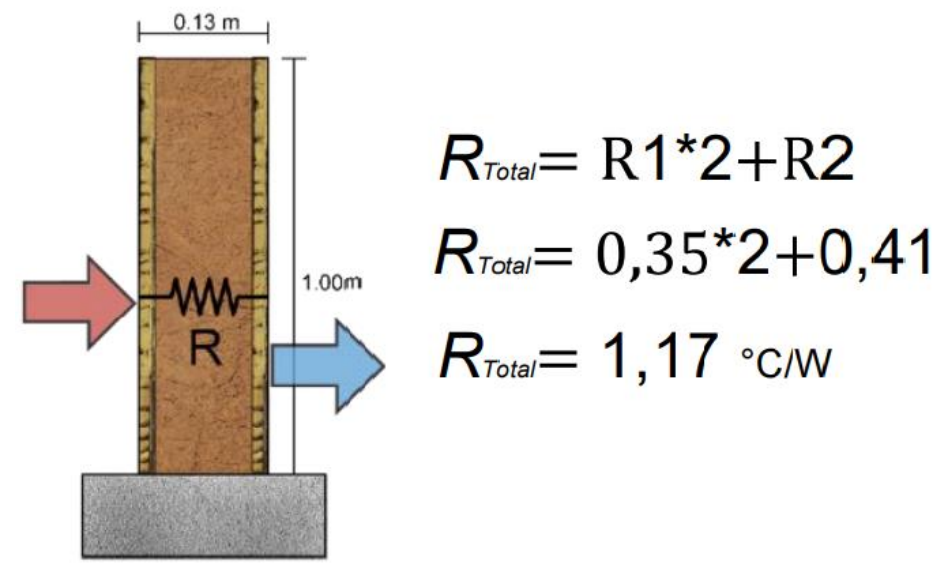

Figura 27 Resistencias totales

Finalmente, con el dato de la resistencia térmica del sistema constructivo del bahareque embutido en tierra con esterilla es posible calcular la transferencia del calor de un muro de $13 \mathrm{~cm}$ de espesor en condiciones climáticas hipotéticas; $36^{\circ} \mathrm{C}$ en el exterior y $23^{\circ} \mathrm{C}$ al interior, dando como resultado $\mathrm{Q}=11,1 \mathrm{~W}$. Posteriormente se realiza la comparación de la transferencia térmica del bahareque tradicional embutido en tierra con esterilla de guadua con otros sistemas constructivos, para 
determinar cuál de ellos tiene menor transferencia de calor y por ende es el más efectivo en términos de confort. Los resultados obtenidos de los otros sistemas constructivos fueron calculados del mismo modo que el del bahareque tradicional, con la diferencia de que el valor de la resistencia fue adquirido del libro de Cengel [8]: La madera con 11,4 W; El panel yeso con 16,5 W; El concreto 17,1W y El ladrillo con 86,7 W

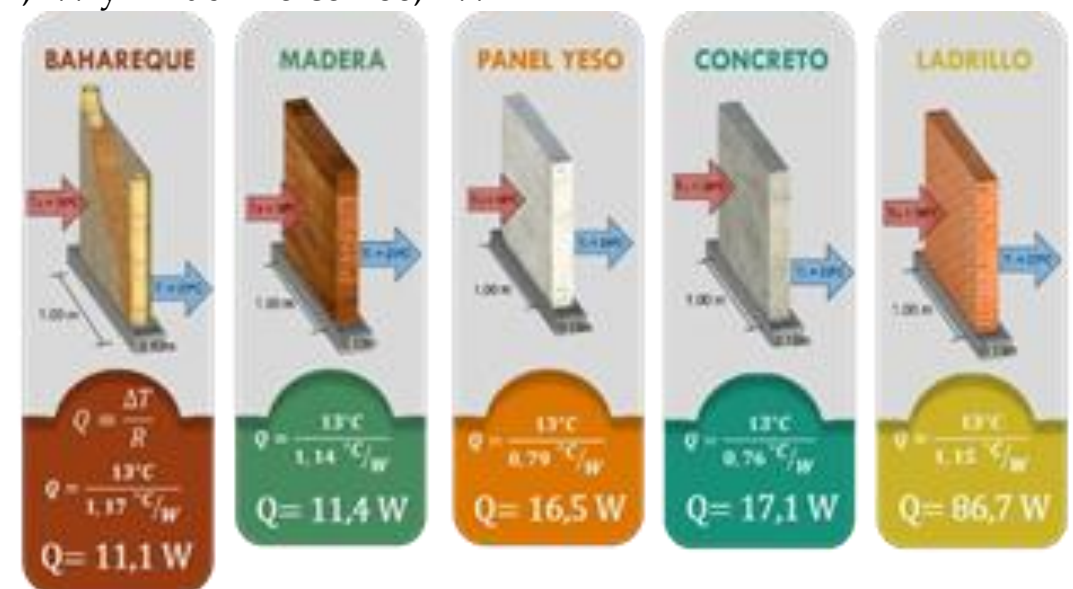

Figura 28 Comparación de transferencia de calor de sistemas constructivos - Elaboración propia. 2018

\section{CONCLUSIONES}

Es importante afirmar que el proceso evolutivo de la técnica de construir con tierra ha sido mayoritariamente empírico, ya que la tradición ha sido transmitida de generación en generación, valiéndose de materia prima local. Por esta razón se evidencian variaciones en los materiales y su utilización en diferentes técnicas constructivas. Del mismo modo, la tierra, al ser un material tan abundante, es infinitamente heterogénea, ya que estando a unos cuantos metros de distancia, puede variar su composición granulométrica. Se identifica, pues, el coeficiente de conductividad térmica de la tierra $0.254 \mathrm{~W} / \mathrm{m} .{ }^{\circ} \mathrm{C}$ y de la esterilla de guadua $0.049 \mathrm{~W} / \mathrm{m} .{ }^{\circ} \mathrm{C}$, probando la gran capacidad de aislamiento térmico y baja conductividad de calor de estos materiales, comparados con otros semejantes o utilizados con mayor frecuencia. Por consiguiente, la unificación de estas dos materias primas da como resultado un sistema constructivo sumamente eficaz para disminuir el paso del calor con un valor de $0.303 \mathrm{~W} / \mathrm{m}$. ${ }^{\circ} \mathrm{C}$. Finalmente se ratifica la efectividad de aislamiento del calor del bahareque embutido en tierra con esterilla, siendo más favorable que el ladrillo, el concreto, el panel yeso y la madera, lo que confirma la posibilidad de disminuir el gasto energético utilizado para proporcionar condiciones térmicas óptimas dentro de edificaciones construidas con materiales naturalmente renovables. Como consecuencia de la investigación anterior, se legitima 
el valor social, cultural, económico y de confort térmico de la construcción con materiales vernáculos como la guadua y la tierra, demostrando la importancia de las tradiciones ancestrales y potenciando el desarrollo de nuevas construcciones sostenibles, respaldadas con una investigación científica que hoy se puede aprovechar para realizar simulaciones virtuales y cálculos teóricos.

\section{REFERENCES}

[1] PNUMA, GEO 5 - Perspectivas del medio ambiente mundial. 2012.

[2] B. Yuste, "Arquitectura de tierra: caracterización de los tipos edificatorios," 2009.

[3] G. Barbeta i Solà, "Mejora de la tierra estabilizada en el desarrollo de una arquitectura sostenible hacia el siglo XXI," p. 163, 2002,

[4] E. Unidos, "I.N.V.E."
[5] E. Unidos, American Standard for Testing and Materials. ASTM, vol. 63. 2007.

[6] E. Unidos, American Association of State Highway and Transportation Ofcials. AASHTO. 2004.

[7] C. Neves and O. Borges Faria, Técnicas de Construccion con tierra. 2011.

[8] Y. Cengel, Transferencia de calor y masa. 1989. 


\title{
CARACTERIZACIÓN TÉRMICA DE ENVOLVENTES ARQUITECTÓNICAS EN ESTERILLA LAMINADA DE GUADUA
}

\section{Thermal characterization of architectural envelopes in Guadua laminated mat}

\author{
Silva, Hector ${ }^{1}$; Herrera, Carlos ${ }^{2}$ \\ ${ }^{1}$ Escuela de Arquitectura, Universidad del Valle \\ ${ }^{2}$ Escuela de Ingeniería Mecánica, Universidad del Valle
}

\section{Resumen}

Hoy en día el confort interno de las edificaciones se soluciona con el uso indiscriminado de sistemas mecánicos de calefacción o enfriamiento de aire que requieren altos consumos de energía. Los materiales de origen vegetal ofrecen importantes ventajas sobre los demás materiales usados en los procesos constructivos, en la medida en que poseen excelentes condiciones de aislamiento térmico. La guadua es un bambú americano de rápido crecimiento, que se auto reproduce, disminuye el impacto ambiental, controla la erosión, captura $\mathrm{CO}^{2} \mathrm{y}$ produce oxígeno [1]. La esterilla laminada de guadua es un material nuevo, resultado de un proceso industrial que la convierte en una opción eficiente como aislante térmico que permite desarrollar envolventes arquitectónicas sostenibles y de importantes cualidades en términos del ahorro energético. La caracterización térmica de la esterilla laminada de guadua se definió a través de la implementación de una fase inicial de pruebas de laboratorio y una fase posterior que efectúa un análisis comparativo con datos de transferencia de calor de tableros contrachapados y materiales de construcción usados como envolventes arquitectónicas. La presente investigación estableció que el coeficiente de conductividad térmica $(\mathrm{k})$ de la esterilla laminada de guadua, es de $0,072 \mathrm{w} /\left(\mathrm{m}^{*} \mathrm{k}\right)$, valor significativo en la medida en que es 1,67 veces mejor aislante térmico que la madera contrachapada $\left(0,120 \mathrm{w} /\left(\mathrm{m}^{*} \mathrm{k}\right)\right)$ y 4,80 veces mejor aislante térmico que el fibrocemento $\left(0,346 \mathrm{w} /\left(\mathrm{m}^{*} \mathrm{k}\right)\right)$. Los resultados obtenidos permiten evidenciar que las fibras vegetales deberán ser motivo de estudio para ser tenidas en cuenta como envolventes arquitectónicas en un mercado que preferentemente hace uso indiscriminado de materiales de alto impacto ambiental.

\footnotetext{
${ }^{1}$ Correo electrónico de contacto: hector.silva@correounivalle.edu.co
} 
Palabras claves: Confort, Esterilla, Guadua, Transferencia Térmica

\section{INTRODUCCIÓN}

El gran problema planteado es la caracterización en términos de confort térmico de la guadua como material de construcción para la ejecución de envolventes arquitectónicas en proyectos de vivienda en Colombia. La Guadua Angustifolia Kunt es una especie de bambú leñoso que crece en forma natural en amplias zonas de Centro y Sur América. [2]

Nuestros antepasados usaron la guadua para convertirla en un aliado importante en el desarrollo de los primeros asentamientos de finales del siglo XVIII y principios del siglo XIX. Desde entonces, la guadua estuvo presente en la construcción de las nuevas ciudades hasta la década del cincuenta, cuando llegaron al país nuevos materiales y tecnologías, lo que ocasionó que la guadua perdiera importancia en el ambiente tecnológico y sólo se siguió usando en construcciones temporales y vivienda campesina. [3]

Existe una alta preocupación por el cambio climático asociado con el aumento de la temperatura global, el deshielo de los polos y los daños a la capa de ozono. Las condiciones de vida del ser humano de hoy son diametralmente mejores que las de hace algunos siglos y se ha aumentado substancialmente la expectativa de vida. Ello también ha generado el aumento de la población mundial a tasas alarmantes. [4]

La sobrepoblación, aunada a la cultura del consumismo, promueve un sector industrial que genera gran cantidad de residuos sólidos, la mayoría de difícil degradación. Además, alimentar a la población mundial no ha sido una tarea fácil; con el pasar del tiempo se han aumentado las áreas de pastoreo y se han ampliado las fronteras agrícolas, poniendo en riesgo las áreas boscosas y la biodiversidad natural. Todo lo anterior contamina el aire, induce un calentamiento de la superficie de la tierra, produce la lluvia acida y origina la desertización de algunas zonas provocando el desplazamiento de grandes cantidades de personas a los centros urbanos.

La arquitectura juega un papel importante en la sostenibilidad ambiental del planeta, toda vez que el crecimiento de la población y la necesidad de mantener niveles de vida adecuados demandan un aumento de la actividad constructiva. Actualmente, la actividad edificatoria consume más del 40\% de la energía del planeta y genera más del 30\% de los gases de efecto 
invernadero, razones más que suficientes para entender que la responsabilidad de la arquitectura en el desempeño climático del planeta essubstancial. [4] Aproximadamente el 50\% de los recursos renovables del planeta son utilizados en los procesos de construcción haciendo de esta una de las actividades menos sostenibles [5]. El enfriamiento, la ventilación y el aislamiento térmico son factores determinantes para el confort en las edificaciones, pero de igual manera son responsables de la emisión significativa de $\mathrm{CO}_{2}$, debido al afán desmedido en generar condiciones óptimas artificiales de habitabilidad, mucho más allá de tener en cuenta las condiciones naturales particulares de clima y las tradiciones tecnológicas involucradas en los procesos constructivos locales.

En los próximos 20 años se duplicarán las emisiones de gases efecto invernadero sino se toman las decisiones apropiadas para mitigar la ineficiente arquitectura de hoy. Si bien, hay muchos factores que influyen dramáticamente en las condiciones climáticas, la arquitectura tiene una responsabilidad fundamental en el origen de los problemas. De allí, la necesidad de entender que debemos propender por diseños, construcciones y materiales sostenibles. Y es precisamente en el ámbito de los materiales de construcción en donde hay mayor preocupación puesto que las tecnologías más comerciales hacen uso de materiales que demandan para su producción grandes cantidades de energía, tales como el acero (que tiene un $25.5 \%$ de participación en el consumo energético en la construcción de $1 \mathrm{~m}^{2}$ ), el cemento (11.7\%) y los productos cerámicos (21.5\%). Contrario a ello las maderas y el bambú requieren para su producción un bajo consumo energético, tan solo del $1.5 \%$ por metro cuadrado de construcción [6].

Asociadas con el alto consumo de energía, las emisiones de $\mathrm{CO}_{2}$ causadas en la producción del cemento y productos cerámicos son significativamente altas comparadas con las asociadas con el uso de la madera y el bambú. Un mayor uso en construcción de un material renovable como la guadua tendería a apreciar el material y crearía incentivos para que los agricultores hagan un mejor mantenimiento de los guaduales y aumenten las áreas de cultivo, con los consecuentes beneficios ambientales para así mitigar y enfrentar de mejor manera el cambio climático.

Similar a la madera, no existe ningún otro material de construcción como el bambú que requiera tan poca energía para su transformación, y cuyas plantaciones capturen $\mathrm{CO}_{2}$ y produzcan oxígeno [1]. Es aquí donde el bambú en general y la guadua en particular, se convierten en una excelente opción como materiales de construcción sustitutos de la madera tradicional, ya que en varios aspectos la superan en términos ambientales. 
Debido a que es una planta de rápido crecimiento, el uso controlado de la guadua no representa un peligro para la naturaleza y ayuda a disminuir el impacto sobre los bosques naturales de maderas tradicionales. Las plantaciones de guadua generan un microclima especial, capturan $\mathrm{CO}_{2}$ de la atmósfera, crecen rápidamente y poseen una alta capacidad para mejorar los suelos y regular las aguas [7]. Por estos motivos, el bambú en general se convierte en un material sustentable, muy favorable para contribuir a la solución del déficit de vivienda en el mundo [8]. A pesar de reconocer las importantes condiciones de la guadua como vegetal y como material de construcción, el uso de tecnologías asociadas a ella en nuestro país aún es muy incipiente y los sistemas constructivos convencionales cubren gran parte del mercado colombiano, dejando a los sistemas constructivos tradicionales con un porcentaje muy bajo dentro del mercado. [9]; Esto también se relaciona

con la efectividad actual de los sistemas industrializados versus la influencia de alto costo de la mano de obra en la construcción vernácula.

Hoy en día, a pesar de entender que la guadua hace parte de los materiales de construcción existentes en nuestro mercado, no existen procesos industriales desarrollados que permitan ofrecer una gama amplia de opciones; El mercado nacional tan solo hace uso de la guadua rolliza, con una transformación muy incipiente, lo cual limita su uso como material de construcción eficiente y amigablemente sostenible.

En ese sentido entonces se requiere un desarrollo técnico que satisfaga las necesidades de un mercado que necesita nuevas opciones de materiales de construcción, efectivos en términos del confort climático, de bajo costo, sostenibles y que permitan el desarrollo de tecnologías apropiadas.

La esterilla laminada de guadua podría satisfacer todas estas necesidades incluyendo su condición ambiental, pero entendiendo que de igual manera hace parte de la historia tecnológica de nuestro país y en ese sentido, respeta tradiciones y costumbres constructivas autóctonas.

\section{MATERIALES Y METODOS}

Con el fin de alcanzar los objetivos propuestos en la presente investigación se implementaron dos fases en las cuales se desarrollaron procesos para determinar las propiedades de la esterilla laminada de guadua como material aislante. 


\section{Fase 1: Pruebas de Laboratorio}

Esta fase toma como referencia principal las determinaciones estipuladas en la Norma ASTM C177-04, denominada "ASTM C177: Standard Test Method for Steady-State Heat Flux Measurements and Thermal Transmission Properties". [10].

De igual manera se tienen en cuenta los procedimientos llevados a cabo en la investigación desarrollada por Kiran, M.C. et al, denominada "Effect of Density on Thermal Conductivity of Bamboo Mat Board", [11], toda vez que hace uso del procedimiento llamado Placa Caliente Resguardada.

Todas las pruebas de laboratorio se ejecutaron en el Laboratorio de Transferencia de Calor de la Escuela de Ingeniería Mecánica de la Universidad del Valle.

Para garantizar que las condiciones internas del laboratorio no influyeran en el resultado de las pruebas, se efectúa una medición de temperatura y humedad relativa en las horas de los días de dichos ensayos con el fin de verificar unas condiciones estables de temperatura y humedad.

Se toma como medida preventiva que las respectivas probetas del laminado de esterilla de guadua se encuentren dispuestos en el laboratorio en mención con una anticipación de más de treinta y un (31) días, condición que permitirá asegurarse que dichos elementos hayan estabilizado su humedad interna con respecto a la humedad del ambiente, antes de dar inicio a las pruebas.

Teniendo en cuenta que la densidad en las fibras vegetales incide directamente en la condición de aislante térmico, se implementan las acciones para tomar los valores de espesor, peso y volumen de cada una de las probetas a desarrollar.

Para determinar el coeficiente de conductividad térmica del laminado de esterilla de guadua se hace uso de un equipo denominado de placa caliente resguardada, el cual está conformado, como su nombre lo indica, por dos placas calientes centrales y dos placas frías ubicadas en la parte exterior.

Con la determinación de las variables ya mencionadas se procede a definir el coeficiente de conductividad térmica de las probetas de Esterilla Laminada de Guadua.

\section{Fase 2: Caracterización del confort y análisis comparativo}

Después de obtener los datos de las pruebas de laboratorio se hace un análisis de dichos valores y se compara con datos de investigaciones recientes efectuadas a tableros contrachapados desarrollados con fibras vegetales. 
De igual manera se hace el análisis comparativo con especímenes de madera, toda vez que este material es el referente más próximo ya que se trata de una fibra vegetal y adicional a ello, existen investigaciones previas muy extensas y desarrolladas.

Teniendo en cuenta que la densidad es una condición importante para determinar condiciones de confort de algunos materiales, se realiza un análisis de las densidades resultantes de las pruebas de laboratorio y se compara con algunas densidades de investigaciones previas realizadas a otros tableros de bambú.

Adicionalmente se hace un análisis comparativo de peso por metro cuadrado y emisión de $\mathrm{CO}_{2}$

\section{RESULTADOS Y ANALISIS DE RESULTADOS}

Los resultados de la presente investigación han determinado que el Coeficiente de Conductividad Térmica

(k) del laminado de esterilla de guadua es de 0,0720 w/(m*k), valor que indica su mejor comportamiento como aislante térmico que el estimado parala madera dura $\left(0,159 \mathrm{w} /\left(\mathrm{m}^{*} \mathrm{k}\right)\right)$ [12], madera suave $\left(0,1150 \mathrm{w} /\left(\mathrm{m}^{*} \mathrm{k}\right)\right)$ [12] y para el tablero contrachapado de madera $\left(0,12 \mathrm{w} /\left(\mathrm{m}^{*} \mathrm{k}\right)\right)$ [12], referencias directas debido a que el origen del material sigue correspondiendo a una fibra vegetal. (Tabla 1)

Teniendo en cuenta el uso más frecuente entre los materiales de cerramientos de las edificaciones, podríamos indicar que el laminado de esterilla de guadua $\left(0,0720 \mathrm{w} /\left(\mathrm{m}^{*} \mathrm{k}\right)\right)$ posee ostensiblemente un mejor comportamiento como aislante térmico que el tablero de fibrocemento $\left(0,3460 \mathrm{w} /\left(\mathrm{m}^{*} \mathrm{k}\right)\right)[13]$ (Tabla 1$)$

En términos de la sostenibilidad es importante mencionar que los tableros de esterilla laminada de guadua provienen de un bambú americano de rápido crecimiento, contrario a la edad de madurez de los tallos de las maderas con los cuales se elaboran los tableros de madera contrachapada.

También es importante indicar que efectuando una comparación entre el tablero de esterilla laminada de guadua (16 mm aprox. de espesor) y el tablero de madera contrachapada (18 mm de espesor), se puede advertir que el tablero de madera contrachapada utiliza cinco capas mientras el tablero de esterilla laminada utiliza solo trescapas. 
En ese sentido se hace claramente entendible que existen muchas ventajas del uso de los tableros de esterilla laminada ya que utiliza menos material, afectando en menor medida los bosques naturales, y adicional a ello, utiliza menor cantidad de adhesivo en su proceso de fabricación, lo cual incide directamente en el menor impacto ambiental que genera el tablero de esterilla laminada de guadua.

En términos de la emisión de $\mathrm{CO}_{2}$ debemos indicar que el tablero de esterilla laminada de guadua emite - 3,25 $\mathrm{Kg} \mathrm{CO}_{2} / \mathrm{Kg}$ [14], valor muy bajo con respecto a la madera contrachapada (0,06 $\mathrm{Kg}$ $\mathrm{CO}^{2} / \mathrm{Kg}$ ) [15] y con respecto a los tableros de fibrocemento $\left(52 \mathrm{Kg} \mathrm{CO}^{2} / \mathrm{Kg}\right.$ ). (Tabla 2)

\section{Tabla 9 Comparación de}

Conductividades Térmicas de distintos

Materiales

\begin{tabular}{|c|c|}
\hline \multirow{2}{*}{ Material } & $\begin{array}{c}\text { Conductividad } \\
\text { Termica }\end{array}$ \\
\cline { 2 - 2 } & $\begin{array}{c}\mathrm{k} \\
\mathrm{w} /\left(\mathrm{m}^{*} \mathrm{k}\right)\end{array}$ \\
\hline $\begin{array}{c}\text { Esterilla Laminada de } \\
\text { Guadua }\end{array}$ & 0,072 \\
\hline $\begin{array}{c}\text { Madera } \\
\text { Contrachapada }\end{array}$ & 0,12 \\
\hline aderas Duras & 0,159 \\
\hline $\begin{array}{c}\text { Maderas Suaves } \\
\text { Tablero de }\end{array}$ & 0,115 \\
\hline Fibrocemento & 0,346 \\
\hline
\end{tabular}

Tabla 10 Comparación de Emisiones de $\mathrm{CO} 2$

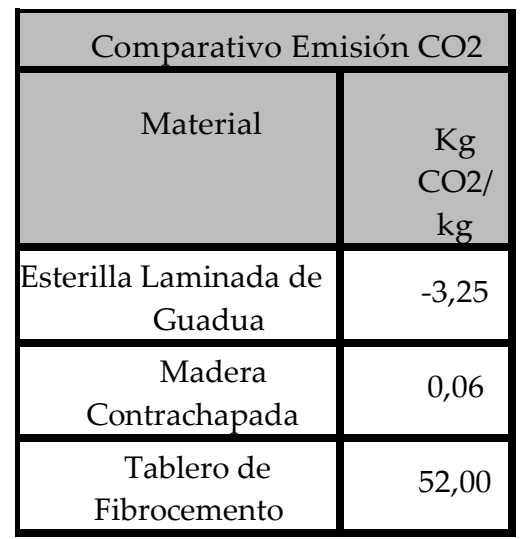

Tabla 11 Comparación de densidad materiales

\begin{tabular}{|c|c|}
\hline Material & Densidad \\
\cline { 2 - 2 } & $\mathrm{Kg} / \mathrm{m} 3$ \\
\hline $\begin{array}{c}\text { Esterilla Laminada } \\
\text { de Guadua }\end{array}$ & 720 \\
\hline $\begin{array}{c}\text { Madera } \\
\text { Contrachapada }\end{array}$ & 545 \\
\hline Maderas Duras & 721 \\
\hline $\begin{array}{c}\text { Maderas } \\
\text { Suaves }\end{array}$ & 513 \\
\hline $\begin{array}{c}\text { Tablero de } \\
\text { Fibrocemento }\end{array}$ & 1625 \\
\hline
\end{tabular}

De igual manera, la densidad tiene una influencia definitiva en la determinación de la conductividad térmica de los materiales. Las maderas contrachapadas poseen una densidad ligeramente más baja que la madera tradicional, seguramente por la existencia de aire entre sus capas y en ese sentido deberá ser materia de estudio posterior la influencia de las posibilidades de alojamiento de aire en las hendiduras de las esterillas. (Tabla 3)

El peso de los materiales de construcción se convierte en un factor importante al momento de tomar la decisión de su uso o aplicación en una edificación, máxime cuando las edificaciones se encuentren en una zona de alto riesgo sísmico, como es la condición de nuestro territorio. En ese sentido entonces los materiales livianos se han convertido en una opción importante e 
inmejorable toda vez que además de no agregar carga muerta a la edificación, permitiría la disminución de las inercias al momento de presentarse un evento sísmico. Por tal razón es importante determinar el peso de la esterilla laminada de guadua, característica establecida en $12,09 \mathrm{Kg} / \mathrm{m}^{2}$. (Tabla 4$)$

Como el uso o aplicación de la esterilla laminada de guadua estará más ligado a las envolventes arquitectónicas es importante efectuar la comparación del peso de la esterilla laminada de guadua con el peso de la madera contrachapada y con el peso de las láminas de fibrocemento, los cuales establecen $11,09 \mathrm{Kg} / \mathrm{m}^{2}$ [16] y $25,33 \mathrm{Kg} / \mathrm{m}^{2}$ [17], respectivamente. (Tabla4)

Estos datos permiten advertir la gran similitud en peso entre la esterilla laminada de guadua y el peso de la madera contrachapada, y la gran diferencia entre estos dos materiales y el peso mayor que tiene el fibrocemento, lo cual certifica una ventaja adicional de los materiales de origen vegetal.

Tabla 12 Comparación Peso

\begin{tabular}{|c|c|}
\hline Material & Peso \\
\cline { 2 - 2 } & $\mathrm{Kg} / \mathrm{m} 2$ \\
\hline $\begin{array}{c}\text { Esterilla Laminada de Guadua }(16 \\
\mathrm{mm})\end{array}$ & 12,09 \\
\hline Madera Contrachapada $(18 \mathrm{~mm})$ & 11,09 \\
\hline Tablero de Fibrocemento $(17 \mathrm{~mm})$ & 25,33 \\
\hline
\end{tabular}

\section{CONCLUSIONES}

La presente investigación permite establecer que el coeficiente de conductividad térmica $(\mathrm{k})$ de un material nuevo, producto de la transformación industrial de la Guadua Angustifolia, denominado esterilla laminada de guadua, es de $0,702 \mathrm{w} /\left(\mathrm{m}^{*} \mathrm{k}\right)$, condición que define su excelente comportamiento como aislante térmico para envolventes arquitectónicas; esta información de igual manera permite identificar que la esterilla laminada de guadua es 1,67 veces mejor aislante térmico que la madera contrachapada $\left(0,120 \mathrm{w} /\left(\mathrm{m}^{*} \mathrm{k}\right)\right)$ y 4,80 veces mejor aislante térmico que el fibrocemento $\left(0,346 \mathrm{w} /\left(\mathrm{m}^{*} \mathrm{k}\right)\right)$. La determinación del coeficiente de conductividad $(\mathrm{k})$ del laminado de esterilla de guadua permitirá que este material se incluya dentro de los programas de 
simulación de confort climático y de esta manera su aplicabilidad sea más efectiva, toda vez que se podrá predecir su comportamiento como envolvente arquitectónica de los proyectos a estudiar. También es importante destacar que el presente análisis permite advertir que es posible la existencia de una relación directa entre materiales sostenibles y envolventes arquitectónicas confortables, sin dejar de lado los criterios técnicos de eficiencia en términos de temperaturas internas y renovación de aire. Las tecnologías vernáculas y tradicionales han desarrollado a través de la historia, criterios informales de adaptación climática, sin embargo, la presente investigación legítima el método científico para determinar las características de los materiales utilizados para las envolventes arquitectónicas, permitiendo a la arquitectura bio climática hacer uso de conocimientos interdisciplinares. Los materiales de origen vegetal, como el laminado de esterilla de guadua, poseen ventajas muy importantes en términos de sostenibilidad en comparación con otros materiales, toda vez que la guadua es un material renovable, adicional a ello, los materiales derivados de la guadua o del Bambú poseen muchísimas ventajas ambientales con respecto a los materiales derivados de la madera. Esta investigación confirma que la esterilla laminada de guadua utilizada como envolvente arquitectónica posee excelentes características como aislante térmico, sin embargo, se deberá establecer desde la protección por diseño, mecanismos que controlen su exposición directa con el sol y el agua, afectación que generaría procesos de mantenimiento exhaustivos. Con esta investigación se comprueba que el uso de la esterilla laminada de guadua como envolvente arquitectónica posibilita excelentes resultados en términos del confort interno de las edificaciones, haciéndola competitiva en el mercado, ambientalmente amigable y con resultados estéticos óptimos, permitiendo el rescate de tradiciones tecnológicas vinculadas con un material local. La efectividad de este material permitirá entender que la Guadua Angustifolia es una opción real para la activación de los procesos agrícolas en el campo, que posibilitará el desarrollo de una naciente industria de la guadua y además, que permitirá entender que estos materiales generan gran interés para el diseño y la construcción de edificaciones sostenibles y de bajo consumo energético.

\section{REFERENCES}

[1] INBAR, Bamboo and Climate Change Mitigation (Vol. 32). Beijing, China. 2010

[2] X, Londoño; G, Camayo; N, Riaño; Y, Lopez; Characterization of the anatomy of Guadua angustifolia culms; Vol. 16; Albany, NY; American Bamboo Society; 2002.

[3] J.E, Robledo; Un Siglo de Bahareque en el Antiguo Caldas;
Bogotá; Ancora editores; 1993. in Transitioning to a Net Neutral Community; Vol. 3; Ottawa, ON; International Journal of Environment 
and Sustainability; 2014.

[7] E, Giraldo; Una Alternativa Sostenible: La Guadua; Segunda Edicion; Armenia, Q. Corporacion Autonoma Regional del Quindio; 2005.

[8] K, De Flander; One laminated bamboo-frame house per hectare per year; Elsiever; 2008.

[9] J, Rios; Sostenibilidad del consumo doméstico de materiales de construcción en Colombia; Vol. 86; Medellín, A.; Lecturas de Economía; 2017.

[10] ASTM International; Standard Test Method for Steady State Heat Flux Measurements and Thermal Transmission Properties by Means of the Guarded Hot Plate Apparatus; Pensilvania, EEUU; American Society for Testing and Materials; 2004.

[11] M, Kiran; Effect of Density on Thermal Conductivity of
Bamboo Mat Board; International Journal of Agriculture and Forestry; 2012.

[12] Y, Cengel; Transferencia de Calor y Masa; Tercera edición; México, DF; McGraw- Hill Interamericana; 2017.

[13] Mexalit. (2.019). www.mexalit.com.mx.

[14] J. R, Becerra; Análisis energético y Ambiental en la producción de Tableros de esterilla de Guadua Laminada; Pereira; Universidad Tecnológica de Pereira; 2013.

[15] A, Salazar; Los Eco Materiales y las V.I.S., Una Visión Sostenible; Medellín, A.; Colegio Mayor de Antioquia; 2012

[16] Pizano S.A; Manual de Producto - Pizano; Bogotá; 2009. Eternit Colombia; Ficha Técnica Eterboard; Bogotá; 2017 


\title{
CLIMATE ZONES AND SUB-ZONES IN THE EQUATORIAL REGION
}

\section{Zonas climáticas y subzonas en la región ecuatorial}

\author{
Clavijo Vergara, Fabio Miguel ${ }^{1}$ \\ ${ }^{1}$ ASHRAE, ASHRAE Colombia, ACAIRE \\ 2 Tecnaire Ltda
}

\begin{abstract}
The challenge to meet optimized energy performance in sustainable buildings looks after improving construction facades criteria to protect interior spaces from incoming sun heat effects, outdoor environmental heat and humidity.

This analysis involves the study of existing outside conditions, which have been addressed by ASHRAE Standard-169 [1] concentrating mainly on outdoor temperature prevailing conditions in climate zones 0 thru 8 across the globe. Little attention has been given to prevailing humidity conditions where ASHRAE considers three sub-zones for humidity ( $\mathrm{A}=\mathrm{Humid}, \mathrm{B}=$ Dry , $\mathrm{C}=$ Marine) while different critical humidity conditions are encountered in Equatorial Region where combined conditions of extreme high temperatures (Zone 0,1$)$ are coincident with extreme humidity (high and low) not considered at this time by ASHRAE.
\end{abstract}

The present research proposes a new approach to redefine sub-zones associated with humidity prevailing conditions across the globe and particularly in the Equatorial Region in order to improve climate data and construction criteria to improve energy performance in buildings. Keywords: Equatorial Region, temperature, humidity, climate zones.

\section{INTRODUCTION}

Tropical regions in the Equatorial belt face challenges to comply with comfort requirements and acceptable energy efficiency performance. Extreme hot and humid climates confront engineers in many ways as compared with prevalent conditions in Northern and Southern Hemispheres thru

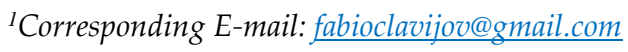


the annual period. These particular Equatorial weather comportments suggest as well innovative approaches thru the architecture, energy and HVAC engineering design process to understand building improvement opportunities in this part of the world.

In this Equatorial region, the annual climate cycle faces HVAC consultant engineers and architects with intensive cooling demand solutions thru the year, which at near sea level, combines typically high temperature outside conditions along with high humidity content. These combined conditions are present across the annual period showing also ample humidity variations thru daily periods.

These particular climate characteristics, set a challenge as to what outdoor conditions should be selected to comply with indoor comfort conditions -Temperature and Humidity- and acceptable energy performance thru the annual operation.

As of today, design outdoor Temperature conditions across the globe are reference under ASHRAE Standard 169-2013 [1] following Climate Zones patterns (zones 0 thru 8) based on temperature prevailing demand across the year and three sub-zones $(A=H u m i d, B=D r y$ ,C=Marine) to reference humidity outside conditions which do not reflect extreme humidity conditions present in the Equatorial Region. This research presents new five (5) Moisture Subzones based on Evaporative cooling applications and resulting air moisture content across different World Equatorial sites defining a set of outside moisture conditions ranging from extremely dry to extremely humid.

This proposed climate zone and moisture sub-zone structure will greatly enhance architects, builders, engineers and investors opportunities and awareness to improve façade construction systems, materials and specifications focusing on the life cycle cost, overall performance and energy efficiency.

\section{Preface}

ASHRAE - American Society of Heating, Refrigerating and Air Conditioning Engineers - has defined thru Standard 169-2013 [1] a World reference of Climate Zones based on annual daily mean temperatures (CDD- Cooling Degree Days and HDD- Heating Degree Days) which allows understanding Air Conditioning (cooling or heating) requirements across every site. 
ASHRAE STD-169-2013 [1] focuses on moisture zones as:

$$
\begin{array}{ll}
\checkmark & \text { A- Humid } \\
\checkmark & \text { B- Dry } \\
\checkmark & \text { C- Marine }
\end{array}
$$

These zones work fairly well in understand humidity issues within Continental United States and by extrapolation to Northern and Southern Hemispheres. Each moisture zone is a result of combined calculations of rain precipitation and mean temperatures across the year. (ASHRAE Std 169-2013, Annex 1-1 [1])

This approach does not bring relevant information when Equatorial Region applications comes to table, at near sea level altitude sites, where humidity reaches extreme high conditions whereas in dessert zones extreme low humidity conditions are found.

This research develops new approaches to define an extended Humidity Sub-zones definitions in order to improve the understanding of combined temperature-moisture outside conditions and the resulting impact on construction systems, materials and specifications in order to improve energy performance and efficiency in buildings across the Equatorial Region.[2][4]

\section{MATERIALS Y METHODS}

The selected method to define humidity sub- zones is based in obtaining the humidity content at design conditions for Evaporation Applications (1\%) as per ASHRAE Standard 169-2013 [1] for any site and corresponding altitude located within Equatorial Region (gr/Kg d.a., grains/lb d.a.). Resulting information provides the most representative moisture content figure among different design conditions including: Cooling, Heating, Humidification, Dehumidification, Enthalpy, Evaporation.

The Equatorial Region is located within latitude $23^{\circ} 26^{\prime} 14^{\prime \prime}$ North and $23^{\circ} 26^{\prime} 14^{\prime \prime}$ South of the Equator line. The map shows the various climate zones as considered by ASHRAE Std-169-2013 [1], and the marking zone denotes the Equatorial region crossing four continents. 


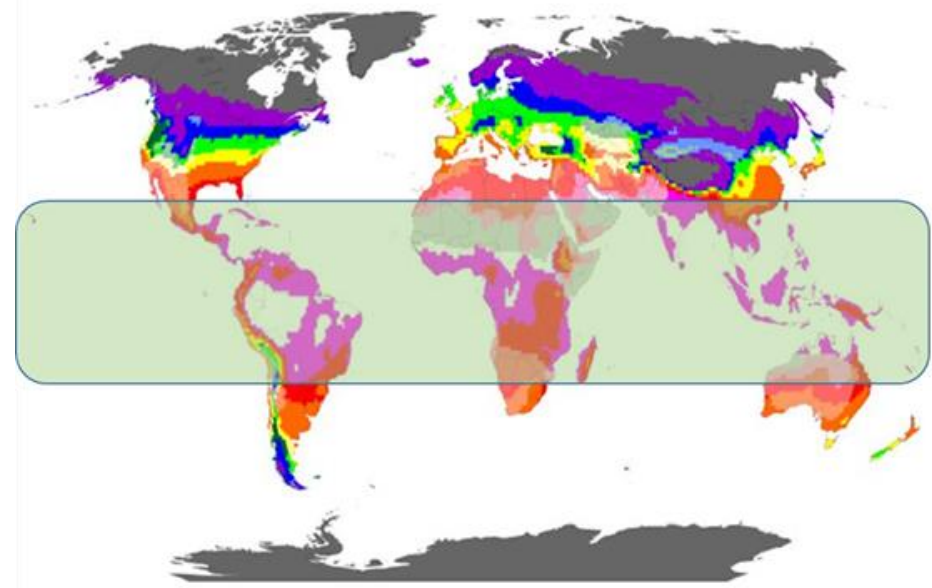

Zone OA Extremely Hot Humid

Zone $0 B$ Extremely Hot Dry

Zone $1 \mathrm{~A}$ Very Hot Humid

Zone $1 \mathrm{~B}$ Very Hot Dry

Zone 2 A Hot Humid

Lone za not Ury

Zone 3 A Warm Humid

Zone 38 Warm Dry

Zone JC Warm Marine

Zone 4 A Mixed Humid

Zone 4B Mixed Dry

Zone $4 C$ Mxed Marine

Zone SA Cool Humid

Zone se cool uny

Zone SC Cool Marine

Zone 6 A Cold Humid

Zone 6B Cold Dry

Zone 7 Very Cold

Zone 8 SubarcticiAretic

Figure 41 New World Climate Zone Map

\section{RESULTS AND ANALYSIS}

ASHRAE STD-169-2013 [1] Temperature Zones have been observed as valid definitions and it shows following ranges of CDD (Cooling Degree Days) for Cooling Applications and HDD (Heating Degree Days) for Heating Applications.

Table 14 ASHRAE International climate zones

\begin{tabular}{|c|c|c|c|}
\hline $\begin{array}{l}\text { INTERNATIONAL } \\
\text { CLIMATE ZONES }\end{array}$ & Name & $\begin{array}{l}\text { Criteria } \\
\mathrm{CDD}^{\circ} 0^{\circ} \mathrm{F}\end{array}$ & $\begin{array}{c}\text { Criteria } \\
\text { HDD65F }\end{array}$ \\
\hline ASHRAE STD-169-2013 & & $\sum$ Diff. day. ${ }^{\circ} \mathrm{F}$ & $\sum$ Diff. day. ${ }^{\circ} F$ \\
\hline ZONE 0 & Extremely hot & $10800<$ CDD50 & - \\
\hline ZONE 1 & Very hot & $9000<C D D 50 \leq 10800$ & - \\
\hline ZONE 2 & Hot & $6300<$ CDD50 $\leq 9000$ & - \\
\hline ZONE 3 & Warm & $0<\operatorname{CDD} 50 \leq 6300$ & $0<\mathrm{HDD} 65 \leq 3600$ \\
\hline ZONE 4 & Mixed & $0<$ CDD $50 \leq 6300$ & \& $3600<$ HDD $65 \leq 5400$ \\
\hline ZONE 5 & Cool & $0<$ CDD50 $\leq 6300$ & $5400<$ HDD $65 \leq 7200$ \\
\hline ZONE 6 & Cold & - & $7200<$ HDD $65 \leq 9000$ \\
\hline ZONE 7 & Very Cold & - & $9000<$ HDD $65 \leq 12600$ \\
\hline ZONE 8 & Subartic/Arctic & - & $12600<\mathrm{HDD} 65$ \\
\hline
\end{tabular}


ASHRAE moisture zones - As of today, ASHRAE [1] [2] definitions and formulas are:

Marine Zone (C)

- MeanTemp. Coldest month $=$ range $27^{\circ} \mathrm{F}$ to $65^{\circ} \mathrm{F}\left(-3^{\circ}\right.$ to $\left.18^{\circ} \mathrm{C}\right)$

- Mean warmest month $<72^{\circ} \mathrm{F}\left(22^{\circ} \mathrm{C}\right)$

- At least 4 months Mean Temperatures $>50^{\circ} \mathrm{F}\left(10^{\circ} \mathrm{C}\right)$

- Monthly heaviest precipitation in cold season is at least 3 times higher than monthly with least precipitation

Dry Zone (B)- (No Marine) and

$P_{\text {in }}<0.44 \times\left(T-19.5^{\circ} \mathrm{F}\right) \quad P_{m m}<20 \times\left(T+7^{\circ} \mathrm{C}\right)$

If $30 \%$ to $70 \%$ of anual precipitation P occurs during the the high sun period.

Where:

$P=$ Annual Precip. (Inches $/ \mathrm{mm}$ )

$T=$ Mean Annual temperature $\left({ }^{\circ} \mathrm{F} /{ }^{\circ} \mathrm{C}\right)$

Figure 42 ASHRAE Moisture zones

ASHRAE calculations evaluate Marine and Dry zones thru above mentioned formulas, leaving the definition of Humid Zones to non-previously assigned zones. Such approach does not consider extremely humid or extremely dry conditions which are frequently found thru the Equatorial Region.

\section{Equatorial Moisture Zones-}

The approach to consider straight moisture content ranges based on available weather data information across the world map looks to be more comprehensible and logic from the architectural and engineering standpoint of view. Such information is readily obtainable thru Climate Design Conditions publications [1] [2] [3] [4] [5]. At the end, the amount of moisture present in every site across the Equatorial Region is what really matters when building and facades construction issues come to discussion and decision. The following table proposes a set of humidity sub-zones for Equatorial Region sites. 
Table 15 Humidity sub-zones for equatorial region sites

\begin{tabular}{|c|c|c|c|}
\hline \multicolumn{4}{|c|}{ MOISTURE SUB-ZONES - EQUATORIAL REGION } \\
\hline Letter & ZONE DENOMINATION & $\begin{array}{l}\text { Specific Humidity } \\
\text { grains/lb d.a. }\end{array}$ & $\begin{array}{l}\text { Specific Humidity } \\
\text { g/Kg d.a. }\end{array}$ \\
\hline V & Extreme dry & $w<70$ & $W<10.00$ \\
\hline $\mathbf{W}$ & Moderate Dry & $70.1 \leq W<95$ & $10.01 \leq W<13.57$ \\
\hline $\mathbf{x}$ & Moderate humid & $95.1 \leq W<120$ & $13.58 \leq W<17.14$ \\
\hline $\mathbf{Y}$ & Very humid & $120.1 \leq W<145$ & $17.15 \leq W<20.71$ \\
\hline $\mathbf{Z}$ & Extremely humid & $145.1 \leq W$ & $20.72 \leq W$ \\
\hline & Criteria: Specific I & dity - 1\% Eva & \\
\hline
\end{tabular}

Exposing above reference table to multiple territories across four (4) Equatorial continents comparing ASHRAE Climate Zones and Moisture sub-zones with Equatorial Temperature zones and Moisture sub-zones, several figures become relevant in the following table that collects weather information from several reference documents. [1] [2] [3] [4] [5]. 
Table 16 equatorial region-climate zones

\begin{tabular}{|c|c|c|c|c|c|c|c|}
\hline EQUATORIAL REGI & $\mathrm{N}$-Climate Zones & & & Specific & Climate Zone & Clim a+Moist & Clim a+Moist. \\
\hline $\begin{array}{c}\text { Clim ate \& Moisture } \\
\text { Country }\end{array}$ & Sites of the World & $\begin{array}{c}\text { Altitude ASL } \\
\text { ft }\end{array}$ & $\begin{array}{c}\text { Altitude } \\
\text { ASL } \\
\text { m }\end{array}$ & $\begin{array}{l}\text { Hum idity } \\
\text { grinb d.a. }\end{array}$ & $\begin{array}{l}\text { ASHRAE } \\
\text { Climate zone } \\
\end{array}$ & $\begin{array}{c}\text { ASHRAE } \\
\text { Zone } \\
\end{array}$ & $\begin{array}{c}\text { Equatorial } \\
\text { Zone } \\
\end{array}$ \\
\hline Colombia & Providencia & 3 & 1 & 158.8 & 0 & $0-A$ & $0-Z$ \\
\hline Colombia & San Andrés & 3 & 1 & 1592 & 0 & O-A & $0-2$ \\
\hline Puerto Rico & San Juán - Luis Muñoz & 8 & 2 & 146.6 & 0 & $0-A$ & $0-z$ \\
\hline Thailand & Bangkok & 9 & 3 & 1682 & 0 & $0-A$ & $0-Z$ \\
\hline Jamaica & Kingston - Norman Manley & 10 & 3 & 157.9 & 0 & $0-A$ & $0-z$ \\
\hline Mexico & Acapulco & 13 & 4 & 151.0 & 0 & $0-A$ & $0-2$ \\
\hline Indonesia & Bali Ngarah Rai & 14 & 4 & 152.3 & 0 & $0-A$ & $0-z$ \\
\hline Singapore & Singapore Changhi & 22 & 7 & 1558 & 0 & $0-A$ & $0-2$ \\
\hline Sri Lanka & Colombo - Bandanariake & 29 & 9 & 151.9 & 0 & $0-A$ & $0-z$ \\
\hline Brazil & Recife & 33 & 10 & 1472 & 0 & O-A & $0-z$ \\
\hline Philippines & Manila & 43 & 13 & 155.3 & 0 & $0-A$ & $0-z$ \\
\hline India & Coimbatore & 52 & 16 & 156.3 & 0 & O-A & $0-Z$ \\
\hline Colombia & Montería & 66 & 20 & 140.8 & 0 & O-A & O-Y \\
\hline Senegal & Dakar-Leopold Se dar Senghor & 85 & 26 & 157.9 & 1 & $1-B$ & $1-z$ \\
\hline Malaysia & Kuala Lumpur & 90 & 27 & 1538 & 0 & $0-A$ & $0-z$ \\
\hline Colombia & Barranquilla & 102 & 31 & 163.9 & 0 & O-A & $0-z$ \\
\hline Panama & Panama City-Tocumen & 135 & 41 & 151.1 & 0 & 0-A & $0-Z$ \\
\hline Colombia & Quibdó & 174 & 53 & 152.3 & 0 & $0-A$ & $0-Z$ \\
\hline Tanzania & Dar Es Salaam & 182 & 55 & 145.0 & 1 & $1-A$ & $1-Y$ \\
\hline Kenya & Mombasa & 200 & 61 & 142.7 & 1 & 1-A & $1-Y$ \\
\hline Venezuela & Simon Bolivar & 235 & 72 & 176.1 & 0 & $0-B$ & $0-Z$ \\
\hline Brazil & Manaus & 267 & 81 & 147.6 & 0 & $0-A$ & $0-Z$ \\
\hline Colombia & Leticia & 276 & 84 & 160.7 & 1 & $1-A$ & $1-z$ \\
\hline Vietnam & Da Nang & 381 & 116 & 176.9 & 1 & $1-\mathrm{A}$ & $1-z$ \\
\hline Colombia & Valledupar & 453 & 138 & 130.9 & 0 & $0-A$ & $0-Y$ \\
\hline India & Nagpur & 1,033 & 315 & 152.5 & 0 & $0-A$ & $0-Z$ \\
\hline Colombia & Villavicencio & 1,388 & 423 & 1178 & 0 & O-A & $0-x$ \\
\hline Colombia & Neiva & 1,440 & 439 & 115.1 & 0 & O-A & $0-X$ \\
\hline Saudi Arabia & Gassim - Prince Abdulaziz & 2,126 & 648 & 70.5 & 1 & $1-B$ & $1-W$ \\
\hline Brazil & Belo Horizonte & 2,589 & 789 & 115.5 & 2 & $2-A$ & $2-x$ \\
\hline Colombia & Cali & 3,153 & 961 & 109.0 & 2 & 2-A & $2-X$ \\
\hline Honduras & Tocontin/Tegucigalpa & 3,294 & 1,004 & 1188 & 2 & $2-A$ & $2-x$ \\
\hline Colombia & Bucaramanga & 3,900 & 1,189 & 123.9 & 2 & 2-A & $2-Y$ \\
\hline Madagascar & Antananarivo - Ivato & 4,198 & 1280 & 128.5 & 3 & 3-A & $3-Y$ \\
\hline Colombia & Pereira & 4,403 & 1,342 & 117.4 & 2 & $2-A$ & $2-x$ \\
\hline Zimbabwe & Harare & 4,887 & 1,490 & 108.4 & 3 & 3-A & $3-x$ \\
\hline Colombia & Medellin & 4,888 & 1,490 & 105.4 & 2 & 2-A & $2-x$ \\
\hline Kenya & Nairobi & 5,327 & 1,624 & 100.9 & 3 & $3-C$ & $3-X$ \\
\hline Colombia & Pasto & 5,958 & 1,816 & 103.1 & 2 & 2-A & $2-x$ \\
\hline Saudi Arabia & Abha & 6,858 & 2,091 & 114.9 & 3 & 3-B & $3-X$ \\
\hline Colombia & Rionegro - Aerop. JM Cordoba & 7,028 & 2,143 & 100.4 & 3 & 3-C & $3-X$ \\
\hline Mexico & Mexico City & 7,316 & 2,230 & 84.9 & 3 & 3-A & $3-W$ \\
\hline Bolivia & Cochabamba & 8,360 & 2,549 & 89.8 & 3 & 3-B & $3-W$ \\
\hline Colombia & Bogotá & 8,360 & 2,549 & 95.0 & 3 & $3-\mathrm{C}$ & $3-W$ \\
\hline Mexico & Toluca & 8466 & 2,581 & 89.2 & 3 & 3-A & $3-W$ \\
\hline Peru & Cusco & 10,860 & 3,311 & 70.7 & 4 & 4-A & $4-W$ \\
\hline Bolivia & La Paz - ElAlto & 13,325 & 4,063 & 68.1 & 5 & 5-A & $5-Y$ \\
\hline & & & & & & & \\
\hline
\end{tabular}




\section{CONCLUSIONS}

Resulting data leads to the following analysis and relevant conclusions:

a) There is a correlation between Altitude and Climate zones- All Climate Zones 0 and 1 sites across the Equatorial Region fall within $0-1000 \mathrm{~m}$ ASL $(0-3280 \mathrm{ft})$.

b) It is consistent the fact that within this altitude range most sites fall in ASHRAE Sub-zone A (Humid) while three (3) sites respond to ASHRAE Sub-zone B (Dry).

c) Those same sites, under the Equatorial Moisture Climate zone frame, report more precise humidity conditions delivering Extremely Humid (Z), Very Humid (Y) cities in most cases, but also pointing moderate humid $(X)$ and moderate dry $(\mathrm{W})$ territories in some other cases.

d) Altitude sites ranging from 1000-2000 m ASL (3280- 6,560 ft) report ASHRAE climate zones hot-humid (2A) and warm-humid (3A). The sample shows the case of Nairobi, Kenya reporting Marine moisture conditions.

e) At above mentioned altitude range under Equatorial moisture criteria moderate humid (X) and very humid (Y) sites are predominant under hot (2) and warm (3) climate zones. This mild conditions call for attention as they will demand less costly construction and façades compared to climate zones 0 and 1 where extremely humid $(Z)$ sites are prediminant.

f) Above $2000 \mathrm{~m}$ ASL (6560 ft ASL) up to $3000 \mathrm{~m}$ ASL (9840 ft ASL) there are prevalent climate zone 3 sites. ASHRAE moisture sub-zones cover all three options $(\mathrm{A}, \mathrm{B}, \mathrm{C})$ while in Equatorial zones the reference calls for moderate dry $(\mathrm{W})$ and moderate humid $(\mathrm{X})$ sites. These findings between these two ways of referring outside moisture conditions do not correlate, while Equatorial Climate zones and sub-zones are more consistent with reported humidity content.

g) Above $3000 \mathrm{~m}$ ASL (9840 ft ASL) territories tends to be moderate dry (W) and extremely dry $(V)$. These conditions would call for construction specifications to keep indoor moisture in houses along with thermal insulation on facades in order to prevent moisture migration thru building structures towards the outdoor environment while avoiding temperature loss towards outdoor environment.

This conclusions suggests the convenience to consider a separate approach for Equatorial Region Climate zones and sub-zones in order to consistently comply and improve comfort requirements, $\mathrm{HVAC}+\mathrm{R}$ systems, temperature and humidity space control, energy and operation performance.

Construction improvement opportunities should be understood based on: life cycle costs, energy 
performance, energy annual costs as well as overall buildings performance.

\section{REFERENCES}

[1] ASHRAE. 2013 Weather Data for building design standards. ANSI/ASHRAE Standard 169-2013.

[2] ASHRAE. 2016 Energy Standard for Buildings except Low Rise Residential Buildings. ANSI/ASHRAE/IES Standard 90.1-2016.

[3] ASHRAE. 2017 Fundamentals 2017.
[4] $\mathrm{ROM})$

[5] Fabio Clavijo. 2016 Condiciones Climáticas de Diseño para sistemas de Climatización, Ventilación y Refrigeración (CVR) dieciséis (16) ciudades de Colombia. ACAIRE.2016 
CONGRESO DE DESARROLLO

SOSTENIBLE

\section{GESTIÓN INTEGRAL DE PROCESOS}




\title{
ANÁLISIS CON ALGORITMO K-MEANS DE LA FUERZA LABORAL EN EL SECTOR COMERCIO COLOMBIANO
}

\section{Algorithm analysis with k-means of the labor force in the Colombian commerce sector}

\author{
Suárez, Ruth ${ }^{1}$; Ladino, Iván; Baquero, Luis \\ Fundación Universitaria Los Libertadores, Grupo de investigación GIDAD
}

\section{Resumen}

El presente estudio se centra en identificar patrones comunes de contratación por modalidad y por género que se dan en el sector comercio colombiano, a través de técnicas de clustering y establecer así una base útil para el diseño de políticas de apoyo a la igualdad de género en temas de contratación laboral, tal como lo establece el objetivo de Desarrollo Sostenible No. 8 Trabajo decente y desarrollo económico. Metodología: Para comprobar la existencia de patrones de comportamientos afines en la preferencia de contratación que permitan clasificar a los subsectores en grupos homogéneos se aplicó el algoritmo de K-means con un valor k=4 obtenido por el método del codo. Resultados: Los resultados obtenidos con el Algoritmo K-Means presentan 4 agrupaciones de subsectores comerciales organizados según la cantidad de personal contratado, y donde se evidencia que el $47 \%$ de los subsectores contratan personal femenino en niveles altos y en algunos casos en proporciones similares a la contratación masculina, sin embargo existen otros sectores donde persiste mayoritariamente el personal masculino.

Keywords: K means, Comercio, Contratación, Género.

\section{INTRODUCCIÓN}

Actualmente hay más mujeres en el mercado laboral que en épocas pasadas, sin embargo, aún existen desigualdades marcadas en términos de contratación laboral [1]. Para el año 2007 el porcentaje de actividad laboral femenina en América Latina y el Caribe era del 48,7\% y para al año 2017 había alcanzado un 50,2\%, sin embargo la brecha laboral aún existe y afecta la calidad de vida no solo de las mujeres sino de sus familias [2], las mujeres que se encuentran vinculadas

${ }^{1}$ Correo electrónico de contacto: rmsuarezc@gmail.com 
laboralmente devengan en promedio un 19\% menos de ingresos que los hombres [3]. Ante esta situación, el Programa de Desarrollo de Naciones Unidas PNUD ha incluido en los 17 objetivos formulados para el desarrollo sostenible a 2030, dos objetivos relacionados con esta problemática; el objetivo 5: Equidad de género y el objetivo 8: Trabajo decente y crecimiento económico.

Las cifras que presenta el objetivo 8 relacionado con Trabajo decente y crecimiento económico, muestran que para 2018 alrededor de 3 de cada 5 trabajadores eran hombres, indicando una brecha en la participación de las mujeres en la fuerza laboral [4]. Con el propósito de reducir esta brecha, el objetivo 8 establece como meta que en el año 2030 se logre empleo pleno y productivo $\mathrm{y}$ trabajo decente para todas las mujeres y los hombres.

En la tabla 1, se presentan los porcentajes contratación laboral por sector y por género en Colombia en los años 2017 y 2018, donde se observa que en sectores como agricultura, construcción, transporte y almacenamiento es evidente la brecha laboral de género, existente entre hombres y mujeres, adicionalmente, se observa que los sectores de manufactura, servicios y comercio actualmente generan más ocupación de mujeres, frente a la cantidad los hombres.

Tabla 13 Vinculación laboral por sector económico y género en Colombia 2017-2018

\begin{tabular}{|c|c|c|c|c|c|c|c|c|c|}
\hline & 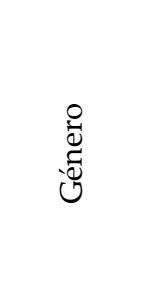 & 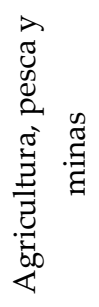 & 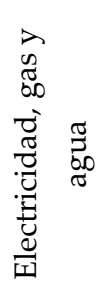 & 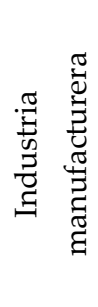 & 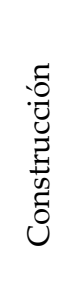 & $\begin{array}{l}\stackrel{0}{0} \\
\dot{\Xi} \\
\tilde{\Xi} \\
0\end{array}$ & 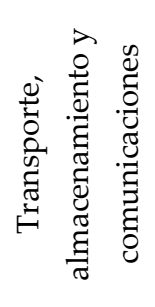 & 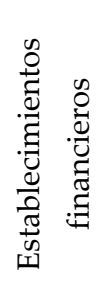 & 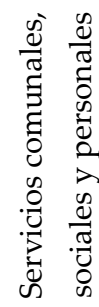 \\
\hline \multirow{3}{*}{2017} & Total & 17,5 & 0,5 & 11,8 & 6,1 & 27,2 & 8,1 & 9,6 & 19,3 \\
\hline & Hombres & 24,4 & 0,7 & 11,2 & 10,1 & 22,3 & 11,8 & 8,3 & 11,2 \\
\hline & Mujeres & 7,9 & 0,3 & 12,5 & 0,6 & 33,9 & 2,9 & 11,3 & 30,6 \\
\hline \multirow{3}{*}{2018} & Total & 17,7 & 0,6 & 12 & 6,2 & 26,8 & 8 & 9,2 & 19,5 \\
\hline & Hombres & 24,6 & 0,7 & 11,3 & 10,1 & 22,5 & 11,6 & 7,8 & 11,3 \\
\hline & Mujeres & 7,9 & 0,3 & 13,1 & 0,8 & 32,8 & 2,9 & 11,1 & 31,1 \\
\hline
\end{tabular}

Fuente: Panorama Laboral, 2019 [3]

El sector comercio agrupa a las empresas que se dedican a la venta y distribución de bienes y productos al por mayor o minorista en centros comerciales, plazas de mercado, entre otros espacios. En Colombia para mayo de 2020, el sector comercio, hoteles y restaurantes generó un 
14\% de los empleos [5] en el caso de Bogotá el sector comercio aportó el 28\% de los empleos [6], por esa razón se buscó identificar las actividades comerciales según el tipo de modalidad en contratación que manejan y la forma de contratación en términos del género, con el fin de identificar los subsectores en los cuales pueden generarse estrategias para el fortalecimiento de habilidades que permitan una mayor participación de mujeres trabajadoras.

Para el desarrollo del estudio se tomaron los datos de la Encuesta Anual de Comercio 2018, en donde se identificaron las variables que hacen relación a las formas de contratación discriminadas por género en los subsectores del comercio colombiano. Para ello se utilizó una técnica de minería de datos denominada Algoritmo K-Means, por medio del cual se analizó la distribución de fuerza laboral en el sector comercial.

\section{Minería de datos y Algoritmo Kmeans}

La minería de datos es un agregado de técnicas que permiten identificar comportamientos y patrones que se encuentran presentes en un conjunto de datos, el resultado del análisis tiene como fin la generación de conocimiento que dé solución a un problema existente [7]. Un aspecto de interés de las técnicas de minería de datos es que se pueden identificar agrupaciones de datos muy grandes con poco conocimiento (a priori) de los patrones que los describen, no requiere supuestos de normalidad previos [8]

Las técnicas más utilizadas en minería de datos son: Métodos de partición, Métodos jerárquicos, Métodos basados en cuadrícula y Métodos basados en densidad [9]. El agrupamiento juega un papel vital en la exploración de datos, la creación de predicciones y para superar las anomalías en los datos. Los grupos que contienen características colaterales idénticas en un conjunto de datos se agrupan utilizando técnicas reiterativas.

El agrupamiento o clustering es una técnica que pertenece a la minería de datos es un tipo de algoritmo de clúster, y es propuesto por J.B.MacQueen [10]. Es conocida como técnica particional de aprendizaje no supervisado, que organiza los datos en clusteres, asegurando que las observaciones correspondientes a cada grupo se encuentren muy cercanas entre ellas, así como bastante separadas de los otros grupos. Como método descriptivo de agrupamiento no necesita conocimiento previo de los datos a analizar, es decir, no cuenta con juicios a priori de los datos [7]. 
El algoritmo K-means, permite la agrupación de un conjunto de datos en subconjuntos cada uno de los cuales tiene definido un valor central (centroide), la agrupación se da en función de su cercanía entre sí de acuerdo con la distancia euclidiana [9]. Cada subconjunto contiene datos similares, en el caso de datos numéricos, la agrupación se da por mínima distancia euclidiana existente entre los datos [11].

La utilidad del algoritmo K-Means está dada por la posibilidad de clasificación y extracción de información relevante, que llevan al descubrimiento de nueva información. Para ello, el algoritmo toma $\mathrm{k}$ como parámetro de entrada y divide un conjunto de datos en k clusters o agrupaciones. El valor medio de cada cluster (centroide) se toma como parámetro de similitud para formar grupos. La media o centro del grupo está formada por la selección aleatoria del objeto k.

Este algoritmo se basa en los siguientes pasos: primero se indica el número de grupos a crear en el estudio los cuales se pueden definir por el método del codo, posteriormente, se obtiene el valor de los centroides para cada grupo creado, a continuación cada individuo es asignado al grupo cuyo centroide es el más cercano por medio del análisis de distancia euclidiana al cuadrado (ESS), por último, los centroides del grupo se actualizan después de cada asignación, hasta asegurar que cada individuo está en el grupo con el centroide más cercano. Este estudio pretende procesar y caracterizar la información obtenida de la encuesta anual de Comercio 2018 (DANE), según la modalidad de contratación del personal discriminado por género.

\section{MATERIALES Y MÉTODOS}

Se toma la base de datos de la Encuesta de anual de comercio 2018 del DANE por ser la información disponible del sector más reciente, en ella se encuentra información correspondiente a 15 subsectores de comercio en Colombia, se da énfasis a la información relacionada con personal contratado por género en 4 modalidades diferentes por subsector. Las variables del estudio son: trabajadores con contratación fija (FIJO_FEM, FINO_MASC), trabajadores con contratación temporal directa (TDIR_FEM, TDIR_MASC), trabajadores con contratación temporal por agencia de empleo (TAGE_FEM, TAGE_MASC)y aprendices (APRE_FEM, APRE_MASC); todos discriminados por género masculino o femenino.

El propósito del estudio es la agrupación de subsectores comerciales según modalidad de contratación del personal, discriminado por género. Para el desarrollo del estudio se utilizó la 
técnica de clúster conocida como Algoritmo K-medias, que permite el análisis de variables cuantitativas organizando la información en grupos que comparten características semejantes; esta técnica se aplicó con medidas de similitud de distancia Euclídea y con formación de grupos por el método del centroide, a través del software $\mathrm{R}$ con el uso de los paquetes FactoMineR y FactoExtra. El software R se desarrolló en 1993 por parte de Robert Gentleman y Ross Ihaka, pertenecientes al Departamento de Estadística de la Universidad de Auckland

\section{RESULTADOS Y ANÁLISIS DE RESULTADOS}

El presente estudio representa una valiosa contribución de para el sector comercio ya que permite identificar patrones comunes de contratación del personal entre los subsectores del sector comercio, identificando aquellos que evidencian desigualdad de género. Los datos tomados de la Encuesta Anual de Comercio 2018 - DANE, fueron organizados en 15 subsectores (individuos) y se contrastaron con 8 variables que presentan la cantidad de trabajadores contratados por género y por modalidad.

Según lo observado en la figura 1.las actividades de comercio de alimentos, farmacéuticos, enseres, y maquinaria, vinculan la mayor cantidad de personas de género masculino y especialmente la vinculación se realiza por contratación fija y no temporal con 51, 43, 33 y 31 hombres vinculados en promedio. La contratación temporal por medio de agencias de empleo se presenta con mayor frecuencia en las actividades de comercio de alimentos, enseres y maquinaria. La contratación temporal por medio de agencias de empleo en el caso de hombres, ser presenta en el comercio de alimentos y enseres principalmente. 


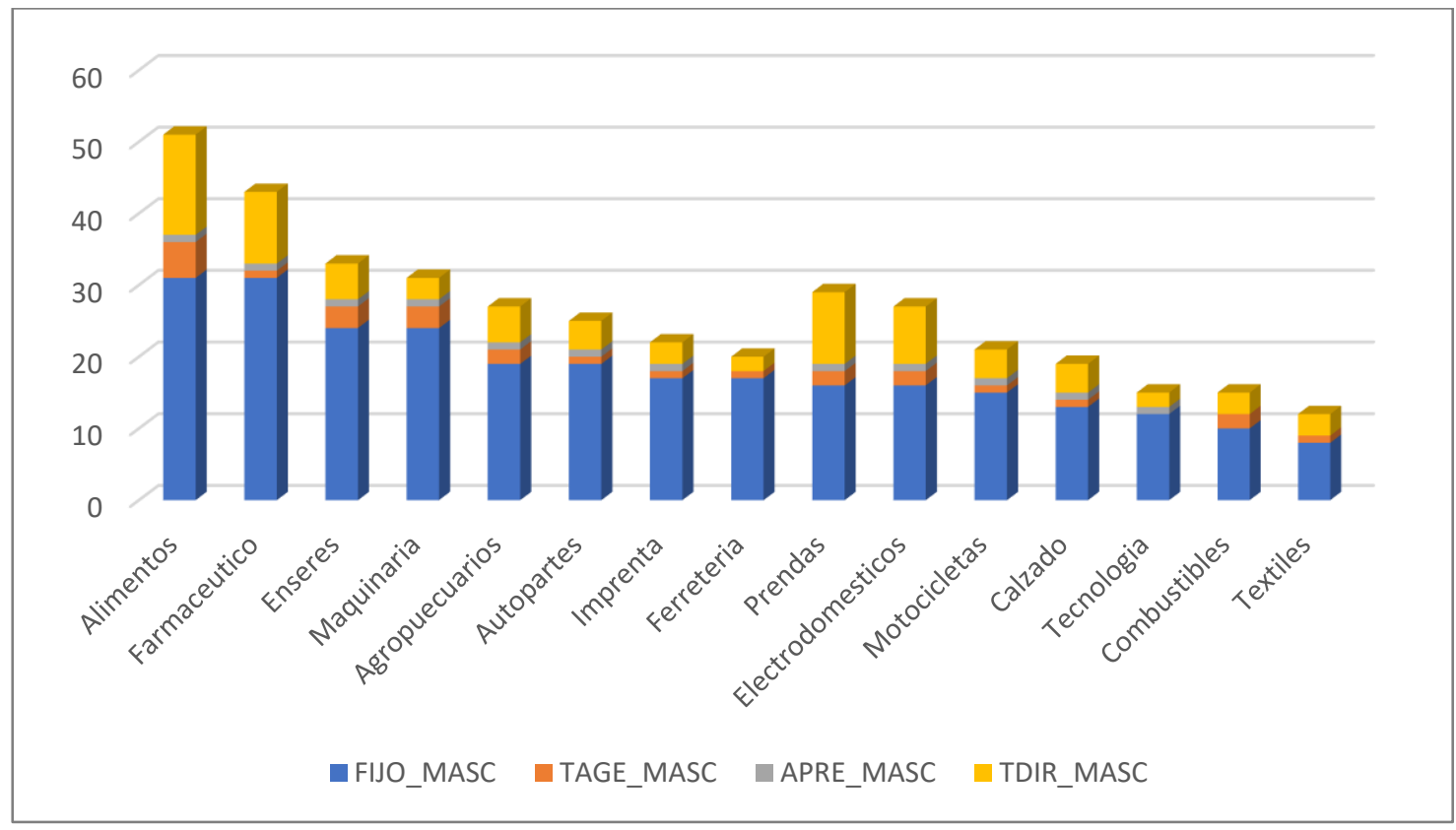

Figura 29 Media de contratación de hombres por modalidad. Fuente: Autores

La figura 2 presenta el promedio de muejres vincualdas laboralmente en las actividades del sector comercio por actividad y por donde modalidad de vinculación. Se destaca allí el comercio de prendas de vestir se tiene a la mayor cantidad de mujeres contratadas 58 en total, distribuidas en contratación fija y temporal directa en su mayoría, es decir, sin intermediacón de agencias de empleo. Le sigue el comercio de alimentos, farmacéutico, enseres y calzado ocn 46, 45 y 41 personas respectivamente. 


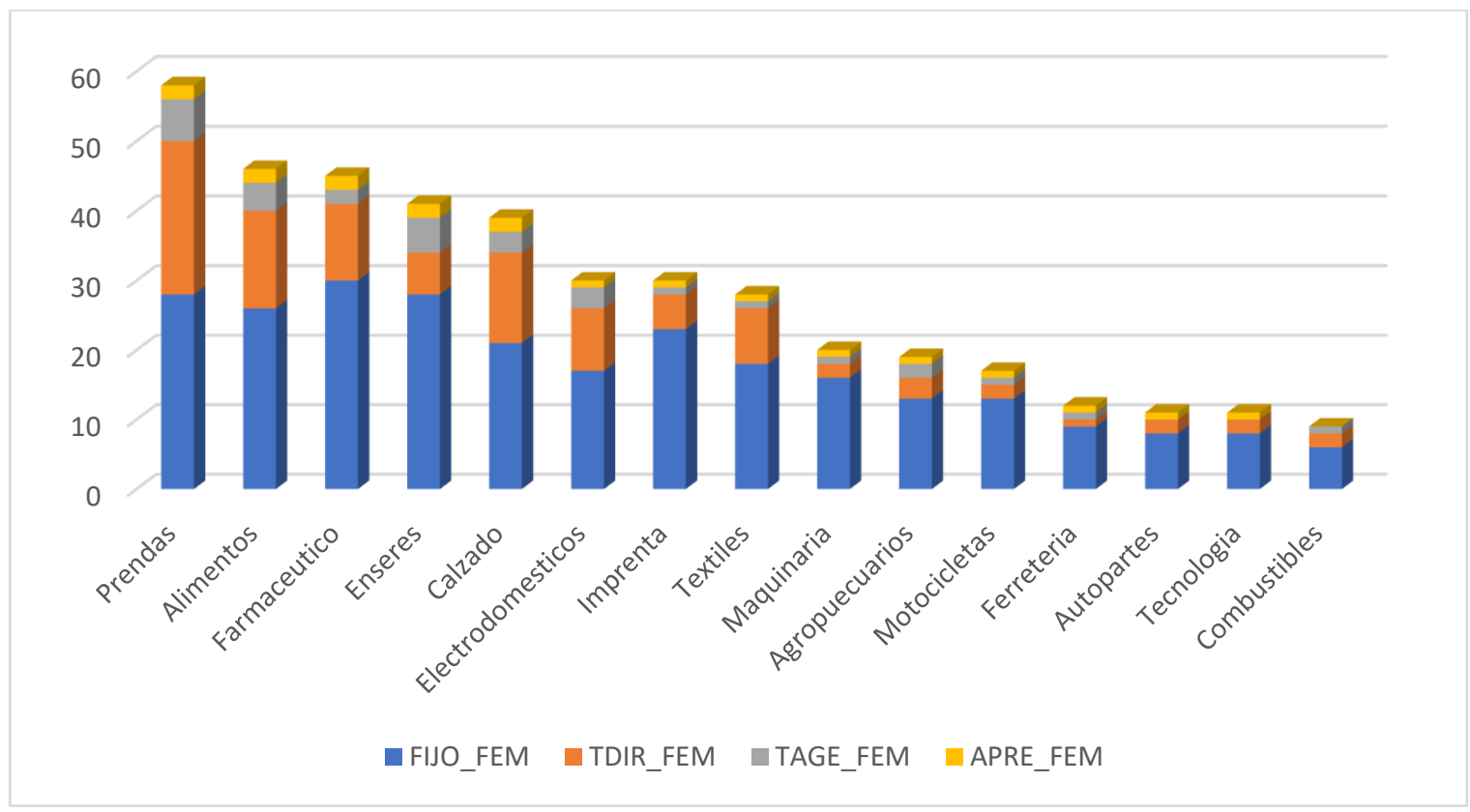

Figura 30 Media de contratación de mujeres por modalidad. Fuente: Autores

Dentro del estudio se utilizó minería de datos, la cual es una técnica muy utilizada en problemas de agrupación sectorial, permite analizar empresas que por su objeto social pertenecen a un determinado subsector, identificando patrones de comportamiento afines con otros subsectores. Se busca entonces, agrupar los subsectores que pertenecen al sector comercio de acuerdo a la forma de vinculación de la fuerza de trabajo discriminada por género.

Inicialmente se realizó el cálculo de la correlación entre las 8 variables, encontrando que las variables que presentan el mayor valor de correlación son: FIJO_FEM y APRE_FEM con un valor de 0.945 , las variables relacionadas con contratación femenina permanente o temporal presentan alta incidencia en la contratación de aprendices del mismo género y en general de mujeres en todas las modalidades de contratación. El menor valor de correlación se dio entre las variables FIJO_MASC y TDIR_FEM con un valor de 0.188, así como FIJO_MASC y TAGE_FEM con un valor de 0.344 , indicando que la cantidad de personal masculino contratado de forma permanente no tiene relación en la contratación femenina en modalidad temporal por agencia y directa.

A fin de establecer el número de grupos adecuado para la realización del análisis se aplicó el método del codo (Elbow method), donde se identificaron 4 clusters $(k=4)$. El valor $\mathrm{k}$ se determinó por el número de clúster a partir del cual la reducción en la suma total de cuadrados internos 
empezó a estabilizarse.

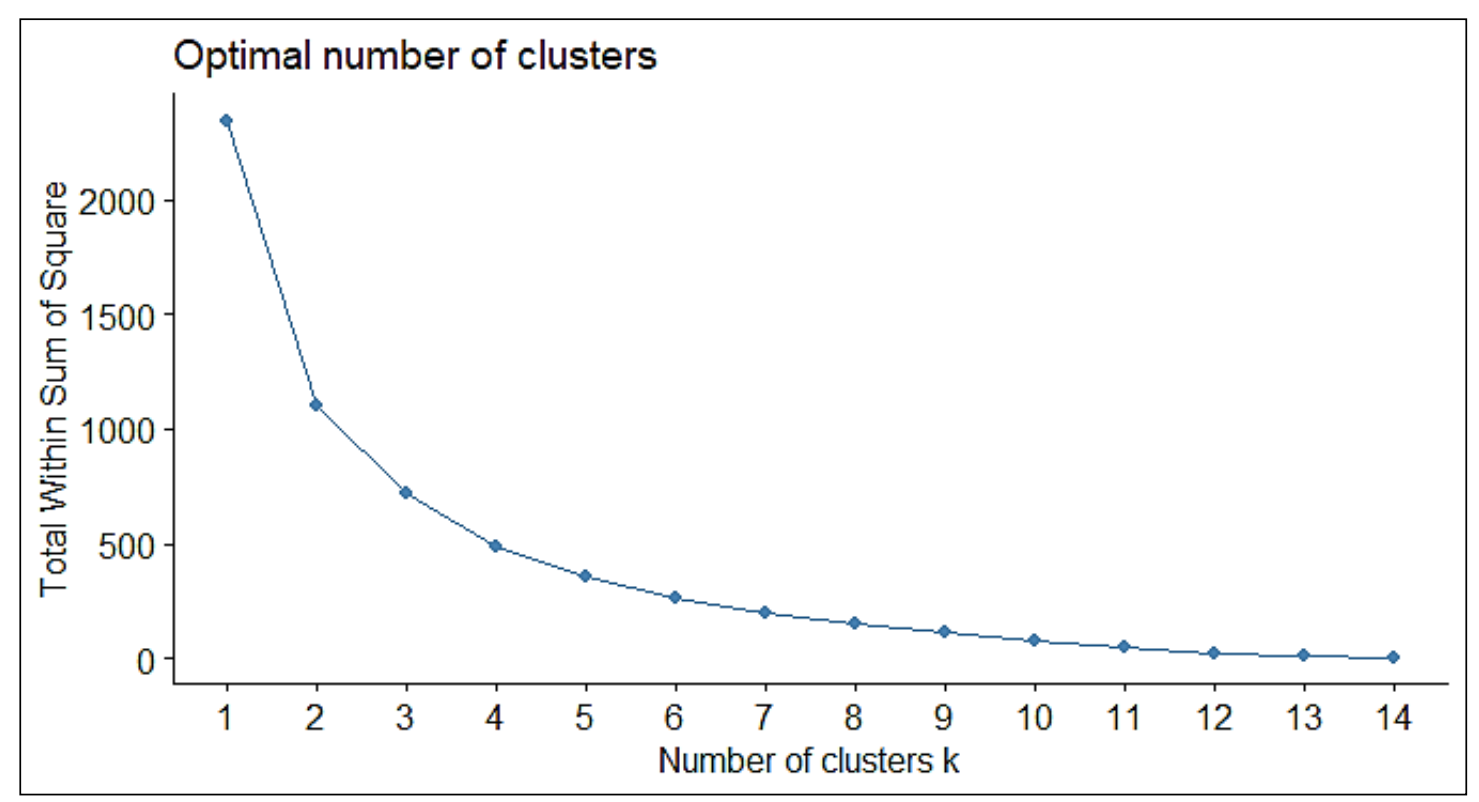

Figura 31 Número óptimo de clusters por método del codo. Fuente: Autores

Dado que el estudio tenía múltiples variables fue preciso realizar una reducción de la dimensionalidad a partir de Análisis de Componentes Principales PCA, de tal manera que las dos primeras componentes generadas describen el comportamiento del grupo de datos en términos de la mayor varianza (combinación lineal), con independencia estadística. La figura 4 presenta las dos primeras componentes principales para el estudio, indicando que la componente 1 (Dim1) contiene el 69,2\% de información de las variables, mientras que la componente 2 (Dim2) contiene el 15,9\% de la información. El total de información de las variables del estudio, compilada en estas dos componentes muestra el $85,1 \%$ del total de la varianza de los datos iniciales. 


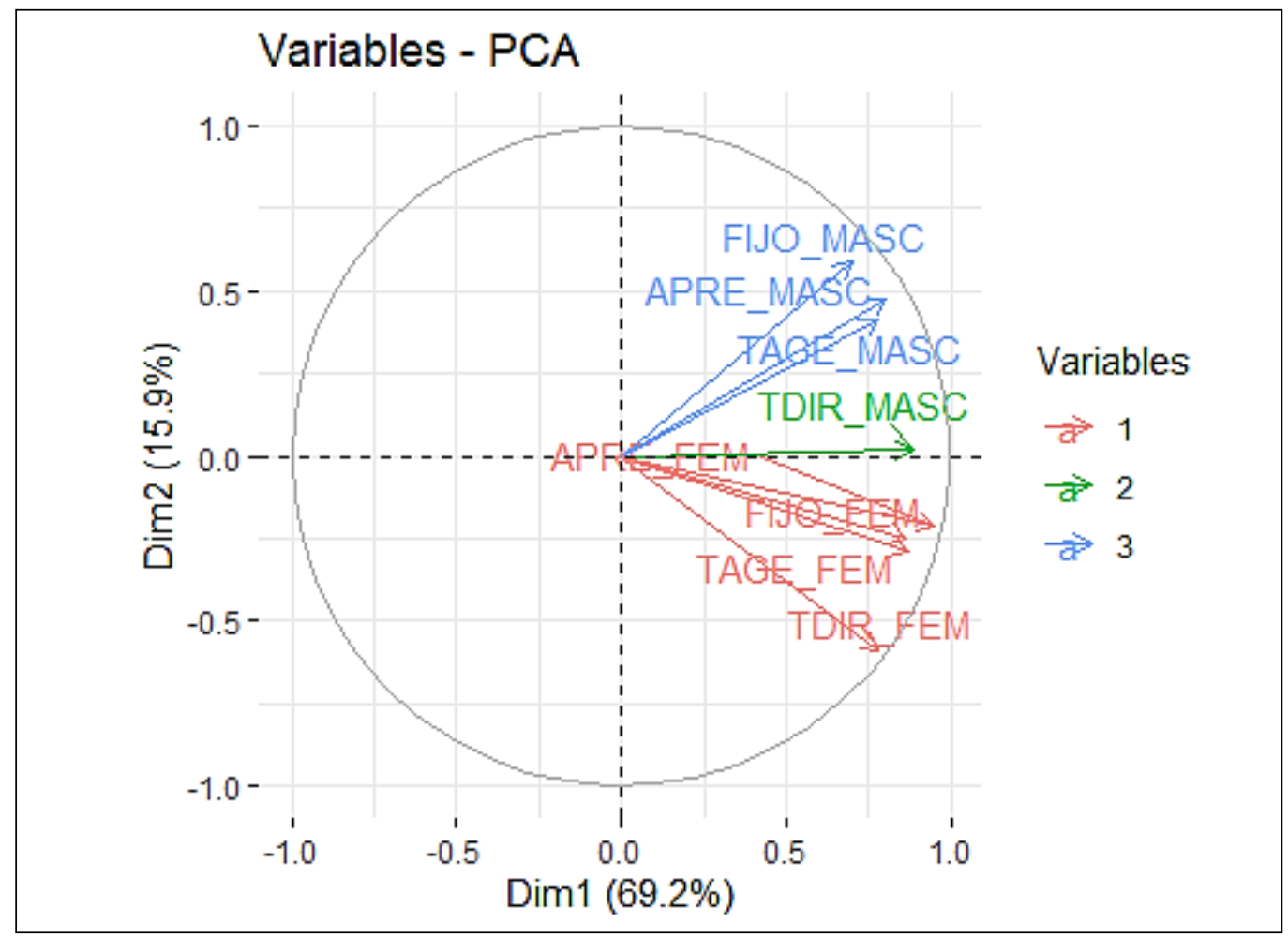

Figura 32 Variables en Análisis de Componentes Principales. Fuente: Autores

La figura 4 muestra que las 8 variables se organizan en tres grupos discriminados por color, donde las variables asociadas a los trabajadores de género masculino se encuentran correlacionadas entre ellas, la misma situación se presenta entre las variables de género femenino, sin embargo, no están relacionadas significativamente entre grupos de variables. Se confirma lo evidenciado en el estudio de correlación: Entre más personal de un género esté contratado, la tendencia es a contratar en otras modalidades personas del mismo género. 


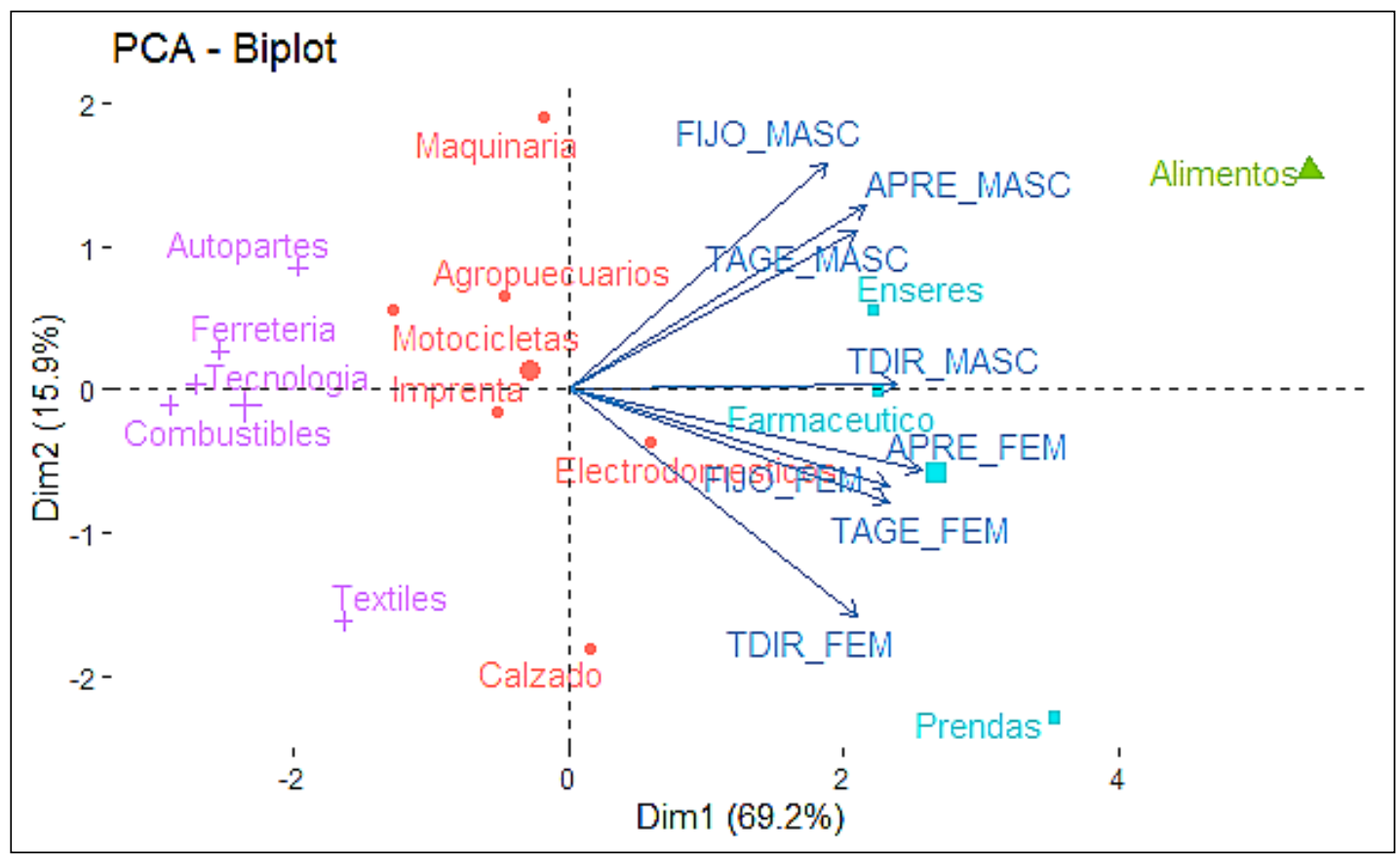

Figura 33 Clusters según modalidad de contratación y género en sector comercio. Fuente: Autores

En el análisis resultante se obtuvieron 4 clusters que están organizados según la cantidad de trabajadores totales que vinculan, el primero con el 6,6\% de la muestra, presenta al comercio de alimentos (verde) con un nivel muy alto de contratación promedio de personal 97 en todas las modalidades: fijos, temporales y aprendices de los dos géneros, con una mayor participación del género masculino 51 frente a 46, según los valores presentado en la tabla 2. El segundo clúster (turquesa) con el $20 \%$ de la muestra agrupa al comercio de enseres, farmacéuticos y prendas de vestir, con un promedio de contratación que oscila entre 72 y 87 trabajadores. Predomina en el comercio de enseres y de farmacéuticos un nivel alto de vinculación para los dos géneros. En el caso del comercio de prendas de vestir predomina un nivel muy alto de contratación femenina en comparación con un nivel medio masculino, especialmente en la modalidad de contratación temporal directa y por medio de agencias de empleo. Los subsectores analizados hacen parte del comercio de artículos de primera necesidad para el consumo en Colombia. 
Tabla 14 Nivel de contratación por género en el sector comercio

\begin{tabular}{|c|c|c|}
\hline \multicolumn{3}{|c|}{ Género Masculino } \\
\hline Mínimo & Máximo & Nivel \\
\hline 44 & 51 & Muy Alto \\
\hline 35 & 46 & Alto \\
\hline 28 & 35 & Medio \\
\hline 19 & 28 & Bajo \\
\hline 12 & 19 & Muy bajo \\
\hline
\end{tabular}

\begin{tabular}{|c|c|c|}
\hline \multicolumn{3}{|c|}{ Género Femenino } \\
\hline Mínimo & Máximo & Nivel \\
\hline 48 & 58 & Muy Alto \\
\hline 38 & 48 & Alto \\
\hline 29 & 38 & Medio \\
\hline 19 & 29 & Bajo \\
\hline 9 & 19 & Muy bajo \\
\hline
\end{tabular}

Fuente: Autores

El tercer clúster identificado (rojo) agrupa el $40 \%$ de los subsectores de la muestra, lo componen el comercio de maquinaria, agropecuario, motocicletas, imprenta, electrodomésticos y calzado. Este clúster tiene un promedio de personas contratadas que oscila entre 38 y 57, es decir cuentan con un nivel de contratación medio-bajo para personal masculino y para personal femenino.

En este clúster se identifica dos patrones de contratación específicos. Para el primer caso los subsectores maquinaria, agropecuario y motocicletas presentan mayor contratación de personal masculino (61\%) en las modalidades de contrato fijo FIJO_MASC, temporal por agencia de empleo TAGE_MASC y de practicantes APRE_MASC, mientras que la contratación de personal femenino es del 39\%, en todas las modalidades de contratación. El segundo caso presenta los subsectores de imprenta, electrodomésticos y calzado, los cuales presentan mayor contratación de personal femenino en todas las modalidades con un $60 \%$ de mujeres frente al $40 \%$ de hombres. Específicamente en el subsector de comercio de calzado se presenta una mayor proporción de mujeres contratadas temporalmente de forma directa.

Se observa un cuarto clúster (morado) en el cual se encuentra el 33,3\% de la muestra, conformada por los subsectores de autopartes, ferretería, tecnología, combustibles y textiles. Se evidencia que estos subsectores en el comercio colombiano son los que cuentan con un nivel bajo de personal contratado en los dos géneros. Teniendo en cuenta la concentración de los subsectores en torno al centroide, se encuentran 4 subsectores con distancias muy cercanas entre sí, a diferencia del subsector Textiles, el cual se encuentra a mayor distancia, situación dada por la preferencia que tiene el subsector de contratar mano de obra femenina en mayor proporción que la masculina; mientras que los demás subsectores del clúster evidencia menor preferencia a la contratación de personal femenino con tan solo un $30 \%$. 
Los resultados muestran que el sector comercio en Colombia presenta mayor tendencia a la contratación en razón del género y a pesar de que realiza contratación de personal femenino en todas las actividades comerciales, continúa prevaleciendo la fuerza laboral masculina en varias modalidades de contratación. Esto concuerda totalmente con las cifras presentadas por el PNUD en relación al objetivo de trabajo decente y desarrollo económico.

\section{CONCLUSIONES}

El estudio permite observar la mayor tendencia en contratación de personal masculino en comparación con el personal femenino en el 53\% de los subsectores comerciales, situación que confirma la necesidad de desarrollar políticas de equidad de género como parte de los objetivos de desarrollo sostenible. Al tratarse de actividades comerciales es necesario implementar una política empresarial con enfoque de género, que permitan la vinculación laboral de un número mayor de personal femenino, como lo es el sistema de gestión en enfoque de género Equipares, impulsado por el Ministerio de trabajo desde el año 2013.

Se obtuvieron 4 agrupaciones de subsectores del comercio colombiano, a partir de la aplicación de la técnica de clustering particionado, bajo el Algoritmo K-means. Encontrándose evidencia de que subsectores relacionados con actividades que en la industria son consideradas exclusivas para hombres, prefieran contratar a personal masculino, a pesar de tratarse en este caso de actividades comerciales.

Debido a que cerca del $70 \%$ de los trabajadores de ambos géneros están vinculados con contratación fija, y el porcentaje de otras modalidades de contratación es significativamente menor, no fue posible ahondar en las preferencias de contratación con el algoritmo K-Means.

A partir de los agrupamientos obtenidos en los subsectores comerciales se pueden definir estrategias de fortalecimiento de competencias en relaciones comerciales para el personal femenino, así como avanzar en la apertura de oportunidades laborales en los subsectores comúnmente conformados en su mayoría por hombres. 
[1] OIT, «La participación laboral de las mujeres aumenta camino a la igualdad aún es largo en América Latir Caribe,» Organización Internacional del trabajo, 8 marz [En línea]. Available: https://www.ilo.org/americas/s prensa/WCMS_619953/lang--es/index.htm. [Último ac junio 2020].

[2] I. Vaca Trigo, «Oportunidades y desafíos para la autono las mujeres en el futuro escenario del trabajo,» Serie: Ası género. Comisión Económica para América Latina y el (CEPAL), vol. 154, no (LC/TS.2019/3), 2019.

[3] OIT, «Panorama laboral para América latina y el C Organización Internacional del Trabajo, Lima, 2019.

[4] PNUD, «Objetivos de desarrollo sostenible,» Programe Naciones Unidas para el Desarrollo, 2015. [En Available:

https://www.undp.org/content/undp/es/home/sustaina development-goals.html. [Último acceso: 2 junio 2020].

[5] DANE, «Mercado laboral según sexo,» Departc Administrativo Nacional de Estadística, 11 mayo 20? línea].

Av

https://www.dane.gov.co/index.php/estadisticas-portema/mercado-laboral/segun-

sexo\#: :text=Para\%20el\%20trimestre\%20enero\%20\%2D arzo,los\%20hombres\%209\%2C8\%25.\&text=Las\%20muj 0j\%C3\%B3venes\%20desocupadas $\% 20 \mathrm{de}, \% 2 \mathrm{C} 7 \% 25 \% 20$ los\%20desocupados.. [Último acceso: 2 junio 2020].

[6] $\mathrm{CCB}$, «Observatorio de la región Bogotá - Cundinar Cámara de Comercio de Bogotá, 2020. [En línea]. Avi https://www.ccb.org.co/observatorio/Economia/Econor dinamica-incluyente-e-innovadora/Mercado-laboral. [ acceso: 2 Junio 2020].

[7] H. Ramírez-Murillo, C. Torres-Pinzón y E. Forero-1 «Estimación del potencial fotovoltaico mediante min datos en cuatro ciudades de Colombia,» TecnoLógicas, no 46, pp. 65-85, 2019.

[8] L. M. Castro Heredia, Y. Carvajal Escobar y Á. J. Ávili «Análisis clúster como técnica de análisis exploratc registros múltipes en datos metereológicos,» Ingeni Recursos Naturales y del Ambiente, nº 11, pp. 11-20, 2012.

[9] A. Preeti, D. Deepali y V. Shipra, «Analysis of K-Means Medoids Algorithm For Big Data,» Procedia Computer vol. 78, pp. 507-512, 2016.

[10] L. Youguo y W. Haiyan, «A Clustering Method Based Means Algorithm,» Physics Procedia, vol. 25, pp. 110 2012.

[11] T. T. Pham, G. A. Lobos y C. Vidal-Silva, «Innovac Minería de Datos para el Tratamiento de Imi Agrupamiento K-media para Conjuntos de Datos de Alargada y su Aplicación en la Agroindustria,» Infor tecnológica, vol. 30, nº 2, pp. 135-142, 2019. 


\title{
MODELO DE GOBERNANZA INTELIGENTE PARA LA GESTIÓN DE PROCESOS EN LAS INSTITUCIONES DE EDUCACIÓN SUPERIOR: CASO UNIVERSIDAD DE SANTANDER
}

\section{Smart governance model for process management in education institutions: case Universidad de Santander}

\author{
Jaimes, Mayra1; Caballero, José Alonso² \\ ${ }^{1}$ Universidad de Santander UDES, Grupo de Desarrollo Experimental y Tecnológico GEDETEC \\ 2Unidades Tecnológicas de Santander UTS, Grupo de Investigación en Producción y Calidad GIPROCAL
}

\section{Resumen}

El concepto de gobernanza ha ganado un espacio protagónico en el discurso de académicos, investigadores y practicantes de los asuntos públicos. Sin embargo, en la práctica sigue siendo un término relativamente desconocido, que se sigue utilizando como sinónimo de gobernabilidad, buen gobierno, y otros conceptos cercanos, pero definitivamente no equivalentes. Es por ello que, el objetivo de este documento consiste en estructurar de un modelo de gobernanza inteligente paras Instituciones de Educación Superior - IES -, basado en fuentes primarias y secundarias, tomando como referencia las particularidades de la Universidad de Santander, con el fin de materializar los esfuerzos teóricos en el área y contrastarlos con la experiencia de la institución. Como resultados, se destacan las coincidencias en cuanto a los principales factores que componen la gobernanza inteligente en las instituciones de educación superior, desde el punto de vista teórico y la experiencia universitaria, logrando proponer un modelo en el cual se consolidan dichos componentes. Se espera que este análisis y el modelo propuesto, funcione como punto de referencia y soporte para académicos y practicantes del área, de modo que se pueda continuar avanzando en un área con grandes oportunidades para su exploración.

Palabras clave: Gobernanza Inteligente, Instituciones de Educación Superior, Universidades Inteligentes, Tecnologías de la Información y la Comunicación.

\footnotetext{
${ }^{2}$ Correo electrónico de contacto: jcaballero@correo.uts.edu.co.
} 


\section{INTRODUCCIÓN}

Los campus universitarios son considerados sistemas complejos, compuestos por numerosos elementos, tales como usuarios, edificios, infraestructura, entre otros, los cuales constantemente interactúan entre sí [1]. Dichos espacios proporcionan diferentes servicios a sus grupos de interés, los cuales se basan en ciertas áreas fundamentales para su desarrollo, de modo que se mantenga un ambiente agradable para su interacción. Dentro de estas áreas fundamentales, la Gobernanza juega un papel de gran importancia, ya que su rango de acción va mucho más allá del análisis tradicional de organización interna, sino a la implementación de Tecnologías de Información y Comunicación (TIC's) para el desarrollo de procesos más efectivos, transparentes, contabilizables y sostenibles [2].

Por otra parte, los campus universitarios representan un ejemplo perfecto de las células urbanas, comparables a las ciudades a pesar de las diferencias en la dimensión y tipo de estructuras. Una de las razones por las cuales los marcos de trabajo de ciudad inteligente son extensibles al estudio de campus inteligentes y viceversa, es porque comparten desafíos comunes en las áreas anteriormente mencionadas [3], lo que permite un intercambio de conceptos entre estos dos espacios territoriales tan diversos [1].

Teniendo en cuenta lo anterior, y los diferentes análisis de factores pertenecientes a campus inteligentes realizados en la Universidad, se busca la estructuración de un modelo a partir de la profundización en el pilar de la gobernanza en universidades inteligentes, tomando como referencia el caso de la Universidad de Santander UDES, sede Bucaramanga, de modo que se identifiquen los principales componentes y prácticas en gobernanza inteligente en el marco de un Smart Campus, los cuales guíen los procesos de decisión, ejecución y evaluación institucionales, aportando un mayor conocimiento en el área y la experiencia de la institución.

\section{MATERIALES Y MÉTODOS.}

La metodología propuesta corresponde a una investigación de tipo descriptiva con enfoque cualitativo, representada en un proceso dividido en tres fases, las cuales son:

- Revisión de literatura: en esta primera etapa se llevará a cabo una revisión de literatura contenida en las bases de datos científicas Web of Science y Scopus, con el fin de establecer el estado actual del tema, determinando así sus principales componentes y metodologías 
de desarrollo

- Diseño y aplicación de herramienta de recopilación de información: teniendo en cuenta los principales componentes identificados en la literatura, se procede a estructurar una herramienta de recopilación de información tipo cuestionario, para su aplicación a los principales actores de la institución a modo de entrevista semiestructurada, con el fin de consolidar sus conocimientos sobre el tema y la institución, para la posterior sistematización de la información.

Contraste de fuentes de información: en esta etapa final, se realizará el contraste de los hallazgos provenientes de las fuentes primarias y secundarias de los dos momentos anteriores, empleando el software de análisis cualitativo Nvivo, con el fin de establecer un modelo de Gobernanza adaptado a la institución, de modo que funcione como línea base para el desarrollo sostenible de los procesos misionales de la universidad.

\section{RESULTADOS}

\section{Revisión de literatura}

Inicialmente se resalta la relación entre un "gobierno inteligente" con la buena gobernanza, en la medida en que este primero busca la toma de medidas de forma transparente, equitativa y participativa, vinculadas al gobierno electrónico para el avance progresivo de la tecnología. Así mismo, [4] relacionan la gobernanza inteligente con la toma de decisiones políticas correctas y su implementación de forma efectiva y eficiente. Por su parte, [5] menciona que la toma de decisiones puede ser innovadora a partir de la vinculación de tecnologías de red, concordando con [6] quienes relacionan la gobernanza inteligente con la recopilación de información sobre gestión a través de sensores o redes de tecnología, buscando que estas nuevas tecnologías fortalezcan la racionalidad del gobierno a la hora de tomar decisiones a través de información más completa y disponible, facilitado la implementación de estas mismas.

Por su parte, [7] concibe la gobernanza inteligente como la colaboración entre departamentos y comunidades, para promover el crecimiento económico a partir del ofrecimiento de operaciones y servicios centrados en los ciudadanos. Así mismo, [8] destacan la gobernanza basada en la comunidad y una mayor conectividad apoyada en la tecnología. De manera similar, [9] presentan la gobernanza inteligente como proactiva y abierta, involucrando todos los actores, buscando maximizar el desempeño socioeconómico y ecológico, con el fin de enfrentar a los efectos negativos externos y alcanzar una mayor sostenibilidad. 
Para [10], la gobernanza inteligente está relacionada con la buena administración y buenas políticas que pueden generar fuertes interacciones, que puedan atender problemas que enfrente la comunidad. Para esto, se requieren de procesos de toma de decisión inteligentes y su respectiva implementación a través de una administración inteligente, soportados en las Tecnologías de Información y Comunicación - TIC - que, a su vez, integren la información, procesos, instituciones e infraestructura física para el servicio a la comunidad. A partir de esta vinculación integral, se logran transformaciones no solo a nivel interno sino externo, generando una mayor incidencia con los resultados obtenidos.

En cuanto [11] señala que la gobernanza inteligente permitirá el mayor acercamiento a una sociedad Smart, al integrar la participación de la comunidad, generando estrategias y políticas que permitan el desarrollo de diferentes áreas como la educación y el transporte, integrando así a ciudadanos, empresarios y otros actores. Este mismo autor, resume la gobernanza inteligente en la interacción de los actores y sus espacios de interacción apoyados en las TIC [12], logrando procesos más eficaces y eficientes, maximizando así los resultados positivos.

Teniendo en cuenta las anteriores definiciones, se destacan los principales componentes como lo son: la participación política, servicios para los ciudadanos y el buen funcionamiento de la administración [13], la promoción de iniciativas inteligentes, el uso de tecnologías en red para la toma de decisiones y su respectiva implementación [14], el uso de nuevos canales de comunicación con los ciudadanos (García-Garrido, 2017), la inclusión social de la comunidad [15], el manejo de un modelo de gobernanza basado en la comunidad y facilitado por nuevas tecnologías [8], así como la interconexión e integración de la información, procesos, instituciones e infraestructura física al servicio de la comunidad [2].

Una vez identificados los principales componentes en la literatura, se procede a la continuación en el desarrollo metodológico.

\section{Diseño y aplicación de herramienta de recopilación de información}

Mediante entrevistas realizadas a algunos funcionarios de la institución, se logró conocer en profundidad el concepto de gobernanza implementada al interior de la misma. Con el fin de obtener una visión global de la situación, se obtuvo la participación de actores pertenecientes a los diferentes procesos misionales de la universidad, como lo son: rector, vicerrectores (de investigaciones, extensión, administrativa y posgrados), al director de planeación institucional, 
una asesora externa de la universidad y el representante de docentes ante el consejo académico. Las preguntas dirigidas a cada uno de estos representantes, tienen como finalidad identificar los criterios y conceptos que se ejecutan en la institución, teniendo como base el concepto de gobernanza y el modelo propuesto previamente.

Una vez consolidada la información se logró identificar aspectos clave como:

- La gobernanza al interior de la institución se centra en tres aspectos considerados fundamentales: la autonomía institucional, la participación en el gobierno corporativo y la rendición de cuentas. Por ello, se apoya en su desarrollo a partir de su estatuto general, reglamentos, plan de desarrollo, así como la participación de estudiantes en los órganos de gobierno y la búsqueda de la calidad de los programas académicos.

- El acercamiento de la institución hacia la práctica de un buen gobierno con financiamiento propio que ejerce el principio de transparencia en la rendición de cuentas con implementación social.

- Se resalta la preocupación por aspectos intraorganizacionales, articulando de manera adecuada el cumplimiento de los logros con calidad. La gobernanza de la institución, se concibe y relaciona con la capacidad de coordinación institucional entre políticas, acciones y estrategias que permiten el engranaje de los objetivos liderados por los directivos de la institución.

- La capacidad de equilibrar un procesamiento educativo a través del marco normativo implementado por la UDES, evidencia una respuesta positiva al sistema universitario, es un reconocimiento encaminado en fortalecer las políticas de desarrollo institucional en el ámbito gubernamental dejando atrás un término ambiguo para transformarlo en un concepto de moda.

- De igual manera, se resalta la importancia de los sistemas informáticos para la gestión de los procesos al interior de la universidad, así como para la comunicación con la comunidad, haciendo uso de las TIC's como, por ejemplo, el Campus Virtual, el cual está muy bien estructurado y ha ido fortaleciendo su articulación con los procesos propios del currículo, y una herramienta como indicador de cumplimiento en el seguimiento a docentes, estudiantes etc; sin embargo, se podría articular con los programas presenciales, para un mejor aprovechamiento en la búsqueda de un mayor impacto para la Universidad.

- A partir de lo anterior, se identifica la búsqueda de estandarizar procesos, a partir de la norma ISO 9001, sin embargo, es necesario que la Universidad sea más eficiente en el manejo de las decisiones administrativas y de sus recursos para la optimización de los 
procesos y la mejora continua de los mismos; así como, desconcentrar la toma de decisiones para generar un flujo continuo de los procesos, ya que tiene procesos muy ágiles y otros que conllevan más tiempo, entre ellos, se tienen procesos muy operativos y estos demandan más trabajo, como: la contratación de profesores tanto nacionales como internacionales y la gestión de nómina para los pagos.

Dados los factores revisados anteriormente, se evidencia que la Institución tiene gran capacidad para desarrollar controles directos e indirectos, planificados y compatibles con la autonomía universitaria creando sistemas competitivos para captar a los estudiantes, docentes y demás recursos, así como presenta oportunidades de mejora que le permitirán aumentar su eficiencia a nivel de procesos. Este planteamiento le permitirá a la institución potenciar su funcionamiento interno, enfocado en el concepto de gobernanza inteligente para una mejor gestión de sus procesos misionales.

\section{Contraste de fuentes de información}

Teniendo en cuenta, tanto la revisión de literatura realizada como la recopilación de información de fuentes primarias a partir de actores claves al interior de la universidad, se logra identificar los principales factores que fundamentan el modelo de gobernanza inteligente que se busca proponer, (Figura 1) el cual estará conformado de la siguiente manera:

- Inclusión social y económica: la institución debe gestionar de la mejor forma sus recursos económicos, así como buscar la financiación de sus actividades relacionadas con investigación, innovación y relación con la sociedad, con el fin de generar una mayor confianza a esta última.

- Tecnologías de la Información y la Comunicación: juegan un papel fundamental en el desarrollo de la gobernanza inteligente, teniendo en cuenta que se recomienda su uso generalizado en la institución, de modo que apoye todos los procesos internos para obtener así información verídica y su circulación lo más rápido posible.

- Gestión de la información: a partir del uso de la tecnología, se puede generar gran cantidad de información que servirá para la generación de nuevas soluciones, buscar la mejora continua y responder de forma rápida y eficiente a situaciones problemáticas, aprendiendo de las mismas.

- Gestión interna efectiva: la toma de decisiones por parte de la institución en todos sus niveles y estamentos debe ser lo más ágil posible, apoyándose en las TIC y la Gestión de 
la Información, de modo que se disminuyan los reprocesos y se aumente la efectividad en los procesos de toma de decisión, aprovechando así al máximo tanto los recursos existentes como el tiempo en la gestión de los mismos.

- Interacción con la comunidad: finalmente, todos los esfuerzos por parte de la gobernanza deben enfocarse en la comunidad universitaria, la vinculación de todos los miembros de la misma en las decisiones que se tomen, que se mantengan informados acerca de lo que sucede en la institución, que puedan involucrarse en los procesos que se desarrollan, que las soluciones sean centradas en la comunidad permitirá un mayor y mejor desarrollo de la organización de manera integral y transversal.

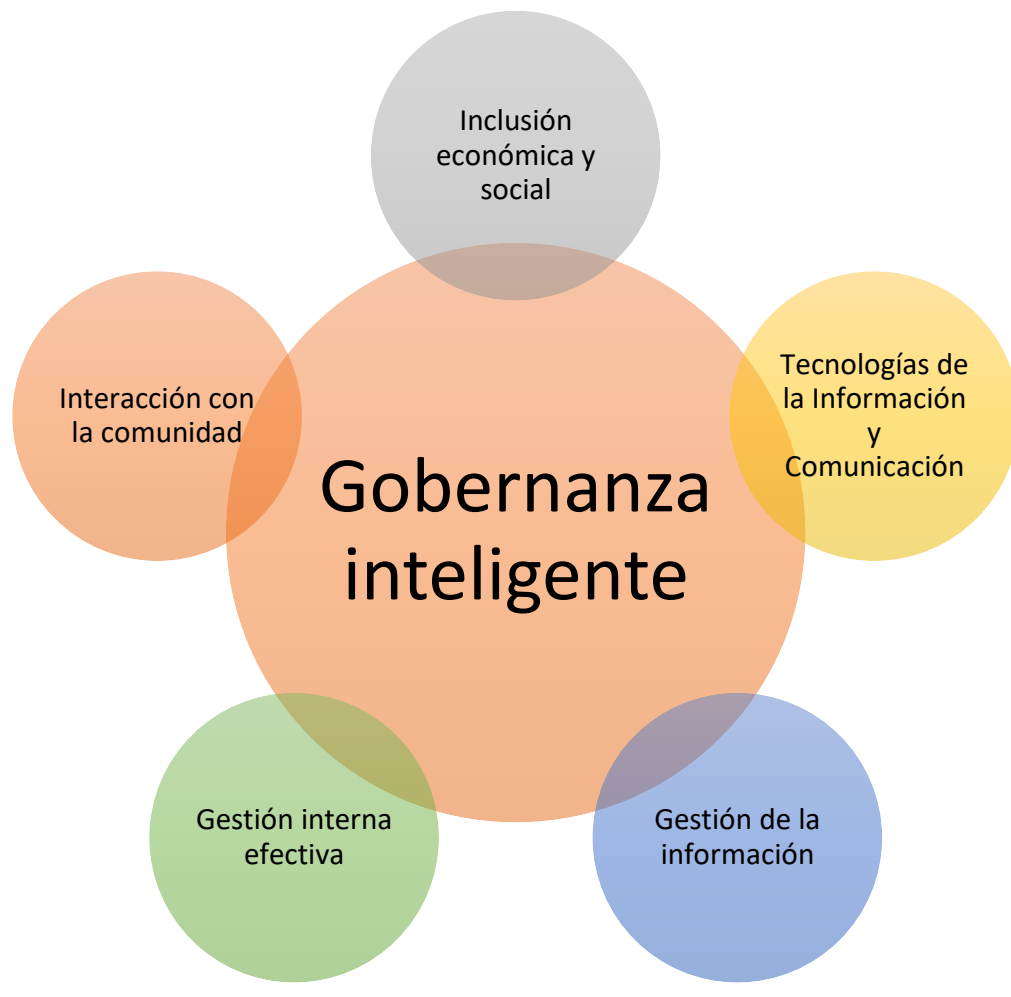

Figura 34 Modelo de Gobernanza Inteligente

\section{CONCLUSIONES}

Definir un modelo de gobernanza que sea apropiado a las necesidades de las Universidades y su círculo social, representa un gran reto. Las orientaciones de gestión directivas derivadas de una estructura institucional ayudan a mejorar el proceso de búsqueda a nuevos saberes con exigencia 
en un orden administrativo, social y académico. Reconocer un modelo de gobernanza que va más allá del gobierno universitario es una tarea ardua de instituciones que han identificado como una gran limitante a la respuesta oportuna y la toma de decisiones por parte de directivos o funcionarios de las instituciones. Así mismo, las aportaciones teóricas de la gobernanza de la presente investigación, permiten exponer una transformación de la universidad, la sociedad y todos los roles de nuevos actores en relación con la gestión de un proceso basado en una gobernanza tradicional. A medida que el tiempo pasa, la gobernanza continuara acreditándose beneficiando las futuras investigaciones en la materia.

\section{REFERENCIAS}

[1] B. Mattoni et al., "A matrix approach to identify and choose efficient strategies to develop the Smart Campus," in 2016 IEEE 16th International Conference on Environment and Electrical Engineering (EEEIC), 2016, pp. 1-6.

[2] J. R. Gil-Garcia, "Towards a smart State? Inter-agency collaboration, information integration, and beyond," Inf. Polity, vol. 17, no. 3, 4, pp. 269-280, 2012.

[3] F. Pagliaro et al., "A roadmap toward the development of Sapienza Smart Campus," in 2016 IEEE 16th International Conference on Environment and Electrical Engineering (EEEIC), 2016, pp. 1-6.

[4] M. Batty et al., "Smart cities of the future," Eur. Phys. J. Spec. Top., vol. 214, no. 1, pp. 481-518, 2012.

[5] N. Walravens, "Mobile business and the smart city: Developing a business model framework to include public design parameters for mobile city services," J. Theor. Appl. Electron. Commer. Res., vol. 7, no. 3, pp. 121135, 2012.

[6] D. Schuurman, B. Baccarne, L. De Marez, and P. Mechant, "Smart ideas for smart cities: Investigating crowdsourcing for generating and selecting ideas for ICT innovation in a city context," J. Theor. Appl. Electron. Commer. Res., vol. 7, no. 3, pp. 49-62, 2012.

[7] L. Bătăgan, "Smart cities and sustainability models," Inform. Econ., vol. 15, no. 3, pp. 80-87, 2011.

[8] R. U. P. Martín and A. C. C. Martínez, "Incidencias de la Tecnología web 2.0 en el contexto de la gobernanza y la gobernabilidad," Iustitia Soc. Rev. Arbitr. Ciencias Jurídicas y Crim., vol. 4, no. 6, pp. 90-116, 2019.

[9] K. Kourtit and P. Nijkamp, "Smart cities in the innovation age," Innov. Eur. J. Soc. Sci. Res., vol. 25, no. 2, pp. 93-95, 2012.

[10] A. Meijer and M. P. R. Bolívar, “Governing the smart city: a review of the literature on smart urban governance," Int. Rev. Adm. Sci., vol. 82, no. 2, pp. 392408,2016

[11] C. E. Jimenez, A. Solanas, and F. Falcone, "Egovernment interoperability: Linking open and smart government," Computer (Long. Beach. Calif)., vol. 47, no. 10, pp. 22-24, 2014.

[12] H. J. Scholl and M. C. Scholl, "Smart governance: A roadmap for research and practice," IConference 2014 Proc., 2014.

[13] R. Giffinger, C. Fertner, H. Kramar, and E. Meijers, "City-ranking of European medium-sized cities," Cent. Reg. Sci. Vienna UT, pp. 1-12, 2007.

[14] S. Deva, "Sustainable good governance and corporations: an analysis of asymmetries," Geo. Int'l Envtl. L. Rev., vol. 18, p. 707, 2005.

[15] A. Caragliu, C. D. Bo, and P. Nijkamp, "Smart Cities in Europe «3rd Central European Conference in Regional Science-CERS," A13 L, vol. 90, p. O18, 2009. 


\title{
MODELO DE METABOLISMO INDUSTRIAL APLICADO A UNA STARTUP DE ALIMENTACIÓN EN CANADÁ
}

\section{Industrial metabolism model applied to a food startup in Canada}

\section{Resumen}

\author{
Torres, Jorge'; Ospina, Miguel \\ Missfresh (Cook It)
}

Uno de los retos de la industria es reducir sus impactos ambientales. En Norteamérica según la US Environmental Protection Agency el 21\% de los impactos corresponde a la industria "polución quieta", de estos el 70\% equivale a PYMES. En Canadá un sector emprendedor que ha ganado mercado son las Startup de alimentación "Listo para comer" - Prêt à manger. Desde 2017 al menos 150 PYMES han sido creadas y una de las críticas son los impactos asociados a la cadena de este negocio tanto en producción, transporte, empaques. Para estudiar la problemática y encontrar una solución a una de las empresas del sector y en el marco de tesis de maestría se propone aplicar un modelo de metabolismo industrial aplicado a Missfresh, PYME de 250 trabajadores, creada en 2016 en Montreal Canadá. La metodología aplicada esta basada en el modelo de metabolismo urbano del profesor Peter Baccini. Al cambiar las variables del modelo urbano y aplicarlo en la empresa durante seis meses. Para este tiempo se planearon tres fases:

\section{Colecta de datos}

2. Análisis y diseño del modelo

3. Aplicación y seguimiento

Cada uno con dos meses de duración. Apoyados de herramientas de producción limpia, plan de circularidad, cultura empresarial para finalmente medir los resultados de progreso y construir el mapa de metabolismo industrial virtual de la empresa que apoyará decisiones futuras. Este modelo Sui Generis es de los pocos de metabolismo industrial aplicados a la industria con reconocimiento tanto en la empresa Missfresh, como en la academia en Montreal.

Palabras clave: Modelo de metabolismo industrial, circularidad, producción limpia, ecodiseño, mapa metabólico

\footnotetext{
${ }^{1}$ Correo electrónico de contacto: Jorge.torres.b@gmail.com
} 


\section{INTRODUCCIÓN}

Como referentes a este trabajo se utilizaron conceptos generales de metabolismo estudiados, inicialmente de manera biológica por el profesor de la Universidad Padua Santorious Santorious en la edad media, posteriormente los concepto de metabolismo científico de la revolución industrial de Herbert Spencer de manera energética como de Karl Marx en la parte de flujo de materiales, apoyados de los estudios de Paul-Emile de Puydt sobre la conexión de "Panarquia" o conexión de los sistemas mediante las variables matemáticas. De manera específica, se utilizó de base el modelo de metabolismo urbano del profesor Peter Baccini de la UTH de Zurich para adaptarlo a un modelo aplicado en la empresa Missfresh. Este trabajo es original ya que no hay antecedentes prácticos de un modelo similar en otras empresas. La problemática es adaptar el modelo y mejorar las prácticas de producción limpia, ecodiseño, circularidad dentro de la empresa y observar los indicadores. El producto final es el modelo aplicado y el resumen del mismo por un mapa metabólico durante el tiempo estudiado con el objetivo de observar el comportamiento de la empresa de manera metabólica.

\section{MATERIALES Y MÉTODOS}

Durante las tres fases de estudio del modelo de metabolismo industrial de flujo de materiales se utilizaron los siguientes materiales o herramientas para la aplicación del modelo:

\section{Colecta de datos}

Inicialmente se diseñó un plan de trabajo por fases. En esta etapa se diseñaron tablas de análisis de colecta diaria de los datos de actividad de la empresa según la distribución del modelo de Baccini de las $4 \mathrm{R}$ en tablas de Excel y los formatos de producción de la compañía. Se verificaron los stocks muertos de la compañía para disminuirlos mediante valorización y fechas de expiración de alimentos. Ejemplo del modelo y la tabla de datos. 


\section{CONGRESO DE DESARROLLD SOSTENIBLE}

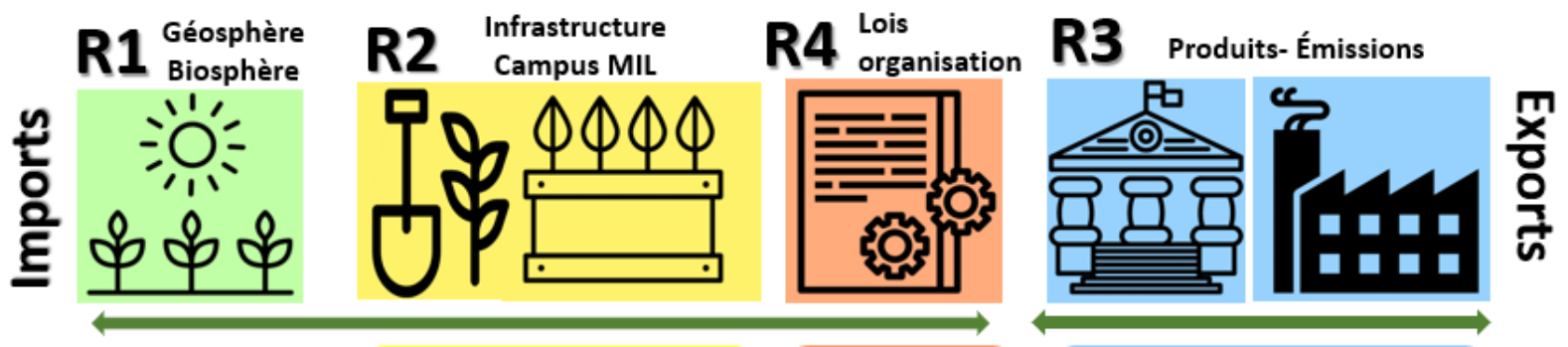

Figura 35 Modelo de Baccini de las 4R: Ingredientes, infraestructura, producción-emisiones-ecodiseño y normas o leyes.

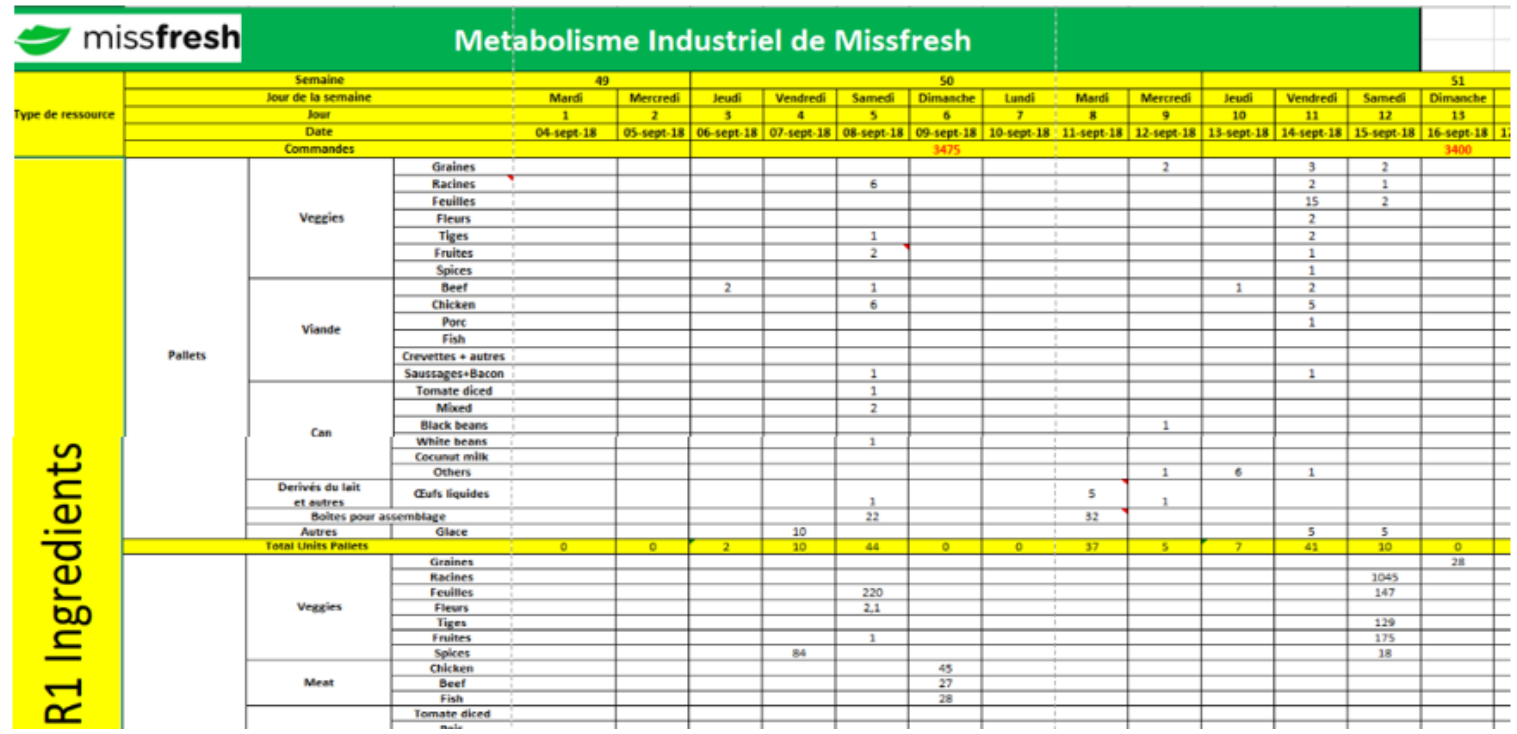

Figura 36 Formato adaptado para análisis de las $4 R$ o recursos por semana.

\section{Análisis y diseño del modelo}

Una vez colectado se procede al análisis de los datos y diseño del modelo. Se hace igualmente distribución de los flujos de materiales en la planta por cantidades y variables en la empresa para identificar las problemáticas de los flujos y los problemas visibles de la empresa de acuerdo a los cuatro recursos del modelo: Ingredientes, infraestructura, producción-emisiones-ecodiseño y finalmente las normas. 
missfresh

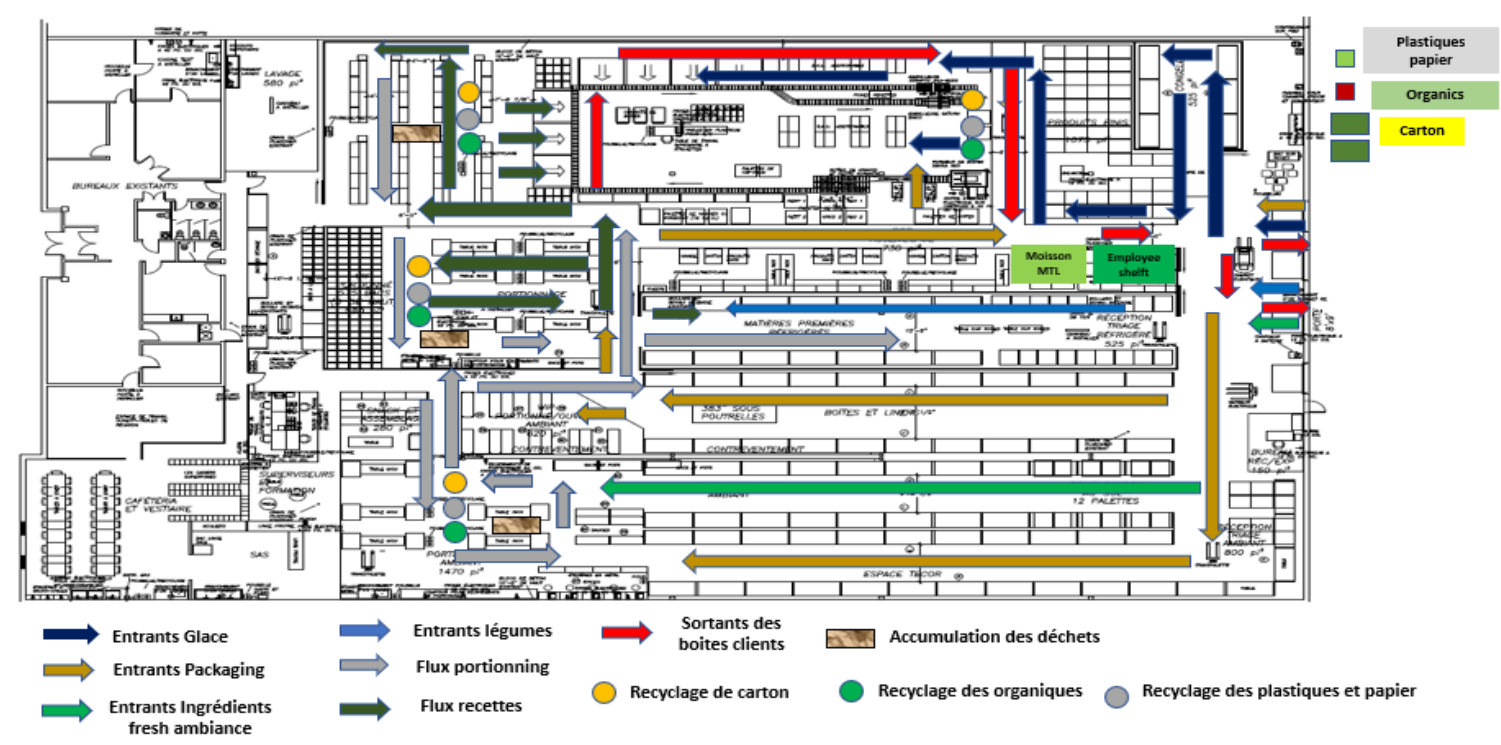

Figura 37 Diagrama de flujo de producción

\section{Aplicación y seguimiento}

Una vez analizados los datos, se hizo un plan de circularidad por cada material de la empresa, se propuso soluciones de ecodiseño mediante propuestas de evaluación de proyectos en hojas de cálculo y seguimiento con los responsables, todo documentado también en material audiovisual. Al final, el mapa metabólico se trabajó en el programa 3DS Max donde se simularon los datos de la segunda semana de septiembre.

\section{RESULTADOS Y ANÁLISIS DE RESULTADOS}

\section{Modelo metabólico final:}

Basado en la experimentación se logró estructurar un primer modelo aplicado a las PYMES. 


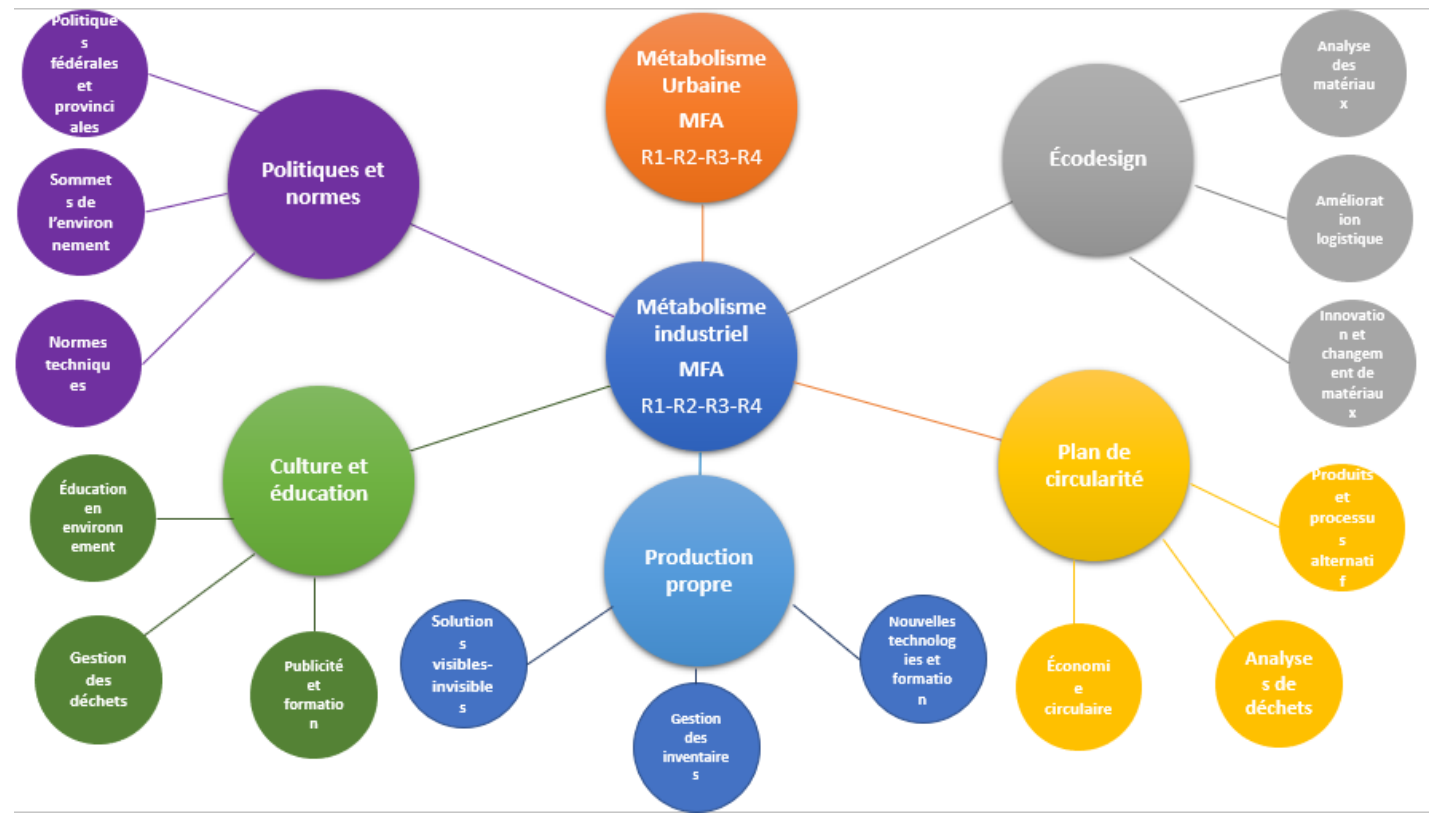

Figura 38 Modelo aplicado a las PYMES

\section{Plan de circularidad:}

A cada problemática en la generación de desechos se propuso un plan de circularidad, donde fueron analizados todas las entradas de material de la compañía y su posterior por problemática, y para cada problemática se plantearon soluciones, un ejemplo el cartón utilizado y la inversión en un compactador para venta de cartón.

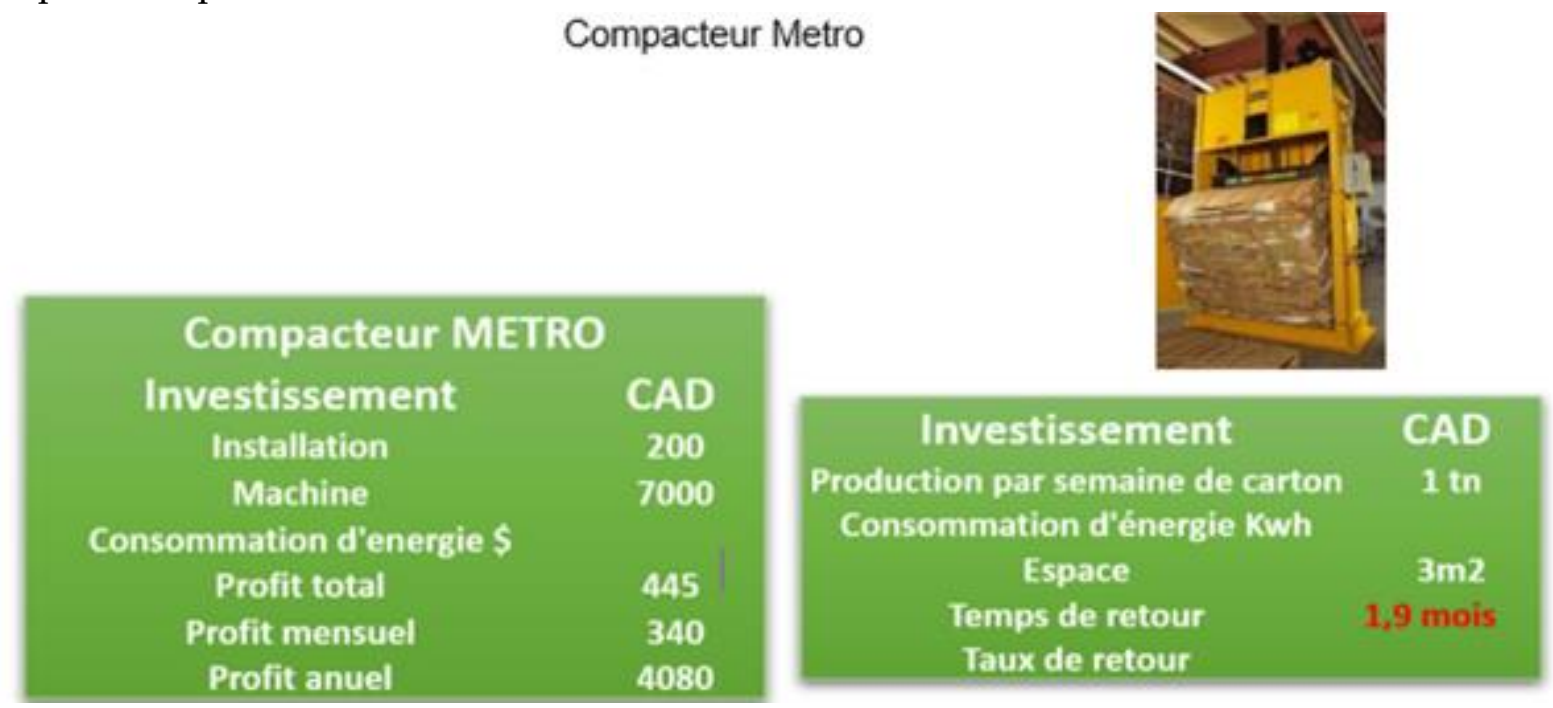

Figura 39 Plan de circularidad 


\section{CONGRESO DE DESARROLLD SOSTENIBLE}

\section{Ecodiseño:}

Se analizaron propuestas de empaque sostenible y reciclable en cada componente, se llegó a una estrategia interna (producción) y externa (cliente) para minimizar los impactos.

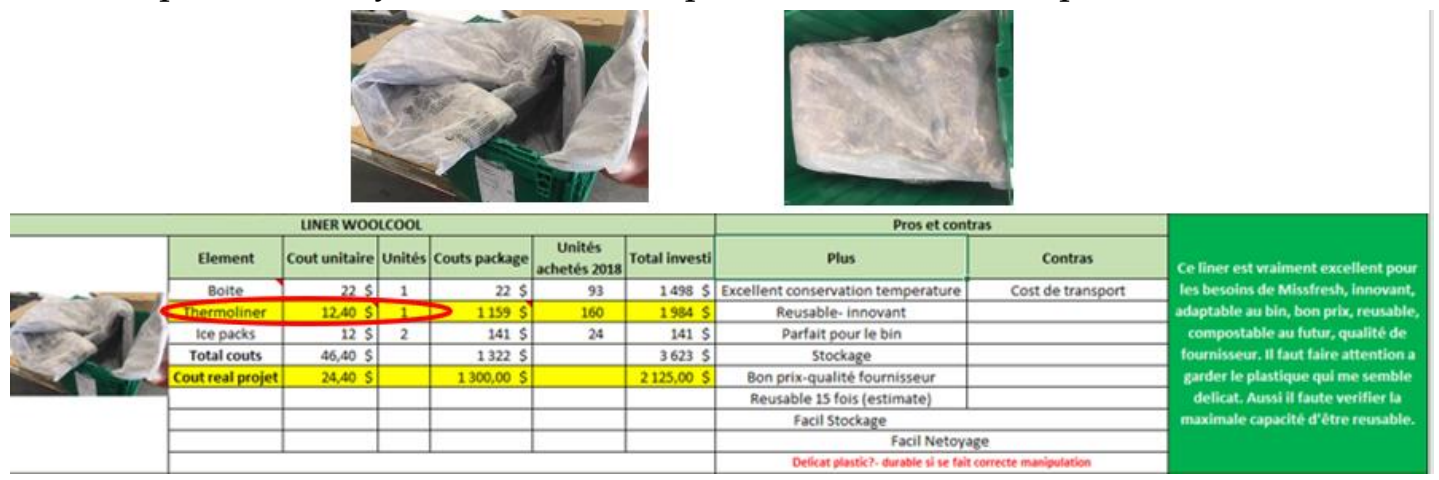

Figura 40 Análisis de propuestas de empaque sostenible y reciclable

\section{Producción limpia:}

Se logró concientizar a la planta de la importancia de producir de manera limpia y separar los desechos para su recirculación interna y venta a empresas de reciclaje. Se formó a los trabajadores en la separación de residuos, limpieza y orden (cada cosa en su sitio) de los puestos de trabajo antes y después de la producción.

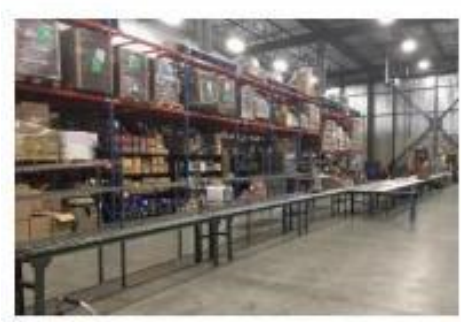

Ligne propre avant et après assemblage

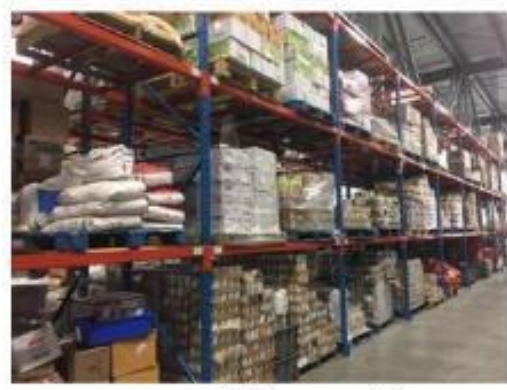

Rack bien organisés

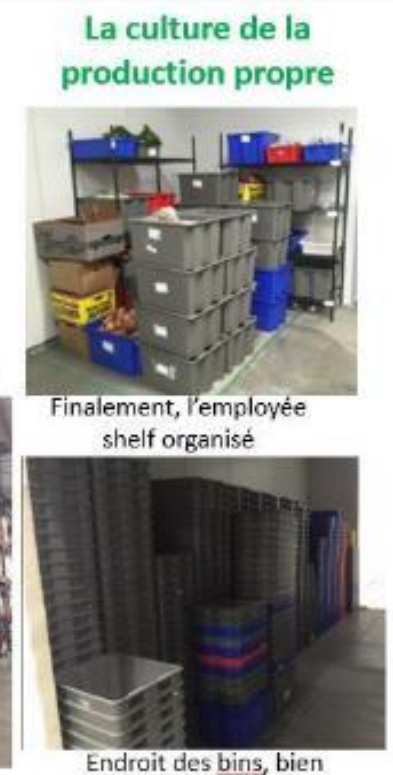

place, organisé et propre

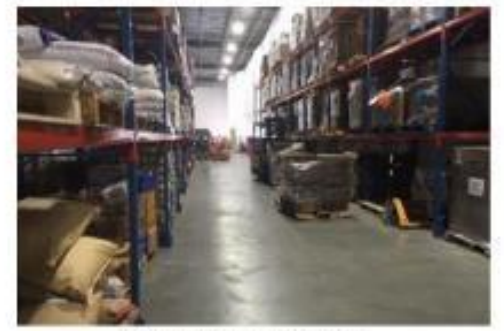

Les couloirs sont libres

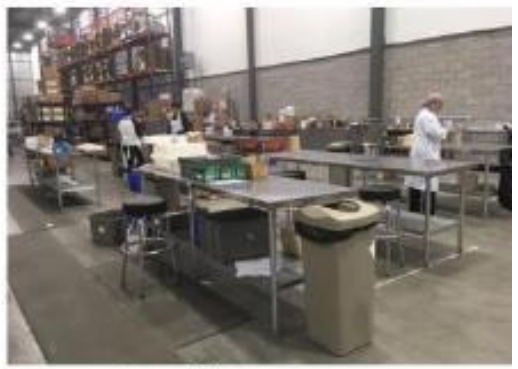

Les tables sont propres

Figura 41 Promoción de la cultura de producción organizada 


\section{CONGRESO DE DESARROLLD SOSTENIBLE}

\section{Mapa metabólico animado:}

Se presentó una simulación de una semana de una animación virtual como mapa metabólico, se deja para segunda fase de investigación adecuar los movimientos durante el tiempo de colección de datos y representación matemática de un modelo.

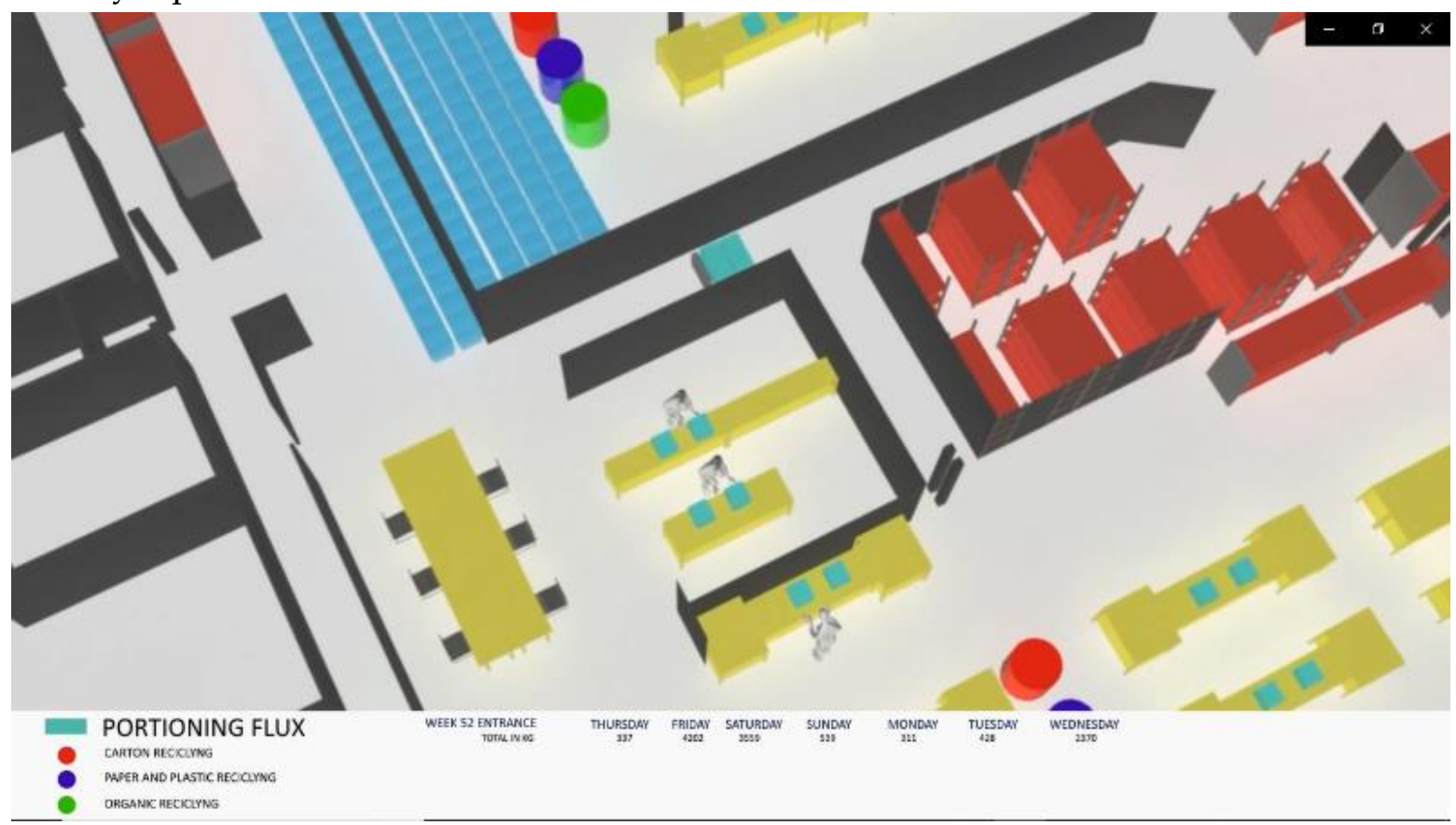

Figura 42 Simulación de un mapa metabólico

Respecto a los resultados de la aplicación del modelo en la empresa se encuentran los siguientes análisis:

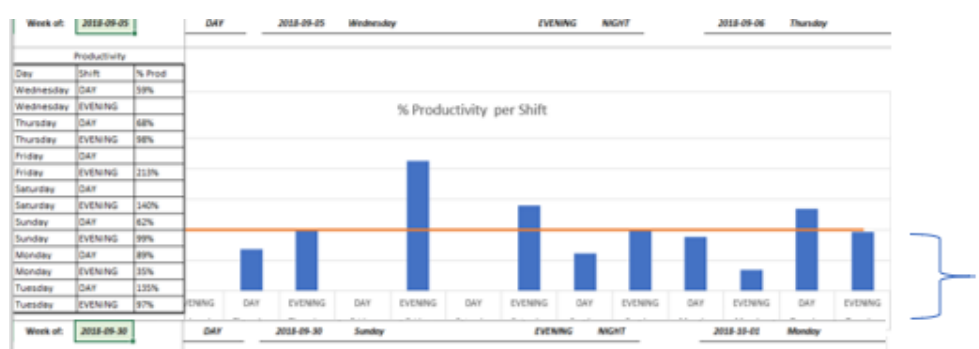

Avant de la stage métabolisme, bas productivité dans quatre quarts dans la semaine

Figura 43 Análisis de la productividad de la empresa durante los meses de Agosto y Septiembre

En general, antes de la pasantía y estudio, la empresa tenía durante los meses de agosto y septiembre una productividad baja por pérdidas de tiempo en la desorganización de la línea de producción durante los horarios de trabajo en la semana. 


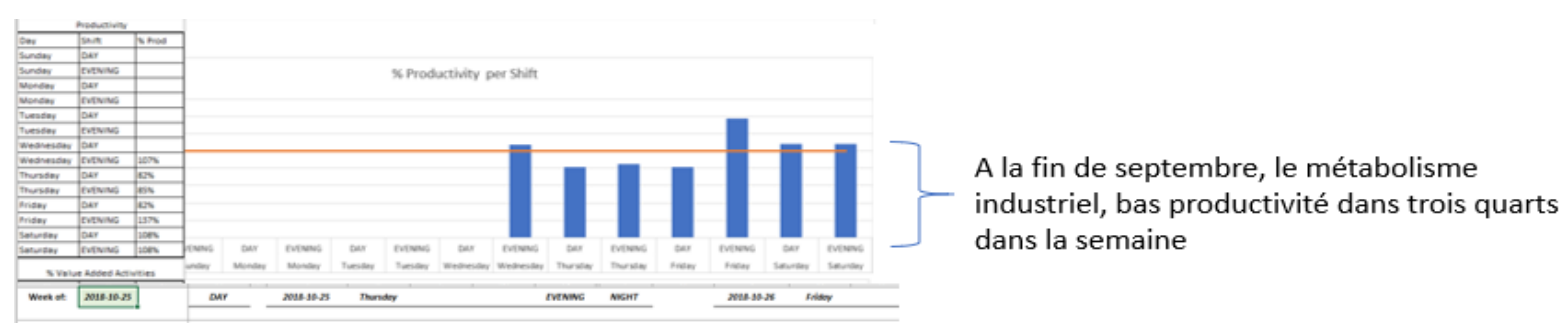

Figura 44 Análisis de la productividad de la empresa después del estudio durante los meses de Agosto y Septiembre

Al final de septiembre y octubre la productividad se estabilizó, se mejoraron los indicadores de tiempo y los empleados tenían más horas para dedicar al mantenimiento y orden de la planta. En ecodiseño Missfresh exploró alternativas de empaque, uno de ellos el de Woolcool en Inglaterra, un liner o protector de comida hecho en lana de oveja que fue ensayado y aplicado con los clientes, completamente reutilizable. Posterior al estudio, Missfresh fue muy atractiva y fue absorbida por su gran competencia y líder en el sector Cook It. Un año después del estudio pasó de 250 empleados a más de 500. Por lo tanto deja de ser una pequeña empresa a consolidarse como mediana compañía. Otro resultado fue que la tesis tuvo una calificación de A, nota alta y muy bien recibida en la comunidad académica y empresarial. Sobre este estudio la Ceo de la época Marie-Eve Prevost fue entrevista por algunos medios de comunicación canadienses. Se planea hacer un grupo de investigación en el tema en la Universidad de Montreal y en McGill University, este trabajo es único en su ramo ya que modelos de metabolismo industrial de flujo de materiales no hay aplicados en el sector.

\section{CONCLUSIONES}

Se deja abierto un campo de investigación en los dos tipos de metabolismo, energético y de flujo de materiales para aplicar a manera de consultoría en PYMEs que busquen reducir su impacto ambiental pues es un modelo aplicado que debe traducirse a futuro en simulaciones animadas para la ayuda de decisiones en la gerencia. De igual forma integrar modelos matemáticos que describan el comportamiento de la producción y disminución de desechos o la eficiencia energética de las empresas. Durante los seis meses de estudio se vio la transformación de la empresa, desde la gerencia que fue más consciente ambientalmente hasta los clientes y medios de comunicación que notaron el cambio. Uno de los cambios significativos es en materia de comunicación entre los departamentos de compras, marketing y producción, estos tres deben ir alineados para mantener la sostenibilidad y los cambios en el modelo de metabolismo. Un modelo empírico de metabolismo industrial es como la receta para mejorar el cuerpo empresarial y hacerlo 
más fuerte y competitivo en el sector, por lo tanto es un útil de consultoría y gerencia para organizar una empresa.

\section{AGRADECIMIENTOS}

A las profesoras de la Universidad de Montreal: Marie-Noelle Carré por inspirar la idea de un modelo de metabolismo en una de sus clases de maestría y a Sara Teitelbamun, directora de la maestria de Desarrollo Sostenible y Medio Ambiente de la Universidad de Montreal. Bajo la tutoría de ambas se logró un muy buen estudio. En Missfresh: Celine Boulos y Marie-Eve Prevost por permitir el estudio en la empresa y adoptar los cambios sugeridos en el estudio, también por apoyar incondicionalmente el proyecto. En Colombia a Guillermo Camacho Caro quien en su libro lo "Importante es el hombre" relaciona un concepto de metabolismo industrial de manera energética. A Miguel Ospina por invitarme a presentar la ponencia conjunta en este congreso y a los organizadores del congreso por dar espacios a la sostenibilidad y la industria.

\section{REFERENCIAS}

[1] B. Peter, Metabolism of the Anthroposphere: Analysis, evaluation, design., Cambridge Massachusetts: MIT Press 2012.

[2] J. Clammer, Cultures of Transition and Sustainability, Culture after Capitalism, Tokyo: United Nations University, 2016.

[3] D. Bourg, Industrial Ecology and Material Flow Analysis. Perspectives on Industrial Ecology, Londres: Routledge, 2003.

[4] C. Kennedy, S. Pincelt et P. Bunje, «The study of urban metabolism and its applications to urban planning and design. Environmental Pollution,» Environmental Pollutior vol. 159, no \%18-9, pp. 1965-1973, 2011.

[5] F. I. Canada, «Récupéré sur Meal kits: convenience vs. packaging,» 2404 2018. [En ligne]. Available: https://www.foodincanada.com/features/meal-kitsconvenience-vs-packaging/. [Accès le 1512 2018]. 


\title{
THE STRATEGIC DIRECTION OF THE ORGANIZATION AND THE MANAGEMENT SYSTEMS. TOWARDS A RESEARCH AGENDA
}

\section{La dirección estratégica de la organización y los sistemas de gestión. Hacia una agenda de investigación}

\author{
Bautista, Sandra1; Peña, Guillermo2; Angarita, Diego ; Pérez, Amable ${ }^{4}$. \\ ${ }^{1}$ Universidad el Bosque, Programa de Ingeniería Industrial, Grupo de investigación GINTEPRO \\ ${ }^{2}$ Universidad Santo Tomás, Grupo de Investigación GEAMEC \\ ${ }^{3}$ Universidad Santo Tomás, Grupo de Investigación CAYPRO \\ ${ }^{4}$ Universidad Santo Tomás, Grupo de Investigación GIFAE
}

\begin{abstract}
The implementation of the environmental, health, security, and quality management (EHSQ) system certifications has been an increasing priority in all types of organizations. Companies have different types of motivations, either to show their success on other occasions as a prerequisite to stay in their sector. However, there are difficulties in relating, or aligning, the strategic direction of the organization with management systems, and even more so, with the integration of these systems. Researching the current state of work on integrated management, strategic direction, and management systems allow us to establish the focal points where research actions are being coordinated, what the perspectives are, and what the main challenges are, to propose a research agenda on the subject.
\end{abstract}

Keywords: Integrated management systems, organizational strategic direction.

\section{Resumen}

La implementación de las certificaciones del sistema de gestión ambiental, de salud, seguridad y calidad (EHSQ) ha sido una prioridad creciente en todo tipo de organizaciones. Las empresas tienen diferentes tipos de motivaciones, por ejemplo, para presentar su éxito previo como un requisito para mantenerse en su sector. Sin embargo, existen dificultades para relacionar, o alinear, la dirección estratégica de la organización con los sistemas de gestión, y más aún, con la

\footnotetext{
${ }^{1}$ Corresponding Email: sbautistar@unbosque.edu.co
} 
integración de estos sistemas. Investigar el estado actual del trabajo en gestión integrada, dirección estratégica y sistemas de gestión nos permite establecer los focos donde se coordinan las acciones de investigación, las perspectivas y los principales desafíos para proponer una agenda de investigación en el tema.

Palabras clave: Sistemas integrados de gestión, dirección estratégica organizacional.

\section{INTRODUCTION}

The implementation of quality certifications such as ISO 9001, environment (14001), and occupational health (OHSAS18801) has been of high importance for many organizations worldwide. By the end of 2017, the International Organization for Standardization or ISO reported a little over one million companies in the world were certified in ISO 9001. Colombia in 2017, has been gaining positions in the world ranking of the 10 countries with the highest growth in ISO 9001 certificates (at the time of the study it was the sixth country that had grown the most, in several certificates, concerning the previous year) and ISO 14001 certificates (the fourth country that had grown the most). Colombia has been climbing up the ranks to currently be the second South American country with the highest number of ISO 9001, ISO 14001, and ISO 27001 certificates. In practice, it has proven difficult to achieve separate management of systems, and there are also challenges in establishing alignment with the strategic direction of the organization.

Therefore, analyzing how an integrated management system can be established requires understanding its research status and what the focal and articulating aspects are. The purpose of this research is to establish the state of scientific research related to the links between the strategic direction of the organization and the management systems, especially those related to the integration of the systems. It proposes to propose a research agenda that, from the guidelines to deepen in nodal focuses and enhancers of the integration of management systems in the organizations, evidencing the binding relationships between them and that are the basis to contribute in a conceptual model of integration.

\section{MATERIALS AND METHODS}

Initially, a literature review is conducted to determine how the scientific community has studied the key connections and challenges between management systems (especially related to quality, health, and environment) and strategic direction. Next, a literature review strategy and the technical tool for mapping and co-occurrence analysis among the selected documents are defined. 
- Literature review strategy: the parameters are shown in Table 1.

Table 15. Definition of parameters for the identification and selection of documents

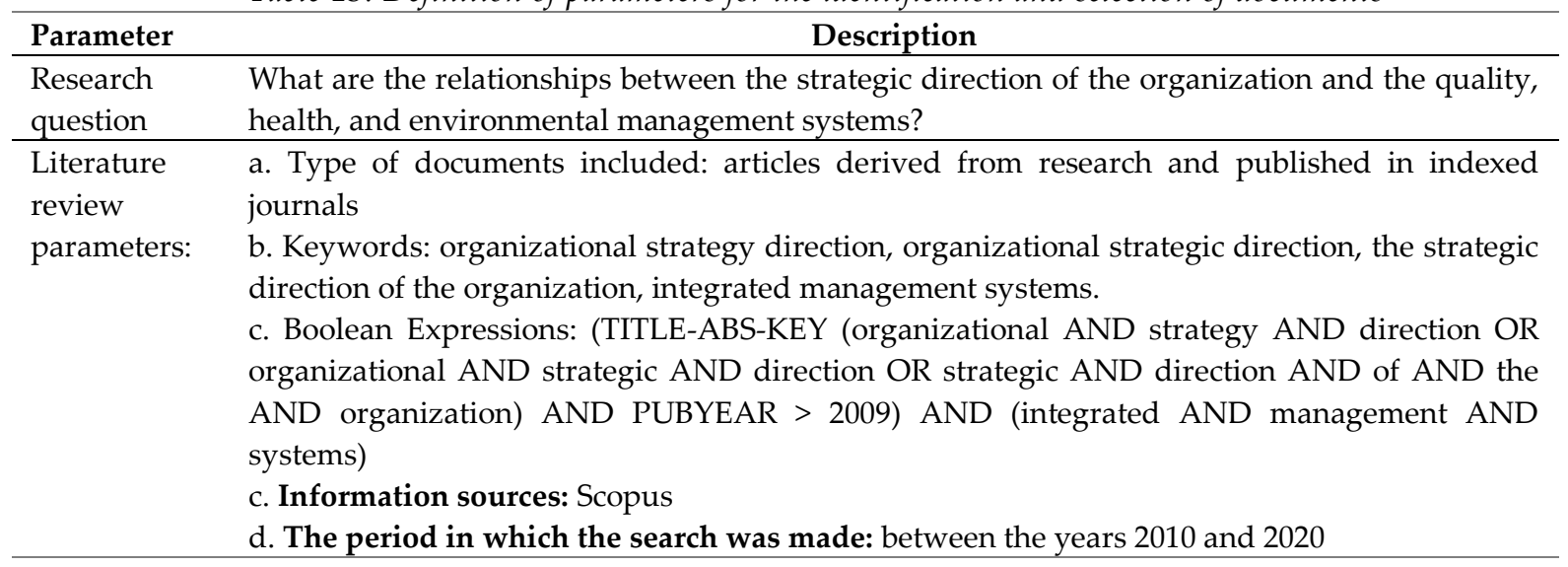

\section{Mapping technique using VOSviewer}

The construction of the map by the VOSviewer consists of three steps; first it calculates a similarity matrix, second it constructs a map, and third it translates, rotates, and mirrors the map [1]. The first step is to calculate a similarity matrix based on the co-occurrence matrix; it uses a measure of similarity known as the force of association as shown in equation (1) and is similar to the proximity index or as the probabilistic affinity index.

$$
s_{i j}=\frac{c_{i j}}{w_{i} w_{j}}
$$

Where cij denotes the number of co-occurrences of articles $i$ and $j$ and where wi and wj denote the total number of occurrences of articles $i$ and $j$ or the total number of co-occurrences of these articles, for further discussion of the strength of the association we refer to [2]. The second step is the construction of a map based on the similarity matrix obtained in step 1 . The general objective of the VOS mapping technique is to minimize a weighted sum of the Euclidean distances squared between all pairs of items. The greater the similarity between two items, the greater the weight of their squared distance in the sum [1]. In mathematical notation, the objective function to be minimized is given by equation (2). Where the vector $x i=(x i 1, x i 2)$ denotes the location of item $i$ on a two-dimensional map and where ||$-||$ denotes the Euclidean standard. The minimization of the target function is performed subject to the restriction equation (3). More information on how the problems of constrained optimization of minimization (equation 2) subject to (equation 3) are developed in [1]. 


$$
V\left(x_{1}, \ldots, x_{n}\right)=\sum_{i<j} s_{i j}\left\|x_{i}-x_{j}\right\|^{2} \quad \quad(2) ; \quad \frac{2}{n(n-1)} \sum_{i<j}\left\|x_{i}-x_{j}\right\|=1
$$

In step 3, the VOSviewer transforms the solution obtained for the optimization problem examined in step 2, the fundamental steps of optimization to ensure that VOSviewer produces consistent results are expanded into [1].

\section{Co-occurrence data analysis}

Research clusters are identified based on density and group view. The density view is represented by the color of a point on the map based on the item density. Let ${ }^{-} \mathrm{d}$ denote the average distance between two items (equation 4 ), the item density $\mathrm{D}(\mathrm{x})$ of a point $\mathrm{x}=(\mathrm{x} 1, \mathrm{x} 2)$ is then defined in equation (5). Where $\mathrm{K}:[0, \infty) \rightarrow[0, \infty)$ denotes a kernel function, $\mathrm{h}>0$ means a parameter called kernel width, and wi represents the weight of item $i$, that is, the total number of occurrences or co-occurrences of item $i$. The kernel function must be non-increasing. VOSviewer uses a Gaussian kernel function given by equation (6) [1].

$$
\bar{d}=\frac{2}{n(n-1)} \sum_{i<j}\left\|x_{i}-x_{j}\right\| \quad(4) ; D(x)=\sum_{i=1}^{n} w_{i} K\left(\left\|x_{i}-x_{j}\right\| /(\bar{d} h)\right)(5) ; \quad K(t)=\exp \left(-t^{2}\right)
$$

The element density is calculated from a point on a separate map for each cluster. The element density of a point $x$ for a cluster $p$, denoted by Dp (x), is defined as equation (7), where Ip(i) denotes an indicator function that is equal to 1 if element $i$ belongs to the cluster $p$ and that is equal to 0 otherwise.

$$
D_{p}(x)=\sum_{i=1}^{n} I_{p}(i) w_{i} K\left(\left\|x_{i}-x_{j}\right\| /(\bar{d} h)\right)
$$

\section{RESULTS AND ANALYSIS OF RESULTS}

Forty-eight documents were selected, including three papers from the year 2020. The following were identified as the main themes business, management, and accounting. Followed by studies from the social sciences, computer science, and decision making. It is observed that interest in working in the area of environmental sciences is beginning to appear, with an article associated with hybrid organizations and the challenges they present for the sustainability of companies. 


\section{Overall cluster analysis:}

A bibliometric analysis of the 48 documents is performed using the VOSviewer software. Each circle in Figure 1 has a color that depends on the density of the items at that point and the relationship to the clusters is presented in Table 2. Cluster 1 (red) represents research on issues related to strategic management and sustainability.

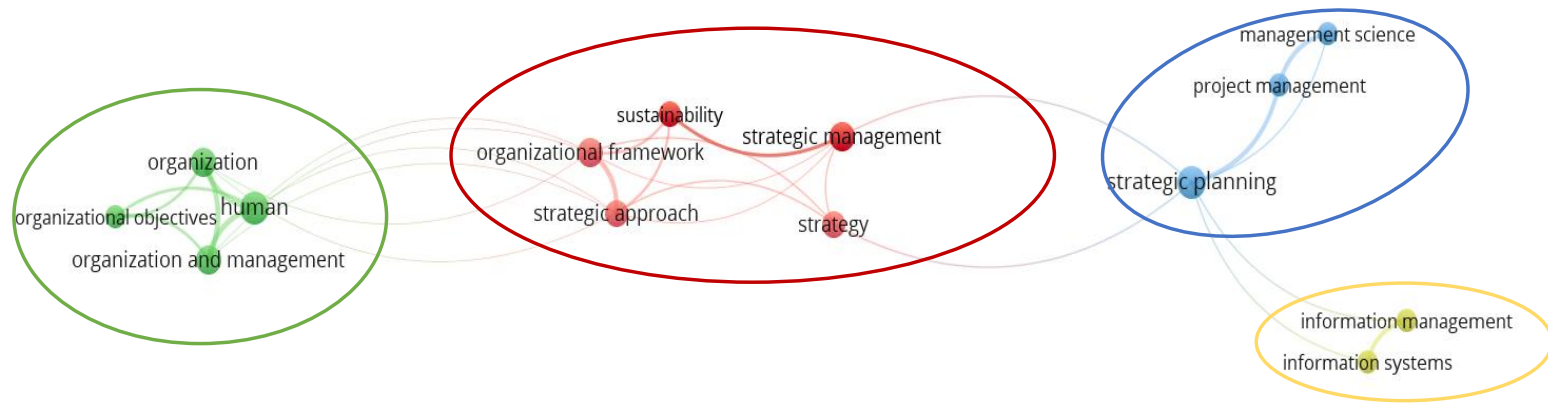

Figure 43 General cluster display of the 48 documents

Table 2. The general cluster of the 48 documents

\begin{tabular}{ll}
\hline Cluster & Subjects \\
\hline Cluster 1 (Red) & $\begin{array}{l}\text { Organizational framework; Strategic approach; Strategic management, Strategy; } \\
\text { Sustainability }\end{array}$ \\
\hline Cluster 2 (green) & Human; Organization; Organization and management; Organizational objectives \\
\hline Cluster 3 (blue) & Management Science; Project management; Strategic planning \\
\hline Cluster 4 (yellow) & Information management; Information systems \\
\hline
\end{tabular}

A more detailed search is conducted within the 48 documents on the relationship between organizational strategies and integrated systems management, obtaining 10 documents. A bibliometric analysis of the keywords defined by the authors was carried out, finding 36 items and six clusters. In Figure 2, yellow cluster 1 shows that the articles that analyze strategic management integrate it with the strategies of human talent cultivation, knowledge management, and resource-based approach. Cluster 2 in blue shows that the study of enterprise risk management is related to the performance of organizations, their competitive advantages, business strategy, and information systems. Cluster 3 can be analyzed from a relevant node that is the corporate strategic planning, in the investigations that work this topic they relate it in a greater way with the tactical planning, the operational planning, and the leadership.

On the other hand, tactical planning is directly linked to sustainable practices, leadership, analyses of the global financial crisis. On the other hand, studies on operational planning directly consider 
aspects associated with decision-making, executive development, and leadership. Other aspects considered in the studies on corporate strategic planning are human resource training and innovation. Cluster 4 shows that the studies on strategic alignment consider mainly the relationships with health information systems and the dynamics of the organization's systems. Cluster 5 shows that researchers working on strategic change in companies include change management, performance management systems, and a balanced scorecard in its analysis. Finally, there is cluster 6, in which the articles analyzed are related to the synergy between sustainable advantages, strategic innovation, and environmental uncertainty.

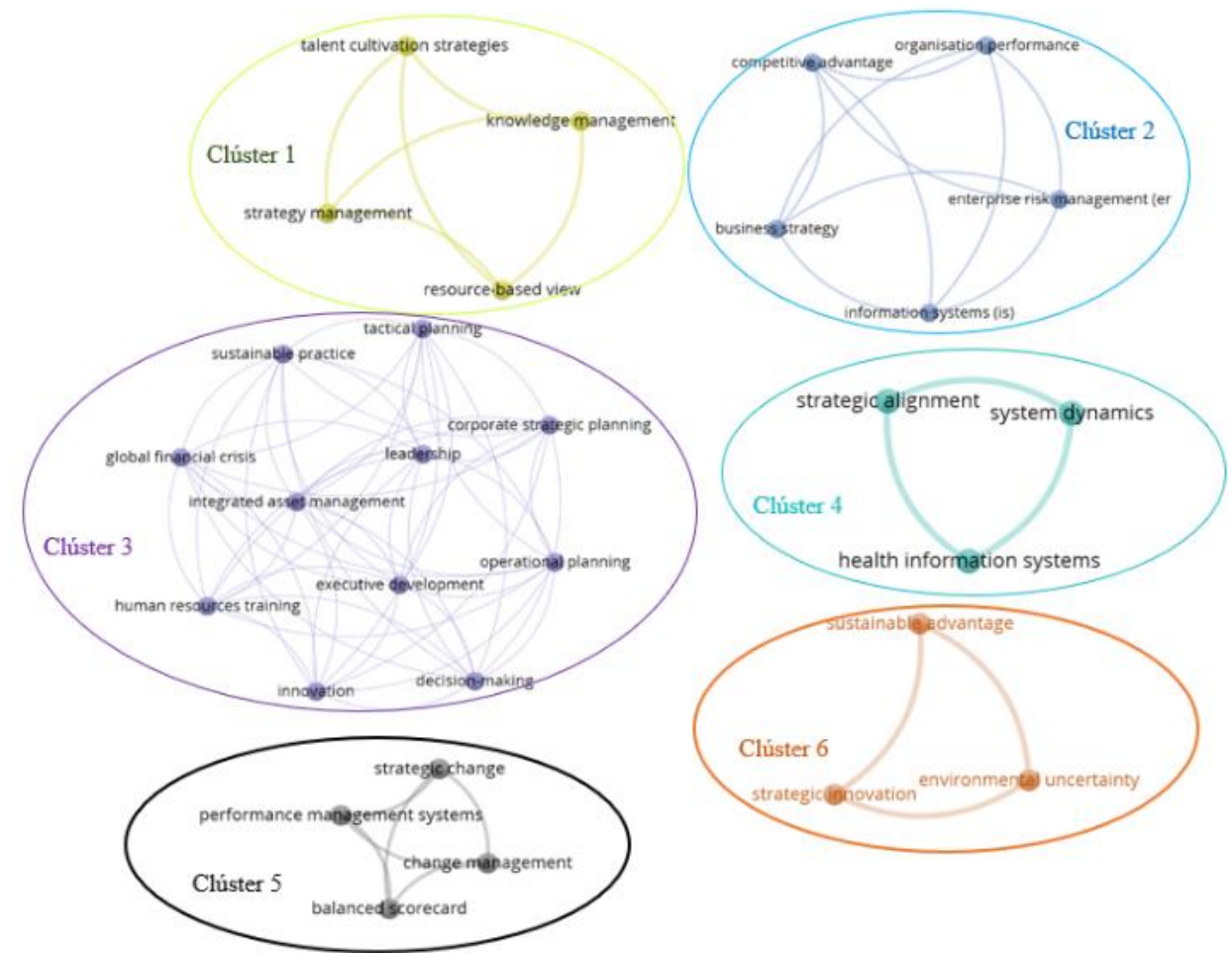

Figure 44 Cluster visualization of the relationship between organizational strategies and integrated systems management

\section{Conceptual links between organizational strategies and integrated systems management:}

based on the correlation analyses, a series of theories and concepts linked to organizational strategy, business management, and auditing or accounting, and integrated systems management were identified. In Table 3, if the theory or concept appears to be related, the value of one (1) is 
placed, otherwise zero (0), it should be noted that the links were established employing figures 1 and 2. When carrying out a cross-sectional analysis of the theories and concepts, linked to organizational strategy, the definition of the organization and its objectives, management, and strategic focus, and information management are highlighted. On the other hand, in what is associated with the integrated management of systems there are eleven concepts, in turn, related to business strategy and human talent. Finally, crosscutting concepts, which have emerged in recent years, such as sustainability, innovation, and knowledge management, are highlighted.

Table 3. Relationship between theories - concepts with organizational strategy and integrated management

\begin{tabular}{|c|c|c|c|}
\hline Theory and concepts associated with: & $\begin{array}{l}\text { Organizational } \\
\text { strategy }\end{array}$ & $\begin{array}{c}\text { Integrated } \\
\text { system } \\
\text { management }\end{array}$ & References \\
\hline Organization and organizational objectives & 1 & 0 & {$[3] ;[4] ;[5] ;[6]$} \\
\hline Strategic management and corporate strategy & 1 & 1 & $\begin{array}{c}{[7] ;[8] ;[9] ;[10] ;[11] ;[12] ;[13] ;} \\
{[14] ;[15]}\end{array}$ \\
\hline Strategic planning & 1 & 1 & {$[10] ;[16] ;[17] ;[18] ;[19] ;[20] ;[7]$} \\
\hline Top management teams & 0 & 0 & {$[14] ;[15]$} \\
\hline Decision making & 0 & 1 & {$[21] ;[22] ;[15]$} \\
\hline $\begin{array}{l}\text { Sustainability, sustainable practices and sustainable } \\
\text { advantage }\end{array}$ & 1 & 1 & [23]; [24]; [25]; [6]; [12]; [20]; [14] \\
\hline Organizational framework & 1 & 0 & [26]; [25]; [27]; [12]; [15] \\
\hline Strategic alignment & 0 & 1 & {$[26] ;[3] ;[28] ;[22] ;[29]$} \\
\hline Strategic approach & 1 & 0 & [26]; [25]; [27]; [12]; [29] \\
\hline Operational planning and integrated asset management & 0 & 1 & {$[30]$} \\
\hline Project management & 1 & 0 & {$[10] ;[17] ;[22]$} \\
\hline Management science & 1 & 0 & {$[17] ;[31] ;[22] ;[14]$} \\
\hline Information management & 1 & 0 & {$[32] ;[33] ;[3] ;[20]$} \\
\hline knowledge management & 0 & 1 & {$[34] ;[28] ;[35] ;[7]$} \\
\hline Innovation, strategic innovation, and innovation management & 0 & 1 & $\begin{array}{c}{[23] ;[36] ;[32] ;[34] ;[37] ;[6] ;[20] ;} \\
{[38] ;[14]}\end{array}$ \\
\hline $\begin{array}{l}\text { Talent cultivation strategies, leadership, and human resources } \\
\text { training }\end{array}$ & 0 & 1 & [34]; [39]; [27]; [5]; [30]; [40] \\
\hline Resource-based view & 0 & 1 & {$[8] ;[25] ;[34] ;[13]$} \\
\hline Organisation performance & 0 & 1 & {$[41]$} \\
\hline Competitive advantage & 0 & 1 & [25]; [42]; [28]; [31]; [35];[19]; [20] \\
\hline Business strategy & 0 & 1 & [23]; [17]; [19]; [20] \\
\hline Change management & 0 & 1 & [43]; [31]; [29] \\
\hline
\end{tabular}

\section{Research agenda proposed:}

Considering the documentary review and the identified relationships it can be established how the management systems are a relevant activity for the organizations. This management can create competitive advantages for companies and contribute to sustainable development. There is an important interest in relating binding concepts that allow articulating the different management systems for their integration. It is therefore relevant to identify key aspects in the processes of integration of management systems, directly related to the organizational strategy. It is proposed 
that the research focus on materializing these concepts in the organizations, defining the methods of implementation and articulation. The relevant issues are presented in Figure 3.

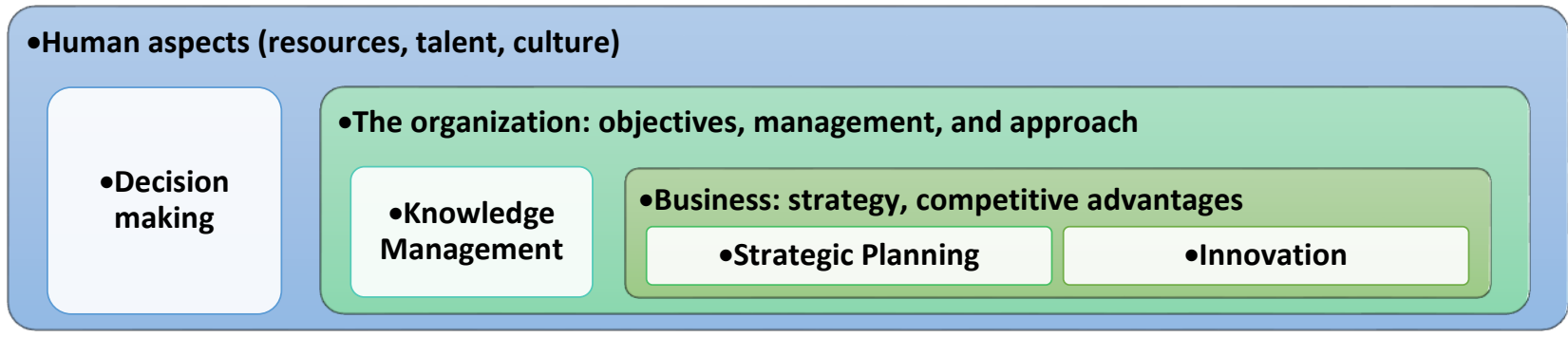

Figure 3. Research approach

\section{CONCLUSIONS}

The relationships between the organization's strategic direction and the quality, health, and environmental management systems, as well as systems integration, has been addressed in more than half of the articles on business, management, and audit issues. The environmental sciences have addressed these connections the least. The four focal clusters of relationships between strategic direction and integrated management systems are the management of organizations with an emphasis on the "human". The second is strategic management linked to sustainability. The third is strategic planning and the fourth is information management. The research trend is towards the relationships between the links of strategic focus and sustainability. Likewise, the organizational aspects that are related to the business culture, and its human talent.

It identifies as transversal concepts between organizational strategy and integrated systems management: strategic management, strategic planning, decision making, sustainability, sustainable practices, sustainable advantage, knowledge management, and innovation.

\section{REFERENCES}

[1] N. J. Eck and L. Waltman, "Software survey: VOSviewer, a computer program for bibliometric mapping," Scientometrics, vol. 84, no. 2, pp. 523-538, 2010.

[2] N. J. van Eck and L. Waltman, "How to Normalize Cooccurrence Data? An Analysis of SomeWell-Known Similarity Measures," J. Am. Soc. Inf. Sci. Technol., vol. 60, no. 8, pp. 1635-1651, 2009.

[3] M. C. A. Odiit, G. K. Mayoka, N. M. Ochara, and A. S. Rwashana, "Alignment of information systems to strategy in the health sector using a systems dynamics approach," in ACM International Conference Proceeding Series, 2014, vol. 28-September-2014, pp. 38-49.

[4] F. Rabbani et al., "Understanding the context of balanced scorecard implementation: A hospital-based case study in Pakistan," Implement. Sci., vol. 6, no. 1, p. 31, Mar. 2011.

[5] J. G. Spicer, P. S. A. Sparacino, and N. E. Larkin, "Developing a Nursing Practice Framework to Align With Strategy," JONA J. Nurs. Adm., vol. 48, no. 9, pp. 
419-421, Sep. 2018.

[6] C. Saunders and D. J. Carter, "Is health systems integration being advanced through Local Health District planning?" Aust. Heal. Rev., vol. 41, no. 2, p. 154, Apr. 2017.

[7] J. M. Bryson, F. S. Berry, and Kaifeng Yang, "The State of Public Strategic Management Research: A Selective Literature Review and Set of Future Directions," $A m$. Rev. Public Adm., vol. 40, no. 5, pp. 495-521, Sep. 2010.

[8] S. Gotteiner, M. Mas-Machuca, and F. Marimon, "Fighting organizational decline: a risk-based approach to organizational anti-aging," Manag. Res. Rev., vol. 42, no. 11, pp. 1259-1277, Nov. 2019.

[9] J. Allison, "Values statements: The missing link between organizational culture, strategic management, and strategic communication," Int. J. Organ. Anal., vol. 27, no. 3, pp. 666-689, Jul. 2019.

[10] L. Bai and Q. Du, "Co-evolution efficacy of project portfolio based on strategic orientation," RAIRO Oper. Res., vol. 52, pp. 645-659, 2018.

[11] R. Krupski, K. Piórkowska, and A. Sus, "Organizational endogenous development: The microfoundations, opportunities, and real options perspective," Argumenta Oeconomica, vol. 38, no. 1, pp. 63-97, 2017.

[12] N. Haigh and A. J. Hoffman, "The New Heretics: Hybrid Organizations and the Challenges They Present to Corporate Sustainability," Organ. Environ., vol. 27, no. 3, pp. 223-241, Sep. 2014.

[13] J. Söderlund and F. Tell, "Strategy and capabilities in the P-form corporation: Linking strategic direction with organizational capabilities," Adv. Strategy. Manag., vol. 28, pp. 235-262, 2011.

[14] Y. L. Chen and D. Y. Li, "Strategic innovation of enterprise: Antecedents, processes and consequences," in 2010 International Conference on Management and Service Science, MASS 2010, 2010.

[15] X. Gimbert, J. Bisbe, and X. Mendoza, "The role of performance measurement systems in strategy formulation processes," Long Range Plann., vol. 43, no. 4, pp. 477-497, Aug. 2010.

[16] M. Taghian, M. J. Polonsky, and C. D'Souza, "Green Marketing Strategies," in An Integrated Approach to Environmental Management, Wiley, 2016, pp. 231-253.

[17] M. Louis, V.W.; Carl, “Applying governance principles to improve agile project success," in IAMOT 2016 - 25th International Association for Management of Technology Conference, Proceedings: Technology - Future Thinking, 2016, pp. 359-376.

[18] K. Akingbola, "Contingency, fit and flexibility of HRM in nonprofit organizations," Empl. Relations, vol. 35, no. 5, pp. 479-494, 2013.

[19] C. Harding, "Systems Engineering Business Context: The Evaluation of the South African Systems
Engineering Industry," 22nd Annu. Int. Symp. Int. Counc. Syst. Eng. INCOSE 2012 8th Bienn. Eur. Syst. Eng. Conf. 2012, EuSEC 2012, vol. 22, no. 1, pp. 800-815, Jul. 2012.

[20] A. Althonayan, J. Keith, and A. Misiura, "Aligning enterprise risk management with business strategy and information systems," in Proceedings of the European, Mediterranean and Middle Eastern Conference on Information Systems - Informing Responsible Management: Sustainability in Emerging Economies, EMCIS 2011, 2011, pp. 109-129.

[21] K. Longfield, B. Smith, R. Gray, L. Ngamkitpaiboon, and N. Vielot, "Putting health metrics into practice: Using the disability-adjusted life year for strategic decision making," BMC Public Health, vol. 13, no. SUPPL 2, p. S2, Jun. 2013.

[22] C. Bai and J. Sarkis, "A grey-based DEMATEL model for evaluating business process management critical success factors," Int. J. Prod. Econ., vol. 146, no. 1, pp. 281-292, Nov. 2013.

[23] D. Chakraborty and W. Biswas, "Articulating the value of human resource planning (HRP) activities in augmenting organizational performance toward a sustained competitive firm," J. Asia Bus. Stud., vol. 14, no. 1, pp. 62-90, Jan. 2020.

[24] M. Arnold and F. Osorio, "Introducción a los Conceptos Básicos de la Teoría General de Sistemas," pp. 40-49, 1998.

[25] Rodrigues and Franco, "The Corporate Sustainability Strategy in Organisations: A Systematic Review and Future Directions," Sustainability, vol. 11, no. 22, p. 6214, Nov. 2019.

[26] M. Sholihah, T. Maezono, Y. Mitake, and Y. Shimomura, "PSS Strategic Alignment: Linking Service Transition Strategy with PSS Business Model," Sustainability, vol. 11, no. 22, p. 6245, Nov. 2019.

[27] S. Carlton and C. E. Mills, "The Student Volunteer Army: a 'repeat emergent' emergency response organization," Disasters, vol. 41, no. 4, pp. 764-787, Oct. 2017.

[28] J. Al-Ammary, "The strategic alignment between knowledge management and information systems strategy: The impact of contextual and cultural factors," J. Inf. Knowl. Manag., vol. 13, no. 1, Mar. 2014.

[29] P. Jayashree and S. J. Hussain, "Aligning change deployment: A Balanced Scorecard approach," Measuring Business Excellence, vol. 15, no. 3. Emerald Group Publishing Limited, pp. 63-85, Aug-2011.

[30] R. F. Stapelberg, “Corporate executive development for integrated assets management in a new global economy," in Engineering Asset Lifecycle Management Proceedings of the 4th World Congress on Engineering Asset Management, WCEAM 2009, 2009, pp. 733-742.

[31] C. Demartini, "The Strategy Formulation Mechanism," 
in Contributions to Management Science, Springer, 2014 pp. 139-149.

[32] N. Kyriakou and E. N. Loukis, "Do strategy, processes, personnel, and technology affect a firm's propensity to adopt cloud computing?: An empirical investigation," J. Enterp. Inf. Manag., vol. 32, no. 3, pp. 517-534, Jun. 2019.

[33] J. M. Simões, C. F. Gomes, and M. M. Yasin, "Changing role of maintenance in business organizations: Measurement versus strategic orientation," Int. J. Prod. Res., vol. 54, no. 11, pp. 3329-3346, Jun. 2016.

[34] P. J. Hsieh, C. C. Chen, and W. Liu, "Integrating talent cultivation tools to enact a knowledge-oriented culture and achieve organizational talent cultivation strategies," Knowl. Manag. Res. Pract., vol. 17, no. 1, pp. 108-124, Jan. 2019.

[35] L. Abdelheq, K. Abdelatif, and H. Mustapha, "The formalization of the knowledge management in the managerial approach in the Algerian firms: Case of the territorial direction of Algeria telecom of Tlemcen," Mediterr. J. Soc. Sci., vol. 4, no. 6, pp. 191-204, Jul. 2013.

[36] N. Chutivongse and N. Gerdsri, "Creating an innovative organization: Analytical approach to developing a strategic roadmap guiding organizational development," J. Model. Manag., vol. 15, no. 1, pp. 5088, Jan. 2020.

[37] E. D. Romanopoulou, V. I. Zilidou, and P. D. Bamidis, "Creating and sustaining a social health care ecosystem: The case of LLM care services in Greece,"
Hell. J. Nucl. Med., vol. 20, no. 2, pp. 40-48, 2017.

[38] A. Savaneviciene and Z. Stankeviciute, "The Interaction between Top Management and Line Managers Implementing Strategic Directions into Praxis," Eng. Econ., vol. 22, no. 4, pp. 412-422, Oct. 2011.

[39] S. Oreg and Y. Berson, "Leaders' impact on organizational change: Bridging theoretical and methodological chasms," Acad. Manag. Ann., vol. 13, no. 1, pp. 272-307, Jan. 2019.

[40] R. Phillips, V. Milligan, and A. Jones, "Integration and social housing in Australia: theory and practice authored by," in AHURI Final Report, 2009.

[41] A. Althonayan, J. Keith, J. P. Morgan, and A. Misiura, "Aligning Enterprise Risk Management with Business Strategy and Information Systems," in Proceedings of the European, Mediterranean and Middle Eastern Conference on Information Systems - Informing Responsible Management: Sustainability in Emerging Economies, EMCIS 2011, 2011, pp. 109-129.

[42] A. K. Schnackenberg, J. Bundy, C. A. Coen, and J. D. Westphal, "Capitalizing on categories of social construction: A review and integration of organizational research on symbolic management strategies," Acad. Manag. Ann., vol. 13, no. 2, pp. 375413, Jul. 2019.

[43] M. Ekstrand and S. Damman, "Management of integrated workplace concepts at multiple locations," Facilities, vol. 35, no. 3-4, pp. 188-201, 2017. 


\title{
TOOL FOR INTERNAL AUDITS IN LOCAL GOVERNMENTS
}

\section{Herramienta para auditorías internas en gobiernos locales}

\author{
Ávila, Fernando; Muñoz, David; Ríos, Margarita1; Camacho, Hernando; Londoño, Diana \\ Universidad Santo Tomás, School of Mechanical Engineering
}

\begin{abstract}
According to the National Planning Department - DNP, in Colombia 972 of the 1101 municipalities are in the sixth category, in the Department of Tolima 43 of the 47 are in the sixth category. Furthermore, within the Internal Control Offices of these municipalities, it has been demonstrated that there is inefficiency in the fulfillment of their roles (strategic leadership, focus on prevention, evaluation of risk management, evaluation and follow-up (internal audits); relationship with external control entities). This occurs due to different causes (methods, processes and personnel). For this reason, this research aims to improve this process, developing a tool to perform comprehensive internal audits in the sixth category municipalities, taking as a reference the following Technical Standards: NTC ISO 19011:2018, NTC ISO 45001:2018, NTC ISO 14001:2015, NTC ISO 18091:2019, articulated with The Audit Guide for Public Entities, Decree 1072 of 2017, Law 99 of 1993, and the Integrated Planning and Management Model - MIPG v2 respectively. Through an investigation with a qualitative approach of exploratory and descriptive character, analyzing the audit process and identifying the compliance and priorities of the aforementioned technical standards versus the legislation, to later design and validate an application that allows to guide the internal auditor of the Mayors' Internal Control Offices of the sixth category municipalities in the fulfillment of their roles. It is important to emphasize that, performing integral audit processes with the support of the proposed tool leads to the following benefits: integrates information for decision making, risk occurrence prevention, favors the achievement of institutional objectives, increases the transparency index, improves planning and resource management, reduces operation time and efforts, reduces over costs due to redundant activities, improves process control, favors legal compliance and other stakeholders, facilitates appropriation (awareness). With the research and development of the tool, it was concluded that in most Latin American countries, there are no structured performance evaluation systems for the municipalities that make up the system, or they are in the initial stages. The sixth category municipalities have shortcomings in the exercise of internal audits due to different internal and
\end{abstract}

${ }^{1}$ Corresponding e-mail: margaritarios@usta.edu.co; mmriosp@gmail.com 
external factors, causing a malpractice of the exercise, which with the help of a tool for planning and conducting comprehensive internal audits, allows the sixth category municipalities to adequately comply with the regulations and provide valuable inputs to senior management for decision making

Keywords: Sixth Category Municipality; Integral Internal Audit; NTC 19011; MIPG

\section{Resumen}

De acuerdo al departamento de planeación nacional DNP, en Colombia 972 de los 1102 municipios son de sexta categoría, en el departamento del Tolima 43 de las 47 áreas son de sexta categoría. Además, dentro de las oficinas de control interno de estos municipios se ha demostrado que existe ineficiencia en el cumplimiento de roles (liderazgo estratégico, enfoque preventivo, evaluación de la gestión de riesgos, evaluación y seguimiento (Auditorías internas), relación con las entidades de control externo) Esto ocurre por diferentes razones (Métodos, procesos y personal). Por esto, esta investigación tiene como objetivo mejorar este proceso, desarrollando una herramienta para realizar auditorías internas integrales en municipios de sexta categoría, tomando como referencia las normas técnicas: NTC ISO 19011:2018, NTC ISO 45001:2018, NTC ISO 14001:2015, NTC ISO 18091:2019, articulado con la guía de autoría para entidades publicas, Decreto 1072 de 2017, Ley 99 de 1993 y el Modelo Integrado de Planificación y Gestión - MIPG v2 respectivamente.

La investigación se desarrollará a través de un enfoque cualitativo de carácter exploratorio y descriptivo, analizando el proceso de auditoría e identificando el cumplimiento y prioridades de las referidas normas técnicas versus la legislación, para posteriormente diseñar y validar una aplicación que permita orientar al auditor interno de los alcaldes y oficinas de control interno de los municipios de sexta categoría en el cumplimiento de sus funciones.

Es importante resaltar que realizar procesos de auditoría integral con el apoyo de la herramienta propuesta conlleva los siguientes beneficios: integra información para la toma de decisiones, prevención de ocurrencia de riesgos, favorece el logro de los objetivos institucionales, aumenta el índice de transparencia, mejora la planificación y los recursos gestión, reduce tiempos y esfuerzos de operación, reduce sobre costos por actividades redundantes, mejora el control de procesos, favorece el cumplimiento legal y otros grupos de interés, facilita la apropiación. Con la investigación y desarrollo de la herramienta se concluyó que en la mayoría de los países latinoamericanos no existen sistemas estructurados de evaluación del desempeño de los municipios que integran el sistema, o se encuentran en etapas iniciales. Los municipios de sexta categoría tienen deficiencias en el ejercicio de las auditorías internas debido a diferentes factores internos y externos, provocando una mala práctica del ejercicio, que, con la ayuda de una herramienta de planificación y realización de auditorías internas integrales, permite a los 
municipios de sexta categoría cumplir adecuadamente con las regulaciones y proporcionar valiosos aportes a la alta dirección para la toma de decisiones.

Palabras clave: Municipios de sexta categoría; Auditoría integral interna; NTC 19011; MIPG

\section{INTRODUCTION}

In Colombia, more than $90 \%$ of municipalities are classified into the sixth category, that is, according to the legal provisions, municipalities with a population of no more than 10000 inhabitants and with a current income of free destination that do not exceed 15.000 minimum monthly wages (SMLMV) [1][2]. The municipality must comply with the legislation that the State issues for its operation, which includes internal audits to measure and evaluate the efficiency, effectiveness and economy of the other control entities.

In this context, performance assessments for municipalities are defined as a process that must be continuous, systematic and periodic. This process assessed quantitatively and qualitatively the degree to which the local authorities achieve the goals of their management, in terms of commitments established within the Municipal Development Plan [3]. In Colombia, the measurement of the Municipal Performance Assessment aims to measure, compare and order municipalities according to their integral performance, understood as management capacity and development results, taking into account their initial endowments, in order to incentivize better management, quality of expenditure and results-oriented investment [4].

For the evaluation of the performance, municipalities in the sixth category have the following technical standards: NTC ISO 19011:2018, NTC ISO 45001:2018, NTC ISO 14001:2015, NTC ISO 18091:2019, the Audit Guide for Public Entities, Decree 1072 of 2017, Law 99 of 1993, and the Integrated Model of Planning and Management-MIPG v2, which is regulated through article 133 of Law 1753 of 2015 that issues the National Development Plan 2014 - 2018. In this law, the Administrative Development System (1998) and the Quality Management System (2003) was integrated into one: The Management System, which must be articulated with the Internal Control System (2005). For the new Management System and its articulation with the Internal Control System, the MIPG v2, adopted in 2012 by Decree 2482, was updated. [5].

Although it is clear that there are regulations that support the monitoring and evaluation process in public entities (Internal Control) and specifically in the Mayors' offices of the municipalities that comprise the Nation, corruption cases, and malpractice have been evidenced over the years in the internal audit process, which can arise for different causes such as: methods, unskilled personnel and inefficient procedures. Additionally, the extensive regulations that the Colombian State issues, which constitute a wide spectrum, can become a cumbersome and confusing practice for the officer when applying them in the municipalities of the different categories, thus losing 
objectivity in the audit and its purpose.

The research group does not identify previous investigations that address the problem posed by the development of a tool that guides the internal control officer of a municipality to conduct comprehensive internal audits that facilitate the application and understanding of the regulations; however, it suggests how this tool can serve as an input to subsequent investigations that help municipalities in this category.

\section{MATERIALS AND METHODS}

The type of research is exploratory, because an application of this type does not currently exist and requires interviews with experts, as well as a pilot test in the Mayor's office of Mariquita Tolima that was structured to validate its contents. The research is carried out in five phases that are described below.

Phase I. Conception of the idea of the application to solve the problem: It consists in problem analysis through a diagnosis of the internal audit process of a sixth-category Mayor's office in accordance with ISO 18091:2019, ISO 19011:2018, the MIPG v2 in the dimension of internal control, and the Audit Guide for Public Entities

Phase II. Design and architecture: This phase aims to model the program according to the identified needs, in a clear language for employees of the Mayor's office, easy to use and consult.

Phase III. Programming: This phase comprises the design and development of the tool programming, which consists of:

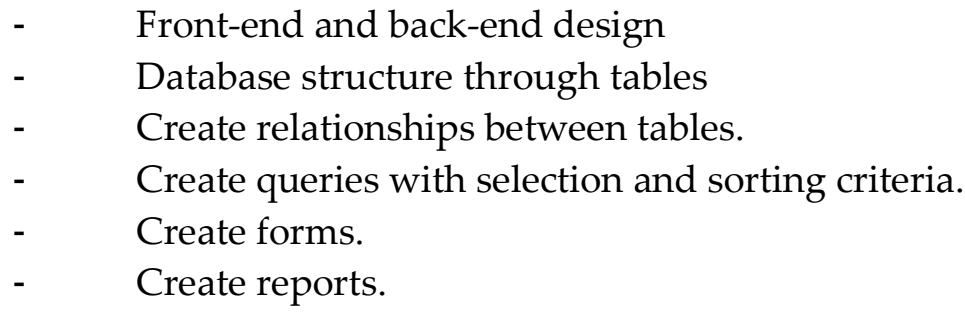

The program is developed under the Cascade methodology, it is so named because of the position of the phases in the development of this one, which seem to cascade "by gravity" towards the following phases. It is the methodological approach that rigorously orders the stages of the process for the development of software, in such a way that the beginning of each stage must wait for the completion of the previous stage. At the end of each stage, the model is designed to carry out a final review, which is responsible for determining whether the project is ready to move on to the next phase. This model was the first to originate and is the basis for all other life cycle 
models. [6]

Phase IV. Application tests and adjustments: This phase makes a parallel in terms of difficulties or shortcomings regarding a typical audit in the municipality of Mariquita, Tolima, in order to apply the necessary adjustments for the tool to be effective.

Phase V. Documentation and maintenance: Both the technical specifications of the tool and step-bystep instructions that help the officials of a Mayor's office to carry out a comprehensive internal audit are documented in guide mode, including the maintenance plan of the tool.

\section{RESULTS AND DISCUSSION}

To generate an effective tool, easy to use and access to the municipalities of the sixth category, the phases proposed for its obtaining were developed as follows:

Phase I. Conception of the idea of the application to solve the problem: Initially, the research team conducted a bibliographic study in which it was possible to determine that, in some Latin American countries, systems for municipal evaluation have been designed and implemented. Mexico, for example, designed and implemented a system of measurement and evaluation of performance, called SEDEM, in 2005 [7] and the Municipal Performance Guide in 2019 [8]. Chile initiated the implementation of the measurement and evaluation of performance in its municipalities. The Latin American and Caribbean Institute for Economic and Social Planning ILPES produced in 2012 the manual "Designing a performance measurement system to evaluate municipal management" [9], as a methodological proposal to measure the performance of municipal management in Latin America. In Colombia, the Integrated Model of Planning and Management, adopted in 2012 by Decree 2482, was updated in 2018. [5]. Additionally, the existence of computer tools to carry out comprehensive audits was researched. Countless commercial software was found, such as: Gensuit Audit Management, Panotica Hidra 4.0, Suite Gesia, among others [10]. This demonstrated that such software is designed for organizations of any kind, without taking into account specific requirements, such as those of a municipality. Therefore, this reveals the feasibility of developing the proposed tool, since it is specifically designed towards the needs of a sixth category municipality and is an innovative product in this sector.

Figure 1 graphically shows how the MIPG v2 are integrated and articulated with the components and elements of internal control, and supported by technical standards, to develop the tool. 


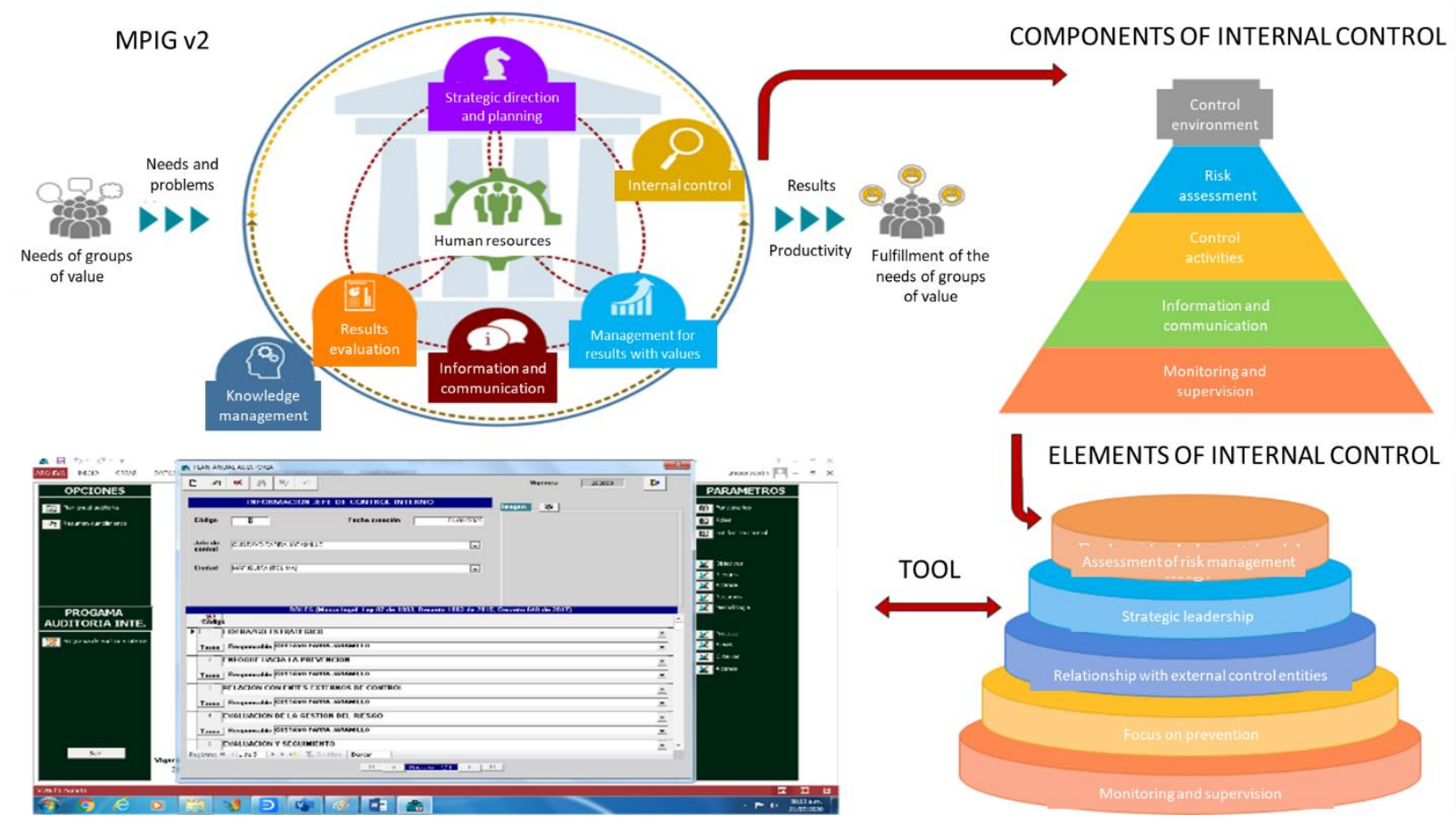

Figure 1. Conception idea of the app

The research team started from the MIPG v2, which consists of 7 dimensions: human management, strategic management and planning, management for results with values, evaluation of results, information and communication, knowledge management, and internal control [11]. The team focused on the seventh dimension, "Internal Control", since with this dimension and the implementation of the policy that integrates it, the objective of the MIPG v2 is fulfilled, that is, to develop an organizational culture based on information, control and evaluation, for decision-making and continuous improvement [12]. The components of this dimension are considered to establish the effectiveness within the entities of the controls designed from the structure of the other dimensions of MIPG v2 and to define the responsibility and authority to control through its scheme of Lines of Defense, and the elements of internal control, since the Heads of Internal Control carry out their work through the roles described. These are the inputs for the design of the tool, taking into account that the Internal Control office is the one that determines the objective, risk, scope, resources, methodology and criteria of the audit [12]. The equipment also includes the technical standards NTC ISO 19011:2018, NTC ISO 45001:2018, NTC ISO 14001:2015, NTC ISO 18091:2019, the Audit Guide for Public Entities, the Decree 1072 of 2017, Law 99 of 1993, and the MIPG v2. These documents are included in order to generate checklists that guide the control managers and allow them to adjust the tool to their needs and make decisions about the benchmarks or criteria to use. 
Phase II. Design and architecture: Considering the problem to be solved, the application is developed in Microsoft Access and the programming language is VBA (Visual Basic for Applications). This language is used to program Windows applications and is included in several Microsoft applications. It also allows the functionalities of an event-oriented language with access to the Windows API. These resources are easily accessible and applicable in the Mayors' offices of sixth category municipalities, as they are compatibles to their systems and commonly used.

The technological requirements to install the application are:

a) OS: Windows XP, Vista, 7, 8, or 10 with 64-bit processor.

b) Memory: 2 GB RAM.

c) Graphics: DirectX 11 Compatible.

d) DirectX: Version 11.

e) Storage: $63 \mathrm{MB}$ of available space.

f) Server Specifications: Windows server

Phase III. Programming: The following activities were carried out for the design and development of the tool:

a. Splitting the database: It consists in creating two databases. The first one is called backend and contains the programming tables exclusively. The second one is front-end and contains the rest of objects: queries, forms, reports, macros and modules (Figure 2).

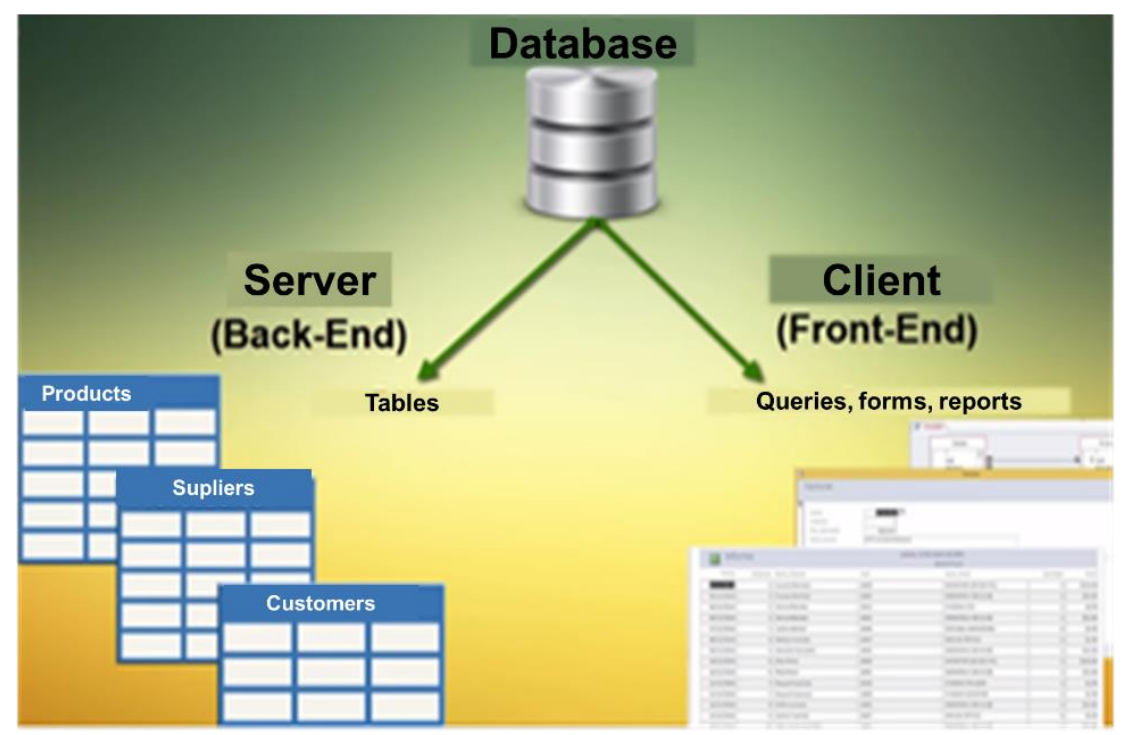

Figure 2. Creation of split database: back-end and front end 
b. Database structure using tables: Tables are the fundamental structure of a database system. In Microsoft Access, a table is an object that stores data in records (rows) and fields (columns).

c. Create relationships between tables: This is an established association between common fields (columns) in two tables. Fields that are related can be called differently, but they must have the same type of data. The relationship allows the Access engine to find data from both tables.

d. Create queries with your selection and sorting criteria: This is a way to find and collect information from one or more tables to obtain detailed information from a database.

e. Create forms: These are elements of Microsoft Access that allow building a communication interface between the user and the database.

f. Create reports: This is an effective way to present data in printed or on-screen format.

Phase IV: Application and Adjustment Tests (phase to run): In this phase, supported by the Mayor of Mariquita, Tolima, the pilot test of the tool will be carried out by developing a comprehensive audit. Additionally, the tool will be presented to expert personnel for analysis and evaluation. Once the results of the application tests are obtained, the corresponding adjustments will be made.

Phase V: Documentation and Maintenance (phase to run): Both the technical requirements of the tool and step by step instructions are documented in guide mode to help the employees of the Mayor's office to carry out a comprehensive internal audit of the municipality. This guide also includes the maintenance plan of the tool.

For this phase, the Internal Risk-Based Audit Guide for Public Entities [8] was taken into account, so that both the language and the structure of the proposed document is in accordance with the aforementioned guide, since this is law enforcement for municipalities. Besides, the guide has similar structure and language to the NTC ISO 19011:2018, thus it is easy for users of the Mayor's office to understand it.

\section{CONCLUSIONS}

In Latin America, it was observed that, in most countries, structured performance assessment systems for their constituent municipalities do not exist or are at the early stages. For example, although Chile and Argentina have already started with the performance assessment process, both countries are still behind Mexico and Colombia, which have structured systems for such measurement. 
The municipalities of the sixth category have shortcomings in the exercise of internal audits for several reasons, such as the cumbersome regulation in Colombia and the poor training of some internal control heads on the subject that causes malpractice of the exercise.

A tool for planning and carrying out comprehensive internal audits allows sixth category municipalities an adequate compliance with the regulations and gives value inputs to the upper management for decision-making.

Once the tool is validated, it will provide sixth category municipalities with a powerful aid for the efficient and effective execution of comprehensive internal audits, providing important inputs for decision making, early detection of malpractice, possible corruption cases.

The tool helps the internal control officials of a municipality to understand the regulations, since it provides correlation matrices between the different regulations, simplifying their interpretation, avoiding reprocessing, and reducing audit times.

The development of a tool to carry out comprehensive internal audits of sixth-category municipalities helps to conduct audits in a simple and practical manner; it is recommended that the phases be continued: Application tests, adjustments, documentation and maintenance, in order to have a tool applicable to the municipalities of the mentioned category.

\section{REFERENCES}

[1] Ruiz, R. F. (2001). Evolución histórica del control. Revista No 5. http://legal.legis.com.co/document/Index?obra=rcontad or\&document=rcontador 7680752a7d50404ce0430a0101 $\underline{51404 c}$

[2] Diagnóstico de la Capacidad Institucional de Municipios de Sexta Categoría, Departamento Administrativo de la Función Pública, 2013.

[3] La evaluación del desempeño a nivel municipal, Olivera, D; Florez, M, Revista Ciencia Administrativa; 2012.

[4] Guía Orientaciones para Realizar la Medición del Desempeño Integral Municipal, DNP; 2017.

[5] Modelo Integrado de Planeación y Gestión, DAFP 2018.

[6] Metodologías de Desarrollo de Software, Maida, Esteban Gabriel ; Pacienzia, Julián, Universidad Católica de Argentina, 2015

[7] Premio Interamericano a la Innovación para la Gestión Pública Efectiva 2013, www.oas.org/dpge 2013.

[8] Guía de Desempeño Municipal, https://www.gob.mx/inafed/acciones-yprogramas/documento-guia-consultiva-de-desempenomunicipal

[9] Manual Diseño de un sistema de medición de desempeño para evaluar la gestión municipal: una propuesta metodológica; Instituto Latinoamericano y del Caribe de Planificación Económica y Social - ILPES; Chile 2012.

[10] Software de auditorías, https://www.capterra.es/directory/30664/audit/softwar $\underline{\mathrm{e}}$ Página Función Pública; https://www.funcionpublica.gov.co/web/mipg; Departamento Administrativo de la Función Pública.

[12] Guía de Auditoría Interna Basada en Riesgos para Entidades Públicas, V4; Dirección de Gestión y Desempeño Institucional; Colombia 2020.

[13] Manual Operativo del Modelo Integrado de Planeación y Gestión; Consejo para la Gestión y el Desempeño Institucional; 2019. 


\title{
DISEÑO DE UN PROTOTIPO PARA EL MANEJO DE DATOS EN EL MUSEO DE LA UNIVERSIDAD DE LA SALLE
}

\section{Prototype design for data management in the museum of the La Salle University}

\author{
Triana, Yesid'; Rodríguez, Diana²; Jaime, Yamile ${ }^{3}$ \\ Universidad de La Salle, Grupo de Investigación GIII
}

\section{Resumen}

Los Sistemas de Información permiten a las organizaciones de cualquier nivel contar con un activo organizado para la toma de decisiones estratégicas y administrativas que redundan en su beneficio y el del entorno. En el caso del Museo de la Universidad de La Salle, este alberga ocho colecciones biológicas; los datos de estas colecciones se registran actualmente en varios archivos en Excel, lo que no facilita su mantenimiento. El cuerpo de investigadores del Museo manifiesta que esta no es la manera más adecuada para trabajar con datos que requieren estar interrelacionados, esto significa que actualmente es necesario un arduo trabajo a la hora de buscar información para respaldar su trabajo.

En razón a lo anterior, el Museo requiere desarrollar un proyecto para implementar un sistema de información soportado en una base de datos relacional, que tenga la capacidad de almacenar los datos de sus colecciones y proveer información de manera organizada y eficaz. Para responder a esta necesidad, se plantearon cuatro fases, que corresponden a: (1) Análisis y diagnóstico de los archivos existentes, (2) Propuesta de diseño para la base de datos mediante un modelo entidad relación, (3) Desarrollo del diseño e implementación del sistema de reportes y consultas (4) Análisis del nuevo sistema mediante el desarrollo de pruebas con los actores del Museo, verificando la optimización de la información.

Se espera que, una vez desarrollado el sistema de información, este permita a los investigadores encontrar los datos requeridos de manera oportuna, facilitando además su mantenimiento al momento de realizar las actualizaciones exigidas por la normatividad que rige los museos

${ }^{1}$ Correo electrónico correspondiente: ytriana46@unisalle.edu.co

${ }^{2}$ Correo electrónico correspondiente: dianadrodriguez51@unisalle.edu.co

${ }^{3}$ Correo electrónico correspondiente: yajaime@unisalle.edu.co 
Palabras claves: Sistemas de información, Base de datos, Colecciones biológicas, Museo de La Salle.

\section{INTRODUCCIÓN}

La misión del Museo es: "contribuir a la recuperación y construcción de identidades (nacional, institucional, personal), hacer memoria de la tradición Lasallista, conservar y preservar por medio de sus colecciones, y proyectos de investigación y divulgación, el patrimonio natural y cultural convirtiéndose en una ventana interpretativa y en agente de cambio social a través de la apropiación social del conocimiento" [1] Lo que Esta investigación en sintonía con los objetivos de desarrollo sostenibles: (4) de una educación con calidad, (14) Conservar y utilizar sosteniblemente los océanos, los mares y los recursos marinos y (15) Gestionar sosteniblemente los bosques, luchar contra la desertificación, detener e invertir la degradación de las tierras, detener la pérdida de biodiversidad.

El Museo de la Universidad de La Salle es un agente de cambio social y desarrollo, inspirado en los principios del Código Profesional Deontología del ICOM [2], así como un promotor de los valores asociados al estudio de la diversidad biológica y cultural de Colombia. Se auto define como un escenario donde el encuentro de saberes y el ejercicio interdisciplinario se convierten en la dinámica propia de su accionar. Su objetivo principal es fomentar la investigación sobre el patrimonio que custodia y reflexionar sobre su quehacer pedagógico y museológico a través del patrimonio que conserva y preserva en sus colecciones. Como centro cultural y de apropiación social de conocimiento promueve actividades para el encuentro entre la ciencia y la comunidad que beneficia, mediante la difusión de la memoria biológica que alberga [3].

Las diversas colecciones con las que cuenta resguardan algunos de los especímenes más antiguas en el país, y otras de estas piezas incluso datan de periodos en los cuales el Museo de la Universidad de La Salle no se había constituido. Entre sus ejemplares se encuentran, en algunos casos, registros únicos de las especies que alguna vez vivieron en las proximidades de Bogotá, Cúcuta, Pamplona, Medellín, Villavicencio, Leticia, Florencia y que probablemente, ya estén extintas localmente Dada la antigüedad de estos ejemplares y la calidad de los mismos, se generaron grandes aportes al estudio del ecosistema colombiano y el Museo se destaca como un foco importante para la exhibición, restauración, conservación e investigación del patrimonio cultural, social y educativo para la transmisión de todo un legado cultural. [4]. 
Actualmente, el Museo se ha visto en la necesidad de clasificar en ocho colecciones sus especímenes, estas son: vertebrados, invertebrados, colecciones con fines científicos, depósito de ejemplares y donación de objetos, centro de documentación, exposiciones permanentes, exposiciones temporales y herbario. Cada una de ellas tienen específicamente su organización de acuerdo a sus respectivas características. Inicialmente, el Museo contaba con un software para el manejo de bases de datos biológicas, Specify, [5], Sin embargo, según los científicos del Museo, este software se volvió obsoleto ya que se debían hacer actualizaciones diarias para mantener al tanto la información de manera simultánea con respecto a las diferentes actividades de seguimiento de transacción de especímenes, enlaces de imágenes para los registros que se llevan a cabo en el Museo y demás procesos que se desarrollan allí [4]. Por lo tanto, los registros de las colecciones se empezaron a llevar en archivos de Excel, sin que esto representara una solución eficaz.

En cuanto a sistemas de información específicos para colecciones museológicas, a nivel internacional, se encuentran entre otros, el sistema Netx [6] plataforma líder en la gestión de activos digitales utilizado en museos como el Metropolitano de Arte de Nueva York (MET), el Museo de Brooklyn, y la Galería Nacional de Arte de Washington DC. Por otra parte, está el sistema Argus de Lucidea que brinda una plataforma web configurable, que permite la entrega de servicios de acuerdo a las necesidades de los usuarios [7]. También se encuentra, Altru, esta solución hace seguimiento asociado a la construcción de relaciones sólidas entre el museo y el visitante, para administrar las operaciones diarias con un sistema de gestión eficiente [8]. Otro software es Museum Space, disponible para ceso a través de la nube; es un software de gestión integral para museos, galerías, bibliotecas, archivos y lugares del patrimonio. Este sistema, contiene módulos para: gestión de la conservación, registro de imágenes, gestión de exposiciones, catalogación, gestión de préstamos, ubicaciones y movimientos, gestión de adquisiciones, gestión de bajas, entre otros [9].

En Colombia, se desarrolló el Prototipo de sistema de Recuperación de Información Artística de Las Exposiciones del Museo de Arte Moderno De Bogotá: Un análisis del Museo como Unidad le Información y la implementación de un Sistema de Información para recuperar las exposiciones del museo como un todo. El sistema permite recuperar la información que los guías brindan a los visitantes sobre el contexto de cada exposición. El sistema evita la pérdida de conocimiento dando un mayor sentido al trabajo de todas las personas que producen información en el Museo de Arte Moderno en Bogotá [10] 


\section{MATERIALES Y MÉTODOS}

Este trabajo se fundamenta en la información existente en la base de datos que actualmente posee el Museo, y en los criterios de los científicos que la manejan, sin llegar a niveles de detalle propios del objeto de estudio de cada uno de ellos. En cuanto la fundamentación técnica, se apoya en los principios de diseño de los SMBD [11], que permiten organizar la información,

Las fases del proyecto se presentan en la Tabla 1 y se describen a continuación:

Fase I: Mediante entrevistas a los científicos de Museo, desarrolladas inicialmente mediante entrevistas abiertas, y luego con entrevistas semidirectivas, se logra tener un conocimiento general del Museo y sus necesidades. En esta fase se aborda el análisis de los archivos de Excel en conde cada uno de ellos alberga una colección diferente. Su contenido está dividido en cuatro (4) hojas las cuales son: introducción, platilla, definiciones y vocabulario. Estos archivos se elaboraron de acuerdo a una clasificación entre zoología, dirigido a los animales, o botánica, dirigido a las plantas. A partir de ahí, los archivos se desarrollaron por subreinos y esto se hace tanto para las colecciones en exhibición como para las que están en bodega y de esta manera poderlas catalogar y relacionar fácilmente la llegada de especímenes nuevos. Las instrucciones acerca de cómo se debe hacer una platilla de documentación de registros biológicos está basada en los estándares de Darwin Core versión (2011-10-26) [12], donde menciona que son por lo general 159 elementos de utilidad a la hora de registrar un espécimen biológico, de los cuales solo cinco de ellos son obligatorios. Cada elemento consta de una columna y de un campo en específico. Como ejemplo de un archivo Excel de las colecciones, se presenta en la Tabla 1 un fragmento del archivo que registra los datos de la colección de ranas.

Tabla 16 Ejemplo del archivo en que se registran los datos de la colección de ranas

Ranos

occurrencell - basisofReco - institutionCo - collectionCo - catalogNumi - type D del registro $b$ Base del registn Código de la ins Código de la co Número de cat Tipo MLS:ran:1 Espécimen pres MLS MLS:ran:10 Espécimen pres MLS ran 1 Objeto físico - modified - language

MLS:ran:100 Espécimen pres MIS ran 10 100 Objeto fisico Modificado Idioma

MLS:ran:1000 Espécimen pres MLS ran Objeto fisico 2015-03-09 ES

MLS:ran:1001 Especimen pres MLS

$\operatorname{ran}$

ran Objeto fisico 15.03.09 ES 2015-05-11 ES

1000 Objeto fisico 2015-04-14 ES

1001 Objeto físico 2015-04-14 ES

De esta manera, el Museo de la Universidad de La Salle es fuente de estudio para la 
implementación de un Sistema de información, que tenga la capacidad de almacenar, modificar o integrar datos una manera eficaz, así como brindar información de acuerdo con los perfiles de usuario identificados en esta investigación. Que incluyen a los diferentes científicos que trabajan en el Museo, y a los visitantes, ya sean investigadores externos, estudiantes o solo visitantes [13]. Lo anterior permite organizar los elementos de manera que se conciba un modelo de datos y se desarrolle un vocabulario especializado para albergar el significado de los datos, lo que permite asegurar la calidad al almacenar los datos de forma organizada y conservar la correcta clasificación según la colección que corresponda, dando como resultado el modelo conceptual y el diccionario de datos. Como ejemplo, en la tabla 2 se presenta un fragmento del vocabulario propio de las colecciones del Museo.

Tabla 17 Vocabulario con las. palabras estratégicas que se deben utilizar a la hora de catalogar un espécimen.

\begin{tabular}{|c|c|c|}
\hline \multirow{7}{*}{ 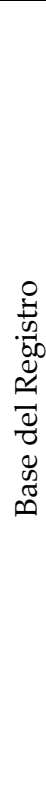 } & \multicolumn{2}{|c|}{ Registro biológico $\quad$ Evento } \\
\hline & Espécimen preservado & $\begin{array}{l}\text { El organismo esta preservado (muerto), vivió dentro de tiempos históricos y } \\
\text { existe (o alguna vez existió) una parte física que podría ser evaluada } \\
\text { nuevamente }\end{array}$ \\
\hline & Espécimen fosilizado & $\begin{array}{l}\text { El organismo vivió dentro de tiempos prehistóricos y existe (o alguna vez } \\
\text { existió) una parte física que podría ser evaluada nuevamente }\end{array}$ \\
\hline & Espécimen vivo & $\begin{array}{l}\text { Existe un espécimen disponible, se encuentra vivo (crece o metaboliza, no es } \\
\text { una parte dominante de un Espécimen preservado) al menos cuando se creó } \\
\text { el recurso }\end{array}$ \\
\hline & Observación humana & $\begin{array}{l}\text { El registro fue documentado sin recolectar evidencia física o digital que } \\
\text { pudiera ser evaluada nuevamente }\end{array}$ \\
\hline & Observación con maquina & $\begin{array}{l}\text { El registro fue documentado recolectando evidencia física o digital que } \\
\text { pudiera ser evaluada nuevamente. Fotografías, video o sonidos }\end{array}$ \\
\hline & $\begin{array}{l}\text { Lista de chequeo } \\
\text { nomenclatura }\end{array}$ & \\
\hline
\end{tabular}

Fase II: Sistematización de la información adquirida, con lo que mediante el diseñó lógico se obtiene como resultado el modelo de datos entidad relación, con la definición de atributos y sus respectivas restricciones, el cual debe validarse con los científicos del Museo. Como ejemplo en la figura 1 se presenta un fragmento del modelo entidad relación para albergar los datos de especímenes del reino anphibia, que entre otros alberga cecilias, ranas, sapos y salamandras. 
Relaciones

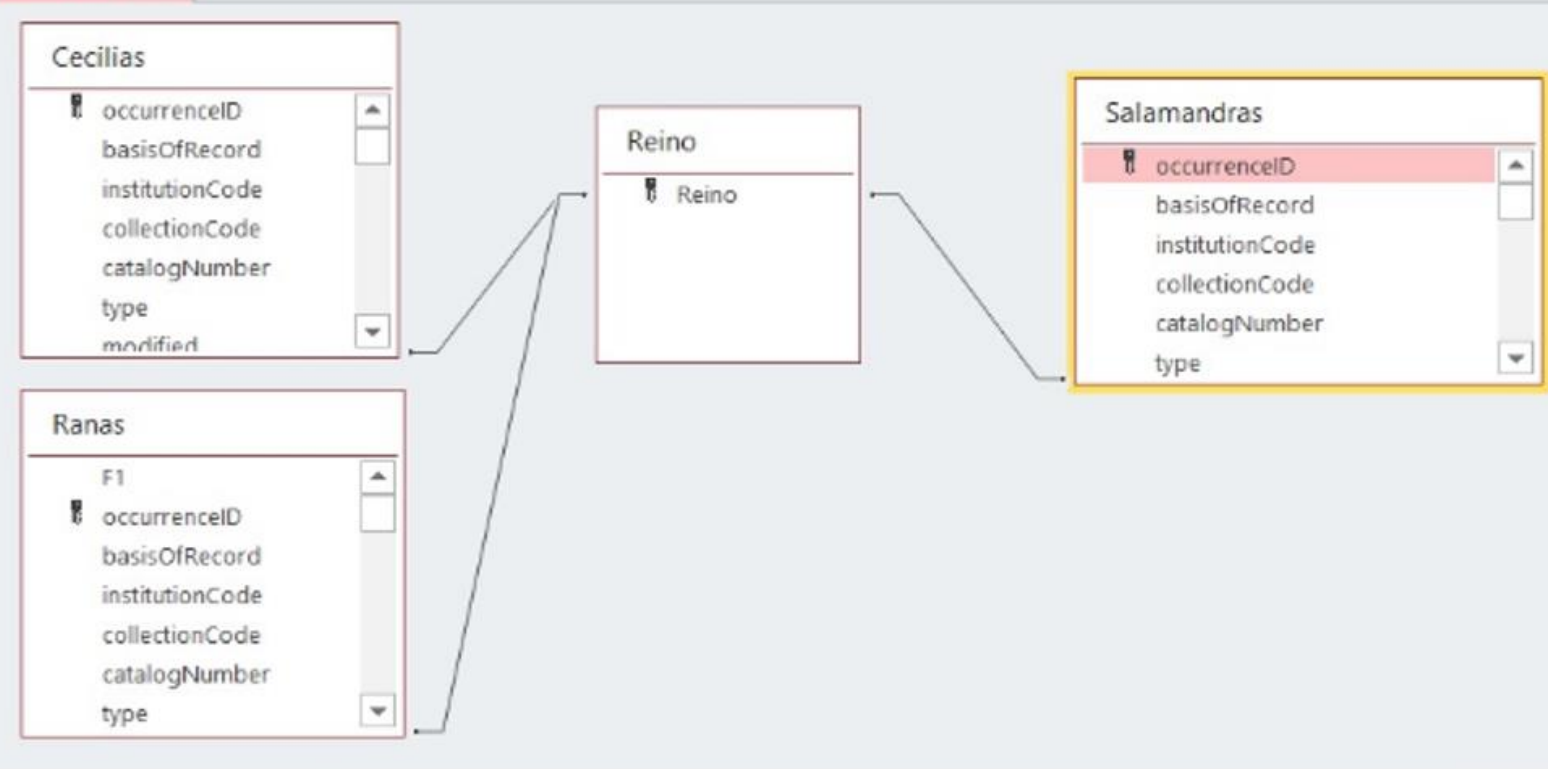

Figura 45 Fragmento de modelo entidad relación para el reino anphibia

Fase III: Implementar el modelo construyendo la base de datos, iniciando por importar los datos desde los archivos de Excel, Una vez que la base de datos reciba el contenido de los archivos de las colecciones, se verificará con los usuarios finales el almacenamiento de los registros en las nuevas tablas. Luego, se procederá a diseñar y programar los formularios y reportes, para lo cual se necesita identificar los diferentes usuarios y construir el documento de diseño de interfaces, con caso de uso y diagramas de secuencia, utilizando el modelado de sistemas de software con el lenguaje unificado de modelado (UML, por sus siglas en inglés, Unified Modeling Language). Luego, el documento de diseño se completará con la definición de la vista lógica de cada usuario. El resultado es la base de datos en Access con las tablas y vistas lógica, que aseguren que la información se disponga de acuerdo con el perfil de cada usuario mediante el desarrollo de formularios y reportes adecuados a cada uno de ellos.

Fase IV: Validar los avances obtenidos de la implementación del nuevo modelo con los usuarios del sistema, para lo cual se plantean dos ciclos de pruebas con diferentes actores. El primero para capacitar a los usuarios y validar el modelo desarrollado, y el segundo ciclo para verificar los ajustes que puedan requerirse como resultado del ciclo inicial de pruebas, de manera que el modelo pueda implementarse para su producción. 


\section{RESULTADOS Y ANÁLISIS DE RESULTADOS}

La información de las colecciones del Museo de la Universidad de la Salle requiere de un sistema de información soportado por una base de datos relacional, la cual se encuentra en construcción. Actualmente, este proyecto está su tercera fase; ya se desarrolló una primera fase del modelo, al obtener en la fase 1 los archivos de Excel que albergan los datos de las colecciones y poder plasmar las entidades y relaciones en el modelo conceptual para la nueva base de datos, así como definir el diccionario de datos para este sistema. Luego, en la fase dos, se construyó el modelo lógico y se definieron los atributos para cada tabla, con sus respectivas restricciones y reglas de validación, lo cual es fundamental para poder implementar el modelo en el motor Microsoft Access y diseñar las interfaces para cada uno de los roles asociados a los científicos, colaboradores y visitantes que puedan requerir información de alguna de las colecciones. En la definición de roles, también se establecieron los permisos para cada uno de ellos, dado que, para asegurar la calidad de la información, solo ciertos roles podrán almacenar, modificar o borrar datos de las tablas. Para cada uno de ellos se desarrollarán formularios, los cuales requieren programar reglas de validación, usando VBA, Visual Basic for Applications, por su sigla en inglés. De otra parte, todos los roles podrán generar reportes mediante consultas previamente definidas, las cuales están siendo desarrolladas en el lenguaje de consultas SQL. Tanto los formularios, como los reportes, van a requerir integrarse en una aplicación organizada, la cual se construirá usando VBA, lo que es fundamental a la hora de adquirir la información por cada usuario. De esta manera, este proyecto ayuda al Museo de la Universidad de la Salle a recolectar, procesar y disponer toda la información de cada uno de los especímenes que alberga de acuerdo con las colecciones correspondientes.

A lo largo del desarrollo del proyecto se han obtenido los siguientes avances:(1) Levantamiento de las políticas de manejo de datos en el Museo de acuerdo a sus necesidades. (2) Definición de los roles de usuario para el futuro sistema. (3) Construcción del diccionario de datos científico y técnico de acuerdo a la Norma Darwin Core. (4) Desarrolló de un primer módulo del sistema correspondiente a la colección de anfibios. Una vez verificado este módulo, la siguiente fase es agregar nuevos módulos para otras colecciones e integrar consultas entre los mismos.

\section{CONCLUSIONES}

La información con la que cuenta el Museo de la Universidad de la Salle esta almacenada de forma inapropiada mediante una serie de archivos Excel. Los datos se registran de acuerdo con la plantilla de documentación de registros biológicos, basada en los estándares de Darwin Core 
versión (2011-10-26) es de utilidad a la hora de registrar un espécimen biológico, lo que garantiza la calidad de los datos en cada archivo. El estándar Darwin Core debe ser respetado a la hora de implementar una nueva base de datos, para lo cual se escogió un modelo relacional, usando la metodología UML, el motor Microsoft Access y el lenguaje de programación VBA para la construcción del modelo. Además, el diseñó incluyó un análisis de seguridad de los datos mediante la identificación de perfiles de usuario a los cuales se les generaron las respectivas vistas lógicas sobre la nueva base de datos. De esta manera, se podrá administrar de manera más eficiente la información que maneja el Museo de La Salle en su día a día.

El nuevo modelo, se construye a partir del descubrimiento de las necesidades de los científicos del Museo integrando en un solo sistema todas las colecciones, adicionalmente el análisis del nuevo sistema incluye a los usuarios externos al Museo como son investigadores externos, estudiantes o visitantes ocasionales. Actualmente, el proyecto se encuentra en la tercera fase de implementación y se requiere que los científicos del Museo, como usuarios principales participen constantemente en la validación de cada una de las fases, implementando algunos de los principios de las metodologías agiles de desarrollo de software.

\section{REFERENCIAS}

[1] M. d. L. Salle, «Mision,» Universidad de La Salle, [En línea]. Available: http://museo.lasalle.edu.co/Museo-de-LaSalle/el-mls/detalle/mision. [Último acceso: 7 10 2020].

[2] Código de Deontología del ICOM para Museos, ICOM, 2017OM.

[3] Museo de La Salle, «Historia,» 27 2020. [En línea]. Available: http://museo.lasalle.edu.co/Museo-de-LaSalle/el-mls/detalle/historia/!

[4] «Acerca del Museo de La Salle,» Museo de La Salle, [En línea]. Available: http://museo.lasalle.edu.co/Museo-de-LaSalle/el-mls. [Último acceso: 105 2020].

[5] «Specify,» Specify, software, [En línea]. Available:

https://www.sustain.specifysoftware.org/. [Último acceso: 165 2020].

[6] I. NetXposure, «netx,» NetXposure, Inc, 2019. [En línea]. Available: https://www.netx.net/. [Último acceso: 0909 2020].

[7] Lucidea, «Argus,» Lucidea, 2020. [En línea].
Available: https://lucidea.com/argus/. [Último acceso: 0909 2020].

[8] I. Blackbaud, «Altru,» Blackbaud, Inc, 2020. [En línea].

Available: https://www.blackbaud.com/products/blackba ud-altru. [Último acceso: 0909 2020].

[9] S. Group, «Museum space,» Sirma Group, 2018. [En línea]. Available: https://museumspace.com/. [Último acceso: 09 09 2020].

[10] M. F. HINESTROSA, «PROTOTIPO DEL SISTEMA DE INFORMACIÓN DE RECUPERACIÓN DE,» Bogotá, 2009.

[11] M. Mannino, Administración de Bases De Datos, Diseño y Desarrollo de aplicaiones, Mexico: McGraw Hill, 2011.

[12] Instituto de Investigación de Recursos Biológicos Alexander von Humboldt. , «Perfil estándar Darwin Core (Adaptación IAvH). Infraestructura Institucional de Datos, Bogotá D.C., Colombia,» 2016. [En línea]. Available: https://sites.google.com/humboldt.org.co/i2dw iki/perfil-est $\%$ C3\%A1ndar-darwin-core. 
[Último acceso: 194 2020].

[13] K. L. J. Laudon, Systemas de Información, New
York: Pearson, 2015. 